\title{
MODFLOW-2000, THE U.S. GEOLOGICAL SURVEY \\ MODULAR GROUND-WATER MODEL - \\ USER GUIDE TO THE OBSERVATION, SENSITIVITY, AND \\ PARAMETER-ESTIMATION PROCESSES AND THREE \\ POST-PROCESSING PROGRAMS
}

By MARY C. HILL, EDWARD R. BANTA, ARLEN W. HARBAUGH, and EVAN R. ANDERMAN

U.S. Geological Survey

Open-File Report 00-184

Prepared in cooperation with the

U.S. Department of Energy 


\section{U.S. DEPARTMENT OF THE INTERIOR \\ BRUCE BABBITT, Secretary \\ U.S. GEOLOGICAL SURVEY \\ Charles G. Groat, Director}

The use of trade, product, industry, or firm names is for descriptive purposes only and does not imply endorsement by the U.S. Government.

For additional information write to:

Regional Research Hydrologist

U.S. Geological Survey

Box 25046, Mail Stop 413

Denver Federal Center

Denver, CO 80225-0046
Copies of this report can be purchased from:

U.S. Geological Survey

Branch of Information Services

Box 25286

Denver, CO 80225-0425 


\section{PREFACE}

This report describes the capabilities and use of the Observation, Sensitivity, and Parameter-Estimation Processes of the computer program MODFLOW-2000, and the postprocessing programs RESAN-2000, YCINT-2000, and BEALE-2000. The documentation of the Parameter-Estimation Process and the post-processing programs presented here includes brief listings of the methods used and detailed descriptions of the required input files and how the output files are typically used. Background for the methods is provided in the report 'Methods and Guidelines for Effective Model Calibration' (Hill, 1998). Hill (1998) provides detailed information on the methods used in the Parameter-Estimation Process and post-processing programs of MODFLOW-2000, and in the universal inverse model UCODE (Poeter and Hill, 1998). Hill (1998) also presents guidelines for conducting the calibration of a model of a complex system, using examples from ground-water modeling to illustrate the ideas presented.

The Observation, Sensitivity, and Parameter-Estimation Processes support many capabilities of MODFLOW-2000's Ground-Water Flow Process, but do not support any of the other processes. The MODFLOW-2000 reports and computer programs supersede MODFLOW96 (Harbaugh and McDonald, 1996) and the MODFLOWP (Hill, 1992) computer program and part of the report. Hill (1998) supersedes all other parts of Hill (1992). The performance of MODFLOW-2000 has been tested in a variety of applications. Future applications, however, might reveal errors that were not detected in the test simulations. Users are requested to notify the U.S. Geological Survey of any errors found in this document or the computer program using the email address available at the web address below. Updates might occasionally be made to both this document and to MODFLOW-2000. Users can check for updates on the Internet at URL http://water.usgs.gov/software/ground_water.html/. 


\section{CONTENTS}

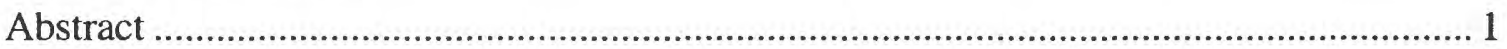

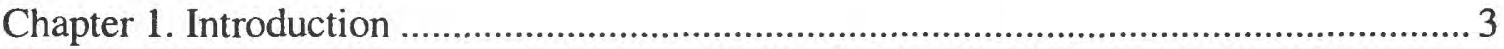

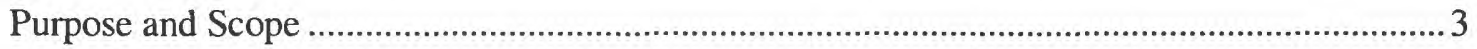

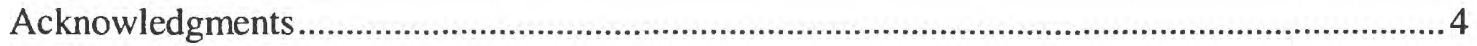

Chapter 2. Overview, Compatibility, and Program Control ................................................ 5

Overview of the Observation, Sensitivity, and Parameter-Estimation Processes .......................... 5

Program Sequence................................................................................................

Parallel-Processing Capability for the Sensitivity Process …………………………………....

Compatibility of the Observation, Sensitivity, and Parameter-Estimation Processes with Other Components of MODFLOW-2000 ……………………................................... 8

Using the Name File to Control Program Execution and Output.............................................. 10

Activation of the Observation, Sensitivity, and Parameter-Estimation Processes and

Definition of Input and Output Files Using File Types ................................................... 10

Contents of the GLOBAL and LIST Output Files ……………………………………….... 13

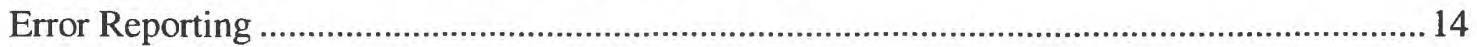

Chapter 3. Inverse Modeling Considerations .................................................................... 15

Guidelines for Effective Model Calibration............................................................................ 15

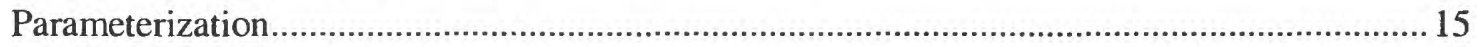

Nonlinearity of the Ground-Water Flow Equation with Respect to Parameters and Consequences for the Sensitivity and Parameter-Estimation Processes ................................ 16

Starting Parameter Values...................................................................................................... 18

Weighting Observations and Prior Information ........................................................................ 18

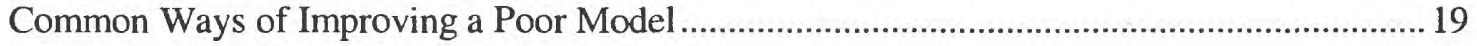

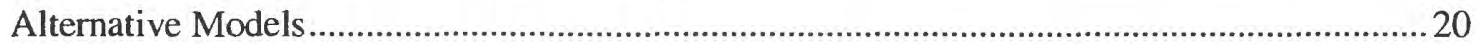

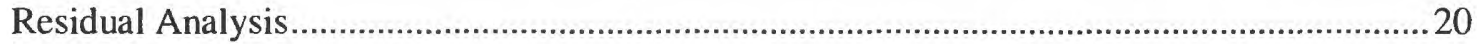

Predictions and Differences, and Their Linear Confidence Intervals and Prediction

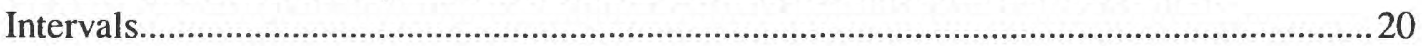

Chapter 4. Observation Process .................................................................................... 23

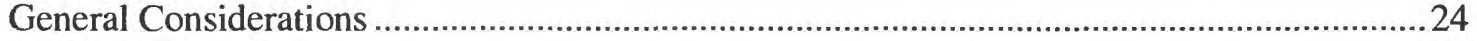

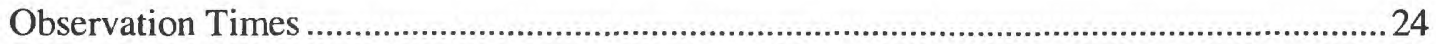

Dry Cells in Convertible Layers at Observation Locations .................................................2 24

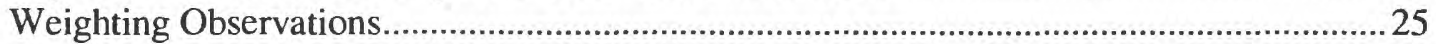

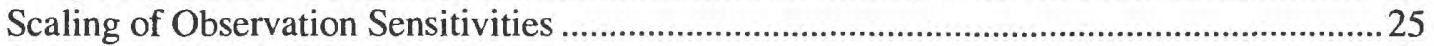

Input File For All Observations ……………………………………………………….....2

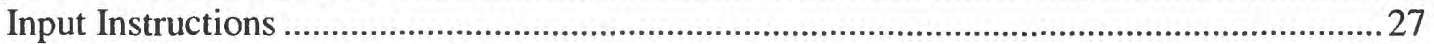

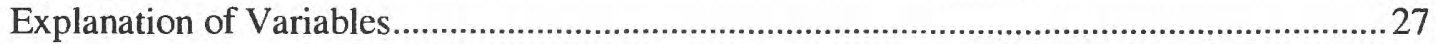

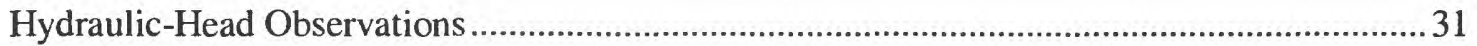


Calculation of Simulated Equivalents to the Observations ................................................. 31

Spatial Interpolation for Hydraulic-Head Observations at Arbitrary Locations ................ 31

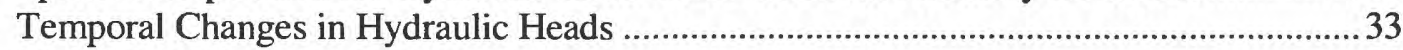

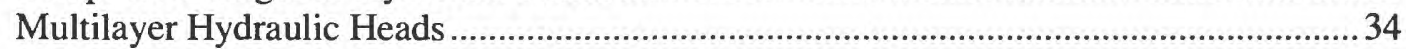

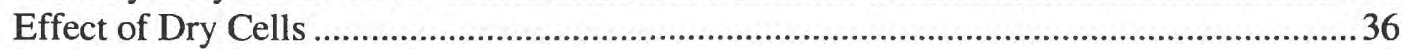

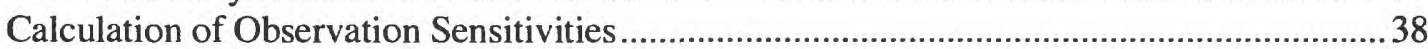

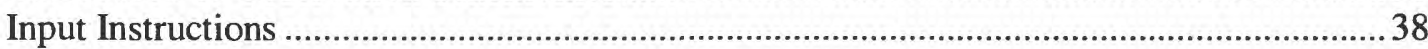

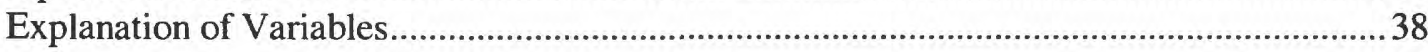

Flow Observations at Boundaries Represented as Head Dependent.......................................41

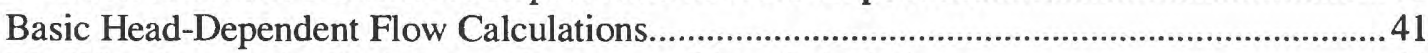

Modifications to the Basic Head-Dependent Flow Calculations ......................................... 44

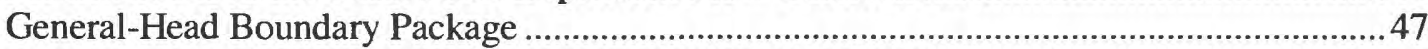

Calculation of Simulated Equivalents to the Observations ............................................47

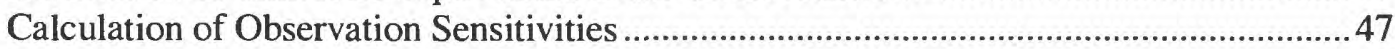

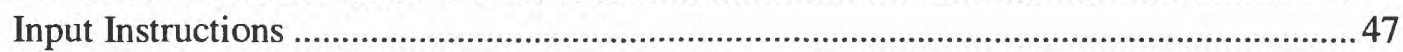

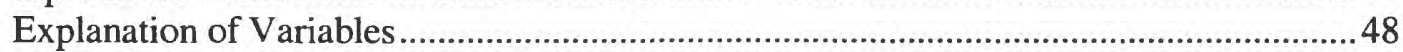

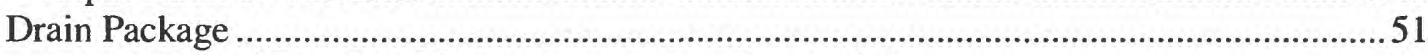

Calculation of Simulated Equivalents to the Observations ..........................................51

Calculation of Observation Sensitivities ........................................................................ 51

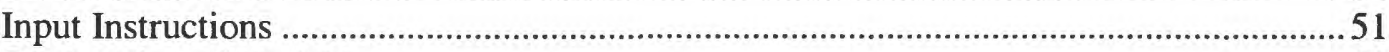

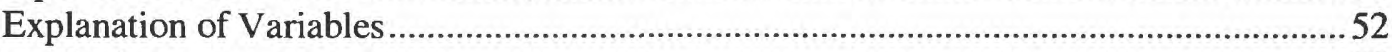

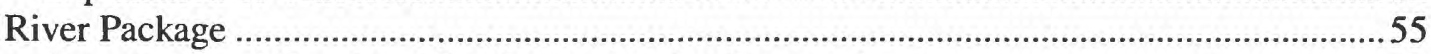

Calculation of Simulated Equivalents to the Observations ............................................55

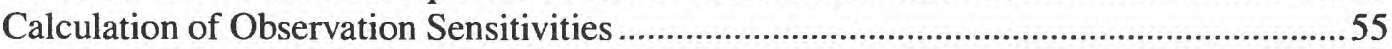

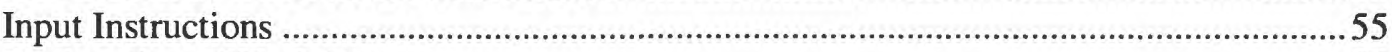

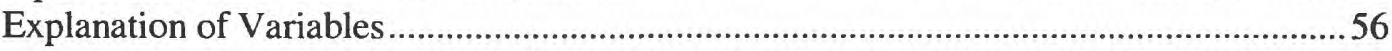

Observations at Cells Having More Than One Head-Dependent Boundary Feature Represented by the Same Package..................................................................................59

Flow Observations at Boundaries Represented as Constant Head..........................................62

Calculation of Simulated Equivalents to the Observations ..................................................62

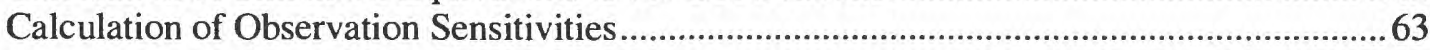

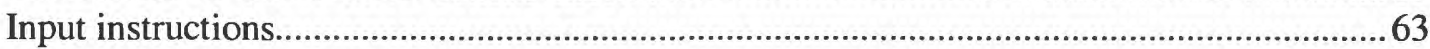

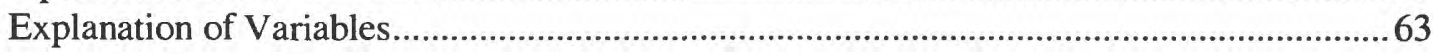

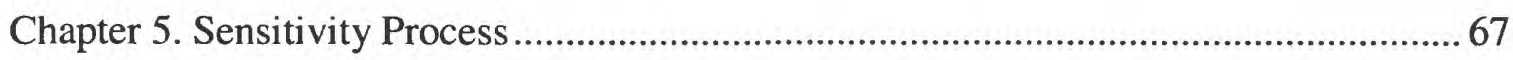

Equations for Grid Sensitivities for Hydraulic Heads Throughout the Model..........................6 67

Solving for Grid Sensitivities for Hydraulic Heads Throughout the Model ............................. 70

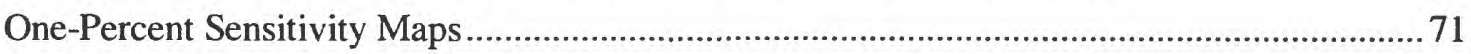

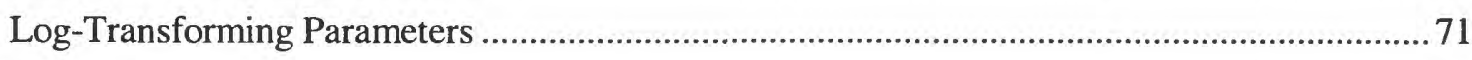

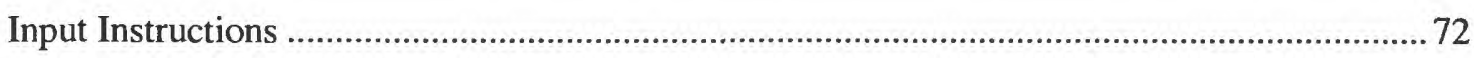

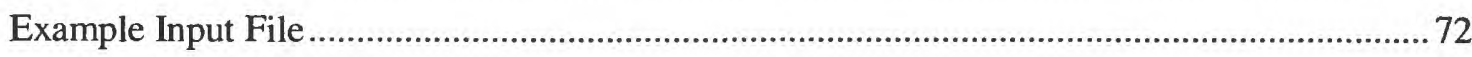

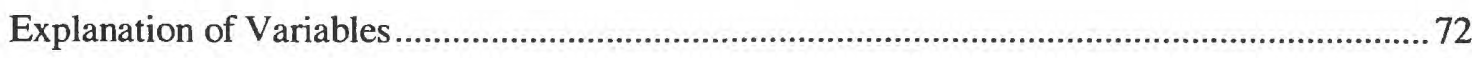

Chapter 6. Parameter-Estimation Process ................................................................ 77

Modified Gauss-Newton Optimization ............................................................................ 77

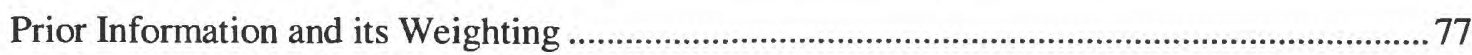




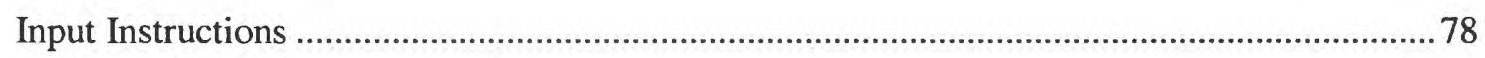

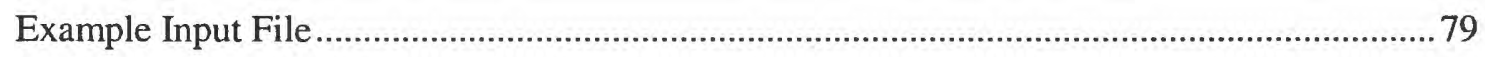

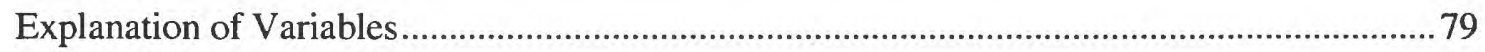

Additional Examples of Prior Information Equations............................................................... 83

Chapter 7. Post-Processing Programs RESAN-2000, YCINT-2000, and BEALE-

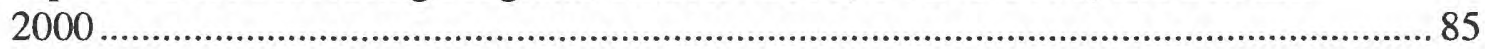

Using RESAN-2000 to Test Weighted Residuals and Identify Influential Observations...........85

Using YCINT-2000 to Calculate Linear Confidence and Prediction Intervals on Predictions and Differences Simulated with Estimated Parameter Values.............................87

Using BEALE-2000 to Test Model Linearity .......................................................................92

Chapter 8. Using Output From MODFLOW-2000 and Post-Processors RESAN-

2000, YCINT-2000, AND BEALE-2000

Output Files from Mode 'Forward with Observations', with or without Parameter

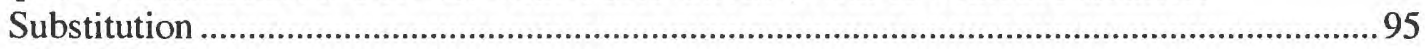

Output Files from Modes 'Parameter Sensitivity' and 'Parameter Sensitivity with

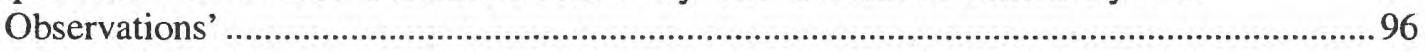

Tables of Sensitivities Produced for all Sensitivity with Observation Modes, the Sensitivity Analysis Mode, and the Parameter-Estimation Mode........................................96

Output Files from Mode 'Sensitivity Analysis' .....................................................................96

Output Files from Mode 'Parameter Estimation' ........................................................................97

Output Files for Residual Analysis and Identifying Influential Observations from

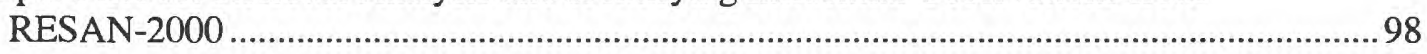

Output Files for Predictions and Differences from YCINT-2000 …....................................... 104

Output Files from Test of Linearity with BEALE-2000 ………........................................... 104

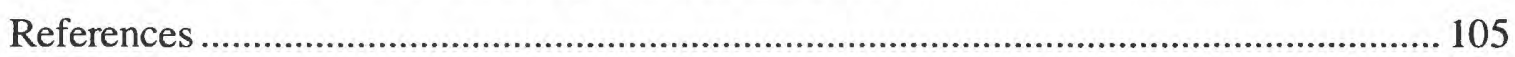

Appendix A. Example Simulations ...................................................................... 108

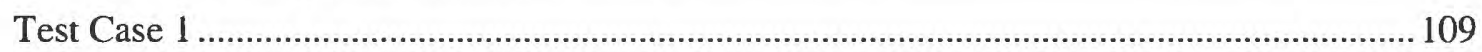

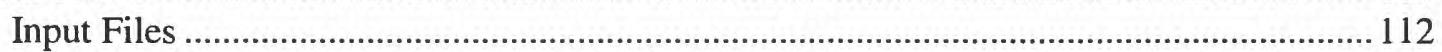

GLOBAL Output File ………………………………………………………………115

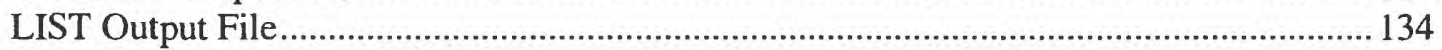

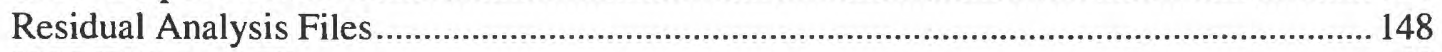

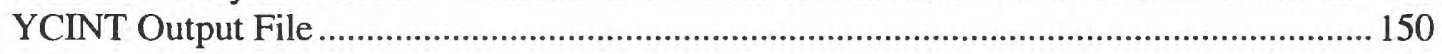

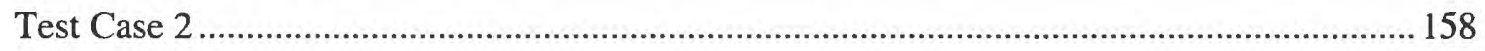

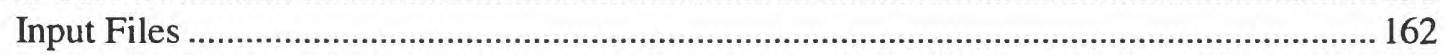

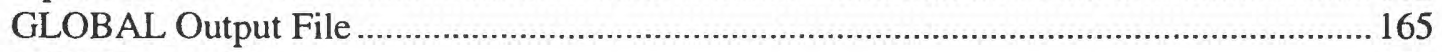

LIST Output File ........................................................................................................ 191

Appendix B. Program Distribution, Installation, and a Hint For Execution ..................... 202

Distributed Files and Directories...................................................................................... 202

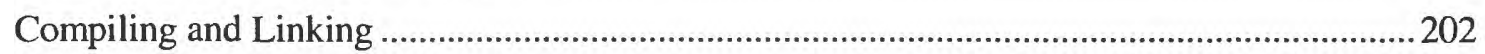




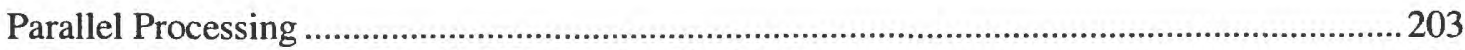

Error Reporting with Parallel Processing Enabled........................................................... 204

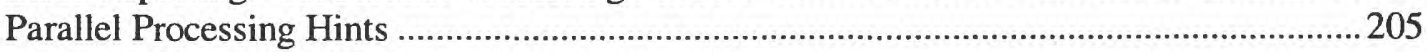

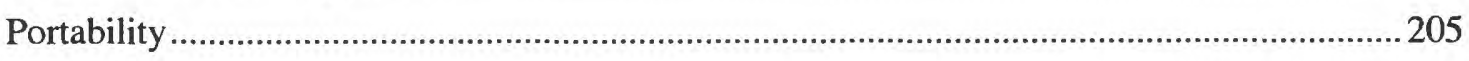

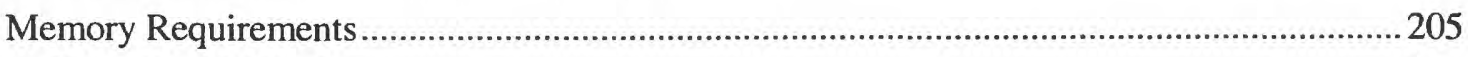

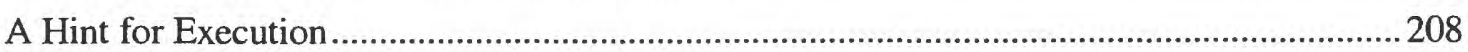

Appendix C. Suggestions For Graphical Interface Design ...................................... 209

\section{FIGURES}

Figure 1: Flowchart showing the major steps of the Ground-Water Flow (GWF), Observation (OBS), Sensitivity (SEN), and Parameter-Estimation (PES) Processes when all are active and LASTX in the PES input file equals zero..........................................6

Figure 2: Locating points within a finite-difference cell using ROFF and COFF. ........................ 33

Figure 3: Calculating the simulated value of hydraulic head for a multilayer observation well.

Figure 4: Situations for which the Observation Process (A) can and (B) cannot produce correct spatial interpolation for the multilayer hydraulic-head observation shown in figure 3 .

Figure 5: Effect of dry cells on interpolation of heads at a hydraulic-head observation location.

Figure 6: Diagram depicting the quantities used to calculate flow between the groundwater system and a surface-water body.

Figure 7: Representation of head-dependent boundary gain or loss observations between two gaging stations, showing the finite-difference cells used to represent the appropriate reach.

Figure 8: The dependence of simulated gains and losses on hydraulic head in the model layer $\left(h_{n}\right)$ in: (A) the General-Head Boundary Package, $(B)$ the Drain Package, and (C) the River Package.

Figure A1: Physical system for test case 1 .

Figure A2: Test case 2 model grid, boundary conditions, observation locations and hydraulic conductivity zonation used in parameter estimation.

\section{TABLES}

Table 1: Compatibility of the Observation, Sensitivity, and Parameter-Estimation

Processes with other components of MODFLOW-2000

Table 2: File types that control the Observation, Sensitivity, and Parameter-Estimation Processes and primary output files.

Table 3: Modes of MODFLOW-2000 produced by activating different combinations of the Observations (OBS), Sensitivity (SEN), and Parameter-Estimation (PES) Processes, the source of parameter values, and commonly used model output. 
Table 4: Guidelines for effective model calibration.

Table 5: Files produced by MODFLOW-2000 when OUTNAM is not "NONE" that are designed for use by plotting routines and other programs.

Table 6: Files produced by the MODFLOW-2000 post-processors RESAN-2000, YCINT-2000, and BEALE-2000 (chapter 7) when OUTNAM is not "NONE".

Table 7: Packages available for representing flow observations as head-dependent boundaries

Table 8: Information contained in the _rs file of table 5, which is produced by

MODFLOW-2000 and used by the post-processing program RESAN-2000

Table 9: Information contained in the _y0 file of table 5, which is produced by MODFLOW-2000 when IYCFLG $=0$ and is used by the post-processing program YCINT-2000.

Table 10: Information contained in the _yl file of table 5, which is produced when IYCFLG $=1$ in the Parameter-Estimation Process input file.

Table 11: Information contained in the _y2 file of table 5, which is produced when IYCFLG $=2$ in the Parameter-Estimation Process input file.

Table 12: Information contained in the _bl file of table 5, which is produced when IBEFLG $=1$ in the Parameter-Estimation Process input.

Table 13: Information contained in the _b2 file of table 5, which is produced by MODFLOW-2000 when IBEFLG=2

Table 14: Residuals and model-fit statistics printed in the GLOBAL and LIST output files when the Observation Process is active ......

Table 15: Parameter statistics printed in the GLOBAL output file when the ParameterEstimation Process is active and IBEFLG $<2$.

Table 16: Using the files created by MODFLOW-2000 that contain data sets for graphical residual analysis

Table 17: Using the files created by RESAN-2000 that contain data sets for graphical residual analysis

Table 18: Regression performance measures printed in the GLOBAL output file when the Parameter-Estimation Process is active and IBEFLG $<2$

Table B1: Contents of the subdirectories distributed with MODFLOW-2000.

Table B2: The sequence of calculations performed by MODFLOW-2000 given nine parameters and (A) three and (B) four computer processors.

Table B3: Arrays and corresponding dimensioning Fortran parameters in MODFLOW2000.

Table B4: Fortran parameters specified in file "param.inc" that could require adjustment for some problems.

Table B5: The files needed to automatically answer MODFLOW-2000's query for the NAME FILE using, as an example, a Windows computer operating system. 


\title{
MODFLOW-2000, \\ THE U.S. GEOLOGICAL SURVEY MODULAR \\ GROUND-WATER MODEL - \\ USER GUIDE TO THE OBSERVATION, SENSITIVITY, AND \\ PARAMETER-ESTIMATION PROCESSES AND THREE POST-PROCESSING PROGRAMS
}

\author{
By Mary C. Hill, Edward R. Banta, Arlen W. Harbaugh, \\ and Evan R. Anderman
}

\begin{abstract}
This report documents the Observation, Sensitivity, and Parameter-Estimation Processes of the ground-water modeling computer program MODFLOW-2000. The Observation Process generates model-calculated values for comparison with measured, or observed, quantities. A variety of statistics is calculated to quantify this comparison, including a weighted least-squares objective function. In addition, a number of files are produced that can be used to compare the values graphically. The Sensitivity Process calculates the sensitivity of hydraulic heads throughout the model with respect to specified parameters using the accurate sensitivity-equation method. These are called grid sensitivities. If the Observation Process is active, it uses the grid sensitivities to calculate sensitivities for the simulated values associated with the observations. These are called observation sensitivities. Observation sensitivities are used to calculate a number of statistics that can be used (1) to diagnose inadequate data, (2) to identify parameters that probably cannot be estimated by regression using the available observations, and (3) to evaluate the utility of proposed new data.

The Parameter-Estimation Process uses a modified Gauss-Newton method to adjust values of user-selected input parameters in an iterative procedure to minimize the value of the weighted least-squares objective function. Statistics produced by the Parameter-Estimation Process can be used to evaluate estimated parameter values; statistics produced by the Observation Process and post-processing program RESAN-2000 can be used to evaluate how accurately the model represents the actual processes; statistics produced by post-processing program YCINT-2000 can be used to quantify the uncertainty of model simulated values.

Parameters are defined in the Ground-Water Flow Process input files and can be used to calculate most model inputs, such as: for explicitly defined model layers, horizontal hydraulic conductivity, horizontal anisotropy, vertical hydraulic conductivity or vertical anisotropy, specific storage, and specific yield; and, for implicitly represented layers, vertical hydraulic conductivity. In addition, parameters can be defined to calculate the hydraulic conductance of the River, General-Head Boundary, and Drain Packages; areal recharge rates of the Recharge Package; maximum evapotranspiration of the Evapotranspiration Package; pumpage or the rate of flow at defined-flux boundaries of the Well Package; and the hydraulic head at constant-head boundaries. The spatial variation of model inputs produced using defined parameters is very flexible, including interpolated distributions that require the summation of contributions from different parameters.
\end{abstract}


Observations can include measured hydraulic heads or temporal changes in hydraulic heads, measured gains and losses along head-dependent boundaries (such as streams), flows through constant-head boundaries, and advective transport through the system, which generally would be inferred from measured concentrations.

MODFLOW-2000 is intended for use on any computer operating system. The program consists of algorithms programmed in Fortran 90, which efficiently performs numerical calculations and is fully compatible with the newer Fortran 95. The code is easily modified to be compatible with FORTRAN 77. Coordination for multiple processors is accommodated using Message Passing Interface (MPI) commands. The program is designed in a modular fashion that is intended to support inclusion of new capabilities. 


\section{Chapter 1. INTRODUCTION}

Despite their apparent utility, formal sensitivity and parameter-estimation methods are used much less than would be expected - sensitivity analyses and calibrations conducted using trial-and-error methods only are much more commonly used in practice. This situation has arisen partly because of difficulties inherent in inverse modeling, which are related to the mathematics used, the complexity of the simulated systems, and the sparsity of data in most situations; and partly due to a lack of effective inverse models that make the inherent and powerful statistical aspects of inverse modeling widely understandable. Recent work (for example, Poeter and Hill, 1997) has clearly demonstrated that inverse modeling, though an imperfect tool, provides capabilities that help modelers take greater advantage of the insight available from their models and data. Expanded use of this technology requires sophisticated computer programs that combine the ability to represent the complexities typical of many ground-water situations with statistical and optimization methods able to reveal the strengths and weaknesses of calibration data and calibrated models.

The program presented in this work incorporates the most accurate method available for calculating sensitivities with a comprehensive set of statistics for model evaluation, as described by Hill (1998), and the newest version of the world's most widely used ground-water flowsimulation program, MODFLOW-2000 (McDonald and Harbaugh, 1988, Harbaugh and McDonald, 1996; Harbaugh and others, 2000). Experience has shown that the accuracy of calculated sensitivities is important to some aspects of the analysis and that the sensitivityequation sensitivity method used in the Sensitivity Process documented in this report produces the most accurate possible sensitivities. As of its publication, MODFLOW-2000 is the only ground-water flow model capable of calculating such accurate sensitivities for systems with typical complexities. The accurate sensitivities are rarely important to nonlinear regression, in which parameter values that produce the closest fit between observed and simulated values are determined. The increased accuracy is crucial, however, in the calculation of some of the statistics used to evaluate the information provided by the observations and the uncertainty of simulated values. Of particular note is that accurate sensitivities are needed to calculate parameter correlation coefficients that are accurate enough to be useful in determining whether the available observations are sufficient to estimate parameters uniquely.

\section{Purpose and Scope}

This report documents how to use the Observation, Sensitivity, and Parameter-Estimation Processes of MODFLOW-2000. The report begins with an overview of these processes, how they relate to the Ground-Water Flow (GWF) Process of MODFLOW-2000, and how these processes work together. The theory behind the Parameter-Estimation Process and the post-processors is described by Hill $(1994,1998)$, and guidelines for pursuing model calibration and uncertainty analysis are described by Hill (1998). Basic ideas from those works are presented briefly in this report. Subsequent sections describe the Observation, Sensitivity, and Parameter-Estimation Processes, with an emphasis on providing detailed input instructions and descriptions of the output files. Appendix A includes selected input and output files for two example problems. Appendix B includes information about obtaining and compiling the code; Appendix C provides suggestions related to construction of a graphical user interface for MODFLOW-2000. Source files for MODFLOW-2000 are available at the Internet address listed in the preface of this report.

Users of this report need to be familiar with the Ground-Water Flow Process of MODFLOW-2000 (McDonald and Harbaugh, 1988, and Harbaugh and others, 2000). Also, although this report is written at an elementary level, some knowledge about basic statistics and the application of nonlinear regression is assumed. For example, it is assumed that the reader is familiar with the terms standard deviation, variance, correlation, sensitivity, optimal parameter 
values, residuals and confidence intervals. Readers who are unfamiliar with these terms need to review a basic statistics book and Hill (1998). Useful references and applications are cited in Hill (1998), including the illustrative example described by Poeter and Hill (1997).

\section{Acknowledgments}

Professor Eileen Poeter of the Colorado School of Mines and of the International Ground-Water Modeling Center shared many comments and insights that greatly matured the ideas upon which MODFLOW-2000 is built. Steen Christensen of Aarhus University, Denmark, kindly integrated his programming of the full weight matrix on observations and prior information into MODFLOWP, and supported its transfer into MODFLOW-2000. Guy Robinson of the Arctic Regions Supercomputing Center, Fairbanks, Alaska, assisted with the parallelization of MODFLOW-2000. Colleague reviews by Dr. Tracy Nishakawa and Dr. Wayne Belcher, both of the U.S Geological Survey, and Professor David Dougherty of the University of Vermont were much appreciated. Conversations with Wen-Hsing Chiang, Jeffrey Davis, Douglas Graham, James Rumbaugh, and Richard Winston over the years also have been very helpful.

This work would not be possible without the pioneering and continuing work of Richard L. Cooley of the U.S. Geological Survey. We are very grateful for his tremendous contribution and encouragement. Claire R. Tiedeman and Richard M. Yager of the U.S Geological Survey identified numerous program errors over many years and provided reflections, advice, insights, and many hours of hard work on innumerable issues. Richard M. Yager of the U.S. Geological Survey contributed the programming in RESAN-2000 to calculate the Cook's D and DFBeta statistics. 


\section{Chapter 2. OVERVIEW, COMPATIBILITY, AND PROGRAM CONTROL}

This section presents an overview of the sequence of calculations performed by MODFLOW-2000 when the Observation, Sensitivity, and Parameter-Estimation Processes all are active and parameters are being estimated. In addition, the example applies when the variable LASTX is set to 0 in the Parameter-Estimation Process (this variable is discussed in the Parameter-Estimation Process chapter of this report). This situation is used because it best illustrates the interactions between these processes and the Ground-Water Flow Process. Other ways of using these processes are discussed in the following chapter. The present chapter also discusses how to activate the Observation, Sensitivity, and Parameter- Estimation Processes, and it discusses the compatibility of these processes with the other capabilities of MODFLOW-2000.

\section{Overview of the Observation, Sensitivity, and Parameter-Estimation Processes}

A generalized flowchart of MODFLOW-2000 is presented in figure 1. This section describes the steps listed in the flowchart and shows how the Observation, Sensitivity, and Parameter-Estimation Processes relate to these steps.

\section{Program Sequence}

MODFLOW-2000 initializes a problem by reading input from the following files: (1) Ground-Water Flow Process input files, which define the ground-water flow simulation and parameters that can be listed in the Sensitivity Process input file; (2) Observation Process input files, which define the observations; (3) Sensitivity Process input file, which lists the parameters for which (a) values are controlled by the Sensitivity Process, (b) sensitivities are to be calculated, and (c) values are to be estimated through the Parameter-Estimation Process; and (4) ParameterEstimation Process input file, which lists values for variables that control the modified GaussNewton nonlinear regression.

Parameter-estimation iterations are used by MODFLOW-2000 to solve the nonlinear regression problems for which MODFLOW-2000 is designed. The regression is nonlinear because the simulated equivalents of observed quantities such as hydraulic head are nonlinear functions of system characteristics that commonly are represented by parameters, such as hydraulic conductivity. The nonlinearity of hydraulic head with respect to hydraulic conductivity is discussed in the next section of this report. In MODFLOW-2000, parameter-estimation iterations begin by using the starting parameter values listed in the Sensitivity Process input file. MODFLOW-2000 proceeds by using these parameter values to calculate hydraulic heads and then to calculate sensitivity-equation sensitivities for selected parameters. For simulations that consist of a single time step, which commonly would be the case for a purely steady-state simulation, this sequence is performed once. For simulations that consist of multiple time steps, the program proceeds through the stress periods and time steps as defined in the discretization file (Harbaugh and others, 2000), calculating first hydraulic heads and then sensitivity-equation sensitivities for each time step. Note that MODFLOW-2000 allows any sequence of steady-state and transient stress periods in a single simulation. At each time step, the Observation Process determines if any observations are applicable and, if so, obtains the information needed to calculate simulated equivalents and observation sensitivities. After all time steps are completed, the simulated values are subtracted from the observed values to produce residuals, and these are 


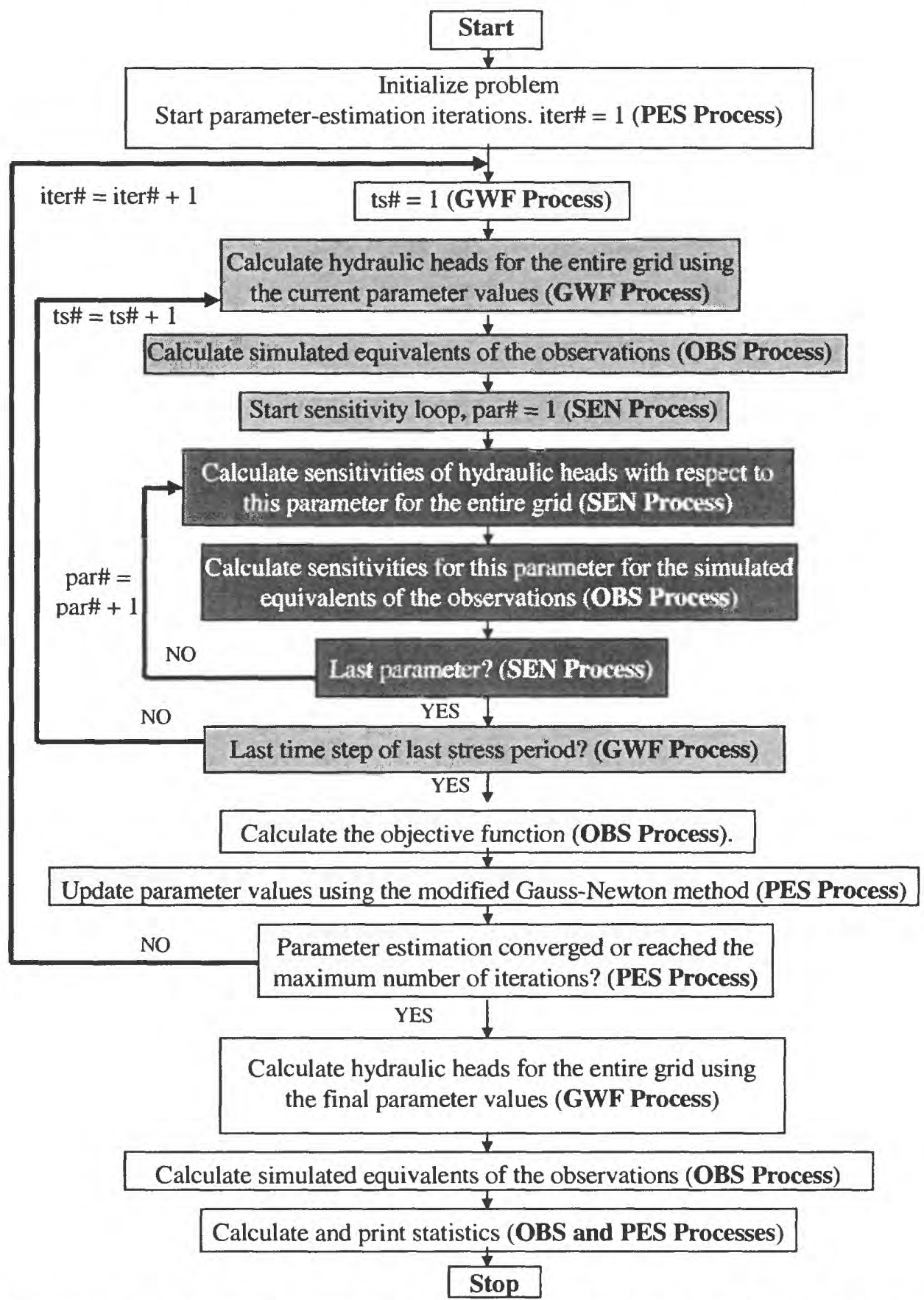

Figure 1: Flowchart showing the major steps of the Ground-Water Flow (GWF), Observation (OBS), Sensitivity (SEN), and Parameter-Estimation (PES) Processes when all are active and LASTX in the PES input file equals zero. iter\# identifies the parameter-estimation iteration, ts\# indicates the time step from the beginning of the simulation, and par\# indicates the parameter. In MODFLOW-2000, a time step is a subdivision of a stress period, which is a period of constant simulated stress. Grey shading is used to emphasize loops. 
weighted, squared, and summed to calculate the least-squares objective function, which is used by the regression to measure model fit to the observations (Hill, 1998, eq. 1). Depending on the value of user-defined variables, various statistics calculated using the sensitivities and the residuals are produced that can be useful in diagnosing problems with the parameter-estimation problem as posed.

Once the residuals and the sensitivities are calculated, they are used by the ParameterEstimation Process to perform one iteration of the modified Gauss-Newton nonlinear regression method to update the parameter values as described by Hill $(1998$, eq. 4$)$. The last step of each parameter-estimation iteration involves comparing two quantities against convergence criteria specified in the Parameter-Estimation Process input file: (1) the largest fractional change in any of the parameter values and (2) the change in the weighted least-squares objective function. If the changes exceed the corresponding convergence criteria and the maximum number of parameterestimation iterations has not been reached, the next parameter-estimation iteration is executed. If either of the calculated changes is less than the corresponding convergence criterion, parameterestimation converges. If convergence is achieved because the changes in the parameter values are small ( 1 above), the parameter values are likely to be the optimal parameter values - that is, the values that produce the best possible match between the simulated and observed values, as measured using the weighted least-squares objective function. If convergence is achieved because the changes in the objective function are small ( 2 above), it is less likely that the estimated parameter values are optimal. In both cases, further analysis is recommended to test the optimality of the solutions. These tests involve starting the regression from a range of starting parameter values. Consistent convergence to parameter values that are close to one another compared to their calculated standard deviations indicates that the parameter estimates are optimal (see the section 'Starting Parameter Values' below).

If parameter estimation does not converge and the maximum number of iterations has not been reached, then the updated parameter values are used in the Ground-Water Flow and Sensitivity Process calculations, and the next parameter-estimation iteration is performed. When parameter estimation converges or the maximum number of iterations has been reached, regression stops, information about the regression and estimated parameters is produced, and the program stops. Generally, parameters will be estimated using MODFLOW-2000 many times within a model calibration as regression is used to test different ideas about what is important in the system.

Once a model is calibrated, it can be used to make predictions for resource management or other purposes. The post-processing program YCINT-2000 can calculate linear confidence and prediction intervals that approximate the likely uncertainty in predictions simulated using the calibrated model and optimized parameter values.

\section{Parallel-Processing Capability for the Sensitivity Process}

MODFLOW-2000 is distributed with a parallel-processing capability for the Sensitivity Process that results in much reduced execution times when calculating sensitivities or performing parameter estimation in a computing environment with multiple processors. In the flowchart shown in figure 1, the parallelization involves the sensitivity loop; the Sensitivity and related Observation Process calculations for each parameter are assigned to different processors for simultaneous execution.

The parallel-processing capability is not enabled in the executable file included in the MODFLOW-2000 distribution. To enable this capability, the program must be modified slightly and recompiled, as described in the 'Parallel Processing' section of Appendix B. 


\section{Compatibility of the Observation, Sensitivity, and Parameter-Estimation Processes with Other Components of MODFLOW-2000}

Since its release by the U.S. Geological Survey in 1984, MODFLOW has provided a foundation upon which substantial development has occurred. The Process and Package structure of MODFLOW-2000 (Harbaugh and others, 2000) allows most of these developments to be viewed in a more cohesive framework than was previously available. Of these developments, table 1 describes the compatibility of the new Observation, Sensitivity, and Parameter-Estimation Processes documented in this report with developments published by the U.S. Geological Survey, as well as MT3DMS, which is public domain and supported by the U.S. Corps of Engineers Waterways Experiment Station.

Other than the Ground-Water Flow Process, the programs listed in table 1 are distributed separately and must be integrated into the program by the user following instructions in the documentation for those components. As the program evolves, compatibilities are likely to change, and such changes will be described in files distributed with MODFLOW-2000.

For some circumstances, MODFLOW-2000 may be applicable, but may lack some system features, parameters, or observations of concern. In such situations, MODFLOW-2000 and other processes such as MOC3D and MT3DMS often can be used in conjunction with UCODE (Poeter and Hill, 1998) or PEST (Doherty, 1994) to accomplish sensitivity analyses, parameter estimation, and uncertainty analysis. In these circumstances, MODFLOW-2000 generally can be used to simplify the substitutions and extractions required by UCODE or PEST. 
Table 1: Compatibility of the Observation, Sensitivity, and Parameter-Estimation Processes with other components of MODFLOW-2000

\begin{tabular}{|c|l|}
\hline MODFLOW-2000 Process & $\begin{array}{c}\text { Compatibility with the Observation, Sensitivity, and Parameter- } \\
\text { Estimation Processes }\end{array}$ \\
\hline $\begin{array}{c}\text { Ground-Water Flow Process } \\
\text { (Harbaugh and others, 2000) }\end{array}$ & $\begin{array}{l}\text { Compatible for the hydraulic-head and flow observation types } \\
\text { discussed in this report and the advective transport observation } \\
\text { type of Anderman and Hill (1997; with changes as described in } \\
\text { the readme file distributed with MODFLOW-2000). Compatible } \\
\text { for all parameter types listed in Harbaugh and others (2000) } \\
\text { except that the compatibility for HK parameters is limited to } \\
\text { calculating interblock transmissivity by harmonic averaging. } \\
\text { There are incompatibilities with the following packages: } \\
\text { Transient Leakage Package' (TLK) (Leake and others, 1994) } \\
\text { Interbed Storage Package } \text { (IBS) (Leake and Prudic, 1991) } \\
\text { Reservoir Package } \text { (RES) (Fenske and others, 1996) }\end{array}$ \\
\hline $\begin{array}{c}\text { MODPATH (Advective } \\
\text { Transport Process) }\end{array}$ & $\begin{array}{l}\text { Hydraulic head and flux output files produced when using the } \\
\text { Observation, Sensitivity, and Parameter-Estimation Processes } \\
\text { can be used by MODPATH (Pollock, 1994). } \\
\text { Advective-transport observations can be represented with the } \\
\text { ADV Package (Anderman and Hill, 1997). }\end{array}$ \\
\hline $\begin{array}{c}\text { GWT (Ground-Water } \\
\text { referred to as MOC3D) } \\
\text { (Konikow and others, 1996) }\end{array}$ & $\begin{array}{l}\text { Does not interfere with the program, but there are no parameters } \\
\text { or observations associated with this process. }\end{array}$ \\
\hline $\begin{array}{c}\text { MT3DMS (Mass Transport } \\
\text { Pith Multiple Species } \\
\text { Process) (Zheng and Wang, } \\
\text { 1998) }\end{array}$ & $\begin{array}{l}\text { Does not interfere with the program, but there are no parameters } \\
\text { or observations associated with this process. }\end{array}$ \\
\hline
\end{tabular}

'If used, sensitivities for layer properties that contribute to vertical leakance will be incorrect. Also, TLK is not compatible with the Layer Property Flow Package and the Hydrogeologic Unit Flow Package (Anderman and Hill, 2000).

${ }^{2}$ Does not interfere, but there are no parameters or observations associated with these packages. 


\section{Using the Name File to Control Program Execution and Output}

The name file of Harbaugh and others (2000) is used to activate capabilities and define input and output files for MODFLOW-2000. Example name files are shown in Appendix A. The name file is composed of comment lines that begin with a "\#" in column one, and non-comment lines. The first variable read from non-comment lines is a "file type" that controls the activation of processes and packages of MODFLOW-2000. The file type is followed by a unit number and a file name. Specific file types need to be listed in the name file to activate the Observation, Sensitivity, and (or) Parameter-Estimation Processes and to establish one or two primary output files. These are described in the following paragraphs. The contents of the primary output files are then described.

\section{Activation of the Observation, Sensitivity, and Parameter-Estimation}

\section{Processes and Definition of Input and Output Files Using File Types}

The file types used to control the Observation, Sensitivity, and Parameter-Estimation Processes and primary output files are listed in table 2. The Observation Process is activated if the file type "OBS" and one or more of the other file types from the Observation Process section of table 2 are listed in the name file. If an "OBS" file is not listed but one or more input files for observation packages are listed, the Observation Process is not activated. This feature enables the user to deactivate the Observation Process simply by commenting out only the "OBS" file line in the name file.

The Sensitivity Process is activated if file type "SEN" is listed in the name file. The Parameter-Estimation Process is activated if file type "PES" is listed. If the Parameter-Estimation Process is active, the Observation and Sensitivity Processes also need to be active.

As noted by Harbaugh and others (2000) and repeated in table 2, each MODFLOW-2000 run produces either one or two primary output files, the GLOBAL and LIST files. Either a GLOBAL or a LIST file must be the first file listed in the name file. If both are listed, the GLOBAL file needs to be listed first, and it needs to be immediately followed by the LIST file. When either the Sensitivity Process is active or both the Sensitivity and Parameter-Estimation Processes are active, often it is helpful to define both primary output files. If only one is defined, the model input discussed below all goes to one file, and the file can become extremely large.

Depending on the file types specified in the name file and the values assigned to certain variables in the Sensitivity Process and Parameter Estimation input files, MODFLOW-2000 can be used in any of eight modes. The modes are listed in table 3, which also identifies whether parameter values used in the model run will be read from the input files for Ground-Water Flow Process packages or from the Sensitivity Process input file, and whether the parameter values will be updated by regression. Table 3 also briefly lists commonly used model output.

To facilitate activating and deactivating processes and packages in the name file, lines in the name file can be "commented out" with an introductory "\#" symbol. 
Table 2: File types that control the Observation, Sensitivity, and Parameter-Estimation Processes and primary output files

[The file types can be listed in any combination of upper and lower case. NOTE: Do not specify units 96 through 99 in the name file because they are reserved for other uses.]

\begin{tabular}{|l|l|}
\hline \multicolumn{1}{|c|}{ File type } & \multicolumn{1}{c|}{$\begin{array}{c}\text { Process or observation, or description of file output } \\
\text { [Documented in this report unless noted] }\end{array}$} \\
\hline $\begin{array}{l}\text { The user must specify a GLOBAL output file or a LIST output file or both. Specifying both is } \\
\text { recommended when using the Sensitivity or Parameter-Estimation Process, and the contents } \\
\text { described below are as generated when both are specified. If only one is specified, the GLOBAL } \\
\text { and LIST output described below is combined and written to the single file, and output is never } \\
\text { erased. This can produce a very long file. }\end{array}$ \\
\hline GLOBAL & $\begin{array}{l}\text { Primary output file. Contains echoed input and summary information about } \\
\text { parameter definitions, model fit, parameter sensitivity, and regression } \\
\text { performance. }\end{array}$ \\
\hline LIST & $\begin{array}{l}\text { Primary output file. Contains information related to the forward and sensitivity } \\
\text { model run(s). If a GLOBAL file also is listed and the Parameter-Estimation } \\
\text { Process is active, the LIST file is erased and rewritten each parameter- } \\
\text { estimation iteration. }\end{array}$ \\
\hline Observation Process input files
\end{tabular}

${ }^{1}$ Documented by Anderman and Hill (1997) 
Table 3: Modes of MODFLOW-2000 produced by activating different combinations of the Observations (OBS), Sensitivity (SEN), and Parameter-Estimation (PES) Processes, the source of parameter values, and commonly used model output

\begin{tabular}{|c|c|c|c|c|c|}
\hline \multicolumn{3}{|c|}{ Active? } & \multirow[t]{2}{*}{ Mode $^{2}$} & \multirow{2}{*}{$\begin{array}{l}\text { Source Of Parameter } \\
\text { Values }\end{array}$} & \multirow{2}{*}{$\begin{array}{l}\text { Commonly Used Model } \\
\text { Output }^{3}\end{array}$} \\
\hline OBS & SEN & PES & & & \\
\hline \multicolumn{6}{|c|}{ Forward Modes } \\
\hline NO & NO & NO & Forward & Package files & $\begin{array}{l}\text { - Head contour maps } \\
\text { - Water budget terms }\end{array}$ \\
\hline YES & NO & NO & $\begin{array}{l}\text { Forward with } \\
\text { Observations }\end{array}$ & Package files & $\begin{array}{l}\text { - Head contour maps } \\
\text { - Water-budget terms } \\
\text { - Fit of simulated equivalents to } \\
\text { observations }\end{array}$ \\
\hline NO & $\mathrm{YES}^{4}$ & NO & $\begin{array}{l}\text { Forward with } \\
\text { Parameter- } \\
\text { Value } \\
\text { Substitution }\end{array}$ & $\begin{array}{l}\text { SEN input file for } \\
\text { parameters listed there }\end{array}$ & - As for "Forward" mode \\
\hline YES & YES $^{4}$ & $\begin{array}{l}\text { NO } \\
\text { or } \\
\text { YES }\end{array}$ & $\begin{array}{l}\text { Forward with } \\
\text { Observations } \\
\text { and Parameter- } \\
\text { Value } \\
\text { Substitution }\end{array}$ & $\begin{array}{l}\text { SEN input file for } \\
\text { parameters listed there }\end{array}$ & $\begin{array}{l}\text { - As for "Forward with } \\
\text { Observations" mode }\end{array}$ \\
\hline \multicolumn{6}{|c|}{ Parameter-Sensitivity Modes } \\
\hline NO & YES & NO & $\begin{array}{l}\text { Parameter } \\
\text { Sensitivity }\end{array}$ & $\begin{array}{l}\text { SEN input file for } \\
\text { parameters listed there }\end{array}$ & $\begin{array}{l}\text { - One-percent scaled sensitivity } \\
\text { maps (Set print flags in SEN file) }\end{array}$ \\
\hline YES & YES & NO & $\begin{array}{l}\text { Parameter } \\
\text { Sensitivity with } \\
\text { Observations }\end{array}$ & $\begin{array}{l}\text { SEN input file for } \\
\text { parameters listed there }\end{array}$ & $\begin{array}{l}\text { - Fit of simulated equivalents to } \\
\text { observations } \\
\text { - One-percent scaled sensitivity } \\
\text { maps (Set print flags in SEN file) } \\
\text { - Composite scaled sensitivities } \\
\text { - Prediction scaled sensitivities }\end{array}$ \\
\hline YES & YES & YES $^{5}$ & $\begin{array}{l}\text { Sensitivity } \\
\text { Analysis }\end{array}$ & $\begin{array}{l}\text { SEN input file for } \\
\text { parameters listed there }\end{array}$ & $\begin{array}{l}\text { - Fit of simulated equivalents to } \\
\text { observations } \\
\text { - Composite scaled sensitivities } \\
\text { - Prediction scaled sensitivities } \\
\text { - Parameter correlation } \\
\text { coefficients }\end{array}$ \\
\hline \multicolumn{6}{|c|}{ Parameter-Estimation Mode } \\
\hline YES & YES & YES & $\begin{array}{l}\text { Parameter } \\
\text { Estimation }\end{array}$ & $\begin{array}{l}\text { First, values from SEN } \\
\text { input file for parameters } \\
\text { listed there; then, values } \\
\text { calculated by regression }\end{array}$ & $\begin{array}{l}\text { - Optimal parameter values or, if } \\
\text { optimal values are not achieved, } \\
\text { data from parameter-estimation } \\
\text { iterations to diagnose problems. }\end{array}$ \\
\hline
\end{tabular}

${ }^{1}$ The processes are made active by being listed in the name file.

${ }^{2}$ The most commonly used modes are shaded. "Forward with Observations and Parameter-Value Substitution" is like PHASE=1 of UCODE; "Sensitivity Analysis" is like PHASE=22;

"Parameter Estimation" is like PHASE=3. UCODE's PHASE=11, which supports plotting of objective-function surfaces, does not have an analog in MODFLOW-2000.

${ }^{3}$ The output and its use are described in detail in chapter 8 .

${ }^{4}$ ISENALL $<0$ in the Sensitivity Process input file.

${ }^{5} \mathrm{MAX}-\mathrm{ITER}=0$ in the Parameter-Estimation Process input file. 


\section{Contents of the GLOBAL and LIST Output Files}

This section describes the output from the Global, Ground-Water Flow, Observation, Sensitivity, and Parameter-Estimation Processes to the GLOBAL and LIST files. Other processes also may print to these files, but those contributions are not discussed here.

When both the GLOBAL and LIST files are defined, and depending on what processes are active, the GLOBAL file contains information related to parameter definitions, model fit, parameter sensitivity, and regression performance; this file likely will be frequently used during model calibration. The LIST output file contains information from the most recent calculation of hydraulic heads and flows and sensitivities. When the Parameter-Estimation Process is active, the LIST output file is erased and rewritten each parameter-estimation iteration. Relating this to the flowchart of figure 1, the LIST output file contains information from the most recent parameterestimation iteration (iter\#).

For the Parameter-Estimation mode (table 3), the GLOBAL output file contains:

1. Information about the array storage needed by the Observation, Sensitivity, and ParameterEstimation packages.

2. Information about the definition of parameters and observations.

3. Observation-sensitivity tables produced using the starting parameter values. The Observation sensitivities are scaled depending on the value of ISCALS specified in the Observation Process input file. The most commonly used possibilities include dimensionless scaled sensitivities and composite scaled sensitivities, and(or) one-percent scaled sensitivities.

4. Parameter values and other information from each parameter-estimation iteration.

5. Observation sensitivity tables produced using the final parameter values. The tables are described under point 1 of this list.

6. Parameter variance-covariance and correlation matrices.

7. Parameter confidence intervals.

8. A comparison of the parameter values and user-defined reasonable upper and lower limits of the parameter value.

9. Summary statistics about model fit to the observations.

The LIST output file contains:

10. Information about the array storage needed by each Ground-Water Flow Process package.

11. Printed arrays of heads for the entire finite-difference grid. The arrays printed depend on the contents of the Ground-Water Flow Process, Basic Package Output Control file (Harbaugh and others, 2000).

12. Tables that list observed and simulated values from the most recent flow simulation, which was performed using the latest set of parameter values.

13. Observation-sensitivity tables calculated using the most recently calculated grid sensitivities. The observation sensitivities are scaled depending on the value of ISCALS specified in the Observation Process input file. Possibilities include dimensionless scaled sensitivities and composite scaled sensitivities, and(or) one-percent scaled sensitivities.

For other modes, the LIST output file is written only once. For the 'Sensitivity Analysis' mode, the GLOBAL output file contains similar information as for the 'Parameter-Estimation' mode. 


\section{Error Reporting}

All versions of MODFLOW have been programmed to recognize certain errors caused by problems related to input data or to the nature of the system of equations being solved. In previous versions of MODFLOW, when such an error was encountered during execution, a message explaining the error was written to the listing file and the program stopped. The addition of support for parallel processing (see the Parallel Processing section of Appendix B) has necessitated a different approach to error reporting, to ensure that the user can determine the cause of an error.

When MODFLOW-2000 encounters such an error, it writes an error message to the GLOBAL file, the LIST file, or both, and the program stops. In addition, for errors that occur in the sensitivity loop or below in the flow chart (fig. 1), MODFLOW-2000 writes the error message to an error file and informs the user of its existence by writing a message to the screen. The error file is named "mf2kerr.p00" if parallel processing is not enabled. Warnings also are written to the error file, but warnings do not cause the program to stop. See the Parallel Processing section of Appendix B for additional discussion of error reporting when parallel processing is enabled. 


\section{Chapter 3. INVERSE MODELING CONSIDERATIONS}

Calibration of models of complex systems commonly is hampered by problems of parameter insensitivity and extreme correlation caused by data that are insufficient to estimate the parameters defined. The utility and limitations of using sensitivity analysis and nonlinear regression methods in the calibration and analysis of complex models are discussed in Hill (1998). In this report, this chapter and chapter 8 briefly present a few key issues and provide suggestions and warnings where they will be most readily available to users. For additional information, see the companion report Hill (1998).

The first section of this chapter lists a set of guidelines that can be thought of as organized common sense for ground-water model calibration with some new perspectives and statistics. The guidelines are discussed in detail in Hill (1998). The following sections discuss a few of the issues from the guidelines that are likely to be of concern. The final section also includes definitions of some terms related to confidence and prediction intervals.

\section{Guidelines for Effective Model Calibration}

There are many opinions about how nonlinear regression can best be applied to the calibration of complex models, and there is not a single set of ideas that is applicable to all situations. It is useful, however, to consider one complete set of guidelines that incorporates many of the methods and statistics available in nonlinear regression, such as those suggested and explained by Hill (1998) and listed in table 4. This approach has been used successfully even with exceptionally complex systems; see D'Agnese and others $(1998,1999)$. Table 4 is presented to introduce and remind the reader of the guidelines, but the brief statements could be misleading. Those who wish to use these guidelines are encouraged to read the discussions here and in Hill (1998).

\section{Parameterization}

Parameterization is the process of identifying the aspects of the simulated system that are to be represented by estimated parameters. Most data sets are limited and, therefore, only support the estimation of relatively few parameters. In most circumstances, it is useful to begin with a simple model and add complexity as warranted by the complexity of the system and the inability of the model to match observed values (Guideline 1 of table 4).

To obtain an accurate model and a tractable calibration problem, data not used directly as observations in the regression need to be incorporated into model construction (Guideline 2 of table 4). For example, in ground-water systems, it is important to respect and use the known hydrogeology of the system, and it is unacceptable to add features to the model to attain model fit if they contradict known hydrogeologic characteristics.

During calibration it may not be possible to estimate all parameters of interest using the available observations. In such circumstances, the suggestions of the section "Common Ways of Improving a Poor Model" in this chapter may be useful. 
Table 4: Guidelines for effective model calibration (from Hill, 1998)

1. Apply the principle of parsimony (start very simple; build complexity incrementally as needed)

2. Use a broad range of information to constrain the problem

3. Maintain a well-posed, comprehensive regression problem

4. Include many kinds of data as observations in the regression

5. Use prior information carefully

6. Assign weights that reflect measurement errors

7. Encourage convergence by making the model more accurate

8. Evaluate model fit

9. Evaluate optimized parameters

10. Test alternative models

11. Evaluate potential new data

12. Evaluate the potential for additional estimated parameters

13. Use confidence and prediction intervals to indicate parameter and prediction uncertainty

14. Formally reconsider model calibration from the perspective of the desired predictions

\section{Nonlinearity of the Ground-Water Flow Equation with Respect to Parameters and Consequences for the Sensitivity and Parameter- Estimation Processes}

Nonlinear regression needs to be used for ground-water flow models because hydraulic head, as the solution of the ground-water flow equation, is related in a nonlinear fashion to many commonly estimated parameters. In contrast, for confined aquifers, the ground-water flow equation can be classified as linear in time and space because hydraulic head is a linear function of time and space. The linearity and nonlinearity of the ground-water flow equation can be illustrated by considering Darcy's Law, the relation upon which the ground-water flow equation is based. The differential form of Darcy's Law as applied to a cylinder filled with a homogeneous, saturated porous media with different, temporally constant hydraulic heads imposed at each end generally is expressed as

$$
Q=-K A \frac{d h}{d X}
$$

where,

$\mathrm{Q}$ is the flow produced by the hydraulic heads being different at each end of the cylinder $\left[\mathrm{L}^{3} / \mathrm{T}\right]$;

$\mathrm{K}$ is the hydraulic conductivity of the saturated porous media $[\mathrm{L} / \mathrm{T}]$; 
$A$ is the cross-sectional area of the cylinder $\left[\mathrm{L}^{2}\right]$;

$\mathrm{X}$ is distance along an axis parallel to the length of the cylinder and, therefore, parallel to the direction of flow [L]; and

$\mathrm{h}$ is hydraulic head at any distance $\mathrm{X}$ along the cylinder $[\mathrm{L}]$.

The derivative is expressed as a partial derivative because $\mathrm{h}$ is considered to be a function of variables $\mathrm{X}, \mathrm{Q}$, and $\mathrm{K}$.

Equation (1) can be solved for the hydraulic head at any distance, $\mathrm{X}$, to achieve:

$$
\mathrm{h}=\mathrm{h}_{\mathrm{o}}-\frac{\mathrm{Q}}{\mathrm{KA}} \mathrm{X}
$$

where $h_{0}$ is the hydraulic head at $X=0$. The derivatives $\partial \mathrm{h} / \partial \mathrm{Q}$ or $\partial \mathrm{h} / \partial \mathrm{K}$ are sensitivities in a parameter-estimation problem in which $\mathrm{Q}$ or $\mathrm{K}$ is being estimated. By using partial derivative notation, the derivatives of equation (2) with respect to $\mathrm{X}, \mathrm{Q}$ and $\mathrm{K}$ are:

$$
\begin{aligned}
& \frac{\partial h}{\partial X}=-\frac{Q}{K A} \\
& \frac{\partial h}{\partial Q}=-\frac{1}{K A} X \\
& \frac{\partial h}{\partial K}=-\frac{Q}{K^{2} A} X
\end{aligned}
$$

The hydraulic head is considered to be a linear function of $\mathrm{X}$ because $\partial \mathrm{h} / \partial \mathrm{X}$ is independent of $\mathrm{X}$. Hydraulic head also is a linear function of $Q$, because $\partial \mathrm{h} / \partial \mathrm{Q}$ is independent of $\mathrm{Q}$. Hydraulic head is considered to be a nonlinear function of $\mathrm{K}$ because $\partial \mathrm{h} / \partial \mathrm{K}$ is a function of $\mathrm{K}$. As in this simple example, sensitivities with respect to flows, such as $Q$, are nearly always functions of aquifer properties; sensitivities with respect to aquifer properties, such as $\mathrm{K}$, are nearly always functions of the aquifer properties and the flows. If $\mathrm{Q}$ and $\mathrm{K}$ are being estimated, both situations make the regression nonlinear.

Parameter transformations sometimes can be used to linearize the relation between observations and parameters. By using the example above and considering the transformations $1 / \mathrm{K}$ and $\ln (\mathrm{K})$, equation 5 would be replaced by:

$$
\begin{aligned}
& \frac{\partial \mathrm{h}}{\partial(1 / \mathrm{K})}=-\frac{\mathrm{X}}{\mathrm{A}} \text { and } \\
& \frac{\partial \mathrm{h}}{\partial(\ln (\mathrm{K}))}=-\frac{\mathrm{X}}{\mathrm{KA}} .
\end{aligned}
$$

For $1 / \mathrm{K}$, the right-hand side is independent of any parameters, making it linear. Ground-waterflow problem sensitivities can sometimes be linearized by redefining parameters in this way, but the prospects become less likely as the flow system becomes more complex, as is common in applications of MODFLOW-2000. For $\ln (\mathrm{K})$, the right-hand side is dependent on $1 / \mathrm{K}$ instead of $1 / \mathrm{K}^{2}$, making it less nonlinear. The Sensitivity Process supports the log transformation because 
besides making the problem more linear, it also prohibits the parameter value from becoming negative.

Model nonlinearity affects parameter estimation and other aspects of model sensitivity analysis, calibration, and uncertainty analysis in a number of ways, as discussed by the companion report Hill $(1998$, p. 4-7, 31, 41-42, 60) and references cited therein. The most obvious effect is the need for the parameter-estimation iterations, as noted in figure 1 , and the enhanced difficulties involved in achieving an optimum set of parameter values. The next most obvious effect is the difficulty in assessing model inaccuracy.

\section{Starting Parameter Values}

Nonlinear regression begins with starting parameter values. There are three aspects of these starting values that are important.

1. Depending on the mode (table 3), the starting parameter values are used to calculate residuals, scaled and composite scaled sensitivities, and(or) parameter correlation coefficients. These statistics are important to diagnose potential problems with the model and the regression and to determine ways of addressing these problems. In most circumstances, it is useful to evaluate these statistics regularly as the model changes during the calibration process. The statistics printed by MODFLOW-2000 are discussed in chapter 8 of this report and in Hill (1998). The latter also includes a discussion of how model nonlinearity affects the analysis.

2. It is sometimes advantageous to change the starting parameter values. As calibration proceeds, parameter values that produce a better model fit than the original starting parameter values are estimated by regression. Updating the original starting parameter values using the new estimated values can reduce execution time because, commonly, fewer regression iterations are required when the starting parameter values produce a closer model fit. In MODFLOW-2000, parameter values for each parameter-estimation iteration are printed to the _b file generated by the Parameter-Estimation Process when OUTNAM of the OBS file is not "NONE" (see Chapter 4). The_b file is written such that its lines can be substituted by the user directly into the Sensitivity Process input file.

3. The starting parameter values can be used to test for the uniqueness of optimized parameter values; that is, the values at which the regression converges. This is accomplished by initiating the regression with different sets of starting values. If the resulting optimized parameter values differ from each other by amounts that are small relative to their calculated standard deviations, the optimization is likely to be unique. If this is not the case, the optimization is not unique. Lack of uniqueness can be caused by a number of factors. If caused by local minima in the objective function, it may be possible to examine the objective function values achieved by the different sets of optimized parameter values and identify a global minimum as the set of optimized parameter values that produces the smallest objective-function value. If non-uniqueness is caused by extreme parameter correlation, the objective-function value for each optimized set of parameters is likely to be similar and at least one pair of parameters will have a correlation coefficient very close to 1.0 or -1.0 . This is demonstrated clearly by the simple test case presented by Poeter and Hill (1997).

\section{Weighting Observations and Prior Information}

Observations and prior information need to be weighted so that (1) the weighted residuals will all be in the same units so that they can be squared and summed in the least-squares objective function and (2) to reflect the relative accuracy of the measurements (Hill, 1998, p. 4, 13-14, 45). Suggestions for determining the weights are presented in the discussion for guideline 6 of Hill 
(1998, p. 46-49). It is suggested there that the assigned weighting reflect the observation errors, and this is assumed in the following discussion.

In general, weighting requires a full weight matrix (Hill, 1998, p. 7, eq. 2), where the diagonals of the weight matrix equal the observation error variances and the off-diagonals equal the covariances. A diagonal weight matrix is strictly valid only if the measurement errors are independent. The weight matrix capabilities of MODFLOW-2000 are different for hydraulic heads and for the other types of observations and for prior information. For hydraulic-head observations, MODFLOW-2000 does not support a full weight matrix, but it does support differencing methods designed to accommodate commonly encountered error correlation. For all other types of observations and prior information, MODFLOW-2000 supports a full weight matrix.

The importance of using a full weight matrix even in the presence of correlated measurement errors is questionable. A published study by Christensen and others (1995) and unpublished numerical investigations by Mary C. Hill (U.S. Geological Survey, written communication, 1996) indicate that typical error correlations have little effect on nonlinear regression, residual analysis, or uncertainty analysis. This, however, is a preliminary conclusion drawn from partial, limited investigation. Further work remains to determine the importance of using full weight matrices in problems typical of ground-water investigations.

When a diagonal weight matrix is assumed, MODFLOW-2000 allows users to specify either the variance, standard deviation, or coefficient of variation of the observations error. This allows the statistic that makes most sense in a given situation to be used. For example, streamflow observation error may be most readily understood based on a percent of the observed value, which can be most easily expressed as a coefficient of variation. Hydraulic head observation error is more often expressed as some number of feet, meters, or centimeters, and is most easily expressed as a standard deviation. More detailed information about determining values for weights is provided in Hill (1998, p. 46-49).

\section{Common Ways of Improving a Poor Model}

Problems, such as insensitivity, extreme correlation of parameters, and poor model fit, are common in model calibration. Possible ways of addressing these problems follow, listed in order of how often the suggestion is most appropriate in practice.

1. Reconsider the model construction, including geometry and hydrogeologic units, discretization, and so on. Regression difficulties and poor model fit can help reveal misconceptions used to construct the model.

2. Modify the defined parameters by adding, omitting, and (or) combining parameters to be estimated. See section "Parameterization" above.

3. Carefully eliminate observations or prior information if available evidence indicates that they are likely to be biased. Do not omit observations just because the model does not fit them well.

4. Adjust weights either for groups of observations and prior information, or perhaps individually. Small changes in the weighting rarely affect regression results, so, in most circumstances, time-consuming repeated runs using slightly different weights should be avoided.

A useful approach is to continually strive to identify and correct inaccuracies in the model construction or the use of observations (this is guideline 7 of table 4). Use the model fit and calculated parameter sensitivities and correlation coefficients to facilitate this process. Nearly 
always, nonlinear regression will converge as the problems are resolved. Additional potential difficulties and their resolutions also are discussed in Hill (1998).

\section{Alternative Models}

The sparse data sets available for the development of most ground-water models often support equally feasible alternative conceptual models, and it is important to evaluate all such models. Equally feasible conceptual models are those that reasonably represent known conditions and yield an acceptable fit to the data with reasonable regression-determined optimal parameter values. All such models need to be used to make predictions and to determine the associated confidence in those predictions. If the various models produce a range of predictions that are different enough to make the appropriate scientific conclusion or management decision unclear, and additional data collection is warranted, statistics of the regression can be used to help identify new data that are most likely to differentiate the models, and thus help to identify those that are not representative of the system.

\section{Residual Analysis}

To judge whether a model is likely to represent a system accurately, it is crucial to analyze the residuals (observed minus simulated values). A complete analysis of residuals includes consideration of summary statistics and consideration of graphs and maps of weighted and unweighted residuals (see section "Graphical Analysis of Model Fit and Related Statistics" and Guideline 8 of Hill, 1998). In the graphical analyses, some departure from ideal patterns may be attributed to the limited number of data and the fitting of the regression. The effect of these contributions can be evaluated by generating random data sets that have the same number of data and characteristics consistent with the fitting of the regression (Cooley and Naff, 1990). Such random data sets can be generated with a MODFLOW-2000 output file and the computer program RESAN-2000, as described in chapter 7 of this report.

\section{Predictions and Differences, and Their Linear Confidence Intervals and Prediction Intervals}

Often ground-water flow models are constructed to assess likely system response under given potential conditions such as increased pumpage or climate change. Predictions for these conditions can be simulated using a calibrated model. MODFLOW-2000, used in conjunction with post-processor YCINT-2000, includes some sophisticated methods of calculating and evaluating predictions of hydraulic heads, flows, and advective transport - that is, the same type of quantities supported by the Observations Process. The purpose of this section is to introduce those methods. Detailed information about YCINT-2000 and how to use MODFLOW-2000 and YCINT-2000 is provided by Hill (1994) and chapters 7 and 8 of this report.

MODFLOW-2000 and YCINT-2000 allow differences to be calculated by subtracting values produced by a base simulation from values produced by a predictive simulation. That is:

(value from predictive simulation) - (value from base simulation) $=$ difference.

Commonly, but not always, the base simulation represents conditions related to the calibration. For a steady-state calibration, the base simulation commonly is equivalent to the calibration conditions; for a transient calibration the base simulation commonly is equivalent to the conditions at the end of the calibration period. In a ground-water example, values of interest 
might be hydraulic heads at the same location before and after additional pumpage is imposed on the system. In this circumstance, the predictive simulation includes the additional pumpage; the base simulation does not. The difference would be the drawdown resulting from the pumpage. The use of differences is discussed further by Hill (1994).

The program YCINT-2000 calculates 95-percent linear confidence and prediction intervals on both predictions and differences, using equations 11 though 17 of Hill (1994). Linear confidence and prediction intervals indicate the uncertainty with which the predictions or differences are determined using the calibrated model. In this context, confidence and prediction intervals can be defined as follows:

Confidence intervals represent the uncertainty in the simulated values that results from the uncertainty in the estimated parameter values. For the purpose of calculating confidence intervals, the uncertainty in the estimated parameter values is expressed by the optimal parameter variance-covariance matrix (Hill, 1998, eq. 28). The validity of the confidence intervals depends on the calibrated model accurately representing the true system, the model being linear, and the weighted residuals being normally distributed.

Prediction intervals include the uncertainty in the estimated parameter values as described for confidence intervals, but also include the effects of the measurement error that is likely to be incurred if the predicted quantity is to be measured. Prediction intervals generally are larger than confidence intervals and need to be used when a measured value is to be compared to the calculated interval.

From these definitions, it is obvious that a single prediction can have an associated confidence interval and prediction interval, and the interval to be used depends on whether or not the effects of measurement error are to be included. The idea of prediction interval is distinct from the predictions, but the similarity of the terms can cause confusion. The terminology needs to be used carefully.

There are several ways to calculate confidence and prediction intervals, depending on how many predictions and differences are to be considered together. The calculations differ only in the critical values used (Hill, 1994, eq. 11-17), which are statistics from standard probability distributions. The probability distributions of concern are the Student-t, Bonferroni-t, and Fdistributions. Tables of the statistics from these distributions were programmed into YCINT2000 , so that the appropriate critical value is determined by the program, based on information provided by the user. Two types of intervals are considered -- individual and simultaneous -- and there are three ways of calculating simultaneous intervals. YCINT-2000 calculates all of the intervals and prints three of them after eliminating one of the simultaneous intervals because it is less accurate than its alternative, as discussed below. Of the three intervals printed, the user needs to choose the appropriate interval for a given application. The intervals and selection criteria are described in the following paragraphs.

Individual intervals apply when only one prediction or difference is of concern. There is only one method of calculating individual linear confidence and prediction intervals (Hill, 1994, eq. 11 and 15), and it is exact if the model is linear and accurate, and the residuals are normally distributed.

Simultaneous intervals apply when the number of predictions and differences of concern exceeds one, or when the interval is calculated on a quantity that is not precisely defined, such as the largest value wherever it occurs within the model.

Different types of simultaneous intervals are appropriate for different circumstances. The names of the possible intervals are "Bonferroni", "Scheffé $\mathrm{d}=\mathrm{k}$ ", and "Scheffé $\mathrm{d}=\mathrm{np}$ ", and all are approximate. If the number of predictions plus differences (represented by k) exceeds one and is 
less than the number of parameters, np, both the approximate Bonferroni and Scheffé $d=k$ simultaneous intervals apply. If $\mathrm{k}$ is greater than $\mathrm{np}$, Scheffé $\mathrm{d}=\mathrm{np}$ simultaneous intervals apply. Both the Bonferroni and Scheffé $\mathrm{d}=\mathrm{k}$ methods tend to produce intervals that are larger than exact intervals would be for a linear, accurate model with normally distributed residuals. In any circumstance, therefore, the smaller of the two intervals needs to be used, and YCINT-2000 only prints the smaller of the two intervals.

If the number of predictions and differences of concern cannot be exactly defined, simultaneous linear confidence and prediction intervals using the approximate Scheffé $d=n p$ method apply. Scheffé $d=n p$ intervals tend to be larger than exact linear intervals would be for a linear, accurate model calculated for the same circumstances.

Although linear confidence and prediction intervals can be useful indicators of the uncertainty with which the prediction or difference has been determined (Christensen and Cooley, 1999), the intervals also can be misleading if interpreted and presented without understanding and correctly representing their underlying assumptions. In particular, the significance level of the intervals as calculated is nominally 5 percent ( 1.0 minus 0.95 for 95 -percent intervals), but depends on the model being linear for parameter values near the optimized parameter values, and on the model accurately representing the system. Model linearity can be tested with the MODFLOW-2000 post processor BEALE-2000; model accuracy is evaluated by analyzing model fit as mentioned in the earlier section "Residual Analysis." The proper use and potential inaccuracies of using linear confidence and prediction intervals for nonlinear problems are discussed by Hill (1994 and 1998) and Christensen and Cooley (1999).

Another common problem occurs when the predictions and differences of interest include types of quantities not included in the observations used to calibrate the model, or the prediction conditions differ dramatically from the calibration conditions. In such a circumstance, confidence and prediction intervals may be useful, but they may not accurately indicate prediction uncertainty and need to be used with caution. 


\section{Chapter 4. OBSERVATION PROCESS}

The Observation Process does the following:

1. Calculates simulated equivalents of the observations using the hydraulic heads for the entire model grid produced by the Ground-Water Flow Process of Harbaugh and others (2000),

2. Compares observed values with the simulated equivalent values, and

3. When used with the Sensitivity Process, calculates observation sensitivities (the derivative of the simulated equivalent values with respect to the parameters) using the sensitivities for the entire model grid from the Sensitivity Process discussed in chapter 5.

The word "observed" is used instead of "measured" to coordinate with common regression terminology (Draper and Smith, 1998). Use of the Observation Process in different possible modes is described in table 3 . Use of the observation sensitivities in sensitivity analysis and regression are described in Hill (1998, p. 14-16, 38-42, 58).

The types of observations supported are listed in table 2 with their associated file types. The observations include hydraulic heads; changes in hydraulic head over time; flows to or from surface-water bodies represented using the General-Head Boundary, Drain, or River Packages; flow to or from a set of constant-head finite-difference cells; and advective transport. Advectivetransport observations are documented by Anderman and Hill (1997); the others are documented in this report.

The tasks of the Observation Process are as follows:

1. Read a file that contains information applicable to all observations.

2. Read observed values and information needed to calculate associated simulated values. This information is provided through input files related to the Ground-Water Flow Process capability chosen by the user to calculate the associated simulated value. For example, if a streamflow gain represented using the River Package is to be used as an observation, an Observation Process River Package (file type RVOB of table 2) input file is needed. If an observed flow is represented using the General-Head Boundary Package, an Observation Process General-Head Boundary Package (file type GBOB of table 2) input file is needed. Information about hydraulic-head observations are specified in an Observation Process Basic Package input file, which has file type HOB (table 2).

3. Calculate the associated simulated equivalents to the observations using the hydraulic heads for the entire grid produced by the Ground-Water Flow Process.

4. If the Sensitivity Process is active, calculate the associated observation sensitivities using the grid sensitivities produced by the Sensitivity Process.

5. Facilitate comparison between the simulated and observed values by calculating statistical measures and by producing files to support graphical comparisons. The statistics and graphical procedures supported are listed by Hill (1998, table 1). The files are named using a file name base, defined by the user in the file mentioned in step 1 above, and a programdefined file name extension, as discussed below. 


\section{General Considerations}

Several issues are common to more than one type of observation, and these are presented in this section. They include defining observation times, coping with observations that are alternatively included and omitted from the regression because of cells of convertible layers becoming dry or wet and head-dependent boundaries becoming disconnected and connected, weighting of observations, and scaling of observation sensitivities.

\section{Observation Times}

For all the Observation Process packages except the Advective-Transport Observation Package, the user identifies the time of an observation by a stress period number (referred to as the reference stress period, IREFSP) and a time offset (TOFFSET). A multiplier (TOMULT) is provided so that the time offsets can be in convenient units. The time of the observation is the time at the beginning of the reference stress period plus the time offset. The time offset may exceed the length of the reference stress period as long as the resulting observation time is not later than the end of the final stress period. This method of specifying observation time can facilitate construction and maintenance of input files for Observation-Process Packages because it can allow the number and length of stress periods and time steps to be changed without changing the observation time definition.

For example, consider a flow system simulated with one steady-state stress period (stress period 1) followed by several transient stress periods (stress periods $2,3, \ldots$ ). In the input files for Observation-Process packages, the user can specify a reference stress period of 2 (the first transient stress period) for all transient observations and define time offsets to identify observation times as the time since the beginning of the transient simulation. In this circumstance, the number and length of transient stress periods and time steps in the Discretization file (Harbaugh and others, 2000) can be changed without changing the Observation-Process input files, as long as the total simulation time is sufficient to include all specified observation times.

Two other issues are important. First, the time unit in the Discretization file need not be the time unit used for TOFFSET because TOMULT can be used as a conversion factor. This allows the observation times to be defined in days, for example, even when the simulation time is in seconds. Second, when an observation time falls within a time step, linear interpolation between the beginning and end of the time step is used to calculate the simulated value.

\section{Dry Cells in Convertible Layers at Observation Locations}

There are two aspects of the Ground-Water Flow Process that can cause an observation to be omitted from at least some parameter-estimation iterations. The first is when finite-difference cells go dry, as can occur for convertible model layers (Harbaugh and others, 2000). The second is when the hydraulic head calculated adjacent to a head-dependent boundary represented by the Drain or River Package falls below a specified level.

When the hydraulic head at a cell in a water-table layer falls below the bottom of the cell, the cell is designated as inactive ("goes dry") and remains inactive through the last time step unless cells in the layer are allowed to be reactivated ("rewet") (see the instructions in Harbaugh and others, 2000 for the Block-Centered Flow or Layer Property Flow Packages, or Anderman and Hill, 2000 for the Hydrogeologic-Unit Flow Package). At a dry cell, hydraulic head is not calculated, and the cell cannot be used to calculate simulated hydraulic heads or head-dependent boundary gains and losses for the parameter-estimation iteration.

For head-dependent boundary reaches, drying of cells generally poses a problem less often because head-dependent boundary cells do not tend to go dry as often as other cells. When 
they do go dry, these cells generally account for only a fraction of a flow observation. Although it is possible for an entire reach associated with an observation to go dry, this is uncommon. No special provisions have been made in the Observation Process to account for observations to be omitted for observed-flow gains or losses represented using head-dependent boundaries.

For constant-head flow observations, dry cells are not a problem because constant-head cells do not go dry.

The effect of omitting observations due to dry cells is that the impetus for changing the parameters to keep the dry areas wet is lost from the parameter-estimation procedure. This loss is unfortunate, but currently there is no practical alternative.

Observations being alternately used and omitted and used again in successive parameterestimation iterations makes it more difficult for parameter estimation to converge. This situation might occur for hydraulic-head observations in convertible layers that go dry and for headdependent boundary gain-and-loss observations. The problem of alternately used and omitted observations can be addressed in the following ways:

1. Eliminate the omitted observations early in the calibration process, and try including them later when the parameter estimates are closer to the final values or the model is closer to its final form.

2. A water-table layer can be simulated as a confined layer using estimated layer thicknesses early in the calibration process, and represented as a water-table layer later when the parameter estimates are closer to the final values or the model is closer to its final form.

3. For head-dependent boundary gain-and-loss observations, small streambed or riverbed thicknesses can aggravate the problem. Increase these thicknesses if such a change is consistent with available field data.

4. Review the representation of the ground-water flow system and make changes if needed. This is the same process that a modeler goes through in a trial-and-error calibration, and its goal is to ensure that the physical system is being represented realistically. Unrealistic representations cause problems in nonlinear-regression parameter estimation just as they cause problems when calibrating by trial and error.

\section{Weighting Observations}

As discussed in chapter 3, observations generally need to be weighted because they have different units or are not equally accurate. MODFLOW-2000 allows either (1) for the weight matrix to be diagonal, so that only the uncertainty of each observation needs to be specified, or (2) except hydraulic-head measurements, the weight matrix can be full, so that the error correlation also can be included. For hydraulic heads, some differencing methods are available to eliminate some types of error correlation. These are discussed below. For (2), the correlations are limited to being specified for observations within each package discussed below, so that, for example, errors in flows represented using the General-Head Boundary Package can be correlated to one another but not to errors in observations represented by other packages. Determining the values for the weighting is discussed in Guideline 6 of Hill (1998), as mentioned in Chapter 3 of this report.

\section{Scaling of Observation Sensitivities}

The observation sensitivities can be scaled to obtain measures of such things as (1) the relative importance of different observations to the estimation of the same parameters, (2) the relative importance of an observation to the estimation of different parameters, and (3) the total amount of information provided by the observations for estimating each parameter. Hill (1998, p. 
14-16) discusses the calculation and use of dimensionless, composite, and one-percent scaled observation sensitivities. The scaled sensitivities produced by MODFLOW-2000 differ from those discussed in Hill (1998) in that the absolute value of the parameter is used and, if the parameter is not log-transformed, BSCAL is used if the parameter value is near zero (see the discussion for BSCAL below).

For log-transformed parameters, a difficulty occurs when the parameter value equals 1.0 so that its log-transformed value, which is used in the scaling, is 0.0 . If this situation occurs, the printed sensitivities equal 0.0 , and a message is printed in the output file. The problem can be corrected by modifying the multipliers of the parameter so that the estimated native value does not equal 1.0. 


\section{Input File For All Observations}

This short input file contains information applicable to all observations, and needs to be included in the name file using file type OBS if any of the input file types for ObservationProcess packages listed in table 2 are included.

\section{Input Instructions}

Input for the Observation Process for all observations is read from a file listed in the name file with "OBS" as the file type (table 2).

0 . [\#Text]

Item 0 is optional and can include as many lines as desired. Each line needs to begin with the "\#" character in the first column.

1. OUTNAM ISCALS (free format)

\section{Explanation of Variables}

Text-is a character string (maximum of 79 characters) that starts in column 2 . Any characters can be included in Text. The "\#" character needs to be in column 1. Text is printed when the file is read and provides an opportunity for the user to include information about the model both in the input file and the associated output file.

OUTNAM - a string of one to 78 nonblank characters. OUTNAM indicates whether or not the output files listed in tables 5 and 6 are produced by MODFLOW-2000 (table 5) or its post-processing programs RESAN-2000, YCINT-2000, or BEALE-2000 (table 6). If "NONE" is specified (can be any combination of upper and lower case), none of the output files are created. Otherwise, the output files are named using OUTNAM as the base followed by a period and the two- or three-character extensions listed in tables 5 and 6. The specification of lower and upper case in OUTNAM is preserved in generating the file-name base. Extensions for files that are intended for use by a post-processor, graphics program, or other program start with an underscore ( $~$ ); extensions for files that are intended to be read by the user start with a number sign (\#). OUTNAM can include a path; constraints imposed by the operating system regarding file names and paths should be considered when specifying OUTNAM. For compatibility with the post-processing programs, the OUTNAM string should not be changed between the separate model runs used to generate the files to be read by the post processors (see header of table 5 and the discussion in chapter 7). 
Table 5: Files produced by MODFLOW-2000 when OUTNAM is not "NONE" that are designed for use by plotting routines and other programs.

[Files are named as OUTNAM followed by a period and an extension that begins with an underscore. For example, if OUTNAM is "gw", file names would be "gw._os" and so on. The files can be used as described later in this report and in table 16. Shading indicates files that probably require special simulations to be produced properly.]

\begin{tabular}{|c|c|}
\hline $\begin{array}{l}\text { Exten- } \\
\text { sion }\end{array}$ & $\begin{array}{l}\text { File contents (The file contents are in the order listed. An * indicates that for each } \\
\text { observation the listed items are followed by the OBSNAM and PLOT-SYMBOL. } \\
\text { The files can be read as space-delimited free format.) }\end{array}$ \\
\hline \multicolumn{2}{|c|}{ If the Observation Process is active: } \\
\hline _os & Unweighted simulated equivalents and observations * \\
\hline Ew & Weighted simulated equivalents and observations * \\
\hline _ws & Weighted simulated equivalents and residuals * \\
\hline r & Unweighted residuals * \\
\hline w & Weighted residuals * \\
\hline $\mathrm{nm}$ & Weighted residuals and probability plotting positions * \\
\hline \multicolumn{2}{|c|}{ If the Sensitivity Process also is active, the following files also are produced: } \\
\hline _sc & Composite scaled sensitivity for each parameter, preceded by the PARNAM. \\
\hline _sd & $\begin{array}{l}\text { Dimensionless scaled sensitivities for each parameter, preceded by OBSNAM and } \\
\text { PLOT-SYMBOL. Repeated for each observation. }\end{array}$ \\
\hline -sl & $\begin{array}{l}\text { One-percent scaled sensitivities for each parameter, preceded by OBSNAM and } \\
\text { PLOT-SYMBOL. Repeated for each observation. }\end{array}$ \\
\hline \multicolumn{2}{|r|}{ If the Parameter-Estimation Process also is active, the following files also are produced: } \\
\hline _ss & $\begin{array}{l}\text { Sum of squared weighted residuals for each type of observation and prior information } \\
\text { and the total; values are listed for all parameter-estimation iterations. }\end{array}$ \\
\hline -pa & $\begin{array}{l}\text { Parameter values for each parameter-estimation iteration, formatted for easy } \\
\text { production of graphs showing parameter values for each iteration. This file is not } \\
\text { produced until the end of the program; to access parameter values as each iteration is } \\
\text { performed, use the _b file described below. }\end{array}$ \\
\hline$-\mathrm{b}$ & $\begin{array}{l}\text { Information from each parameter-estimation iteration, including parameter values } \\
\text { formatted for easy substitution into the Sensitivity Process input file, sum of squared } \\
\text { weighted residuals, maximum calculated fractional parameter change and the } \\
\text { associated parameter number (as listed in the SEN file), and value of the Marquardt } \\
\text { parameter. }\end{array}$ \\
\hline rs & Input file for post-processing program RESAN-2000 (see chapter 7) \\
\hline$-\mathrm{y} 0$ & $\begin{array}{l}\text { Input file for post-processing program YCINT-2000 (see chapter 7). Produced when } \\
\text { IYCFLG }=0 \text {. }\end{array}$ \\
\hline -yl & $\begin{array}{l}\text { Input file for post-processing program YCINT-2000 (see chapter 7). Produced when } \\
\text { IYCFLG }=1 \text {. }\end{array}$ \\
\hline$-y^{2}$ & $\begin{array}{l}\text { Optional input file for post-processing program YCINT-2000 (see chapter 7). } \\
\text { Produced when IYCFLG }=2 \text {. }\end{array}$ \\
\hline -b1 & $\begin{array}{l}\text { One of two input files for post-processing program BEALE-2000 (see chapter 7). } \\
\text { Produced when IBEFLG }=1 \text {. }\end{array}$ \\
\hline _b2 & $\begin{array}{l}\text { The second input file for the post-processing program BEALE- } 2000 \text { (see chapter } 7 \text { ). } \\
\text { Produced when IBEFLG }=2 \text {. }\end{array}$ \\
\hline
\end{tabular}


Table 6: Files produced by the MODFLOW-2000 post-processors RESAN-2000, YCINT-2000, and BEALE-2000 (chapter 7) when OUTNAM is not "NONE".

[File names are the base specified by OUTNAM followed by a period and an extension. For example, if OUTNAM is defined as "gwmodel," the file names would be gwmodel._rd, gwmodel.\#yc, and so on. Files with extensions that start with an underscore (_) are designed to facilitate plotting; files with extensions that start with a \# are designed to be read by the modeler. Use these files as described later in this report and in table 16.]

\begin{tabular}{|c|l|}
\hline $\begin{array}{c}\text { Exten- } \\
\text { sion }\end{array}$ & $\begin{array}{c}\text { File contents (The '_files contain the items listed in the order listed. An * } \\
\text { indicates that for each observation the listed items are followed by the OBSNAM } \\
\text { and PLOT-SYMBOL. All '_-' files can be read as space-delimited free format.) }\end{array}$ \\
\hline If the post-processing program RESANP is executed: \\
\hline \#rs & Main output file. \\
\hline rd & Ordered uncorrelated deviates and probability plotting positions. * \\
\hline rg & Ordered correlated deviates and probability plotting positions. * \\
\hline rc & Cook's D statistic for each observation. * \\
\hline rb & $\begin{array}{l}\text { DFBeta statistics for each parameter, preceded by OBSNAM and PLOT- } \\
\text { SYMBOL. Repeated for each observation. }\end{array}$ \\
\hline If the post-processing program YCINT-2000 is executed: \\
\hline \#yc & $\begin{array}{l}\text { For the listed predictions or differences, this file contains the linear, 95-percent } \\
\text { confidence and prediction intervals. Individual and simultaneous intervals are } \\
\text { included. }\end{array}$ \\
\hline _yp & $\begin{array}{l}\text { Confidence and prediction intervals on predictions. Title lines describing the type } \\
\text { of interval are followed by data lines for each prediction. Data lines include lower } \\
\text { limit, upper limit, prediction, and standard deviation. * }\end{array}$ \\
\hline _yd & $\begin{array}{l}\text { Confidence and prediction intervals on differences. Title lines describing the type } \\
\text { of interval are followed by data lines for each difference. Data lines include lower } \\
\text { limit, upper limit, difference, and standard deviation. * }\end{array}$ \\
\hline If the post-processing program BEALE-2000 is executed: \\
\hline \#be & The modified Beale's measure statistic and auxiliary information. \\
\hline
\end{tabular}

ISCALS-Controls printing of the observation-sensitivity tables in the primary output files. Creation of the _sc, _sd, and _s1 files is not affected by ISCALS. (ISCALS typically is specified as 1,2, or 3. Unscaled sensitivities are rarely of interest.) The different types of sensitivities are discussed in Hill (1998, p. 14-16, 33, 38-40, 62-64).

ISCALS $<0$, No observation-sensitivity tables are printed, but a table showing composite scaled sensitivity for each parameter is printed.

ISCALS $=0$, Unscaled sensitivities are printed.

ISCALS $=1$, Dimensionless scaled sensitivities are printed. Sensitivities are scaled by multiplying by the parameter value and the square-root of the weight, which produces dimensionless numbers. If the parameter value is less than BSCAL, which is read from the Sensitivity Process input file for each parameter listed there, the parameter value is replaced by BSCAL for the scaling. The resulting values are dimensionless and equal the number of observation error standard deviations that the simulated value would change given a one-percent change in the parameter value, times 100. Composite scaled sensitivities also are printed. 
ISCALS $=2$, One-percent sensitivities are printed. Sensitivities are scaled by multiplying by the parameter value and dividing by 100 . If the parameter value is less than BSCAL, which is read from the Sensitivity Process input file for each parameter listed there, the parameter value is replaced by BSCAL for the scaling. The onepercent scaled sensitivities are designed to have the same dimensions as the observations and to equal the amount the associated simulated value will change given a one-percent change in the parameter value.

ISCALS $=3$, Both dimensionless and one-percent sensitivities are printed. 


\section{Hydraulic-Head Observations}

The Hydraulic-Head Observation part of the Basic Package of MODFLOW-2000 supports specification of observations that are hydraulic heads at any location and time. For locations that are not at cell centers within layers and for times that are not at the beginning or end of a time step, interpolation is used to obtain simulated equivalent values.

Two options are included in the Hydraulic-Head Observation part of the Basic Package. The first option supports observations of temporal changes in head, where simulated equivalents are calculated as a simulated hydraulic head minus the hydraulic head simulated for the first observation listed at the same location. The advantage of using temporal changes (differences) as observations is that time-invariant errors, such as errors in well elevation, are removed. The disadvantage is that sensitivities generally are smaller when using changes in head, rather than heads, as observations. When estimating parameters, the advantage results in an observation that is expected to be more accurate and, therefore, a larger weight for the observation is defined; the disadvantage results in smaller sensitivities and tends to reduce the effect of the observation on parameter estimation. Whether differencing results in the observation contributing to model calibration or not depends on how the advantage of the increased accuracy compares to the disadvantage of the decreased sensitivity. Thus, whether differencing is advantageous or not is problem dependent.

The second option supports observations that are multilayer, in that they reflect the hydraulic head calculated in more than one model layer. For observations that are vertically between model cell centers, this capability can be used to interpolate simulated hydraulic heads from adjoining layers. Or, this capability can be used when the observation well is open to the subsurface system in more than one model layer. The formulation for multilayer wells presented in this version of MODFLOW-2000 is elementary; the user specifies the fractional contribution to be applied to the hydraulic head in the layers involved. A more sophisticated approach would involve calculating these coefficients, but this capability is not included in MODFLOW-2000.

\section{Calculation of Simulated Equivalents to the Observations}

This section describes the spatial interpolation performed by MODFLOW-2000, the differencing performed to calculate observations of temporal changes in hydraulic head, the calculation of simulated equivalents for multilayer hydraulic-head observations and how the interpolation is affected by dry cells. The temporal interpolation used for hydraulic-head observations is as described above in the section 'Observation Times'.

\section{Spatial Interpolation for Hydraulic-Head Observations at Arbitrary Locations}

The finite-difference method calculates hydraulic heads at the center of each active finitedifference cell. Observation wells, however, rarely are located at cell centers and might not be screened throughout the entire thickness represented by the model layer. To account for observation wells located away from cell centers, simulated hydraulic heads at observation locations need to be calculated by interpolating within the two-dimensional plane of a single layer. Six locations (A-F) for which hydraulic heads might need to be interpolated are shown in figure 2. Exact interpolation of hydraulic heads, in which the interpolated hydraulic heads would correspond to the hydraulic heads simulated using a locally very fine numerical grid, is not generally possible for block-centered finite-difference methods. This is because hydraulic properties are defined for cells that do not extend between locations where hydraulic head is calculated (McDonald and Harbaugh, 1988). For example, interpolation for locations B, C, D, E, or $\mathrm{F}$ in figure 2 could require as many as four different hydraulic-conductivity values, and, for 
this complicated case, no exact interpolation method is available. Geometric interpolation methods that ignore the variations in hydraulic conductivity, however, are available. In this report, geometric interpolation based on linear, finite-element basis functions is used.

Linear one-dimensional basis functions (equivalent to linear interpolation) are used for locations, such as B and E in figure 2, which are adjacent to two inactive cells or are exactly between adjoining cell centers; triangular basis functions are used for locations such as $\mathrm{C}$ and $\mathrm{F}$ in figure 2 , which are within a triangle formed by the centers of three neighboring cells because the fourth neighboring cell is inactive; and quadrilateral basis functions are used for locations such as $\mathrm{D}$ in figure 2, which are within a rectangle formed by the centers of four active cells. All basis functions are calculated using local coordinates that are specified by the user and define the observation location within a cell relative to the cell center. These local coordinates are a row offset, ROFF, and a column offset, COFF, that range in value from -0.5 to +0.5 , with 0.0 indicating that there is no offset. Use of ROFF and COFF is illustrated in figure 2. Note that $\mathrm{ROFF}$ is negative in the direction of decreasing row numbers, and COFF is negative in the direction of decreasing column numbers.

The basis functions used are described in numerous texts and are not discussed in this report. They are equivalent to the one-dimensional simplex, two-dimensional simplex, and quadratic-element basis functions of Segerlind (1976, p. 24, 28, and 258), and the triangular "archetypal" and rectangular-element basis functions of Wang and Anderson (1982, p. 119 and 153). Wang and Anderson (1982) do not discuss a linear, one-dimensional basis function.

Errors introduced by using geometric interpolation might become substantial when the hydraulic properties of neighboring cells are different and cell dimensions are large. At such locations, the differences between observed and simulated hydraulic heads might be inaccurate and could produce inaccurate parameter estimates. This problem would be characterized by larger than expected differences between observed and simulated hydraulic heads. 


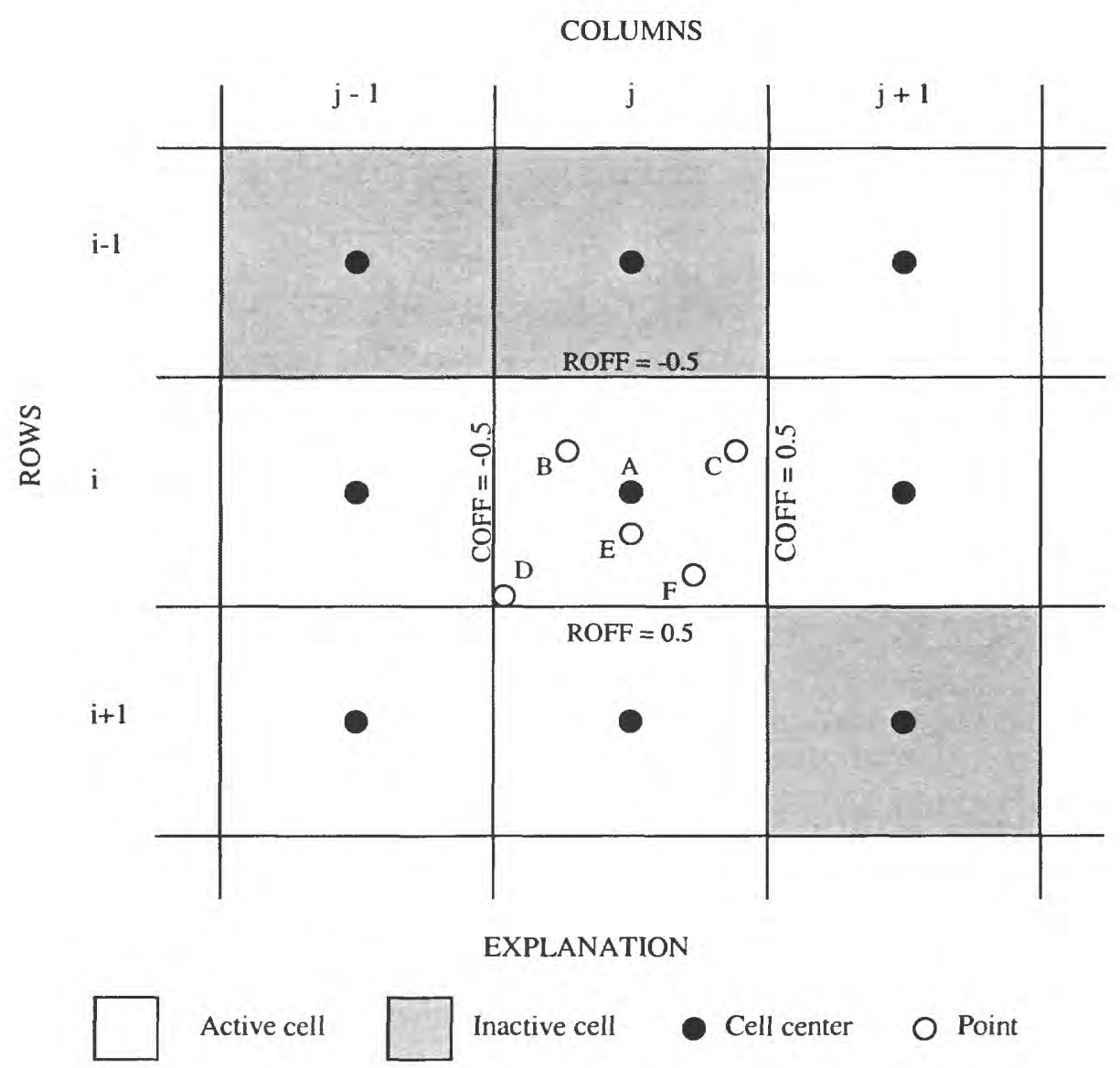

\begin{tabular}{|c|c|c|}
\hline POINT & ROFF & COFF \\
\hline A (Cell center) & 0.0 & 0.0 \\
\hline B & -0.25 & -0.25 \\
\hline C & -0.2 & 0.4 \\
\hline D & 0.45 & -0.45 \\
\hline E & 0.25 & 0.0 \\
\hline F & 0.4 & 0.25 \\
\hline
\end{tabular}

Figure 2: Locating points within a finite-difference cell using ROFF and COFF.

To account for observation wells with screened or open intervals that do not correspond with a model layer, interpolation between hydraulic heads simulated in different model layers is needed. For this situation, the multilayer capability described below can be used to define vertical interpolation.

\section{Temporal Changes in Hydraulic Heads}

In many circumstances in ground-water problems, it is more effective to match changes in hydraulic head over time than to match the hydraulic heads themselves. The classic situation is matching drawdown caused by pumpage, but it is useful in other situations as well. In the 
MODFLOW-2000 Observation Process, the temporal change is calculated as a specified hydraulic head minus the first hydraulic head specified for that location. The first hydraulic head at a location is included as a hydraulic head in the regression. The advantage of matching temporal changes in hydraulic head is that errors that are constant in time, such as the well elevation, are expunged. Hydraulic heads are interpolated spatially as in figure 2 before subtraction.

\section{Multilayer Hydraulic Heads}

If an observation well is screened over intervals that represent more than one model layer, and the observed hydraulic head or change in hydraulic head is affected by all screened intervals, then the associated simulated value is a weighted average of the hydraulic heads or changes in hydraulic head calculated for each of the layers involved. The simulated value is calculated by multiplying the hydraulic head or change in hydraulic head in each layer by a userspecified proportion and then summing the results, as shown in figure 3 . The proportions generally are assigned using the thickness screened within each layer and the local hydraulic properties. A more realistic representation of this problem would be produced by calculating the proportions that are based on the flow-system and hydraulic properties, but the Hydraulic Head Observation part of the Observation Process currently does not support this approach.

Interpolation for multilayer hydraulic heads can be complicated because neighboring cells needed for the interpolation can be active or inactive, depending on the layer. In general, this means that the coefficients used for interpolation would be different for different layers, but the Observation Process does not support this option. In the Observation Process, the interpolation is defined using the IBOUND array (McDonald and Harbaugh, 1988, p. 4-2) of the first layer listed for the multilayer hydraulic-head observation (see item 4 under Input Instructions). Thus, for each neighboring cell that is inactive in any of the other model layers, the cell in the same row and column in the first layer listed needs to be inactive. If no one layer contains a complete set of inactive cells, correct interpolation cannot be accomplished. This is illustrated in figure 4. 
Chapter 4. OBSERVATION PROCESS

\begin{tabular}{l|l|l} 
Ground surface & \\
\hline Model layer 1 & & \\
\hline Model layer 2 & & \multicolumn{2}{|c}{$\mathrm{h}_{2}$} \\
\hline Model layer 3 & $\mathrm{h}_{3}$ \\
\hline Model layer 4 & & $\mathrm{h}_{4}$ \\
\hline
\end{tabular}

$$
y^{\prime}=p_{2} h_{2}+p_{3} h_{3}+p_{4} h_{4}
$$

$y^{\prime}$ is the simulated equivalent of an observed hydraulic head in the well.

$h_{2}, h_{3}$, and $h_{4}$ are calculated heads at the observation location in layers 2,3 , and 4 .

$\mathrm{p}_{1}, \mathrm{p}_{2}$, and $\mathrm{p}_{3}$ are proportions defined by the user.

The proportions need to be positive numbers and need to sum to 1.0 for each well.

Figure 3: Calculating the simulated value of hydraulic head for a multilayer observation well. 
(A)

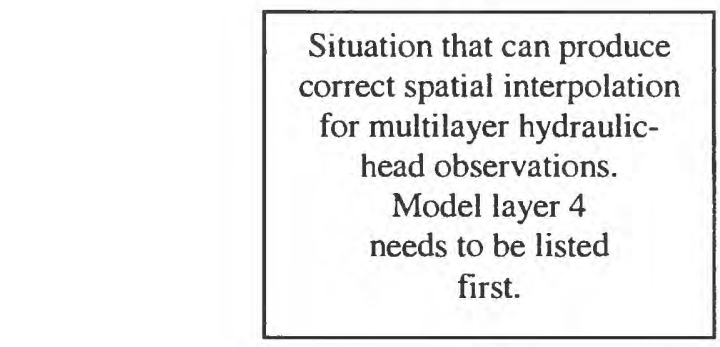

Model layer 2

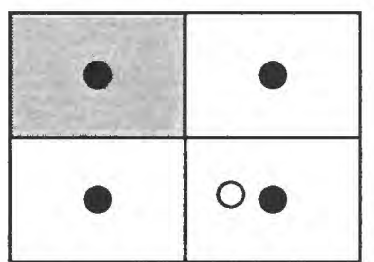

Model layer 3

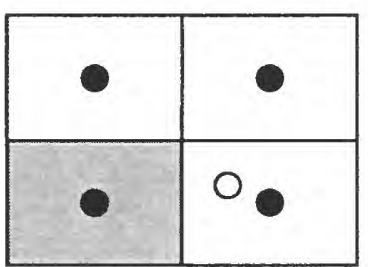

Model layer 4

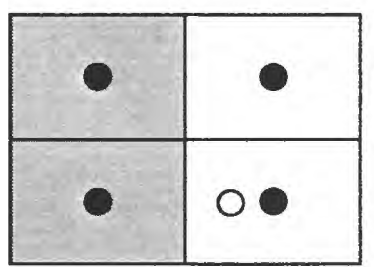

(B)

Situation that can NOT produce correct spatial interpolation for multilayer hydraulic-head observations.

No layer has inactive cells that correspond to the inactive cells in all other layers.
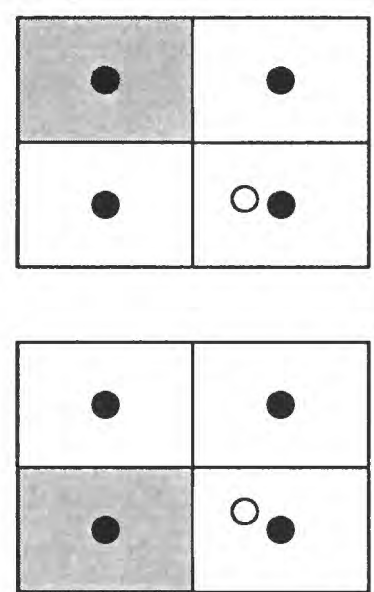

Active cell

Inactive cell

- Cell center

O Observation well

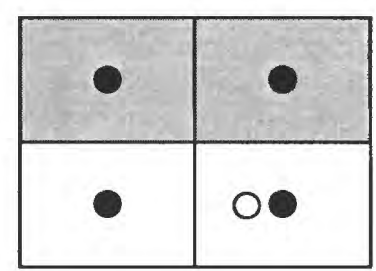

Figure 4: Situations for which the Observation Process (A) can and (B) cannot produce correct spatial interpolation for the multilayer hydraulic-head observation shown in figure 3.

\section{Effect of Dry Cells}

Problems are more severe when cells go dry at or adjacent to hydraulic-head observation locations; the three problem situations are shown in figure 5 and described in the following text. 


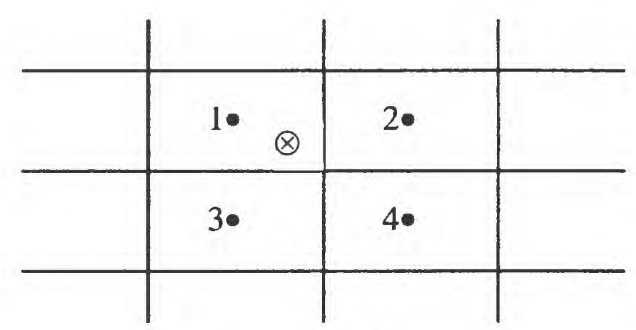

EXPLANATION

$\otimes$ Observation location

- Finite-difference cell center

\begin{tabular}{|c|c|c|}
\hline $\begin{array}{c}\text { Layers of } \\
\text { observation }\end{array}$ & Dry cells & Consequence \\
\hline Single layer & 2,3 , and(or) 4 & Recalculate interpolation \\
\hline Multilayer & 2,3, and(or) 4 & Omit observation \\
\hline Either & 1 & Omit observation \\
\hline
\end{tabular}

Figure 5: Effect of dry cells on interpolation of heads at a hydraulic-head observation location.

First, if the observation is single layer and an adjacent cell that is used in the interpolation goes dry, then the dry cell usually can be omitted from the interpolation without introducing too much error into the interpolated value. This procedure was adopted in the Observation Process.

Second, if the observation is multilayer and cells used for interpolation in one or more layers go dry, then the proportions used to weight the hydraulic heads from those layers probably are no longer valid. Although the cells could be omitted from the interpolation for the layers involved and a simulated hydraulic head analogous to the observed value could be calculated, the problem with the proportions cannot easily be resolved. In the Observation Process, multilayer observations are omitted from the objective function if any cells used in the interpolation go dry.

Third, if the observation is single layer or multilayer and the cell containing the observation location goes dry in any of the layers involved, then the observation is omitted from the parameter-estimation procedure.

In addition, if the observation occurs within a time step, as described in the earlier section "Observation Times", it is omitted if any cell involved in the interpolation is dry for either time step involved; if temporal changes in hydraulic head are used in the regression and the interpolation changes for any of the heads involved, the observation also is omitted. The effect of omitting the observations for the last two situations is that the impetus for changing the parameters to keep the dry areas wet is lost from the parameter-estimation procedure. This loss is unfortunate, but, at this point, no practical alternative exists.

Any cells that go dry are reactivated at the beginning of each parameter-estimation iteration, and the original interpolation and number of observations are reinstated. 


\section{Calculation of Observation Sensitivities}

For hydraulic-head observations, the sensitivities of simulated equivalents to the observations are calculated from the grid sensitivities produced by the Sensitivity Process. The calculations are the same as those described above except that the observation sensitivity replaces the simulated equivalent, and grid sensitivities from the Sensitivity Process replace the hydraulic heads from the Ground-Water Flow Process. As mentioned previously, observation sensitivities are used in sensitivity analysis and regression as discussed by Hill (1998, p. 14-16, 38-42, 58).

\section{Input Instructions}

Input for the Head-Observation Package is read from a file that is specified with "HOB" as the file type in the name file (table 2).

0 . [\#Text]

Item 0 is optional and can include as many lines as desired. Each line needs to begin with the "\#" character in the first column.

1. NH MOBS MAXM (free format)

2. TOMULTH EVH (free format)

Read sufficient repetitions of item 3 and, optionally, items 4 through 6 to obtain NH head or change-in-head observations.

3. OBSNAM LAYER ROW COLUMN IREFSP TOFFSET ROFF COFF HOBS STATISTIC STAT-FLAG PLOT-SYMBOL (free format)

If LAYER is less than zero, hydraulic heads from multiple layers are combined to calculate a simulated value. The number of layers equals the absolute value of LAYER, or |LAYER|. Sufficient repetitions of item 4 are read to define the contributions from each layer. The order of the layers needs to be specified according to the method presented in figure 4.

4. $\operatorname{MLAY}(1), \operatorname{PR}(1), \operatorname{MLAY}(2), \operatorname{PR}(2), \ldots, \operatorname{MLAY}(|\operatorname{LAYER}|)$, $\mathrm{PR}$ (|LAYER|) (free format)

If IREFSP in item 3 is less than zero, read item 5.

5. ITT (free format)

If IREFSP in item 3 is less than zero, read item 6 for each of |IREFSP| observation times

6. OBSNAM IREFSP TOFFSET HOBS STATh STATdd STAT-FLAG PLOTSYMBOL (free format)

\section{Explanation of Variables}

Text-is a character string (maximum of 79 characters) that starts in column 2 . Any characters can be included in Text. The "\#" character needs to be in column 1. Text is printed when the file is read and provides an opportunity for the user to include information about the model both in the input file and the associated output file.

$\mathrm{NH}$ - is the number of head (or change in head) observations.

MOBS - is the number of the NH observations that are multilayer.

MAXM-is the maximum number of layers used for any of the MOBS observations. 
TOMULTH - is the time-offset multiplier for head observations [-- or T/T]. The product of TOMULTH and TOFFSET must produce a time value in units consistent with other model input. TOMULTH can be dimensionless or can be used to convert the units of TOFFSET to the time unit used in the simulation.

$\mathrm{EVH}$ - is the input error variance multiplier for hydraulic-head observations and is used to calculate the weights as described below in the calculation of STATISTIC. EVH makes it easy to change the weights uniformly for all hydraulic-head observations.

OBSNAM - is a string of 1 to 12 nonblank characters used to identify the observation. The identifier need not be unique; however, identification of observations in the output files is facilitated if each observation is given a unique OBSNAM.

LAYER - is the layer index of the cell in which the head observation is located. If LAYER is less than zero, hydraulic heads from multiple layers are combined to calculate a simulated value. The number of layers equals the absolute value of LAYER, or |LAYER|.

ROW - is the row index of the cell in which the head observation is located.

COLUMN - is the column index of the cell in which the head observation is located.

IREFSP - is the stress period to which the observation time is referenced. The reference point is the beginning of the specified stress period. If the value of IREFSP read in item 3 is negative, there are observations at |IREFSP| times -- item 5 is read and |IREFSP| repetitions of item 6 are read. Also, if IREFSP is negative, values of OBSNAM, HOBS, and STATISTIC read in item 3 are ignored and values read in item 6 are used.

TOFFSET - is the time from the beginning of stress period IREFSP to the time of the observation [T]. TOFFSET must be in units such that the product of TOMULTH (in item 2 above) and TOFFSET is in time units consistent with other model input. TOFFSET and TOMULTH from the HOB file and values of PERLEN, NSTP, and TSMULT from the Discretization file (Harbaugh and others, 2000) are used to determine the stress period, time step, and time during the time step for the observation. To specify that an observation is for a steady-state model solution, specify IREFSP as the stress-period number of the steady-state stress period, and specify TOFFSET such that the product TOMULTH $\times$ TOFFSET is less than or equal to PERLEN for the stress period; if PERLEN is zero, set TOFFSET to zero. If the observation falls within a time step, the simulated equivalent is calculated by linearly interpolating between heads at the beginning and end of the time step. If the first stress period is transient and the observation falls within the first time step of the stress period, the head from the beginning of the time step is determined by using the initial head distribution specified in the Basic Package input file.

ROFF-is the row offset used to locate the observation within a finite-difference cell (fig. 2).

COFF - is the column offset used to locate the observation within a finite-difference cell (fig. 2).

HOBS-is the observed hydraulic head [L]. In item 6, this needs to be hydraulic head even when ITT $=2$ in item 5; the program will perform the required subtraction.

STATISTIC - is the value from which the observation weight is calculated as determined using STAT-FLAG.

STAT-FLAG-is a flag identifying what STATISTIC is and how the observation weight is calculated.

STAT-FLAG $=0$, STATISTIC is a scaled variance $\left[\mathrm{L}^{2}\right]$, weight $=1 /($ STATISTIC $\times$ EVH),

STAT-FLAG $=1$, STATISTIC is a scaled standard deviation $[\mathrm{L}]$, weight $=$ $1 /\left(\right.$ STATISTIC $^{2} \times$ EVH $)$, and $^{2}$ 
STAT-FLAG $=2$, STATISTIC is a scaled coefficient of variation [--], weight $=$ $1 /\left[(\text { STATISTIC } \times \text { HOBS })^{2} \times\right.$ EVH $]$.

PLOT-SYMBOL_is an integer that is written to output files intended for graphical analysis to allow control of the symbols used to plot data.

MLAY(I)-is the $\mathrm{I}^{\text {th }}$ layer number for a multilayer head observation.

PR(I) - is the proportion of the simulated hydraulic head in layer MLAY(I) that is used to calculate simulated multilayer head. The sum of all PR values for a given observation needs to equal 1.0 .

ITT - is a flag that identifies whether head or changes in head are to be used as observations.

ITT $=1$ : The observed hydraulic heads are used as observations.

ITT $=2$ : The initial observed hydraulic head and subsequent changes in head (for example, drawdown) are used as observations. Changes in head are calculated internally from the hydraulic-head values listed in item 6 , so the HOBS values specified in item 6 need to be hydraulic heads.

STATh-is the value from which the weight is calculated if the observation is hydraulic head. STAT-FLAG is used to identify what STATh is and how the weight is calculated, as for STATISTIC.

STATdd-is the value from which the weight is calculated if the observation is the temporal change in hydraulic head. STAT-FLAG is used to identify what STATdd is and how the weight is calculated, as for STATISTIC. 


\section{Flow Observations at Boundaries Represented as Head Dependent}

Flow observations often are related to surface-water bodies such as streams and lakes. The physics of such flows often are best represented using one of the three head-dependent boundary packages of MODFLOW-2000, the General-Head Boundary, Drain, or River Package. For the three packages, figure 6 depicts how the ground-water/surface-water interaction is conceptualized, and shows all of the variables that may be included in the calculations. Not all the variables shown are used in all the packages mentioned. For all packages, the variables $K_{n}, A_{n}$, and $\mathrm{D}_{\mathrm{n}}$ are combined to form conductance terms, and these are specified in the package input file. Details of the calculations are presented below.

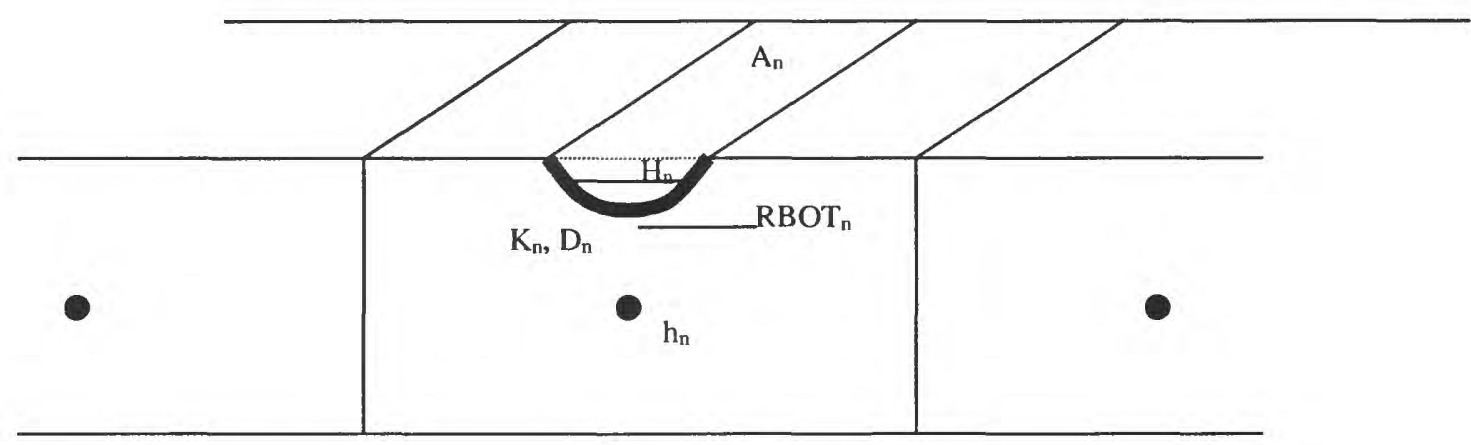

EXPLANATION

$A_{n} \quad$ Area of the water-body within finite difference cell $n$

$D_{n} \quad$ Thickness of the water-body bed within finitedifference cell $n$

$\mathrm{K}_{\mathrm{n}} \quad$ Hydraulic conductivity of the water-body bed within finite-difference cell $n$

$h_{n} \quad$ Calculated hydraulic head for finite-difference cell $n$
$\mathrm{H}_{\mathrm{n}} \quad$ Water level in the water body within finite-difference cell $\mathrm{n}$, or, for the Drain Package, the elevation of the drain

$\mathrm{RBOT}_{\mathrm{n}}$ Elevation of the bottom of the waterbody bed

Finite-difference cell center

Figure 6: Diagram depicting the quantities used to calculate flow between the ground-water system and a surface-water body.

\section{Basic Head-Dependent Flow Calculations}

In many circumstances, flow between a single finite-difference cell representing the ground-water system and the surface-water body (such as a lake or stream) is calculated as:

$$
q_{n}=C_{n}\left(H_{n}-h_{n}\right)=\frac{K_{n} A_{n}}{D_{n}}\left(H_{n}-h_{n}\right)
$$

where, 
$\mathrm{q}_{\mathrm{n}} \quad$ is the simulated flow rate at one cell $\left(\mathrm{L}^{3} / \mathrm{T}\right)$ (negative for flow out of the groundwater system);

$\mathrm{C}_{n} \quad$ is the conductance of the material separating the surface-water body from the ground-water system and is defined as $K_{n} A_{n} / D_{n}\left(L^{2} / T\right)$;

$\mathrm{K}_{\mathrm{n}} \quad$ is the hydraulic conductivity $(\mathrm{L} / \mathrm{T})$ of, for example, the riverbed or lakebed;

$\mathrm{D}_{\mathrm{n}} \quad$ is the thickness $(\mathrm{L})$ of the water-body bed within the finite-difference cell;

$A_{n} \quad$ is the area of the water body within the finite-difference cell $\left(L^{2}\right)$;

$h_{n} \quad$ is the calculated hydraulic head for finite-difference cell $n(L)$; and

$\mathrm{H}_{\mathrm{n}}$ is the water level in the water body within finite-difference cell $\mathrm{n}$, or, for the Drain Package, the elevation of the drain $(\mathrm{L})$.

A flow observation commonly is represented by a group of cells, as in figure 7. Summing over nqcl cells, the simulated equivalent to the observation equals:

$$
y^{\prime}=\sum_{n=1}^{n g c l} f_{n} q_{n}
$$

where,

$y^{\prime} \quad$ is the simulated equivalent to a measured gain or loss,

$\mathrm{f}_{\mathrm{n}} \quad$ is a user-defined multiplicative factor, and

nqcl is the number of wells in the group.

Generally $f_{n}=1.0$. However, using figure 7 as an example, if gaging sites for $Q_{1}$ or $Q_{2}$ or both are located within a cell instead of at the edges, $\mathrm{f}_{\mathrm{n}}$ needs to be less than 1.0 so that only part of the simulated flow for the cell is included in $\mathrm{y}^{\prime}$. 


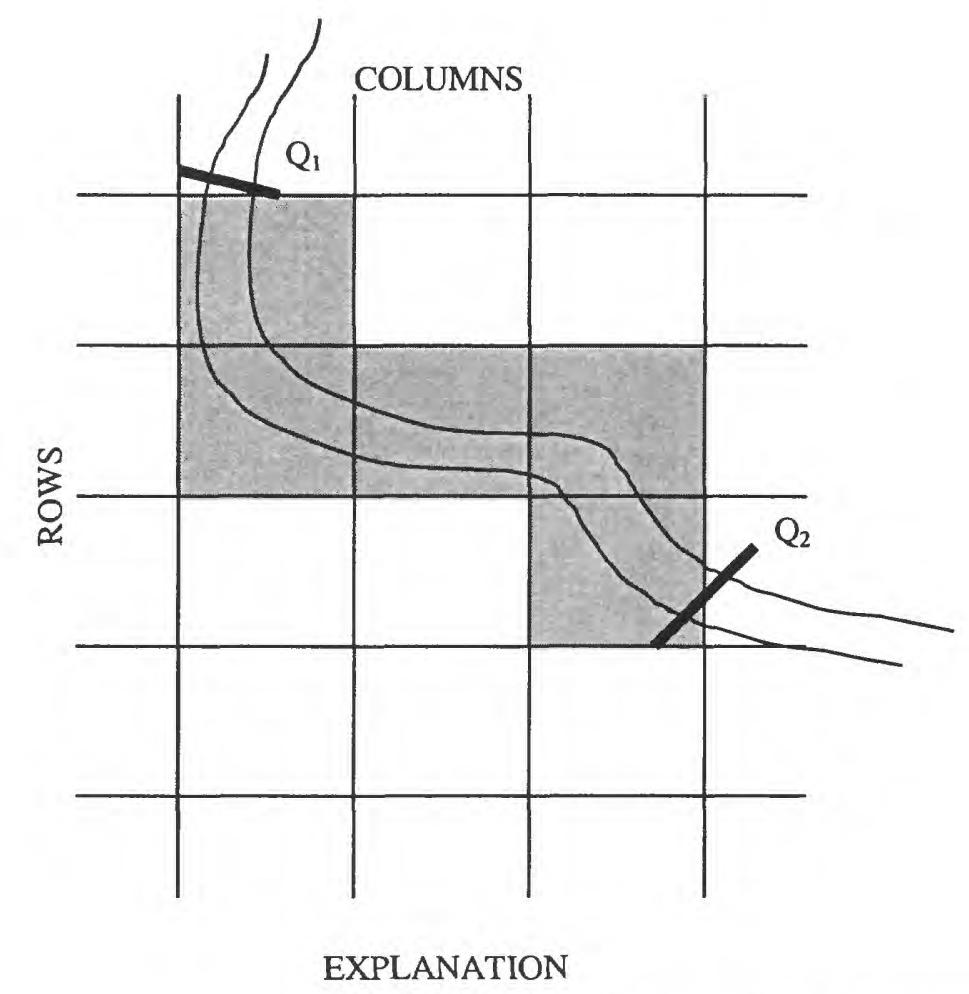

Finite-difference cell

Finite-difference cell used to represent the reach between $\mathrm{Q}_{1}$ and $\mathrm{Q}_{2}$ in the model

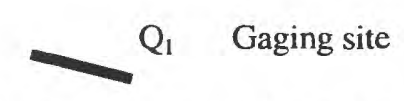

Figure 7: Representation of head-dependent boundary gain or loss observations between two gaging stations, showing the finite-difference cells used to represent the appropriate reach.

Substituting equation 8 into equation 9 makes each term of the sum $f_{n} q_{n}=f_{n} C_{n}\left(H_{n}-h_{n}\right)$. The simulated hydraulic head $h_{n}$ generally is a function of all of the parameters. $C_{n}$ is a function of any parameters used to calculate $C_{n}$. In MODFLOW-2000, the terms $f_{n}$ and $H_{n}$ cannot be defined using parameters; calculating sensitivities for or estimating these terms requires using UCODE or PEST. Taking the derivative with respect to parameter $b_{\ell}$ of equation 9 after substituting equation 8 yields

$$
\frac{\partial \mathrm{y}^{\prime}}{\partial \mathrm{b}_{\ell}}=\sum_{\mathrm{n}=1}^{\mathrm{nc}} \mathrm{f}_{\mathrm{n}} \frac{\partial \mathrm{q}_{\mathrm{n}}}{\partial \mathrm{b}_{\ell}}=\sum_{\mathrm{n}=1}^{\mathrm{nc}} \mathrm{f}_{\mathrm{n}}\left(-\mathrm{C}_{\mathrm{n}} \frac{\partial \mathrm{h}_{\mathrm{n}}}{\partial \mathrm{b}_{\ell}}+\frac{\partial \mathrm{C}_{\mathrm{n}}}{\partial \mathrm{b}_{\ell}}\left(\mathrm{H}_{\mathrm{n}}-\mathrm{h}_{\mathrm{n}}\right)\right) .
$$

The derivative $\partial h_{n} / \partial b_{\ell}$ is calculated by the Sensitivity Process and is available to the Observation Process; all other terms are calculated within the Observation Process. Equation 10 is the basic equation used to calculate observation sensitivities for all head-dependent flow observations. Exceptions occur, however, for all packages except the GHB Package, as described below. 


\section{Modifications to the Basic Head-Dependent Flow Calculations}

The Ground-Water Flow Process capabilities of the three MODFLOW-2000 packages used to simulate head-dependent boundaries are documented in McDonald and Harbaugh (1988) and Harbaugh and others (2000) and are described briefly in table 7. The relation between calculated flow and calculated hydraulic head in the ground-water system for each package is shown in figure 8. 
Table 7: Packages available for representing flow observations as head-dependent boundaries [All are documented in McDonald and Harbaugh (1988) and Harbaugh and others (2000)]

\begin{tabular}{|c|c|}
\hline Package Name & Basic Features \\
\hline $\begin{array}{l}\text { General-Head } \\
\text { Boundary }\end{array}$ & $\begin{array}{c}\text { Flow at each cell is calculated using equation } 8 \text { for all values of the simulated } \\
\text { hydraulic head. }\end{array}$ \\
\hline Drain & $\begin{array}{l}\text { Operates same as the General-Head Boundary Package except that the flow } \\
\text { equals zero if the simulated hydraulic head is less than the reference hydraulic } \\
\text { head, } \mathrm{H}_{n} \text { of equation } 8 \text { and figure } 6 \text {. }\end{array}$ \\
\hline River & $\begin{array}{c}\text { Operates same as the General-Head Boundary Package except that the flow is } \\
\text { constant for all values of the simulated hydraulic head that are lower than the } \\
\text { bottom of the water body bed, RBOT of figure } 6 \text {. }\end{array}$ \\
\hline
\end{tabular}

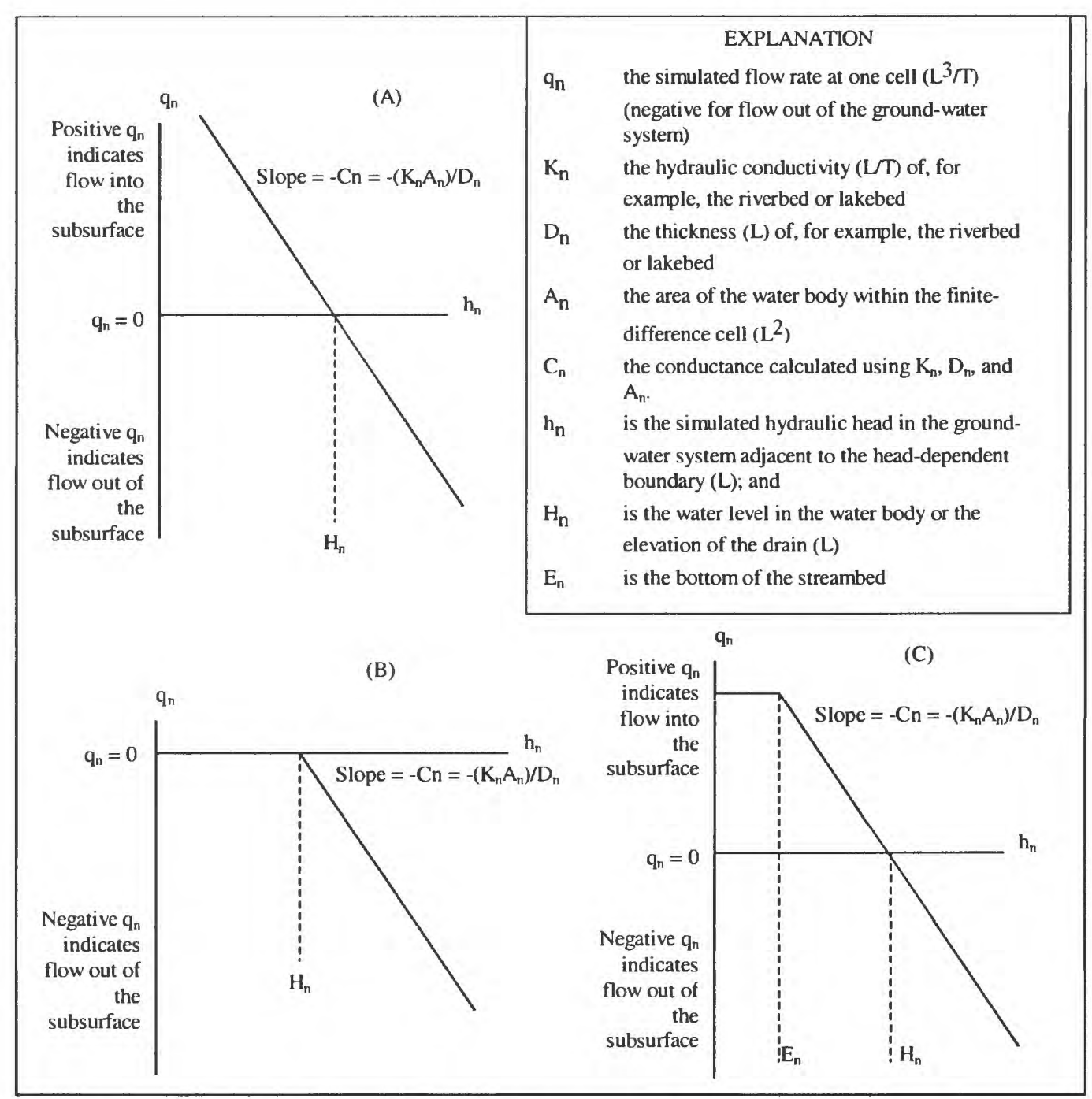

Figure 8: The dependence of simulated gains and losses on hydraulic head in the model layer $\left(\mathrm{h}_{\mathrm{n}}\right)$ in: (A) the General-Head Boundary Package, (B) the Drain Package, and (C) the River Package. 
Flows calculated as shown in equation 8 produce the calculated sensitivities of equation 10. The exceptions shown in figure 8 , listed in table 7 , and described in more detail in the following sections, all have the effect of removing $h_{n}$ from the calculation of flow, and removing $\partial h_{n} / \partial b_{\ell}$ from the calculation of sensitivity. For example, in the River Package if $h_{n}$ falls beneath $H_{n}$ , $\mathrm{q}_{\mathrm{n}}=\mathrm{C}_{\mathrm{n}}\left(\mathrm{H}_{\mathrm{n}}-\mathrm{RBOT}\right)$ and $\partial \mathrm{q}_{\mathrm{n}} / \partial \mathrm{b}_{\ell}=\left(\partial \mathrm{C}_{\mathrm{n}} / \partial \mathrm{b}_{\ell}\right)\left(\mathrm{H}_{\mathrm{n}}-\mathrm{RBOT}_{\mathrm{n}}\right)$; in the Drain Package if $h_{n}$ falls beneath $\mathrm{H}_{\mathrm{n}}, \mathrm{q}_{\mathrm{n}}=0.0$ and $\partial \mathrm{q}_{\mathrm{n}} / \partial \mathrm{b}_{\ell}=0.0$. For all parameters except, sometimes, for those used to calculate $\mathrm{C}_{n}$, this produces a zero contribution from the cell to the observation sensitivity, thereby diminishing the effect of the flow on parameter estimation.

Thus, the restrictions shown in table 7 all result in the simulated flow between the ground-water and the stream being controlled more by factors other than the estimated parameters. The importance of the parameters to the simulated equivalent value is thus diminished, and the measured flow becomes less useful in their estimation. If the problem can be posed to diminish such external factors, the flow is more useful to the regression. Posing the problem with this in mind can save much time and frustration if the outside factors are likely to dominate for some sets of parameter values, but probably not by parameter values that represent the system accurately.

For example, if springs are present, use of the Drain Package might be suggested because, as in reality, the simulated spring flow will be zero if the simulated hydraulic head in the ground-water system is too low. If, however, the spring is flowing under calibration conditions, using the Drain Package means that the spring-flow observation will be eliminated from the regression if the simulated water level is too low for some set of parameter values, thus removing any motivation for the regression to change parameter values such that that spring will again flow. Alternatively, using the General-Head Boundary Package will keep the spring in the regression for all parameter values, constantly exerting influence on the regression to match the observed spring flow.

Given the effects of the exceptions on sensitivities, often the best package to use, at least in early regression runs, is the General-Head Boundary Package. Once optimal or near-optimal parameter values are found, other packages then can be used. The most advantageous approach for any given situation, however, depends on the circumstances involved, and needs careful consideration. 


\section{General-Head Boundary Package}

The Ground-Water Flow Process capabilities of the General-Head Boundary Package are documented in McDonald and Harbaugh (1988) and Harbaugh and others (2000).

\section{Calculation of Simulated Equivalents to the Observations}

The General-Head Boundary Package uses equations 8 and 9 to calculate flows that are simulated equivalents to the observations.

\section{Calculation of Observation Sensitivities}

Observation sensitivities for flows represented using the General-Head Boundary Package are calculated as shown in equation 10. As discussed above in the section "Modifications to the Basic Head-Dependent Flow Calculations", these sensitivities have the advantage of always depending on the simulated hydraulic head. When estimating parameters by nonlinear regression, it is important to maintain the dependence on hydraulic head, if possible. To avoid the exceptions of table 7, it is often advisable to use the General-Head Boundary Package to represent flow observations during at least the first regression runs even if one of the other packages is used in the final model.

As mentioned previously, observation sensitivities are used in sensitivity analysis and regression as discussed by Hill (1998, p. p. 14-16, 38-42, 58).

\section{Input Instructions}

Input for the General-Head-Boundary Observation Package is read from a file that is specified with "GBOB" as the file type listed in the name file (table 2).

0. [\#Text]

Item 0 is optional and can include as many lines as desired. Each line needs to begin with the "\#" character in the first column.

1. NQGB NQCGB NQTGB (free format)

2. TOMULTGB EVFGB IOWTQGB (free format)

Read items 3, 4, and 5 for each of NQGB groups of cells for which general-headboundary observations are to be specified.

3. NQOBGB NQCLGB (free format)

Read item 4 for each of NQOBGB observation times for this group of cells. STATISTIC and STAT-FLAG are ignored if IOWTQGB is greater than zero.

4. OBSNAM IREFSP TOFFSET HOBS STATISTIC STAT-FLAG PLOT-SYMBOL (free format)

Read item 5 for each of $\mid$ NQCLGB $\mid$ cells in this group.

5. LAYER ROW COLUMN FACTOR (free format)

Read items 6 and 7 if IOWTQGB is greater than 0.

6. FMTIN IPRN (free format)

7. WTQ $(1,1), \mathrm{WTQ}(1,2), \mathrm{WTQ}(1,3), \ldots, \mathrm{WTQ}(1, \mathrm{NQTGB})$ (format: FMTIN) $\operatorname{WTQ}(2,1)$, WTQ $(2,2), \operatorname{WTQ}(2,3), \ldots . \operatorname{WTQ}(2$, NQTGB $)$ 
$\cdots$

WTQ (NQTGB , 1) , WTQ (NQTGB , 2) , WTQ (NQTGB, 3), . . , WTQ (NQTGB, NQTGB)

\section{Explanation of Variables}

Text-is a character string (maximum of 79 characters) that starts in column 2 . Any characters can be included in Text. The "\#” character needs to be in column 1. Text is printed when the file is read and provides an opportunity for the user to include information about the model both in the input file and the associated output file.

NQGB - is the number of cell groups for which general-head-boundary observations are listed. A group consists of the cells needed to represent one flow measurement (eq. 9).

NQCGB - is greater than or equal to the total number of cells in all cell groups. NQCGB must be greater than or equal to the sum of all |NQCLGB|.

NQTGB - is the total number of general-head-boundary observations for all cell groups. NQTGB must equal the sum of all NQOBGB, which are specified in repetitions of item 3 in the input file.

TOMULTGB -is the time-offset multiplier for general-head-boundary observations [-- or T/T]. The product of TOMULTGB and TOFFSET must produce a time value in units consistent with other model input. TOMULTGB can be dimensionless or can be used to convert the units of TOFFSET to the time unit used in the simulation.

EVFGB - is the error variance multiplier for observations represented by the General-Head Boundary Package and is used to calculate the weights as described below in the explanation of STATISTIC. EVFGB makes it easy to change the weights uniformly for all flow observations represented using the General-Head Boundary Package.

IOWTQGB - is a flag that indicates that the variance-covariance matrix on general-headboundary observations is to be read into array WTQ of item 7. If IOWTQGB equals zero, weights are calculated using STATISTIC of item 4; if it is greater than zero, items 6 and 7 are read and used to calculate the weights.

NQOBGB - is the number of times at which flows are observed for the group of cells.

NQCLGB - is a flag, and the absolute value of NQCLGB is the number of cells in the group. If NQCLGB is less than zero, FACTOR $=1.0$ for all cells in the group.

OBSNAM - is a string of 1 to 12 nonblank characters used to identify the observation.

IREFSP - is the reference stress period to which the observation time is referenced. The reference point is the beginning of the stress period. 
TOFFSET - is the time from the beginning of stress period IREFSP to the time of the observation [T]. TOFFSET must be in units such that the product of TOMULTGB and TOFFSET is in time units consistent with other model input. TOFFSET and TOMULTGB from the GBOB file and values of PERLEN, NSTP, and TSMULT from the Discretization file (Harbaugh and others, 2000) are used to determine the stress period, time step, and time during the time step for the observation. To specify that an observation is for a steadystate model solution, specify IREFSP as the stress-period number of the steady-state stress period, and specify TOFFSET such that the product TOMULTGB $\times$ TOFFSET is less than or equal to PERLEN for the stress period; if PERLEN is zero, set TOFFSET to zero. If the observation falls within a time step, the simulated equivalent is calculated by linearly interpolating between values for the beginning and end of the time step. If the first stress period is transient and the observation falls within the first time step, the simulated equivalent from the end of the time step is used because no flow from the beginning of the time step is available for interpolation.

HOBS-is the observed general-head-boundary gain (if HOBS is negative) or loss (if HOBS is positive) $\left[\mathrm{L}^{3} / \mathrm{T}\right]$. The terms "gain" and "loss" are from the perspective of the surfacewater body, so that gains occur when water leaves the ground-water system, and losses occur when water flows into the ground-water system.

STATISTIC - is the value from which the weight for the observation is calculated as determined using STAT-FLAG. STATISTIC is ignored if IOWTQGB is greater than zero, in which case WTQ of item 7 is used to define the weighting.

STAT-FLAG-is a flag identifying what STATISTIC is and how the weight is calculated. STAT-FLAG is ignored if IOWTQGB is greater than zero.

STAT-FLAG $=0$, STATISTIC is a scaled variance $\left[\left(\mathrm{L}^{3} / \mathrm{T}\right)^{2}\right]$, weight $=1 /($ STATISTIC $\times$ EVFGB),

STAT-FLAG $=1$, STATISTIC is a scaled standard deviation $\left[\mathrm{L}^{3} / \mathrm{T}\right]$, weight $=$ $1 /\left(\right.$ STATISTIC $^{2} \times$ EVFGB $)$, and $^{2}$

STAT-FLAG $=2$, STATISTIC is a scaled coefficient of variation [--], weight $=$ $1 /\left[(\text { STATISTIC } \times \text { HOBS })^{2} \times\right.$ EVFGB].

PLOT-SYMBOL-is an integer that will be written to output files intended for graphical analysis to allow control of the symbols used when plotting data.

LAYER - is the layer index of a general-head-boundary cell included in the cell group.

ROW-is the row index of a general-head-boundary cell included in the cell group.

COLUMN-is the column index of a general-head-boundary cell included in the cell group.

FACTOR - is the portion of the simulated gain or loss in the cell that is included in the total simulated gain or loss for this cell group ( $f_{n}$ of eq. 9 ).

FMTIN - is the Fortran format to be used in reading each line of the variance-covariance matrix used to calculate the weighting. The format needs to be enclosed in parentheses and needs to accommodate real numbers. 
IPRN - is a flag identifying the format with which the variance-covariance matrix is printed. If IPRN is less than zero, the matrix is not printed.

Permissible values of IPRN and corresponding formats are:

\begin{tabular}{|c|c|c|c|}
\hline \multicolumn{2}{|c|}{ Output requires more than 80 columns } & \multicolumn{2}{|c|}{ Output fits in 80 columns } \\
\hline IPRN & FORMAT & IPRN & FORMAT \\
\hline 1 & $10 \mathrm{G} 12.3$ & 6 & $5 \mathrm{G} 12.3$ \\
\hline 2 & $10 \mathrm{G} 12.4$ & 7 & $5 \mathrm{G} 12.4$ \\
\hline 3 & $9 \mathrm{G} 12.5$ & 8 & $5 \mathrm{G} 12.5$ \\
\hline 4 & $8 \mathrm{G} 13.6$ & 9 & $4 \mathrm{G} 13.6$ \\
\hline 5 & $8 \mathrm{GI} 14.7$ & 10 & $4 \mathrm{G} 14.7$ \\
\hline
\end{tabular}

WTQ-is an NQTGB by NQTGB array containing the variance-covariance matrix on generalhead-boundary flow observations $\left[\left(\mathrm{L}^{3} / \mathrm{T}\right)^{2}\right]$. For elements WTQ(I,J), if $I \neq J, W T Q(I, J)$ is the covariance between observations I and $\mathrm{J}$; if $\mathrm{I}=\mathrm{J}$, WTQ $(\mathrm{I}, \mathrm{J})$ is the variance of observation I. Note that the variance-covariance matrix is symmetric, but the entire matrix (upper and lower parts) must be entered. 


\section{Drain Package}

The Ground-Water Flow Process capabilities of the Drain Package are documented in McDonald and Harbaugh (1988) and Harbaugh and others (2000).

\section{Calculation of Simulated Equivalents to the Observations}

In the Drain Package, flow at each finite-difference cell specified is calculated as in equation 8 except for cells in which the simulated hydraulic head $\left(h_{n}\right)$ falls below $H_{n}$ of figure 6 . For these cells the flow is set to zero, so that the Drain Package never allows flow into the ground-water system. The relation between flow and hydraulic heads is as depicted in figure $8 \mathrm{C}$. Mathematically, for finite-difference cell $\mathrm{n}$, this is expressed as:
$\mathrm{q}_{\mathrm{n}}=\mathrm{C}_{\mathrm{n}}\left(\mathrm{h}_{\mathrm{n}}-\mathrm{H}_{\mathrm{n}}\right)$
$h_{n}>H_{n}$
$\mathrm{q}_{\mathrm{n}}=0.0$
$\mathrm{h}_{\mathrm{n}} \leq \mathrm{H}_{\mathrm{n}}$

If a measured gain to the surface-water body is represented using more than one finitedifference cell, the calculation is summed for the cells involved, using equation 9 .

\section{Calculation of Observation Sensitivities}

In the Drain Package, observation sensitivities are calculated as they are for the GeneralHead Boundary Package (using eq. 10) except when $h_{n} \leq H_{n}$, the flow equals zero. In this situation, the observation sensitivity also equals zero because no incremental change in any of the parameter values will change the simulated flow. If all of the cells representing an observation are similarly disconnected, the sensitivity related to the entire observation will be zero, and there will be no motivation for the regression to fit the observation.

As mentioned previously, observation sensitivities are used in sensitivity analysis and regression as discussed by Hill (1998, p. 14-16, 38-42, 58).

\section{Input Instructions}

Input for the Drain Observation Package is read from a file that is specified with "DROB" as the file type listed in the name file (table 2).

\section{0 . [ \#Text]}

Item 0 is optional and can include as many lines as desired. Each line needs to begin with the "\#" character in the first column.

1. NQDR NQCDR NQTDR (free format)

2. TOMULTDR EVFDR IOWTQDR (free format)

Read items 3, 4, and 5 for each of NQDR groups of cells for which drain observations are to be specified. 
3. NQOBDR NQCLDR (free format)

Read item 4 for each of NQOBDR observation times for this group of cells. STATISTIC and STAT-FLAG are ignored if IOWTQDR is greater than zero.

4. OBSNAM IREFSP TOFFSET HOBS STATISTIC STAT-FLAG PLOT-SYMBOL (free format)

Read item 5 for each cell in this group; the number of cells equals the absolute value of NQCLDR from item 3.

5. Layer Row Column Factor (free format)

Read items 6 and 7 if IOWTQDR is greater than 0 .

6. FMTIN IPRN (free format)

7. $\mathrm{WTQ}(1,1), \mathrm{WTQ}(1,2), \mathrm{WTQ}(1,3), \ldots, \operatorname{WTQ}(1, \operatorname{NQTDR})$ (format: FMTIN) $\operatorname{WTQ}(2,1), \operatorname{WTQ}(2,2), \operatorname{WTQ}(2,3), \ldots, \operatorname{WTQ}(2, \operatorname{NQTDR})$

...

WTQ (NQTDR, 1) , WTQ (NQTDR, 2) , WTQ (NQTDR, 3) , . . , WTQ (NQTDR, NQTDR)

\section{Explanation of Variables}

Text-is a character string (maximum of 79 characters) that starts in column 2 . Any characters can be included in Text. The "\#" character needs to be in column 1. Text is printed when the file is read and provides an opportunity for the user to include information about the model both in the input file and the associated output file.

NQDR-is the number of cell groups for which drain observations are listed. A group consists of the cells needed to represent one flow measurement (eq. 9).

NQCDR-is greater than or equal to the total number of cells in all cell groups. NQCDR must be greater than or equal to the sum of all |NQCLDR|.

NQTDR - is the total number of drain observations for all cell groups. NQTDR must equal the sum of all NQOBDR, which are specified in repetitions of item 3 in the input file.

TOMULTDR - is the time-offset multiplier for drain observations [-- or T/T]. The product of TOMULTDR and TOFFSET must produce a time value in units consistent with other model input. TOMULTDR can be dimensionless or can be used to convert the units of TOFFSET to the time unit used in the simulation.

EVFDR - is the error variance multiplier for observations represented by the Drain Package, and is used to calculate the weights as described below in the explanation of STATISTIC.

EVFDR makes it easy to change the weights uniformly for all flow observations represented using the Drain Package.

IOWTQDR - is a flag that indicates that the variance-covariance matrix on drain observations is to be read into array WTQ of item 7. If IOWTQDR equals zero, weights are calculated using STATISTIC of item 4; if it is greater than zero, items 6 and 7 are read and used to calculate the weights.

NQOBDR - is the number of times at which flows are observed for the group of cells.

NQCLDR - is a flag, and the absolute value of NQCLDR is the number of cells in the group. If NQCLDR is less than zero, FACTOR $=1.0$ for all cells in the group.

OBSNAM-is a string of 1 to 12 nonblank characters used to identify the observation.

IREFSP-is the reference stress period to which the observation time is referenced. The reference point is the beginning of the stress period. 
TOFFSET - is the time from the beginning of stress period IREFSP to the time of the observation

[T]. TOFFSET must be in units such that the product of TOMULTDR and TOFFSET is in time units consistent with other model input. TOFFSET and TOMULTDR from the DROB file and values of PERLEN, NSTP, and TSMULT from the Discretization file (Harbaugh and others, 2000) are used to determine the stress period, time step, and time during the time step for the observation. To specify that an observation is for a steadystate model solution, specify IREFSP as the stress-period number of the steady-state stress period, and specify TOFFSET such that TOMULTDR $\times$ TOFFSET is less than or equal to PERLEN for the stress period; if PERLEN is zero, set TOFFSET to zero. If the observation falls within a time step, the simulated equivalent is calculated by linearly interpolating between values for the beginning and end of the time step. If the first stress period is transient and the observation falls within the first time step, the simulated equivalent from the end of the time step is used because no flow from the beginning of the time step is available for interpolation.

HOBS-is the observed drain-boundary flow $\left[\mathrm{L}^{3} / \mathrm{T}\right]$. For the Drain Package only negative values of HOBS are expected. Negative values indicate flow out of the ground-water system.

STATISTIC - is the value from which the weight for the observation is calculated as determined using STAT-FLAG. STATISTIC is ignored if IOWTQDR is greater than zero, in which case WTQ of item 7 is used to define the weighting.

STAT-FLAG-is a flag identifying what STATISTIC is and how the weight is calculated. STAT-FLAG is ignored if IOWTQDR is greater than zero.

STAT-FLAG $=0$, STATISTIC is a scaled variance $\left[\left(\mathrm{L}^{3} / \mathrm{T}\right)^{2}\right]$, weight $=1 /($ STATISTIC $\times$ EVFDR)

STAT-FLAG $=1$, STATISTIC is a scaled standard deviation $\left[\mathrm{L}^{3} / \mathrm{T}\right]$, weight $=$ $1 /\left(\right.$ STATISTIC $^{2} \times$ EVFDR $^{-}$

STAT-FLAG $=2$, STATISTIC is a scaled coefficient of variation [-- $]$, weight $=$ $1 /\left[\left(\right.\right.$ STATISTIC $\left.\times \mathrm{HOBS}^{2} \times \mathrm{EVFDR}\right]$

PLOT-SYMBOL_-is an integer that will be written to output files intended for graphical analysis to allow control of the symbols used when plotting data.

LAYER - is the layer index of a drain cell included in the cell group.

ROW - is the row index of a drain cell included in the cell group.

COLUMN-is the column index of a drain cell included in the cell group.

FACTOR - is the portion of the simulated drain flow in the cell that is included in the total simulated drain flow for this cell group ( $f_{n}$ of eq. 9 ).

FMTIN - is the Fortran format to be used in reading each line of the variance-covariance matrix used to calculate the weighting. The format needs to be enclosed in parentheses and needs to accommodate real numbers. 
IPRN-is a flag identifying the format in which the variance-covariance matrix is printed. If IPRN is less than zero, the matrix is not printed. Permissible values of IPRN and corresponding formats are:

\begin{tabular}{|c|c|c|c|}
\hline \multicolumn{2}{|c|}{ Output requires more than 80 columns } & \multicolumn{2}{|c|}{ Output fits in 80 columns } \\
\hline IPRN & FORMAT & IPRN & FORMAT \\
\hline 1 & $10 \mathrm{G} 12.3$ & 6 & $5 \mathrm{G} 12.3$ \\
\hline 2 & $10 \mathrm{G} 12.4$ & 7 & $5 \mathrm{G} 12.4$ \\
\hline 3 & $9 \mathrm{G} 12.5$ & 8 & $5 \mathrm{G} 12.5$ \\
\hline 4 & $8 \mathrm{G} 13.6$ & 9 & $4 \mathrm{G} 13.6$ \\
\hline 5 & $8 \mathrm{G} 14.7$ & 10 & $4 \mathrm{G} 14.7$ \\
\hline
\end{tabular}

WTQ - is an NQTDR by NQTDR array containing the variance-covariance matrix on drain observations $\left[\left(\mathrm{L}^{3} / \mathrm{T}\right)^{2}\right]$. For elements WTQ $(I, J)$, if $I \neq J, W T Q(I, J)$ is the covariance between observations I and $\mathrm{J}$; if $\mathrm{I}=\mathrm{J}, \mathrm{WTQ}(\mathrm{I}, \mathrm{J})$ is the variance of observation $\mathrm{I}$. Note that the variance-covariance matrix is symmetric, but the entire matrix (upper and lower parts) must be entered. 


\section{River Package}

The Ground-Water Flow Process capabilities of the River Package are documented in McDonald and Harbaugh (1988) and Harbaugh and others (2000).

\section{Calculation of Simulated Equivalents to the Observations}

In the River Package, flow at each finite-difference cell specified is calculated using equation 8 except when the hydraulic heads falls below RBOT. The relation between flow and hydraulic head is depicted in figure 8A. Mathematically, for finite-difference cell $n$, this is expressed as:

$$
\begin{array}{ll}
\mathrm{q}_{\mathrm{n}}=\mathrm{C}_{\mathrm{n}}\left(\mathrm{H}_{\mathrm{n}}-\mathrm{h}_{\mathrm{n}}\right) & \mathrm{h}_{\mathrm{n}}>\mathrm{RBOT}_{\mathrm{n}} \\
\mathrm{q}_{\mathrm{n}}=\mathrm{C}_{\mathrm{n}}\left(\mathrm{H}_{\mathrm{n}}-\mathrm{RBOT}_{\mathrm{n}}\right) & \mathrm{h}_{\mathrm{n}}<\text { RBOT }_{\mathrm{n}}
\end{array}
$$

If a measured gain to the surface-water body is represented using more than one finitedifference cell, the calculation is summed for the cells involved, as in equation 9.

\section{Calculation of Observation Sensitivities}

In the River Package, sensitivities are calculated as they are for the General-Head Boundary Package (eq. 10) except when $h_{n} \leq$ RBOT $_{n}$ (eq. 12). For any cell at which this condition occurs, the contribution to equation 10 is replaced by:

$$
\frac{\partial \mathrm{q}_{\mathrm{n}}}{\partial \mathrm{b}_{\ell}}=\frac{\partial \mathrm{C}_{\mathrm{n}}}{\partial \mathrm{b}_{\ell}}\left(\mathrm{H}_{\mathrm{n}}-\mathrm{RBOT}_{\mathrm{n}}\right) .
$$

The sensitivity equals zero for all parameters except those used to calculate $C_{n}$. As the number of cells characterized by this condition increases, the sensitivity related to the entire observation will diminish. If all cells are affected, the observation will affect the regression only through the parameters affecting $\mathrm{C}_{\mathrm{n}}$.

As mentioned previously, observation sensitivities are used in sensitivity analysis and regression as discussed by Hill (1998, p. p. 14-16, 38-42, 58).

\section{Input Instructions}

Input for the River Observation Package is read from a file that is specified with "RVOB" as the file type (table 2).

0 . [\#Text]

Item 0 is optional and can include as many lines as desired. Each line needs to begin with the "\#" character in the first column.

1. NQRV NQCRV NQTRV (free format)

2. TOMULTRV EVFRV IOWTQRV (free format) 
Read items 3, 4, and 5 for each of NQRV groups of cells for which river observations are to be specified.

3. NQOBRV NQCLRV (free format)

Read item 4 for each of NQOBRV observation times for this group of cells. STATISTIC and STAT-FLAG are ignored if IOWTQRV is greater than zero.

4. OBSNAM IREFSP TOFFSET HOBS STATISTIC STAT-FLAG PLOT-SYMBOL (free format)

Read item 5 for each cell in this group; the number of cells is equal to the absolute value of NQCLRV read in item 3.

5. LAYER ROW COLUMN FACTOR (free format)

Read items 6 and 7 if IOWTQRV is greater than 0.

6. FMTIN IPRN (free format)

7. WTQ $(1,1), \operatorname{WTQ}(1,2), \operatorname{WTQ}(1,3), \ldots, \operatorname{WTQ}(1, \operatorname{NQTRV})$ (format: FMTIN)

$\operatorname{WTQ}(2,1), \operatorname{WTQ}(2,2), \operatorname{WTQ}(2,3), \ldots, \operatorname{WTQ}(2, \operatorname{NQTRV})$

...

WTQ (NQTRV, 1), WTQ (NQTRV, 2) , WTQ (NQTRV, 3) , . . , WTQ (NQTRV, NQTRV)

\section{Explanation of Variables}

Text-is a character string (maximum of 79 characters) that starts in column 2 . Any characters can be included in Text. The "\#" character needs to be in column 1. Text is printed when the file is read and provides an opportunity for the user to include information about the model both in the input file and the associated output file.

NQRV - is the number of cell groups for which river observations are listed. A group consists of the cells needed to represent one flow measurement (eq. 9).

NQCRV - is greater than or equal to the total number of cells in all cell groups. NQCRV must be greater than or equal to the sum of all of the cells listed in all cell groups; that is, NQCRV needs to exceed the sum of the absolute values of all of the NQCLRV variables in the repetitions of item 3 .

NQTRV - is the total number of river observations for all cell groups. NQTRV must equal the sum of all NQOBRV, which are specified in repetitions of item 3 in the input file.

TOMULTRV - is the time-offset multiplier for river observations [-- or T/T]. The product of TOMULTRV and TOFFSET must produce a time value in units consistent with other model input. TOMULTRV can be dimensionless or can be used to convert the units of TOFFSET to the time unit used in the simulation.

EVFRV - is the error variance multiplier for river observations, and is used to calculate the weights as described below in the explanation of STATISTIC. EVFRV makes it easy to change the weights uniformly for all flow observations represented using the River Package.

IOWTQRV - is a flag that indicates that the variance-covariance matrix on river observations used to calculate the weighting is to be read into array WTQ of item 7. If IOWTQRV equals zero, weights are calculated using STATISTIC of item 4; if it is greater than zero, items 6 and 7 are read and used to calculate the weights.

NQOBRV - is the number of times at which flows are observed for the group of cells.

NQCLRV - is a flag, and the absolute value of NQCLRV is the number of cells in the group. If NQCLRV is less than zero, FACTOR $=1.0$ for all cells in the group. 
OBSNAM-is a string of 1 to 12 nonblank characters used to identify the observation.

IREFSP - is the reference stress period to which the observation time is referenced. The reference point is the beginning of the stress period.

TOFFSET - is the time offset of the observation, from the beginning of stress period IREFSP [T]. TOFFSET must be in units such that the product of TOMULTRV and TOFFSET is in time units consistent with other model input. TOFFSET and TOMULTRV from the RVOB file and values of PERLEN, NSTP, and TSMULT from the Discretization file (Harbaugh and others, 2000) are used to determine the stress period, time step, and time during the time step for the observation. To specify that an observation is for a steadystate model solution, specify IREFSP as the stress-period number of the steady-state stress period, and specify TOFFSET such that TOMULTRV $\times$ TOFFSET is less than or equal to PERLEN for the stress period; if PERLEN is zero, set TOFFSET to zero. If the observation falls within a time step, the simulated equivalent is calculated by linearly interpolating between values for the beginning and end of the time step. If the first stress period is transient and the observation falls within the first time step, the simulated equivalent from the end of the time step is used because no flow from the beginning of the time step is available for interpolation.

HOBS-is the observed river-boundary gain (if HOBS is negative) or loss (if HOBS is positive) $\left[\mathrm{L}^{3} / \mathrm{T}\right]$. The terms "gain" and "loss" are from the perspective of the surface-water body, so that gains occur when water leaves the ground-water system, and losses occur when water flows into the ground-water system.

STATISTIC - is the value from which the weight for the observation is calculated as determined using STAT-FLAG. STATISTIC is ignored if IOWTQRV is greater than zero, in which case WTQ of item 7 is used to define the weighting.

STAT-FLAG-is a flag identifying what STATISTIC is and how the weight is calculated. STAT-FLAG is ignored if IOWTQRV is greater than zero.

STAT-FLAG $=0$, STATISTIC is a scaled variance $\left[\left(\mathrm{L}^{3} / \mathrm{T}\right)^{2}\right]$, weight $=1 /($ STATISTIC $\times$ EVFRV),

STAT-FLAG $=1$, STATISTIC is a scaled standard deviation $\left[\mathrm{L}^{3} / \mathrm{T}\right]$, weight $=$ $1 /\left(\right.$ STATISTIC $^{2} \times$ EVFRV $)$, and $^{2}$

STAT-FLAG $=2$, STATISTIC is a scaled coefficient of variation [--], weight $=$ $1 /\left[(\text { STATISTIC } \times \text { HOBS })^{2} \times\right.$ EVFRV].

PLOT-SYMBOL - is an integer that will be written to output files intended for graphical analysis to allow control of the symbols used when plotting data.

LAYER - is the layer index of a river cell included in the cell group.

ROW-is the row index of a river cell included in the cell group.

COLUMN-is the column index of a river cell included in the cell group.

FACTOR - is the portion of the simulated gain or loss in the cell that is included in the total simulated gain or loss for this cell group ( $f_{n}$ of eq. 9 ).

FMTIN-is the Fortran format to be used in reading each line of the full variance-covariance matrix used to calculate the weighting. The format needs to be enclosed in parentheses and needs to accommodate real numbers.

IPRN - is a flag identifying the format in which the matrix is printed. If IPRN is less than zero, the matrix is not printed. Permissible values of IPRN and corresponding formats are:

\begin{tabular}{|c|c|c|c|}
\hline \multicolumn{2}{|c|}{ Output requires more than 80 columns } & \multicolumn{2}{|c|}{ Output fits in 80 columns } \\
\hline IPRN & FORMAT & IPRN & FORMAT \\
\hline
\end{tabular}




\begin{tabular}{|c|c|c|c|}
1 & $10 \mathrm{G} 12.3$ & 6 & $5 \mathrm{G} 12.3$ \\
\hline 2 & $10 \mathrm{G} 12.4$ & 7 & $5 \mathrm{G} 12.4$ \\
\hline 3 & $9 \mathrm{G} 12.5$ & 8 & $5 \mathrm{G} 12.5$ \\
\hline 4 & $8 \mathrm{G} 13.6$ & 9 & $4 \mathrm{G} 13.6$ \\
\hline 5 & $8 \mathrm{G} 14.7$ & 10 & $4 \mathrm{G} 14.7$ \\
\hline
\end{tabular}

WTQ - is an NQTRV by NQTRV array containing the variance-covariance matrix on river observations $\left[\left(\mathrm{L}^{3} / \mathrm{T}\right)^{2}\right]$. For elements WTQ $(I, J)$, if $I \neq J, W T Q(I, J)$ is the covariance between observations $I$ and $J$; if $I=J, W T Q(I, J)$ is the variance of observation I. Note that the variance-covariance matrix is symmetric, but the entire matrix (upper and lower parts) must be entered. 


\section{Observations at Cells Having More Than One Head-Dependent Boundary}

\section{Feature Represented by the Same Package}

The Ground-Water Flow Process allows multiple head-dependent boundary specifications in a single finite-difference cell in the same package. For example, two canals most appropriately represented by the Drain Package may cross an area such that they would be represented using the same finite difference cell, as designated by its layer, row and column. Hence, that layer, row, and column would be listed twice in the Drain Package input file.

To accumulate the information needed to define the simulated equivalent of an observation and its sensitivities, the Observation Process uses an observation cell list from the applicable Observation Process input file, which defines an observation cell group, and additional information specified in the corresponding Ground-Water Flow Process input file. The information for each cell is accumulated by matching cells listed in the Observation Process input file with those listed in the Ground-Water Flow Process input file. For the General-Head Boundary, Drain, and River Packages documented in this work, features match when the cell's layer, row, and column match. As long as the cell occurs only once in each list of cells, no problem occurs. If the list of cells used to define the observation cell group includes a feature at a cell where more than one feature is defined for the stress period in which the observation occurs in the Ground-Water Flow input file for the same package, a procedure is needed to ensure that the correct feature is included in the simulated equivalent. In MODFLOW-2000, the following sequential matching procedure is used.

If a cell is listed once in the observation cell group, the simulated equivalent for the observation includes flow calculated only for the first occurrence of the cell, as listed in the Ground-Water Flow Process input file for the package of concern for the stress period in which the observation occurs. Note that the stress period in which the observation occurs may be the reference stress period for the observation, or a later stress period, depending on the length of the reference stress period and the values of the time-offset multiplier and the variable TOFFSET. The listing order of cells in the Ground-Water Flow Process input file is determined as follows: all non-parameter cells are listed before all parameter-controlled cells for a given stress period, and the order in which parameters are listed in the head-dependent boundary flow input file for each stress period determines the listing order of parameter-controlled cells. Within the list of cells controlled by a parameter, the order is determined by the cell list in the parameter definition specified near the top of the Ground-Water Flow Process input file.

When a cell in an observation cell group is to be associated with the second or later occurrence of the cell in the Ground-Water Flow Process input for a given stress period, the observation cell group needs to include two or more occurrences of the cell, where the number of occurrences corresponds to the sequential occurrence of the feature sought. Occurrences of the cell for which the flow calculated by the Ground-Water Flow Process is not to contribute to the flow observation need to be specified with $F A C T O R=0.0$ (see preceding sections for explanation of FACTOR). For each observation cell group, the program starts at the first cell listed for the stress period in the Ground-Water Flow Process input file and searches for a match for the first cell in the observation cell group. After a match is found, appropriate calculations are done and the search for a match for the next cell in the observation cell group begins, starting at the feature following the feature matching the previous cell in the observation cell group. When the end of the list for the stress period in the Ground-Water Flow process input file is reached, the search continues at the beginning of the list. This can be confusing and care is needed to obtain the desired results. Searching and matching continues in this fashion until all cells in the observation cell group are matched. For the next observation cell group, the search starts at the beginning of the list for the stress period in the Ground-Water Flow process input file. 
Understanding this search logic is necessary when determining the order in which cells are listed in an observation cell group to ensure that observation cells are matched as intended with features listed for the Ground-Water Flow Process. When the features simulated by a particular package change from one stress period to the next, the list of cells in an observation cell group may not apply appropriately to both stress periods. In this situation, multiple cell groups may need to be defined to specify flow observations in different stress periods.

As an example, consider a model for an area where a series of springs discharge water from intervals at different elevations in an aquifer. For this model, the Drain Package is used and three drain features are specified in each of three finite-difference cells, for a total of nine features. All features are defined using parameters. One parameter is used to simulate three drain features, in rows 5,6 , and 7 of column 6 ; the elevations of these drain features are 20, 22, and 24 in this model. A second parameter is used to simulate drain features in the same three cells, each having an elevation of 30 . A third parameter is used to simulate drain features in the same three cells; the elevation is 45 at the first two cells, and 47 at the third cell. For this model, the GroundWater Flow Process Drain Package input file, listed with file type DRN in the name file, is as follows:

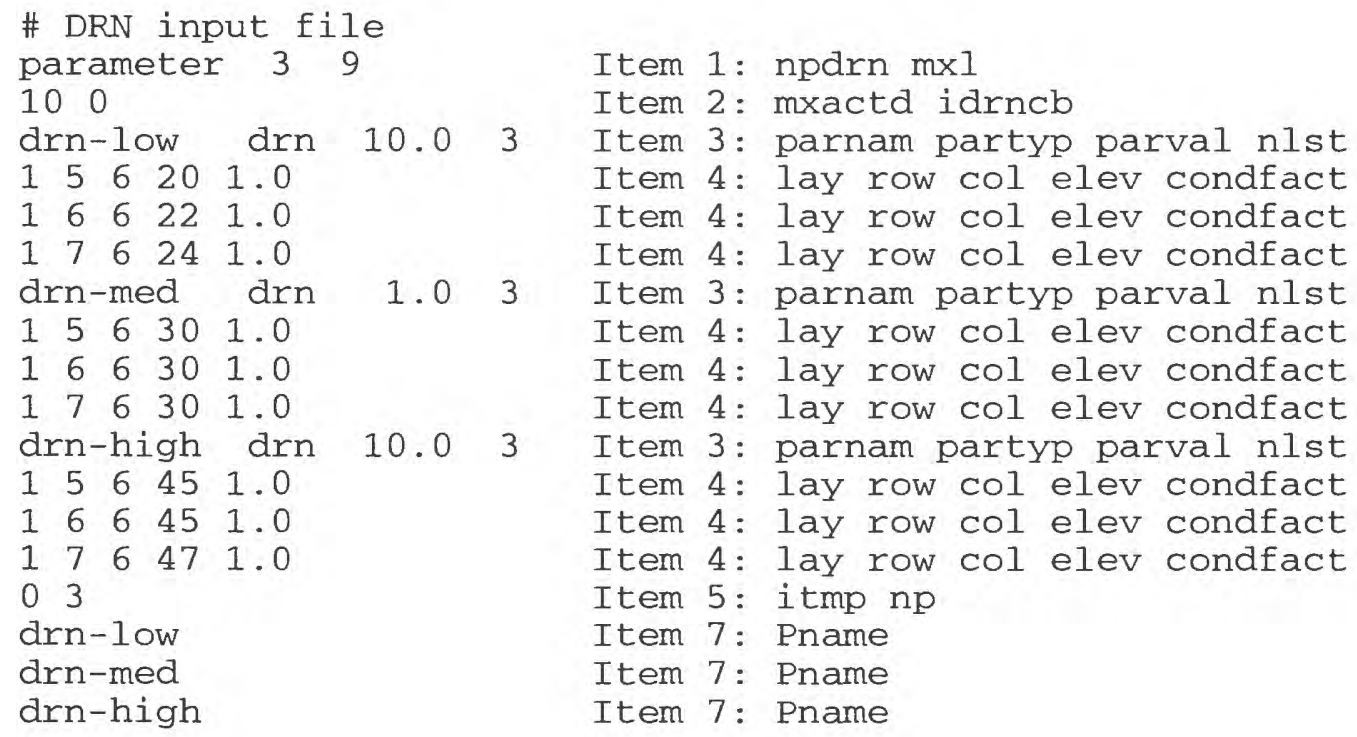

Observations of flow from the springs are represented such that the drain features in rows 5 and 6 at elevations 20 and 22 are associated with observations named D-low-5 and D-low-6, respectively; all the drain features in row 7 are together associated with an observation named D7 , the drain features in rows 5 and 6 at elevation 30 are together associated with an observation named D-med-56, and the springs in rows 5 and 6 at elevation 45 are associated with an observation named D-high-56. The following DROB file correctly associates the five observations with the nine drain features: 


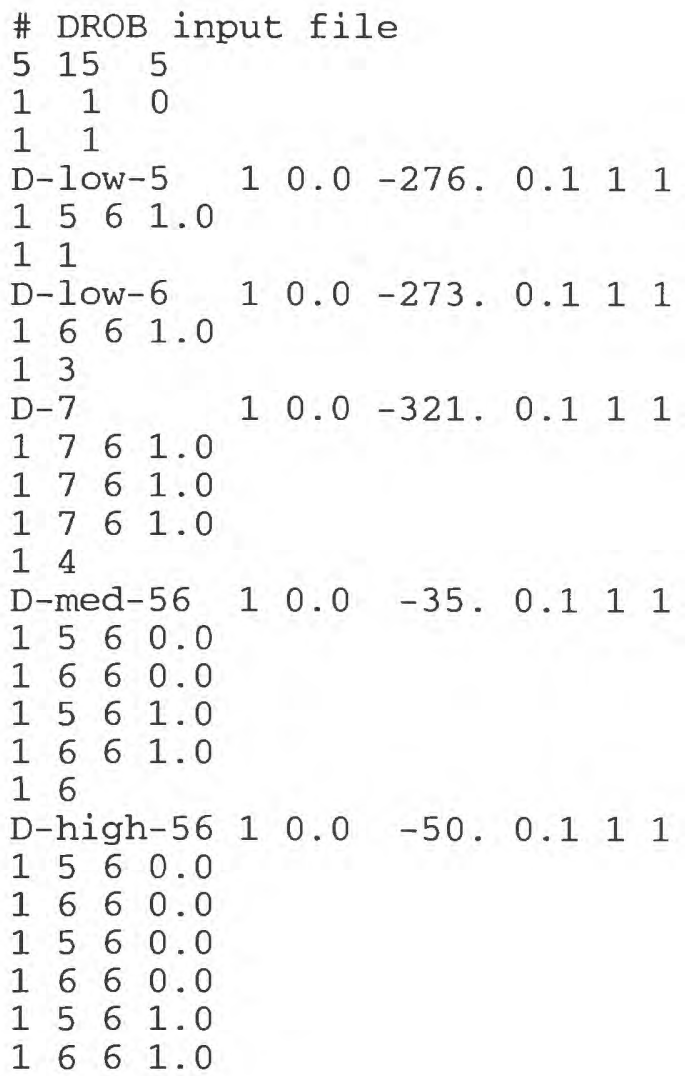

Item 1: NQDR NQCDR NQTDR

Item 2: TOMULTDR EVFDR IOWTQDR

Item 3: NQOBDR NQCLDR

Item 4

Item 5: LAY ROW COL FACTOR

Item 3: NQOBDR NQCLDR

Item 4

Item 5: LAY ROW COL FACTOR

Item 3: NQOBDR NQCLDR

Item 4

Item 5: LAY ROW COL FACTOR

Item 5: LAY ROW COL FACTOR

Item 5: LAY ROW COL FACTOR

Item 3: NQOBDR NQCLDR

Item 4

Item 5: LAY ROW COL FACTOR

Item 5: LAY ROW COL FACTOR

Item 5: LAY ROW COL FACTOR

Item 5: LAY ROW COL FACTOR

Item 3: NQOBDR NQCLDR

Item 4

Item 5: LAY ROW COL FACTOR

Item 5: LAY ROW COL FACTOR

Item 5: LAY ROW COL FACTOR

Item 5: LAY ROW COL FACTOR

Item 5: LAY ROW COL FACTOR

Item 5: LAY ROW COL FACTOR 


\section{Flow Observations at Boundaries Represented as Constant Head}

At cells defined as constant head, the model calculates flow to and from the cell as needed to maintain the constant head. If anything is known about the likely flow rate, it is important to include it to constrain model calibration. These flows can be included through the Constant-Head Flow Observation part of the Basic Package described here.

\section{Calculation of Simulated Equivalents to the Observations}

Consider a constant-head cell located at finite-difference cell $\mathrm{n}$. Like all finite-difference cells in a three-dimensional grid, the constant-head cell has six faces; these faces will be numbered 1 through 6 , and will be designated using $\mathrm{p}$. If the cell adjacent to side p exists and is active, the flow through cell face $\mathrm{p}$ of the constant-head cell can be calculated as:

$$
\mathrm{q}_{\mathrm{n}, \mathrm{p}}=\mathrm{C}_{\mathrm{n}, \mathrm{p}}\left(\mathrm{H}_{\mathrm{n}}-\mathrm{h}_{\mathrm{n}, \mathrm{p}}\right)
$$

where

$\mathrm{q}_{\mathrm{n}, \mathrm{p}} \quad$ is the simulated flow rate through cell face $\mathrm{p}\left(\mathrm{L}^{3} / \mathrm{T}\right)$ (negative for flow into the constant-head cell);

$\mathrm{C}_{\mathrm{n}, \mathrm{p}}$ is the conductance of the material separating the center of the constant-head finite-difference cell from the center of the cell adjacent to side $\mathrm{p}\left[\mathrm{L}^{2} / \mathrm{T}\right]$;

$h_{n} \quad$ is the hydraulic head in neighboring cell $\mathrm{p}(\mathrm{L})$; and

$\mathrm{H}_{n} \quad$ is the specified hydraulic head in the constant-head cell (L).

To calculate the total flow to or from one constant-head cell, the flow through each face for which the neighboring cell exists and is not constant head needs to be accumulated. That is,

$\mathrm{q}_{\mathrm{n}}=\sum_{\mathrm{p}=1}^{6} \mathrm{q}_{\mathrm{n} . \mathrm{p}}$ (omit flow through sides with adjacent cells that are constant head, inactive, or nonexistent)

where $\mathrm{q}_{n}$ is the flow into (-) or out (+) of the constant-head cell.

A constant-head flow observation commonly is represented by a group of constant-head cells. Summing over nqcl cells, the simulated equivalent to the observation equals:

$$
y^{\prime}=\sum_{n=1}^{n q c l} f_{n} q_{n}
$$

where

$f_{n} \quad$ is a user-defined multiplicative factor.

Generally $f_{n}=1.0$. However, $f_{n}$ needs to be less than 1.0 if only part of the flow calculated for the cell is to be included in the simulated equivalent to the observation. 


\section{Calculation of Observation Sensitivities}

In equation 14 , the calculated hydraulic head, $h_{n}$, generally is a function of all of the parameters, and $C_{n}$ is a function of any parameters used to calculate $C_{n}$. Also, $H_{n}$ can be a function of parameters in MODFLOW-2000 if the Constant-Head Boundary Package (Harbaugh and others, 2000) is used. Taking the derivative of equation 14 with respect to parameter $b_{\ell}$ yields

$$
\frac{\partial \mathrm{q}_{\mathrm{n}, \mathrm{p}}}{\partial \mathrm{b}_{\ell}}=\mathrm{C}_{\mathrm{n}}\left(\frac{\partial \mathrm{H}_{\mathrm{n}}}{\partial \mathrm{b}_{\ell}}-\frac{\partial \mathrm{h}_{\mathrm{n}}}{\partial \mathrm{b}_{\ell}}\right)+\frac{\partial \mathrm{C}_{\mathrm{n}}}{\partial \mathrm{b}_{\ell}}\left(\mathrm{H}_{\mathrm{n}}-\mathrm{h}_{\mathrm{n}}\right)
$$

The summations of equations 15 and 16 are then applied to obtain the sensitivity; that is, $\partial \mathrm{y}^{\prime} / \partial \mathrm{b}_{\ell}$ the derivative of the simulated equivalent, $\mathrm{y}^{\prime}$, with respect to parameter $\mathrm{b}_{\ell}$.

As mentioned previously, observation sensitivities are used in sensitivity analysis and regression as discussed by Hill (1998, p. p. 14-16, 38-42, 58).

\section{Input instructions}

Input for the Constant-Head Flow Observation Package is read from a file that is specified with "CHOB" as the file type listed in the name file (table 2).

0 . [\#Text]

Item 0 is optional and can include as many lines as desired. Each line needs to begin with the "\#" character in the first column.

1. NQCH NQCCH NQTCH (free format)

2. TOMULTCH EVFCH IOWTQCH (free format)

Read items 3, 4, and 5 for each of NQCH groups of cells for which constant-head flow observations are to be specified.

3. $\mathrm{NQOBCH} \mathrm{NQCLCH}$ (free format)

Read item 4 for each of NQOBCH observation times for this group of cells. STATISTIC and STAT-FLAG are ignored if IOWTQCH is greater than zero.

4. OBSNAM IREFSP TOFFSET HOBS STATISTIC STAT-FLAG PLOT-SYMBOL (free format)

Read item 5 for each of $|\mathrm{NQCLCH}|$ cells in this group.

5. LAYER ROW COLUMN FACTOR (free format)

Read items 6 and 7 if IOWTQCH is greater than 0.

6. FMTIN IPRN (free format)

7. WTQ $(1,1), \mathrm{WTQ}(1,2), \mathrm{WTQ}(1,3), \ldots, \mathrm{WTQ}(1, \mathrm{NQTCH})$ (format: FMTIN) WTQ $(2,1)$, WTQ $(2,2)$, WTQ $(2,3), \ldots$. WTQ $(2$, NQTCH $)$ ...

WTQ $(\mathrm{NQTCH}, 1)$, WTQ $(\mathrm{NQTCH}, 2), \mathrm{WTQ}(\mathrm{NQTCH}, 3), \ldots$ WTQ (NQTCH, NQTCH)

\section{Explanation of Variables}

Text-is a character string (maximum of 79 characters) that starts in column 2 . Any characters can be included in Text. The "\#” character needs to be in column 1. Text is printed when the file is read and provides an opportunity for the user to include information about the model both in the input file and the associated output file. 
$\mathrm{NQCH}$ - is the number of cell groups for which constant-head flow observations are listed. A group consists of the cells needed to represent one flow measurement (eq. 9).

$\mathrm{NQCCH}-$ is greater than or equal to the total number of cells in all groups. NQCCH must be greater than or equal to the sum of all |NQCLCH|.

NQTCH -is the total number of constant-head flow observations for all cell groups. NQTCH must equal the sum of all $\mathrm{NQOBCH}$, which are specified in repetitions of item 3 in the input file.

TOMULTCH -is the time-offset multiplier for constant-head flow observations [-- or T/T]. The product of TOMULTCH and TOFFSET must produce a time value with units that are consistent with the other model input. TOMULTCH can be dimensionless or can be used to convert the units of TOFFSET to the time unit used in the simulation.

EVFCH - is the error variance multiplier for constant-head flow observations, and is used to calculate the weights as described below in the explanation of STATISTIC. EVFCH makes it easy to change the weights uniformly for all constant-head flow observations.

IOWTQCH-is a flag that indicates that the variance-covariance matrix on constant-head flow observations used to calculate the weighting is to be read into array WTQ. If IOWTQCH equals zero, weights are assigned using STATISTIC of item 4; if it is greater than zero, items 6 and 7 are read.

NQOBCH-is the number of times at which flows are observed for the group of constant-head cells.

NQCLCH - is a flag, and the absolute value of NQCLCH is the number of cells in the group. If NQCLCH is less than zero, FACTOR $=1.0$ for all cells in the group.

OBSNAM-is a string of 1 to 12 nonblank characters used to identify the observation.

IREFSP - is the reference stress period to which the observation time is referenced. The reference point is the beginning of this stress period.

TOFFSET - is the time offset of the observation, from the beginning of stress period IREFSP [T]. TOFFSET must be in units such that the product of TOMULTCH and TOFFSET is in time units consistent with other model input. TOFFSET and TOMULTCH from the CHOB file and values of PERLEN, NSTP, and TSMULT from the Discretization file (Harbaugh and others, 2000) are used to determine the stress period, time step, and time during the time step for the observation. To specify that an observation is for a steadystate model solution, specify IREFSP as the stress-period number of the steady-state stress period, and specify TOFFSET such that the product TOMULTCH $\times$ TOFFSET is less than or equal to PERLEN for the stress period; if PERLEN is zero, set TOFFSET to zero. If the observation falls within a time step, the simulated equivalent is calculated by linearly interpolating between values for the beginning and end of the time step. If the first stress period is transient and the observation falls within the first time step, the simulated equivalent from the end of the time step is used because no flow from the beginning of the time step is available for interpolation. 
HOBS-is the observed constant-head flow into (positive) or out of (negative) the system $\left[\mathrm{L}^{3} / \mathrm{T}\right]$.

STAT - is the value from which the weight for the observation is calculated, as determined using STAT-FLAG. STATISTIC is ignored if IOWTQCH is greater than zero, in which case WTQ of item 7 is used to define the weighting.

STAT-FLAG-is a flag identifying what STATISTIC is and how the weight is calculated.

STAT-FLAG is ignored if IOWTQCH is greater than zero.

STAT-FLAG $=0$, STATISTIC is a scaled variance $\left[\left(\mathrm{L}^{3} / \mathrm{T}\right)^{2}\right]$, weight $=1 /($ STATISTIC $\times$ EVFCH),

STAT-FLAG $=1$, STATISTIC is a scaled standard deviation $\left[\mathrm{L}^{3} / \mathrm{T}\right]$, weight $=$ $1 /\left(\right.$ STATISTIC $^{2} \times$ EVFCH$)$, and

STAT-FLAG $=2$, STATISTIC is a scaled coefficient of variation $[--]$, weight $=$ $1 /\left[(\text { STATISTIC } \times \text { HOBS })^{2} \times\right.$ EVFCH $]$.

PLOT-SYMBOL - is an integer that will be written to output files intended for graphical analysis to allow control of the symbols used when plotting data.

LAYER - is the layer index of a constant-head cell included in the cell group.

ROW-is the row index of a constant-head cell included in the cell group.

COLUMN -is the column index of a constant-head cell included in the cell group.

FACTOR - is the portion of the simulated flow for the cell that is included in the total simulated flow for this cell group ( $f_{n}$ of eq. 16).

FMTIN-is the Fortran format to be used in reading each line of the variance-covariance matrix used to calculate the weighting. The format needs to be enclosed in parentheses and needs to accommodate real numbers.

IPRN - is a flag identifying the format in which the variance-covariance matrix is printed. If IPRN is less than zero, the matrix is not printed. Permissible values of IPRN and corresponding formats are:

\begin{tabular}{|c|c||c|c|}
\hline \multicolumn{2}{|c|}{ Output requires more than 80 columns } & \multicolumn{2}{c|}{ Output fits in 80 columns } \\
\hline IPRN & FORMAT & IPRN & FORMAT \\
\hline 1 & $10 \mathrm{G} 12.3$ & 6 & $5 \mathrm{G} 12.3$ \\
\hline 2 & $10 \mathrm{G} 12.4$ & 7 & $5 \mathrm{G} 12.4$ \\
\hline 3 & $9 \mathrm{G} 12.5$ & 8 & $5 \mathrm{G} 12.5$ \\
\hline 4 & $8 \mathrm{G} 13.6$ & 9 & $4 \mathrm{G} 13.6$ \\
\hline 5 & $8 \mathrm{G} 14.7$ & 10 & $4 \mathrm{G} 14.7$ \\
\hline
\end{tabular}

WTQ - is an NQTCH by NQTCH array containing the variance-covariance matrix on constanthead flow observations $\left[\left(\mathrm{L}^{3} / \mathrm{T}\right)^{2}\right]$. For elements WTQ(I,J), if I $\neq \mathrm{J}, \mathrm{WTQ}(\mathrm{I}, \mathrm{J})$ is the covariance between observations I and J; if $\mathrm{I}=\mathrm{J}, \mathrm{WTQ}(\mathrm{I}, \mathrm{J})$ is the variance of observation I. The variance-covariance matrix is symmetric, but the entire matrix (upper and lower parts) must be entered. 


\section{Chapter 5. SENSITIVITY PROCESS}

Use of the Sensitivity Process in different possible modes is described in table 3. Use of the grid sensitivities are described in Hill (1998, p. 16, 55).

MODFLOW-2000 calculates sensitivities for hydraulic head throughout the model using the sensitivity-equation method, which has been discussed by Yeh (1986) among others, and is described below. The increased accuracy of the sensitivity-equation method over perturbation methods generally has little effect on the sensitivity-analysis and nonlinear-regression calculations described by Hill (1998). It can, however, have an enormous effect on calculated parameter correlation coefficients and may affect calculated inferential statistics, such as confidence intervals. The increased accuracy is important, but comes at great effort. The programming required to calculate sensitivities generally is at least as much as the programming needed to solve the forward problem. When possible, it is advantageous to use inverse models, such as MODFLOW-2000, that can calculate sensitivity-equation sensitivities. Otherwise, programs such as UCODE (Poeter and Hill, 1998) and PEST (Doherty, 1994) can be used, but the inherent limitations in some of the results need to be understood and accommodated. In particular, parameter correlation coefficients will not reliably identify parameters that are extremely correlated and, therefore, cannot be estimated uniquely given the problem as posed.

Sensitivities can be calculated for any of the parameters discussed by Harbaugh and others (2000). For parameters designated in the Layer Property Flow Package as horizontal hydraulic conductivity (PARTYP = HK), vertical hydraulic conductivity $(\mathrm{VK})$, vertical anisotropy (VANI), and vertical hydraulic conductivity of an implicitly defined confining bed (VKCB), however, sensitivity-equation sensitivities can only be calculated if the horizontal interblock transmissivities are calculated using harmonic averaging.

\section{Equations for Grid Sensitivities for Hydraulic Heads Throughout the Model}

In MODFLOW-2000, sensitivities are first calculated for all hydraulic heads throughout the entire grid using the sensitivity-equation method. The equation used to solve for sensitivities is derived by taking the derivative of the ground-water flow equation with respect to each parameter of interest. For this purpose, it is convenient to write the ground-water flow equation in matrix form, as presented by McDonald and Harbaugh (1988, p. 2-26, eq. 27), but with the storage terms separated out from the stress terms on the right-hand side. This produces a steadystate (time step $\mathrm{m}=0$ ) equation of the form:

$$
\underline{A}(0) \underline{h}(0)=\underline{f}(0) . \quad m=0
$$

Transient (time step $\mathrm{m}>0$ ) equations for confined layers are of the form:

$$
\underline{\mathrm{A}}(\mathrm{m}) \underline{\mathrm{h}}(\mathrm{m})=\underline{\mathrm{B}}(\mathrm{m}) \underline{\mathrm{h}}(\mathrm{m}-1)+\underline{\mathrm{f}}(\mathrm{m}) \quad \mathrm{m}>0
$$

where extra terms required for convertible layers are omitted for simplicity. Underlined capital letters indicate matrices and underlined lower-case letters indicate vectors. The symbols used in equations 18 and 19 are as follows.

$\mathrm{m} \quad$ is the time step 
$\underline{\mathrm{A}}(\mathrm{m})=\frac{-\underline{\mathrm{S}}(\mathrm{m})}{\Delta \mathrm{t}(\mathrm{m})}+\underline{\mathrm{K}}+\underline{\mathrm{P}}(\mathrm{m}) \quad\left[\mathrm{L}^{2} / \mathrm{T}\right]$

$\underline{S}(m)$ is a diagonal matrix of specific storage multiplied by cell volume, or specific yield multiplied by cell area, depending on whether the layer is confined or, if the layer is convertible, the hydraulic head $\left[\mathrm{L}^{2}\right]$;

$\Delta t(m)$ is the length of time step $\mathrm{m}[\mathrm{T}]$;

$\underline{\mathrm{K}}$ is a matrix of horizontal and vertical conductances $\left[\mathrm{L}^{2} / \mathrm{T}\right]$;

$\underline{\mathrm{P}}(\mathrm{m})$ is a diagonal matrix of conductances at head-dependent boundaries $\left[\mathrm{L}^{2} / \mathrm{T}\right]$;

$\underline{h}(\mathrm{~m})$ is a vector of hydraulic heads at the end of time step $\mathrm{m}$ for all nodes in the finitedifference grid [L];

$\underline{f}(m)$ is a vector containing the $-Q_{i, j, k}$ terms of McDonald and Harbaugh, (1988, p. 2-26, eq. 26) $\left[\mathrm{L}^{3} / \mathrm{T}\right]$

$\underline{\mathrm{B}}(\mathrm{m})=\underline{\mathrm{S}}(\mathrm{m}) / \Delta \mathrm{t}(\mathrm{m}) ;$ and

$\underline{A}(0), \underline{h}(0)$, and $\underline{f}(0)$ equal $\underline{A}(m), \underline{h}(m)$, and $\underline{f}(m)$ at steady-state, when $m=0$.

The right-hand sides of equations 18 and 19 are equivalent to vector $\{\mathrm{q}\}$ of McDonald and Harbaugh (1988, p. 2-26, eq. 27).

For the transient equations, the initial conditions are:

$\underline{\mathrm{h}}(0)=\underline{\mathrm{H}}$,

where $\underline{\mathrm{H}}$ is a distribution of hydraulic heads over the grid. Often the initial hydraulic heads are calculated steady-state hydraulic heads that are consistent with the hydraulic properties of the transient model. MODFLOW-2000 can use a single model run to first calculate steadystate hydraulic heads, and then to use these as the initial hydraulic heads for a subsequent simulation composed of transient and possibly interspersed steady-state stress periods (Harbaugh and others, 2000).

To produce equations for sensitivity-equation sensitivities, take the derivative of equations 18 through 20 with respect to $\mathrm{b}_{\ell}$, apply rule for taking the derivative of a product, and rearrange the terms. Equation 18 then yields the sensitivity equation for steady-state systems,

$$
\underline{\mathrm{A}}(0) \frac{\partial \underline{\mathrm{h}}(0)}{\partial \mathrm{b}_{\ell}}=-\frac{\partial \underline{\mathrm{A}}(0)}{\partial \mathrm{b}_{\ell}} \underline{\mathrm{h}}(0)+\frac{\partial \underline{\mathrm{f}}(0)}{\partial \mathrm{b}_{\ell}} . \quad \mathrm{m}=0
$$

Equation 19 yields the sensitivity equation for transient time step $\mathrm{m}$,

$$
\begin{array}{rlr}
\underline{\mathrm{A}}(\mathrm{m}) \frac{\partial \underline{\mathrm{h}}(\mathrm{m})}{\partial \mathrm{b}_{\ell}}= & -\frac{\partial \underline{\mathrm{A}}(\mathrm{m})}{\partial \mathrm{b}_{\ell}} \underline{\mathrm{h}}(\mathrm{m})+\frac{\partial \underline{\mathrm{f}}(\mathrm{m})}{\partial \mathrm{b}_{\ell}}+ & \mathrm{m} \geq 1 \\
& +\frac{\partial \underline{\mathrm{B}}(\mathrm{m})}{\partial \mathrm{b}_{\ell}} \underline{\mathrm{h}}(\mathrm{m}-1)+\underline{\mathrm{B}}(\mathrm{m}) \frac{\partial \underline{\mathrm{h}}(\mathrm{m}-1)}{\partial \mathrm{b}_{\ell}} . &
\end{array}
$$


Boundary and initial conditions for the system are:

$$
\begin{aligned}
& \left.\frac{\partial \underline{\mathrm{h}}}{\partial \mathrm{b}_{\ell}}\right|_{\Gamma_{1}}=0, \\
& \left.\frac{\partial \mathrm{H}}{\partial \mathrm{b}_{\ell}}\right|_{\Gamma_{2}}=0 \text {, and } \\
& \frac{\partial \underline{\mathrm{h}}(0)}{\partial \mathrm{b}_{\ell}}=\frac{\partial \underline{\mathrm{H}}}{\partial \mathrm{b}_{\ell}} .
\end{aligned}
$$

The $\Gamma_{1}$ are constant-head boundaries. Equation 23a applies unless $b_{\ell}$ is used to define the hydraulic-head along a constant-head boundary. In that situation, $\partial \underline{h} / \partial \mathrm{b}$, along the boundary is calculated from the relation between the constant heads and $b_{\ell}$.

The $\Gamma_{2}$ are head-dependent boundaries and $\mathrm{H}$ is the constant-head on one side of the boundary. MODFLOW-2000 does not support $\mathrm{H}$ being a function of the parameters, so $23 \mathrm{~b}$ always applies.

In the initial conditions of equation $23 \mathrm{c}, \underline{\mathrm{H}}$ is a distribution of hydraulic heads over the grid. If $\underline{H}$ is the solution from a preceding steady-state stress period, $\partial \underline{H} / \partial b_{\ell}$ generally is not zero and is calculated for the preceding stress period by the Sensitivity Process. If $\underline{H}$ is specified by the user, it does not depend on any estimated parameters and all elements of $\partial \underline{H} / \partial b_{\varepsilon}$ are zero for all parameters.

By using equations 21 to 23 , the sensitivities for hydraulic heads throughout the model, $\partial \underline{\mathrm{h}} / \partial \mathrm{b}_{\ell}$, for each time step can be calculated for all parameters before progressing to the next time step. With this method, $\underline{A}$ is formulated once for each time step, and solutions of hydraulic heads and sensitivities for all parameters are saved for use in the next time step. This procedure is followed by MODFLOW-2000, as shown in figure 1 .

If any model layer is convertible, at least some of the conductance terms of matrix $\underline{A}$ are functions both of the parameter values, $\underline{b}$, and of hydraulic heads, $\underline{h}(\underline{b})$, where $\underline{b}$ is a vector of the parameters and $b_{\ell}$ is one element of $\underline{b}$. In this circumstance, the first term on the right-hand side of equations 21 and 22 needs to be expanded using the chain rule. The resulting equation is solved using iterative updating using solver iterations as suggested by Shah and others (1978) to avoid solving a problem with an unsymmetric matrix. An iterative solver such as PCG2 (Hill, 1990) needs to be used. Specifying the solver iteration using $r$ and noting that the other terms of the equations are the same for all solver iterations, the equation for steady-state problems with convertible layers is:

$$
\begin{aligned}
\underline{\mathrm{A}}(0)\left(\frac{\partial \underline{\mathrm{h}}(0)}{\partial \mathrm{b}_{\ell}}\right)^{\mathrm{r}}= & -\frac{\partial \underline{\mathrm{A}}(0)}{\partial \mathrm{b}_{\ell}} \underline{\mathrm{h}}(0)+\frac{\partial \underline{\mathrm{f}}(0)}{\partial \mathrm{b}_{\ell}}- & \mathrm{m}=0 \\
& -\frac{\partial \underline{\mathrm{A}}(0)}{\partial \underline{\mathrm{h}}(0)}\left(\frac{\partial \underline{\mathrm{h}}(0)}{\partial \mathrm{b}_{\ell}}\right)^{\mathrm{r}-1} \underline{\mathrm{h}}(0) &
\end{aligned}
$$


and the equation for transient problems is:

$$
\begin{array}{rlr}
\underline{\mathrm{A}}(\mathrm{m}) \frac{\partial \underline{\mathrm{h}}(\mathrm{m})}{\partial \mathrm{b}_{\ell}}= & -\frac{\partial \underline{\mathrm{A}}(\mathrm{m})}{\partial \mathrm{b}_{\ell}} \underline{\mathrm{h}}(\mathrm{m})+\frac{\partial \underline{\mathrm{f}}(\mathrm{m})}{\partial \mathrm{b}_{\ell}}+ & \\
& +\frac{\partial \underline{\mathrm{B}}(\mathrm{m})}{\partial \mathrm{b}_{\ell}} \underline{\mathrm{h}}(\mathrm{m}-1)+\underline{\mathrm{B}}(\mathrm{m}) \frac{\partial \underline{\mathrm{h}}(\mathrm{m}-1)}{\partial \mathrm{b}_{\ell}}- & \mathrm{m}>0 \\
& -\frac{\partial \underline{\mathrm{A}}(\mathrm{m})}{\partial \underline{\mathrm{h}}(\mathrm{m})}\left(\frac{\partial \underline{\mathrm{h}}(\mathrm{m})}{\partial \mathrm{b}_{\ell}}\right)^{\mathrm{r}-1} \underline{\mathrm{h}}(\mathrm{m}) .
\end{array}
$$

By using index notation to clarify the multiplication involved, the last term of equation 24 and 25 (where $m=0$ in equation 24 ) equals the vector:

$$
-\frac{\partial \underline{\mathrm{A}}(\mathrm{m})}{\partial \underline{\mathrm{h}}(\mathrm{m})}\left(\frac{\partial \underline{\mathrm{h}}(\mathrm{m})}{\partial \mathrm{b}_{\ell}}\right)^{\mathrm{r}-1} \underline{\mathrm{h}}(\mathrm{m})=\frac{\partial \mathrm{A}_{i j}(\mathrm{~m})}{\partial \underline{\mathrm{h}}_{\mathrm{n}}(\mathrm{m})}\left(\frac{\partial \mathrm{h}_{n}(\mathrm{~m})}{\partial \mathrm{b}_{\ell}}\right)^{\mathrm{r}-1} \mathrm{~h}_{\mathrm{j}}(\mathrm{m}) . \quad \mathrm{m} \geq 0
$$

\section{Solving for Grid Sensitivities for Hydraulic Heads Throughout the Model}

When sensitivities are calculated, the solver selected to solve the ground-water flow equation for hydraulic heads also is used to solve the sensitivity equation for sensitivities of hydraulic head throughout the grid with respect to each parameter. The equations above can be solved using the following packages available with MODFLOW-2000 (Harbaugh and others, 2000): the direct (DE4; Harbaugh, 2000) and preconditioned conjugate gradient (PCG2; Hill, 1990) solvers. The strongly implicit solver (SIP; McDonald and Harbaugh, 1988) generally does not work well for solving sensitivities because it would require different values of the seed to solve sensitivities for different parameters; the slice-successive overrelaxation solver (SOR; McDonald and Harbaugh, 1988) tends to be slower than the other solvers. The DE4 and the PCG2 solvers have some characteristics that are important to the solution of sensitivities, and these are described in the following paragraphs.

The DE4 solver generally is slower than the PCG2 solver for most practical problems, but when solving for sensitivities the DE4 solver has the nice characteristic that the matrix decomposition from the hydraulic-head solution for a time step can be used to solve sensitivities for that time step for all of the parameters. This is a consequence of the $\underline{A}$ matrix on the left-hand side of equations 18 and 20, and equations 19 and 22 being identical for any time step. Only the right-hand sides change to calculate sensitivities. To take advantage of this, the call to the DE4 solver for sensitivities has some arguments hardwired. Thus, it is sometimes useful to try the DE4 solver for problems that involve solving for sensitivities even though the DE4 solver is somewhat slower than PCG2 for solving hydraulic heads.

Sensitivity-equation sensitivities for different parameters might vary from each other and from hydraulic-head values by many orders of magnitude. As a result, the convergence criteria specified for hydraulic heads in the solver input file, such as HCLOSE and RCLOSE in PCG2 (Hill, 1990) and HCLOSE in DE4 (Harbaugh, 1995), are unlikely to be applicable to the 
sensitivity solutions. MODFLOW-2000 addresses this problem by calculating unique convergence criteria for the sensitivities of each parameter. For parameter $b_{f}$, the convergence criteria are calculated by dividing the hydraulic-head convergence criteria by $\left(\left|\mathrm{b}_{\ell}^{0}\right| \times 100\right)$, where $\mathrm{b}_{\ell}{ }_{\ell}$ is the parameter value specified in the Sensitivity Process input file. For PCG2, for example, the convergence criteria would be HCLOSE $/\left(\left|\mathrm{b}_{\ell}{ }^{0}\right| \times 100\right)$ and RCLOSE $/\left(\left|\mathrm{b}_{\ell}{ }^{0}\right| \times 100\right)$.

\section{One-Percent Sensitivity Maps}

When the Sensitivity Process is active, and ISENALL $>0$ or ISENALL $=0$ and at least one ISENS $>0$ (see the input instructions below), MODFLOW-2000 uses the parameter values and designations listed in the Sensitivity Process input file and the sensitivity-equation method to calculate, for the entire grid, hydraulic-head sensitivities $\left(\partial \mathrm{h}_{\mathrm{n}} / \partial \mathrm{b}_{\ell}\right)$. When printed or saved, these arrays of sensitivities are scaled by multiplying by the absolute value of the parameter value divided by 100 . An exception occurs if the absolute value of the parameter value is less than BSCAL. In this circumstance, BSCAL is used to scale the sensitivities. These are called arrays of one-percent scaled sensitivities because they approximate the change in simulated hydraulic head resulting from a one-percent increase in the parameter value (Hill, 1998, p. 15-16). The printing and saving of these arrays are controlled by the variables IPRINTS, ISENSU, ISENPU, and ISENFM of the SEN file (see below), and the arrays can be contoured just as arrays of hydraulic heads can be contoured. The resulting one-percent scaled sensitivity contour maps can be used to identify locations of large one-percent scaled sensitivities, where observations of hydraulic heads are likely to be most valuable for model calibration.

\section{Log-Transforming Parameters}

As discussed by Hill (1998, p. 12), log-transforming parameters can encourage convergence of parameter estimation, and can be used to prevent parameter estimates, confidence interval limits, and values used for the modified Beale's measure (see chapter 7) from becoming negative. MODFLOW-2000 allows all parameters related to hydraulic conductivity and storage to be log-transformed, but it does not allow parameters of the Well, Recharge, or Evapotranspiration Package to be log-transformed.

MODFLOW-2000 has been designed to make the log-transformation of parameters as transparent to the user as possible because the native, untransformed values are more meaningful in most circumstances. Hill (1998, p. 12-13) describes three situations for which the logtransformation is not transparent, but MODFLOW-2000 has been programmed to make the first of these less troublesome. This situation is described in the following paragraph; the other two situations are as described in Hill (1998, p. 12-13), and are not discussed here.

The first situation occurs when prior information is defined on a log-transformed parameter. In MODFLOW-2000, prior information is defined at the bottom of the ParameterEstimation Process input file; this problem is discussed here because the flags for logtransforming parameters are specified in the Sensitivity Process input file. When defining prior information of log-transformed parameters, the statistic that quantifies the reliability of the prior parameter value can be specified relative either to the native or to the log-transformed parameter value. If the statistic is specified relative to the native value and is not a variance, the program calculates the variance relative to the native value. From the variance relative to the native value, the program calculates the variance relative to the log-transformed value of the parameter, using the equation (Benjamin and Cornell, 1970, p. 267):

$$
\sigma_{\ln b}^{2}=\ln \left[\left(\sigma_{b} / b\right)^{2}+1\right]
$$


where $b$ is the mean of the log-normal distribution attributed to the prior estimate.

\section{Input Instructions}

Input for the Sensitivity Process is read from a file that is specified with "SEN" as the file type in the name file (table 2).

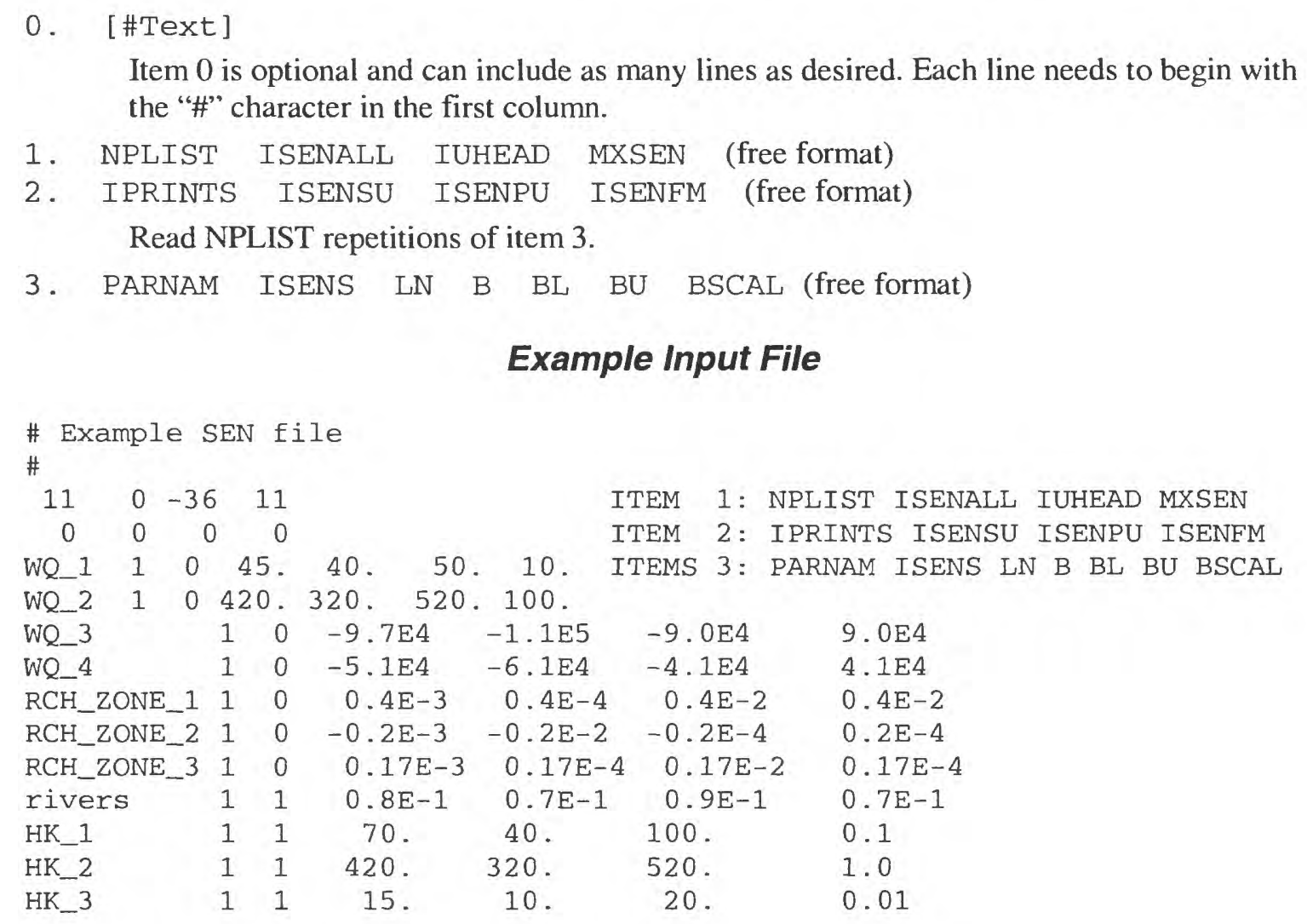

\section{Example Input File}

$\#$

\begin{tabular}{|c|c|c|c|c|c|c|c|}
\hline 11 & 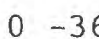 & & 11 & & & ITEM $1: \mathrm{N}$ & NPLIST ISENALL IUHEAD MXSEN \\
\hline 0 & 0 & 0 & 0 & & & ITEM & IPRINTS ISENSU ISENPU ISENFM \\
\hline WQ_1 & 0 & & 5. & 40 . & 10. & ITEMS $3: \mathrm{P}$ & PARNAM ISENS LN B BL BU BSCAL \\
\hline WQ 2 & 1 & 42 & & 320. & 100. & & \\
\hline WQ_3 & & 1 & 0 & $-9.7 \mathrm{E} 4$ & $-1.1 \mathrm{E} 5$ & $-9.0 \mathrm{E} 4$ & $9.0 \mathrm{E} 4$ \\
\hline WQ_4 & & 1 & 0 & $-5.1 E 4$ & $-6.1 \mathrm{E4}$ & $-4.1 E 4$ & $4.1 \mathrm{E} 4$ \\
\hline RCH_zO & NE_1 1 & 1 & 0 & $0.4 \mathrm{E}-3$ & $0.4 \mathrm{E}-4$ & $0.4 \mathrm{E}-2$ & $0.4 \mathrm{E}-2$ \\
\hline RCH_zO & NE_2 & 1 & 0 & $-0.2 \mathrm{E}-3$ & $-0.2 E-2$ & $-0.2 E-4$ & $0.2 \mathrm{E}-4$ \\
\hline RCH_ZO & NE_3 3 & 1 & 0 & $0.17 E-3$ & $0.17 E-4$ & $0.17 \mathrm{E}-2$ & $0.17 \mathrm{E}-4$ \\
\hline rivers & & 1 & 1 & $0.8 \mathrm{E}-1$ & $0.7 \mathrm{E}-1$ & $0.9 \mathrm{E}-1$ & $0.7 \mathrm{E}-1$ \\
\hline HK_1 & & 1 & 1 & 70 . & 40. & 100. & 0.1 \\
\hline HK_2 & & 1 & 1 & 420. & 320. & 520 . & 1.0 \\
\hline HK_3 & & 1 & 1 & 15. & 10. & 20. & 0.01 \\
\hline
\end{tabular}

In this example, WQ_1, WQ_2, WQ_3, WQ_4, RCH_ZONE_1, RCH_ZONE_2, RCH_ZONE_3, rivers, HK_1, HK_2, and HK_3 are the names of parameters defined in Ground-Water Flow Process input files. The Sensitivity Process matches parameter names with those defined in the Ground-Water Flow Process input files in a case-insensitive manner.

\section{Explanation of Variables}

Text-is a character string (maximum of 79 characters per line) that starts in column 2 . Any characters can be included in Text. The "\#" character needs to be in column 1 . Text is printed when the file is read and provides an opportunity for the user to include information about the model both in the input file and the associated output file.

NPLIST - is the number of named parameters listed in the Sensitivity Process input file. Parameters need to be listed in this file for sensitivities to be calculated and for the parameter value to be estimated by the Parameter-Estimation Process, but whether or not the sensitivities are calculated or the parameter is estimated depends on the value of ISENALL and ISEN, as described below. 
ISENALL - is a flag that can override values of ISENS listed for each parameter in the input file and can deactivate the Parameter-Estimation Process.

ISENALL $=0$, use the ISENS flags listed for each parameter.

ISENALL > 0, set ISENS to 1 for all listed parameters and deactivate the ParameterEstimation Process if it is active. Use this option to evaluate sensitivities for all listed parameters without losing the notation that governs which parameters are to be estimated.

ISENALL $<0$, Set ISENS to 0 for all listed parameters so that no sensitivities are calculated, but use the parameter values specified in this file. Deactivate the Parameter-Estimation Process if a PES file is listed in the name file. This option might be used, for example, to perform a forward model run using parameter values from an intermediate parameter-estimation iteration.

IUHEAD - is a flag that allows the user to choose between using scratch (temporary) files or memory for storage of grid sensitivities. If IUHEAD $>0$, it is also the first of a series of file unit numbers.

IUHEAD $>0$, one temporary scratch file is opened for each of MXSEN parameters. File unit numbers in the range IUHEAD through IUHEAD+MXSEN-1 are used for the scratch files and may not be used in the name file. This range of unit numbers also must not include the numbers 96 through 99 , because these unit numbers are reserved for other uses.

IUHEAD $\leq 0$, sensitivities are stored in memory. If the program has been converted to comply with FORTRAN 77 standards, the amount of allocated storage may need to be modified; see the 'Memory Requirements' section of Appendix B. When the parallel computing capability is used, IUHEAD needs to be less than or equal to zero.

MXSEN-is the maximum number of parameters for which sensitivities are to be calculated. MXSEN needs to equal or exceed the number of parameters for which ISENS is greater than zero. If ISENALL >0, MXSEN needs to equal or exceed NPLIST; the program will stop if this condition is not satisfied. If IUHEAD $\leq 0$, make MXSEN as small as possible to reduce the computer memory requirements.

IPRINTS - is a flag that indicates how the saving and printing of sensitivity arrays are controlled if ISENSU and (or) ISENPU, below, are greater than zero. The arrays are saved (to unit ISENSU) and printed (to unit ISENPU) only when the Sensitivity Process is active and the Parameter-Estimation Process is inactive. The sensitivities are for parameters for which ISENS $>0$ unless ISENALL $>0$, in which case the sensitivities are for all parameters listed in the Sensitivity Process input file. These are arrays for the entire grid and can be contoured to obtain sensitivity maps. They are one-percent scaled sensitivities; that is, they are scaled by multiplying by the parameter value and dividing by 100 . An exception is described under BSCAL.

IPRINTS $=0$, Printing of sensitivity arrays is controlled by the IHDDFL and Hdpr variables of the Output Control option of the Basic Package, which also control printing of heads in the Ground-Water Flow Process.

IPRINTS $=1$, Print and save sensitivity arrays for all model layers and all time steps.

ISENSU - is a flag that controls whether sensitivity arrays are to be saved and, if so, to what file. The file format is controlled by the user through the Output Control input file. The output is written as text if CHEDFM is defined in the Output Control input file, and is binary otherwise. The file needs to be opened in the NAME file with the appropriate file type (Harbaugh and others, 2000). 
ISENSU $=0$, sensitivity arrays are not saved.

ISENSU > 0 , sensitivity arrays are saved on unit ISENSU.

ISENPU - is a flag identifying whether sensitivity arrays are to be printed and, if so, to what file. Sensitivity arrays are written as text to unit ISENPU using format number ISENFM (see below). ISENPU can be set equal to the GLOBAL file unit number, the LIST file unit number, or another unit opened in the name file using the DATA file type. To avoid inadvertently producing enormous output files, sensitivity arrays are only printed when the Parameter-Estimation Process is not active.

ISENPU $=0$, sensitivity arrays are not printed.

ISENPU $>0$, sensitivity arrays are printed on unit ISENPU.

ISENFM - is a code indicating the format for printing sensitivity arrays as described for ISENPU. If ISENFM is less than zero, the arrays are not printed. Permissible values of ISENFM and corresponding formats are:

\begin{tabular}{|c|c|c|c|}
\hline ISENFM & FORMAT & ISENFM & FORMAT \\
\hline 0 & $10 \mathrm{G} 11.4$ & 11 & $20 \mathrm{~F} 5.4$ \\
\hline 1 & $11 \mathrm{G} 10.3$ & 12 & $10 \mathrm{G} 11.4$ \\
\hline 2 & $9 \mathrm{G} 13.6$ & 13 & $10 \mathrm{~F} 6.0$ \\
\hline 3 & $15 \mathrm{~F} 7.1$ & 14 & $10 \mathrm{~F} 6.1$ \\
\hline 4 & $15 \mathrm{~F} 7.2$ & 15 & $10 \mathrm{~F} 6.2$ \\
\hline 5 & $15 \mathrm{~F} 7.3$ & 16 & $10 \mathrm{~F} 6.3$ \\
\hline 6 & $15 \mathrm{~F} 7.4$ & 17 & $10 \mathrm{~F} 6.4$ \\
\hline 7 & $20 \mathrm{~F} 5.0$ & 18 & $10 \mathrm{~F} 6.5$ \\
\hline 8 & $20 \mathrm{~F} 5.1$ & 19 & $5 \mathrm{G} 12.5$ \\
\hline 9 & $20 \mathrm{~F} 5.2$ & 20 & $6 \mathrm{G} 11.4$ \\
\hline 10 & $20 \mathrm{~F} 5.3$ & 21 & $7 \mathrm{G} 9.2$ \\
\hline
\end{tabular}

PARNAM - is a parameter name (up to 10 nonblank characters) that matches one of the parameter names specified in input for one of the Ground-Water Flow Process packages. Matching is performed in a case-insensitive manner.

ISENS-is a flag identifying whether or not sensitivities are to be calculated for parameter PARNAM. If the PES process is active, ISENS also identifies whether or not the parameter is to be estimated by regression. If ISENALL, above, is not zero, ISENS is ignored.

ISENS $\leq 0$, Sensitivities are not calculated and the parameter is not estimated.

ISENS $>0$, Sensitivities are calculated and, if the PES process is active, the parameter is estimated by regression.

LN-is a flag identifying whether parameter PARNAM is to be log-transformed for parameter estimation.

$\mathrm{LN} \leq 0$, Estimate the native, untransformed parameter.

LN $>0$, Estimate the log transform of the parameter.

For B, BL, BU, and BSCAL below, enter the values related to the native, untransformed parameter, even if $\mathrm{LN}$ is greater than zero. 
B-is the starting value for parameter PARNAM. This value always replaces the value listed in the Flow-Process package input file. See the section "Starting Parameter Values" for a discussion of things to consider about starting parameter values.

$\mathrm{BL}$ - is the minimum reasonable parameter value for parameter PARNAM. BL does not restrict the estimated parameter value. BL is printed in the output to facilitate comparison with the estimated value.

BU-is the maximum reasonable parameter value for parameter PARNAM. BU does not restrict the estimated parameter value. $\mathrm{BU}$ is printed in the output to facilitate comparison with the estimated value.

BSCAL - is an alternate scaling factor for parameter PARNAM, and always needs to be a positive number. In MODFLOW-2000, dimensionless and one-percent scaled sensitivities are calculated using the scaling discussed in Hill (1998, p. 14-17, eq. 8, 9, and 11), except that the absolute value of the current parameter value is used. When the parameter value equals 0.0 , however, which can occur for parameters that are not logtransformed, this scaling results in scaled sensitivities that equal 0.0. MODFLOW-2000 accommodates this situation using BSCAL. If the absolute value of the parameter is less than BSCAL, BSCAL is used in the scaling. The best value to use for BSCAL is problem dependent. Good choices are the smallest (in absolute value) reasonable value of the parameter or a value two to three orders of magnitude smaller than the value specified by B. If the smallest reasonable value is 0.0 , a reasonable non-zero value needs to be used. BSCAL has no effect on the scaled sensitivities for log-transformed parameters. 


\section{Chapter 6. PARAMETER-ESTIMATION PROCESS}

The parameter-estimation mode of MODFLOW-2000 is activated as indicated in table 3. MODFLOW-2000 estimates parameters using nonlinear regression, as discussed by Seber and Wild (1989), introduced into the ground-water literature by Cooley $(1977,1979,1982,1983 \mathrm{a}, \mathrm{b}$, 1985), Yeh and Yoon (1981), Yeh (1986), and Cooley and Naff (1990). Many of the ideas are adapted from linear regression, as presented by Draper and Smith (1998), among others. In MODFLOW-2000, the least-squares objective function is minimized by the modified GaussNewton method described in detail in the companion report by Hill (1998). That document is intended to be used in conjunction with the present report, so the methods used are simply listed below. The methods are followed by instructions for preparing the Parameter-Estimation Process input file.

\section{Modified Gauss-Newton Optimization}

The Parameter-Estimation Process uses the sum of squared, weighted residuals objective function (Hill, 1998, eq. 1), which also is called the least-squares objective function, to evaluate the fit of simulated to observed dependent-variable values (hydraulic heads, flows, and advective transport) and of parameter values to prior information. The contribution of the observed dependent variables to the objective function is calculated by the Observation Process, using results from the Ground-Water Flow Process; the contribution of prior information to the objective function is calculated by the Parameter-Estimation Process. Using the modified GaussNewton method, implemented as described in Hill (1998, p. 7-13, eq. 4; p. 77-82, Appendix B), the Parameter-Estimation Process attempts to determine a set of parameter values that are optimal in that they produce a minimum value of the least-squares objective function. For each parameter for all observations, the modified Gauss-Newton method requires sensitivities, which are calculated by the Observation Process using the results of the Sensitivity Process. The coordination of the Observation, Sensitivity, and Parameter-Estimation Processes was presented in figure 1 .

\section{Prior Information and its Weighting}

Prior information is information about parameter values that is independent of the observations used in the regression. Prior information is included in the weighted least-squares objective function along with the observations (Hill, 1998, p. 4), and can be thought of as a penalty function that encourages fitted parameter values to be close to their expected values. Care is needed in using prior information in ground-water problems because issues of scale and nonlinearity can make it unclear how the prior information actually relates to model values (Hill and others, 1998; Guadagnini and Neuman, 1999). Suggestions for using prior information are discussed in guideline 4 of Hill (1998, p. 43), which is 'Use prior information carefully'.

Like observations, prior information needs to be weighted. If the weighting reflects the uncertainty of the data upon which the prior information is based, the regression and calculated measures of model uncertainty fall within a Bayesian framework.

It is not uncommon to weight prior information to reflect greater certainty than is supportable by the data to achieve a solution to a regression problem; at the extreme, parameter values may be set and not allowed to be modified by the regression. If prior information is weighted to reflect greater certainty than is supportable by the data, the prior information needs to be categorized as regularization and calculated measures of model uncertainty will indicate greater certainty than is warranted (Backus, 1988). 
Like the Observation Process, the Parameter-Estimation Process allows the user to specify prior information uncertainty using statistics that are interpreted to be variances, standard deviation, or coefficients of variation, and the program calculates weights using the statistics. For parameters that are log-transformed, the statistics can be associated either with the native or logtransformed parameter. The regression needs the latter, and equation 27 is used to calculate the statistics associated with native values, if specified, to those associated with the log-transformed parameters.

\section{Input Instructions}

Input for the Parameter-Estimation Process is read from a file that is specified with file type "PES" in the name file (table 2).

0 . [\#Text ]

Item 0 is optional and can include as many lines as desired. Each line needs to begin with the "\#" character in the first column.

1. MAX-ITER MAX-CHANGE TOL SOSC (free format)

Item 1 includes variables that are most often changed by the user.

2. IBEFLG IYCFLG IOSTAR NOPT NFIT SOSR RMAR RMARM IAP (free format)

Item 2 includes variables that control the modified Gauss-Newton calculations and are sometimes changed by the user.

3. IPRCOV IPRINT LPRINT (free format)

Item 3 includes variables that control printing.

4. CSA FCONV LASTX (free format)

Item 4 includes variables that control the modified Gauss-Newton calculations and are rarely changed by the user.

5. NPNG IPR MPR (free format)

Item 5 includes variables that indicate whether additional items need to be read. The additional possible items are as follows.

If NPNG is greater than zero, read item 6 once.

6. PARNEG (1), PARNEG (2), . . , PARNEG (NPNG) (free format)

If IPR is greater than zero, read item IPR repetitions of item 7. Parameters that appear in item 7 may not appear in item 10.

7. NIPRNAM BPRI PLOT-SYMBOL (free format)

If IPR is greater than zero, read items 8 and 9 once. The size of item 9 depends on IPR

8. IWTP (free format)

9. $\operatorname{WTP}(1,1), \operatorname{WTP}(1,2), \ldots, \operatorname{WTP}(1, \operatorname{IPR})$ (free format)

$\operatorname{WTP}(2,1), \operatorname{WTP}(2,2), \ldots, \operatorname{WTP}(2, \operatorname{IPR}) \quad$ (new line; free format)

...

$\operatorname{WTP}(I P R, 1), W T P(I P R, 2), \ldots, W T P(I P R, I P R) \quad$ (new line; free format)

If MPR is greater than zero, read MPR repetitions of item 10. Examples are shown after the next section. Parameters that appear in item 7 may not appear in item 10.

10. EQNAM PRM $"="$ [SIGN] [COEF "*"] PNAM [SIGN [COEF "*"] PNAM [SIGN...]] "STAT" STATP STAT-FLAG PLOT-SYMBOL 


\section{Chapter 6. PARAMETER-ESTIMATION PROCESS}

(free format: maximum of 200 characters; one or more spaces must separate all words, numbers, and symbols)

\section{Example Input File}

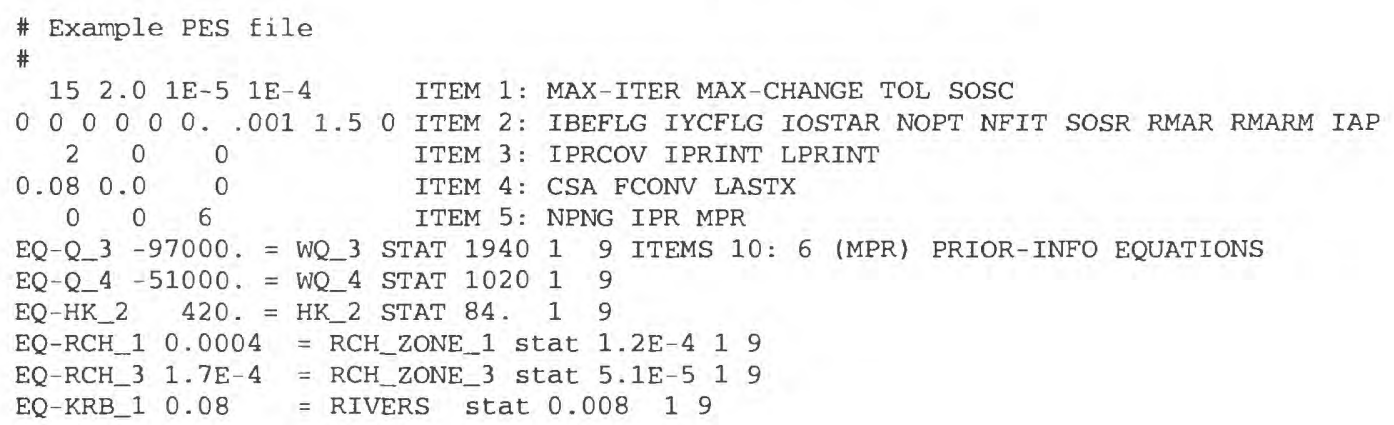

In this example, WQ_3, WQ_4, HK_2, RCH_ZONE_1, RCH_ZONE_3, and RIVERS are parameter names, which need to be defined in input files for Ground-Water Flow Process packages and need to be listed with ISENS $>0$ in the Sensitivity Process input file. The program matches parameter names among the various input files in a case-insensitive manner. EQ-Q_3, $\mathrm{EQ}-\mathrm{Q} \_4, \mathrm{EQ}-\mathrm{HK} \_2, \mathrm{EQ}-\mathrm{RCH} \_1, \mathrm{EQ}-\mathrm{RCH} \_3$, and $\mathrm{EQ}-\mathrm{KRB} \_1$ are names assigned to the 6 (MPR) prior-information equations.

\section{Explanation of Variables}

Text-is a character string (maximum of 79 characters) that starts in column 2 . Any characters can be included in Text. The "\#" character needs to be in column 1. Text is printed when the file is read and provides an opportunity for the user to include information about the model both in the input file and the associated output file.

MAX-ITER-is the maximum number of parameter-estimation iterations. If MAX-ITER $=0$, the program calculates the variance-covariance matrix on parameters and related statistics (the parameter correlation coefficients generally are of most interest) using the starting parameter values from the Sensitivity Process input file, and parameter estimation stops after one iteration.

MAX-CHANGE-is the maximum fractional change for parameter values in one iteration (Hill, 1998, eq. 5, p. 9). MAX-CHANGE commonly equals 2.0, or less if parameter values are unstable during parameter-estimation iterations.

TOL-is the parameter-estimation closure criteria, as a fractional change in parameter values (Hill, 1998, eq. 7, p.12). TOL commonly equals 0.01 . Larger values often are used during preliminary calibration efforts; values as small as 0.001 may be used for theoretical work.

SOSC - is the second convergence criterion discussed in Hill (1998, p. 12). If SOSC $\neq 0.0$, parameter estimation will converge if the least-squares objective function does not decrease by more than SOSC $\times 100$ percent over two parameter-estimation iterations. SOSC usually equals 0.0. Typical nonzero values of SOSC are 0.01 and 0.05 .

IBEFLG-is a flag that controls the generation of files to be used as input to the post-processing program BEALE-2000, which tests model linearity.

$\mathrm{IBEFLG}=0$, no file for BEALE-2000 is produced.

$\mathrm{IBEFLG}=1$, the _bl file of tables 5 and 12 is produced. 
$\mathrm{IBEFLG}=2$, the _b2 file of tables 5 and 13 is produced. Production of this file may require MODFLOW-2000 input files that differ from the files used for model calibration, as discussed in chapter 7 .

IYCFLG-is a flag that controls the generation of files to be used as input to the post-processing program YCINT-2000, which calculates confidence and prediction intervals on simulated equivalents to observations.

IYCFLG $=0$, The _y0 file of tables 5 and 9 is produced. Production of this file may require MODFLOW-2000 input files that differ from the files used for model calibration.

IYCFLG $=1$, The _y 1 file of tables 5 and 10 is produced. Production of this file may require MODFLOW-2000 input files that differ from the files used for model calibration, as discussed in chapter 7. Sensitivities for the predicted quantities are calculated, but the calculations related to nonlinear regression and the variancecovariance matrix on parameters are not made.

IYCFLG $=2$, The _y2 file of tables 5 and 11 is produced. This file is needed if confidence and prediction intervals on differences (eq. 7) are to be calculated. Production of this file may require MODFLOW-2000 input files that differ from the files used for model calibration, as discussed in chapter 7. Sensitivities for the predicted quantities are calculated, but the calculations related to nonlinear regression and the variance-covariance matrix on parameters are not made.

IOSTAR - is a flag that controls printing to the screen. If IOSTAR equals one, printing to the screen is suppressed. Usually IOSTAR $=0$.

NOPT - is a flag identifying whether or not to include matrix $\underline{R}$ of equation (B1) in equation (4a), as described in Hill $(1998$, p. 8, 78). Regression may converge in fewer iterations with NOPT $=1$ for problems with large residuals and a large degree of nonlinearity.

NFIT - is the number of Gauss-Newton iterations (when NOPT equals 1 ) after which matrix $\underline{R}$ of equation (Bl) is included in equation (4a) of Hill $(1998$, p. 8,79$)$.

SOSR - is a criterion for using $\underline{R}$ of equation (B1) in equation (4a) of Hill (1998, p. 8,78). Matrix $\underline{R}$ is used if the percentage change in the sum of squared, weighted residuals does not exceed SOSR*100 in two parameter-estimation iterations. Usually SOSR equals 0.0.

RMAR - is used along with RMARM to calculate the Marquardt parameter, if its use is indicated based on CSA of item 4. The calculation of the Marquardt parameter described by Hill $\left(1998\right.$, p. 9) is expressed as $m_{s}^{\text {new }}=R M A R M \times m_{r}^{\text {old }}+R M A R$. Typically, $R M A R=0.001$.

RMARM - is used along with RMAR to calculate the Marquardt parameter, if its use is indicated based on CSA of item 4. The calculation of the Marquardt parameter described by Hill $(1998, p .9)$ is expressed as $m_{r}^{\text {new }}=R M A R M \times m_{r}^{\text {old }}+$ RMAR. Typically, RMARM $=1.5$.

IAP - is a flag identifying whether, for log-transformed parameters, MAX-CHANGE applies to the native parameter value or to the log transform of the parameter value. Generally, IAP $=0$.

IAP $=0$, MAX-CHANGE applies to the native parameter value.

$\mathrm{IAP}=1$, MAX-CHANGE applies to the log transform of the parameter value.

IPRCOV - is a format code for printing of variance-covariance and correlation matrices.

Permissible values of IPRCOV and corresponding formats are:

\begin{tabular}{|c|c|c|c|}
\hline IPRCOV & FORMAT & IPRCOV & FORMAT \\
\hline 1 & $11 \mathrm{G} 10.3$ & 6 & $6 \mathrm{G} 10.3$ \\
\hline
\end{tabular}




\begin{tabular}{|c|c|c|c|}
\hline 2 & $10 \mathrm{G} 11.4$ & 7 & $5 \mathrm{G} 11.4$ \\
\hline 3 & $9 \mathrm{G} 12.5$ & 8 & $5 \mathrm{G} 12.5$ \\
\hline 4 & $8 \mathrm{G} 13.6$ & 9 & $4 \mathrm{G} 13.6$ \\
\hline 5 & $8 \mathrm{G} 14.7$ & 10 & $4 \mathrm{G} 14.7$ \\
\hline
\end{tabular}

IPRINT - is a flag that controls printing of various statistics computed for each parameterestimation iteration, including simulated equivalents, unweighted and weighted residuals, observation sensitivities, summary statistics for residuals by observation type, scaled least-squares matrix of the Gauss-Newton method, and scaled gradient vector of the objective function.

IPRINT $=0$, the statistics are printed at the first and last parameter-estimation iterations.

IPRINT $>0$, the statistics are printed at each iteration. Also, a summary of parameter values and statistics for all parameter-estimation iterations is printed in the GLOBAL output file.

LPRINT - is a flag that controls printing of eigenvalues and eigenvectors.

LPRINT $=0$, eigenvalues and eigenvectors are not printed.

LPRINT > 0 , if parameter estimation converges, eigenvalues and eigenvectors are printed.

CSA - is the search-direction adjustment parameter used in the Marquardt procedure. Usually equals 0.08 .

FCONV -is a flag and a value used to allow coarser solver convergence criteria for early parameter-estimation iterations. If FCONV equals zero, coarser convergence criteria are not used. Commonly, $\mathrm{FCONV}=0.0$; typical nonzero values would be 5.0 or 10.0 , and these can produce much smaller execution times in some circumstances.

LASTX - is a flag that controls calculation of the sensitivities used to calculate the parameter variance-covariance matrix when parameter estimation converges.

LASTX $=0$, sensitivities from the last parameter-estimation iteration are used to calculate the variance-covariance matrix. The program proceeds as in figure 1 .

LASTX $>0$, sensitivities are recalculated using the final parameter estimates and are used to calculate the variance-covariance matrix.

NPNG-is the number of parameters of type HK, VK, VANI, VKCB, SS, SY, or EVT that can have negative values. This is useful for some interpolation methods in which, for example, deviations from a base value are calculated, where the deviations can be positive or negative. An example of such a method is described by Keidser and Rosbjerg (1991). If NPNG is greater than zero, item 6 is read.

IPR - is the number of parameters included in the full variance-covariance matrix used to weight the prior information.

MPR - is the number of prior-information equations to be used in the regression.

PARNEG-is an array of NPNG names of parameters of type HK, VK, VANI, VKCB, SS, SY, or EVT that can have negative values. This may be the case when the second kriging method discussed in Hill (1992, p. 125) is used.

NIPRNAM - is the name of one of the IPR parameters for which a variance-covariance matrix for prior parameter estimates is to be read.

BPRI-is the prior estimate for parameter NIPRNAM. 
PLOT-SYMBOL-is an integer that will be written to output files intended for graphical analysis to allow control of the symbols used when plotting data related to the prior information.

IWTP - is a flag identifying how the weight matrix for correlated prior information is to be calculated using the values in array WTP specified in item 9.

IWTP $=0$, WTP is a variance-covariance matrix. The diagonal terms of this matrix are the variances of the prior information, the off-diagonals are the covariances. Diagonal term WTP(I,I) is the variance for the parameter designated by NIPRNAM(I); off-diagonal term WTP(I,J) is the covariance for the parameters designated by NIPRNAM(I) and NIPRNAM(J). For parameters specified as being log-transformed in the Sensitivity Process input file, the corresponding WTP elements are interpreted as being relative to the log-transformed value (using log base 10 ). The weight matrix is calculated by taking the inverse of the array specified in item 9.

IWTP $=1$, WTP is a matrix of coefficients of variation (the standard deviation divided by the prior information value) and correlation coefficients. The diagonal terms are the coefficients of variation of the prior information; off-diagonals are the correlation coefficients and vary in value from -1.0 to +1.0 . Diagonal term WTP(I,I) is the coefficients of variation for the parameter designated by NIPRNAM(I); off-diagonal term WTP(I,J) is the correlation coefficients for the parameters designated by NIPRNAM(I) and NIPRNAM(J). For parameters specified as being logtransformed in the Sensitivity Process input file, the corresponding WTP elements are interpreted as being relative to the log-transformed value (using log base 10). The weight matrix is calculated by in two steps. First, the coefficients of variation and correlation coefficients are used to calculate variances and covariances, and then the inverse of the variance-covariance matrix is calculated. In calculating variances, the coefficients of variation are multiplied by the prior information values specified in item 7 , or the $\log _{10}$ of that value for log-transformed parameters; if the value equals zero, 1.0 is used instead.

WTP-is an IPR by IPR array containing statistics used to calculate the weight matrix for correlated prior information. The statistics specified depends on the value of IWTP. Note that the matrix is symmetric, but the entire matrix (upper and lower parts) must be entered.

EQNAM - is a user-supplied name (up to 10 nonblank characters) for a prior-information equation.

PRM - is the prior estimate for prior-information equation EQNAM. PRM always needs to be specified as a native, untransformed value. That is, even if the parameter is specified as being log-transformed in the Sensitivity Process input file, here PRM needs to be the untransformed value. The program will calculate the log-transformed value.

$"="$ indicates that an equal sign (without quotes) must be entered literally.

SIGN-is either "+" or "-" (entered without quotes). The SIGN before the first PARNAM is assumed to be "+" unless otherwise indicated.

$\mathrm{COEF}$ - is the coefficient for the parameter following the "*" in prior-information equation EQNAM. COEF can be specified with or without a decimal point and can be specified in scientific notation.

"*"-_indicates that an asterisk (without quotes) must be entered literally if a value for COEF is entered. 
PNAM - is a parameter name (up to 10 nonblank characters) as specified in the SEN file. If the parameter is designated in the Sensitivity Process input file as being log-transformed (LN greater than 0 ), the prior-information equation may contain only one parameter name. If a prior-information equation contains no log-transformed parameters, the equation may contain any number of terms, where each term is defined by the sequence: SIGN [COEF "*"] PNAM.

"STAT"-indicates that the word "STAT" (without quotes) must be entered literally, although it may be in any combination of upper- and lowercase letters.

STATP — is the value from which the weight for prior-information equation EQNAM is calculated, as determined using STAT-FLAG. If a parameter is specified as being logtransformed in the Sensitivity Process input file, STATP may be specified relative either to the native value or to the log-transformed value (using log base 10), depending on the value of STAT-FLAG.

STAT-FLAG-is a flag identifying how the weight for prior-information equation EQNAM is to be calculated. This depends both on whether the user chooses to specify the variance, standard deviation, or coefficient of variation, and whether, for log-transformed parameters, the user chooses to specify the statistic related to the native, untransformed parameter, or to the transformed parameter.

STAT-FLAG $=0$, STATP is the variance associated with PRM, and is related to the native prior value. Weight $=1 /$ STATP unless the parameter is defined as logtransformed in the Sensitivity Process input file, in which case equation 27 is used to convert STATP (which equals $\sigma_{b}^{2}$ of equation 27) to $\sigma_{\ln b}^{2}$, and weight $=1 / \sigma_{\ln b^{\circ}}^{2}$

STAT-FLAG $=1$, STATP is the standard deviation associated with PRM, and is related to the native prior value. Weight $=1 /$ STATP $^{2}$ unless the parameter value is defined as log-transformed in the Sensitivity Process input file, in which case equation 27 is used to convert STATP (which equals $\sigma_{\mathrm{b}}$ of equation 27) to $\sigma_{\ln \mathrm{b}}^{2}$, and weight $=1 / \sigma_{\ln b}^{2}$.

STAT-FLAG $=2$, STATP is the coefficient of variation associated with PRM, and is related to the native prior value. Weight $=1 /(\text { STATP } \times \text { PRM })^{2}$ unless the parameter is defined as log-transformed in the Sensitivity Process input file, in which case equation 27 is used to convert STATP (which equals $\sigma_{b} /$ b of equation 27), to $\sigma_{\ln b}^{2}$, and weight $=$ $1 / \sigma_{\mathrm{In} b^{*}}^{2}$

STAT-FLAG $=10$, STATP is the variance associated with the $\log$ (base 10) transform of PRM; weight $=1 /\left[\right.$ STATP $\left.\times 2.3026^{2}\right]$.

STAT-FLAG $=11$, STATP is the standard deviation associated with the log (base 10) transform of PRM; weight $=1 /\left[\mathrm{STATP}^{2} \times 2.3026^{2}\right]$.

STAT-FLAG $=12$, STATP is the coefficient of variation associated with the log (base 10) transform of PRM; weight $=1 /\left[\left(\text { STATP } \times \log _{10}(\mathrm{PRM})\right)^{2} \times 2.3026^{2}\right]$.

\section{Additional Examples of Prior Information Equations} the form:

As noted above, each of MPR prior-information equations needs to be designated using

EQNAM PRM $"="$ [SIGN]
PNAM $[$ [SIGN...] ]


Because this expression can be difficult to decipher, a few additional examples are provided here.

First, consider a recharge parameter named RCH1 (in $\mathrm{m} / \mathrm{d}$ ) for which field data are available. As described by Hill (1998, guideline 6, p. 45), the available information needs to be expressed in probabilistic terms to assign prior information for the regression. Upon further consideration, perhaps it is decided that the field data indicate that the recharge is about $22 \mathrm{~cm} / \mathrm{yr}$, and that the standard deviation of this estimate is $5 \mathrm{~cm} / \mathrm{yr}$. If this prior information is given the name PRCH1, for prior information on $\mathrm{RCH} 1$, the line in the Parameter-Estimation Process input file would be:

PRCH1 $22=36500 *$ RCH1 STAT 5.014

The coefficient 36500 converts the units of the model parameter to the units of the prior information, assuming 365 days per year. Alternatively, the units of the parameter could have been adjusted when the parameter was defined. The latter approach often is useful to avoid confusion concerning units. The remaining elements in the example are the required key word "STAT", the value of 5.0 used for weighting, which the " 1 " indicates is the standard deviation of the native, untransformed value (note that recharge parameters are not allowed to be logtransformed), and a PLOT-SYMBOL of 4. The PLOT-SYMBOL plays no functional role in MODFLOW-2000, but is written whenever data about the prior information is written so that it can be used to control plot symbols. For example, often all prior information is represented with the same plot symbol to aid interpretation of residual plots (see, for example, Hill, 1998, p. 61).

As a second example, consider hydraulic-conductivity parameter $\mathrm{K} 1$ for which pumpingtest data and a geologic depositional interpretation are available. Given this information, it is concluded that $\mathrm{Kl}$ equals about $10 \mathrm{~m} / \mathrm{d}$, and that this value is accurate to within an order of magnitude. This can be represented correctly if $\mathrm{K} 1$ is specified as being log-transformed in the Sensitivity-Process input file. The appropriate standard deviation can be determined using the method described by Hill (1998, p. 48) to be 0.5 , by assuming (1) that there is a 95 percent chance that the true value falls within an order of magnitude of the estimate, and (2) that the uncertainty in the log-transformed value is adequately represented by a normal probability distribution. Letting EQNAM be the string PK1 (for "Prior on K1"), this prior information would be defined in the Parameter-Estimation input file as:

PK1 $10=$ K1 STAT $0.5 \quad 11 \quad 5$

where here the plot-symbol is 5 .

As a third example, consider a storage coefficient that has been determined from a pumping test in which the screened interval completely intersects material which is represented by two model layers thought to have distinct values of specific storage which are represented by parameters S1 and S2. The local thicknesses of the two model layers are 20 and 30 meters. The storage coefficient value determined from the pumping test is 0.02 and is thought to be accurate to within a factor of 10 , as in the last example. The user would like this prior information to be plotted using the same symbol as the prior information defined in the last example. Letting EQNAM be the string PS1\&2 (for "Prior on specific storage 1 and 2"), this prior information would be defined in the Parameter-Estimation input file as:

$\operatorname{PS} 1 \& 20.02=20 * \mathrm{~S} 1+30 * \mathrm{~S} 2$ STAT 0.5115 


\section{Chapter 7. POST-PROCESSING PROGRAMS RESAN-2000, YCINT-2000, AND BEALE-2000}

Thorough analysis of a calibrated model requires that the match achieved to the observations be evaluated and presented to the users. In addition, it often is useful to evaluate the relative dominance of the different observations in parameter estimation. Finally, when model predictions are to be used for resource management, remediation planning, and so on, the uncertainty of the predictions needs to be communicated along with the predictions themselves. To address these issues, three post-processing programs are provided as part of MODFLOW2000 , and their use is described in this document. Additional information about the analyses and statistics are provided in cited references, and especially in Hill (1994 and 1998).

The descriptions provided here include short statements of the purpose of the program, descriptions of the input files, all of which are produced by MODFLOW-2000, and a listing of the steps that need to be followed to execute the program. Use of the output files produced by MODFLOW-2000, including the post-processing programs, is discussed in chapter 8.

\section{Using RESAN-2000 to Test Weighted Residuals and Identify Influential Observations}

RESAN-2000 performs two functions, as described below. RESAN-2000 is the most commonly used of the post-processing programs. Often it is advisable to include it in the script or macro being used to execute MODFLOW-2000 so that the results are always available.

The first function performed by RESAN-2000 is to test the weighted residuals for acceptable deviations from being independent (lacking any correlation) and normally distributed, as suggested by Draper and Smith (1998), Cooley and Naff (1990), and Hill (1998, p. 24). Deviations are characterized using normal probability graphs of the weighted residuals (produced using the _nm file of tables 5 and 16) and normal probability graphs of generated random numbers (produced using the files with extensions_rd and_rg of tables 6 and 17). Two types of generated random numbers are considered: (1) independent and (2) correlated as expected for the weighted residuals considering the regression performed. Correlated weighted residuals can result from the fitting process of the regression.

The weighted-residual test needs to be conducted if the weighted residuals normal probability graph, produced using the _nm file, does not approximate a straight line (Hill, 1998, p. 23-24). Greater deviations from a straight line indicates a greater chance that the weighted residuals cannot be considered as random and normally distributed. A statistic, $\mathrm{R}_{\mathrm{N}}{ }^{2}$ (Hill. 1998, eq. 25; critical values are listed in Appendix D), printed in the GLOBAL file is useful; values of $\mathrm{R}_{\mathrm{N}}^{2}$ that are too much less than 1.0 indicate that the weighted residuals are less likely to be independent and normally distributed. A message printed in the GLOBAL output file compares the calculated value of $R_{N}^{2}$ to the appropriate critical values and states the conclusion to be drawn from this comparison. To test the weighted residuals, RESAN-2000 needs to be executed only if the weighted residuals deviate significantly from being normally and independently distributed, as indicated by small values of $\mathrm{R}_{\mathrm{N}}{ }^{2}$ and normal probability graphs on which the points do not fall on a straight line. 
The second function of RESAN-2000 is to calculate statistics that can be used to identify observations that are influential in the regression. The statistics calculated are Cook's D and DFBetas, which are described by Beasley and others (1980) and Cook and Weisberg (1982), and applied to the development of a ground-water model by Yager (1998).

To produce the _w and _rs input files used by RESAN-2000, MODFLOW-2000 needs to be run in the Sensitivity Analysis or Parameter-Estimation mode (table 3) and OUTNAM in the Observation Process input file must be specified as a string other than "NONE". The _rs file contains the information listed in table 8; the contents of the _w file are listed in table 5 . RESAN2000 can then be executed and will ask for the name of the name file used to execute MODFLOW-2000, which it will use to find the _rs and _w files.

The number of sets of random deviates (NSETS) in the _rs file (table 8) is set to four by MODFLOW-2000, which should be sufficient. Additional sets may be desired, however, to conclusively test a set of residuals. In such circumstances, the value of NSETS needs to be changed in the _rs file and RESAN-2000 needs to be executed. Sequential executions of RESAN2000 will not correctly produce random numbers for additional sets.

Table 8: Information contained in the _rs file of table 5, which is produced by MODFLOW-2000 and used by the post-processing program RESAN-2000

\begin{tabular}{|c|c|c|c|}
\hline Item & Format & Variables & Description \\
\hline 1 & $6 \mathrm{I} 5, \mathrm{I} 10, \mathrm{~F} 13.0$ & $\begin{array}{l}\text { NPE, ND, } \\
\text { NH, NQT, } \\
\text { MPR, IPR, } \\
\text { NSETS, } \\
\text { NRAN, } \\
\text { VAR }\end{array}$ & $\begin{array}{l}\text { Number of estimated parameters, } \\
\text { number of observations, } \\
\text { number of head observations, } \\
\text { number of observations other } \\
\text { than heads, } \\
\text { number of prior information } \\
\text { equations, number of prior with } \\
\text { a full weight matrix, } \\
\text { number of sets of random } \\
\text { deviates } \\
\text { number for random number } \\
\text { generator, } \\
\text { calculated error variance }\end{array}$ \\
\hline 2 & $6(\mathrm{~A} 10,1 \mathrm{X})$ & PARNAM & Parameter names \\
\hline 3 & $16 \mathrm{~F} 13.0$ & $\mathrm{COV}(\mathrm{NP}, \mathrm{NP})$ & $\begin{array}{l}\text { Parameter variance-covariance } \\
\text { matrix }\end{array}$ \\
\hline 4 & $16 \mathrm{~F} 13.0$ & $\mathrm{WT}(\mathrm{NH})$ & $\begin{array}{l}\text { Weights for the head } \\
\text { observations }\end{array}$ \\
\hline 5 & $16 \mathrm{~F} 13.0$ & WQ(NQT,NQT) & $\begin{array}{l}\text { Full weight matrix for } \\
\text { observations other than heads }\end{array}$ \\
\hline 6 & $16 \mathrm{~F} 13.0$ & $\mathrm{X}(\mathrm{NP}, \mathrm{ND})$ & $\begin{array}{l}\text { Sensitivities for all parameters } \\
\text { and observations }\end{array}$ \\
\hline 7 & $16 \mathrm{~F} 13.0$ & $\begin{array}{l}\text { PRM(NP,I), } \\
\text { WP(I), I=1,MPR }\end{array}$ & $\begin{array}{l}\text { Coefficients and weights for the } \\
\text { prior information equations. }\end{array}$ \\
\hline 8 & $16 \mathrm{~F} 13.0$ & NIPR(IPR) & $\begin{array}{l}\text { Parameters with prior } \\
\text { information with a full weight } \\
\text { matrix. }\end{array}$ \\
\hline 9 & $16 \mathrm{~F} 13.0$ & WTPS(IPR,IPR) & $\begin{array}{l}\text { Square-root of the full weight } \\
\text { matrix for prior information }\end{array}$ \\
\hline
\end{tabular}




\section{Using YCINT-2000 to Calculate Linear Confidence and Prediction Intervals on Predictions and Differences Simulated with Estimated Parameter Values}

Predictions produced by a calibrated model should be reported with an evaluation of prediction uncertainty. Use of regression in model calibration, as supported by MODFLOW2000 , provides clear methods by which the uncertainty with which the parameters are estimated can be propagated into measures of uncertainty for the predictions. If the model is designed such that the defined parameters include those aspects of the system that are both least well known and are most important to predictions, the measures of uncertainty discussed here are likely to closely approximate actual prediction uncertainty. Suggestions for defining parameters are discussed in guideline 3 of Hill (1998, p. 38).

To facilitate the analysis of uncertainty, post-processing program YCINT-2000, which is distributed with MODFLOW-2000, can be used to calculate 95-percent linear confidence and prediction intervals on predicted values and differences calculated using predicted values. The advantages and disadvantages of using these linear intervals to quantify model uncertainty are discussed by Hill (1998, p. 29-31) and references cited therein. The situations in which the different types of intervals are applicable are discussed in Hill $(1998$, p. 29-31) and in chapters 3 and 8 of this report.

YCINT-2000 calculates confidence and prediction intervals on simulated values that are equivalent to the types of values that can be represented by the Observation Process. Simulated quantities might be hydraulic head, temporal change in hydraulic head, streamflow gain or loss, flow to or from a constant-head boundary, or advective transport. The model run(s) used to generate the simulated values may simulate, for example, potential future pumpage, a climatechange scenario, and so on. The capabilities of the Observation Process are used in special runs of MODFLOW-2000 to produce the input files for YCINT-2000.

The intervals of interest generally need to be produced in a single execution of YCINT2000. YCINT-2000 requires two input files, and it also requires a third if intervals are to be constructed on differences (eq. 7). The first two input files are the _y0 and _y 1 files; the third is the _y2 file (all are listed in table 5). To produce these files, OUTNAM needs to be specified as a string other than 'NONE' in the Observation Process input file for all runs and needs to be the same for the runs that produce the _y0, _y 1 , and _y2 files.

Generally the _y 1 and _y 2 files, and sometimes the _y0 file, need to be produced using extra runs of MODFLOW-2000. The reasons for requiring extra runs are as follows.

1. For the _y0 file, an extra run is needed if anything related to the parameters differs from the calibration run. Parameters need to be represented differently than during calibration when calculating confidence and prediction intervals if the parameters were (a) held constant or (b) assigned prior information with smaller statistics than supportable by independent measurements. While these methods can be valid ways to constrain the estimated parameter values sufficiently to attain a stable regression during model calibration, it is important that the actual uncertainty in the parameters be included in the calculation of confidence and prediction intervals (Hill, 1998, p. 17, 25-26, 31, fig. 16). Parameters that were held constant need to be activated, and appropriate prior information applied if warranted by independently available data. Parameters assigned prior information with statistics that were smaller than supportable by independent data need to be assigned statistics that are consistent with the independent data.

2. For the _y 1 and _y 2 files, Observation Process input file(s) are used to define the predictions of interest instead of observations. These are different files than those used for model 
calibration and they define predictions, not observations. As discussed in chapter 3 , the number of predictions listed is used to determine the critical value of the simultaneous confidence and prediction intervals, so that the number of predictions included is important if simultaneous intervals are to be used.

3. For the _yl and _y2 files, prediction conditions often are different than calibration conditions. The prediction conditions generally are imposed through changes in GroundWater Flow Process input files. For example, changes in pumpage can be imposed using the Well Package input file and changes in areal recharge caused by climate change can be imposed using the Recharge Package input file. Uncertainty in parameters characterizing such stresses can be included in the calculation of confidence and prediction intervals.

The contents of the _y0,_y 1, and_y2 files are listed in tables 9, 10, and 11. Generally these input files are not accessed by the user.

Table 9: Information contained in the _y0 file of table 5, which is produced by MODFLOW-2000 when IYCFLG $=0$ and is used by the post-processing program YCINT-2000.

\begin{tabular}{|c|c|c|c|}
\hline Item & Format & Variables & Description \\
\hline 1 & free & NDCALIB & Number of observations used in regression \\
\hline 2 & free & MPR & Number of prior-information equations \\
\hline 3 & free & IPR & $\begin{array}{c}\text { Number of parameters included in the full variance- } \\
\text { covariance matrix used to weight the prior } \\
\text { information }\end{array}$ \\
\hline 4 & free & IDIF & $\begin{array}{c}\text { Flag indicating whether intervals are to be } \\
\text { calculated on differences (1 for differences, } \\
\text { otherwise 0) }\end{array}$ \\
\hline 5 & Free & PARNAM(NVAR) & Parameter names (NVAR is read from the_yl file) \\
\hline 6 & 16 F13.0 & C(NVAR,NVAR) & Parameter variance-covariance array \\
\hline
\end{tabular}


Table 10: Information contained in the _yl file of table 5, which is produced when IYCFLG=1 in the Parameter-Estimation Process input file. Production of this file may require a MODFLOW-2000 run that differs from the model-calibration runs; see text.

\begin{tabular}{|c|c|c|c|}
\hline Item & Format & Variables & Description \\
\hline 1 & 4110 & $\begin{array}{c}\text { NVAR, NINT, } \\
\text { NH, IFSTAT }\end{array}$ & $\begin{array}{c}\text { Number of parameters, number of intervals, number } \\
\text { of intervals on heads, flag indicating whether to read } \\
\text { user-specified critical values (item 2) }\end{array}$ \\
\hline 2 & Free & $\begin{array}{c}\text { STATIND, } \\
\text { STATSF, } \\
\text { FSTATSI, } \\
\text { FSTATKGTNP }\end{array}$ & $\begin{array}{c}\text { Item 2 is read only if IFSTAT > 0. User-specified } \\
\text { critical values for: individual intervals, finite } \\
\text { number of simultaneous intervals, undefined } \\
\text { number of simultaneous intervals, simultaneous } \\
\text { prediction intervals when K > NP }\end{array}$ \\
\hline 3 & $6(\mathrm{~A} 12,1 \mathrm{X})$ & PREDNAM(NINT) & Name assigned to each prediction \\
\hline 4 & 1615 & ISYM(NINT) & Plot symbol associated with each prediction \\
\hline 5 & $6 \mathrm{~F} 13.0$ & PRED(NINT) & Simulated value of the prediction \\
\hline 6 & $8 \mathrm{~F} 10.0$ & V(NH) & $\begin{array}{r}\text { Variance of the error with which the predicted heads } \\
\text { could be measured. }\end{array}$ \\
\hline 7 & $8 \mathrm{~F} 10.0$ & WQ(NDMH) & $\begin{array}{r}\text { Variance of the error for predictions other than } \\
\text { heads (NDMH = NINT }- \text { NH) }\end{array}$ \\
\hline 8 & $6 \mathrm{~F} 13.0$ & X(NVAR,NINT) & $\begin{array}{c}\text { Sensitivities of the prediction quantities with respect } \\
\text { to the parameters. }\end{array}$ \\
\hline
\end{tabular}

Table 11: Information contained in the _y2 file of table 5, which is produced when IYCFLG $=2$ in the Parameter-Estimation Process input file. Production of this file may require a MODFLOW-2000 run that differs from the model-calibration runs; see text.

\begin{tabular}{|c|c|c|c|}
\hline Item & Format & Variables & Description \\
\hline 1 & $6(\mathrm{~A} 12,1 \mathrm{X})$ & PREDNAM(NINT) & Name assigned to each base quantity \\
\hline 2 & $16 \mathrm{I} 5$ & ISYM1(NINT) & Plot symbol associated with each base quantity \\
\hline 3 & $6 \mathrm{~F} 13.0$ & PRED(NINT) & Simulated value of base quantity \\
\hline 4 & $8 \mathrm{~F} 10.0$ & $\mathrm{~V}(\mathrm{NH})$ & $\begin{array}{c}\text { Variance of the error with which the base quantity } \\
\text { heads could be measured. }\end{array}$ \\
\hline 5 & $8 \mathrm{~F} 10.0$ & WQ(NDMH) & $\begin{array}{c}\text { Variance of the error for base quantities other than } \\
\text { heads (NDMH = NINT - NH) }\end{array}$ \\
\hline 6 & $6 \mathrm{~F} 13.0$ & $\mathrm{X}(\mathrm{NVAR}, \mathrm{NINT})$ & $\begin{array}{c}\text { Sensitivities of the base quantities with respect to } \\
\text { the parameters. }\end{array}$ \\
\hline
\end{tabular}


To use YCINT-2000, first generate an _y0 file using steps 1 through 6.

1. Make sure the appropriate parameter values are in the Sensitivity Process input file. Often this requires that the final calibrated parameter values from the _b file be substituted into the Sensitivity Process input file. Also, often more parameters are active for calculating confidence and prediction intervals than for regression, and prior information on some of the unestimated parameters may need to be defined in the Parameter-Estimation Process input file for the additional parameters (see Hill, 1998, p. 25). Finally, prior information in the Parameter-Estimation input file may need to have different statistic values specified than those used for model calibration. This occurs when the statistic used for calibration indicates more certainty in the prior value than can be justified given the available data.

2. In the Observation Process input file set OUTNAM to a string other than 'NONE'.

3. Activate the Parameter-Estimation Process. Set IYCFLG $=0$. IBEFLG in the ParameterEstimation Process input file may be specified as 0 or 1, but not 2. MAX-ITER may be set to 0 .

4. In all other respects the input files for MODFLOW-2000 need to be the same as they were for calibration.

5. Execute MODFLOW-2000.

6. Once the _y0 file is created, it needs to be edited if differences and confidence and prediction intervals for differences are to be calculated. In this circumstance, the variable IDIF needs to be changed from 0 to 1 ; IDIF is read from item 4 of the _y0 file in free format.

Next, generate an_yl file using steps 7 through 11 .

7. If not done in step 1 to generate the _y0 file, substitute the final calibrated parameter values from the _b file into the Sensitivity Process input file.

8. In the Parameter-Estimation Process input file, set IYCFLG $=1$ to generate an $\_y 1$ file. IBEFLG may be specified as 0 or 1 , but not 2 . Set MAX-ITER $=0$ so the final calibrated parameter values listed in the Sensitivity Process input file remain unchanged.

9. Modify the observation package files (for example, files with file type HOB, DROB, and so forth; see table 2) to define only the quantities for which confidence and prediction intervals are to be calculated.

10. Change input files for flow-process packages to represent the stresses, boundary conditions, and so on of the system for which predictions are being made.

11. Execute MODFLOW-2000.

To have YCINT calculate confidence or prediction intervals on differences (done when IDIF is set to 1 in the _y0 file, as described in step 6 above), an _y 2 file is needed and can be generated using steps 12 through 16.

12. Use the same Sensitivity Process input file used to generate the _yl file.

13. Use the same observation package files (for example, HOB, DROB, and so forth) used to generate the _yl file.

14. In the Parameter-Estimation Process input file, set IYCFLG $=2$ to generate an _y2 file. Set MAX-ITER $=0$ so the final calibrated parameter values in the Sensitivity Process input file remain unchanged. 
15. Modify the flow-process package files to define the base conditions.

16. Execute MODFLOW-2000.

17. Finally, execute YCINT.

Output will be to files with extensions \#yc,_yp, and_yd (table 6).

A potential problem occurs when a combination of predictions and differences is included in the $\mathrm{k}$ values. This is because critical values for the Bonferroni and Scheffé $\mathrm{d}=\mathrm{k}$ intervals for predictions are determined by YCINT-2000 using the number of predictions, and the critical values for differences using the number of differences. This problem can be resolved by defining enough predictions or differences to make the number used to determine the critical values the desired number $\mathrm{k}$, and then ignoring results that are not of interest. 


\section{Using BEALE-2000 to Test Model Linearity}

The linear intervals produced by YCINT-2000 can accurately reflect the uncertainty of the simulated values only if the model is sufficiently linear (Seber and Wild, 1989; Cooley and Naff, 1990; Hill, 1994; Hill, 1998, p. 31-32). Model nonlinearity can be tested using the modified Beale's measure presented by Cooley and Naff (1990) and also discussed by Hill (1994); the modified Beale's measure should be reported for all calibrated models. Ground-water models are nearly always nonlinear with respect to estimated parameter values, as discussed in chapter 2 of this report. Although the modified Gauss-Newton optimization method and many of the statistical methods calculated by MODFLOW-2000 and discussed by Hill (1998) are useful even for problems which are quite nonlinear, more stringent requirements on linearity are needed for the linear confidence and prediction intervals produced by YCINT-2000 (discussed below) to adequately represent uncertainty. The modified Beale's measure can indicate the possible severity of the problem.

The modified Beale's measure indicates nonlinearity of the confidence region of the parameters and does not directly measure nonlinearity of the confidence and prediction intervals. One consequence of this is that it can be misleading if the predictive quantities are substantially different from the observed quantities used in the regression, or if predictive ground-water flow conditions are substantially different than calibration conditions. No better indicator of nonlinearity is available at this time, however, so the modified Beale's measure is suggested.

The modified Beale's measure is calculated using two output files produced by MODFLOW-2000 and the post-processing program BEALE-2000. The two files are the _bl and _b2 files of table 5; the contents of these files are shown in tables 12 and 13 . If circumstance 3 above for YCINT-2000 applies, the _bl file needs to be produced by the same run of MODFLOW-2000 used to produce the _y0 file. File_b2 needs to be produced by a separate run of MODFLOW-2000. OUTNAM needs to be specified as a string other than 'NONE' in the Observation Process input file for both runs; the string must be the same for the runs that produce the _b1 and _b2 files.

To use BEALE-2000, first generate an _bl file as follows:

1. In the Parameter-Estimation Process input file, set IBEFLG $=1$.

2. Substitute the final calibrated parameter values from the _ b file into the Sensitivity Process input file.

3. Execute MODFLOW-2000. This run will generate an_bl file.

Next, generate an_b2 file as follows:

4. In the Parameter-Estimation Process input file, set IBEFLG $=2$.

5. Execute MODFLOW-2000. In this run, the _b1 file produced in step 3 is read and an _b2 file is generated.

6. Finally, execute BEALEP. Output is to a file with extension \#be (table 6).

The modified Beale's measure is printed near the bottom of the file along with critical values. This information can be used to determine whether the calibrated model is roughly linear, intermediate, or nonlinear, with respect to the observations used for model calibration. The rest of the information in the \#be file can be used to detect which observations and parameters contribute most to the nonlinearity. 
Table 12: Information contained in the _bl file of table 5, which is produced when IBEFLG=1 in the Parameter-Estimation Process input. This file is read by MODFLOW-2000 when IBEFLG=2 and by BEALE-2000.

\begin{tabular}{|c|c|c|c|}
\hline Item & Format & Variables & Description \\
\hline 1 & $\begin{array}{l}5 \mathrm{I} 10,1 \mathrm{X} \\
\text { F14.0 }\end{array}$ & $\begin{array}{l}\text { NPE, ND, } \\
\text { NDMH, } \\
\text { MPR, } \\
\text { IPR } \\
\text { VAR }\end{array}$ & $\begin{array}{l}\text { Number of estimated parameters and } \\
\text { observations, } \\
\text { number of observations with full } \\
\text { weighting, } \\
\text { number of prior-information equations, } \\
\text { prior information with a full weight } \\
\text { matrix, and } \\
\text { the calculated error variance }\end{array}$ \\
\hline 2 & $6(\mathrm{~A} 10,1 \mathrm{X})$ & PARNAM(NPE) & Parameter names \\
\hline 3 & $16 \mathrm{~F} 13.0$ & BOPT(NPE) & Optimized parameter values \\
\hline 4 & $6(\mathrm{~A} 12,1 \mathrm{X})$ & OBSNAM(ND) & Observation names \\
\hline 5 & $16 \mathrm{~F} 13.0$ & $\mathrm{H}(\mathrm{ND})$ & $\begin{array}{l}\text { Simulated equivalents of the } \\
\text { observations calculated using the } \\
\text { optimized parameter values }\end{array}$ \\
\hline 6 & $6 \mathrm{~F} 13.0$ & HOBS(ND) & Observed values. \\
\hline 7 & $8 \mathrm{~F} 10.0$ & WT(NH) & $\begin{array}{l}\text { Weights for the head observations }(\mathrm{NH}= \\
\mathrm{ND}-\mathrm{NDMH})\end{array}$ \\
\hline 8 & $8 \mathrm{~F} 10.0$ & WTQ(NDMH,NDMH) & $\begin{array}{l}\text { Weight matrix for the observations other } \\
\text { than heads }\end{array}$ \\
\hline 9 & $6 \mathrm{~F} 13.0$ & $\mathrm{X}(\mathrm{NPE}, \mathrm{ND})$ & $\begin{array}{l}\text { Sensitivities for all parameters and } \\
\text { observations. }\end{array}$ \\
\hline 10 & $8 \mathrm{~F} 10.0$ & $\begin{array}{l}\text { PRM(NPE,J),WP(J), } \\
\quad \mathrm{J}=1, \mathrm{MPR}\end{array}$ & $\begin{array}{l}\text { Prior information equation coefficients } \\
\text { and weights }\end{array}$ \\
\hline 11 & $8 \mathrm{IIO}$ & NIPR(IPR) & $\begin{array}{l}\text { List of parameters for which prior } \\
\text { information has a full weight matrix. }\end{array}$ \\
\hline 12 & $8 \mathrm{~F} 13.0$ & BPRI(IPR) & Prior information values \\
\hline 12 & $8 \mathrm{~F} 13.0$ & WTP(IPR,IPR) & Full weight matrix \\
\hline 13 & $8 \mathrm{IIO}$ & LN(NPE) & $\begin{array}{l}\text { Flag indicating whether each parameter } \\
\text { is log-transformed }\end{array}$ \\
\hline 14 & $8 \mathrm{~F} 13.0$ & BBEA(NPE) & $\begin{array}{l}\text { Sets of parameter values used to } \\
\text { calculate Beale's measure. } 2 \times \text { NPE sets } \\
\text { of parameter values are listed. }\end{array}$ \\
\hline
\end{tabular}


Table 13: Information contained in the _b2 file of table 5, which is produced by MODFLOW2000 when IBEFLG=2. To generate this file, an _bl file must have been produced by a previous MODFLOW-2000 run.

\begin{tabular}{|c|c|c|c|}
\hline Item & Format & Variables & Description \\
\hline \multicolumn{3}{|c|}{ The following two items are repeated $2 \times$ NPE times } \\
\hline 1 & $8 \mathrm{~F} 13.0$ & B(NPE) & $\begin{array}{c}\text { Parameter values for one of the } 2 \times \\
\text { NPE sets of parameter values to be } \\
\text { considered in the modified Beale's } \\
\text { measure. }\end{array}$ \\
\hline 2 & $6 \mathrm{~F} 13.0$ & FC(ND) & $\begin{array}{c}\text { Simulated equivalents of the } \\
\text { observations used in the regression, } \\
\text { calculated using the preceding set of } \\
\text { parameter values. }\end{array}$ \\
\hline
\end{tabular}




\section{Chapter 8. USING OUTPUT FROM MODFLOW-2000 AND POST-PROCESSORS RESAN-2000, YCINT-2000, AND BEALE- 2000}

MODFLOW-2000 and its post-processing programs provide substantial flexibility in performance, as indicated by the modes listed in table 3 . A large number of output files can be produced, as shown in tables 5 and 6 . This chapter describes how these files commonly are used given different modes of MODFLOW-2000 execution and different post-processors.

The primary MODFLOW-2000 model output files are the files defined using file types GLOBAL and LIST of table 2. In the following discussion it is assumed that both primary output files are defined. If only one file is defined, all output will be printed in that file. If the Sensitivity Process or both the Sensitivity and Parameter-Estimation Processes are active, the file may be extremely big; for these situations, definition of both files is recommended.

\section{Output Files from Mode 'Forward with Observations', with or without \\ Parameter Substitution}

As noted in table 3, a forward run can be achieved four ways with MODFLOW-2000, depending on whether or not observations are defined and whether parameter values from the Ground-Water Flow Process input files or the Sensitivity Process input file are used. If the Sensitivity Process is active, the parameter values listed in the Sensitivity Process input file are used in the forward run. This discussion assumes observations are listed; the parameter values could come from either source.

For a forward simulation, the MODFLOW-2000 Ground-Water Flow Process calculates hydraulic heads once using the specified parameter values. The output files produced include the GLOBAL and LIST files and, if OUTNAM is not "NONE" in the Observation Process input file, the files listed in the top section of table 5. The GLOBAL and LIST files need to be used to check for errors in the forward simulation and the definition of observations; some of the files listed in tables 5 and 16 also can be useful.

After executing MODFLOW-2000, the LIST file includes the table and statistics described in the top of table 14. This information is repeated in the GLOBAL file, except that only summary information is presented from the tables of observations, simulated values, and residuals. The weighted residuals also are included in several of the files listed in tables 5 and 16. The weighted residuals reflect the model fit given the expected accuracy of the observations, the existing model configuration, the parameter values used, and ideas about how to calculate the equivalent simulated values being compared with the observations. Large discrepancies between simulated and observed values need to be investigated and may indicate, for example, that there is a data input error, or a conceptual error in the model configuration or in the calculation of the simulated values. Inspection of these values for the forward model run with observations and correction of obvious problems can eliminate many hours of frustration. Use of the _ws file to graph weighted residuals against weighted simulated values will clearly show whether there are large discrepancies between observed and simulated values. If there are large discrepancies, it is important to investigate whether they are caused by errors in the Ground-Water Flow Process input files or in how the equivalent simulated values are being calculated. It is essential for MODFLOW-2000 to perform correctly for this simulation. Proceeding with errors will result in an invalid regression and wasted time. 


\section{Output Files from Modes 'Parameter Sensitivity' and 'Parameter Sensitivity with Observations'}

The output that is unique to the two Parameter-Sensitivity modes (table 3) are arrays of one-percent scaled sensitivities for the entire grid (defined and discussed in Chapter 5); these arrays can be mapped and contoured. Though not often used quantitatively, these maps enhance understanding of the influence of different parameters on the calculation of hydraulic head. As the number of parameters, model layers, and time steps increases, the number of possible maps can be overwhelming, but judicious map production can produce important insights into system dynamics.

\section{Tables of Sensitivities Produced for all Sensitivity with Observation Modes, the Sensitivity Analysis Mode, and the Parameter-Estimation Mode}

Depending on the value specified for variable ISCALS in the Observation Process input file, tables of dimensionless and composite scaled sensitivities and (or) one percent scaled sensitivities are printed in the GLOBAL output file and in the _sc, _sd, and _sl files (table 5). For the Parameter-Estimation mode, in which nonlinear regression is performed, the GLOBAL file includes tables of sensitivities calculated using both the starting and final parameter values; the _sc, _sd, and_sl files contain sensitivities calculated using the final parameter values. The use of dimensionless, composite, and one-percent scaled sensitivities is discussed in Hill (1998), and briefly summarized in the following paragraphs.

Dimensionless scaled sensitivities can be used to determine which observations are likely to be most important to the estimation of each parameter. They often do not, however, identify observations that reduce parameter correlation, because these observations may not have large dimensionless scaled sensitivities. Bar charts of dimensionless scaled sensitivities readily indicate the observations with the largest dimensionless scaled sensitivities.

Composite scaled sensitivities can be used to evaluate whether the available observations are likely to provide adequate information to allow estimation of defined parameters, and are generally plotted using a bar chart. Plotting of such bar charts routinely during model calibration is important because both the nonlinearity of the sensitivities and the scaling result in composite scaled sensitivities that will change. These changes become important if they indicate that a parameter included in the estimation can no longer be supported by the observations, or a previously excluded parameter probably can be estimated given the updated version of the model. It is important to include both estimated and unestimated parameters in bar charts of composite scaled sensitivities when published.

One-percent sensitivities from model runs constructed as described for the YCINT postprocessor are the most convenient sensitivities from which to calculate prediction scaled sensitivities. Depending on the situation, they can sometimes be used directly as prediction scaled sensitivities, but they sometimes need additional scaling. As for the other scaled sensitivities, it is often useful to plot them on bar charts, as in Hill and others (in press).

\section{Output Files from Mode 'Sensitivity Analysis'}

This mode is achieved by specifying MAX-ITER as zero in the Parameter-Estimation Process input file. The unique benefit of this mode is that the statistics needed for the sensitivity analysis described by Hill (1998), scaled sensitivities and parameter correlation coefficients, are calculated using the parameter values listed in the Sensitivity Process input file. These statistics 
are calculated for parameters with ISENS $>0$, without proceeding through a series of parameterestimation iterations. If defined, the statistics are printed in the GLOBAL output file.

The scaled sensitivities produced by the Sensitivity Analysis Mode were discussed in the preceding section. The parameter correlation coefficients that are produced can be used to identify highly correlated parameter pairs. The presence of highly correlated parameters can be problematic during parameter estimation because of the difficulty of determining unique values for highly correlated parameters. The correlation coefficients calculated by MODFLOW-2000 are accurate because the sensitivity-equation method produces sensitivities that are accurate to four or five significant digits. Parameter correlation coefficients produced using perturbation sensitivities, as is done using UCODE, do not tend to be as reliable.

\section{Output Files from Mode 'Parameter Estimation'}

MODFLOW-2000 performs nonlinear regression and produces the GLOBAL and LIST files when the Parameter-Estimation Process is active, ISENALL $=0$ in the Sensitivity-Process input file, and IBEFLG $<2$ in the Parameter-Estimation Process input file. If OUTNAM is not defined as "NONE" in the Observation Process input file for all observations, all files of table 16 are produced by MODFLOW-2000. If executed, RESAN-2000 produces the first five files of table 6. Often it is useful to set up batch files such that RESAN-2000 is routinely executed after MODFLOW-2000 so that these files are routinely produced.

Another file likely to be accessed by the user is the _b file (table 5), which contains the parameter values for each parameter-estimation iteration in a format suitable for substitution into the Sensitivity Process input file. Values from this file can be used to replace the starting parameter values in the Sensitivity Process input file to achieve the goals discussed in chapter 3 in the section "Starting Parameter Values". In brief, these values might be changed to (1) investigate simulated equivalents to the observations and observations sensitivities calculated with parameter values from intermediate parameter-estimation iterations, and (2) start the regression using values from the final or intermediate parameter-estimation iterations that are likely to be closer to the optimal parameter values than the previous starting values. The second use of the values listed in the _ b file often reduces execution time.

The GLOBAL file includes information about the regression and indicates whether or not the regression converged. In either case, the GLOBAL file lists the statistics described in tables 14,15 , and 18. A sample GLOBAL file from a regression is included in Appendix A of this report. The best way to become familiar with the file is to review that example and the comments in tables 14,15 , and 18 .

Residual analysis can be accomplished using the statistics listed in table 14 and the files listed in table 16. Examples of the files with their contents labeled are shown in Appendix A. File names listed in table 16 with two letters in the extension include two columns of values and generally are used to create $x-y$ plots. File names listed in table 16 with a single letter in the extension contain only one column of values and generally are used to create maps, temporal plots, or higher-dimensional images of residuals. Each line includes the information related to one observation or piece of prior information. In all files, each line lists the OBSNAM or, for prior information, the EQNAM. Each line also lists the PLOT-SYMBOL. Comments about how to use the generated graphs are presented in table 15. Additional discussion can be found in Hill (1998) and references cited therein.

During most model calibration, MODFLOW-2000 regression runs will be executed many times as various aspects of the model are changed to test hypotheses about the system. Once a satisfactory set of parameter estimates is obtained, predictions can be calculated, linear confidence and prediction intervals can be calculated to provide an indication of the prediction 
uncertainty, and the linearity of the model at the optimized parameter values can be evaluated. The model output related to these capabilities is described in the following sections.

\section{Output Files for Residual Analysis and Identifying Influential Observations from RESAN-2000}

The RESAN-2000 program produces five files with extensions \#rs, _rd, _rg, _rc, and _rb. The \#rs file details some intermediate steps of the program and rarely needs to be accessed.

The _rd and _rg files contain sets of generated random numbers. The number of values in each set equals the number of weighted residuals (including values for all observations and prior information). The _rd file contains uncorrelated values; the _rg file contains values correlated to match the correlations produced through the regression. The _rd and_rg files are comprised of lines that contain the generated random number followed by a normal probability plotting position that is adjusted so that it can be plotted on an arithmetic axis (Hill, 1994); the lines are ordered from largest to smallest generated value within each of the 4 sets. On each line the generated random numbers and plotting positions are followed by the OBSNAM and PLOTSYMBOL from the associated observation. The values from the _rd and _rg files typically are presented as normal probability graphs along with similar graphs produced using the _nm file.

One Cook's D statistic is calculated for each observation and these are contained in the _rc file (table 6). The Cook's D statistics can be conveniently presented in a bar chart with the sequential observation number on the horizontal axis, or plotted on a map. Large values identify observations that, if omitted, would cause the greatest changes in the set of estimated parameter values.

DFBeta statistics are calculated for every observation, for every parameter, and are listed in the _rb file (table 6). Large values identify observations that are influential in the estimation of the parameter. Values for each parameter can be presented in a bar chart or on a map. 


\section{Chapter 8. USING OUTPUT FROM MODFLOW-2000 AND POST-PROCESSORS \\ RESAN-2000, YCINT-2000, AND BEALE-2000}

Table 14: Residuals and model-fit statistics printed in the GLOBAL and LIST output files when the Observation Process is active

[Summarized from Hill (1998, sections "Graphical Analysis of Model Fit and Related Statistics" and "Statistical Measures of Model Fit"); see example output file in Appendix A of this report.]

\begin{tabular}{|c|c|}
\hline $\begin{array}{l}\text { Statistic as labeled in the } \\
\text { GLOBAL and LIST } \\
\text { output files }\end{array}$ & Comments \\
\hline \multicolumn{2}{|c|}{ The following table is in the LIST file; largest and smallest residuals are repeated in the GLOBAL file. } \\
\hline $\begin{array}{l}\text { Table of observations, } \\
\text { simulated values, residuals, } \\
\text { and weighted residuals }\end{array}$ & $\begin{array}{l}\text { Residuals are calculated as the observations minus the simulated values. Use } \\
\text { this table to investigate model fit for individual observations. }\end{array}$ \\
\hline \multicolumn{2}{|c|}{ The following information is repeated in both the GLOBAL and LIST files } \\
\hline $\begin{array}{l}\text { MAXIMUM WEIGHTED } \\
\text { RESIDUAL } \\
\text { MINIMUM WEIGHTED } \\
\text { RESIDUAL } \\
\end{array}$ & $\begin{array}{l}\text { The maximum and minimum weighted residuals indicate where the worst fit } \\
\text { occurs, and often reveals gross errors. }\end{array}$ \\
\hline $\begin{array}{l}\text { AVERAGE WEIGHTED } \\
\text { RESIDUAL }\end{array}$ & $\begin{array}{l}\text { An average weighted residual near zero is needed for an unbiased model fit } \\
\text { (usually satisfied if regression converges). }\end{array}$ \\
\hline $\begin{array}{l}\# \text { RESIDUALS }>=0 \\
\# \text { RESIDUALS }<0\end{array}$ & $\begin{array}{l}\text { The number of positive and negative residuals indicates whether the model } \\
\text { fit is consistently high or low. Preferably, the two values are about equal. }\end{array}$ \\
\hline NUMBER OF RUNS & $\begin{array}{l}\text { Number of sequences of residuals with the same sign (+ or }-) \text {. Too few or } \\
\text { too many runs can indicate model bias. The related statistic is printed and } \\
\text { interpreted. Hill }(1998, \text { p. } 22) \text { explains the test. }\end{array}$ \\
\hline \multicolumn{2}{|c|}{ The following are printed in the GLOBAL file if the PES Process is active. } \\
\hline $\begin{array}{l}\text { LEAST-SQUARES OBJ FUNC } \\
\text { (DEP.VAR. ONLY) } \\
\text { (W/ PARAMETERS) }\end{array}$ & $\begin{array}{l}\text { Weighted least-squares objective function value. Given randomly distribut- } \\
\text { ed residuals and the same observations and weight matrix, a lower value of } \\
\text { the least-squares objective function indicates a closer model fit to the data. }{ }^{2}\end{array}$ \\
\hline $\begin{array}{l}\text { CALCULATED ERROR } \\
\text { VARIANCE }\end{array}$ & $\begin{array}{l}\text { Given randomly distributed residuals, smaller values are desirable. Values } \\
\text { less than } 1.0 \text { indicate that the model generally fits the data better than is } \\
\text { consistent with the statistics used to weight observations and prior } \\
\text { information; values greater than } 1.0 \text { indicate that the fit is worse. (Hill, 1998, } \\
\text { Guideline 6) }\end{array}$ \\
\hline $\begin{array}{l}\text { STANDARD ERROR OF THE } \\
\text { REGRESSION }\end{array}$ & The square root of the calculated error variance. \\
\hline $\begin{array}{l}\text { CORRELATION COEFFICIENT } \\
\text { W/ PARAMETERS }\end{array}$ & R of Hill (1998, p. 21). Values below about 0.9 indicate poor model fit. \\
\hline $\begin{array}{l}\text { MAX LIKE OBJ FUNC } \\
\text { AIC } \\
\text { BIC }\end{array}$ & $\begin{array}{l}\text { The maximum likelihood objective function, and the AIC and BIC statistics. } \\
\text { Given randomly distributed residuals, lower values indicate better model fit. }\end{array}$ \\
\hline $\begin{array}{l}\text { ORDERED WEIGHTED } \\
\text { RESIDUALS }\end{array}$ & The weighted residuals are ordered smallest to largest. ${ }^{2}$ \\
\hline $\begin{array}{l}\text { CORRELATION BETWEEN } \\
\text { ORDERED WEIGHTED } \\
\text { RESIDUALS AND NORMAL } \\
\text { ORDER STATISTICS }\end{array}$ & $\begin{array}{l}\mathrm{R}_{\mathrm{N}}{ }^{2} \text { of Hill (1998). Values above the critical values listed in Hill (1998, } \\
\text { Appendix D) and printed in the GLOBAL file indicate independent, normal } \\
\text { weighted residuals, and that the points listed in the nm file (table 5) are } \\
\text { likely to fall on a straight line. }\end{array}$ \\
\hline
\end{tabular}

${ }^{1}$ THIS FONT is used for labels taken directly from the output

${ }^{2}$ To allow detection of poor fit to one type of regression data, these statistics are calculated both for (a) the observed dependent variables (the observations) and (b) the observations and prior information. 


\section{Chapter 8. USING OUTPUT FROM MODFLOW-2000 AND POST-PROCESSORS}

RESAN-2000, YCINT-2000, AND BEALE-2000

Table 15: Parameter statistics printed in the GLOBAL output file when the Parameter-Estimation Process is active and IBEFLG $<2$.

[Summarized from Hill (1998, section "Parameter Statistics" and Guidelines 3 and 9); see example file in Appendix A of this report]

\begin{tabular}{|c|c|}
\hline $\begin{array}{l}\text { Parameter statistic or } \\
\text { characteristic }^{\prime}\end{array}$ & Function of item in interpreting results \\
\hline $\begin{array}{l}{ }^{2} \text { DIMENSIONLESS SCALED } \\
\text { SENSITIVITIES (SCALED } \\
\text { BY } B^{*}(\text { WT**.5)) }\end{array}$ & $\begin{array}{l}\text { Indicates the importance of an observation to the estimation of a parameter } \\
\text { or, conversely, the sensitivity of the simulated equivalent of the observation } \\
\text { to the parameter. These values are listed in a table with a row for each } \\
\text { observation and a column for each parameter. }\end{array}$ \\
\hline $\begin{array}{l}{ }^{2} \text { COMPOSITE SCALED } \\
\text { SENSITIVITIES } \\
((\text { SUM OF THE SQUARED } \\
\text { VALUES) } / \text { ND })^{* \star} .5\end{array}$ & $\begin{array}{l}\text { Indicates the information content of all of the observations for the } \\
\text { estimation of a parameter. Printed at the end of the scaled sensitivity table. } \\
\text { Values less than } 0.01 \text { times the largest value indicate parameters with much } \\
\text { less information, and that the regression is likely to have trouble } \\
\text { converging. }\end{array}$ \\
\hline $\begin{array}{l}{ }^{2} \text { ONE-PERCENT SCALED } \\
\text { SENSITIVITIES (SCALED } \\
\text { BY B/100) }\end{array}$ & $\begin{array}{l}\text { These scaled sensitivities have the dimensions of the observations, which } \\
\text { can sometimes be useful. For example, for prediction scaled sensitivities. }\end{array}$ \\
\hline $\begin{array}{l}\text { Parameter covariance } \\
\text { matrix }\end{array}$ & $\begin{array}{l}\text { The diagonal terms of this matrix are variances, the off-diagonal terms are } \\
\text { covariances. These values are used to calculate the statistics listed below. }\end{array}$ \\
\hline \multicolumn{2}{|c|}{ The statistics in this box are printed in a table labeled "PARAMETER SUMMARY". } \\
\hline Parameter values & $\begin{array}{l}\text { When parameter estimation converges, these are the optimized parameter } \\
\text { values and the items listed below in this table constitute a linear uncertainty } \\
\text { analysis of the optimized parameter values. Unreasonable optimal values } \\
\text { may indicate a problem with the observations or the model. }\end{array}$ \\
\hline $\begin{array}{l}\text { Parameter standard } \\
\text { deviations }\end{array}$ & $\begin{array}{l}\text { Standard deviations on optimized parameter values indicate the precision } \\
\text { with which the values are estimated. }\end{array}$ \\
\hline $\begin{array}{l}\text { Parameter coefficients of } \\
\text { variation }\end{array}$ & $\begin{array}{l}\text { Provides a dimensionless measure of the precision with which the } \\
\text { parameters are estimated which can be used to compare the precision of } \\
\text { parameters with different dimensions. }\end{array}$ \\
\hline $\begin{array}{l}\text { Parameter } 95 \% \text { linear } \\
\text { individual confidence } \\
\text { Intervals }^{4}\end{array}$ & $\begin{array}{l}\text { Given normally distributed residuals, reasonable optimized parameter } \\
\text { values, a satisfactory model fit, and a linear model, linear confidence } \\
\text { intervals are likely to reflect the uncertainty of the optimal parameter } \\
\text { values. Model linearity is tested using post-processing program BEALE- } \\
2000 .\end{array}$ \\
\hline $\begin{array}{l}\text { Parameter correlation } \\
\text { coefficients }\end{array}$ & $\begin{array}{l}\text { For any set of parameter values, absolute values larger than about } 0.95 \text { may } \\
\text { indicate that two or more parameters cannot be uniquely estimated. Explore } \\
\text { uniqueness by varying starting parameter values and checking for changes } \\
\text { in optimized parameter values, as described in the output file. }\end{array}$ \\
\hline \multicolumn{2}{|c|}{$\begin{array}{l}\text { THIS FONT is used for labels taken directly from the output } \\
{ }^{2} \text { Printing controlled by ISCALS in the input file for all observations. } \\
{ }^{3} \text { For log-transformed parameters, the parameter value and associated confidence intervals are } \\
\text { calculated and printed first as log-transformed values, and next as native values. The native } \\
\text { values generally are of most interest. } \\
{ }^{4} \text { Calculated as usual; see eq. } 28 \text { of Hill (1998). }\end{array}$} \\
\hline
\end{tabular}


Table 16: Using the files created by MODFLOW-2000 that contain data sets for graphical residual analysis

[Summarized from Hill (1998, section "Graphical Analysis of Model Fit and Related Statistics"); selected example annotated files are presented in Appendix A.]

\begin{tabular}{|c|c|c|}
\hline $\begin{array}{c}\text { File- } \\
\text { name }^{1}\end{array}$ & Intended graph or analysis & Comments $^{2}$ \\
\hline \multicolumn{3}{|c|}{ Files produced by MODFLOW-2000 when the Observation Process is active and OUTNAM $\neq$ 'NONE' } \\
\hline _os & $\begin{array}{l}\text { Observed versus simulated } \\
\text { values }\end{array}$ & $\begin{array}{l}\text { Ideally, points lie along a line with a slope of } 1.0 \text {. Uneven } \\
\text { spreading along the length of the line does not necessarily } \\
\text { indicate problems because the values are not weighted. }\end{array}$ \\
\hline _WW & $\begin{array}{l}\text { Weighted observed versus } \\
\text { weighted simulated values. }\end{array}$ & $\begin{array}{l}\text { Ideally, points lie along a line with a slope of } 1.0 \text {. Different } \\
\text { slope or uneven spreading may indicate problems. }\end{array}$ \\
\hline -ws & $\begin{array}{l}\text { Weighted residuals versus } \\
\text { weighted simulated values. } \\
\text { Traditionally, plot weighted } \\
\text { simulated values on the } \mathrm{x} \text { axis. }\end{array}$ & $\begin{array}{l}\text { Ideally, the points are evenly distributed above and below } \\
\text { the weighted residual zero axis, which indicates random } \\
\text { weighted residuals. Uneven spreading along the zero axis } \\
\text { may indicate problems. }\end{array}$ \\
\hline$-r$ & $\begin{array}{l}\text { The residuals listed in this file } \\
\text { can be plotted against any } \\
\text { independent variable of interest. }\end{array}$ & $\begin{array}{l}\text { Possible displays include plotting values from a single } \\
\text { location against time on an } x-y \text { graph, on maps, on three- } \\
\text { dimensional images of a contaminant plume, and on maps } \\
\text { representing different times. Useful to display model fit, but } \\
\text { use of unweighted residuals means that large values may not } \\
\text { indicate problems. }\end{array}$ \\
\hline$-\mathrm{W}$ & $\begin{array}{l}\text { The weighted residuals listed can } \\
\text { be plotted as suggested for the_r } \\
\text { file }\end{array}$ & $\begin{array}{l}\text { Plots should be random; test using a runs test. Individual } \\
\text { extreme values and areas of consistent negative or positive } \\
\text { values are likely to indicate problems. They should be } \\
\text { closely examined and the model corrected if possible. }\end{array}$ \\
\hline nm & $\begin{array}{l}\text { Normal probability graph of the } \\
\text { weighted residuals. The } \\
\text { probability values are } \\
\text { transformed so that they plot on } \\
\text { an arithmetic scale. }\end{array}$ & $\begin{array}{l}\text { Ideally, the weighted residuals fall along a straight line. If } \\
\text { not, possibilities include: (1) The apparent nonrandomness } \\
\text { results from limited number of values or from the regression } \\
\text { itself, which can be tested using the _rd and _rg files, (2) } \\
\text { problems are indicated. }^{3}\end{array}$ \\
\hline _Sc & $\begin{array}{l}\text { Bar chart of composite scaled } \\
\text { sensitivities with PARNAM on } \\
\text { the horizontal axis. }\end{array}$ & $\begin{array}{l}\text { Large values indicate better support by the regression data. } \\
\text { Aspects of the system associated with large values perhaps } \\
\text { can be represented with more parameters. }\end{array}$ \\
\hline _sd & $\begin{array}{l}\text { Bar charts of dimensionless } \\
\text { scaled sensitivities for each } \\
\text { parameter with the sequential } \\
\text { observation number on the } \\
\text { horizontal axis. }\end{array}$ & $\begin{array}{l}\text { A parameter with large composite scaled sensitivity and } \\
\text { many large dimensionless scaled sensitivities is probably } \\
\text { more reliably estimated than a parameter with a large } \\
\text { composite scaled sensitivity and one large dimensionless } \\
\text { scaled sensitivity because the error of the single important } \\
\text { observation is propagated directly into the estimate. }\end{array}$ \\
\hline _sl & $\begin{array}{l}\text { Often used as prediction scaled } \\
\text { sensitivities. }\end{array}$ & $\begin{array}{l}\text { Use to compare the importance of different parameters to } \\
\text { simulated values. }\end{array}$ \\
\hline
\end{tabular}

${ }^{1}$ File names are formed using OUTNAM from the Observation Package input file for all observations, followed by a period and the extensions listed here and in tables 5 and 6 .

${ }^{2}$ The phrase "indicate problems" means that the circumstance described indicates that the processes represented by the data may not be adequately modeled.

${ }^{3}$ For examples, see Hill (1998) and references cited therein. 
Table 17: Using the files created by RESAN-2000 that contain data sets for graphical residual analysis

[Summarized from Hill (1998, section "Graphical Analysis of Model Fit and Related Statistics").]

\begin{tabular}{|c|l|l|}
\hline $\begin{array}{c}\text { File- } \\
\text { name }^{1}\end{array}$ & \multicolumn{1}{|c|}{ Intended graph or analysis } & \multicolumn{1}{c|}{ Comments $^{2}$} \\
\hline$\_$rd & $\begin{array}{l}\text { Normal probability graph of random } \\
\text { numbers. }\end{array}$ & $\begin{array}{l}\text { Demonstrates the deviation from a straight line } \\
\text { caused by the limited number of plotted values. }\end{array}$ \\
\hline$\_$rg & $\begin{array}{l}\text { Normal probability graph of correlated } \\
\text { random numbers. }\end{array}$ & $\begin{array}{l}\text { Demonstrates the deviation from a straight line } \\
\text { caused by the limited number of weighted } \\
\text { residuals and by the regression fitting of the data. }\end{array}$ \\
\hline$\_$rc & $\begin{array}{l}\text { Bar chart of the Cook's D statistics with the } \\
\text { sequential observation number of the } \\
\text { horizontal axis, or maps of the study area } \\
\text { with the statistic plotted at the observation } \\
\text { location. }\end{array}$ & $\begin{array}{l}\text { Large values identify observations that, if omitted, } \\
\text { would result in greater changes to the set of } \\
\text { parameter estimates. }\end{array}$ \\
\hline$\_$rb & $\begin{array}{l}\text { Bar charts of DFBeta statistics for each } \\
\text { parameter with the sequential observation } \\
\text { number of the horizontal axis, or maps of } \\
\text { the study area with the statistic plotted at the } \\
\text { observation location. }\end{array}$ & $\begin{array}{l}\text { Large values identify observations with the most } \\
\text { influence on each parameter estimate. }\end{array}$ \\
\hline
\end{tabular}

${ }^{1}$ File names are formed using OUTNAM from the Observation Package input file for all observations, followed by a period and the extensions listed here and in tables 5 and 6 . ${ }^{2}$ The phrase "indicate problems" means that the circumstance described indicates that the processes represented by the data may not be adequately modeled.

${ }^{3}$ For examples, see Hill (1998) and references cited therein. 


\section{Chapter 8. USING OUTPUT FROM MODFLOW-2000 AND POST-PROCESSORS \\ RESAN-2000, YCINT-2000, AND BEALE-2000}

Table 18: Regression performance measures printed in the GLOBAL output file when the Parameter-Estimation Process is active and IBEFLG $<2$

[These measures are printed for each parameter-estimation iteration; see example file in Appendix A of this report]

\begin{tabular}{|l|l|}
\hline $\begin{array}{c}\text { Performance measure as } \\
\text { labeled in the GLOBAL } \\
\text { file }\end{array}$ & \multicolumn{1}{c|}{ Comments } \\
\hline MARQUARDT PARAMETER & $\begin{array}{l}\text { Used as described in Hill (1998, eq. 4). Non-zero values indicate an ill- } \\
\text { conditioned problem. }\end{array}$ \\
\hline $\begin{array}{l}\text { DAMPING PARAMETER } \\
\text { (RANGE 0 TO 1) }\end{array}$ & $\begin{array}{l}\text { The damping parameter of eq. 4 of Hill (1998). Values less than 1.0 } \\
\text { indicate that the maximum fractional parameter change exceeded the } \\
\text { MAX-CHANGE value specified in the Parameter-Estimation Process } \\
\text { input file, or that oscillation control was active (Hill, 1998, Appendix B). }\end{array}$ \\
\hline $\begin{array}{l}\text { MAX. FRACTIONAL PAR. } \\
\text { CHANGE }\end{array}$ & $\begin{array}{l}\text { Maximum fractional change calculated for any parameter in the } \\
\text { parameter-estimation iteration. The fractional change is always relative to } \\
\text { the native parameter value, even if the parameter is log-transformed (Hill, } \\
\text { 1998, Appendix B). When this value is less than the user specified TOL } \\
\text { of file Parameter-Estimation Process input file, the regression converges. }\end{array}$ \\
\hline $\begin{array}{l}\text { MAX. FRAC. CHANGE } \\
\text { OCCURRED FOR PAR. } \\
\text { "PARNAM }\end{array}$ & $\begin{array}{l}\text { The parameter for which the maximum fractional change occurs. If the } \\
\text { regression does not converge, the parameters listed here are likely to be } \\
\text { contributing to the problem. }\end{array}$ \\
\hline
\end{tabular}

'THIS FONT is used for labels taken directly from the output 


\section{Output Files for Predictions and Differences from YCINT-2000}

YCINT-2000 can print predictions and differences (eq. 7), and 95-percent linear confidence and prediction intervals on the predictions and differences, as described above in the section "Predictions and differences and their linear confidence and prediction intervals" of chapter 3 and in chapter 7 . The sequence of runs needed is described in chapter 7 . The YCINT2000 output files are named using OUTNAM for the base and the extension \#yc, _yp or _yd. The tables in the output file are labeled, indicating the type of confidence or prediction interval included in the table. The labels used are:

INDIVIDUAL 95\% CONFIDENCE INTERVALS

$\mathrm{k}$ SIMULTANEOUS 95\% CONFIDENCE INTERVALS, ( $\mathrm{k}$ is replaced by a number) and

\section{UNDEFINED NUMBER OF SIMULTANEOUS 95\% CONFIDENCE INTERVALS}

The first label is followed by individual confidence intervals. The second label is followed by the Bonferroni or $d=k$ Scheffé (if $k$ is larger than NP, the label will read $d=N P$ Scheffé) confidence intervals, whichever are smaller (Bonferroni are used when they are equal). These are labeled as:

\section{BONFERRONI CONFIDENCE INTERVALS ARE USED}

or

\section{SCHEFFÉ CONFIDENCE INTERVALS ARE USED}

The last of the three labels is followed by $\mathrm{d}=\mathrm{NP}$ Scheffé confidence intervals.

If the _ $y 2$ file is produced as described in chapter 7 and IDIF $=1$ in the _ $y 0$ file, YCINT2000 calculates 95 -percent linear confidence and prediction intervals on predictions and differences, as described above in the section "Predictions and differences and their linear confidence and prediction intervals" of chapter 3. In this situation, the YCINT-2000 output file contains both predictions and differences and their intervals. An example YCINT-2000 output file is presented in Appendix A.

The theory for calculating confidence and prediction intervals is discussed by Hill (1994). The linearity assumption of these confidence and prediction intervals needs to be evaluated using BEALE-2000.

\section{Output Files from Test of Linearity with BEALE-2000}

BEALE-2000 calculates the modified Beale's measure of model linearity (Cooley and Naff, 1990; Hill, 1994, p. 47) and statistics that indicate the magnitude of the nonlinearity of each parameter. When regression is performed and IBEFLG is set to 1, MODFLOW-2000 produces an bl output file, which is then used by MODFLOW-2000 in a separate run with IBEFLG $=2$ to produce an _b2 file. Generally, the user does not access these two files. BEALE-2000 uses the _b2 file to produce the BEALE-2000 output file, an example of which is distributed electronically with MODFLOW-2000. Information related to interpretation of the output is included in the file. Hill (1994) explains the modified Beale's measure and the information printed in the BEALE2000 output file. 


\section{REFERENCES}

Anderman, E.R., and Hill, M.C., 1997, Advective-transport observation (ADV) package, a computer program for adding advective-transport observations of steady-state flow fields to the three-dimensional ground-water flow parameter-estimation model MODFLOWP: U.S. Geological Survey Open-File Report 97-14,67 p.

2000, Documentation of the Hydrogeologic-Unit Flow (HUF) Package for the U.S. Geological Survey modular ground-water model MODFLOW-2000: U.S. Geological Survey.

Backus, G.E., 1988, Bayesian inference in geomagnetism: Geophysical Journal, v. 92 , p. 125-142.

Beasley, D.A., Kuh, Edwin, and Welsch, R.E., 1980, Regression diagnostics, Identifying influential data and sources of collinearity: New York, Wiley Series in Probability and mathematical statistics, $292 \mathrm{p}$.

Benjamin, J.R., and Cornell, C.A., 1970, Probability, statistics, and decision for civil engineers: New York, McGraw-Hill, 684 p.

Christensen, Steen, and Cooley, R.L., 1999, Evaluation of confidence intervals for a steady-state leaky aquifer model: Advances in Water Resources, Special Section on Model Calibration and Reliability Evaluation, v. 22, no. 8, p. 807-817.

Cook, R.D., and Weisberg, Sanford, 1982, Residuals and influence in regression: New York, Chapman and Hall, 230 p.

Cooley, R.L., 1977, A method for estimating parameters and assessing reliability for models of steady-state groundwater flow 1 . Theory and numerical methods: Water Resources Research, v. 13, no. 2, p. 318-324.

1979, A method for estimating parameters and assessing reliability for models of steadystate groundwater flow 2. Application of statistical analysis: Water Resources Research, v. 15 , no. 3 , p. $603-617$.

1982, Incorporation of prior information on parameters into nonlinear regression groundwater flow models-- 1. Theory: Water Resources Research, v. 18, no. 4, p. 965-976. 1983a, Incorporation of prior information on parameters into nonlinear regression groundwater flow models--2. Applications: Water Resources Research, v. 19, no. 3, p. 662-676.

1983b, Some new procedures for numerical solution of variably saturated flow problems: Water Resources Research, v. 19, no. 5, p. 1271-1285.

1985. A comparison of several methods of solving nonlinear regression groundwater flow problems: Water Resources Research, v. 21, no. 10, p. 1525-1538.

Cooley, R.L., and R.L. Naff, 1990, Regression modeling of ground-water flow: U.S. Geological Survey Techniques of Water Resources Investigations, book 3, chap. B4. 232 p.

D'Agnese, F.A. Faunt, C.C., Hill, M.C, and Turner, A.K., 1999, Death Valley regional groundwater flow model calibration using optimal parameter estimation methods and geoscientific information systems: Advances in Water Resources, Special Section on Groundwater Model Calibration and Reliability, v. 22, no. 8, p. 777-790.

D’Agnese, F.A., Faunt, C.C., Turner, A.K, and Hill, M.C., 1998, Hydrogeologic evaluation and numerical simulation of the Death Valley Regional ground-water flow system, Nevada and California: U.S. Geological Survey Water-Resources Investigations Report 96-4300, 124 p.

Doherty, J., 1994, PEST: Corinda, Australia, Watermark Computing, 122 p.

Draper, N.R., and Smith, Harry, 1998, Applied regression analysis (3rd ed.): New York, John Wiley and Sons, $706 \mathrm{p}$.

Fenske, J.P., Leake, S.A., and Prudic, D.E., 1996. Documentation of a computer program (RES1) to simulate leakage from reservoirs using the modular finite-difference ground-water flow model (MODFLOW): U. S. Geological Survey Open-File Report 96-364, 51 p. 
Guadagnini, Alberto, and Neuman, S.P., 1999, Nonlocal and localized analyses of conditional mean steady state flow in bounded, nonuniform domains: Water Resources Research, v. 35, no. 10, p. 2999-3039.

Harbaugh, A.W., Banta, E.R., Hill, M.C., and McDonald, M.G., 2000, MODFLOW-2000, the U.S. Geological Survey modular ground-water model - user guide to modularization concepts and the ground-water flow process: U.S. Geological Survey Open-File Report 00-92, 121 p.

Harbaugh, A.W., and McDonald, M.G., 1996, User's documentation for MODFLOW-96, an update to the U.S. Geological Survey Modular Finite Difference Ground-Water Flow Model: U.S. Geological Survey Open-File Report 96-485, 56 p.

Hill, M.C., 1990, Preconditioned Conjugate Gradient 2 (PCG2), A computer program for solving ground-water flow equations: U.S. Geological Survey Water-Resources Investigations Report 98-4048, p. 43. 1992, A computer program (MODFLOWP) for estimating parameters of a transient, threedimensional, ground-water flow model using nonlinear regression: U.S. Geological Survey Open-File Report 91-484, 358 p.

1994, Five computer programs for testing weighted residuals and calculating linear confidence and prediction intervals on results from the ground-water parameter estimation computer program MODFLOWP: U.S. Geological Survey Open-File Report 93-481, 81 p. 1998, Methods and guidelines for effective model calibration: U.S. Geological Survey Water-Resources Investigations Report 98-4005, 90 p.

Hill, M.C., Cooley, R.L., and Pollock, D.W., 1998, A controlled experiment in ground-water flow model calibration: Ground Water, v. 36, no. 3, p. 520-535.

Hill, M.C., D'Agnese, F.A., and Faunt, C.C., in press, Guidelines for model calibration and application to simulation of flow in the Death Valley regional ground-water system: in Fritz Stauffer, Wolfgang Kinzelbach, Karel Kovar, and E. Hoehn, eds, Proceedings of the 1999 Model CARE Conference, Zurich, Switzerland, September, 1999, IAHS Publication no. 265.

Keidser, Allan, and Rosbjerg, Dan, 1991, A comparison of four inverse approaches to groundwater flow and transport parameter identification: Water Resources Research, v. 27, no. 9, p. 2219-2232.

Konikow, L.F., Goode, D.J., and Hornberger, G.Z., 1996, A three-dimensional method-ofcharacteristics solute-transport model (MOC3D): U.S. Geological Survey Water-Resources Investigations Report 96-4267, $87 \mathrm{p}$.

Leake, S.A., Leahy, P.P., and Navoy, A.S., 1994, Documentation of a computer program to simulate leakage from confining units using the modular finite-difference ground-water flow model: U.S. Geological Survey Open-File Report 94-59, 70 p.

Leake, S.A., and Prudic, D.E., 1991, Documentation of a computer program to simulate aquifersystem compaction using the modular finite-difference ground-water flow model: U.S. Geological Survey Techniques of Water Resources Investigations, book 6, chap. A2, 68 p.

McDonald, M.G., and Harbaugh, A.W., 1998, A modular three-dimensional finite-difference ground-water flow model: U.S. Geological Survey Techniques of Water-Resources Investigations, book 6, chap. A1, $586 \mathrm{p}$.

Pacheco, P.S., 1997, Parallel programming with MPI: San Francisco, Morgan Kaufmann Publishers, $418 \mathrm{p}$.

Poeter, E. P., and Hill, M.C., 1997, Inverse models: A necessary next step in groundwater modeling: Ground Water, v. 35(2), p. 250-260. 1998, Documentation of UCODE, A computer code for universal inverse modeling: U.S. Geological Survey Water-Resources Investigations Report 98-4080, 116 p.

Pollock, D.W., 1994, User's Guide for MODPATH/MODPATH-PLOT, Version 3: A particle tracking post-processing package for MODFLOW, the U.S. Geological Survey finite- 
difference ground-water flow model: U.S. Geological Survey Open-File Report 94-464, $6 \mathrm{ch}$.

Seber, G.A.F., and Wild, C.J., 1989, Nonlinear regression: New York, John Wiley, 768 p.

Segerlind, L.J., 1976, Applied finite element analysis: New York, John Wiley and Sons, 422 p.

Shah, P.C., Gavalas, G.R., and Seinfeld, J.H., 1978, Error analysis in history matching - The optimum level of parameterization: Journal of the Society of Petroleum Engineers, v. 18, no. 3, p. 219-228.

Wang, H.F., and Anderson, M.P., 1982, Introduction to groundwater modeling. Finite difference and finite element: New York, W.H. Freeman, 237 p.

Yager, R.M., 1998, Detecting influential observations in nonlinear regression modeling of groundwater flow: Water Resources Research, v. 34, no. 7, p. 1623-1633.

Yeh, W.W-G., 1986, Review of parameter identification procedures in ground-water hydrology-The inverse problem: Water Resources Research, v. 22, no. 2, p. 95-108.

Yeh, W.W-G., and Yoon, Y.S., 1981, Aquifer parameter identification with optimum dimension in parameterization: Water Resources Research, v. 17, no. 3, p. 664-672.

Zheng, Chunmiao, and Wang, P.P., 1998, MT3DMS, a Modular Multi-Species Transport Model, Documentation and User's Guide: U.S. Army Corps of Engineers Waterways Experiment Station Technical Publication, 203 p. 


\section{APPENDIX A. EXAMPLE SIMULATIONS}

The test cases presented in this section are intended to provide example input and output files and examples of some of the graphs of weighted residuals. Suggestions for how to conduct ground-water model calibration are presented in the guidelines of Hill (1998).

Output from two test cases is presented below. Test case 1 involves a two-layer system with a simple hydraulic-conductivity distribution; test case 2 has a more complicated hydraulic conductivity distribution. The two test cases use different packages to represent flow observations.

For test cases 1 and 2, input and output files from the Parameter-Estimation mode (table 3) are presented. Output from these and other test cases and modes is provided in electronic files with distributions of MODFLOW-2000 (see the Distributed Files and Directories section of Appendix B). 


\section{Test Case 1}

The physical system for test case 1 is shown in figure A1. The system consists of two confined aquifers separated by a confining unit. Each aquifer is $50 \mathrm{~m}$ thick, and the confining unit is $10 \mathrm{~m}$ thick. The river is treated as a head-dependent boundary that is hydraulically connected to aquifer 1 . Recharge from the hillside adjoining the system is treated as a headdependent boundary that is hydraulically connected to aquifers 1 and 2 at the boundary farthest from the river.

Stresses on the system include (1) areal recharge to aquifer 1 in the area near the stream (zone 1) and in the area farther from the stream (zone 2), and (2) pumpage from wells completed in each of the two layers. Pumpage from aquifer 1 is assumed to equal pumpage from aquifer 2.

Observations of head and river-flow gain are available for comparison with steady-and transient-state model results. The river is represented using MODFLOW-2000's River Package.

For the finite-difference method, the system is discretized into square $1,000-\mathrm{m}$ by 1,000 $\mathrm{m}$ cells, so that the grid has 18 rows and 18 columns. Time discretization for the model run is specified to simulate a period of steady-state conditions with no pumpage followed by a transientstate period with a constant rate of pumpage. The steady-state period is simulated with one stress period having one time step. The transient period is simulated with four stress periods: the first three are 1, 3, and 6 days long, and each has one time step; the fourth is 272.8 days long and has 9 time steps, and each time-step length is 1.2 times the length of the previous time-step length.

The parameters that define aquifer properties are shown in figure $\mathrm{Al}$ and listed in tables $\mathrm{A} 1$ and A2. The hydraulic conductivity of aquifer 2 is known to increase with distance from the river. The variation is simulated using the multiplier-array capability of MODFLOW-2000. In this case, a multiplier array is defined to represent a step function and contains the value 1.0 in columns 1 and 2, 2.0 in columns 3 and 4 , and so on to the value 9.0 in columns 17 and 18; this multiplier array is referenced in the definition of parameter HK_2 in the input file for the Layer Property Flow Package. For this test case, parameters SS_1 and SS_2 are defined such that their values are storage coefficients. SS_1 and SS_2 are divided by the aquifer thickness (using a multiplier array defined to be the inverse of the aquifer thickness) to produce the specific-storage values expected by the Layer Property Flow Package.

The river is simulated using the River Package to designate 18 river cells in column 1 of layer 1; the head in the river is $100 \mathrm{~m}$. The conductance of the riverbed for each cell is calculated as $\left(\left[\mathrm{L}_{\mathrm{RB}} \times \mathrm{W}_{\mathrm{RB}} / \mathrm{b}_{\mathrm{RB}}\right] \times \mathrm{K} \_\mathrm{RB}\right)$, where, for each cell, $\mathrm{L}_{\mathrm{RB}}$ is the length of the river, $\mathrm{W}_{\mathrm{RB}}$ is the width of the river, and $b_{R B}$ is the thickness of the riverbed. $K \_R B$ is a parameter defined to be the hydraulic conductivity of the riverbed material, so that the quantity $\left[\mathrm{L}_{\mathrm{RB}} \times \mathrm{W}_{\mathrm{RB}} / \mathrm{b}_{\mathrm{RB}}\right]$ is listed as Condfact for each cell in the input file for the River Package (Harbaugh and others, 2000). For this system, $\mathrm{L}_{\mathrm{RB}}=1000 \mathrm{~m}, \mathrm{~W}_{\mathrm{RB}}=10 \mathrm{~m}$, and $\mathrm{b}_{\mathrm{RB}}=10 \mathrm{~m}$ at each river cell, so all Condfact values equal $1000 \mathrm{~m}$.

Ground-water flow into the system from the adjoining hillside is represented using the General-Head Boundary Package. Thirty-six general-head-boundary cells are specified in column 18 of layers 1 and 2, each having an external head of $350 \mathrm{~m}$ and a hydraulic conductance of $1 \times 10^{-7} \mathrm{~m}^{2} / \mathrm{s}$.

Recharge in zone 1 (RCH_1) applies to cells in columns 1 through 9, recharge in zone 2 ( $\left.\mathrm{RCH}_{2} 2\right)$ applies to cells in columns 10 through 18 . A multiplier array defined as a constant is referenced in the definitions of the recharge parameters to convert the recharge rates from units of $\mathrm{cm} / \mathrm{yr}$ to $\mathrm{m} / \mathrm{s}$. 


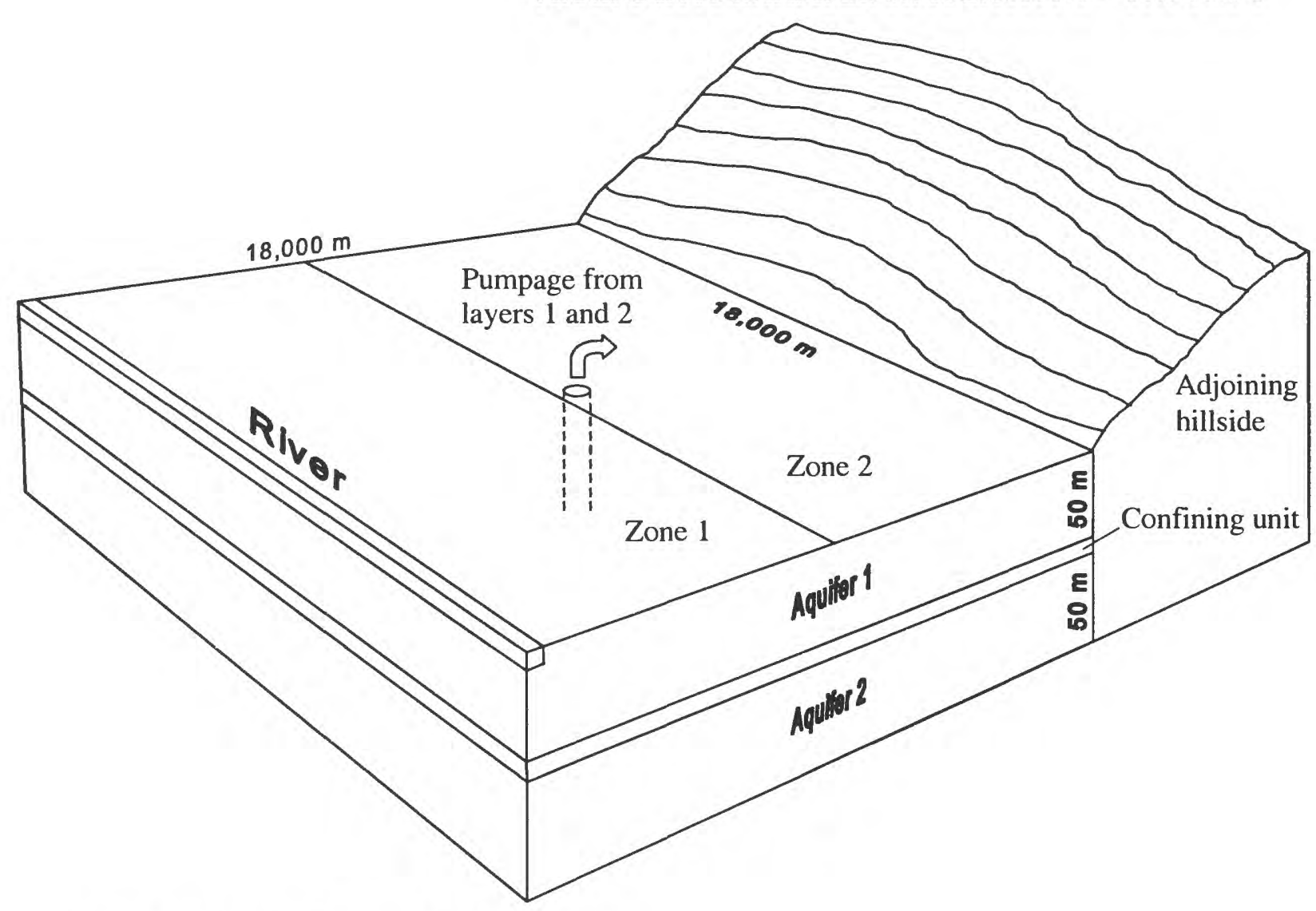

Figure A1: Physical system for test case 1

Table A1: Parameters defined for test case 1, starting and true parameter values, and the values estimated using the data with errors added. The associated output file tcl.glo is presented in this appendix. [m, meter; s, second; cm, centimeter; yr, year]

\begin{tabular}{|c|c|c|c|c|}
\hline $\begin{array}{l}\text { Parameter } \\
\text { name }\end{array}$ & Description & $\begin{array}{l}\text { Starting } \\
\text { Value }\end{array}$ & $\begin{array}{l}\text { Estimated } \\
\text { Value }\end{array}$ & $\begin{array}{l}\text { True } \\
\text { Value }\end{array}$ \\
\hline WELLS_TR & $\begin{array}{l}\text { Pumping rate in each of layers } 1 \text { and } 2 \\
\left(\mathrm{~m}^{3} / \mathrm{s}\right)\end{array}$ & -1.10 & -1.07 & -1.00 \\
\hline RCH_ZONE_1 & Recharge rate in zone $1(\mathrm{~cm} / \mathrm{yr})$ & 63.1 & 34.1 & 31.6 \\
\hline $\mathrm{RCH}_{\text {ZZONE_2 }}$ ZON & Recharge rate in zone $2(\mathrm{~cm} / \mathrm{yr})$ & 31.5 & 50.5 & 47.3 \\
\hline RIVERS & $\begin{array}{l}\text { Hydraulic conductivity of the riverbed } \\
(\mathrm{m} / \mathrm{s})\end{array}$ & $1.20 \times 10^{-3}$ & $1.38 \times 10^{-3}$ & $1.00 \times 10^{-3}$ \\
\hline SS_1 & $\begin{array}{l}\text { Storage coefficient of aquifer } 1 \\
\text { (dimensionless) }\end{array}$ & $1.30 \times 10^{-3}$ & $1.14 \times 10^{-3}$ & $2.00 \times 10^{-3}$ \\
\hline HK_1 & $\begin{array}{l}\text { Hydraulic conductivity of aquifer } 1 \\
(\mathrm{~m} / \mathrm{s})\end{array}$ & $3.00 \times 10^{-4}$ & $4.26 \times 10^{-4}$ & $4.00 \times 10^{-4}$ \\
\hline VERT_K_CB & $\begin{array}{l}\text { Vertical hydraulic conductivity of the } \\
\text { confining layer }(\mathrm{m} / \mathrm{s})\end{array}$ & $1.00 \times 10^{-7}$ & $2.17 \times 10^{-7}$ & $2.00 \times 10^{-7}$ \\
\hline SS_2 & $\begin{array}{l}\text { Storage coefficient of aquifer } 2 \\
\text { (dimensionless) }\end{array}$ & $2.00 \times 10^{-4}$ & $6.20 \times 10^{-5}$ & $2.00 \times 10^{-6}$ \\
\hline HK_2 & $\begin{array}{l}\text { Hydraulic conductivity of aquifer } 2 \\
\text { under the river }(\mathrm{m} / \mathrm{s})\end{array}$ & $4.00 \times 10^{-5}$ & $4.82 \times 10^{-5}$ & $4.40 \times 10^{-5}$ \\
\hline \multicolumn{2}{|c|}{ Sum of squared, weighted residuals (--) } & 268,000 & 36.5 & \\
\hline
\end{tabular}

Table A2: Parameters defined for test case 1, starting and true parameter values, and the values estimated using the data without errors added. This is from the set of data files with file 
name base "tc1-true" distributed with MODFLOW-2000. [m, meter; s, second; $\mathrm{cm}$, centimeter; yr, year]

\begin{tabular}{|c|c|c|c|c|}
\hline $\begin{array}{l}\text { Parameter } \\
\text { name }\end{array}$ & Description & $\begin{array}{l}\text { Starting } \\
\text { Values }\end{array}$ & $\begin{array}{c}\text { Estimated } \\
\text { Values }\end{array}$ & $\begin{array}{c}\text { True } \\
\text { Values }\end{array}$ \\
\hline WELLS_TR & $\begin{array}{l}\text { Pumping rate in each of layers } 1 \text { and } 2 \\
\left(\mathrm{~m}^{3} / \mathrm{s}\right)\end{array}$ & -1.10 & -1.00 & -1.00 \\
\hline RCH_ZONE_1 & Recharge rate in zone $1(\mathrm{~cm} / \mathrm{yr})$ & 60 & 31.6 & 31.6 \\
\hline $\mathrm{RCH}_{\text {_ZONE_2 }}$ & Recharge rate in zone $2(\mathrm{~cm} / \mathrm{yr})$ & 30 & 47.3 & 47.3 \\
\hline RIVERS & $\begin{array}{l}\text { Hydraulic conductivity of the riverbed } \\
(\mathrm{m} / \mathrm{s})\end{array}$ & $1.20 \times 10^{-3}$ & $1.00 \times 10^{-3}$ & $1.00 \times 10^{-3}$ \\
\hline SS_1 & $\begin{array}{l}\text { Storage coefficient of aquifer } 1 \\
\text { (dimensionless) }\end{array}$ & $1.30 \times 10^{-3}$ & $1.00 \times 10^{-3}$ & $1.00 \times 10^{-3}$ \\
\hline HK_1 & $\begin{array}{l}\text { Hydraulic conductivity of aquifer } 1 \\
(\mathrm{~m} / \mathrm{s})\end{array}$ & $3.00 \times 10^{-4}$ & $4.00 \times 10^{-4}$ & $4.00 \times 10^{-4}$ \\
\hline VERT_K_CB & $\begin{array}{l}\text { Vertical hydraulic conductivity of the } \\
\text { confining layer }(\mathrm{m} / \mathrm{s})\end{array}$ & $1.00 \times 10^{-7}$ & $2.00 \times 10^{-7}$ & $2.00 \times 10^{-7}$ \\
\hline SS_2 & $\begin{array}{l}\text { Storage coefficient of aquifer } 2 \\
\text { (dimensionless) }\end{array}$ & $2.00 \times 10^{-4}$ & $1.00 \times 10^{-4}$ & $1.00 \times 10^{-4}$ \\
\hline HK_2 & $\begin{array}{l}\text { Hydraulic conductivity of aquifer } 2 \\
\text { under the river }(\mathrm{m} / \mathrm{s})\end{array}$ & $4.00 \times 10^{-5}$ & $4.40 \times 10^{-5}$ & $4.40 \times 10^{-5}$ \\
\hline \multicolumn{2}{|c|}{ Sum of squared, weighted residuals (--) } & 269,000 & $1.75 \times 10^{-3}$ & \\
\hline
\end{tabular}

The pumpage is simulated using the Well Package. Wells are located at the center of the cells at row 9, column 10; there is one well is in each layer and both wells have the same pumping rate. The parameter Q_1\&2 specifies the pumping rate for each of the wells.

The parameter values estimated using observations with and without noise added to the observations are listed in tables $\mathrm{A} 1$ and $\mathrm{A} 2$, which were presented at the beginning of the previous section of this report. The results without noise added to the observations are presented to demonstrate that the regression estimated the true parameter values when the model matched the synthetic system used to generate the observations and no noise was added to the observations. This constitutes a test of the regression algorithm, and it can be seen that all parameter values were correctly estimated. Selected input and output files from the run with noisy observations are presented in the following sections. 


\section{Input Files}

For the Parameter-Estimation mode, MODFLOW-2000 needs to be run with a name file that includes file types OBS, SEN, and PES (table 3). The hydraulic-head observations are listed in an HOB file (that is, a file with file type HOB in the name file), and the flow observations are listed in an RVOB file; the file name extensions used for these files are ohd and orv so that they will be together and with the OBS file when files in the directory are listed alphabetically. All input files for test case 1 are listed in the file tcl.nam, which is listed below. Parameters are defined in the input files for the packages to which the parameters apply (Harbaugh and others, 2000); parameter values are obtained from the SEN file.

For this test case, head observations are listed in the tc l.ohd file, and flow observations for boundaries simulated using the River Package are listed in the tcl.orv file. In repetitions of item 5 in the HOB file, ITT=2, so that initial hydraulic heads and subsequent changes in hydraulic head are used as observations. The Observation Process input files are as follows:

Name File (tc1.nam)

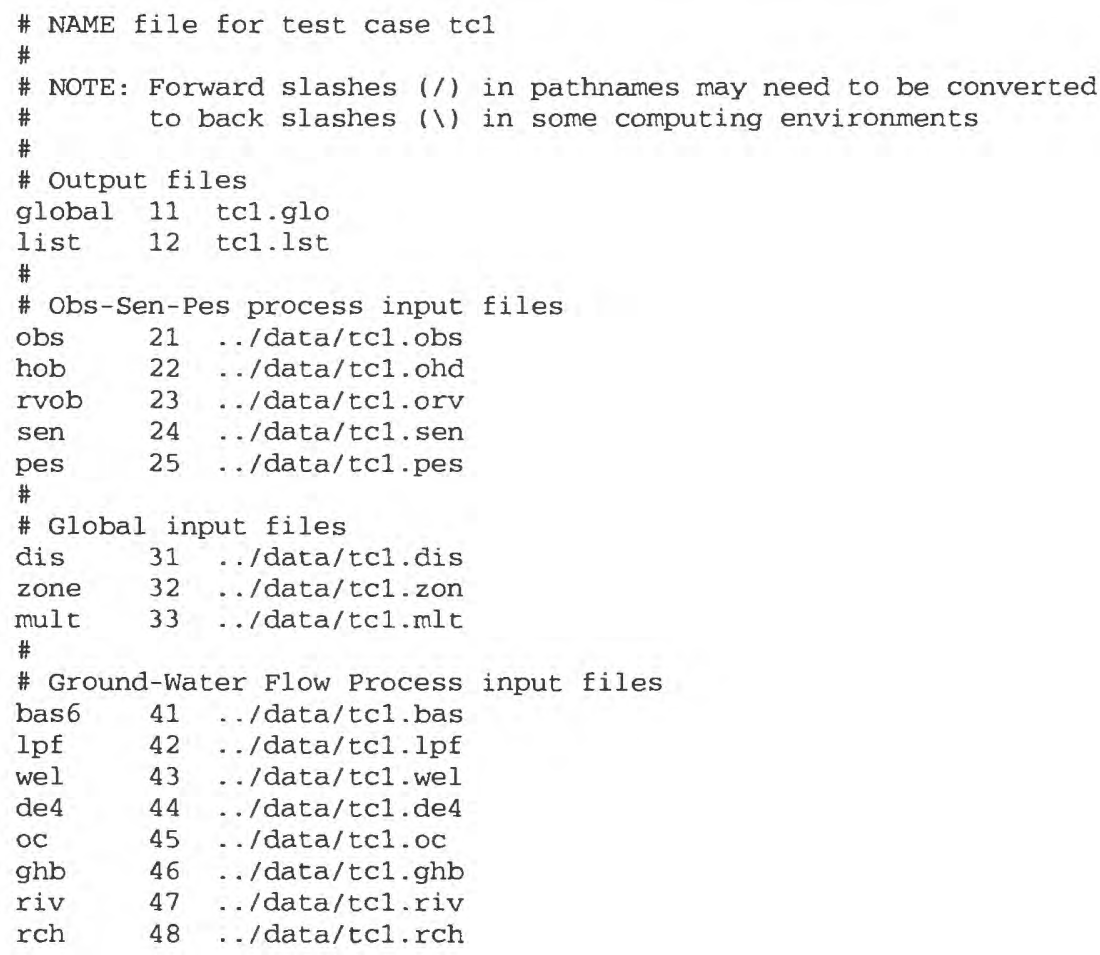

\section{OBS file (tc1.obs):}

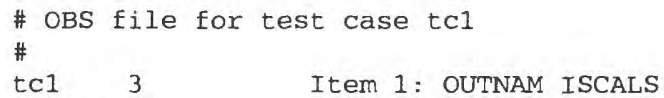

HOB file (tcl.ohd):

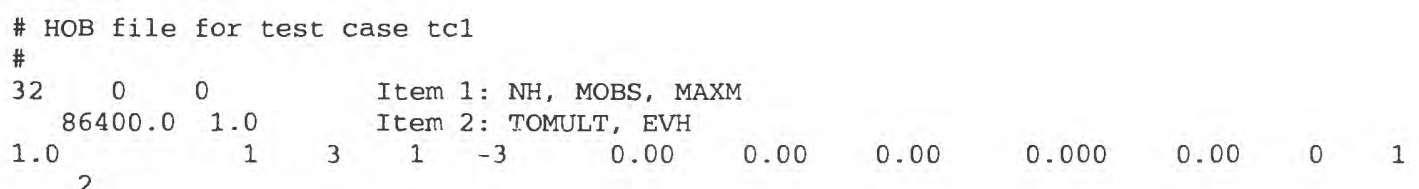




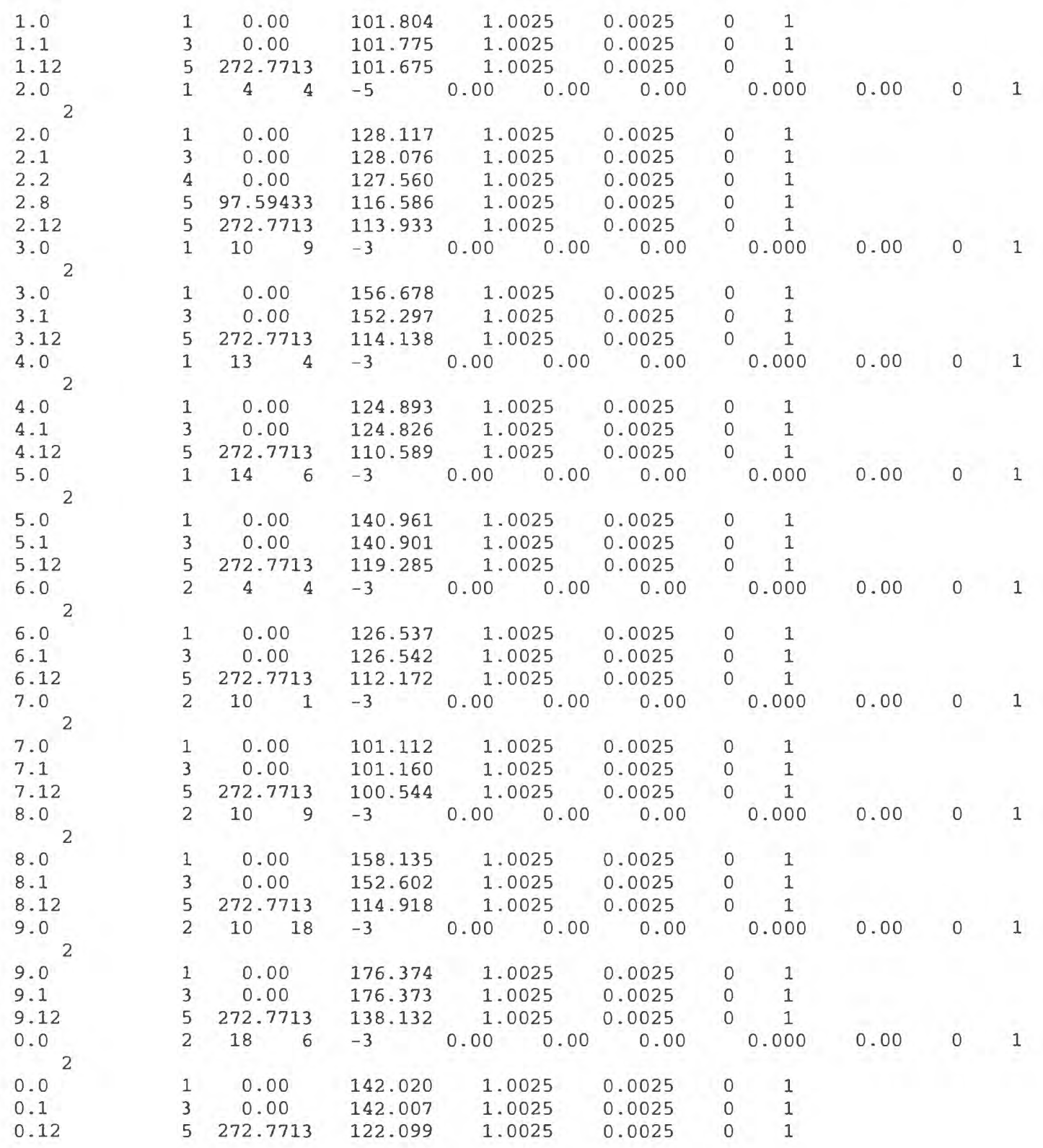

\section{RVOB file (tcl.orv):}

\# RVOB file for test case tcl

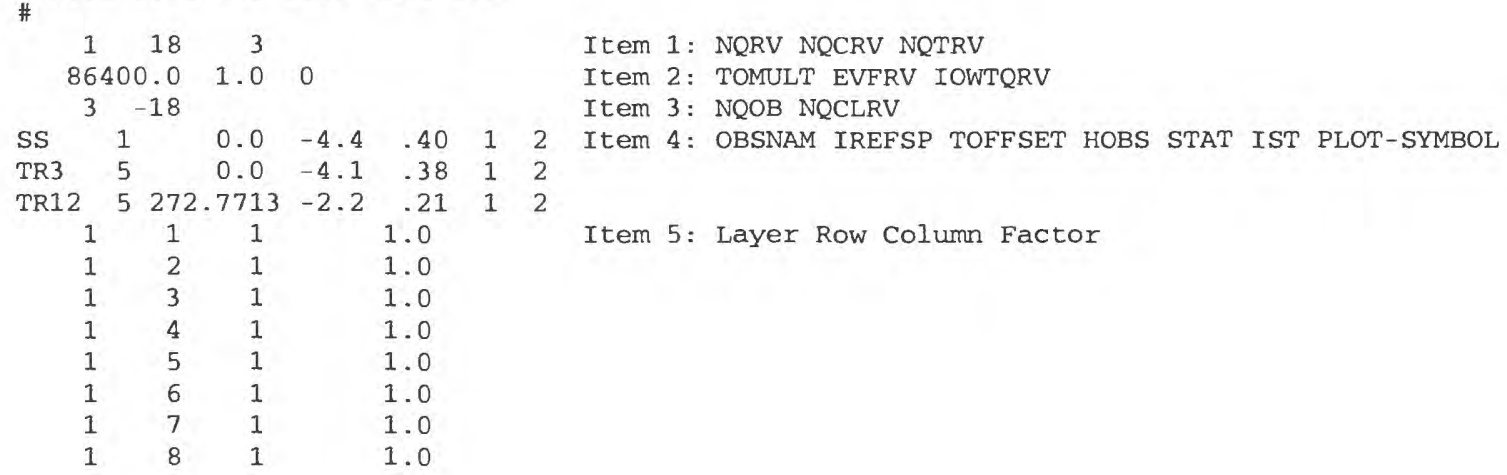




$\begin{array}{rrrr}1 & 9 & 1 & 1.0 \\ 1 & 10 & 1 & 1.0 \\ 1 & 11 & 1 & 1.0 \\ 1 & 12 & 1 & 1.0 \\ 1 & 13 & 1 & 1.0 \\ 1 & 14 & 1 & 1.0 \\ 1 & 15 & 1 & 1.0 \\ 1 & 16 & 1 & 1.0 \\ 1 & 17 & 1 & 1.0 \\ 1 & 18 & 1 & 1.0\end{array}$

Starting estimates for parameters are listed in the Sensitivity Process input file. The Sensitivity Process input file also controls which parameters are analyzed for sensitivity.

SEN file (tc 1.sen):

\# SEN file for test case tc1

\begin{tabular}{|c|c|c|c|c|c|c|c|}
\hline 9 & 0 & -40 & 9 & & & & ITEM 1: NPLIST ISENALL IUHEAD MXSEN \\
\hline 0 & 0 & 12 & 20 & & & & ITEM 2: IPRINTS ISENSU ISENPU ISENFM \\
\hline WELLS_TR & 1 & 0 & -1.1 & -1.4 & -0.80 & $.1 \mathrm{E}-2$ & ITEMS 3: PARNAM ISENS LN B BL BU BSCAL \\
\hline RCH_ZONE_1 & 1 & 0 & 63.072 & 20.0 & 100.0 & $.1 \mathrm{E}-1$ & \\
\hline RCH_ZONE_2 & 1 & 0 & 31.536 & 10.0 & 50.0 & $.1 E-1$ & \\
\hline RIVERS & 1 & 1 & 1. $2 \mathrm{E}-3$ & 1. $2 \mathrm{E}-4$ & 1. $2 \mathrm{E}-2$ & $.1 E-5$ & \\
\hline SS_1 & 1 & 1 & $1.3 E-3$ & 1. $3 E-4$ & 1. $3 E-2$ & $.1 E-6$ & \\
\hline HK__ 1 & 1 & 1 & $3.0 E-4$ & $3.0 \mathrm{E}-5$ & $3.0 E-3$ & $.1 \mathrm{E}-5$ & \\
\hline VERT_K_CB & 1 & 1 & 1. $0 \mathrm{E}-7$ & 1. $0 \mathrm{E}-8$ & 1. $0 \mathrm{E}-6$ & $.1 E-9$ & \\
\hline SS_2 & 1 & 1 & $2.0 E-4$ & $2.0 \mathrm{E}-5$ & $2.0 E-3$ & $.1 E-7$ & \\
\hline HK_2 & 1 & 1 & $4.0 E-5$ & $4.0 \mathrm{E}-6$ & $4.0 E-4$ & $.1 E-6$ & \\
\hline
\end{tabular}

PES file (tc1.pes):

\# PES file for test case tcl

\#

$\begin{array}{llllll}10 & 2.0 & 0.01 & 0.0 & \text { ITEM 1: ITMXP DMAX TOL SOSC }\end{array}$

$\begin{array}{llllllllllll}0 & 0 & 0 & 0 & 0 & 0.0 & 0.001 & 1.5 & 0 & \text { ITEM } & 2: & \text { IBEFLG IYCFLG IOSTAR NOPT NFIT SOSR RMAR RMARM IAP }\end{array}$

$\begin{array}{rrrl}6 & 0 & 0 & \text { ITEM } 3: \text { IPRCOV IPRINT LPRI } \\ 0.08 & 0.0 & 0 & \text { ITEM } 4: \text { CSA FCONV LASTX } \\ 0 & 0 & 0 & \text { ITEM 5: NRNG IPR MPR }\end{array}$ 


\section{APPENDIX A. EXAMPLE SIMULATIONS - Test Case 1 - GLOBAL Output File}

\section{GLOBAL Output File}

File tc1.glo:

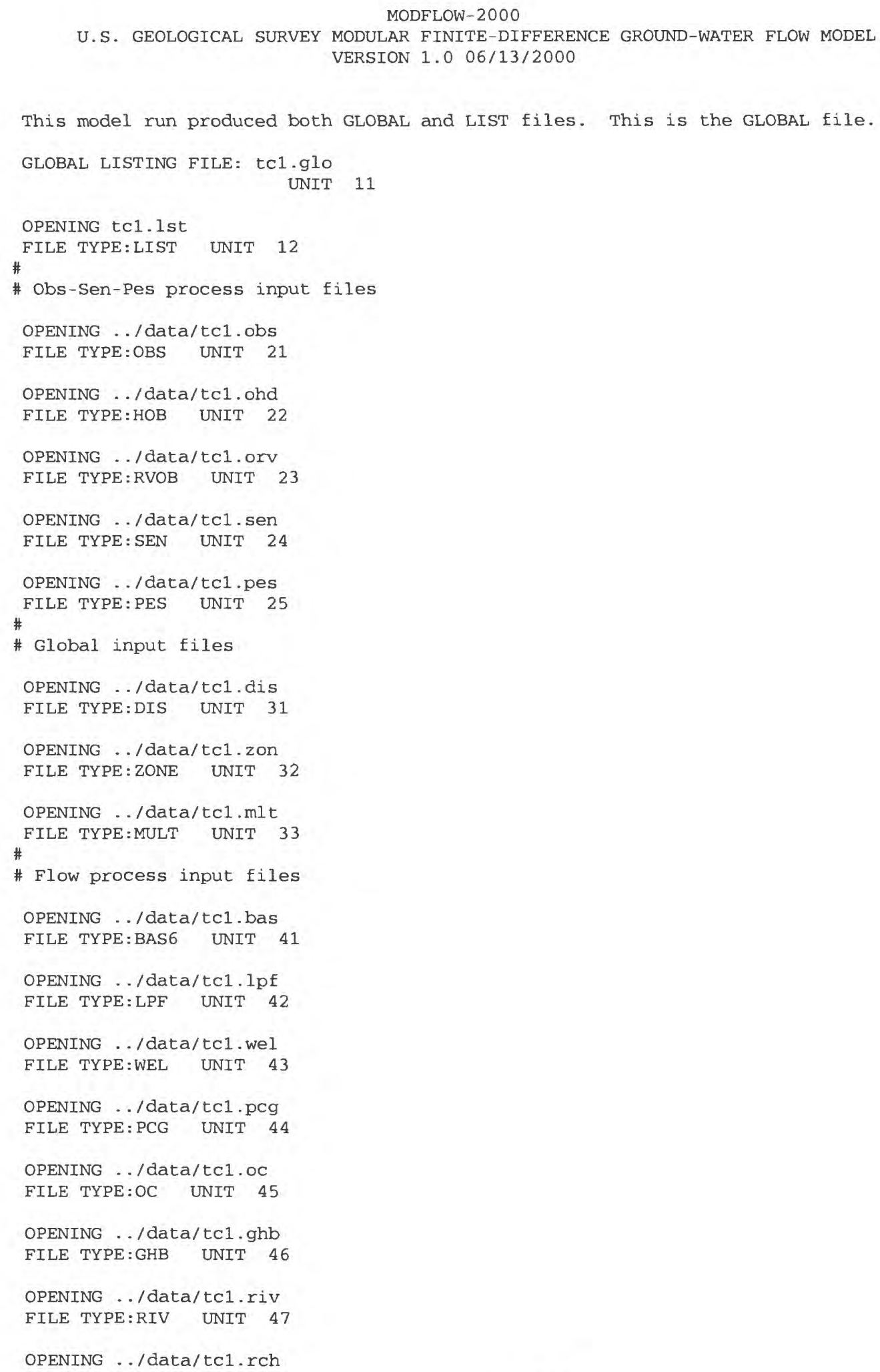




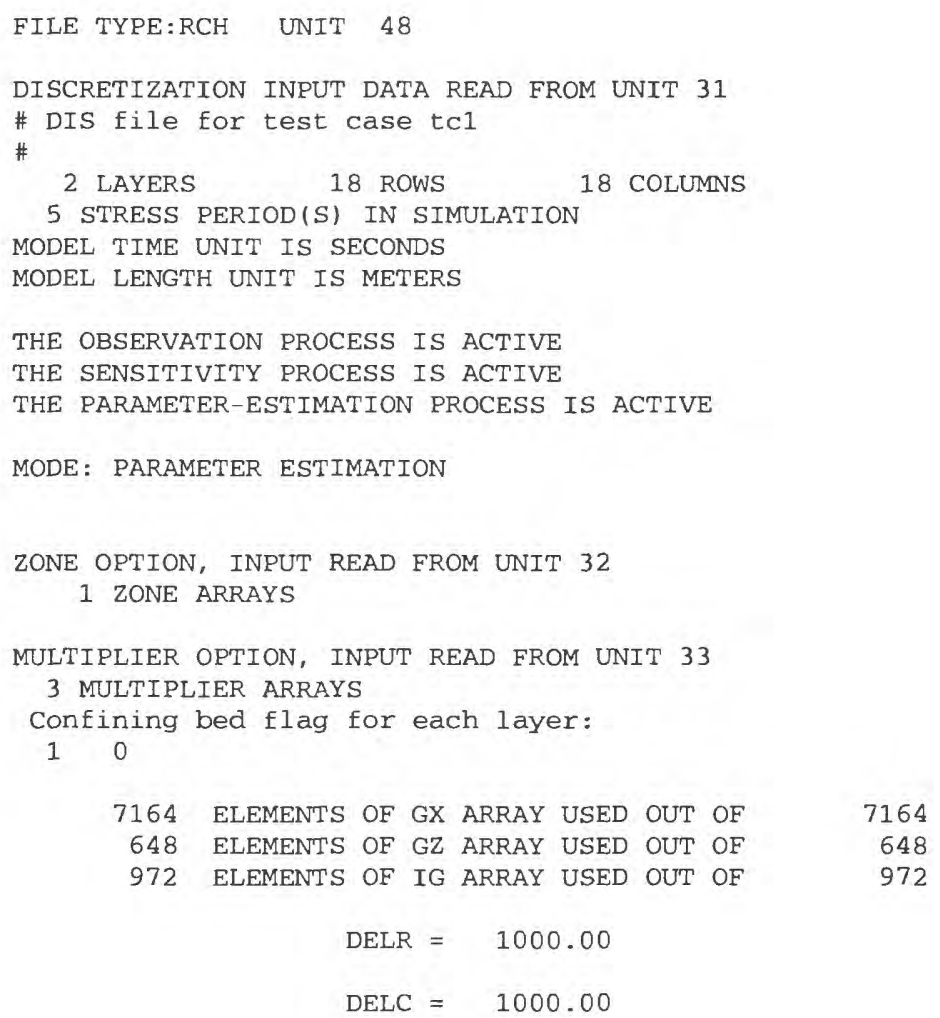

\begin{tabular}{ccccc} 
STRESS PERIOD & LENGTH & TIME STEPS & MULTIPLIER FOR DELT & SS FLAG \\
\hline 1 & 1.000000 & 1 & 1.000 & SS \\
2 & 87162.00 & 1 & 1.000 & TR \\
4 & 261486.0 & 1 & 1.000 & TR \\
4 & 522972.0 & 1 & 1.000 & TR \\
5 & $2.3567440 \mathrm{E}+07$ & 9 & 1.200 & TR
\end{tabular}

COMBINED STEADY-STATE AND TRANSIENT SIMULATION

MULT. ARRAY : MULTARR_3

READING ON UNIT 33 WITH FORMAT: (18F3.0)

\begin{tabular}{|c|c|c|c|c|c|c|c|c|c|c|}
\hline & 1 & 2 & 3 & 4 & 5 & 6 & 7 & 8 & \multirow[t]{2}{*}{9} & \multirow[t]{2}{*}{10} \\
\hline & 11 & 12 & 13 & 14 & 15 & 16 & 17 & 18 & & \\
\hline \multirow[t]{2}{*}{1} & 1.0 & 1.0 & 2.0 & 2.0 & 3.0 & 3.0 & 4.0 & 4.0 & \multirow[t]{2}{*}{5.0} & \multirow[t]{2}{*}{5.0} \\
\hline & 6.0 & 6.0 & 7.0 & 7.0 & 8.0 & 8.0 & 9.0 & 9.0 & & \\
\hline \multirow[t]{2}{*}{2} & 1.0 & 1.0 & 2.0 & 2.0 & 3.0 & 3.0 & 4.0 & 4.0 & \multirow[t]{2}{*}{5.0} & \\
\hline & 6.0 & 6.0 & 7.0 & 7.0 & 8.0 & 8.0 & 9.0 & 9.0 & & \\
\hline
\end{tabular}




$\begin{array}{lllllllllll}17 & 1.0 & 1.0 & 2.0 & 2.0 & 3.0 & 3.0 & 4.0 & 4.0 & 5.0 & 5.0 \\ & 6.0 & 6.0 & 7.0 & 7.0 & 8.0 & 8.0 & 9.0 & 9.0 & & \\ & 1.0 & 1.0 & 2.0 & 2.0 & 3.0 & 3.0 & 4.0 & 4.0 & 5.0 & 5.0 \\ & 6.0 & 6.0 & 7.0 & 7.0 & 8.0 & 8.0 & 9.0 & 9.0 & & \end{array}$

MULT. ARRAY : mlt_rch $=3.170979 \mathrm{E}-10$

MULT. ARRAY: FIFTIETH $=2.000000 \mathrm{E}-02$

ZONE ARRAY: ZONES_1

READING ON UNIT 32 WITH FORMAT: (9I8)

\begin{tabular}{|c|c|c|c|c|c|c|c|c|c|c|c|c|c|c|c|c|c|c|}
\hline & 1 & 2 & 3 & 4 & 5 & 6 & 7 & 8 & 9 & 10 & 11 & 12 & 13 & 14 & 15 & 16 & 17 & 18 \\
\hline 1 & 1 & 1 & 1 & 1 & 1 & 1 & 1 & 1 & 1 & 2 & 2 & 2 & 2 & 2 & 2 & 2 & 2 & 2 \\
\hline 2 & 1 & 1 & 1 & 1 & 1 & 1 & 1 & 1 & 1 & 2 & 2 & 2 & 2 & 2 & 2 & 2 & 2 & 2 \\
\hline 3 & 1 & 1 & 1 & 1 & 1 & 1 & 1 & 1 & 1 & 2 & 2 & 2 & 2 & 2 & 2 & 2 & 2 & 2 \\
\hline 4 & 1 & 1 & 1 & 1 & 1 & 1 & 1 & 1 & 1 & 2 & 2 & 2 & 2 & 2 & 2 & 2 & 2 & 2 \\
\hline 5 & 1 & 1 & 1 & 1 & 1 & 1 & 1 & 1 & 1 & 2 & 2 & 2 & 2 & 2 & 2 & 2 & 2 & 2 \\
\hline 6 & 1 & 1 & 1 & 1 & 1 & 1 & 1 & 1 & 1 & 2 & 2 & 2 & 2 & 2 & 2 & 2 & 2 & $Z$ \\
\hline 7 & 1 & 1 & 1 & 1 & 1 & 1 & 1 & 1 & 1 & 2 & 2 & 2 & 2 & 2 & 2 & 2 & 2 & 2 \\
\hline 8 & 1 & 1 & 1 & 1 & 1 & 1 & 1 & 1 & 1 & 2 & 2 & 2 & 2 & 2 & 2 & 2 & 2 & $\&$ \\
\hline 9 & 1 & 1 & 1 & 1 & 1 & 1 & 1 & 1 & 1 & 2 & 2 & 2 & 2 & 2 & 2 & 2 & 2 & 2 \\
\hline 10 & 1 & 1 & 1 & 1 & 1 & 1 & 1 & 1 & 1 & 2 & 2 & 2 & 2 & 2 & 2 & 2 & 2 & 2 \\
\hline 11 & 1 & 1 & 1 & 1 & 1 & 1 & 1 & 1 & 1 & 2 & 2 & 2 & 2 & 2 & 2 & 2 & 2 & 2 \\
\hline 12 & 1 & 1 & 1 & 1 & 1 & 1 & 1 & 1 & 1 & 2 & 2 & 2 & 2 & 2 & 2 & 2 & 2 & 2 \\
\hline 13 & 1 & 1 & 1 & 1 & 1 & 1 & 1 & 1 & 1 & 2 & 2 & 2 & 2 & 2 & 2 & 2 & 2 & \\
\hline 14 & 1 & 1 & 1 & 1 & 1 & 1 & 1 & 1 & 1 & 2 & 2 & 2 & 2 & 2 & 2 & 2 & 2 & 2 \\
\hline 15 & 1 & 1 & 1 & 1 & $I$ & 1 & 1 & 1 & 1 & 2 & 2 & 2 & 2 & 2 & 2 & 2 & 2 & 2 \\
\hline 16 & 1 & 1 & 1 & 1 & 1 & 1 & 1 & 1 & 1 & 2 & 2 & 2 & 2 & 2 & 2 & 2 & 2 & \\
\hline 17 & 1 & 1 & 1 & 1 & 1 & 1 & 1 & 1 & 1 & 2 & 2 & 2 & 2 & 2 & 2 & 2 & 2 & \\
\hline 18 & 1 & 1 & 1 & 1 & 1 & 1 & 1 & 1 & 1 & 2 & 2 & 2 & 2 & 2 & 2 & 2 & 2 & \\
\hline
\end{tabular}

LPF1 -- LAYER PROPERTY FLOW PACKAGE, VERSION $1,1 / 11 / 2000$ INPUT READ FROM UNIT 42

\# LPF input file for test case tc1

HEAD AT CELLS THAT CONVERT TO DRY $=-999.00$

7 Named Parameters

LAYER FLAGS:

\begin{tabular}{|c|c|c|c|c|c|}
\hline LAYER & LAYTYP & LAYAVG & CHANI & LAYVKA & LAYWET \\
\hline 1 & 0 & 0 & $1.000 \mathrm{E}+00$ & 1 & 0 \\
\hline 2 & 0 & 0 & 1. $.000 E+00$ & 1 & 0 \\
\hline
\end{tabular}

INTERPRETATION OF LAYER FLAGS:

\begin{tabular}{cccccc} 
LAYER TYPE & $\begin{array}{c}\text { INTERBLOCK } \\
\text { TRANSMISSIVITY } \\
\text { (LAYTYP) }\end{array}$ & $\begin{array}{c}\text { HORIZONTAL } \\
\text { (LNISOTROPY } \\
\text { (LHANI) }\end{array}$ & $\begin{array}{c}\text { DATA IN } \\
\text { ARRAY VKA } \\
\text { (LAYVKA) }\end{array}$ & $\begin{array}{c}\text { WETTABILITY } \\
\text { (LAYWET) }\end{array}$ \\
\hline 1 & CONFINED & HARMONIC & $1.000 \mathrm{E}+00$ & ANISOTROPY & NON-WETTABLE \\
2 & CONFINED & HARMONIC & $1.000 \mathrm{E}+00$ & ANISOTROPY & NON-WETTABLE
\end{tabular}

2268 ELEMENTS IN X ARRAY ARE USED BY LPF

12 ELEMENTS IN IX ARRAY ARE USED BY LPF

PCG2 -- CONJUGATE GRADIENT SOLUTION PACKAGE, VERSION $2.4,12 / 29 / 98$

MAXIMUM OF 1 CALLS OF SOLUTION ROUTINE

MAXIMUM OF 50 INTERNAL ITERATIONS PER CALL TO SOLUTION ROUTINE

MATRIX PRECONDITIONING TYPE : 1

2044 ELEMENTS IN X ARRAY ARE USED BY PCG

350 ELEMENTS IN IX ARRAY ARE USED BY PCG

1296 ELEMENTS IN Z ARRAY ARE USED BY PCG 
SEN1BAS6 - SENSITIVITY PROCESS, VERSION 1.0, 10/15/98

INPUT READ FROM UNIT 24

NUMBER OF PARAMETER VALUES TO BE READ FROM SEN FILE: 9

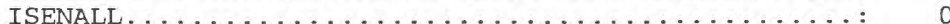

SENSITIVITIES WILL BE STORED IN MEMORY

FOR UP TO 9 PARAMETERS

1026 ELEMENTS IN X ARRAY ARE USED FOR SENSITIVITIES

648 ELEMENTS IN Z ARRAY ARE USED FOR SENSITIVITIES

18 ELEMENTS IN IX ARRAY ARE USED FOR SENSITIVITIES

PES1BAS6 - PARAMETER-ESTIMATION PROCESS, VERSION 1.0, 07/22/99

INPUT READ FROM UNIT 25

\# PES file for test case tc1

\#

MAXIMUM NUMBER OF PARAMETER-ESTIMATION ITERATIONS (MAX-ITER) $=10$

MAXIMUM PARAMETER CORRECTION (MAX-CHANGE) $\ldots$

CLOSURE CRITERION (TOL)

SUM OF SQUARES CLOSURE CRITERION (SOSC) $\ldots \ldots \ldots$

FLAG TO GENERATE INPUT NEEDED BY BEALE-2000 (IBEFLG) $\ldots \ldots$

FLAG TO GENERATE INPUT NEEDED BY YCINT-2000 (IYCFLG) $\ldots \ldots$

OMIT PRINTING TO SCREEN (IF = 1 ) (IOSTAR) -........ = 0

ADJUST GAUSS-NEWTON MATRIX WITH NEWTON UPDATES $(I F=1)($ NOPT) $=0$

NUMBER OF FLETCHER-REEVES ITERATIONS (NFIT) -

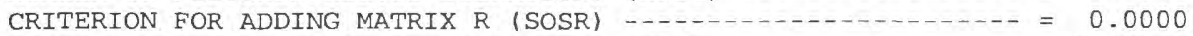

INITIAL VALUE OF MARQUARDT PARAMETER (RMAR)

MARQUARDT PARAMETER MULTIPLIER (RMARM) $\ldots \ldots \ldots$

APPLY MAX-CHANGE IN REGRESSION SPACE (IF $=1$ ) (IAP) $\ldots \ldots$

FORMAT CODE FOR COVARIANCE AND CORRELATION MATRICES (IPRCOV) = 6

PRINT PARAMETER-ESTIMATION STATISTICS

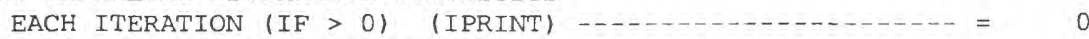

PRINT EIGENVALUES AND EIGENVECTORS OF

COVARIANCE MATRIX (IF > 0 ) (LPRINT)

SEARCH DIRECTION ADJUSTMENT PARAMETER (CSA)

MODIFY CONVERGENCE CRITERIA (IF > 0) (FCONV) $\ldots . . . . .$.

CALCULATE SENSITIVITIES USING FINAL

PARAMETER ESTIMATES (IF > O) (LASTX) $\ldots$

NUMBER OF USUALLY POS. PARAMETERS THAT MAY BE NEG (NPNG) -..- = 0

NUMBER OF PARAMETERS WITH CORRELATED PRIOR INFORMATION $($ IPR $)=0$

NUMBER OF PRIOR-INFORMATION EQUATIONS (MPR) ............. =

232 ELEMENTS IN $X$ ARRAY ARE USED FOR PARAMETER ESTIMATION

295 ELEMENTS IN Z ARRAY ARE USED FOR PARAMETER ESTIMATION

12 ELEMENTS IN IX ARRAY ARE USED FOR PARAMETER ESTIMATION

OBS1BAS6 -- OBSERVATION PROCESS, VERSION 1.0, 4/27/99

INPUT READ FROM UNIT 21

\# OBS file for test case tc1

OBSERVATION GRAPH-DATA OUTPUT FILES

WILL BE PRINTED AND NAMED USING THE BASE: tC1

DIMENSIONLESS SCALED OBSERVATION SENSITIVITIES WILL BE PRINTED

HEAD OBSERVATIONS -- INPUT READ FROM UNIT 22

NUMBER OF HEADS....................... 32

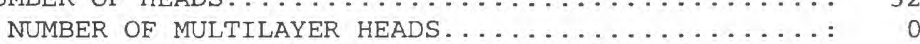

MAXIMUM NUMBER OF LAYERS FOR MULTILAYER HEADS . . . : : 0 
OBSIRIV6 -- OBSERVATION PROCESS (RIVER FLOW OBSERVATIONS)

VERSION 1.0, 10/15/98

INPUT READ FROM UNIT 23

NUMBER OF FLOW-OBSERVATION RIVER-CELL GROUPS...... 1

NUMBER OF CELLS IN RIVER-CELL GROUPS......... 18

NUMBER OF RIVER-CELL FLOWS. . . . . . . . . . 3

1543 ELEMENTS IN X ARRAY ARE USED FOR OBSERVATIONS

20 ELEMENTS IN Z ARRAY ARE USED FOR OBSERVATIONS

339 ELEMENTS IN IX ARRAY ARE USED FOR OBSERVATIONS

COMMON ERROR VARIANCE FOR ALL OBSERVATIONS SET TO:

1.000

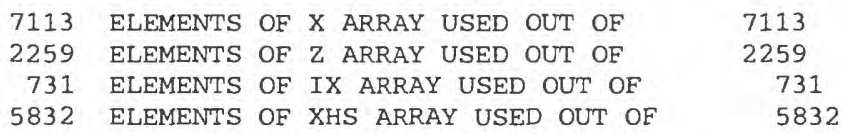

INFORMATION ON PARAMETERS LISTED IN SEN FILE

\begin{tabular}{|c|c|c|c|c|c|c|}
\hline NAME & ISENS & LN & $\begin{array}{l}\text { VALUE IN SEN } \\
\text { INPUT FILE }\end{array}$ & $\begin{array}{l}\text { LOWER } \\
\text { REASONABLE } \\
\text { LIMIT }\end{array}$ & $\begin{array}{c}\text { UPPER } \\
\text { REASONABLE } \\
\text { LIMIT }\end{array}$ & $\begin{array}{l}\text { ALTERNATE } \\
\text { SCALING } \\
\text { FACTOR }\end{array}$ \\
\hline$-\cdots-----$ & ---- & -- & $-\cdots-1-1$ & $-\cdots-1-1$ & $-\cdots-\cdots$ & $---1---1$ \\
\hline WELLS_TR & 1 & 0 & -1.1000 & -1.4000 & -0.80000 & $0.10000 \mathrm{E}-02$ \\
\hline RCH_ZONE_I & 1 & 0 & 63.072 & 30.000 & 80.000 & $0.10000 \mathrm{E}-01$ \\
\hline RCH_ZONE_2 & 1 & 0 & 31.536 & 20.000 & 60.000 & $0.10000 \mathrm{E}-01$ \\
\hline RIVERS & 1 & 1 & $0.12000 \mathrm{E}-02$ & $0.12000 \mathrm{E}-03$ & $0.12000 \mathrm{E}-01$ & $0.10000 \mathrm{E}-05$ \\
\hline SS_1 & 1 & 1 & $0.13000 \mathrm{E}-02$ & $0.13000 \mathrm{E}-03$ & $0.13000 \mathrm{E}-01$ & $0.10000 \mathrm{E}-05$ \\
\hline HK_1 & 1 & 1 & $0.30000 \mathrm{E}-03$ & $0.30000 \mathrm{E}-04$ & $0.30000 E-02$ & $0.10000 \mathrm{E}-06$ \\
\hline VERT_K_CB & 1 & 1 & $0.10000 \mathrm{E}-06$ & $0.10000 \mathrm{E}-07$ & $0.10000 \mathrm{E}-05$ & $0.10000 \mathrm{E}-09$ \\
\hline SS_2 & 1 & 1 & $0.20000 \mathrm{E}-03$ & $0.20000 \mathrm{E}-04$ & $0.20000 \mathrm{E}-02$ & $0.10000 \mathrm{E}-06$ \\
\hline HK_2 & 1 & 1 & $0.40000 \mathrm{E}-04$ & $0.40000 \mathrm{E}-05$ & $0.40000 \mathrm{E}-03$ & $0.10000 \mathrm{E}-07$ \\
\hline
\end{tabular}

FOR THE PARAMETERS LISTED IN THE TABLE ABOVE, PARAMETER VALUES IN INDIVIDUAL PACKAGE INPUT FILES ARE REPLACED BY THE VALUES FROM THE SEN INPUT FILE. THE ALTERNATE SCALING FACTOR IS USED TO SCALE SENSITIVITIES IF IT IS LARGER THAN THE PARAMETER VALUE IN ABSOLUTE VALUE AND THE PARAMETER IS NOT LOG-TRANSFORMED.

F STATISTIC FOR BEALE'S MEASURE SET TO (FSTAT)

2.2700

HEAD OBSERVATION VARIANCES ARE MULTIPLIED BY:

1.000

OBSERVED HEAD DATA -- TIME OFFSETS ARE MULTIPLIED BY: 86400.

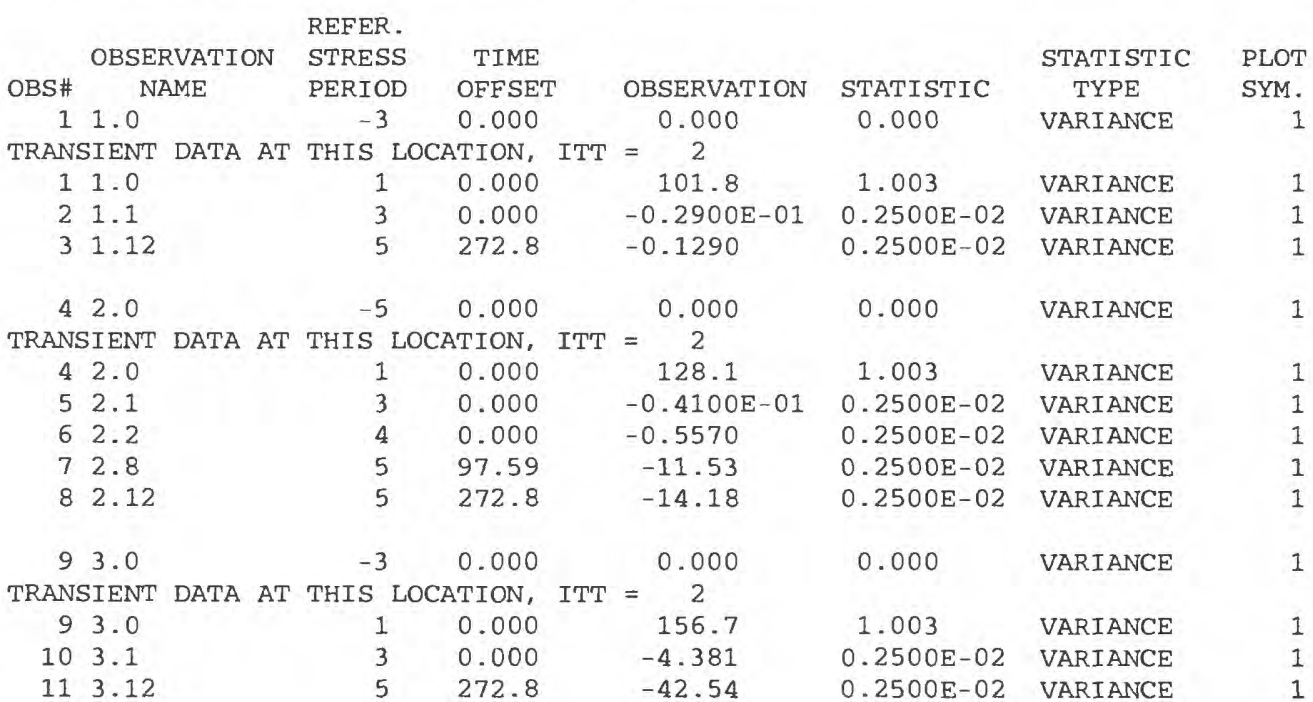


APPENDIX A. EXAMPLE SIMULLATIONS - Test Case 1 - GLOBAL Output File

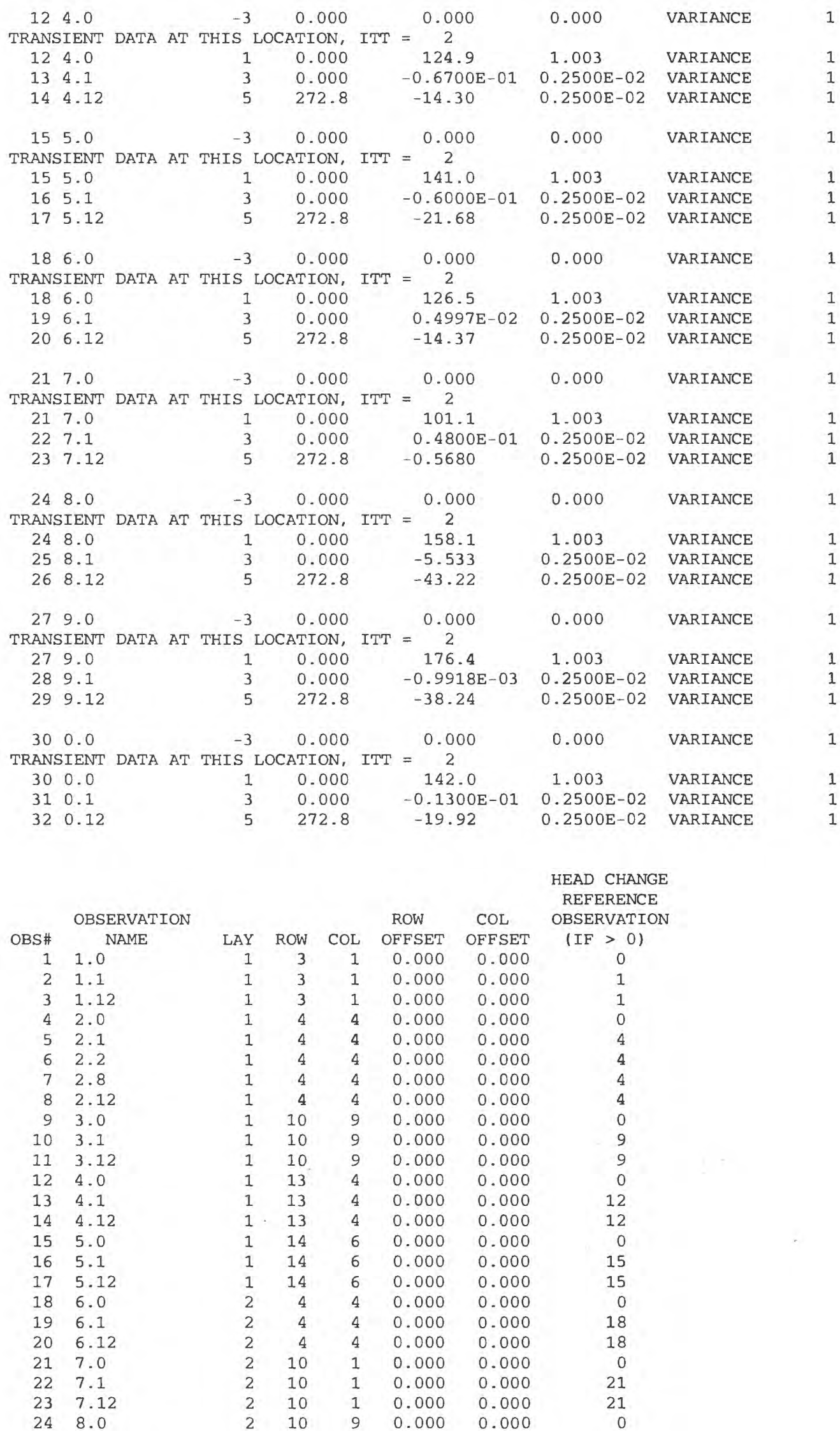


APPENDIX A. EXAMPLE SIMULATIONS - Test Case 1 - GLOBAL Output File

$\begin{array}{llrrrrrr}25 & 8.1 & 2 & 10 & 9 & 0.000 & 0.000 & 24 \\ 26 & 8.12 & 2 & 10 & 9 & 0.000 & 0.000 & 24 \\ 27 & 9.0 & 2 & 10 & 18 & 0.000 & 0.000 & 0 \\ 28 & 9.1 & 2 & 10 & 18 & 0.000 & 0.000 & 27 \\ 29 & 9.12 & 2 & 10 & 18 & 0.000 & 0.000 & 27 \\ 30 & 0.0 & 2 & 18 & 6 & 0.000 & 0.000 & 0 \\ 31 & 0.1 & 2 & 18 & 6 & 0.000 & 0.000 & 30 \\ 32 & 0.12 & 2 & 18 & 6 & 0.000 & 0.000 & 30\end{array}$

RIVER-CELL FLOW OBSERVATION VARIANCES ARE MULTIPLIED BY:

1.000

OBSERVED RIVER-CELL FLOW DATA

-- TIME OFFSETS ARE MULTIPLIED BY: 86400.

GROUP NUMBER: 1 BOUNDARY TYPE: RIV NUMBER OF CELLS IN GROUP: -18 NUMBER OF FLOW OBSERVATIONS : 3

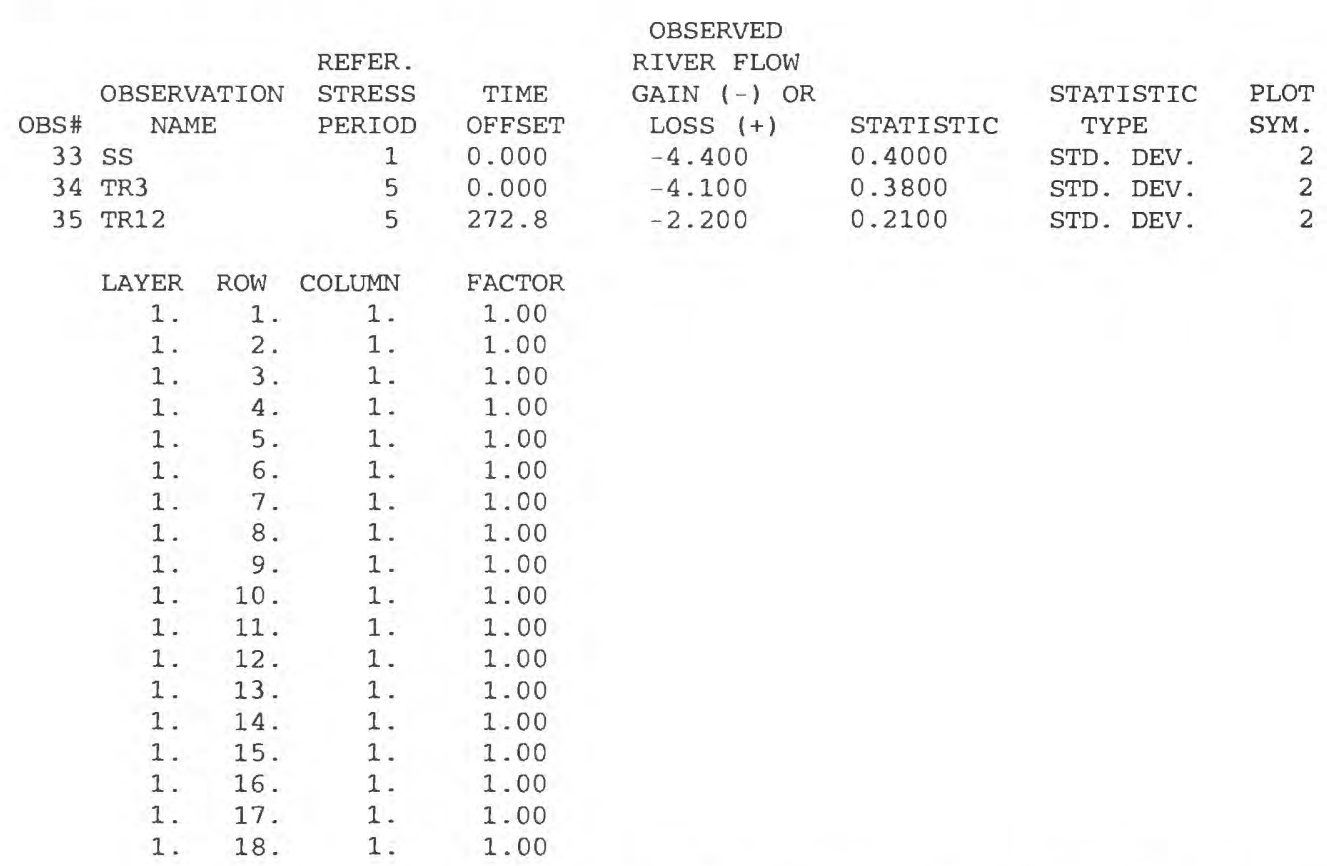

SOLUTION BY THE CONJUGATE-GRADIENT METHOD

MAXIMUM NUMBER OF CALLS TO PCG ROUTINE MAXIMUM ITERATIONS PER CALL TO PCG MATRIX PRECONDITIONING TYPE $=$

RELAXATION FACTOR (ONLY USED WITH PRECOND. TYPE 1 ) = PARAMETER OF POLYMOMIAL PRECOND. $=2$ (2) OR IS CALCULATED : HEAD CHANGE CRITERION FOR CLOSURE = RESIDUAL CHANGE CRITERION FOR CLOSURE = PCG HEAD AND RESIDUAL CHANGE PRINTOUT INTERVAL = PRINTING FROM SOLVER IS LIMITED(1) OR SUPPRESSED $(>1)=$ DAMPING PARAMETER $=$

1
50
1
$0.10000 \mathrm{E}+01$
2
$0.10000 \mathrm{E}-04$
$0.10000 \mathrm{E}-04$
999
2
$0.10000 \mathrm{E}+01$

$\begin{array}{lcc}\text { CONVERGENCE } & \text { CRITERIA FOR } & \text { SENSITIVITIES } \\ \text { PARAMETER } & \text { HCLOSE } & \text { RCLOSE } \\ \text { WELLS_TR } & 0.90909 \mathrm{E}-07 & 0.90909 \mathrm{E}-07 \\ \text { WCL_TONE_1 } & 0.15855 \mathrm{E}-08 & 0.15855 \mathrm{E}-08 \\ \text { RCH_ZONE } & 0.31710 \mathrm{E}-08 \\ \text { RCH_ZONE_2 } & 0.31710 \mathrm{E}-08 & 0.317 \\ \text { RIVERS } & 0.83333 \mathrm{E}-04 & 0.83333 \mathrm{E}-04 \\ \text { SS_1 } & 0.76923 \mathrm{E}-04 & 0.76923 \mathrm{E}-04 \\ \text { HK_I } & 0.33333 \mathrm{E}-03 & 0.33333 \mathrm{E}-03 \\ \text { VERT_K_CB } & 1.0000 & 1.0000 \\ \text { SS_2 } & 0.50000 \mathrm{E}-03 & 0.50000 \mathrm{E}-03 \\ \text { HK_2 } & 0.25000 \mathrm{E}-02 & 0.25000 \mathrm{E}-02\end{array}$




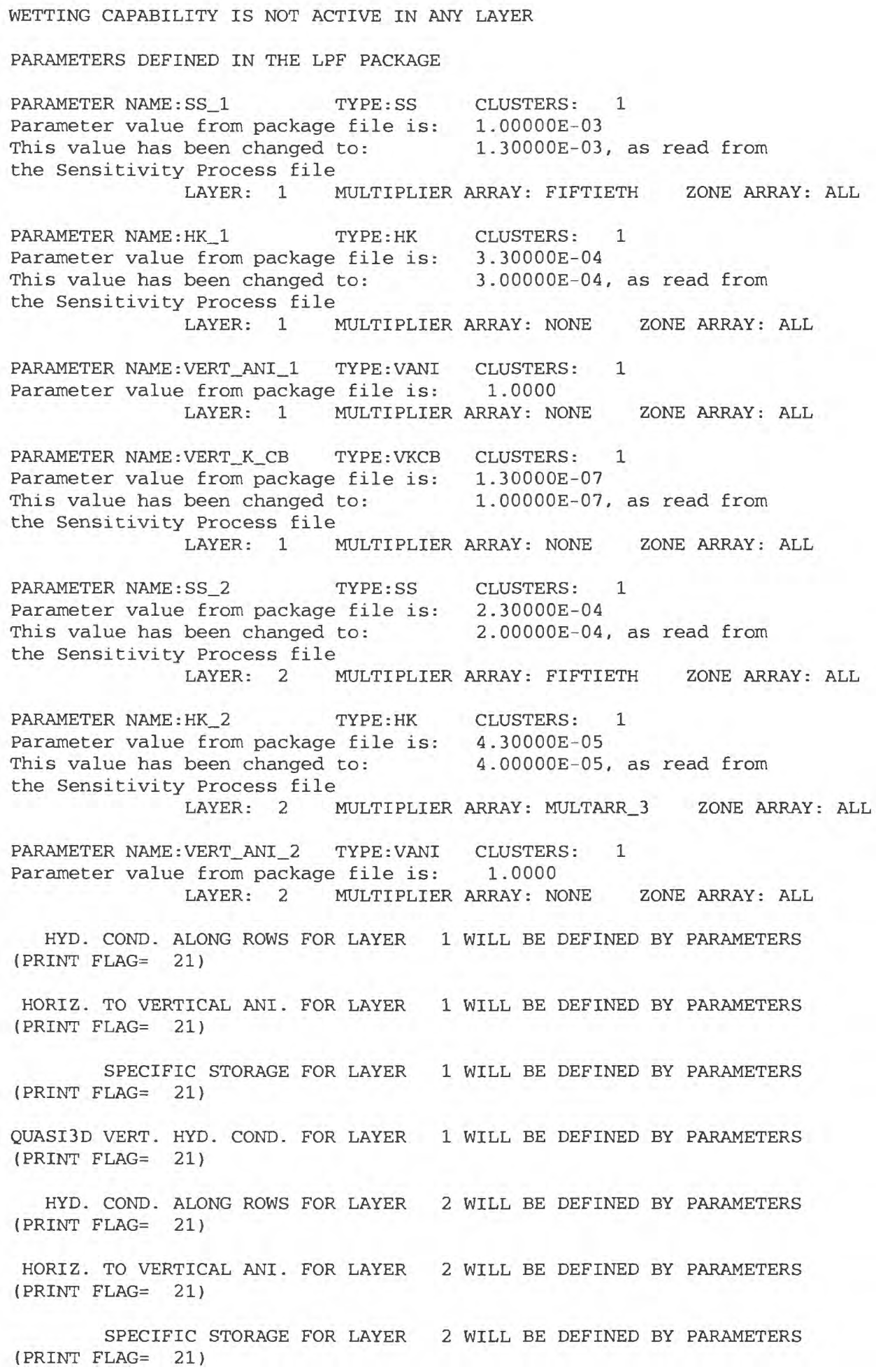


APPENDIX A. EXAMPLE SIMULATIONS - Test Case 1 - GLOBAL Output File

1 Well parameters

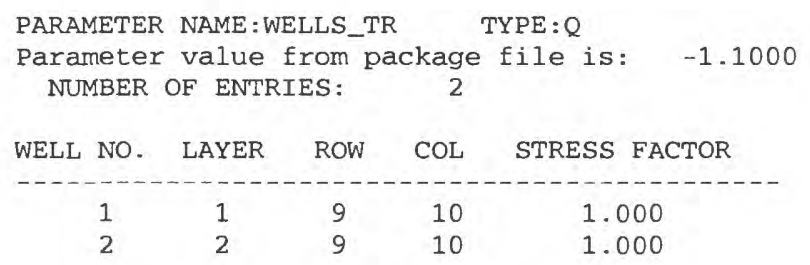

1 River parameters

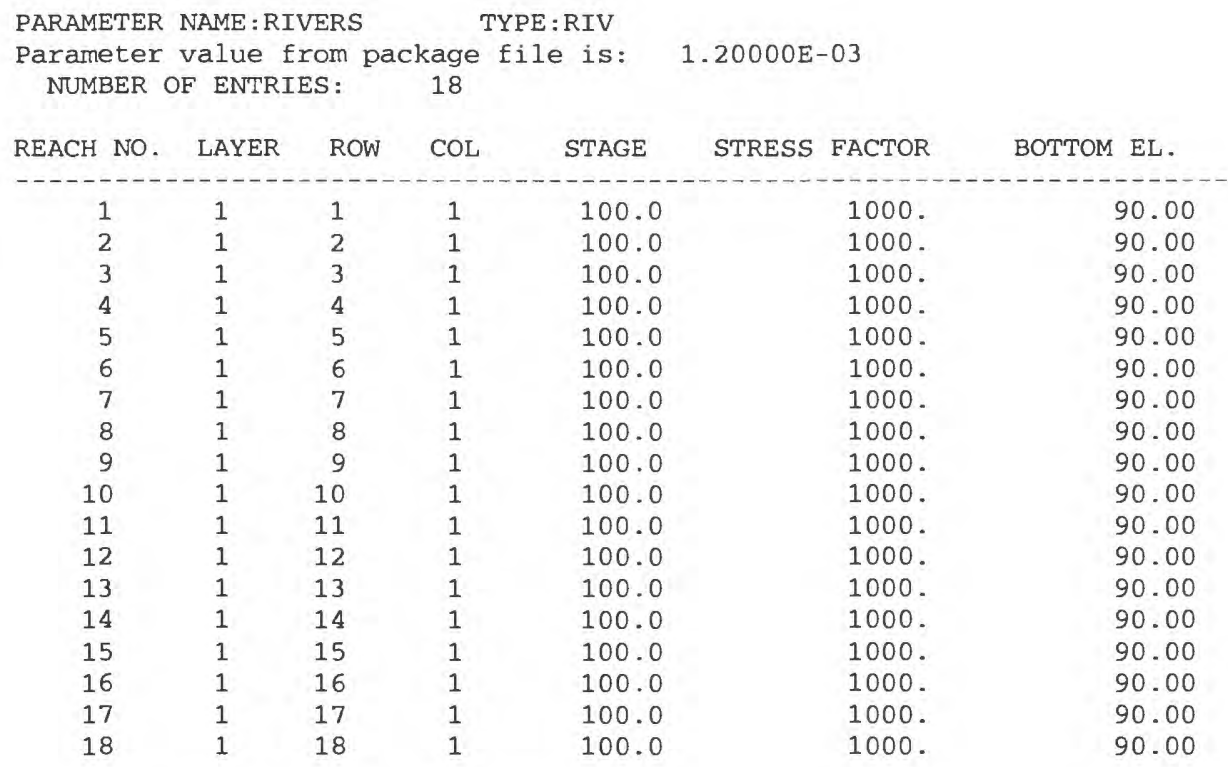

0 GHB parameters

2 Recharge parameters

PARAMETER NAME:RCH_ZONE_1 TYPE:RCH CLUSTERS: 1

Parameter value from package file is: 63.072

MULTIPLIER ARRAY: mlt_rch ZONE ARRAY: zones_1 ZONE VALUES: 1

PARAMETER NAME:RCH_ZONE_2 TYPE:RCH CLUSTERS: 1

Parameter value from package file is:

MULTIPLIER ARRAY: mlt_rch ZONE ARRAY: zones_1 ZONE VALUES:

11 PARAMETERS HAVE BEEN DEFINED IN ALL PACKAGES.

(SPACE IS ALLOCATED FOR 500 PARAMETERS.) 
APPENDIX A. EXAMPLE SIMULATIONS - Test Case 1 - GLOBAL Output File

OBSERVATION SENSITIVITY TABLE(S) FOR PARAMETER-ESTIMATION ITERATION 1

DIMENSIONLESS SCALED SENSITIVITIES (SCALED BY $B^{*}\left(W T^{* *} .5\right)$ )

\begin{tabular}{|c|c|c|c|c|c|c|}
\hline BS \# & $\begin{array}{l}\text { PARAMETER: } \\
\text { OBSERVATION }\end{array}$ & WELLS_TR & RCH_ZONE_1 & RCH_ZONE_2 & RIVERS & SS_1 \\
\hline 1 & 1.0 & 0.00 & 0.150 & $0.749 \mathrm{E}-01$ & -1.51 & 0.00 \\
\hline 2 & 1.1 & $0.262 \mathrm{E}-03$ & $0.294 \mathrm{E}-06$ & $0.147 \mathrm{E}-06$ & $0.237 \mathrm{E}-02$ & $0.573 \mathrm{E}-02$ \\
\hline 3 & 1.12 & 1.81 & $0.294 \mathrm{E}-06$ & $0.147 \mathrm{E}-06$ & 12.2 & 2.22 \\
\hline 4 & 2.0 & 0.00 & 24.0 & 15.3 & -1.51 & 0.00 \\
\hline 5 & 2.1 & 0.190 & $0.376 \mathrm{E}-04$ & 0.00 & $0.224 \mathrm{E}-03$ & 3.31 \\
\hline 6 & 2.2 & 5.52 & 0.00 & 0.00 & $0.271 \mathrm{E}-01$ & 66.5 \\
\hline 7 & 2.8 & 259. & 0.00 & $-0.188 E-04$ & 6.43 & 784. \\
\hline 8 & 2.12 & 375. & $0.376 \mathrm{E}-04$ & $0.188 \mathrm{E}-04$ & 11.4 & 447. \\
\hline 9 & 3.0 & 0.00 & 38.3 & 35.9 & -1.51 & 0.00 \\
\hline 10 & 3.1 & 73.3 & 0.00 & 0.00 & $0.401 \mathrm{E}-05$ & 394. \\
\hline 11 & 3.12 & $0.112 \mathrm{E}+04$ & $0.752 \mathrm{E}-04$ & 0.00 & 10.1 & 975. \\
\hline 12 & 4.0 & 0.00 & 24.0 & 15.3 & -1.51 & 0.00 \\
\hline 13 & 4.1 & 0.325 & $0.376 \mathrm{E}-04$ & 0.00 & $0.309 \mathrm{E}-03$ & 5.28 \\
\hline 14 & 4.12 & 377. & $0.376 E-04$ & $0.188 \mathrm{E}-04$ & 11.2 & 447. \\
\hline 15 & 5.0 & 0.00 & 32.9 & 24.1 & -1.51 & 0.00 \\
\hline 16 & 5.1 & 0.736 & $0.752 \mathrm{E}-04$ & $0.376 \mathrm{E}-04$ & $0.381 \mathrm{E}-04$ & 10.5 \\
\hline 17 & 5.12 & 569. & $0.752 \mathrm{E}-04$ & $0.752 \mathrm{E}-04$ & 10.6 & 690. \\
\hline 18 & 6.0 & 0.00 & 24.0 & 15.6 & -1.51 & 0.00 \\
\hline 19 & 6.1 & 0.249 & $0.376 \mathrm{E}-04$ & 0.00 & $0.235 \mathrm{E}-03$ & 3.60 \\
\hline 20 & 6.12 & 384. & $0.376 \mathrm{E}-04$ & $0.376 \mathrm{E}-04$ & 11.3 & 456. \\
\hline 21 & 7.0 & 0.00 & 1.82 & 1.04 & -1.51 & 0.00 \\
\hline 22 & 7.1 & $0.229 \mathrm{E}-01$ & 0.00 & 0.00 & $0.893 \mathrm{E}-02$ & 0.347 \\
\hline 23 & 7.12 & 27.6 & 0.00 & $0.235 \mathrm{E}-05$ & 13.2 & 30.9 \\
\hline 24 & 8.0 & 0.00 & 37.8 & 36.1 & -1.51 & 0.00 \\
\hline 25 & 8.1 & 116. & 0.00 & $0.752 \mathrm{E}-04$ & $0.601 \mathrm{E}-05$ & 275 . \\
\hline 26 & 8.12 & $0.115 E+04$ & 0.00 & $0.752 \mathrm{E}-04$ & 10.1 & 975. \\
\hline 27 & 9.0 & 0.00 & 38.1 & 52.1 & -1.51 & 0.00 \\
\hline 28 & 9.1 & 1.01 & $0.752 \mathrm{E}-04$ & $0.752 \mathrm{E}-04$ & $-0.200 \mathrm{E}-05$ & 9.93 \\
\hline 29 & 9.12 & 990. & $0.752 \mathrm{E}-04$ & $0.752 \mathrm{E}-04$ & 9.24 & $0.132 \mathrm{E}+04$ \\
\hline 30 & 0.0 & 0.00 & 32.6 & 24.4 & -1.51 & 0.00 \\
\hline 31 & 0.1 & $0.859 \mathrm{E}-01$ & 0.00 & $0.376 \mathrm{E}-04$ & $0.160 \mathrm{E}-04$ & 1.44 \\
\hline 32 & 0.12 & 523. & 0.00 & $0.752 \mathrm{E}-04$ & 10.4 & 695. \\
\hline 33 & sS & 0.00 & -8.10 & -4.05 & $-0.476 \mathrm{E}-03$ & 0.00 \\
\hline 34 & TR3 & -0.375 & -8.53 & -4.26 & $0.162 E-01$ & -3.25 \\
\hline \multirow[t]{3}{*}{35} & TR12 & -9.51 & -15.4 & -7.71 & $0.313 \mathrm{E}-01$ & -11.4 \\
\hline & \multirow[t]{2}{*}{ COMPOSITE } & & & F THE SQUA & D VALUES & $* * .5$ \\
\hline & & 365. & 15.7 & 14.4 & 6.04 & 416. \\
\hline
\end{tabular}

DIMENSIONLESS SCALED SENSITIVITIES (SCALED BY B* (WT**.5))

$\begin{array}{rllcll} & \text { PARAMETER: } & \text { HK_1 } & \text { VERT_K_CB } & \text { SS_2 } & \text { HK_2 } \\ \text { OBS \# } & \text { OBSERVATION } & & & & \\ 1 & 1.0 & 0.904 \mathrm{E}-04 & 0.829 \mathrm{E}-06 & 0.00 & 0.391 \mathrm{E}-04 \\ 2 & 1.1 & -0.605 \mathrm{E}-02 & 0.914 \mathrm{E}-03 & 0.198 \mathrm{E}-02 & -0.404 \mathrm{E}-02 \\ 3 & 1.12 & -2.30 & -0.198 \mathrm{E}-01 & 0.439 & -1.02 \\ 4 & 2.0 & -270 . & -4.57 & 0.00 & -55.4 \\ 5 & 2.1 & -2.15 & 0.489 & 1.11 & -2.08 \\ 6 & 2.2 & -33.1 & 2.57 & 15.5 & -24.2 \\ 7 & 2.8 & 930 . & 8.76 & 155 . & 70.7 \\ 8 & 2.12 & 0.210 \mathrm{E}+04 & 22.8 & 88.2 & 362 . \\ 9 & 3.0 & -470 . & -7.94 & 0.00 & -159 . \\ 10 & 3.1 & 14.9 & -112 . & 83.7 & 94.5 \\ 11 & 3.12 & 0.569 \mathrm{E}+04 & -70.6 & 192 . & 0.260 \mathrm{E}+04 \\ 12 & 4.0 & -270 . & -4.57 & 0.00 & -55.4 \\ 13 & 4.1 & -3.33 & 0.688 & 1.74 & -3.11 \\ 14 & 4.12 & 0.211 \mathrm{E}+04 & 21.2 & 88.2 & 370 . \\ 15 & 5.0 & -377 . & -6.35 & 0.00 & -101 . \\ 16 & 5.1 & -5.93 & 1.24 & 3.55 & -6.19 \\ 17 & 5.12 & 0.302 \mathrm{E}+04 & 27.9 & 136 . & 745 . \\ 18 & 6.0 & -271 . & -10.2 & 0.00 & -54.2 \\ 19 & 6.1 & -2.13 & 1.95 & 1.85 & -3.72 \\ 20 & 6.12 & 0.213 \mathrm{E}+04 & 165 . & 90.6 & 306 . \\ 21 & 7.0 & -19.0 & -38.3 & 0.00 & 21.1 \\ 22 & 7.1 & -0.229 & 0.367 & 0.194 & -0.484\end{array}$


APPENDIX A. EXAMPLE SIMULATIONS - Test Case 1 - GLOBAL Output File

$\begin{array}{ll}23 & 7.12 \\ 24 & 8.0 \\ 25 & 8.1 \\ 26 & 8.12 \\ 27 & 9.0 \\ 28 & 9.1 \\ 29 & 9.12 \\ 30 & 0.0 \\ 31 & 0.1 \\ 32 & 0.12 \\ 33 & \text { SS } \\ 34 & \text { TR3 } \\ 35 & \text { TR12 }\end{array}$

149.
-467.
73.4
$0.544 \mathrm{E}+04$
-540.
-3.69
$0.456 \mathrm{E}+04$
-375.
-0.855
$0.276 \mathrm{E}+04$
$-0.488 \mathrm{E}-02$
3.62
12.1

378.

$-2.15$

6.18

-218 .

0.00

-162 .

456.

239.

95.5

$-0.934$

194.

0.00

6.74

$0.289 \mathrm{E}+04$

11.8

262.

-236 .

$-15.7$

$-5.32$

0.00

$0.195 \mathrm{E}+04$

0.913

0.765

$-103$.

$-1.74$

528.

128.

$-0.457 \mathrm{E}-04$ $-0.596 \mathrm{E}-02$

$-0.211 \mathrm{E}-02$

12.1

0.180

$-0.709$

1.26

4.83

COMPOSITE SCALED SENSITIVITIES ((SUM OF THE SQUARED VALUES)/ND)**. 5

$0.181 \mathrm{E}+04 \quad 120$.

91.4

762 .

PARAMETER COMPOSITE SCALED SENSITIVITY

$\begin{array}{ll}\text { WELLS_TR } & 3.65327 \mathrm{E}+02 \\ \text { RCH_ZONE_1 } & 1.56750 \mathrm{E}+01 \\ \text { RCH_ZONE_2 } & 1.44247 \mathrm{E}+01 \\ \text { RIVERS } & 6.04314 \mathrm{E}+00 \\ \text { SS_1 } & 4.16105 \mathrm{E}+02 \\ \text { HK_1 } & 1.81300 \mathrm{E}+03 \\ \text { VERT_K_CB } & 1.19648 \mathrm{E}+02 \\ \text { SS_2 } & 9.14184 \mathrm{E}+01 \\ \text { HK_2 } & 7.62215 \mathrm{E}+02\end{array}$




\section{APPENDIX A. EXAMPLE SIMULATIONS - Test Case 1 - GLOBAL Output File}

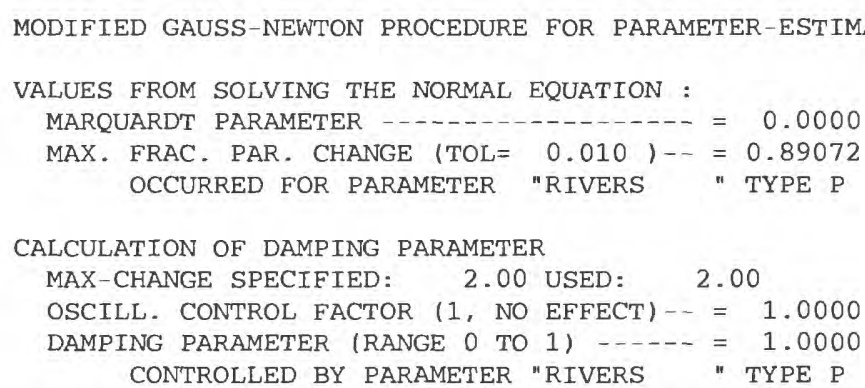

UPDATED ESTIMATES OF REGRESSION PARAMETERS :

$\begin{array}{llllll}\text { WELLS_TR } & \text { RCH_ZONE_1 } & \text { RCH_ZONE_2 } & \text { RIVERS } & \text { SS_1 } & \text { HK_1 } \\ \text { VERT_K_CB } & \text { SS_2 } & \text { HK_2 } & & & \\ -1.0735 & 34.148 & 50.437 & 0.97333 \mathrm{E}-03 & 0.11385 \mathrm{E}-02 & 0.42532 \mathrm{E}-03 \\ 0.21625 \mathrm{E}-06 & 0.62074 \mathrm{E}-04 & 0.48222 \mathrm{E}-04 & & \end{array}$

SUMS OF SQUARED, WEIGHTED RESIDUALS:

ALL DEPENDENT VARIABLES: 40.711

DEP. VARIABLES PLUS PARAMETERS: 40.711

MODIFIED GAUSS-NEWTON PROCEDURE FOR PARAMETER-ESTIMATION ITERATION NO. = 4

VALUES FROM SOLVING THE NORMAL EQUATION :

MARQUARDT PARAMETER _.... $=0.0000$

MAX. FRAC. PAR. CHANGE $(T O L=0.010)--=0.34347$

OCCURRED FOR PARAMETER "RIVERS " TYPE P

CALCULATION OF DAMPING PARAMETER

MAX-CHANGE SPECIFIED: 2.00 USED: 2.00

OSCILL. CONTROL FACTOR $(1 \text {, NO EFFECT })_{--}^{-=} 1.0000$

DAMPING PARAMETER (RANGE 0 TO 1 ) $\ldots \ldots=1.0000$ CONTROLLED BY PARAMETER "RIVERS "TYPE P

UPDATED ESTIMATES OF REGRESSION PARAMETERS :

$\begin{array}{llllll}\text { WELLS_TR } & \text { RCH_ZONE_1 } & \text { RCH_ZONE_2 } & \text { RIVERS } & \text { SS_1 } & \text { HK_1 } \\ \text { VERT_K_CB } & \text { SS_2 } & \text { HK_2 } & & & \\ -1.0741 & 34.123 & 50.479 & 0.13076 \mathrm{E}-02 & 0.11383 \mathrm{E}-02 & 0.42554 \mathrm{E}-03 \\ 0.21654 \mathrm{E}-06 & 0.61866 \mathrm{E}-04 & 0.48234 \mathrm{E}-04 & & \end{array}$

SUMS OF SQUARED, WEIGHTED RESIDUALS:

ALL DEPENDENT VARIABLES: 36.592

DEP. VARTABLES PLUS PARAMETERS: 36.592

MODIFIED GAUSS-NEWTON PROCEDURE FOR PARAMETER-ESTIMATION ITERATION NO. = 5

VALUES FROM SOLVING THE NORMAL EQUATION :

MARQUARDT PARAMETER $\ldots \ldots . . . \ldots 000$

MAX. FRAC. PAR. CHANGE (TOL $=0.010)--=0.55254 \mathrm{E}-01$

OCCURRED FOR PARAMETER "RIVERS " TYPE P

CALCULATION OF DAMPING PARAMETER

MAX-CHANGE SPECIFIED: 2.00 USED: 2.00

OSCILL. CONTROL FACTOR (1, NO EFFECT) $--=1.0000$

DAMPING PARAMETER (RANGE 0 TO 1) $\ldots . .=1.0000$ CONTROLLED BY PARAMETER "RIVERS "TYPE P

UPDATED ESTIMATES OF REGRESSION PARAMETERS :

$\begin{array}{llllll}\text { WELLS_TR } & \text { RCH_ZONE_1 } & \text { RCH_ZONE_2 } & \text { RIVERS } & \text { SS_1 } & \text { HK_1 } \\ \text { VERT_K_CB } & \text { SS_2 } & \text { HK_2 } & & & \\ -1.0741 & 34.119 & 50.484 & 0.13799 \mathrm{E}-02 & 0.11383 \mathrm{E}-02 & 0.42555 \mathrm{E}-03 \\ 0.21651 \mathrm{E}-06 & 0.61945 \mathrm{E}-04 & 0.48236 \mathrm{E}-04 & & \end{array}$




\section{APPENDIX A. EXAMPLE SIMULATIONS - Test Case 1 - GLOBAL Output File}

SUMS OF SQUARED, WEIGHTED RESIDUALS:

ALL DEPENDENT VARIABLES: 36.505

DEP. VARIABLES PLUS PARAMETERS: 36.505

MODIFIED GAUSS-NEWTON PROCEDURE FOR PARAMETER-ESTIMATION ITERATION NO. =

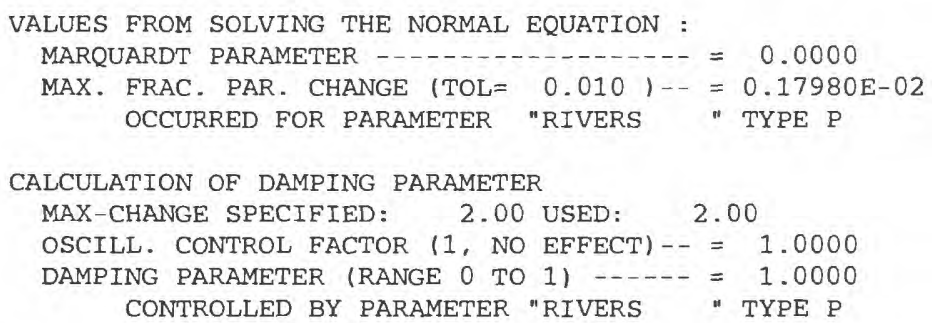

UPDATED ESTIMATES OF REGRESSION PARAMETERS :

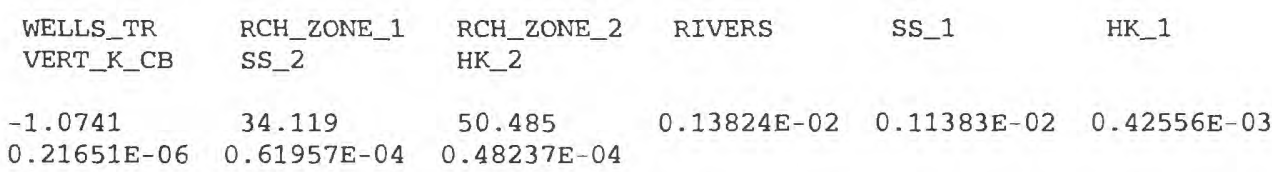

\begin{tabular}{|c|c|c|c|c|c|c|}
\hline OBS \# & $\begin{array}{l}\text { PARAMETER: } \\
\text { OBSERVATION }\end{array}$ & WELLS_TR & RCH_ZONE_1 & RCH_ZONE_2 & RIVERS & SS_1 \\
\hline 1 & 1.0 & 0.00 & $0.705 \mathrm{E}-01$ & 0.104 & -1.15 & 0.00 \\
\hline 2 & 1.1 & $0.168 \mathrm{E}-02$ & $0.159 \mathrm{E}-06$ & 0.00 & $0.111 \mathrm{E}-01$ & $0.354 \mathrm{E}-01$ \\
\hline 3 & 1.12 & 1.66 & 0.00 & 0.00 & 10.9 & 0.800 \\
\hline 4 & 2.0 & 0.00 & 9.34 & 17.6 & -1.15 & 0.00 \\
\hline 5 & 2.1 & 0.674 & $0.203 \mathrm{E}-04$ & $0.301 \mathrm{E}-04$ & $0.203 E-02$ & 11.6 \\
\hline 6 & 2.2 & 10.9 & $0.203 \mathrm{E}-04$ & $0.602 \mathrm{E}-04$ & 0.105 & 118. \\
\hline 7 & 2.8 & 231. & 0.00 & $0.301 \mathrm{E}-04$ & 7.65 & 521. \\
\hline 8 & 2.12 & 284 . & 0.00 & $0.301 \mathrm{E}-04$ & 10.8 & 134. \\
\hline 9 & 3.0 & 0.00 & 15.0 & 42.0 & -1.15 & 0.00 \\
\hline 10 & 3.1 & 86.4 & $0.203 \mathrm{E}-04$ & 0.00 & $0.128 \mathrm{E}-03$ & 407. \\
\hline 11 & 3.12 & 852 . & 0.00 & 0.00 & 10.4 & 295. \\
\hline 12 & 4.0 & 0.00 & 9.34 & 17.6 & -1.15 & 0.00 \\
\hline 13 & 4.1 & 1.03 & $0.203 E-04$ & $0.301 \mathrm{E}-04$ & $0.263 E-02$ & 16.2 \\
\hline 14 & 4.12 & 285: & 0.00 & $0.301 \mathrm{E}-04$ & 10.7 & 134. \\
\hline 15 & 5.0 & 0.00 & 12.9 & 28.0 & -1.15 & 0.00 \\
\hline 16 & 5.1 & 1.97 & $0.203 \mathrm{E}-04$ & 0.00 & $0.514 \mathrm{E}-03$ & 27.8 \\
\hline 17 & 5.12 & 433. & 0.00 & 0.00 & 10.5 & 207. \\
\hline 18 & 6.0 & 0.00 & 9.33 & 17.8 & -1.15 & 0.00 \\
\hline 19 & 6.1 & 0.767 & $0.203 E-04$ & 0.00 & $0.216 \mathrm{E}-02$ & 12.4 \\
\hline 20 & 6.12 & 287. & $0.203 E-04$ & $0.301 \mathrm{E}-04$ & 10.8 & 135. \\
\hline 21 & 7.0 & 0.00 & 0.452 & 0.749 & -1.15 & 0.00 \\
\hline 22 & 7.1 & $0.547 \mathrm{E}-01$ & 0.00 & 0.00 & $0.436 \mathrm{E}-01$ & 0.865 \\
\hline 23 & 7.12 & 13.0 & $-0.636 \mathrm{E}-06$ & 0.00 & 11.9 & 5.75 \\
\hline 24 & 8.0 & 0.00 & 14.9 & 42.1 & -1.15 & 0.00 \\
\hline 25 & 8.1 & 111. & $0.203 \mathrm{E}-04$ & $0.602 \mathrm{E}-04$ & $0.147 \mathrm{E}-03$ & 356. \\
\hline 26 & 8.12 & 862 . & 0.00 & $0.602 \mathrm{E}-04$ & 10.4 & 295. \\
\hline 27 & 9.0 & 0.00 & 15.0 & 61.7 & -1.15 & 0.00 \\
\hline 28 & 9.1 & 2.01 & $0.203 \mathrm{E}-04$ & $0.120 \mathrm{E}-03$ & $0.196 \mathrm{E}-05$ & 26.7 \\
\hline 29 & 9.12 & 767. & 0.00 & $0.120 \mathrm{E}-03$ & 10.1 & 404 \\
\hline 30 & 0.0 & 0.00 & 12.8 & 28.2 & -1.15 & 0.00 \\
\hline 31 & 0.1 & 0.334 & $0.203 E-04$ & $0.602 \mathrm{E}-04$ & $0.224 \mathrm{E}-03$ & 6.51 \\
\hline 32 & 0.12 & 398. & 0.00 & $0.602 \mathrm{E}-04$ & 10.3 & 208. \\
\hline 33 & ss & 0.00 & -4.38 & -6.48 & $-0.942 \mathrm{E}-03$ & 0.00 \\
\hline 34 & TR3 & -0.759 & -4.61 & -6.82 & $0.275 \mathrm{E}-01$ & -5.70 \\
\hline 35 & TR12 & -9.98 & -8.35 & -12.3 & $0.123 \mathrm{E}-01$ & -4.73 \\
\hline
\end{tabular}


COMPOSITE SCALED SENSITIVITIES ((SUM OF THE SQUARED VALUES)/ND) * * 5

$$
\begin{array}{lllll}
279 . & 6.26 & 17.0 & 5.89 & 174 .
\end{array}
$$

DIMENSIONLESS SCALED SENSITIVITIES (SCALED BY $B^{*}\left(W T^{* *} .5\right)$ )

\begin{tabular}{|c|c|c|c|c|c|}
\hline OBS \# & $\begin{array}{l}\text { PARAMETER: } \\
\text { OBSERVATION }\end{array}$ & HK_1 & VERT_K_CB & SS_2 & HK_2 \\
\hline 1 & 1.0 & $0.640 \mathrm{E}-04$ & $0.247 E-06$ & 0.00 & $0.284 \mathrm{E}-04$ \\
\hline 2 & 1.1 & $-0.329 E-01$ & $0.336 \mathrm{E}-02$ & $0.374 \mathrm{E}-02$ & $-0.156 E-01$ \\
\hline 3 & 1.12 & -0.707 & $0.163 \mathrm{E}-02$ & $0.622 \mathrm{E}-01$ & -0.336 \\
\hline 4 & 2.0 & -180 & -1.33 & 0.00 & -34.5 \\
\hline 5 & 2.1 & -6.12 & 1.07 & 1.19 & -4.32 \\
\hline 6 & 2.2 & -39.4 & 2.89 & 9.99 & -26.5 \\
\hline 7 & 2.8 & $0.105 E+04$ & 5.91 & 40.6 & 128. \\
\hline 8 & 2.12 & $0.177 E+04$ & 10.4 & 10.4 & 322. \\
\hline 9 & 3.0 & -351 & -2.45 & 0.00 & -115. \\
\hline 10 & 3.1 & 104. & -58.0 & 36.0 & 129. \\
\hline 11 & 3.12 & $0.474 E+04$ & -42.2 & 22.9 & $0.195 E+04$ \\
\hline 12 & 4.0 & -180 & -1.33 & 0.00 & -34.5 \\
\hline 13 & 4.1 & -8.21 & 1.43 & 1.67 & -5.71 \\
\hline 14 & 4.12 & $0.178 E+04$ & 9.78 & 10.4 & 327. \\
\hline 15 & 5.0 & -263. & -1.75 & 0.00 & -66.2 \\
\hline 16 & 5.1 & -12.5 & 2.47 & 2.89 & -9.75 \\
\hline 17 & 5.12 & $0.260 E+04$ & 13.1 & 16.1 & 625. \\
\hline 18 & 6.0 & -181. & -4.85 & 0.00 & -33.3 \\
\hline 19 & 6.1 & -6.30 & 2.89 & 1.56 & -5.94 \\
\hline 20 & 6.12 & $0.179 E+04$ & 67.4 & 10.5 & 298. \\
\hline 21 & 7.0 & -7.18 & -14.7 & 0.00 & 8.49 \\
\hline 22 & 7.1 & -0.496 & 0.789 & 0.121 & -0.789 \\
\hline 23 & 7.12 & 71.9 & 161. & 0.449 & -93.7 \\
\hline 24 & 8.0 & -350 & -1.99 & 0.00 & -116 \\
\hline 25 & 8.1 & 142. & 328. & 65.7 & 117. \\
\hline 26 & 8.12 & $0.466 E+04$ & 157. & 23.0 & $0.203 E+04$ \\
\hline 27 & 9.0 & -441 & 0.961 & 0.00 & -196 \\
\hline 28 & 9.1 & -9.32 & 12.2 & 3.80 & -19.1 \\
\hline 29 & 9.12 & $0.417 E+04$ & 33.5 & 31.5 & $0.162 E+04$ \\
\hline 30 & 0.0 & -263. & -3.55 & 0.00 & -66.0 \\
\hline 31 & 0.1 & -3.48 & 1.64 & 0.819 & -3.67 \\
\hline 32 & 0.12 & $0.241 E+04$ & 54.3 & 16.2 & 492. \\
\hline 33 & SS & $-0.397 \mathrm{E}-02$ & $-0.159 \mathrm{E}-04$ & 0.00 & $-0.176 \mathrm{E}-02$ \\
\hline 34 & TR3 & 5.61 & $-0.273 E-01$ & -0.465 & 1.62 \\
\hline 35 & TR12 & 4.40 & $0.329 E-01$ & -0.368 & 1.63 \\
\hline
\end{tabular}

COMPOSITE SCALED SENSITIVITIES ((SUM OF THE SQUARED VALUES)/ND) ** . 5

$$
\begin{array}{llll}
0.156 \mathrm{E}+04 & 70.2 & 17.1 & 577 .
\end{array}
$$

PARAMETER COMPOSITE SCALED SENSITIVITY

$\begin{array}{ll}\text { WELLS_TR } & 2.78824 \mathrm{E}+02 \\ \text { RCH_ZONE_1 } & 6.26061 \mathrm{E}+00 \\ \text { RCH_ZONE_2 } & 1.69894 \mathrm{E}+01 \\ \text { RIVERS } & 5.89316 \mathrm{E}+00 \\ \text { SS_1 } & 1.73844 \mathrm{E}+02 \\ \text { HK_1 } & 1.56268 \mathrm{E}+03 \\ \text { VERT_K_CB } & 7.01678 \mathrm{E}+01 \\ \text { SS_2 } & 1.71389 \mathrm{E}+01 \\ \text { HK_2 } & 5.76536 \mathrm{E}+02\end{array}$

FINAL PARAMETER VALUES AND STATISTICS:

PARAMETER NAME(S) AND VALUE(S):

$\begin{array}{clllll}\text { WELLS_TR } & \text { RCH_ZONE_1 } & \text { RCH_ZONE_2 } & \text { RIVERS } & \text { SS_1 } & \text { HK_1 } \\ \text { VERT_K_CB } & \text { SS_2 } & \text { HK_2 } & & \\ -0.107 E+01 & 0.341 E+02 & 0.505 \mathrm{E}+02 & 0.138 \mathrm{E}-02 & 0.114 \mathrm{E}-02 & 0.426 \mathrm{E}-03 \\ 0.217 \mathrm{E}-06 & 0.620 \mathrm{E}-04 & 0.482 \mathrm{E}-04 & & \\ \text { SUMS OF SQUARED WEIGHTED RESIDUALS: } & & \\ \text { OBSERVATIONS } & \text { PRIOR INFO } & \text { TOTAL } & \\ \text { 36.5 } & 0.00 & 36.5 & \end{array}$


SELECTED STATISTICS FROM MODIFIED GAUSS-NEWTON ITERATIONS

\begin{tabular}{ccccc} 
& \multicolumn{2}{c}{ MAX. PARAMETER CALC. CHANGE } & MAX. CHANGE & DAMPING \\
ITER. & PARNAM & MAX. CHANGE & ALLOWED & PARAMETER \\
\hline 1 & VERT_K_CB & 0.866000 & 2.00000 & 1.0000 \\
2 & RIVERS & 1.44000 & 2.00000 & 1.0000 \\
3 & RIVERS & 0.891000 & 2.00000 & 1.0000 \\
4 & RIVERS & 0.343000 & 2.00000 & 1.0000 \\
5 & RIVERS & $0.553000 \mathrm{E}-01$ & 2.00000 & 1.0000 \\
6 & RIVERS & $0.180000 \mathrm{E}-02$ & 2.00000 & 1.0000
\end{tabular}

SUMS OF SQUARED WEIGHTED RESIDUALS FOR EACH ITERATION

\begin{tabular}{rccc}
\multicolumn{4}{c}{ SUMS OF SQUARED WEIGHTED RESIDUALS } \\
ITER. & OBSERVATIONS & PRIOR INFO. & TOTAL \\
1 & $0.26760 \mathrm{E}+06$ & 0.0000 & $0.26760 \mathrm{E}+06$ \\
2 & 1124.0 & 0.0000 & 1124.0 \\
3 & 63.230 & 0.0000 & 63.230 \\
4 & 40.711 & 0.0000 & 40.711 \\
5 & 36.592 & 0.0000 & 36.592 \\
6 & 36.505 & 0.0000 & 36.505 \\
FINAL & 36.503 & 0.0000 & 36.503
\end{tabular}

*** PARAMETER ESTIMATION CONVERGED BY SATISFYING THE TOL CRITERION ** *

\begin{tabular}{|c|c|c|c|c|c|c|}
\hline \multicolumn{7}{|c|}{ COVARIANCE MATRIX FOR THE PARAMETERS } \\
\hline & $\begin{array}{l}\text { WELLS_TR } \\
\text { VERT_K_CB }\end{array}$ & $\begin{array}{l}\text { RCH_ZONE_1 } \\
\text { SS_2 }\end{array}$ & $\begin{array}{l}\mathrm{RCH} \_Z O N E \_2 \\
\mathrm{HK} \_2\end{array}$ & RIVERS & SS_1 & HK_I 1 \\
\hline WELLS_TR & $\begin{array}{r}5.978 E-03 \\
-5.620 E-03\end{array}$ & $\begin{array}{l}-4.435 \mathrm{E}-02 \\
-4.828 \mathrm{E}-03\end{array}$ & $\begin{array}{l}0.349 \\
-5.606 \mathrm{E}-03\end{array}$ & $-5.390 \mathrm{E}-03$ & $-5.631 \mathrm{E}-03$ & $-5.551 \mathrm{E}-03$ \\
\hline RCH_ZONE_1 & $\begin{array}{r}-4.435 \mathrm{E}-02 \\
4.295 \mathrm{E}-02\end{array}$ & $\begin{array}{c}14.4 \\
5.471 \mathrm{E}-02\end{array}$ & $\begin{array}{l}-4.72 \\
3.756 \mathrm{E}-02\end{array}$ & $2.395 \mathrm{E}-02$ & $3.950 \mathrm{E}-02$ & $4.248 \mathrm{E}-02$ \\
\hline RCH_ZONE_2 & $\begin{array}{r}-0.349 \\
0.323\end{array}$ & $\begin{array}{l}-4.72 \\
0.316\end{array}$ & $\begin{array}{r}24.6 \\
0.329\end{array}$ & 0.345 & 0.326 & 0.324 \\
\hline RIVERS & $\begin{array}{l}-5.390 E-03 \\
-2.169 E-02\end{array}$ & $\begin{array}{l}2.395 \mathrm{E}-02 \\
0.168\end{array}$ & $\begin{array}{l}0.345 \\
1.169 \mathrm{E}-02\end{array}$ & 0.350 & $-5.833 E-03$ & $2.270 \mathrm{E}-03$ \\
\hline SS_1 & $\begin{array}{r}-5.631 E-03 \\
8.615 E-03\end{array}$ & $\begin{array}{r}3.950 E-02 \\
-2.603 E-02\end{array}$ & $\begin{array}{l}0.326 \\
5.243 E-03\end{array}$ & $-5.833 E-03$ & $7.524 \mathrm{E}-03$ & $5.208 \mathrm{E}-03$ \\
\hline HK_1 & $\begin{array}{r}-5.551 \mathrm{E}-03 \\
5.302 \mathrm{E}-03\end{array}$ & $\begin{array}{l}4.248 E-02 \\
4.500 E-03\end{array}$ & $\begin{array}{l}0.324 \\
5.097 E-03\end{array}$ & $2.270 \mathrm{E}-03$ & $5.208 \mathrm{E}-03$ & $5.198 \mathrm{E}-03$ \\
\hline VERT_K_CB & $\begin{array}{r}-5.620 \mathrm{E}-03 \\
1.371 \mathrm{E}-02\end{array}$ & $\begin{array}{r}4.295 E-02 \\
-4.703 E-02\end{array}$ & $\begin{array}{l}0.323 \\
4.967 \mathrm{E}-03\end{array}$ & $-2.169 E-02$ & $8.615 E-03$ & $5.302 \mathrm{E}-03$ \\
\hline SS_2 & $\begin{array}{l}-4.828 E-03 \\
-4.703 E-02\end{array}$ & $\begin{array}{l}5.471 \mathrm{E}-02 \\
0.454\end{array}$ & $\begin{array}{l}0.316 \\
5.454 \mathrm{E}-03\end{array}$ & 0.168 & $-2.603 E-02$ & $4.500 \mathrm{E}-03$ \\
\hline HK_2 & $\begin{array}{r}-5.606 \mathrm{E}-03 \\
4.967 \mathrm{E}-03\end{array}$ & $\begin{array}{l}3.756 \mathrm{E}-02 \\
5.454 \mathrm{E}-03\end{array}$ & $\begin{array}{l}0.329 \\
5.565 \mathrm{E}-03\end{array}$ & $1.169 \mathrm{E}-02$ & $5.243 E-03$ & $5.097 \mathrm{E}-03$ \\
\hline
\end{tabular}


PARAMETER SUMMARY

\begin{tabular}{|c|c|c|c|c|c|}
\hline $\begin{array}{l}\text { PARAMETER: } \\
*=\text { LOG TRNS: }\end{array}$ & WELLS_TR & RCH_ZONE_1 & RCH_ZONE_2 & $\underset{*}{\text { RIVERS }}$ & SS_1 \\
\hline UPPER $95 \%$ C.I. & $-9.15 E-01$ & $4.19 \mathrm{E}+01$ & $6.07 \mathrm{E}+01$ & $-2.33 E+00$ & $-2.87 E+00$ \\
\hline FINAL VALUES & $-1.07 E+00$ & 3. $41 E+01$ & $5.05 E+01$ & $-2.86 E+00$ & $-2.94 E+00$ \\
\hline LOWER $95 \%$ C.I. & $-1.23 E+00$ & $2.63 E+01$ & $4.03 E+01$ & $-3.39 E+00$ & $-3.02 E+00$ \\
\hline STD. DEV. & $7.73 \mathrm{E}-02$ & $3.79 \mathrm{E}+00$ & $4.96 \mathrm{E}+00$ & $2.57 \mathrm{E}-01$ & $3.77 \mathrm{E}-02$ \\
\hline COEF. OF VAR. & $\begin{array}{l}\text { D. DEV. / } \\
7.20 \mathrm{E}-02\end{array}$ & $\begin{array}{l}\text { INAL VALUE); } \\
1.11 \mathrm{E}-01\end{array}$ & $\begin{array}{l}"--" \text { IF F } \\
9.82 \mathrm{E}-02\end{array}$ & $\begin{array}{r}\text { INAL VALUE } \\
8.98 \mathrm{E}-02\end{array}$ & $\begin{aligned}= & 0.0 \\
& 1.28 \mathrm{E}-02\end{aligned}$ \\
\hline
\end{tabular}

PARAMETER VALUES IN "REGRESSION" SPACE --- LOG TRANSFORMED AS APPLICABLE

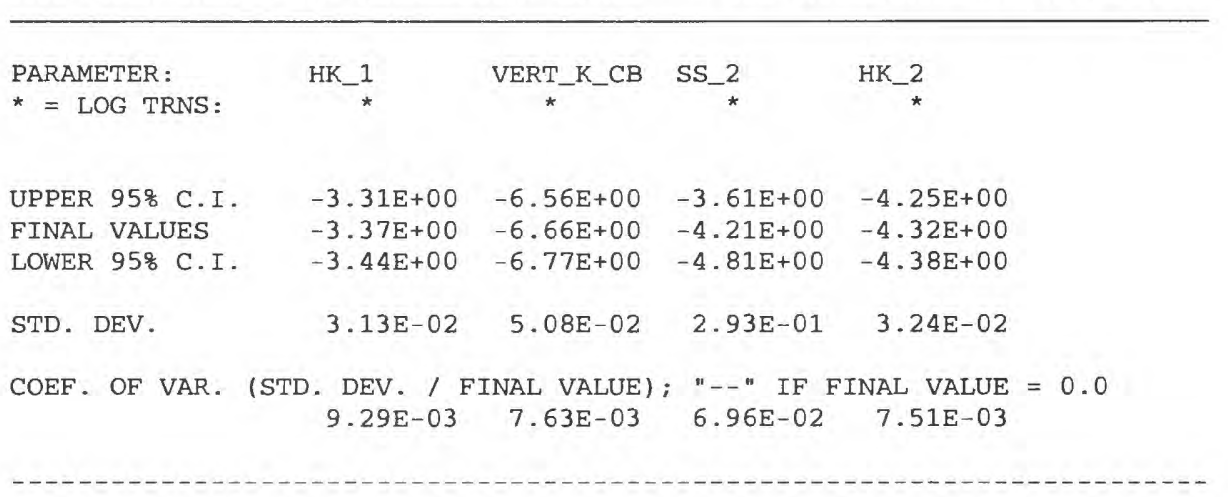

PHYSICAL PARAMETER VALUES --- EXP10 OF LOG TRANSFORMED PARAMETERS

\begin{tabular}{|c|c|c|c|c|c|}
\hline $\begin{array}{l}\text { PARAMETER: } \\
*=\text { LOG TRNS: }\end{array}$ & WELLS_TR & RCH_ZONE_1 & RCH_ZONE_2 & * RIVERS & * SS_1 \\
\hline UPPER $95 \%$ C.I. & $-9.15 E-01$ & $4.19 \mathrm{E}+01$ & $6.07 E+01$ & $4.66 \mathrm{E}-03$ & $1.36 \mathrm{E}-03$ \\
\hline FINAL VALUES & $-1.07 E+00$ & $3.41 \mathrm{E}+01$ & $5.05 E+01$ & $1.38 \mathrm{E}-03$ & $1.14 \mathrm{E}-03$ \\
\hline LOWER $95 \%$ C.I. & $-1.23 E+00$ & $2.63 E+01$ & $4.03 E+01$ & $4.10 \mathrm{E}-04$ & $9.52 \mathrm{E}-04$ \\
\hline REASONABLE & & & & & \\
\hline $\begin{array}{l}\text { UPPER LIMIT } \\
\text { REASONABLE }\end{array}$ & $-8.00 \mathrm{E}-01$ & $8.00 \mathrm{E}+01$ & $6.00 \mathrm{E}+01$ & $1.20 \mathrm{E}-02$ & $1.30 \mathrm{E}-02$ \\
\hline LOWER LIMIT & $-1.40 \mathrm{E}+00$ & $3.00 \mathrm{E}+01$ & $2.00 E+01$ & $1.20 \mathrm{E}-04$ & $1.30 \mathrm{E}-04$ \\
\hline ESTIMATE ABOVE (1) & & & & & \\
\hline $\begin{array}{l}\text { BELOW }(-1) \text { LIMITS } \\
\text { ENTIRE CONF. INT. }\end{array}$ & 0 & 0 & 0 & 0 & 0 \\
\hline ABOVE (1) BELOW (-1) & 0 & 0 & 0 & 0 & 0 \\
\hline
\end{tabular}




\begin{tabular}{|c|c|c|c|c|}
\hline PARAMETER: & HK_1 & VERT_K_CB & SS_2 & HK_2 \\
\hline * = LOG TRNS: & * & * & * & * \\
\hline UPPER 95\% C.I. & $4.94 \mathrm{E}-04$ & $2.75 E-07$ & $2.48 \mathrm{E}-04$ & $5.62 \mathrm{E}-05$ \\
\hline FINAL VALUES & $4.26 E-04$ & $2.17 \mathrm{E}-07$ & $6.20 \mathrm{E}-05$ & $4.82 E-05$ \\
\hline LOWER $95 \%$ C.I. & $3.67 \mathrm{E}-04$ & $1.70 \mathrm{E}-07$ & $1.55 \mathrm{E}-05$ & $4.14 \mathrm{E}-05$ \\
\hline REASONABLE & & & & \\
\hline $\begin{array}{l}\text { UPPER LIMIT } \\
\text { REASONABLE }\end{array}$ & $3.00 \mathrm{E}-03$ & $1.00 \mathrm{E}-06$ & $2.00 \mathrm{E}-03$ & $4.00 E-04$ \\
\hline LOWER LIMIT & $3.00 E-05$ & $1.00 \mathrm{E}-08$ & $2.00 \mathrm{E}-05$ & $4.00 E-06$ \\
\hline
\end{tabular}

ESTIMATE ABOVE (1) BELOW (-1) LIMITS ENTIRE CONF. INT. ABOVE (1) BELOW (-1)

$\begin{array}{llll}0 & 0 & 0 & 0 \\ 0 & 0 & 0 & 0\end{array}$

\begin{tabular}{|c|c|c|c|c|c|c|}
\hline & $\begin{array}{l}\text { WELLS_TR } \\
\text { VERT_K_CB }\end{array}$ & $\begin{array}{l}\text { RCH_ZONE_1 } \\
\text { SS_2 }\end{array}$ & $\begin{array}{l}\mathrm{RCH} \_Z O N E \_2 \\
\mathrm{HK} \_2\end{array}$ & RIVERS & SS_1 & HK_1 \\
\hline & $\ldots \ldots \ldots$ & . . & $\ldots$ & & & \\
\hline WELLS_TR & $\begin{array}{r}1.00 \\
-0.621\end{array}$ & $\begin{array}{l}-0.151 \\
-9.266 E-02\end{array}$ & $\begin{array}{l}-0.911 \\
-0.972\end{array}$ & -0.118 & -0.840 & -0.996 \\
\hline RCH_ZONE_1 & $\begin{array}{l}-0.151 \\
9.679 \mathrm{E}-02\end{array}$ & $\begin{array}{l}1.00 \\
2.142 \mathrm{E}-02\end{array}$ & $\begin{array}{r}-0.251 \\
0.133\end{array}$ & $1.069 \mathrm{E}-02$ & 0.120 & 0.155 \\
\hline RCH_ZONE_2 & $\begin{array}{r}-0.911 \\
0.556\end{array}$ & $\begin{array}{l}-0.251 \\
9.448 \mathrm{E}-02\end{array}$ & $\begin{array}{r}1.00 \\
0.891\end{array}$ & 0.118 & 0.759 & 0.906 \\
\hline RIVERS & $\begin{array}{l}-0.118 \\
-0.313\end{array}$ & $\begin{array}{l}1.069 \mathrm{E}-02 \\
0.421\end{array}$ & $\begin{array}{l}0.118 \\
0.265\end{array}$ & 1.00 & -0.114 & $5.326 \mathrm{E}-02$ \\
\hline SS_1 & $\begin{array}{r}-0.840 \\
0.848\end{array}$ & $\begin{array}{r}0.120 \\
-0.445\end{array}$ & $\begin{array}{l}0.759 \\
0.810\end{array}$ & -0.114 & 1.00 & 0.833 \\
\hline HK_1 & $\begin{array}{r}-0.996 \\
0.628\end{array}$ & $\begin{array}{l}0.155 \\
9.262 \mathrm{E}-02\end{array}$ & $\begin{array}{l}0.906 \\
0.948\end{array}$ & $5.326 \mathrm{E}-02$ & 0.833 & 1.00 \\
\hline VERT_K_CB & $\begin{array}{r}-0.621 \\
1.00\end{array}$ & $\begin{array}{l}9.679 \mathrm{E}-02 \\
-0.596\end{array}$ & $\begin{array}{l}0.556 \\
0.569\end{array}$ & -0.313 & 0.848 & 0.628 \\
\hline SS_2 & $\begin{array}{l}-9.266 E-02 \\
-0.596\end{array}$ & $\begin{array}{l}2.142 \mathrm{E}-02 \\
1.00\end{array}$ & $\begin{array}{l}9.448 \mathrm{E}-02 \\
0.108\end{array}$ & 0.421 & -0.445 & $9.262 \mathrm{E}-02$ \\
\hline HK_2 & $\begin{array}{r}-0.972 \\
0.569\end{array}$ & $\begin{array}{l}0.133 \\
0.108\end{array}$ & $\begin{array}{r}0.891 \\
1.00\end{array}$ & 0.265 & 0.810 & 0.948 \\
\hline
\end{tabular}

THE CORRELATION OF THE FOLLOWING PARAMETER PAIRS $>=.95$

$\begin{array}{llc}\text { PARAMETER } & \text { PARAMETER } & \text { CORRELATION } \\ \text { WELLS_TR } & \text { HK_1 } & -1.00 \\ \text { WELLS_TR } & \text { HK_2 } & -0.97\end{array}$

THE CORRELATION OF THE FOLLOWING PARAMETER PAIRS IS BETWEEN .90 AND .95 PARAMETER PARAMETER CORRELATION

WELLS_TR RCH_ZONE_2 $\quad-0.91$

RCH_ZONE_2 HK_1 0.91

HK_ 1 HK 2

THE CORRELATION OF THE FOLLOWING PARAMETER PAIRS IS BETWEEN .85 AND .90 PARAMETER PARAMETER CORRELATION $\begin{array}{lll}\text { RCH_ZONE_2 HK_2 } & 0.89\end{array}$

CORRELATIONS GREATER THAN 0.95 COULD INDICATE THAT THERE IS NOT ENOUGH INFORMATION IN THE OBSERVATIONS AND PRIOR USED IN THE REGRESSION TO ESTIMATE PARAMETER VALUES INDIVIDUALLY.

TO CHECK THIS, START THE REGRESSION FROM SETS OF INITIAL PARAMETER VALUES

THAT DIFFER BY MORE THAT TWO STANDARD DEVIATIONS FROM THE ESTIMATED

VALUES. IF THE RESULTING ESTIMATES ARE WELL WITHIN ONE STANDARD DEVIATION OF THE PREVIOUSLY ESTIMATED VALUE, THE ESTIMATES ARE PROBABLY 


\section{APPENDIX A. EXAMPLE SIMULATIONS - Test Case 1 - GLOBAL Output File}

DETERMINED INDEPENDENTLY WITH THE OBSERVATIONS AND PRIOR USED IN THE REGRESSION. OTHERWISE, YOU MAY ONLY BE ESTIMATING THE RATIO OR SUM OF THE HIGHLY CORRELATED PARAMETERS.

THE INITIAL PARAMETER VALUES ARE IN THE SEN FILE.

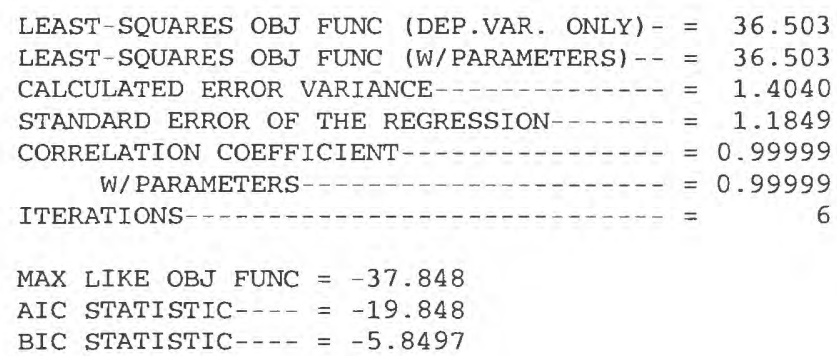

ORDERED DEPENDENT-VARIABLE WEIGHTED RESIDUALS

NUMBER OF RESIDUALS INCLUDED: 35

$\begin{array}{rcccccc}-2.21 & -2.10 & -1.23 & -1.07 & -0.927 & -0.897 & -0.666 \\ -0.578 & -0.452 & -0.375 & -0.368 & -0.312 & -0.265 & -0.146 \\ -0.133 & -0.110 & -0.903 \mathrm{E}-01 & 0.320 \mathrm{E}-01 & 0.407 \mathrm{E}-01 & 0.470 \mathrm{E}-01 & 0.745 \mathrm{E}-01 \\ 0.163 & 0.237 & 0.451 & 0.774 & 0.867 & 0.997 & 1.01 \\ 1.02 & 1.08 & 1.12 & 1.63 & 1.63 & 1.99 & 2.25\end{array}$

SMALLEST AND LARGEST DEPENDENT-VARIABLE WEIGHTED RESIDUALS

\begin{tabular}{rlr}
\multicolumn{3}{r}{ SMALLEST WEIGHTED RESIDUALS } \\
$\begin{array}{rlr} & \text { OBSERVATION } & \text { WEIGHTED } \\
\text { OBS\# } & \text { NAME } & \text { RESIDUAL } \\
26 & 8.12 & -2.2066 \\
12 & 4.0 & -2.0970 \\
10 & 3.1 & -1.2280 \\
14 & 4.12 & -1.0667 \\
3 & 1.12 & -0.92697\end{array}$
\end{tabular}

\begin{tabular}{rll}
\multicolumn{2}{r}{ LARGEST WEIGHTED } & RESIDUALS \\
& OBSERVATION & WEIGHTED \\
OBS\# & NAME & RESIDUAL \\
29 & 9.12 & 2.2488 \\
28 & 9.1 & 1.9852 \\
23 & 7.12 & 1.6312 \\
1 & 1.0 & 1.6273 \\
4 & 2.0 & 1.1230
\end{tabular}

CORRELATION BETWEEN ORDERED WEIGHTED RESIDUALS AND NORMAL ORDER STATISTICS $($ EQ.38 OF TEXT) $=0.978$

COMMENTS ON THE INTERPRETATION OF THE CORRELATION BETWEEN

WEIGHTED RESIDUALS AND NORMAL ORDER STATISTICS:

The critical value for correlation at the $5 \%$ significance level is 0.943

IF the reported CORRELATION is GREATER than the $5 \%$ critical value, ACCEPT the hypothesis that the weighted residuals are INDEPENDENT AND NORMALLY DISTRIBUTED at the 5\% significance level. The probability that this conclusion is wrong is less than $5 \%$.

IF the reported correlation IS LESS THAN the $5 \%$ critical value REJECT the, hypothesis that the weighted residuals are INDEPENDENT AND NORMALLY DISTRIBUTED at the $5 \%$ significance level.

The analysis can also be done using the $10 \%$ significance level. The associated critical value is 0.952

parameter estimation converged by satisfying the tol Criterion * * 


\section{LIST Output File}

File tc1.lst:

MODFLOW-2000

U.S. GEOLOGICAL SURVEY MODULAR FINITE-DIFFERENCE GROUND-WATER FLOW MODEL VERSION $1.0 \quad 06 / 13 / 2000$

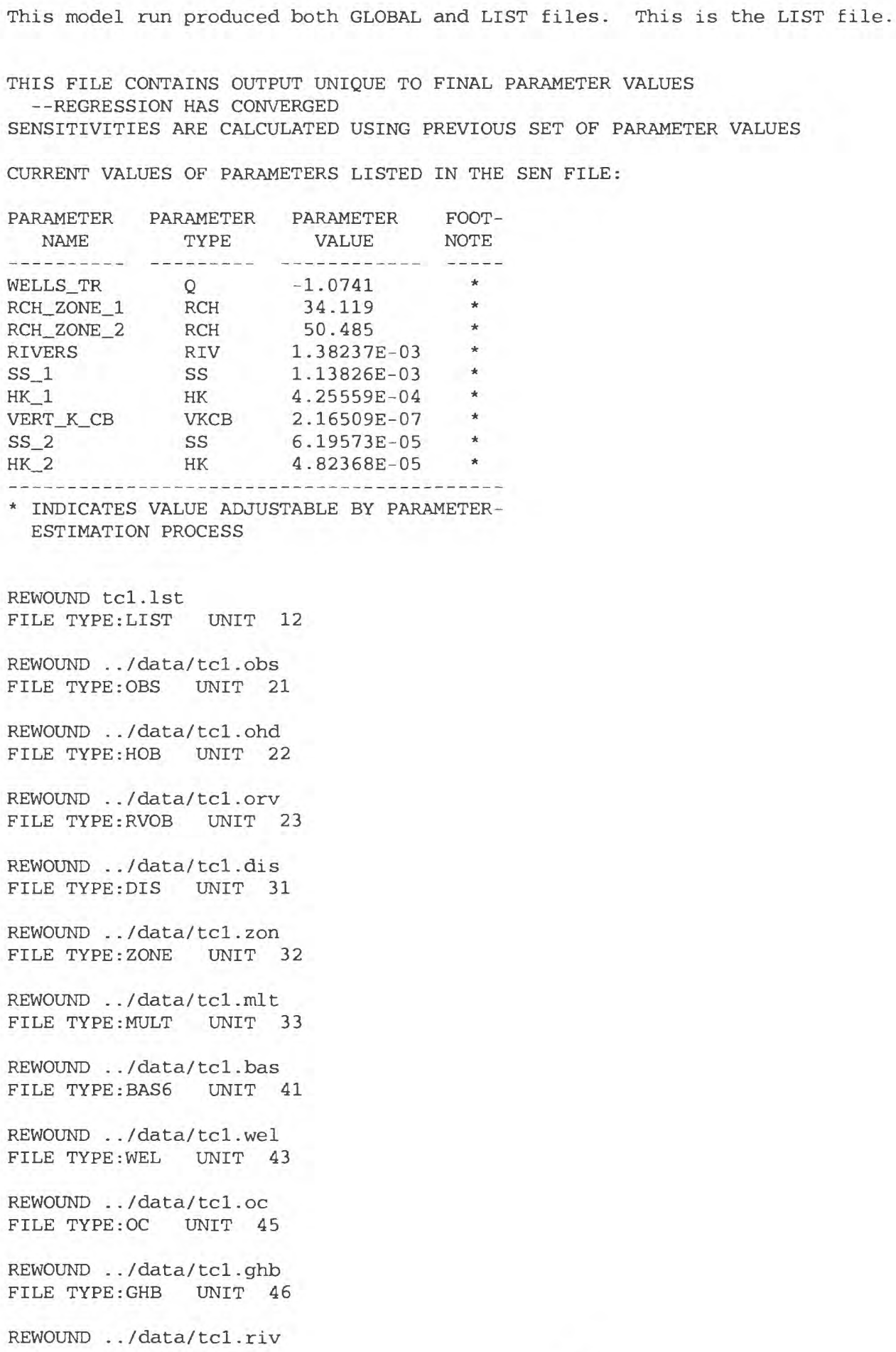




\section{APPENDIX A. EXAMPLE SIMULATIONS - Test Case 1 - LIST Output File}

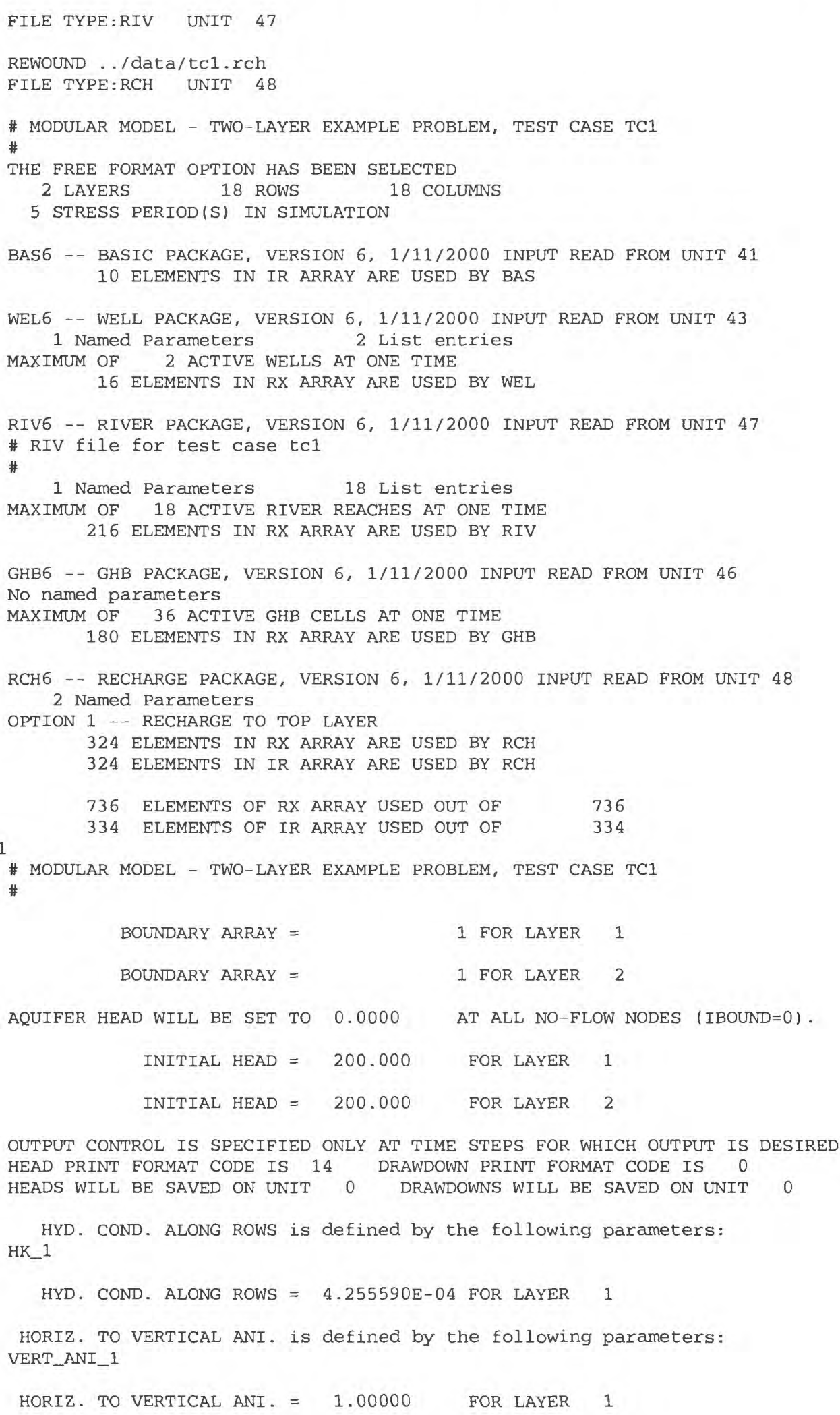


SPECIFIC STORAGE is defined by the following parameters:

SS_1

SPECIFIC STORAGE $=2.276525 \mathrm{E}-05$ FOR LAYER 1

QUASI3D VERT. HYD. COND. is defined by the following parameters: VERT_K_CB

QUASI3D VERT. HYD. COND. $=2.165091 \mathrm{E}-07$ FOR LAYER 1 HK_2

HYD. COND. ALONG ROWS is defined by the following parameters:

HYD. COND. ALONG ROWS FOR LAYER 2

\begin{tabular}{|c|c|c|c|c|c|c|c|}
\hline & 1 & 2 & 3 & 4 & 5 & 6 & 7 \\
\hline & 8 & 9 & 10 & 11 & 12 & 13 & 14 \\
\hline & 15 & 16 & 17 & 18 & & & \\
\hline 1 & $4.82 \mathrm{E}-05$ & $4.82 \mathrm{E}-05$ & $9.65 E-05$ & $9.65 E-05$ & $1.45 \mathrm{E}-04$ & $1.45 \mathrm{E}-04$ & $1.93 \mathrm{E}-04$ \\
\hline & $1.93 E-04$ & $2.41 \mathrm{E}-04$ & $2.41 E-04$ & $2.89 E-04$ & $2.89 \mathrm{E}-04$ & $3.38 \mathrm{E}-04$ & 3. $38 \mathrm{E}-04$ \\
\hline & $3.86 \mathrm{E}-04$ & $3.86 \mathrm{E}-04$ & $4.34 E-04$ & $4.34 \mathrm{E}-04$ & & & \\
\hline 2 & $4.82 E-05$ & $4.82 \mathrm{E}-05$ & $9.65 E-05$ & $9.65 E-05$ & $1.45 E-04$ & $1.45 \mathrm{E}-04$ & $1.93 \mathrm{E}-04$ \\
\hline & $1.93 \mathrm{E}-04$ & $2.41 \mathrm{E}-04$ & $2.41 \mathrm{E}-04$ & $2.89 E-04$ & $2.89 \mathrm{E}-04$ & $3.38 \mathrm{E}-04$ & $3.38 \mathrm{E}-04$ \\
\hline & $3.86 \mathrm{E}-04$ & $3.86 \mathrm{E}-04$ & $4.34 \mathrm{E}-04$ & $4.34 \mathrm{E}-04$ & & & \\
\hline & & & & - & & & \\
\hline & & & & - & & & \\
\hline & & & & • & & & \\
\hline & & & & & & & \\
\hline 17 & $4.82 \mathrm{E}-05$ & $4.82 E-05$ & $9.65 \mathrm{E}-05$ & $9.65 \mathrm{E}-05$ & $1.45 \mathrm{E}-04$ & $1.45 \mathrm{E}-04$ & $1.93 \mathrm{E}-04$ \\
\hline & $1.93 \mathrm{E}-04$ & $2.41 \mathrm{E}-04$ & $2.41 \mathrm{E}-04$ & $2.89 E-04$ & $2.89 E-04$ & $3.38 \mathrm{E}-04$ & $3.38 \mathrm{E}-04$ \\
\hline & $3.86 \mathrm{E}-04$ & $3.86 \mathrm{E}-04$ & $4.34 \mathrm{E}-04$ & $4.34 \mathrm{E}-04$ & & & \\
\hline 18 & $4.82 \mathrm{E}-05$ & $4.82 E-05$ & $9.65 \mathrm{E}-05$ & $9.65 E-05$ & $1.45 \mathrm{E}-04$ & $1.45 \mathrm{E}-04$ & $1.93 \mathrm{E}-04$ \\
\hline & $1.93 E-04$ & $2.41 \mathrm{E}-04$ & $2.41 \mathrm{E}-04$ & $2.89 E-04$ & $2.89 E-04$ & $3.38 \mathrm{E}-04$ & $3.38 \mathrm{E}-04$ \\
\hline & $3.86 \mathrm{E}-04$ & $3.86 \mathrm{E}-04$ & $4.34 E-04$ & $4.34 \mathrm{E}-04$ & & & \\
\hline
\end{tabular}

HORIZ. TO VERTICAL ANI. is defined by the following parameters: VERT_ANI_2

HORIZ. TO VERTICAL ANI. $=1.00000$ FOR LAYER 2

SPECIFIC STORAGE is defined by the following parameters:

SS_2

SPECIFIC STORAGE $=1.239146 \mathrm{E}-06$ FOR LAYER 2

1

STRESS PERIOD NO. 1, LENGTH $=1.000000$
NUMBER OF TIME STEPS $=1$
MULTIPLIER FOR DELT $=1.000$
INITIAL TIME STEP SIZE $=1.000000$

0 WELLS

\begin{tabular}{ccccccc}
$\begin{array}{l}\text { Parameter: } \\
\text { REACH NO. }\end{array}$ & RIVERS & & & \\
\\
\hline 1 & 1 & 1 & 1 & 100.0 & 1.382 & 90.00 \\
2 & 1 & 2 & 1 & 100.0 & 1.382 & 90.00 \\
3 & 1 & 3 & 1 & 100.0 & 1.382 & 90.00 \\
4 & 1 & 4 & 1 & 100.0 & 1.382 & 90.00 \\
5 & 1 & 5 & 1 & 100.0 & 1.382 & 90.00 \\
6 & 1 & 6 & 1 & 100.0 & 1.382 & 90.00 \\
7 & 1 & 7 & 1 & 100.0 & 1.382 & 90.00 \\
8 & 1 & 8 & 1 & 100.0 & 1.382 & 90.00 \\
9 & 1 & 9 & 1 & 100.0 & 1.382 & 90.00
\end{tabular}




\section{APPENDIX A. EXAMPLE SIMULATIONS - Test Case 1 - LIST Output File}

$\begin{array}{lllllll}10 & 1 & 10 & 1 & 100.0 & 1.382 & 90.00 \\ 11 & 1 & 11 & 1 & 100.0 & 1.382 & 90.00 \\ 12 & 1 & 12 & 1 & 100.0 & 1.382 & 90.00 \\ 13 & 1 & 13 & 1 & 100.0 & 1.382 & 90.00 \\ 14 & 1 & 14 & 1 & 100.0 & 1.382 & 90.00 \\ 15 & 1 & 15 & 1 & 100.0 & 1.382 & 90.00 \\ 16 & 1 & 16 & 1 & 100.0 & 1.382 & 90.00 \\ 17 & 1 & 17 & 1 & 100.0 & 1.382 & 90.00 \\ 18 & 1 & 18 & 1 & 100.0 & 1.382 & 90.00\end{array}$

18 RIVER REACHES

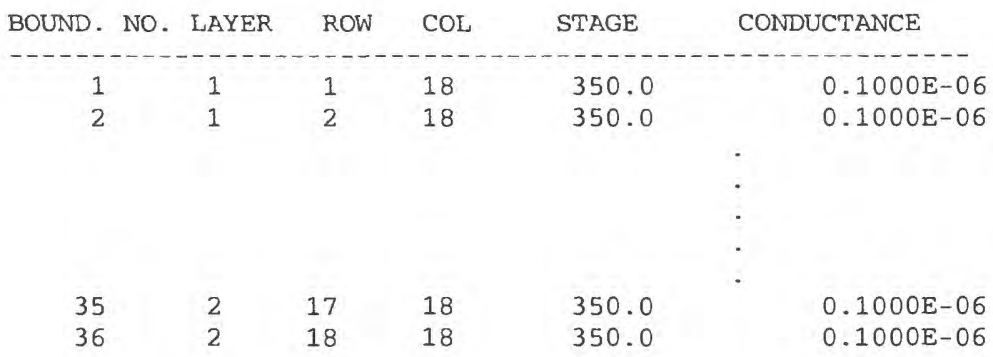

36 GHB CELLS

RECH array defined by the following parameters:

Parameter: RCH_ZONE_1

Parameter: RCH_ZONE_2

RECHARGE

\begin{tabular}{|c|c|c|c|c|c|c|}
\hline & 1 & 2 & 3 & 4 & 5 & 6 \\
\hline & 7 & 8 & 9 & 10 & 11 & 12 \\
\hline & 13 & 14 & 15 & 16 & 17 & 18 \\
\hline 1 & $1.0819 \mathrm{E}-08$ & $1.0819 \mathrm{E}-08$ & $1.0819 \mathrm{E}-08$ & $1.0819 \mathrm{E}-08$ & $1.0819 \mathrm{E}-08$ & $1.0819 \mathrm{E}-08$ \\
\hline & $1.0819 \mathrm{E}-08$ & $1.0819 \mathrm{E}-08$ & $1.0819 \mathrm{E}-08$ & 1. $6009 \mathrm{E}-08$ & 1. $6009 \mathrm{E}-08$ & $1.6009 \mathrm{E}-08$ \\
\hline & $1.6009 \mathrm{E}-08$ & $1.6009 \mathrm{E}-08$ & $1.6009 E-08$ & $1.6009 \mathrm{E}-08$ & $1.6009 E-08$ & $1.6009 \mathrm{E}-08$ \\
\hline 2 & $1.0819 \mathrm{E}-08$ & $1.0819 \mathrm{E}-08$ & $1.0819 E-08$ & $1.0819 \mathrm{E}-08$ & $1.0819 \mathrm{E}-08$ & $1.0819 \mathrm{E}-08$ \\
\hline & $1.0819 \mathrm{E}-08$ & $1.0819 \mathrm{E}-08$ & $1.0819 \mathrm{E}-08$ & $1.6009 \mathrm{E}-08$ & $1.6009 \mathrm{E}-08$ & $1.6009 \mathrm{E}-08$ \\
\hline & $1.6009 \mathrm{E}-08$ & $1.6009 \mathrm{E}-08$ & $1.6009 E-08$ & $1.6009 \mathrm{E}-08$ & $1.6009 \mathrm{E}-08$ & $1.6009 \mathrm{E}-08$ \\
\hline & & & & . & & \\
\hline & & & & - & & \\
\hline & & & & . & & \\
\hline & & & & - & & \\
\hline 17 & $1.0819 \mathrm{E}-08$ & $1.0819 \mathrm{E}-08$ & $1.0819 \mathrm{E}-08$ & $1.0819 \mathrm{E}-08$ & $1.0819 \mathrm{E}-08$ & $1.0819 E-08$ \\
\hline & $1.0819 \mathrm{E}-08$ & $1.0819 \mathrm{E}-08$ & $1.0819 \mathrm{E}-08$ & $1.6009 \mathrm{E}-08$ & $1.6009 \mathrm{E}-08$ & $1.6009 E-08$ \\
\hline & $1.6009 \mathrm{E}-08$ & $1.6009 \mathrm{E}-08$ & $1.6009 \mathrm{E}-08$ & $1.6009 \mathrm{E}-08$ & $1.6009 \mathrm{E}-08$ & $1.6009 \mathrm{E}-08$ \\
\hline 18 & $1.0819 \mathrm{E}-08$ & $1.0819 \mathrm{E}-08$ & $1.0819 \mathrm{E}-08$ & $1.0819 \mathrm{E}-08$ & $1.0819 \mathrm{E}-08$ & $1.0819 \mathrm{E}-08$ \\
\hline & $1.0819 \mathrm{E}-08$ & $1.0819 \mathrm{E}-08$ & $1.0819 \mathrm{E}-08$ & $1.6009 \mathrm{E}-08$ & 1. $6009 \mathrm{E}-08$ & $1.6009 \mathrm{E}-08$ \\
\hline & $1.6009 \mathrm{E}-08$ & $1.6009 \mathrm{E}-08$ & $1.6009 \mathrm{E}-08$ & $1.6009 \mathrm{E}-08$ & $1.6009 \mathrm{E}-08$ & 1. $6009 \mathrm{E}-08$ \\
\hline
\end{tabular}

SOLVING FOR HEAD

OUTPUT CONTROL FOR STRESS PERIOD 1 TIME STEP 1

PRINT HEAD FOR ALL LAYERS

1

HEAD IN LAYER 1 AT END OF TIME STEP 1 IN STRESS PERIOD 1

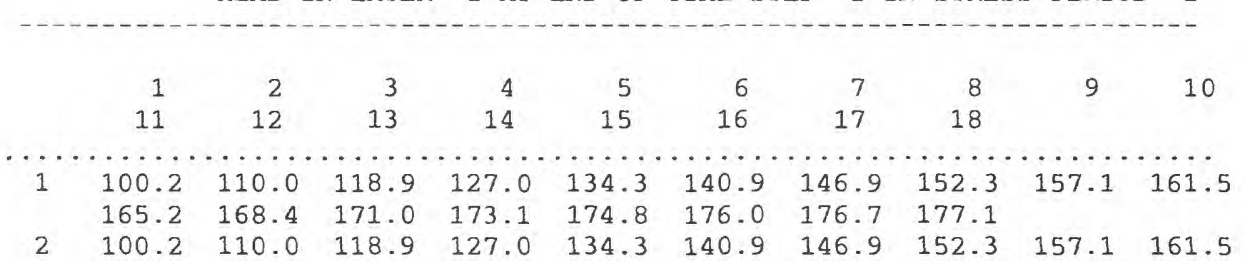




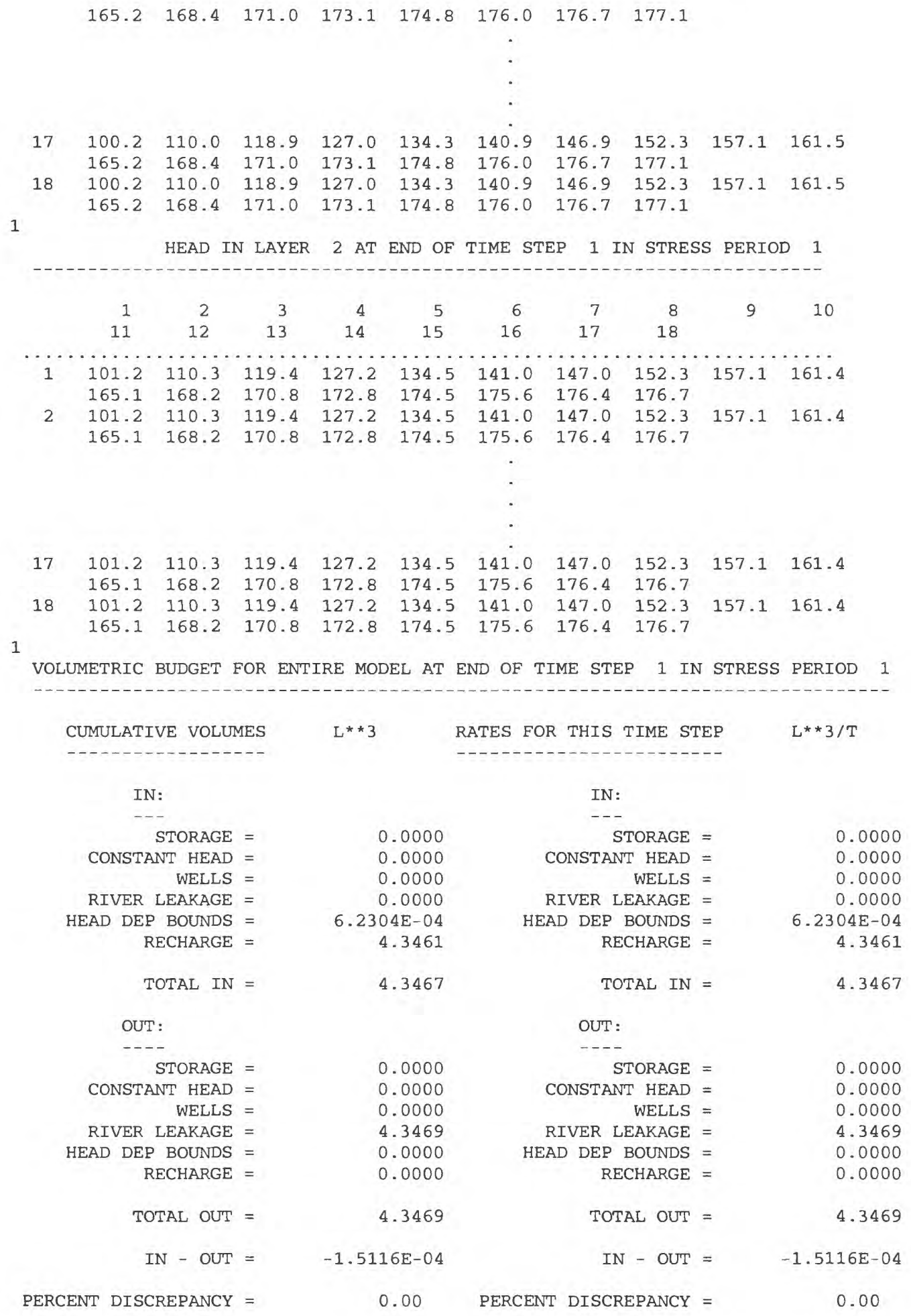




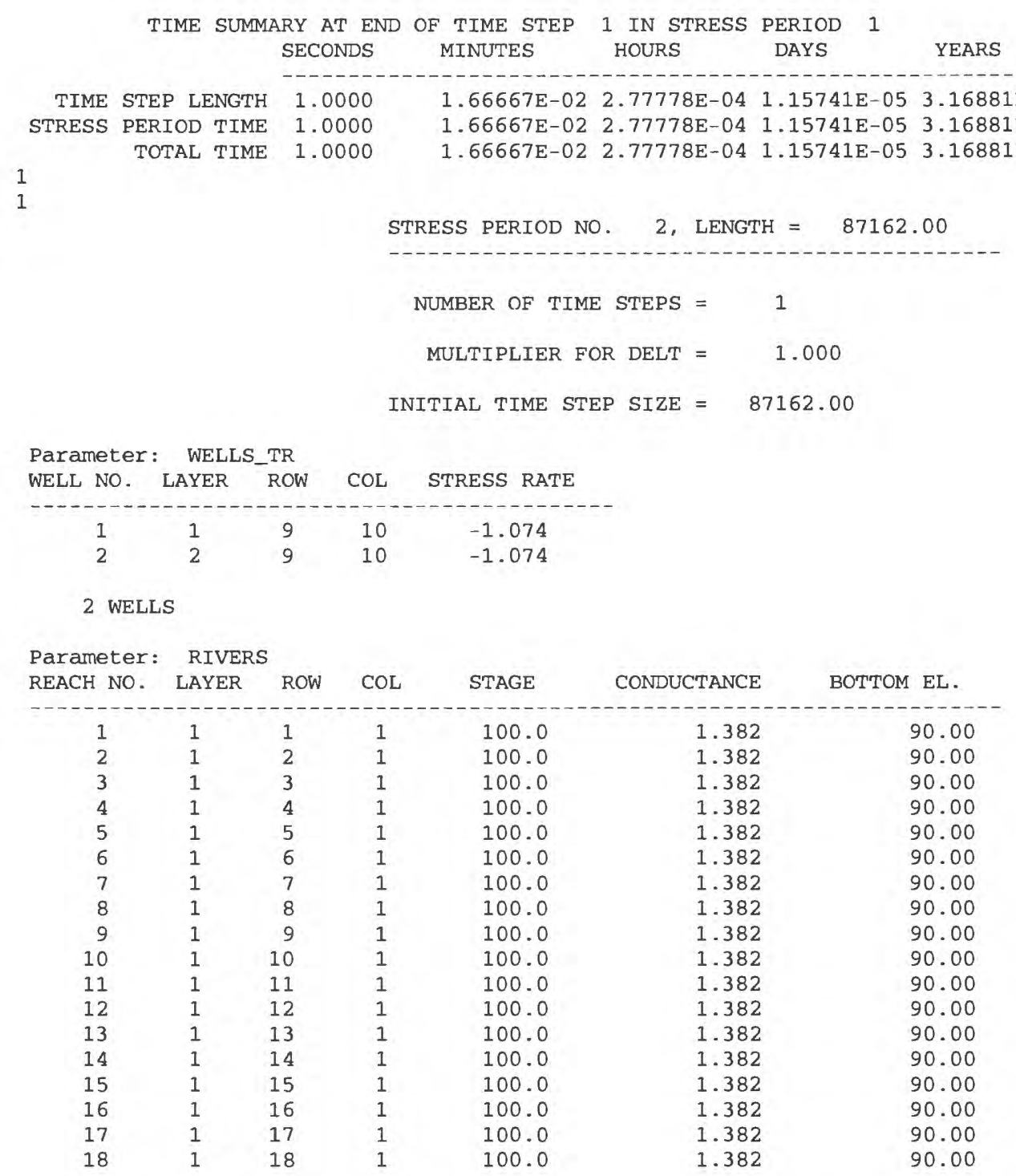

18 RIVER REACHES

REUSING NON-PARAMETER GHB CELLS FROM LAST STRESS PERIOD

36 GHB CELLS

REUSING RECH FROM LAST STRESS PERIOD

SOLVING FOR HEAD

OUTPUT CONTROL FOR STRESS PERIOD 2 TIME STEP 1

PRINT HEAD FOR ALL LAYERS

1

HEAD IN LAYER 1 AT END OF TIME STEP 1 IN STRESS PERIOD 2

\begin{tabular}{|c|c|c|c|c|c|c|c|c|c|c|}
\hline & 1 & 2 & 3 & 4 & 5 & 6 & 7 & 8 & \multirow[t]{2}{*}{9} & \multirow[t]{2}{*}{10} \\
\hline & 11 & 12 & 13 & 14 & 15 & 16 & 17 & 18 & & \\
\hline \multirow[t]{2}{*}{1} & 100.2 & 110.0 & 118.9 & 127.0 & 134.3 & 140.9 & 146.9 & 152.2 & \multirow[t]{2}{*}{157.1} & \multirow[t]{2}{*}{161.4} \\
\hline & 165.2 & 168.3 & 171.0 & 173.1 & 174.7 & 175.9 & 176.7 & 177.1 & & \\
\hline \multirow[t]{2}{*}{2} & 100.2 & 110.0 & 118.9 & 127.0 & 134.3 & 140.9 & 146.8 & 152.2 & \multirow[t]{2}{*}{157.0} & \multirow[t]{2}{*}{161.4} \\
\hline & 165.1 & 168.3 & 170.9 & 173.1 & 174.7 & 175.9 & 176.7 & 177.1 & & \\
\hline
\end{tabular}




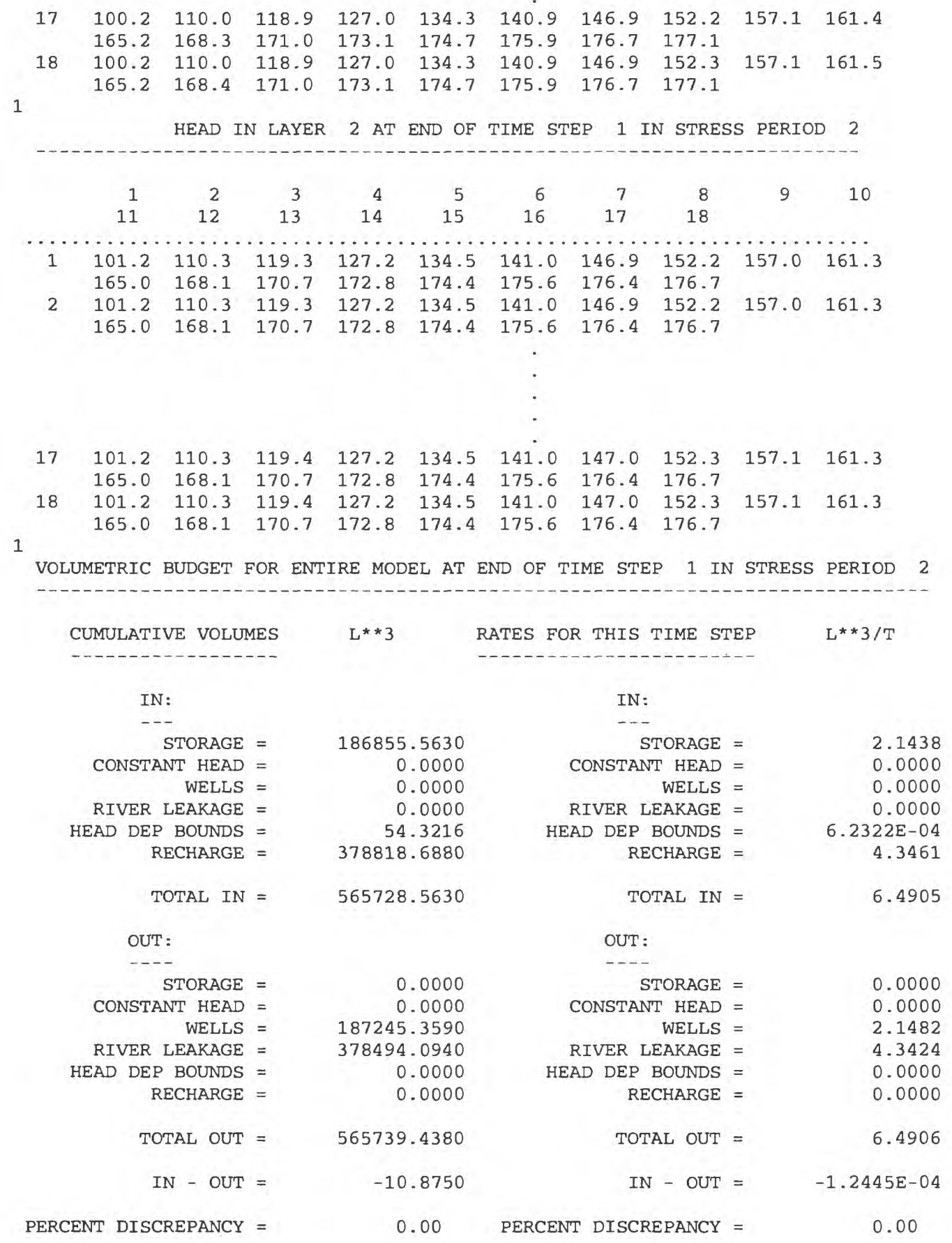


APPENDIX A. EXAMPLE SIMULATIONS - Test Case 1 - LIST Output File

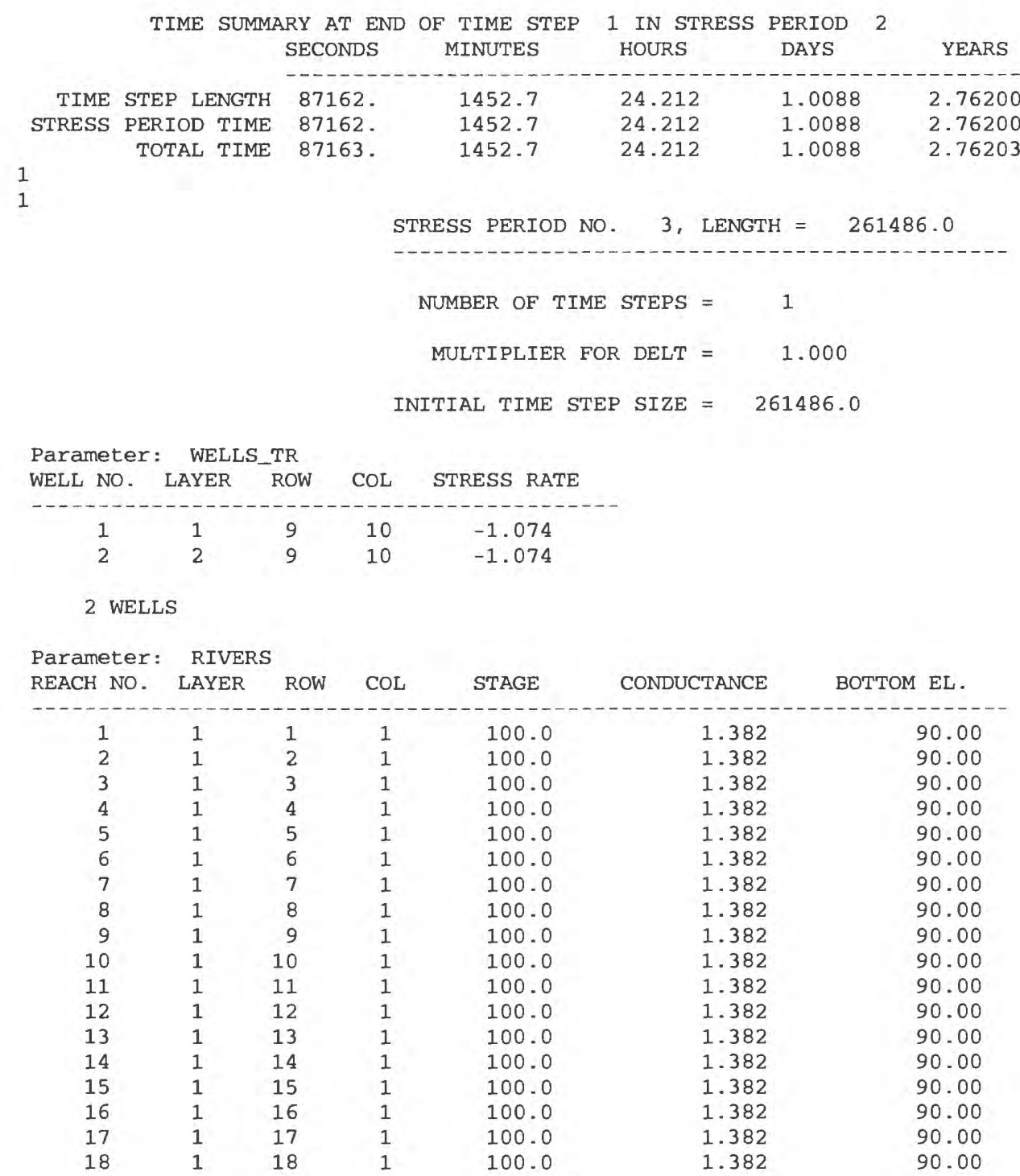

18 RIVER REACHES

REUSING NON-PARAMETER GHB CELLS FROM LAST STRESS PERIOD

36 GHB CELLS

REUSING RECH FROM LAST STRESS PERIOD

SOLVING FOR HEAD

NO OUTPUT CONTROL FOR STRESS PERIOD 3 TIME STEP 1

1

VOLUMETRIC BUDGET FOR ENTIRE MODEL AT END OF TIME STEP 1 IN STRESS PERIOD 3

\begin{tabular}{|c|c|c|c|}
\hline CUMULATIVE VOLUMES & $L * * 3$ & RATES FOR THIS TIME STEP & $L * * 3 / T$ \\
\hline IN: & & IN: & \\
\hline$\ldots$ & & --- & \\
\hline STORAGE $=$ & 728107.0630 & STORAGE $=$ & 2.0699 \\
\hline CONSTANT HEAD = & 0.0000 & CONSTANT HEAD $=$ & 0.0000 \\
\hline WELLS $=$ & 0.0000 & WELLS $=$ & 0.0000 \\
\hline RIVER LEAKAGE = & 0.0000 & RIVER LEAKAGE = & 0.0000 \\
\hline
\end{tabular}




\section{APPENDIX A. EXAMPLE SIMULATIONS - Test Case 1 - LIST Output File}

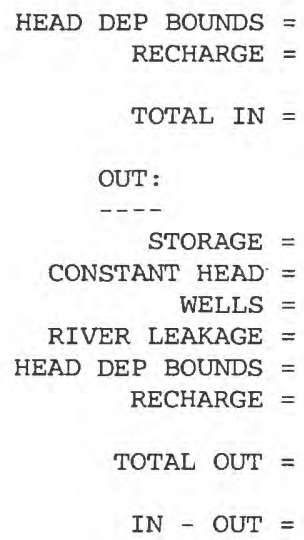

PERCENT DISCREPANCY =

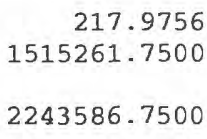

$$
\begin{array}{r}
0.0000 \\
0.0000 \\
748981.4380 \\
1494636.8800 \\
0.0000 \\
0.0000 \\
2243618.2500 \\
-31.5000
\end{array}
$$

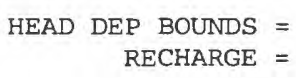

0.00

PERCENT DISCREPANCY =
$6.2586 \mathrm{E}-04$

4.3461

6.4166

0.0000

0.0000

2.1482

4.2685

0.0000

0.0000

6.4167

$-7.9155 E-05$

0.00

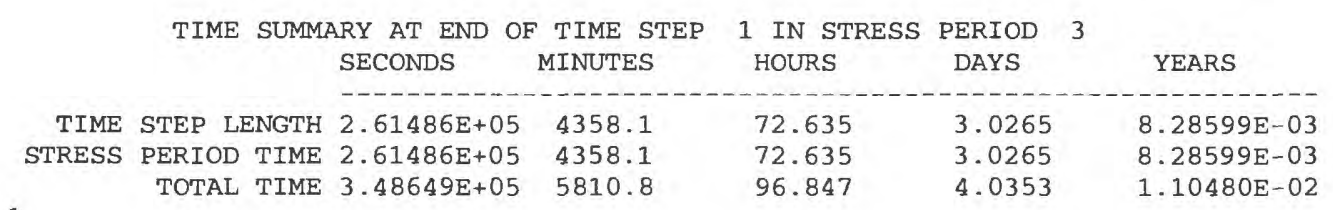

1

1

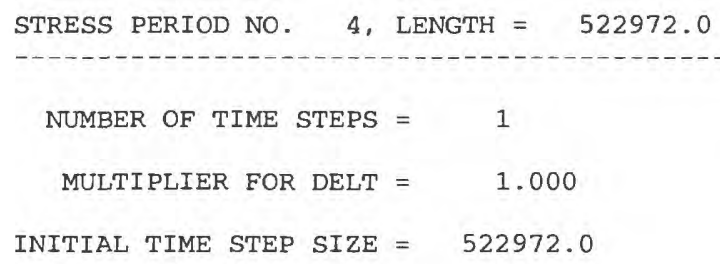

\begin{tabular}{|c|c|c|c|c|c|c|}
\hline 1 & 1 & 1 & 1 & 100.0 & 1.382 & 90.00 \\
\hline 2 & 1 & 2 & 1 & 100.0 & 1.382 & 90.00 \\
\hline 3 & 1 & 3 & 1 & 100.0 & 1.382 & 90.00 \\
\hline 4 & 1 & 4 & 1 & 100.0 & 1.382 & 90.00 \\
\hline 5 & 1 & 5 & 1 & 100.0 & 1.382 & 90.00 \\
\hline 6 & 1 & 6 & 1 & 100.0 & 1.382 & 90.00 \\
\hline 7 & 1 & 7 & 1 & 100.0 & 1.382 & 90.00 \\
\hline 8 & 1 & 8 & 1 & 100.0 & 1.382 & 90.00 \\
\hline 9 & 1 & 9 & 1 & 100.0 & 1.382 & 90.00 \\
\hline 10 & 1 & 10 & 1 & 100.0 & 1.382 & 90.00 \\
\hline 11 & 1 & 11 & 1 & 100.0 & 1.382 & 90.00 \\
\hline 12 & 1 & 12 & 1 & 100.0 & 1.382 & 90.00 \\
\hline 13 & 1 & 13 & 1 & 100.0 & 1.382 & 90.00 \\
\hline 14 & 1 & 14 & 1 & 100.0 & 1.382 & 90.00 \\
\hline 15 & 1 & 15 & 1 & 100.0 & 1.382 & 90.00 \\
\hline 16 & 1 & 16 & 1 & 100.0 & 1.382 & 90.00 \\
\hline 17 & 1 & 17 & 1 & 100.0 & 1.382 & 90.00 \\
\hline 18 & 1 & 18 & 1 & 100.0 & 1.382 & 90.00 \\
\hline
\end{tabular}

2 WELLS

Parameter: RIVERS REACH NO. LAYER ROW COL STAGE CONDUCTANCE BOTTOM EL. 


\section{APPENDIX A. EXAMPLE SIMULATIONS - Test Case 1 - LIST Output File}

18 RIVER REACHES

REUSING NON-PARAMETER GHB CELLS FROM LAST STRESS PERIOD

36 GHB CELLS

REUSING RECH FROM LAST STRESS PERIOD

SOLVING FOR HEAD

NO OUTPUT CONTROL FOR STRESS PERIOD 4 TIME STEP 1

VOLUMETRIC BUDGET FOR ENTIRE MODEL AT END OF TIME STEP 1 IN STRESS PERIOD 4

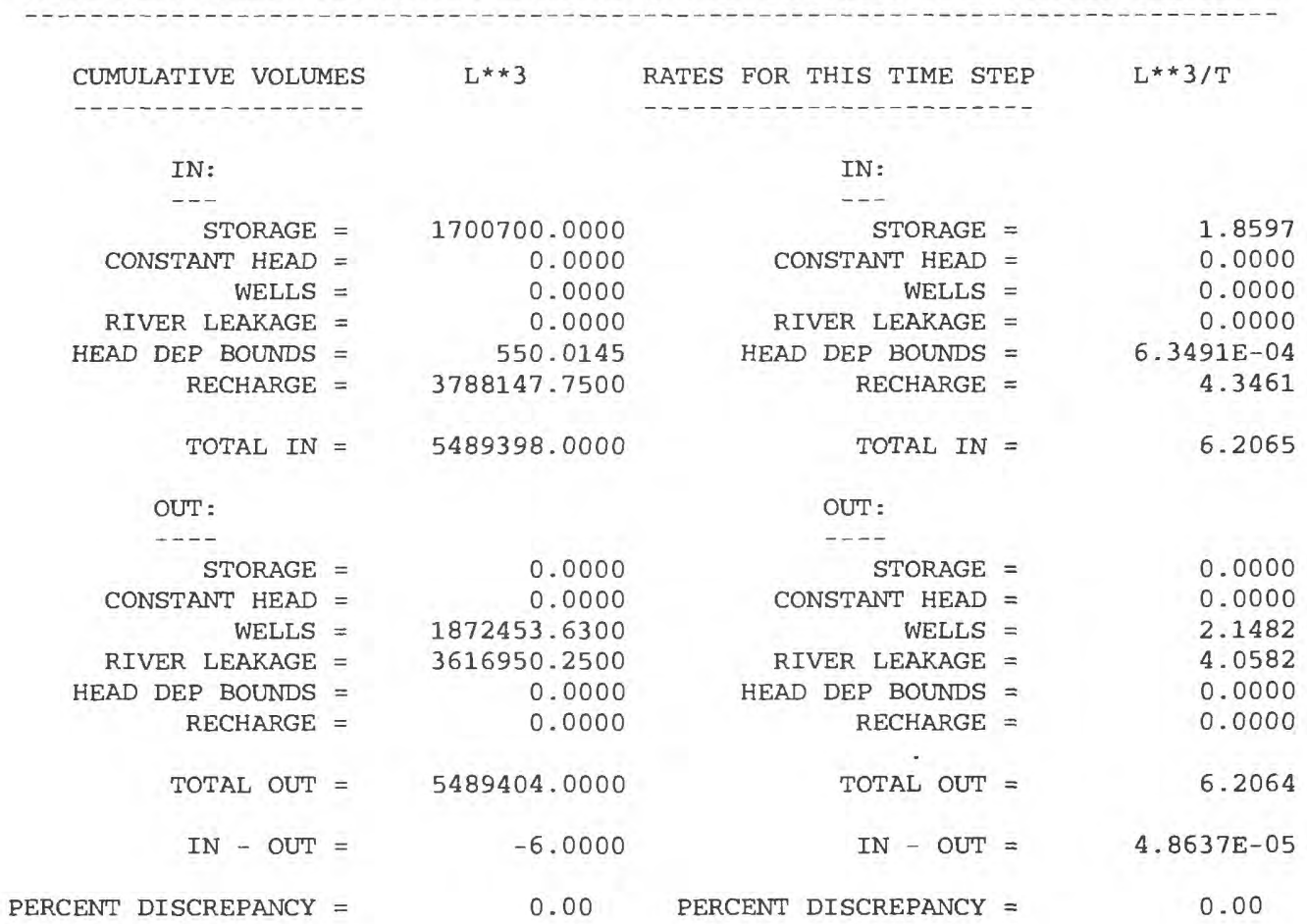

\begin{tabular}{|c|c|c|c|c|c|c|}
\hline & TIME SUMM & $\begin{array}{l}\text { ARY AT END O } \\
\text { SECONDS }\end{array}$ & $\begin{array}{l}\text { F TIME STEP } \\
\text { MINUTES }\end{array}$ & $\begin{array}{l}1 \text { IN STRESS } \\
\text { HOURS }\end{array}$ & $\begin{array}{l}\text { PERIOD } \\
\text { DAYS }\end{array}$ & YEARS \\
\hline TIME & STEP LENGTH & $5.22972 E+05$ & 8716.2 & 145.27 & 6.0529 & $1.65720 \mathrm{E}-02$ \\
\hline TRESS & PERIOD TIME & $5.22972 E+05$ & 8716.2 & 145.27 & 6.0529 & $1.65720 \mathrm{E}-02$ \\
\hline & TOTAL TIME & $8.71621 \mathrm{E}+05$ & 14527. & 242.12 & 10.088 & $2.76200 \mathrm{E}-02$ \\
\hline
\end{tabular}
1

1

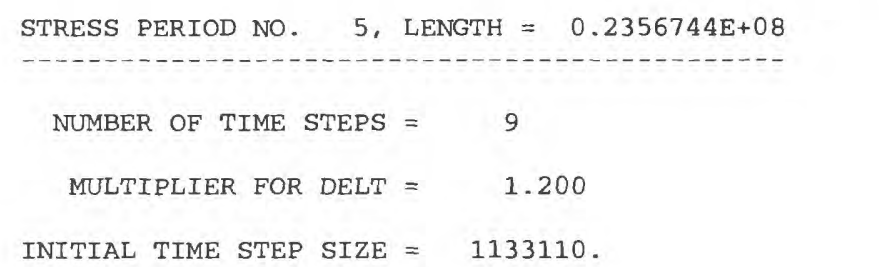




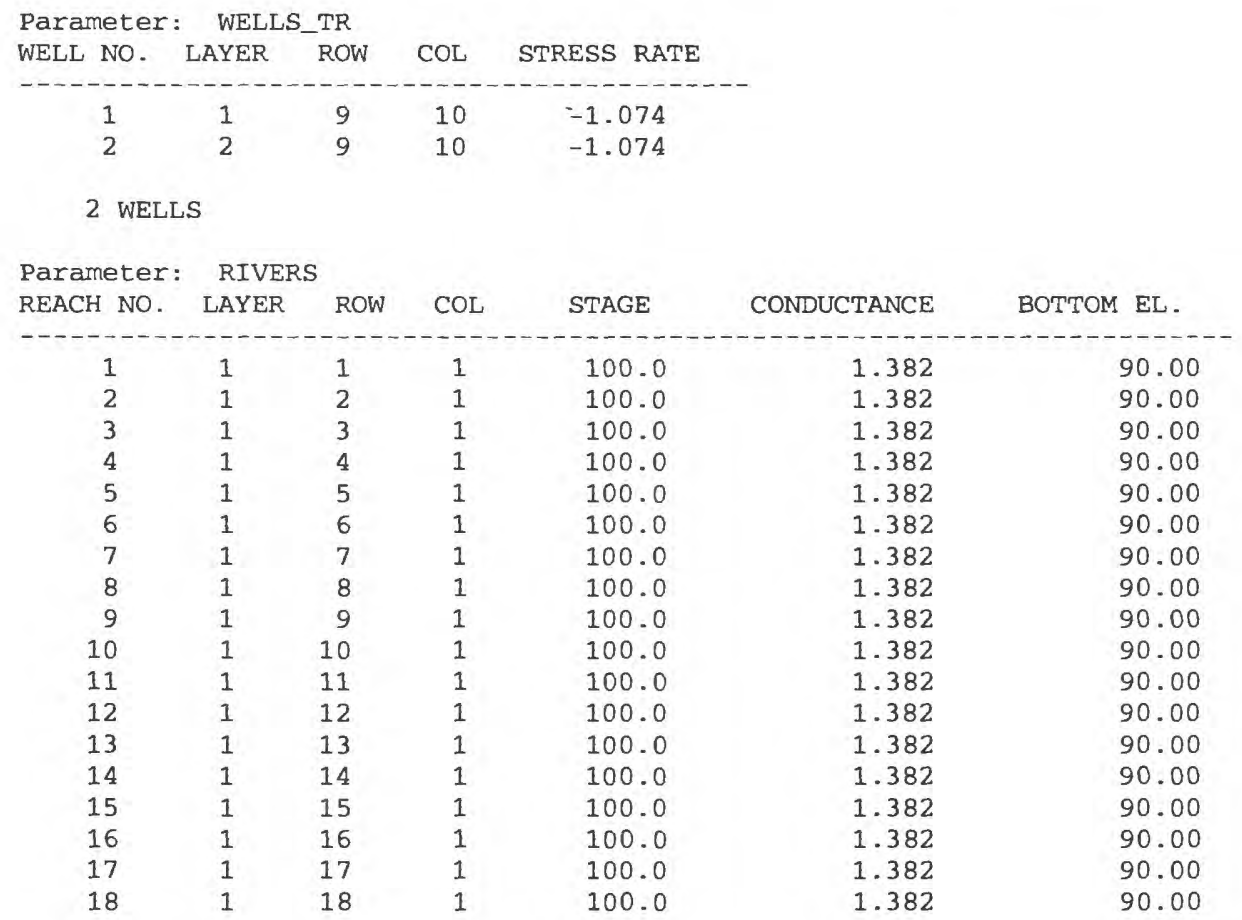

18 RIVER REACHES

REUSING NON-PARAMETER GHB CELLS FROM LAST STRESS PERIOD

36 GHB CELLS

REUSING RECH FROM LAST STRESS PERIOD

SOLVING FOR HEAD

NO OUTPUT CONTROL FOR STRESS PERIOD 5 TIME STEP 1

SOLVING FOR HEAD

NO OUTPUT CONTROL FOR STRESS PERIOD 5 TIME STEP 2

SOLVING FOR HEAD

NO OUTPUT CONTROL FOR STRESS PERIOD 5 TIME STEP 3

SOLVING FOR HEAD

NO OUTPUT CONTROL FOR STRESS PERIOD 5 TIME STEP 4

SOLVING FOR HEAD

NO OUTPUT CONTROL FOR STRESS PERIOD 5 TIME STEP 5

SOLVING FOR HEAD

NO OUTPUT CONTROL FOR STRESS PERIOD 5 TIME STEP 6

SOLVING FOR HEAD

NO OUTPUT CONTROL FOR STRESS PERIOD 5 TIME STEP 7

SOLVING FOR HEAD

NO OUTPUT CONTROL FOR STRESS PERIOD 5 TIME STEP 8

SOLVING FOR HEAD 
OUTPUT CONTROL FOR STRESS PERIOD 5 TIME STEP 9 PRINT HEAD FOR ALL LAYERS HEAD IN LAYER 1 AT END OF TIME STEP 9 IN STRESS PERIOD 5

\begin{tabular}{|c|c|c|c|c|c|c|c|c|c|c|}
\hline & 1 & 2 & 3 & 4 & 5 & 6 & 7 & 8 & \multirow[t]{2}{*}{9} & \multirow[t]{2}{*}{10} \\
\hline & 11 & 12 & 13 & 14 & 15 & 16 & 17 & 18 & & \\
\hline \multirow[t]{2}{*}{1} & 100.1 & 105.2 & 109.6 & 113.4 & 116.9 & 119.9 & 122.6 & 125.1 & \multirow[t]{2}{*}{127.5} & \multirow[t]{2}{*}{129.8} \\
\hline & 131.9 & 133.8 & 135.5 & 137.0 & 138.1 & 139.0 & 139.6 & 139.9 & & \\
\hline \multirow[t]{6}{*}{2} & 100.1 & 105.1 & 109.5 & 113.3 & 116.7 & 119.7 & 122.3 & 124.8 & \multirow[t]{6}{*}{127.2} & \multirow[t]{6}{*}{129.5} \\
\hline & 131.6 & 133.6 & 135.3 & 136.8 & 138.0 & 138.9 & 139.5 & 139.8 & & \\
\hline & & & & & & . & & & & \\
\hline & & & & & & - & & & & \\
\hline & & & & & & - & & & & \\
\hline & & & & & & & & & & \\
\hline \multirow[t]{2}{*}{17} & 100.1 & 105.4 & 110.1 & 114.2 & 117.8 & 121.0 & 123.9 & 126.5 & \multirow[t]{2}{*}{129.0} & \multirow[t]{2}{*}{131.4} \\
\hline & 133.5 & 135.4 & 137.0 & 138.4 & 139.5 & 140.3 & 140.9 & 141.2 & & \\
\hline \multirow[t]{2}{*}{18} & 100.1 & 105.4 & 110.2 & 114.3 & 117.9 & 121.2 & 124.1 & 126.8 & \multirow[t]{2}{*}{129.3} & \multirow[t]{2}{*}{131.7} \\
\hline & 133.8 & 135.6 & 137.2 & 138.6 & 139.7 & 140.5 & 141.0 & 141.3 & & \\
\hline
\end{tabular}

1 HEAD IN LAYER 2 AT END OF TIME STEP 9 IN STRESS PERIOD 5

\begin{tabular}{|c|c|c|c|c|c|c|c|c|c|c|}
\hline & 1 & 2 & 3 & 4 & 5 & 6 & 7 & 8 & \multirow[t]{2}{*}{9} & \multirow[t]{2}{*}{10} \\
\hline & 11 & 12 & 13 & 14 & 15 & 16 & 17 & 18 & & \\
\hline \multirow{3}{*}{$\cdots$} & . & . & . & . & $\ldots \ldots$ & $\ldots \ldots$ & $\ldots \ldots$ & $\ldots \ldots$ & & \multirow{3}{*}{129.7} \\
\hline & 100.6 & 105.3 & 109.8 & 113.5 & 116.9 & 119.8 & 122.6 & 125.1 & \multirow{2}{*}{127.4} & \\
\hline & 131.7 & 133.6 & 135.2 & 136.7 & 137.8 & 138.7 & 139.3 & 139.5 & & \\
\hline \multirow[t]{2}{*}{2} & 100.6 & 105.3 & 109.7 & 113.4 & 116.7 & 119.6 & 122.3 & 124.7 & \multirow[t]{2}{*}{127.1} & \multirow[t]{2}{*}{129.3} \\
\hline & 131.4 & 133.3 & 135.0 & 136.5 & 137.7 & 138.6 & 139.2 & 139.4 & & \\
\hline
\end{tabular}

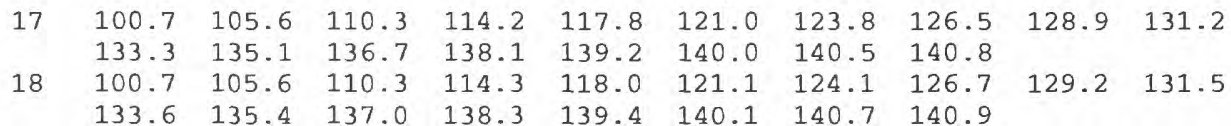

\begin{tabular}{|c|c|c|c|}
\hline CUMULATIVE VOLUMES & $\mathrm{L} * \star 3$ & RATES FOR THIS TIME STEP & $\mathrm{L} * * 3 / \mathrm{T}$ \\
\hline IN: & & IN: & \\
\hline--- & & --- & \\
\hline STORAGE = & 10783088.0000 & STORAGE $=$ & $5.1269 E-02$ \\
\hline CONSTANT HEAD = & 0.0000 & CONSTANT HEAD = & 0.0000 \\
\hline WELLS = & 0.0000 & WELLS $=$ & 0.0000 \\
\hline RIVER LEAKAGE = & 0.0000 & RIVER LEAKAGE = & 0.0000 \\
\hline HEAD DEP BOUNDS = & 17838.7734 & HEAD DEP BOUNDS = & $7.5785 E-04$ \\
\hline RECHARGE = & 106214520.0000 & RECHARGE = & 4.3461 \\
\hline
\end{tabular}




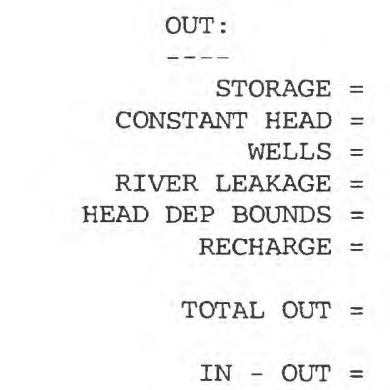

PERCENT DISCREPANCY =

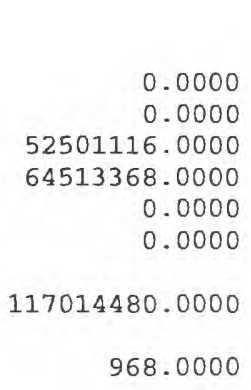

0.00
OUT :

STORAGE $=$

CONSTANT HEAD $=$ WELLS =

RIVER LEAKAGE =

HEAD DEP BOUNDS = RECHARGE =

TOTAL OUT =

$I N-$ OUT $=$

PERCENT DISCREPANCY =
0.0000

0.0000

2.1482

2. 2498

0.0000

0.0000

4.3980

8. $1539 E-05$

0.00

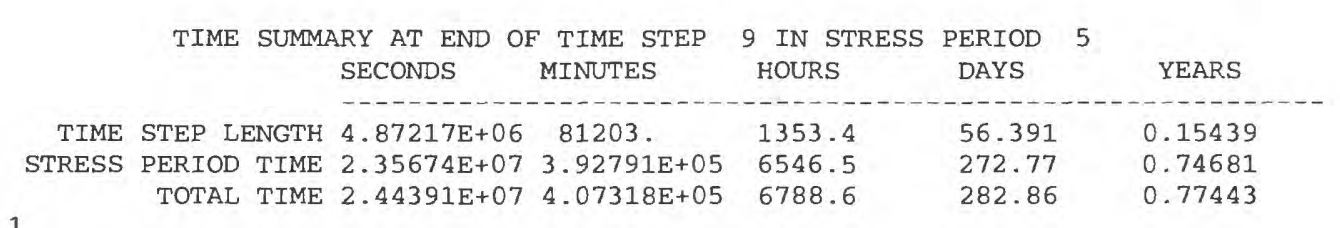

DATA AT HEAD LOCATIONS

\begin{tabular}{|c|c|c|c|c|c|c|}
\hline OBS\# & $\begin{array}{l}\text { OBSERVATION } \\
\text { NAME }\end{array}$ & $\begin{array}{l}\text { MEAS } \\
\text { HEAD }\end{array}$ & $\begin{array}{l}\text { CALC. } \\
\text { HEAD }\end{array}$ & RESIDUAL & WEIGHT $^{*}$ * .5 & $\begin{array}{l}\text { WEIGHTED } \\
\text { RESIDUAL }\end{array}$ \\
\hline 1 & 1.0 & 101.804 & 100.175 & 1.63 & 0.999 & 1.63 \\
\hline 2 & 1.1 & -0.029 & 0.000 & $-0.289 E-01$ & 20.0 & -0.578 \\
\hline 3 & 1.12 & -0.129 & -0.083 & $-0.463 E-01$ & 20.0 & -0.927 \\
\hline 4 & 2.0 & 128.117 & 126.993 & 1.12 & 0.999 & 1.12 \\
\hline 5 & 2.1 & -0.041 & -0.034 & $-0.729 E-02$ & 20.0 & -0.146 \\
\hline 6 & 2.2 & -0.557 & -0.544 & $-0.132 \mathrm{E}-01$ & 20.0 & -0.265 \\
\hline 7 & 2.8 & -11.531 & -11.554 & $0.226 \mathrm{E}-01$ & 20.0 & 0.451 \\
\hline 8 & 2.12 & -14.184 & -14.192 & $0.815 E-02$ & 20.0 & 0.163 \\
\hline 9 & 3.0 & 156.678 & 157.131 & -0.453 & 0.999 & -0.452 \\
\hline 10 & 3.1 & -4.381 & -4.320 & $-0.614 \mathrm{E}-01$ & 20.0 & -1.23 \\
\hline 11 & 3.12 & -42.540 & -42.594 & $0.539 \mathrm{E}-01$ & 20.0 & 1.08 \\
\hline 12 & 4.0 & 124.893 & 126.993 & -2.10 & 0.999 & -2.10 \\
\hline 13 & 4.1 & -0.067 & -0.051 & $-0.156 \mathrm{E}-01$ & 20.0 & -0.312 \\
\hline 14 & 4.12 & -14.304 & -14.251 & $-0.533 E-01$ & 20.0 & -1.07 \\
\hline 15 & 5.0 & 140.961 & 140.914 & $0.471 \mathrm{E}-01$ & 0.999 & $0.470 \mathrm{E}-01$ \\
\hline 16 & 5.1 & -0.060 & -0.099 & $0.387 \mathrm{E}-01$ & 20.0 & 0.774 \\
\hline 17 & 5.12 & -21.676 & -21.658 & $-0.184 \mathrm{E}-01$ & 20.0 & -0.368 \\
\hline 18 & 6.0 & 126.537 & 127.204 & -0.667 & 0.999 & -0.666 \\
\hline 19 & 6.1 & 0.005 & -0.038 & $0.434 \mathrm{E}-01$ & 20.0 & 0.867 \\
\hline 20 & 6.12 & -14.365 & -14.367 & $0.204 \mathrm{E}-02$ & 20.0 & $0.407 \mathrm{E}-01$ \\
\hline 21 & 7.0 & 101.112 & 101.202 & $-0.904 E-01$ & 0.999 & $-0.903 E-01$ \\
\hline 22 & 7.1 & 0.048 & -0.003 & $0.507 \mathrm{E}-01$ & 20.0 & 1.01 \\
\hline 23 & 7.12 & -0.568 & -0.650 & $0.816 \mathrm{E}-01$ & 20.0 & 1.63 \\
\hline 24 & 8.0 & 158.135 & 157.114 & 1.02 & 0.999 & 1.02 \\
\hline 25 & 8.1 & -5.533 & -5.535 & $0.160 \mathrm{E}-02$ & 20.0 & $0.320 \mathrm{E}-01$ \\
\hline 26 & 8.12 & -43.217 & -43.107 & -0.110 & 20.0 & -2.21 \\
\hline 27 & 9.0 & 176.374 & 176.750 & -0.376 & 0.999 & -0.375 \\
\hline 28 & 9.1 & -0.001 & -0.100 & $0.993 E-01$ & 20.0 & 1.99 \\
\hline 29 & 9.12 & -38.242 & -38.354 & 0.112 & 20.0 & 2.25 \\
\hline 30 & 0.0 & 142.020 & 141.022 & 0.998 & 0.999 & 0.997 \\
\hline 31 & 0.1 & -0.013 & -0.017 & $0.372 \mathrm{E}-02$ & 20.0 & $0.745 E-01$ \\
\hline 32 & 0.12 & -19.921 & -19.876 & $-0.449 E-01$ & 20.0 & -0.897 \\
\hline
\end{tabular}

STATISTICS FOR HEAD RESIDUALS :

MAXIMUM WEIGHTED RESIDUAL : 2.25 OBS\# 29

MINIMUM WEIGHTED RESIDUAL : -2.21 OBS\# 26

AVERAGE WEIGHTED RESIDUAL : 0.109 
APPENDIX A. EXAMPLE SIMULATIONS - Test Case 1 - LIST Output File

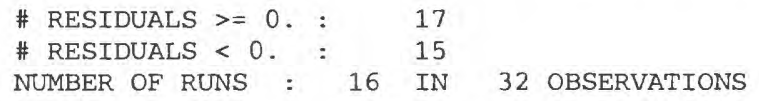

SUM OF SQUARED WEIGHTED RESIDUALS (HEADS ONLY) 36.417

DATA FOR FLOWS REPRESENTED USING THE RIVER PACKAGE

\begin{tabular}{|c|c|c|c|c|c|c|}
\hline OBS\# & $\begin{array}{c}\text { OBSERVATION } \\
\text { NAME }\end{array}$ & $\begin{array}{l}\text { MEAS. } \\
\text { FLOW }\end{array}$ & $\begin{array}{l}\text { CALC. } \\
\text { FLOW }\end{array}$ & RESIDUAL & WEIGHT* * .5 & $\begin{array}{l}\text { WEIGHTED } \\
\text { RESIDUAL }\end{array}$ \\
\hline 33 & SS & -4.40 & -4.35 & $-0.531 E-01$ & 2.50 & -0.133 \\
\hline 34 & TR3 & -4.10 & -4.06 & $-0.418 \mathrm{E}-01$ & 2.63 & -0.110 \\
\hline 35 & TR12 & -2.20 & -2.25 & $0.498 \mathrm{E}-01$ & 4.76 & 0.237 \\
\hline
\end{tabular}

STATISTICS FOR RIVER FLOW RESIDUALS :

MAXIMUM WEIGHTED RESIDUAL : 0.237 OBS\# 35

MINIMUM WEIGHTED RESIDUAL :-0.133 OBS\# 33

AVERAGE WEIGHTED RESIDUAL :-0.187E-02

\# RESIDUALS $>=0$. : 1

\# RESIDUALS $<0$ : : 2

NUMBER OF RUNS : 2 IN 3 OBSERVATIONS

SUM OF SQUARED WEIGHTED RESIDUALS (RIVER FLOWS ONLY) $0.85940 E-01$

SUM OF SQUARED WEIGHTED RESIDUALS (ALL DEPENDENT VARIABLES) 36.503

STATISTICS FOR ALL RESIDUALS :

AVERAGE WEIGHTED RESIDUAL : $0.999 \mathrm{E}-01$

\# RESIDUALS $>=0$ : : 18

\# RESIDUALS < 0 . : 17

NUMBER OF RUNS : 17 IN 35 OBSERVATIONS

INTERPRETTING THE CALCULATED RUNS STATISTIC VALUE OF -0.339

NOTE: THE FOLLOWING APPLIES ONLY IF

\# RESIDUALS $>=0$. IS GREATER THAN 10 AND

\# RESIDUALS < 0 . IS GREATER THAN 10

THE NEGATIVE VALUE MAY INDICATE TOO FEW RUNS:

IF THE VALUE IS LESS THAN - 1.28, THERE IS LESS THAN A 10 PERCENT CHANCE THE VALUES ARE RANDOM,

IF THE VALUE IS LESS THAN - 1.645, THERE IS LESS THAN A 5 PERCENT CHANCE THE VALUES ARE RANDOM,

IF THE VALUE IS LESS THAN -1.96, THERE IS LESS THAN A 2.5 PERCENT CHANCE THE VALUES ARE RANDOM. 


\section{Residual Analysis Files}

\begin{tabular}{|c|c|c|c|c|c|c|c|}
\hline \multicolumn{4}{|c|}{ tc 1._os } & \multicolumn{4}{|c|}{ tcl._ww } \\
\hline \multicolumn{2}{|c|}{ unweighted } & \multirow{2}{*}{\multicolumn{2}{|c|}{$\begin{array}{l}\text { plot observation } \\
\text { variable name }\end{array}$}} & \multicolumn{2}{|c|}{ weighted } & \multirow{2}{*}{\multicolumn{2}{|c|}{$\begin{array}{l}\text { plot observation } \\
\text { variable name }\end{array}$}} \\
\hline $\begin{array}{l}\text { simulated of } \\
\text { value }\end{array}$ & $\begin{array}{l}\text { bservation varia } \\
\text { value }\end{array}$ & & & $\begin{array}{l}\begin{array}{c}\text { simulated } \\
\text { value }\end{array} \\
\end{array}$ & $\begin{array}{l}\text { bservation varial } \\
\text { value }\end{array}$ & & \\
\hline 100.1747 & 101.8040 & 1 & 1.0 & 100.0497 & 101.6770 & 1 & 1.0 \\
\hline$-0.8392334 \mathrm{E}-04$ & $-0.2899933 \mathrm{E}-01$ & 1 & 1.1 & $-0.1678467 \mathrm{E}-02$ & -0.5799866 & 1 & 1.1 \\
\hline$-0.8264923 E-01$ & -0.1289978 & 1 & 1.12 & -1.652985 & -2.579956 & 1 & 1.12 \\
\hline 126.9926 & 128.1170 & 1 & 2.0 & 126.8342 & 127.9572 & 1 & 2.0 \\
\hline$-0.3370667 E-01$ & $-0.4100037 \mathrm{E}-01$ & 1 & 2.1 & -0.6741333 & -0.8200073 & 1 & 2.1 \\
\hline-0.5437775 & -0.5570068 & 1 & 2.2 & -10.87555 & -11.14014 & 1 & 2.2 \\
\hline-11.55357 & -11.53101 & 1 & 2.8 & -231.0713 & -230.6201 & 1 & 2.8 \\
\hline-14.19215 & -14.18401 & 1 & 2.12 & -283.8431 & -283.6801 & 1 & 2.12 \\
\hline 157.1305 & 156.6780 & 1 & 3.0 & 156.9345 & 156.4825 & 1 & 3.0 \\
\hline-4.319595 & -4.380997 & 1 & 3.1 & -86.39191 & -87.61993 & 1 & 3.1 \\
\hline-42.59389 & -42.53999 & 1 & 3.12 & -851.8777 & -850.7999 & 1 & 3.12 \\
\hline 126.9926 & 124.8930 & 1 & 4.0 & 126.8342 & 124.7372 & 1 & 4.0 \\
\hline$-0.5142212 \mathrm{E}-01$ & $-0.6700134 \mathrm{E}-01$ & 1 & 4.1 & -1.028442 & -1.340027 & 1 & 4.1 \\
\hline-14.25066 & -14.30400 & 1 & 4.12 & -285.0133 & -286.0800 & 1 & 4.12 \\
\hline 140.9139 & 140.9610 & 1 & 5.0 & 140.7381 & 140.7851 & 1 & 5.0 \\
\hline$-0.9869385 E-01$ & $-0.5999756 \mathrm{E}-01$ & 1 & 5.1 & -1.973877 & -1.199951 & 1 & 5.1 \\
\hline-21.65761 & -21.67599 & 1 & 5.12 & -433.1522 & -433.5199 & 1 & 5.12 \\
\hline 127.2039 & 126.5370 & 1 & 6.0 & 127.0452 & 126.3791 & 1 & 6.0 \\
\hline$-0.3836823 E-01$ & $0.4997253 \mathrm{E}-02$ & 1 & 6.1 & -0.7673645 & $0.9994507 \mathrm{E}-01$ & 1 & 6.1 \\
\hline-14.36704 & -14.36501 & 1 & 6.12 & -287.3409 & -287.3001 & 1 & 6.12 \\
\hline 101.2024 & 101.1120 & 1 & 7.0 & 101.0761 & 100.9858 & 1 & 7.0 \\
\hline$-0.2738953 E-02$ & $0.4800415 \mathrm{E}-01$ & 1 & 7.1 & $-0.5477905 E-01$ & 0.9600830 & 1 & 7.1 \\
\hline-0.6495590 & -0.5680008 & 1 & 7.12 & -12.99118 & -11.36002 & 1 & 7.12 \\
\hline 157.1141 & 158.1350 & 1 & 8.0 & 156.9180 & 157.9377 & 1 & 8.0 \\
\hline-5.534592 & -5.532990 & 1 & 8.1 & -110.6918 & -110.6598 & 1 & 8.1 \\
\hline-43.10667 & -43.21700 & 1 & 8.12 & -862.1333 & -864.3399 & 1 & 8.12 \\
\hline 176.7497 & 176.3740 & 1 & 9.0 & 176.5291 & 176.1539 & 1 & 9.0 \\
\hline-0.1002502 & $-0.9918213 E-03$ & 1 & 9.1 & -2.005005 & $-0.1983643 E-01$ & 1 & 9.1 \\
\hline-38.35443 & -38.24199 & 1 & 9.12 & -767.0886 & -764.8398 & 1 & 9.12 \\
\hline 141.0217 & 142.0200 & 1 & 0.0 & 140.8458 & 141.8428 & 1 & 0.0 \\
\hline$-0.1672363 E-01$ & $-0.1300049 \mathrm{E}-01$ & 1 & 0.1 & -0.3344727 & -0.2600098 & 1 & 0.1 \\
\hline-19.87614 & -19.92101 & 1 & 0.12 & -397.5229 & -398.4201 & 1 & 0.12 \\
\hline-4.346939 & -4.400000 & 2 & ss & 250.4367 & 254.5100 & 2 & SS \\
\hline-4.058173 & -4.100000 & 2 & TR3 & $-0.2208509 E-03$ & $-0.7631402 \mathrm{E}-01$ & 2 & TR3 \\
\hline-2.249796 & -2.200000 & 2 & TR12 & -0.3935678 & -0.6142752 & 2 & TR12 \\
\hline
\end{tabular}




\begin{tabular}{|c|c|c|c|c|c|c|}
\hline \multicolumn{4}{|c|}{ tc 1._ws } & \multicolumn{3}{|c|}{ tcl._r } \\
\hline \multicolumn{2}{|l|}{ weighted } & \multirow{2}{*}{\multicolumn{2}{|c|}{$\begin{array}{l}\text { plot observation } \\
\text { variable name }\end{array}$}} & \multirow{2}{*}{$\begin{array}{l}\text { unweighted } \\
\text { residual }\end{array}$} & \multirow{2}{*}{$\begin{array}{c}\text { plot } \\
\text { variable }\end{array}$} & \multirow{2}{*}{$\begin{array}{l}\text { observation } \\
\text { name }\end{array}$} \\
\hline $\begin{array}{l}\text { simulated } \\
\text { value }\end{array}$ & $\begin{array}{l}\text { residual } \\
\text { value }\end{array}$ & & & & & \\
\hline 100.0497 & 1.627270 & 1 & 1.0 & 1.629303 & 1 & 1.0 \\
\hline$-0.1678467 \mathrm{E}-02$ & -0.5783081 & 1 & 1.1 & $-0.2891541 E-01$ & 1 & 1.1 \\
\hline-1.652985 & -0.9269714 & 1 & 1.12 & $-0.4634857 \mathrm{E}-01$ & 1 & 1.12 \\
\hline 126.8342 & 1.122964 & 1 & 2.0 & 1.124367 & 1 & 2.0 \\
\hline-0.6741333 & -0.1458740 & 1 & 2.1 & $-0.7293701 \mathrm{E}-02$ & 1 & 2.1 \\
\hline-10.87555 & -0.2645874 & 1 & 2.2 & $-0.1322937 \mathrm{E}-01$ & 1 & 2.2 \\
\hline-231.0713 & 0.4512024 & 1 & 2.8 & $0.2256012 \mathrm{E}-01$ & 1 & 2.8 \\
\hline-283.8431 & 0.1629639 & 1 & 2.12 & $0.8148193 \mathrm{E}-02$ & 1 & 2.12 \\
\hline 156.9345 & -0.4519653 & 1 & 3.0 & -0.4525299 & 1 & 3.0 \\
\hline-86.39191 & -1.228027 & 1 & 3.1 & $-0.6140137 \mathrm{E}-01$ & 1 & 3.1 \\
\hline-851.8777 & 1.077881 & 1 & 3.12 & $0.5389404 \mathrm{E}-01$ & 1 & 3.12 \\
\hline 126.8342 & -2.097020 & 1 & 4.0 & -2.099640 & 1 & 4.0 \\
\hline-1.028442 & -0.3115845 & 1 & 4.1 & $-0.1557922 \mathrm{E}-01$ & 1 & 4.1 \\
\hline-285.0133 & -1.066742 & 1 & 4.12 & $-0.5333710 \mathrm{E}-01$ & 1 & 4.12 \\
\hline 140.7381 & $0.4702987 \mathrm{E}-01$ & 11 & 5.0 & $0.4708862 \mathrm{E}-01$ & 1 & 5.0 \\
\hline-1.973877 & 0.7739258 & 1 & 5.1 & $0.3869629 \mathrm{E}-01$ & 1 & 5.1 \\
\hline-433.1522 & -0.3677368 & 1 & 5.12 & $-0.1838684 \mathrm{E}-01$ & 1 & 5.12 \\
\hline 127.0452 & -0.6660305 & 1 & 6.0 & -0.6668625 & 1 & 6.0 \\
\hline-0.7673645 & 0.8673096 & 1 & 6.1 & $0.4336548 \mathrm{E}-01$ & 1 & 6.1 \\
\hline-287.3409 & $0.4074097 \mathrm{E}-01$ & 11 & 6.12 & $0.2037048 E-02$ & 1 & 6.12 \\
\hline 101.0761 & $-0.9031076 E-01$ & 11 & 7.0 & $-0.9042358 E-01$ & 1 & 7.0 \\
\hline$-0.5477905 E-01$ & 1.014862 & 1 & 7.1 & $0.5074310 \mathrm{E}-01$ & 1 & 7.1 \\
\hline-12.99118 & 1.631165 & 1 & 7.12 & $0.8155823 \mathrm{E}-01$ & 1 & 7.12 \\
\hline 156.9180 & 1.019661 & 1 & 8.0 & 1.020935 & 1 & 8.0 \\
\hline-110.6918 & $0.3204346 E-01$ & 11 & 8.1 & $0.1602173 \mathrm{E}-02$ & 1 & 8.1 \\
\hline-862.1333 & -2.206573 & 1 & 8.12 & -0.1103287 & 1 & 8.12 \\
\hline 176.5291 & -0.3752027 & 1 & 9.0 & -0.3756714 & 1 & 9.0 \\
\hline-2.005005 & 1.985168 & 1 & 9.1 & $0.9925842 \mathrm{E}-01$ & 1 & 9.1 \\
\hline-767.0886 & 2.248840 & 1 & 9.12 & 0.1124420 & 1 & 9.12 \\
\hline 140.8458 & 0.9970149 & 1 & 0.0 & 0.9982605 & 1 & 0.0 \\
\hline-0.3344727 & $0.7446289 \mathrm{E}-01$ & 1 & 0.1 & $0.3723 i 45 E-02$ & 1 & 0.1 \\
\hline-397.5229 & -0.8972168 & 1 & 0.12 & $-0.4486084 \mathrm{E}-01$ & 1 & 0.12 \\
\hline 250.4367 & -0.1326525 & 2 & ss & $-0.5306101 \mathrm{E}-01$ & 2 & ss \\
\hline$-0.2208509 E-03$ & -0.1100703 & 2 & TR3 & $-0.4182673 \mathrm{E}-01$ & 2 & TR3 \\
\hline-0.3935678 & 0.2371232 & 2 & TR12 & $0.4979587 \mathrm{E}-01$ & 2 & TR12 \\
\hline
\end{tabular}




\section{YCINT Output File}

YCINT-2000

MODFLOW-2000 POST-PROCESSING PROGRAM TO CALCULATE

LINEAR CONFIDENCE AND PREDICTION INTERVALS

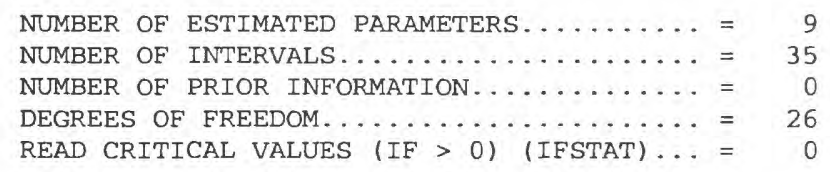

INTERVALS ARE NOT CALCULATED ON DIFFERENCES

VARIANCE-COVARIANCE MATRIX FOR ESTIMATED PARAMETERS

\begin{tabular}{|c|c|c|c|c|c|}
\hline & $\begin{array}{l}\text { WELLS_TR } \\
\text { HK_1 }\end{array}$ & $\begin{array}{l}\text { RCH_ZONE_1 } \\
\text { VERT_K_CB }\end{array}$ & $\begin{array}{l}\mathrm{RCH} \text { ZZONE_2 } \\
\mathrm{SS} \_2\end{array}$ & $\begin{array}{l}\text { RIVERS } \\
\text { HK_2 }\end{array}$ & SS_1 \\
\hline WELLS TR & 5.9 & $76 \mathrm{E}-02$ & -0.3 & -5.38 & $-5.63054 \mathrm{E}-03$ \\
\hline WELLS_TR & $-5.55136 E-03$ & $-5.61987 \mathrm{E}-03$ & $-4.82822 \mathrm{E}-03$ & $-5.60601 \mathrm{E}-03$ & \\
\hline \multirow[t]{2}{*}{ RCH_ZONE_1 } & $-4.43476 E-02$ & 14.368 & -4.7221 & $2.39544 \mathrm{E}-02$ & 3.9500 \\
\hline & $4.24800 \mathrm{E}-02$ & $4.29512 \mathrm{E}-02$ & $5.47146 \mathrm{E}-02$ & $3.75563 E-02$ & \\
\hline \multirow[t]{2}{*}{ RCH_ZONE_2 } & -0.34906 & -4.7221 & 24.555 & 0.34516 & 0.32633 \\
\hline & 0.32359 & 0.32279 & 0.31551 & 0.32945 & \\
\hline \multirow[t]{2}{*}{ RIVERS } & $-5.38978 \mathrm{E}-03$ & $2.39544 \mathrm{E}-02$ & 0.34516 & 0.34952 & $-5.83255 E-03$ \\
\hline & $2.26997 \mathrm{E}-03$ & $-2.16912 \mathrm{E}-02$ & 0.16755 & $926 E-02$ & \\
\hline \multirow[t]{2}{*}{ SS_1 } & $-5.63054 \mathrm{E}-03$ & $3.95006 \mathrm{E}-02$ & 0.32633 & $-5.83255 E-03$ & 7.52 \\
\hline & $5.20787 \mathrm{E}-03$ & $8.61473 E-03$ & $-2.60320 E-02$ & $5.24299 \mathrm{E}-03$ & \\
\hline \multirow[t]{2}{*}{ HK_1 } & $-5.55136 \mathrm{E}-03$ & $4.24800 \mathrm{E}-02$ & 0.32359 & $2.26997 \mathrm{E}-03$ & $5.20787 \mathrm{E}-03$ \\
\hline & 5.19 & $7 E-03$ & $4.49986 \mathrm{E}-03$ & E-03 & \\
\hline \multirow[t]{2}{*}{ VERT_K_CB } & $-5.61987 E-03$ & $4.29512 E-02$ & 0.32279 & $-2.16912 \mathrm{E}-02$ & $8.61473 \mathrm{E}-03$ \\
\hline & $5.30197 \mathrm{E}-03$ & $1.37066 \mathrm{E}-02$ & $-4.70266 \mathrm{E}-02$ & $4.96660 \mathrm{E}-03$ & \\
\hline \multirow[t]{2}{*}{ SS_2 } & $-4.82822 \mathrm{E}-03$ & $5.47146 \mathrm{E}-02$ & 0.31551 & 0.16755 & $-2.60320 \mathrm{E}-02$ \\
\hline & $4.49986 \mathrm{E}-03$ & $-4.70266 \mathrm{E}-02$ & 0.45417 & $355 E-03$ & \\
\hline \multirow[t]{2}{*}{ HK_2 } & $-5.60601 E-03$ & $3.75563 \mathrm{E}-02$ & 0.32945 & $1.16926 \mathrm{E}-02$ & $5.24299 \mathrm{E}-03$ \\
\hline & & 13 & & $04 \mathrm{E}-$ & \\
\hline
\end{tabular}

VALUES COMPUTED WITH OPTIMUM PARAMETERS FOR PREDICTIVE CONDITIONS OBSERVATION

\begin{tabular}{rlllll} 
NO. & \multicolumn{1}{c}{ NAME } & VALUE & NO. & \multicolumn{1}{c}{ NAME } & VALUE \\
1 & 1.0 & 100.22 & 19 & 6.1 & $-.12451 \mathrm{E}-01$ \\
2 & 1.1 & $-.22888 \mathrm{E}-04$ & 20 & 6.12 & -19.185 \\
3 & 1.12 & $-.90614 \mathrm{E}-01$ & 21 & 7.0 & 102.87 \\
4 & 2.0 & 139.33 & 22 & 7.1 & $-.11520 \mathrm{E}-02$ \\
5 & 2.1 & $-.94757 \mathrm{E}-02$ & 23 & 7.12 & -1.3807 \\
6 & 2.2 & -.27621 & 24 & 8.0 & 173.96 \\
7 & 2.8 & -12.963 & 25 & 8.1 & -5.8096 \\
8 & 2.12 & -18.771 & 26 & 8.12 & -57.255 \\
9 & 3.0 & 174.36 & 27 & 9.0 & 190.30 \\
10 & 3.1 & -3.6657 & 28 & 9.1 & $-.50507 \mathrm{E}-01$ \\
11 & 3.12 & -56.237 & 29 & 9.12 & -49.512 \\
12 & 4.0 & 139.33 & 30 & 0.0 & 157.04 \\
13 & 4.1 & $-.16266 \mathrm{E}-01$ & 31 & 0.1 & $-.43030 \mathrm{E}-02$ \\
14 & 4.12 & -18.849 & 32 & 0.12 & -26.129 \\
15 & 5.0 & 157.13 & 33 & SS & -4.8606 \\
16 & 5.1 & $-.36789 \mathrm{E}-01$ & 34 & TR3 & -4.7182 \\
17 & 5.12 & -28.462 & 35 & TR12 & -2.8632 \\
18 & 6.0 & 139.63 & & &
\end{tabular}


APPENDIX A. EXAMPLE SIMULATIONS - Test Case 1 - YCINT Output File

SENSITIVITIES FOR OPTIMUM PARAMETERS FOR PREDICTIVE CONDITIONS

\begin{tabular}{|c|c|c|c|c|c|}
\hline PARAMETER & 1.0 & 1.1 & 1.12 & 2.0 & 2.1 \\
\hline-------- & ---------- & $---\cdots------$ & $-\cdots+\cdots----$ & $---\cdots \cdots$ & $-\cdots+\cdots-\cdots$ \\
\hline WELLS_TR & 0.0000 & $0.11931 E-04$ & $0.82373 E-01$ & 0.0000 & $0.86195 E-02$ \\
\hline RCH_ZONE_1 & $0.23781 E-02$ & $0.23283 E-09$ & $0.23283 E-09$ & 0.38114 & $0.29802 E-07$ \\
\hline RCH_ZONE_2 & $0.23780 \mathrm{E}-02$ & $0.23283 E-09$ & $0.23283 E-09$ & 0.48464 & 0.0000 \\
\hline RIVERS & -0.22503 & $0.17583 E-04$ & $0.90586 E-01$ & -0.22502 & $0.16689 \mathrm{E}-05$ \\
\hline SS_1 & 0.0000 & $0.43096 \mathrm{E}-04$ & $0.16717 \mathrm{E}-01$ & 0.0000 & $0.24942 \mathrm{E}-01$ \\
\hline HK_1 & $0.11153 E-04$ & $-0.37269 E-04$ & $-0.14183 E-01$ & $-33 \cdot 343$ & $-0.13233 E-01$ \\
\hline VERT_K_CB & $0.51517 \mathrm{E}-07$ & $0.28342 E-05$ & $-0.61570 E-04$ & -0.28418 & $0.15171 E-02$ \\
\hline SS_2 & 0.0000 & $0.11629 \mathrm{E}-04$ & $0.25782 \mathrm{E}-02$ & 0.0000 & $0.65209 E-02$ \\
\hline HK_2 & $0.38645 E-05$ & $-0.19960 E-04$ & $-0.50321 E-02$ & -5.4763 & $-0.10268 E-01$ \\
\hline PARAMETER & 2.2 & 2.8 & 2.12 & 3.0 & 3.1 \\
\hline$\ldots---\cdots \cdots$ & 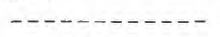 & $-\cdots+\cdots-\cdots--$ & $\ldots \ldots$ & --- & $-\cdots$ \\
\hline WELLS_TR & 0.25110 & 11.785 & 17.065 & 0.0000 & 3.3325 \\
\hline RCH_ZONE_1 & 0.0000 & 0.0000 & $0.29802 E-07$ & 0.60822 & 0.0000 \\
\hline $\mathrm{RCH}$ ZZONE_2 & 0.0000 & $-0.29802 E-07$ & $0.29802 E-07$ & 1.1409 & 0.0000 \\
\hline RIVERS & $0.20175 E-03$ & $0.47841 \mathrm{E}-01$ & $0.84483 E-01$ & -0.22501 & $0.29802 E-07$ \\
\hline SS_1 & 0.50050 & 5.8967 & 3.3654 & 0.0000 & 2.9643 \\
\hline HK_1 & -0.20394 & 5.7307 & 12.946 & -57.960 & $0.91614 \mathrm{E}-01$ \\
\hline VERT_K_CB & $0.79712 \mathrm{E}-02$ & $0.27161 E-01$ & $0.70868 E-01$ & -0.49334 & -0.34792 \\
\hline SS_2 & $0.91114 E-01$ & 0.91182 & 0.51774 & 0.0000 & 0.49137 \\
\hline HK_2 & -0.11964 & 0.34885 & 1.7861 & -15.677 & 0.4 \\
\hline PARAMETER & 3.12 & 4.0 & 4.1 & 4.12 & 5.0 \\
\hline$-\cdots-\cdots---$ & $\cdots+---\cdots$ & --------- & $---------\cdots$ & ----------- & ----------- \\
\hline WELLS_TR & 51.125 & 0.0000 & $0.14780 E-01$ & 17.135 & 0.0000 \\
\hline RCH_ZONE_1 & $0.59605 E-07$ & 0.38114 & $0.29802 E-07$ & $0.29802 E-07$ & 0.52303 \\
\hline $\mathrm{RCH}$ ZZONE_2 & 0.0000 & 0.48464 & 0.0000 & $0.29802 \mathrm{E}-07$ & 0.76518 \\
\hline RIVERS & $0.74748 \mathrm{E}-01$ & -0.22502 & $0.22948 E-05$ & $0.83549 E-01$ & -0.22502 \\
\hline SS_1 & 7.3393 & 0.0000 & $0.39760 E-01$ & 3.3649 & 0.0000 \\
\hline HK_1 & 35.072 & -33.343 & $-0.20504 \mathrm{E}-01$ & 12.989 & -46.549 \\
\hline VERT_K_CB & -0.21915 & -0.28418 & $0.21347 E-02$ & $0.65906 E-01$ & -0.39434 \\
\hline SS_2 & 1.1275 & 0.0000 & $0.10225 E-01$ & 0.51767 & 0.0000 \\
\hline HK_2 & 12.840 & -5.4763 & $-0.15364 E-01$ & 1.8267 & -9.9592 \\
\hline PARAMETER & 5.1 & 5.12 & 6.0 & 6.1 & 6.12 \\
\hline$-\cdots------$ & ----------- & $---------\cdots$ & $\cdots-\cdots$ & $----\cdots-----$ & ---- \\
\hline WELLS_TR & $0.33450 E-01$ & 25.874 & 0.0000 & $0.11322 \mathrm{E}-01$ & 17.441 \\
\hline RCH_ZONE_1 & $0.59605 E-07$ & $0.59605 E-07$ & 0.38054 & $0.29802 E-07$ & $0.29802 \mathrm{E}-07$ \\
\hline RCH_ZONE_2 & $0.59605 E-07$ & $0.11921 E-06$ & 0.49537 & 0.0000 & $0.59605 E-07$ \\
\hline RIVERS & $0.28312 \mathrm{E}-06$ & $0.78590 E-01$ & -0.22502 & $0.17434 \mathrm{E}-05$ & $0.84330 E-01$ \\
\hline SS_1 & $0.79206 \mathrm{E}-01$ & 5.1917 & 0.0000 & $0.27079 E-01$ & 3.4280 \\
\hline HK_1 & $-0.36560 E-01$ & 18.628 & -33.411 & $-0.13145 E-01$ & 13.118 \\
\hline VERT_K_CB & $0.38443 E-02$ & $0.86688 E-01$ & -0.63570 & $0.60395 E-02$ & 0.51133 \\
\hline SS_2 & $0.20848 E-01$ & 0.79817 & 0.0000 & $0.10833 E-01$ & 0.53172 \\
\hline HK_2 & $-0.30542 E-01$ & 3.6760 & -5.3574 & $-0.18354 E-01$ & 1.5101 \\
\hline PARAMETER & 7.0 & 7.1 & 7.12 & 8.0 & 8.1 \\
\hline$-\cdots---\cdots$ & --------- & $-------\cdots--$ & ------- & $--\cdots-\cdots$ & $-\cdots--\cdots$ \\
\hline WELLS_TR & 0.0000 & $0.10388 E-02$ & 1. 2552 & 0.0000 & 5.2815 \\
\hline RCH_ZONE_1 & $0.28925 \mathrm{E}-01$ & 0.0000 & 0.0000 & 0.59968 & 0.0000 \\
\hline RCH_ZONE_2 & $0.33070 \mathrm{E}-01$ & 0.0000 & $0.37253 E-08$ & 1.1451 & $0.11921 E-06$ \\
\hline RIVERS & -0.22503 & $0.66414 \mathrm{E}-04$ & $0.98170 E-01$ & -0.22501 & $0.44704 E-07$ \\
\hline SS_1 & 0.0000 & $0.26073 E-02$ & 0.23228 & 0.0000 & 2.0702 \\
\hline HK_1 1 & -2.3438 & $-0.14117 \mathrm{E}-02$ & 0.91641 & -57.591 & 0.45263 \\
\hline VERT_K_CB & -2.3818 & $0.11380 \mathrm{E}-02$ & 1.1719 & -0.13355 & 1.4141 \\
\hline SS_2 & 0.0000 & $0.11391 \mathrm{E}-02$ & $0.36305 E-01$ & 0.0000 & 1.4010 \\
\hline HK_2 2 & 2.0830 & $-0.23913 E-02$ & -1.0744 & -16.000 & 0.47168 \\
\hline
\end{tabular}


APPENDIX A. EXAMPLE SIMULATIONS - Test Case 1 - YCINT Output File

\begin{tabular}{|c|c|c|c|c|c|}
\hline PARAMETER & 8.12 & 9.0 & 9.1 & 9.12 & 0.0 \\
\hline WELLS TR & 52.050 & 0.0000 & $0.45916 \mathrm{E}-01$ & 45.010 & 0.0000 \\
\hline RCH_ZONE_1 & 0.0000 & 0.60469 & $0.59605 \mathrm{E}-07$ & $0.59605 \mathrm{E}-07$ & 0.51712 \\
\hline RCH_ZONE_2 & $0.11921 \mathrm{E}-06$ & 1.6529 & $0.11921 E-06$ & $0.11921 \mathrm{E}-06$ & 0.77410 \\
\hline RIVERS & $0.74732 \mathrm{E}-01$ & -0.22500 & $-0.14901 E-07$ & $0.68669 \mathrm{E}-01$ & -0.22501 \\
\hline SS_1 & 7.3381 & 0.0000 & $0.74713 \mathrm{E}-01$ & 9.9250 & 0.0000 \\
\hline HK_1 & 33.552 & -66.697 & $-0.22751 E-01$ & 28.101 & -46.337 \\
\hline VERT $K C B$ & 0.87644 & $-0.58038 \mathrm{E}-01$ & $0.36563 \mathrm{E}-01$ & 0.23718 & -0.33066 \\
\hline SS_2 & 1.1365 & 0.0000 & $0.39552 \mathrm{E}-01$ & 1.5355 & 0.0000 \\
\hline HK_2 & 14.274 & -23.307 & $-0.77578 \mathrm{E}-01$ & 9.6387 & -10.144 \\
\hline PARAMETER & 0.1 & 0.12 & ss & TR3 & TR12 \\
\hline$\ldots \ldots \ldots$ & $-\ldots-\ldots-1--$ & $\ldots-\ldots$ & - & $--1-1-1-1$ & $-\ldots-\ldots-1,-$ \\
\hline WELLS_TR & $0.39053 \mathrm{E}-02$ & 23.753 & 0.0000 & -0.12945 & -1.8158 \\
\hline RCH_ZONE_1 & 0.0000 & 0.0000 & $-0.51368 \mathrm{E}-01$ & $-0.51368 \mathrm{E}-01$ & $-0.51368 E-01$ \\
\hline RCH_ZONE_2 & $0.59605 \mathrm{E}-07$ & $0.11921 \mathrm{E}-06$ & $-0.51364 \mathrm{E}-01$ & $-0.51364 \mathrm{E}-01$ & $-0.51364 \mathrm{E}-01$ \\
\hline RIVERS & $0.11921 E-06$ & $0.77196 \mathrm{E}-01$ & $-0.28326 \mathrm{E}-04$ & $0.91489 \mathrm{E}-03$ & $0.97869 \mathrm{E}-03$ \\
\hline SS 1 & $0.10829 E-01$ & 5.2268 & 0.0000 & -0.18607 & -0.36107 \\
\hline $\mathrm{HK}_{-1}$ & $-0.52719 \mathrm{E}-02$ & 17.008 & $-0.24074 \mathrm{E}-03$ & 0.16935 & 0.31302 \\
\hline VERT $K$ CB & $0.28307 \mathrm{E}-02$ & 0.39728 & $-0.11332 E-05$ & $-0.14054 \mathrm{E}-03$ & $0.23405 E-02$ \\
\hline SS_2 & $0.44915 \mathrm{E}-02$ & 0.81014 & 0.0000 & $-0.31652 \mathrm{E}-01$ & $-0.55684 \mathrm{E}-01$ \\
\hline $\mathrm{HK} 2$ & $-0.85840 E-02$ & 2.6070 & $-0.83480 E-04$ & $0.47272 \mathrm{E}-01$ & 0.10022 \\
\hline
\end{tabular}

INDIVIDUAL 95\% CONFIDENCE INTERVALS

UNCERTAINTY ON EACH PREDICTION IS CONSIDERED SEPARATELY

IF SIMULTANEOUS UNCERTAINTY IS DESIRED, GO TO NEXT TABLE

95\% CONFIDENCE INTERVALS INDICATE THAT THERE IS 95\% PROBABILITY THAT THE ACTUAL VALUE WILL BE WITHIN THE INDICATED RANGE

CRITICAL VALUE FOR THE INTERVALS $=2.0560$

\begin{tabular}{|c|c|c|c|c|c|}
\hline & OBSERVATION & SIMULATED & & & \\
\hline NO. & NAME & VALUE & STD. DEV. & CONFIDENCE & INTERVAL \\
\hline 1 & 1.0 & 100.225 & 1.00771 & 98.1532 & 102.297 \\
\hline 2 & 1.1 & $-0.228882 E-04$ & 20.0000 & -41.1200 & 41.1200 \\
\hline 3 & 1.12 & $-0.906143 E-01$ & 20.0001 & -41.2108 & 41.0295 \\
\hline 4 & 2.0 & 139.331 & 1.22016 & 136.822 & 141.840 \\
\hline 5 & 2.1 & $-0.947571 E-02$ & 20.0000 & -41.1295 & 41.1105 \\
\hline 6 & 2.2 & -0.276215 & 20.0000 & -41.3963 & 40.8439 \\
\hline 7 & 2.8 & -12.9634 & 20.0032 & -54.0900 & 28.1632 \\
\hline 8 & 2.12 & $-18 \cdot 7714$ & 20.0012 & -59.8938 & 22.3510 \\
\hline 9 & 3.0 & 174.363 & 1.21891 & 171.857 & 176.869 \\
\hline 10 & 3.1 & -3.66571 & 20.0013 & -44.7884 & 37.4569 \\
\hline 11 & 3.12 & -56.2375 & 20.0060 & -97.3699 & $;-15.1051$ \\
\hline 12 & 4.0 & 139.331 & 1.22016 & 136.822 & ; 141.840 \\
\hline 13 & 4.1 & $-0.162659 \mathrm{E}-01$ & 20.0000 & -41.1363 & 41.1037 \\
\hline 14 & 4.12 & -18.8487 & 20.0012 & -59.9711 & 22.2737 \\
\hline 15 & 5.0 & 157.132 & 1.24335 & 154.576 & 159.688 \\
\hline 16 & 5.1 & $-0.367889 \mathrm{E}-01$ & 20.0000 & -41.1568 & 41.0832 \\
\hline 17 & 5.12 & -28.4615 & 20.0026 & -69.5869 & 12.6639 \\
\hline 18 & 6.0 & 139.632 & 1.20428 & 137.156 & 142.108 \\
\hline 19 & 6.1 & $-0.124512 \mathrm{E}-01$ & 20.0000 & -41.1325 & 41.1075 \\
\hline 20 & 6.12 & -19.1850 & 20.0009 & -60.3069 & 21.9369 \\
\hline 21 & 7.0 & 102.868 & 1.01789 & 100.775 & 104.961 \\
\hline 22 & 7.1 & $-0.115204 \mathrm{E}-02$ & 20.0000 & -41.1212 & 41.1188 \\
\hline 23 & 7.12 & -1.38068 & 20.0002 & -42.5010 & 39.7397 \\
\hline 24 & 8.0 & 173.956 & 1.22484 & 171.438 & 176.474 \\
\hline 25 & 8.1 & -5.80960 & 20.0137 & -46.9578 & 35.3386 \\
\hline 26 & 8.12 & -57.2549 & 20.0044 & -98.3840 & $;-16.1258$ \\
\hline 27 & 9.0 & 190.300 & 2.14651 & 185.887 & ; 194.713 \\
\hline 28 & 9.1 & $-0.505066 \mathrm{E}-01$ & 20.0000 & -41.1705 & 41.0695 \\
\hline 29 & 9.12 & -49.5115 & 20.0091 & -90.6502 & $;-8.37278$ \\
\hline 30 & 0.0 & 157.041 & 1.21930 & 154.534 & 159.548 \\
\hline
\end{tabular}


APPENDIX A. EXAMPLE SIMULATIONS - Test Case 1 - YCINT Output File

$\begin{array}{lllllll}31 & 0.1 & -0.430298 E-02 & 20.0000 & -41.1243 & ; & 41.1157 \\ 32 & 0.12 & -26.1288 & 20.0023 & -67.2536 & ; & 14.9960 \\ 33 & \text { SS } & -4.86063 & 0.487635 & -5.86321 & ; & -3.85805 \\ 34 & \text { TR3 } & -4.71819 & 0.465964 & -5.67621 & ;-3.76017 \\ 35 & \text { TR12 } & -2.86323 & 0.262060 & -3.40202 & ;-2.32443\end{array}$

35 SIMULTANEOUS 95\% CONFIDENCE INTERVALS

UNCERTAINTY ON EACH PREDICTION IS CONSIDERED JOINTLY

IF UNCERTAINTY OVER AN AREA IS DESIRED, GO TO NEXT TABLE

95\% CONFIDENCE INTERVALS INDICATE THAT THERE IS

95\% PROBABILITY THAT THE ACTUAL VALUE WILL BE WITHIN THE INDICATED RANGE

SCHEFFE CONFIDENCE INTERVALS ARE USED

CRITICAL VALUE FOR THE INTERVALS $=3.5800$

\begin{tabular}{|c|c|c|c|c|c|}
\hline NO. & $\begin{array}{l}\text { OBSERVATION } \\
\text { NAME }\end{array}$ & $\begin{array}{l}\text { SIMULATED } \\
\text { VALUE }\end{array}$ & STD. DEV. & CONFIDENCE & INTERVAL \\
\hline 1 & 1.0 & 100.225 & 1.00771 & 96.6174 & 103.833 \\
\hline 2 & 1.1 & $-0.228882 E-04$ & 20.0000 & -71.6000 & 71.6000 \\
\hline 3 & 1.12 & $-0.906143 E-01$ & 20.0001 & -71.6909 & 71.5096 \\
\hline 4 & 2.0 & 139.331 & 1.22016 & 134.963 & 143.699 \\
\hline 5 & 2.1 & $-0.947571 E-02$ & 20.0000 & -71.6095 & 71.5905 \\
\hline 6 & 2.2 & -0.276215 & 20.0000 & -71.8763 & 71.3239 \\
\hline 7 & 2.8 & -12.9634 & 20.0032 & -84.5750 & 58.6482 \\
\hline 8 & 2.12 & -18.7714 & 20.0012 & -90.3756 & 52.8328 \\
\hline 9 & 3.0 & 174.363 & 1.21891 & 169.999 & 178.727 \\
\hline 10 & 3.1 & -3.66571 & 20.0013 & -75.2703 & 67.9389 \\
\hline 11 & 3.12 & -56.2375 & 20.0060 & -127.859 & 15.3841 \\
\hline 12 & 4.0 & 139.331 & 1.22016 & 134.963 & 143.699 \\
\hline 13 & 4.1 & $-0.162659 \mathrm{E}-01$ & 20.0000 & -71.6163 & 71.5837 \\
\hline 14 & 4.12 & -18.8487 & 20.0012 & -90.4529 & 52.7555 \\
\hline 15 & 5.0 & 157.132 & 1.24335 & 152.681 & 161.583 \\
\hline 16 & 5.1 & $-0.367889 E-01$ & 20.0000 & -71.6368 & 71.5632 \\
\hline 17 & 5.12 & -28.4615 & 20.0026 & -100.071 & 43.1479 \\
\hline 18 & 6.0 & 139.632 & 1.20428 & 135.321 & 143.943 \\
\hline 19 & 6.1 & $-0.124512 E-01$ & 20.0000 & -71.6124 & 71.5875 \\
\hline 20 & 6.12 & -19.1850 & 20.0009 & -90.7883 & 52.4183 \\
\hline 21 & 7.0 & 102.868 & 1.01789 & 99.2240 & 106.512 \\
\hline 22 & 7.1 & $-0.115204 E-02$ & 20.0000 & -71.6012 & 71.5988 \\
\hline 23 & 7.12 & $-1 \cdot 38068$ & 20.0002 & -72.9813 & 70.2199 \\
\hline 24 & 8.0 & 173.956 & 1.22484 & 169.571 & 178.341 \\
\hline 25 & 8.1 & -5.80960 & 20.0137 & -77.4587 & 65.8395 \\
\hline 26 & 8.12 & -57.2549 & 20.0044 & -128.871 & 14.3610 \\
\hline 27 & 9.0 & 190.300 & 2.14651 & 182.615 & 197.985 \\
\hline 28 & 9.1 & $-0.505066 \mathrm{E}-01$ & 20.0000 & -71.6505 & 71.5495 \\
\hline 29 & 9.12 & -49.5115 & 20.0091 & $-121 \cdot 144$ & 22.1211 \\
\hline 30 & 0.0 & 157.041 & 1.21930 & 152.676 & 161.406 \\
\hline 31 & 0.1 & $-0.430298 E-02$ & 20.0000 & -71.6043 & 71.5957 \\
\hline 32 & 0.12 & -26.1288 & 20.0023 & -97.7371 & 45.4795 \\
\hline 33 & SS & -4.86063 & 0.487635 & -6.60636 & $;-3.11490$ \\
\hline 34 & TR3 & -4.71819 & 0.465964 & -6.38634 & $;-3.05004$ \\
\hline 35 & TR12 & -2.86323 & 0.262060 & -3.80140 & $;-1.92506$ \\
\hline
\end{tabular}




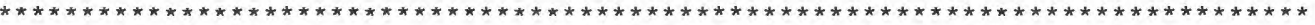

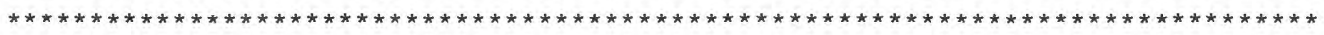

UNDEFINED NUMBER OF SIMULTANEOUS 95\% CONFIDENCE INTERVALS

UNCERTAINTY IS CONSIDERED OVER AN AREA (I.E. AN INFINITE NUMBER OF POINTS)

95\% CONFIDENCE INTERVALS INDICATE THAT THERE IS

95\% PROBABILITY THAT THE ACTUAL VALUE WILL BE

WITHIN THE INDICATED RANGE

CRITICAL VALUE FOR THE INTERVALS $=4.5200$

\begin{tabular}{|c|c|c|c|c|c|}
\hline & OBSERVATION & SIMULATED & & & \\
\hline 0. & NAME & VALUE & STD. DEV. & CONFIDENCE & INTERVAL \\
\hline 1 & 1.0 & 100.225 & 1.00771 & 95.6702 & 104.780 \\
\hline 2 & 1.1 & $-0.228882 E-04$ & 20.0000 & -90.3991 & 90.3991 \\
\hline 3 & 1.12 & $-0.906143 E-01$ & 20.0001 & -90.4901 & 90.3088 \\
\hline 4 & 2.0 & 139.331 & 1.22016 & 133.816 & 144.846 \\
\hline 5 & 2.1 & $-0.947571 \mathrm{E}-02$ & 20.0000 & -90.4086 & 90.3896 \\
\hline 6 & 2.2 & -0.276215 & 20.0000 & -90.6755 & 90.1231 \\
\hline 7 & 2.8 & -12.9634 & 20.0032 & -103.377 & 77.4503 \\
\hline 8 & 2.12 & -18.7714 & 20.0012 & -109.176 & 71.6330 \\
\hline 9 & 3.0 & 174.363 & 1.21891 & 168.854 & 179.872 \\
\hline 10 & 3.1 & -3.66571 & 20.0013 & -94.0706 & 86.7392 \\
\hline 11 & 3.12 & -56.2375 & 20.0060 & -146.664 & 34.1889 \\
\hline 12 & 4.0 & 139.331 & 1.22016 & 133.816 & 144.846 \\
\hline 13 & 4.1 & $-0.162659 E-01$ & 20.0000 & -90.4154 & 90.3828 \\
\hline 14 & 4.12 & -18.8487 & 20.0012 & -109.253 & 71.5557 \\
\hline 15 & 5.0 & 157.132 & 1.24335 & 151.512 & 162.752 \\
\hline 16 & 5.1 & $-0.367889 E-01$ & 20.0000 & -90.4359 & 90.3623 \\
\hline 17 & 5.12 & -28.4615 & 20.0026 & -118.872 & 61.9494 \\
\hline 18 & 6.0 & 139.632 & 1.20428 & 134.189 & 145.075 \\
\hline 19 & 6.1 & $-0.124512 \mathrm{E}-01$ & 20.0000 & -90.4116 & 90.3867 \\
\hline 20 & 6.12 & -19.1850 & 20.0009 & -109.588 & 71.2182 \\
\hline 21 & 7.0 & 102.868 & 1.01789 & 98.2672 & 107.469 \\
\hline 22 & 7.1 & $-0.115204 \mathrm{E}-02$ & 20.0000 & -90.4003 & 90.3980 \\
\hline 23 & 7.12 & -1.38068 & 20.0002 & -91.7805 & 89.0192 \\
\hline 24 & 8.0 & 173.956 & 1.22484 & 168.420 & 179.492 \\
\hline 25 & 8.1 & -5.80960 & 20.0137 & -96.2707 & 84.6515 \\
\hline 26 & 8.12 & -57.2549 & 20.0044 & -147.674 & 33.1643 \\
\hline 27 & 9.0 & 190.300 & 2.14651 & 180.598 & 200.002 \\
\hline 28 & 9.1 & $-0.505066 \mathrm{E}-01$ & 20.0000 & -90.4497 & 90.3487 \\
\hline 29 & 9.12 & -49.5115 & 20.0091 & -139.952 & 40.9288 \\
\hline 30 & 0.0 & 157.041 & 1.21930 & 151.530 & 162.552 \\
\hline 31 & 0.1 & $-0.430298 E-02$ & 20.0000 & -90.4034 & 90.3948 \\
\hline 32 & 0.12 & -26.1288 & 20.0023 & -116.538 & 64.2808 \\
\hline 33 & SS & -4.86063 & 0.487635 & -7.06472 & $;-2.65654$ \\
\hline 34 & TR3 & -4.71819 & 0.465964 & -6.82433 & $;-2.61205$ \\
\hline 35 & TR12 & -2.86323 & 0.262060 & -4.04773 & $;-1.67873$ \\
\hline
\end{tabular}


APPENDIX A. EXAMPLE SIMULATIONS - Test Case 1 - YCINT Output File

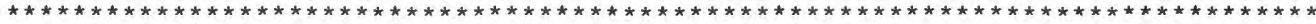

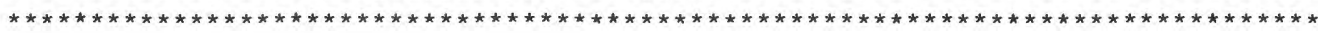

INDIVIDUAL $95 \%$ PREDICTION INTERVALS

UNCERTAINTY ON EACH PREDICTION IS CONSIDERED SEPARATELY

IF SIMULTANEOUS UNCERTAINTY IS DESIRED, GO TO NEXT TABLE

PREDICTION INTERVALS INCLUDE MEASUREMENT ERROR,

I.E. GIVEN THE VARIANCE LISTED IN THE

OBSERVATION INPUT FILES USED TO DEFINE THE

PREDICTIONS, THERE IS A 95\% PROBABILITY

THAT THE MEASUREMENT WILL FALL WITHIN THE

INDICATED RANGE

CRITICAL VALUE FOR THE INTERVALS $=2.0560$

\begin{tabular}{|c|c|c|c|c|c|}
\hline & OBSERVATION & SIMULATED & & & \\
\hline No. ${ }_{1}$ & $1.0^{\text {NAME }}$ & $\begin{array}{l}\text { VALUE } \\
100.225\end{array}$ & $\begin{array}{c}\text { STD. DEV. } \\
1.00771\end{array}$ & $\begin{array}{l}\text { PREDICTION } \\
98.1532\end{array}$ & $\begin{array}{l}\text { J INTERVAL } \\
i \quad 102.297\end{array}$ \\
\hline 2 & 1.1 & $-0.228882 E-04$ & 20.0000 & -41.1200 & 41.1200 \\
\hline 3 & 1.12 & $-0.906143 E-01$ & 20.0001 & -41.2108 & 41.0295 \\
\hline 4 & 2.0 & 139.331 & 1.22016 & 136.822 & 141.840 \\
\hline 5 & 2.1 & $-0.947571 E-02$ & 20.0000 & -41.1295 & 41.1105 \\
\hline 6 & 2.2 & -0.276215 & 20.0000 & -41.3963 & 40.8439 \\
\hline 7 & 2.8 & -12.9634 & 20.0032 & -54.0900 & 28.1632 \\
\hline 8 & 2.12 & -18.7714 & 20.0012 & -59.8938 & 22.3510 \\
\hline 9 & 3.0 & 174.363 & 1.21891 & 171.857 & 176.869 \\
\hline 10 & 3.1 & -3.66571 & 20.0013 & -44.7884 & 37.4569 \\
\hline 11 & 3.12 & -56.2375 & 20.0060 & -97.3699 & $;-15.1051$ \\
\hline 12 & 4.0 & 139.331 & 1.22016 & 136.822 & 141.840 \\
\hline 13 & 4.1 & $-0.162659 \mathrm{E}-01$ & 20.0000 & -41.1363 & 41.1037 \\
\hline 14 & 4.12 & -18.8487 & 20.0012 & -59.9711 & 22.2737 \\
\hline 15 & 5.0 & 157.132 & 1.24335 & 154.576 & 159.688 \\
\hline 16 & 5.1 & $-0.367889 E-01$ & 20.0000 & -41.1568 & 41.0832 \\
\hline 17 & 5.12 & -28.4615 & 20.0026 & -69.5869 & 12.6639 \\
\hline 18 & 6.0 & 139.632 & 1.20428 & 137.156 & 142.108 \\
\hline 19 & 6.1 & $-0.124512 \mathrm{E}-01$ & 20.0000 & -41.1325 & 41.1075 \\
\hline 20 & 6.12 & -19.1850 & 20.0009 & -60.3069 & 21.9369 \\
\hline 21 & 7.0 & 102.868 & 1.01789 & 100.775 & 104.961 \\
\hline 22 & 7.1 & $-0.115204 \mathrm{E}-02$ & 20.0000 & -41.1212 & 41.1188 \\
\hline 23 & 7.12 & -1.38068 & 20.0002 & -42.5010 & 39.7397 \\
\hline 24 & 8.0 & 173.956 & 1.22484 & 171.438 & 176.474 \\
\hline 25 & 8.1 & -5.80960 & 20.0137 & -46.9578 & 35.3386 \\
\hline 26 & 8.12 & -57.2549 & 20.0044 & -98.3840 & $;-16.1258$ \\
\hline 27 & 9.0 & 190.300 & 2.14651 & 185.887 & 194.713 \\
\hline 28 & 9.1 & $-0.505066 \mathrm{E}-01$ & 20.0000 & -41.1705 & 41.0695 \\
\hline 29 & 9.12 & -49.5115 & 20.0091 & -90.6502 & $;-8.37278$ \\
\hline 30 & 0.0 & 157.041 & 1.21930 & 154.534 & 159.548 \\
\hline 31 & 0.1 & $-0.430298 E-02$ & 20.0000 & -41.1243 & 41.1157 \\
\hline 32 & 0.12 & -26.1288 & 20.0023 & -67.2536 & 14.9960 \\
\hline 33 & SS & -4.86063 & 0.487635 & -5.86321 & $;-3.85805$ \\
\hline 34 & TR3 & -4.71819 & 0.465964 & -5.67621 & $;-3.76017$ \\
\hline 35 & TR12 & -2.86323 & 0.262060 & -3.40202 & $;-2.32443$ \\
\hline
\end{tabular}




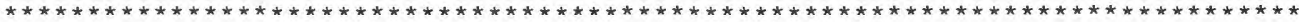

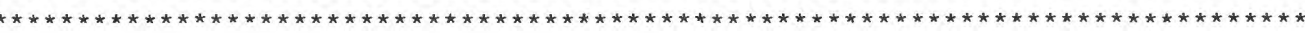

35 SIMULTANEOUS 95\% PREDICTION INTERVALS

UNCERTAINTY ON EACH PREDICTION IS CONSIDERED JOINTLY

IF UNCERTAINTY OVER AN AREA IS DESIRED, GO TO NEXT TABLE

PREDICTION INTERVALS INCLUDE MEASUREMENT ERROR,

I.E. GIVEN THE VARIANCE LISTED IN THE

OBSERVATION INPUT FILES USED TO DEFINE THE

PREDICTIONS, THERE IS A 95\% PROBABILITY

THAT THE MEASUREMENT WILL FALL WITHIN THE

INDICATED RANGE

SCHEFFE PREDICTION INTERVALS ARE USED

CRITICAL VALUE FOR THE INTERVALS $=3.5800$

\begin{tabular}{|c|c|c|c|c|c|}
\hline & OBSERVATION & SIMULATED & & & \\
\hline NO. & NAME & VALUE & STD. DEV. & PREDICTION & J INTERVAL \\
\hline 1 & 1.0 & 100.225 & 1.00771 & 96.6174 & 103.833 \\
\hline 2 & 1.1 & $-0.228882 E-04$ & 20.0000 & -71.6000 & 71.6000 \\
\hline 3 & 1.12 & $-0.906143 \mathrm{E}-01$ & 20.0001 & -71.6909 & 71.5096 \\
\hline 4 & 2.0 & 139.331 & 1.22016 & 134.963 & 143.699 \\
\hline 5 & 2.1 & $-0.947571 \mathrm{E}-02$ & 20.0000 & -71.6095 & 71.5905 \\
\hline 6 & 2.2 & -0.276215 & 20.0000 & -71.8763 & 71.3239 \\
\hline 7 & 2.8 & -12.9634 & 20.0032 & -84.5750 & 58.6482 \\
\hline 8 & 2.12 & -18.7714 & 20.0012 & -90.3756 & 52.8328 \\
\hline 9 & 3.0 & 174.363 & 1.21891 & 169.999 & 178.727 \\
\hline 10 & 3.1 & -3.66571 & 20.0013 & -75.2703 & 67.9389 \\
\hline 11 & 3.12 & -56.2375 & 20.0060 & -127.859 & 15.3841 \\
\hline 12 & 4.0 & 139.331 & 1.22016 & 134.963 & 143.699 \\
\hline 13 & 4.1 & $-0.162659 \mathrm{E}-01$ & 20.0000 & -71.6163 & 71.5837 \\
\hline 14 & 4.12 & -18.8487 & 20.0012 & -90.4529 & 52.7555 \\
\hline 15 & 5.0 & 157.132 & 1.24335 & 152.681 & 161.583 \\
\hline 16 & 5.1 & $-0.367889 \mathrm{E}-01$ & 20.0000 & -71.6368 & 71.5632 \\
\hline 17 & 5.12 & -28.4615 & 20.0026 & -100.071 & 43.1479 \\
\hline 18 & 6.0 & 139.632 & 1.20428 & 135.321 & 143.943 \\
\hline 19 & 6.1 & $-0.124512 \mathrm{E}-01$ & 20.0000 & -71.6124 & 71.5875 \\
\hline 20 & 6.12 & -19.1850 & 20.0009 & -90.7883 & 52.4183 \\
\hline 21 & 7.0 & 102.868 & 1.01789 & 99.2240 & 106.512 \\
\hline 22 & 7.1 & $-0.115204 \mathrm{E}-02$ & 20.0000 & -71.6012 & 71.5988 \\
\hline 23 & 7.12 & -1.38068 & 20.0002 & -72.9813 & 70.2199 \\
\hline 24 & 8.0 & 173.956 & 1.22484 & 169.571 & 178.341 \\
\hline 25 & 8.1 & -5.80960 & 20.0137 & -77.4587 & 65.8395 \\
\hline 26 & 8.12 & -57.2549 & 20.0044 & -128.871 & 14.3610 \\
\hline 27 & 9.0 & 190.300 & 2.14651 & 182.615 & 197.985 \\
\hline 28 & 9.1 & $-0.505066 \mathrm{E}-01$ & 20.0000 & -71.6505 & 71.5495 \\
\hline 29 & 9.12 & -49.5115 & 20.0091 & -121.144 & 22.1211 \\
\hline 30 & 0.0 & 157.041 & 1.21930 & 152.676 & 161.406 \\
\hline 31 & 0.1 & $-0.430298 E-02$ & 20.0000 & -71.6043 & 71.5957 \\
\hline 32 & 0.12 & -26.1288 & 20.0023 & -97.7371 & 45.4795 \\
\hline 33 & SS & -4.86063 & 0.487635 & -6.60636 & $;-3.11490$ \\
\hline 34 & TR3 & -4.71819 & 0.465964 & -6.38634 & $;-3.05004$ \\
\hline 35 & TR12 & -2.86323 & 0.262060 & -3.80140 & $;-1.92506$ \\
\hline
\end{tabular}


APPENDIX A. EXAMPLE SIMULATIONS - Test Case 1 - YCINT Output File

UNDEFINED NUMBER OF SIMULTANEOUS 95\% PREDICTION INTERVALS

UNCERTAINTY IS CONSIDERED OVER AN AREA (I.E. AN INFINITE NUMBER OF POINTS)

PREDICTION INTERVALS INCLUDE MEASUREMENT ERROR,

I.E. GIVEN THE VARIANCE LISTED IN THE

OBSERVATION INPUT FILES USED TO DEFINE THE

PREDICTIONS, THERE IS A 95\% PROBABILITY

THAT THE MEASUREMENT WILL FALL WITHIN THE

INDICATED RANGE

CRITICAL VALUE FOR THE INTERVALS $=4.5200$

\begin{tabular}{|c|c|c|c|c|c|}
\hline & OBSERVATION & SIMULATED & & & \\
\hline NO: & NAME & VALUE & STD. DEV. & PREDICTION & N INTERVAL \\
\hline 1 & 1.0 & 100.225 & 1.00771 & 95.6702 & 104.780 \\
\hline 2 & 1.1 & $-0.228882 E-04$ & 20.0000 & -90.3991 & 90.3991 \\
\hline 3 & 1.12 & $-0.906143 E-01$ & 20.0001 & -90.4901 & 90.3088 \\
\hline 4 & 2.0 & 139.331 & 1.22016 & 133.816 & 144.846 \\
\hline 5 & 2.1 & $-0.947571 E-02$ & 20.0000 & -90.4086 & 90.3896 \\
\hline 6 & 2.2 & -0.276215 & 20.0000 & -90.6755 & 90.1231 \\
\hline 7 & 2.8 & -12.9634 & 20.0032 & -103.377 & 77.4503 \\
\hline 8 & 2.12 & -18.7714 & 20.0012 & -109.176 & 71.6330 \\
\hline 9 & 3.0 & 174.363 & 1.21891 & 168.854 & 179.872 \\
\hline 10 & 3.1 & -3.66571 & 20.0013 & -94.0706 & 86.7392 \\
\hline 11 & 3.12 & -56.2375 & 20.0060 & -146.664 & 34.1889 \\
\hline 12 & 4.0 & 139.331 & 1.22016 & 133.816 & 144.846 \\
\hline 13 & 4.1 & $-0.162659 E-01$ & 20.0000 & -90.4154 & 90.3828 \\
\hline 14 & 4.12 & -18.8487 & 20.0012 & -109.253 & 71.5557 \\
\hline 15 & 5.0 & 157.132 & 1.24335 & 151.512 & 162.752 \\
\hline 16 & 5.1 & $-0.367889 E-01$ & 20.0000 & -90.4359 & 90.3623 \\
\hline 17 & 5.12 & -28.4615 & 20.0026 & -118.872 & 61.9494 \\
\hline 18 & 6.0 & 139.632 & 1.20428 & 134.189 & 145.075 \\
\hline 19 & 6.1 & $-0.124512 E-01$ & 20.0000 & -90.4116 & 90.3867 \\
\hline 20 & 6.12 & -19.1850 & 20.0009 & -109.588 & 71.2182 \\
\hline 21 & 7.0 & 102.868 & 1.01789 & 98.2672 & 107.469 \\
\hline 22 & 7.1 & $-0.115204 E-02$ & 20.0000 & -90.4003 & 90.3980 \\
\hline 23 & 7.12 & -1.38068 & 20.0002 & -91.7805 & 89.0192 \\
\hline 24 & 8.0 & 173.956 & 1.22484 & 168.420 & 179.492 \\
\hline 25 & 8.1 & -5.80960 & 20.0137 & -96.2707 & 84.6515 \\
\hline 26 & 8.12 & -57.2549 & 20.0044 & -147.674 & 33.1643 \\
\hline 27 & 9.0 & 190.300 & 2.14651 & 180.598 & 200.002 \\
\hline 28 & 9.1 & $-0.505066 E-01$ & 20.0000 & -90.4497 & 90.3487 \\
\hline 29 & 9.12 & -49.5115 & 20.0091 & -139.952 & 40.9288 \\
\hline 30 & 0.0 & 157.041 & 1.21930 & 151.530 & 162.552 \\
\hline 31 & 0.1 & $-0.430298 E-02$ & 20.0000 & -90.4034 & 90.3948 \\
\hline 32 & 0.12 & -26.1288 & 20.0023 & -116.538 & 64.2808 \\
\hline 33 & SS & -4.86063 & 0.487635 & -7.06472 & $;-2.65654$ \\
\hline 34 & TR3 & -4.71819 & 0.465964 & -6.82433 & $;-2.61205$ \\
\hline 35 & TR12 & -2.86323 & 0.262060 & -4.04773 & $;-1.67873$ \\
\hline
\end{tabular}




\section{Test Case 2}

The calculations of the Observation, Sensitivity, and Parameter-Estimation Processes were tested using a problem designed to include features relevant to a typical complex threedimensional MODFLOW-2000 model. A synthetic problem was used so that everything is known about the system and parameter values. This approach allows analysis not possible with field data.

The model grid shown in figure A2 has a uniform grid spacing of $1500 \mathrm{~m}$ in the horizontal and has 247 active cells in each of three layers. Layers 1, 2, and 3 have a constant thickness of $500 \mathrm{~m}, 750 \mathrm{~m}$, and $1500 \mathrm{~m}$, respectively. Hydraulic conductivity is divided into four zones, each of which is present in the middle layer and three of which are present in the top and bottom layers (fig. A2). Constant-head boundaries comprise portions of the western and eastern boundaries, with no flow across the remaining boundaries. Head-dependent boundaries representing springs are simulated using both the Drain and General-Head Boundary Packages. Wells are present at selected nodes, with pumpage at rates ranging from 100 to $200 \mathrm{~m}^{3} / \mathrm{d}$. 

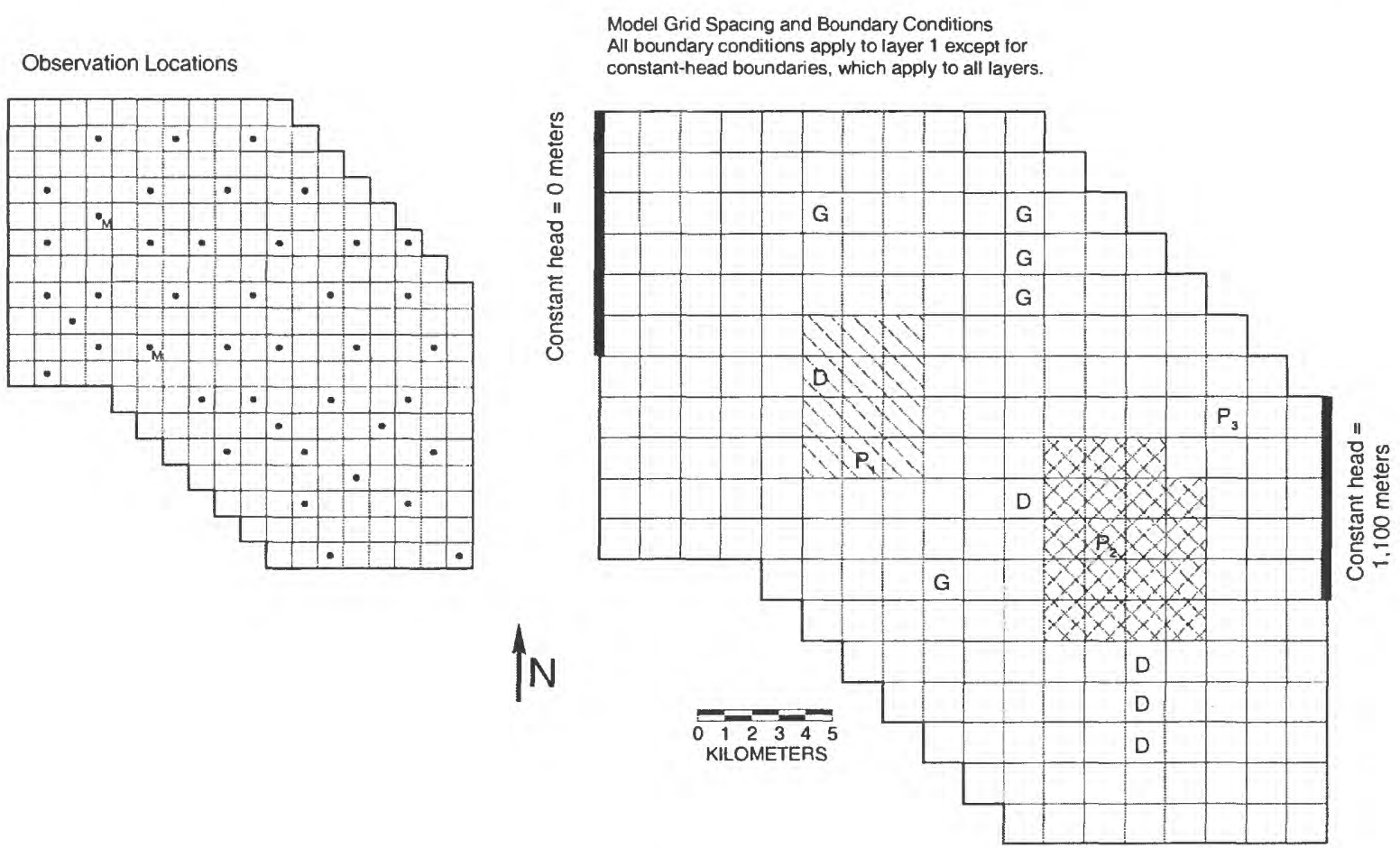

Hydraulic Conductivity Zones

Layer 1

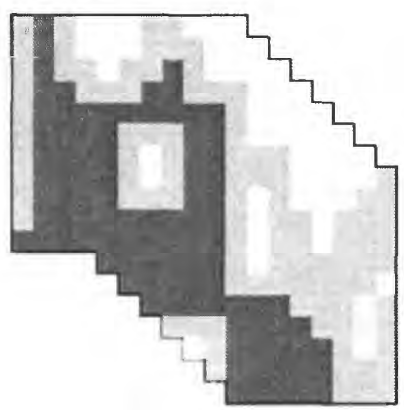

AREAL RECHARGE

EVAPOTRANSPIRATION

GENERAL-HEAD BOUNDARY

DRAIN

PUMPAGE
Layer 2

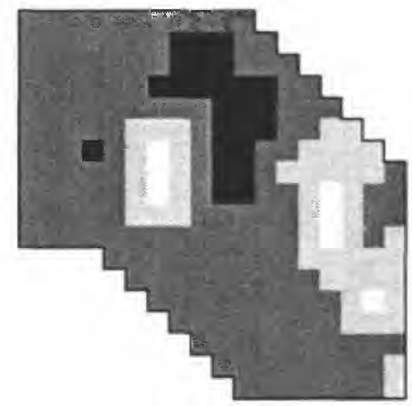

EXPLANATION

- HEAD OBSERVATION

- MUlti-layer head observation
Layer 3

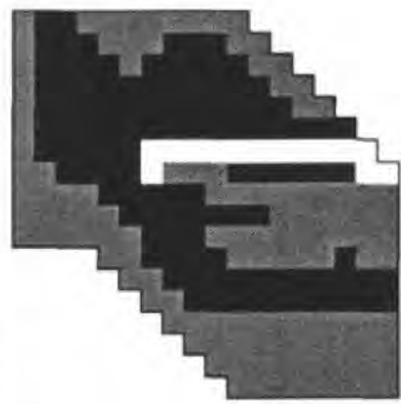

$\square 1$
$\square$
$\mathrm{K} 2$
$\mathrm{~K} 3$
$\mathrm{~K} 4$

Figure A2: Test case 2 model grid, boundary conditions, observation locations and hydraulic conductivity zonation used in parameter estimation (modified from Anderman and Hill, 1997). 
Ten parameters were identified for inclusion in the parameter estimation and are described in table $\mathrm{A} 3$ along with their true (assigned) values. The observations used in the parameter estimation were generated by running the model with the true parameter values. The locations of the 42 "observed" hydraulic heads are shown in figure A2. The flows simulated at the head-dependent boundaries (fig. A2) also were used as observations for the parameter estimation. The model used in the regression exactly matched the model used to generate the values used as observations, the observations had no noise added, and sufficient observations were included to allow estimation of all parameter values. In this ideal situation, the estimated parameter values are expected to match the true parameter values to several significant digits. If this is accomplished, it suggests that the observation sensitivities are calculated correctly and that the regression is performing correctly. This is similar to the test performed for test case 1 , with those results presented in table A2. The results of the present test are shown in table A3. 
Table A3: Labels, descriptions, and estimated values for the parameters for Test Case 2. [m, meter; d, day].

\begin{tabular}{|c|c|c|c|c|c|}
\hline Label & Description & Units & $\begin{array}{l}\text { Starting } \\
\text { Value }\end{array}$ & $\begin{array}{l}\text { Estimated } \\
\text { Value }\end{array}$ & $\begin{array}{l}\text { True } \\
\text { Value }\end{array}$ \\
\hline $\mathrm{HK}_{-} 1$ & $\begin{array}{l}\text { Hydraulic conductivity of zone } 1 \text { (see } \\
\text { figure A2) }\end{array}$ & $\mathrm{m} / \mathrm{d}$ & 1.5 & 1.00 & 1.00 \\
\hline HK_2 & $\begin{array}{l}\text { Hydraulic conductivity of zone } 2 \text { (see } \\
\text { figure } A 2 \text { ) }\end{array}$ & $\mathrm{m} / \mathrm{d}$ & $5.00 \times 10^{-3}$ & $1.00 \times 10^{-2}$ & $1.00 \times 10^{-2}$ \\
\hline HK_3 & $\begin{array}{l}\text { Hydraulic conductivity of zone } 3 \text { (see } \\
\text { figure A2) }\end{array}$ & $\mathrm{m} / \mathrm{d}$ & $1.20 \times 10^{-4}$ & $1.00 \times 10^{-4}$ & $1.00 \times 10^{-4}$ \\
\hline HK_4 & $\begin{array}{l}\text { Hydraulic conductivity of zone } 4 \text { (see } \\
\text { figure } \mathrm{A} 2 \text { ) }\end{array}$ & $\mathrm{m} / \mathrm{d}$ & $2.00 \times 10^{-6}$ & $1.00 \times 10^{-6}$ & $1.00 \times 10^{-6}$ \\
\hline ANIV_12 & Vertical anisotropy of layers 1 and 2 & -- & 1.00 & 4.00 & 4.00 \\
\hline ANIV_3 & Vertical anisotropy of layer 3 & -- & 10.00 & 1.00 & 1.00 \\
\hline RCHRAT & $\begin{array}{l}\text { Areal recharge rate applied to the area } \\
\text { shown in figure A2 }\end{array}$ & $\mathrm{m} / \mathrm{d}$ & $4.40 \times 10^{-4}$ & $3.10 \times 10^{-4}$ & $3.10 \times 10^{-4}$ \\
\hline ETMAX & $\begin{array}{l}\text { Maximum evapotranspiration rate } \\
\text { applied to area shown in figure } \mathrm{A} 2\end{array}$ & $\mathrm{~m} / \mathrm{d}$ & $3.00 \times 10^{-4}$ & $4.00 \times 10^{-4}$ & $4.00 \times 10^{-4}$ \\
\hline C_GHB & $\begin{array}{l}\text { Conductance of head-dependent } \\
\text { boundaries represented using the } \\
\text { General-Head Boundary Package ( } G \text { in } \\
\text { figure A2). }\end{array}$ & $\mathrm{m}^{2} / \mathrm{d}$ & 0.500 & 1.00 & 1.00 \\
\hline C_DRN & $\begin{array}{l}\text { Conductance of the head-dependent } \\
\text { boundaries represented using the Drain } \\
\text { Package (D in figure A2). }\end{array}$ & $\mathrm{m}^{2} / \mathrm{d}$ & 2.00 & 0.999 & 1.00 \\
\hline
\end{tabular}




\section{$\underline{\text { Input Files }}$}

For this test case, input files for the Observation, Sensitivity, and Parameter-Estimation Processes are listed in the NAME file; as a result, MODFLOW-2000 runs in the mode "Parameter Estimation." Observations include measurements of head and measurements of flow to features simulated using the Drain and General Head Boundary Packages. All input files for test case 2 are listed in the file tc2.nam, which is included with the MODFLOW-2000 distribution. Parameters are defined in the input files for the packages to which the parameters apply (Harbaugh and others, 2000).

Head observations are listed in the tc2.ohd file. Flow observations are listed in the tc2.odr file for features simulated using the Drain Package and in the tc2.ogb file for features simulated using the General Head Boundary Package. The Observation Process input files are as follows:

OBS file (tc2.obs):

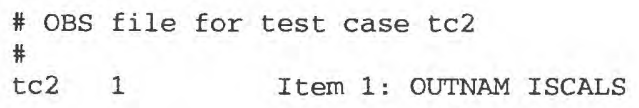

HOB file (tc2.ohd):

\begin{tabular}{|c|c|c|c|c|c|c|c|c|c|c|c|c|}
\hline $\begin{array}{r}42 \\
1.0\end{array}$ & $\begin{array}{ll}2 & 2 \\
2 & 1\end{array}$ & 3 & 0 & & & & & & & & & \\
\hline W2L & & 1 & 2 & 4 & 1 & 0. & 0. & 0. & 979.029 & 5. & 1 & 1 \\
\hline WL2 & & 1 & 2 & 7 & 1 & 0 . & 0 . & 0. & 1015.113 & 5. & 1 & 1 \\
\hline WL2 & & 1 & 2 & 10 & 1 & 0. & 0 . & 0 . & 1186.494 & 5. & 1 & 1 \\
\hline WL4 & & 1 & 4 & 2 & 1 & 0. & 0 . & 0. & 291.694 & 5. & 1 & 1 \\
\hline WL4 & & 1 & 4 & 6 & 1 & 0. & 0 . & 0 . & 964.356 & 5. & 1 & 1 \\
\hline WL4 & & 1 & 4 & 9 & 1 & 0. & 0. & 0. & 1176.542 & 5. & 1 & 1 \\
\hline WL4 & & 1 & 4 & 12 & 1 & 0. & 0 . & 0. & 1192.363 & 5. & 1 & 1 \\
\hline WL5 & & -3 & 5 & 4 & 1 & 0. & 0. & 0. & 760.721 & 5. & 1 & 1 \\
\hline 1 & 0.34 & 2 & 0.33 & 3 & 0.33 & & & & & & & \\
\hline WL 6 & & 1 & 6 & 2 & 1 & 0. & 0. & 0. & 188.804 & 5. & 1 & 1 \\
\hline WL6 & & 1 & 6 & 6 & 1 & 0. & 0. & 0. & 892.570 & 5. & 1 & 1 \\
\hline WL 6 & & 1 & 6 & 8 & 1 & 0 . & 0 . & 0. & 906.942 & 5. & 1 & 1 \\
\hline WL6 & & 1 & 6 & 11 & 1 & 0. & 0 . & 0 . & 1201.148 & 5. & 1 & 1 \\
\hline WL6 & & 1 & 6 & 14 & 1 & 0. & 0 . & 0 . & 1197.885 & 5. & 1 & 1 \\
\hline WL6 & & 1 & 6 & 16 & 1 & 0. & 0. & 0 . & 1198.344 & 5. & 1 & 1 \\
\hline WL8 & & 1 & 8 & 2 & 1 & 0. & 0. & 0. & 209.993 & 5. & 1 & 1 \\
\hline WL8 & & 1 & 8 & 4 & 1 & 0. & 0 . & 0 . & 642.477 & 5. & 1 & 1 \\
\hline WL8 & & 1 & 8 & 7 & 1 & 0. & 0 . & 0 . & 1014.458 & 5. & 1 & 1 \\
\hline WL8 & & 1 & 8 & 10 & 1 & 0. & 0. & 0 . & 1233.051 & 5. & 1 & 1 \\
\hline WL8 & & 1 & 8 & 13 & 1 & 0. & 0. & 0. & 1256.783 & 5. & 1 & 1 \\
\hline WL8 & & 1 & 8 & 16 & 1 & 0 . & 0. & 0. & 1200.920 & 5. & 1 & 1 \\
\hline WL9 & & 1 & 9 & 3 & 1 & 0 . & 0. & 0 . & 444.975 & 5. & 1 & 1 \\
\hline WL10 & & 1 & 10 & 4 & 1 & 0. & 0. & 0 . & 635.430 & 5. & 1 & 1 \\
\hline WL10 & & -3 & 10 & 6 & 1 & 0. & 0. & 0. & 941.034 & 5. & 1 & 1 \\
\hline 1 & 0.34 & 2 & 0.33 & 3 & 0.33 & & & & & & & \\
\hline WL10 & & 1 & 10 & 9 & 1 & 0. & 0. & 0. & 1107.806 & 5. & 1 & 1 \\
\hline WL10 & & 1 & 10 & 11 & 1 & 0. & 0. & 0. & 1395.352 & 5. & 1 & 1 \\
\hline WL10 & & 1 & 10 & 14 & 1 & 0. & 0 . & 0. & 1276.801 & 5. & 1 & 1 \\
\hline WL10 & & 1 & 10 & 17 & 1 & 0 . & 0 . & 0 . & 1159.089 & 5. & 1 & 1 \\
\hline WL11 & & 1 & 11 & 2 & 1 & 0. & 0. & 0. & 336.394 & 5. & 1 & 1 \\
\hline WL12 & & 1 & 12 & 8 & 1 & 0. & 0. & 0. & 1062.879 & 5. & 1 & 1 \\
\hline WL12 & & 1 & 12 & 10 & 1 & 0 . & 0 . & 0. & 1312.105 & 5. & 1 & 1 \\
\hline WL12 & & 1 & 12 & 13 & 1 & 0. & 0. & 0. & 1479.199 & 5. & 1 & 1 \\
\hline WL12 & & 1 & 12 & 16 & 1 & 0 . & 0 . & 0. & 1218.503 & 5. & 1 & 1 \\
\hline WL13 & & 1 & 13 & 11 & 1 & 0. & 0. & 0 . & 1482.972 & 5. & 1 & 1 \\
\hline WL13 & & 1 & 13 & 15 & 1 & 0. & 0. & 0. & 1314.911 & 5. & 1 & 1 \\
\hline WL14 & & 1 & 14 & 9 & 1 & 0. & 0. & 0. & 1225.021 & 5. & 1 & 1 \\
\hline WL14 & & 1 & 14 & 12 & 1 & 0. & 0. & 0. & 1404.986 & 5. & 1 & 1 \\
\hline
\end{tabular}




\begin{tabular}{|c|c|c|c|c|c|c|c|c|c|c|}
\hline WL14 & 1 & 14 & 17 & 1 & 0 . & 0. & 0. & 1193.007 & 5. & 1 \\
\hline WL15 & 1 & 15 & 14 & 1 & 0 . & 0 . & 0 . & 1219.002 & 5. & 1 \\
\hline WL16 & 1 & 16 & 12 & 1 & 0 . & 0. & 0 . & 1262.521 & 5. & 1 \\
\hline WL16 & 1 & 16 & 16 & 1 & 0. & 0. & 0 . & 1197.466 & 5. & 1 \\
\hline WL18 & 1 & 18 & 13 & 1 & 0 . & 0. & 0. & 1234.803 & 5. & 1 \\
\hline WL18 & 1 & 18 & 18 & 1 & 0 . & 0. & 0. & 1194.097 & 5. & 1 \\
\hline
\end{tabular}

\section{DROB file (tc2.odr):}

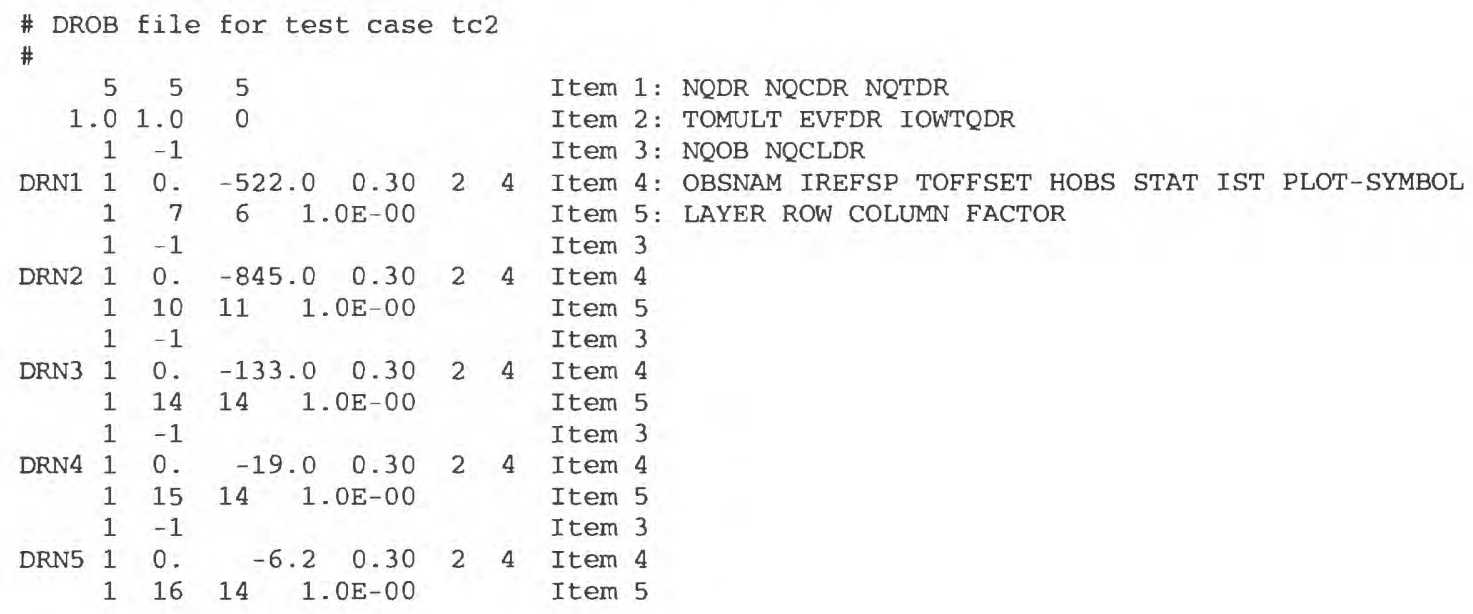

GBOB file (tc2.ogb):

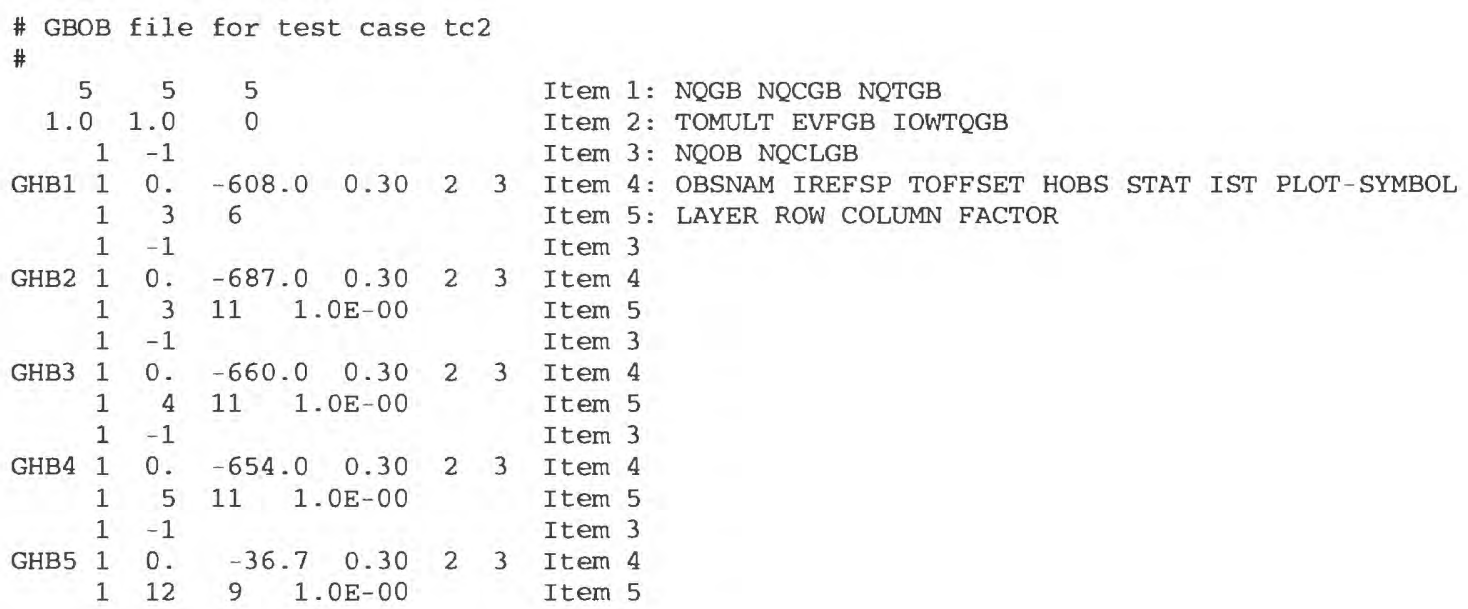

Starting parameter estimates are listed in the Sensitivity Process input file. The Sensitivity Process input file also controls which parameters are to be estimated.

SEN file (tc2.sen):

\# SEN file for test case tc2 $\#$

\begin{tabular}{|c|c|c|c|c|c|c|}
\hline 10 & 0 & -4 & 10 & & & \\
\hline 0 & 0 & & 0 & & & \\
\hline HK_1 & 1 & 0 & 1.50 & -1.4 & -0.8 & $1.0 \mathrm{E}-3$ \\
\hline HK_2 & 1 & 0 & $0.5 E-2$ & 2. $0 \mathrm{E}-9$ & 2. $0 \mathrm{E}-7$ & 1. $0 \mathrm{E}-5$ \\
\hline $\mathrm{HK}_{3}$ & 1 & 0 & 1. $2 \mathrm{E}-4$ & 1. $0 \mathrm{E}-9$ & 1. $0 \mathrm{E}-7$ & 1. $0 \mathrm{E}-7$ \\
\hline HK_4 & 1 & 0 & $2.0 \mathrm{E}-6$ & 1. $2 \mathrm{E}-4$ & 1. $2 \mathrm{E}-2$ & $1.0 \mathrm{E}-9$ \\
\hline ANIV_12 & 1 & 0 & 1.0 & 1. $3 E-4$ & 1. $3 E-2$ & $1.0 \mathrm{E}-3$ \\
\hline ANIV_3 & 1 & 0 & 10.0 & $3.0 \mathrm{E}-5$ & $3.0 E-3$ & 1. $.0 \mathrm{E}-2$ \\
\hline RCHRAT & 1 & 0 & $4.4 E-4$ & 4. $0 \mathrm{E}-6$ & $4.0 \mathrm{E}-4$ & 1. $0 \mathrm{E}-7$ \\
\hline
\end{tabular}

ITEM 1: NPLIST ISENALL IUHEAD MXSEN ITEM 2: IPRINTS ISENSU ISENPU ISENFM ITEM 3: PARNAM ISENS LN B BL BU BSCAL 
ETMAX 1 O $3.0 \mathrm{E}-4$ 4.0E-6 4.0E-4 $1.0 \mathrm{E}-7$

$\begin{array}{lllllll}\text { C_GHB } \quad 1 & 0 & 0.5 & 2.0 \mathrm{E}-5 & 2.0 \mathrm{E}-3 & 1.0 \mathrm{E}-4\end{array}$

C_DRN $10 \quad 2.0 \quad 1.0 E-8 \quad 1.0 E-6 \quad 1.0 E-3$

Parameter estimation is controlled by the Parameter-Estimation Process input file.

PES file (tc2.pes):

\# PES file for test case tc2

$\begin{array}{lllll}30 & 2.0 & 0.01 & 0.0\end{array}$

ITEM 1: MAX-ITER MAX-CHANGE TOL SOSC

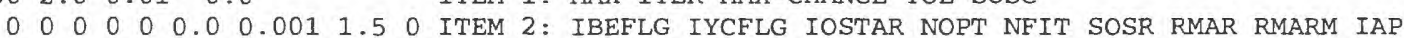

800

0.080 .00

$\begin{array}{lll}0 & 0 & 0\end{array}$

ITEM 3: IPRCOV IPRINT LPRINT

ITEM 4: CSA FCONV LASTX

ITEM 5: NPNG IPR MPR 


\section{GLOBAL Output File}

The GLOBAL and LIST output files for test case 2 are listed on the following pages. The GLOBAL file, tc2.glo, follows:

MODFLOW-2000

U.S. GEOLOGICAL SURVEY MODULAR FINITE-DIFFERENCE GROUND-WATER FLOW MODEL VERSION $1.0 \quad 06 / 13 / 2000$

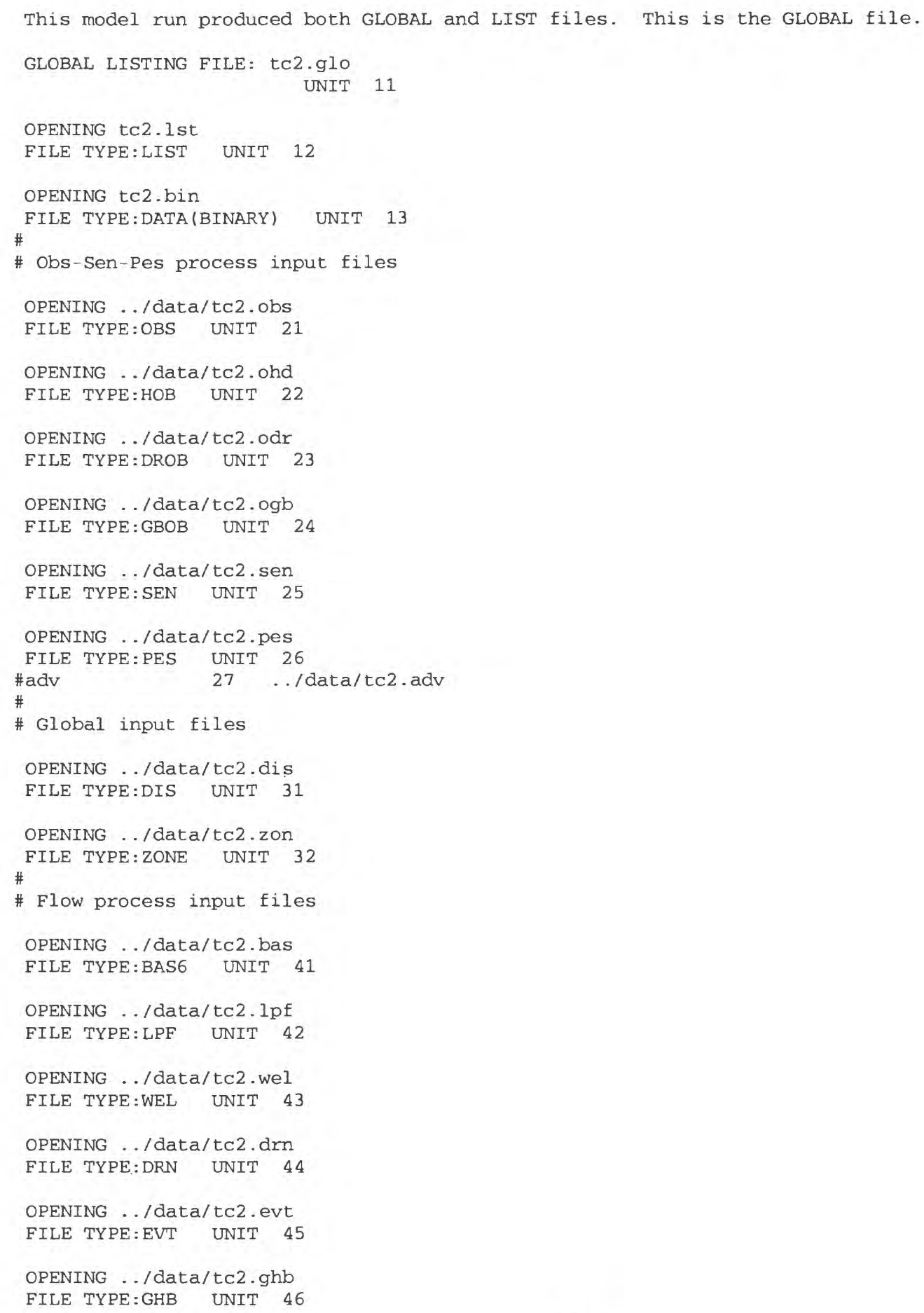




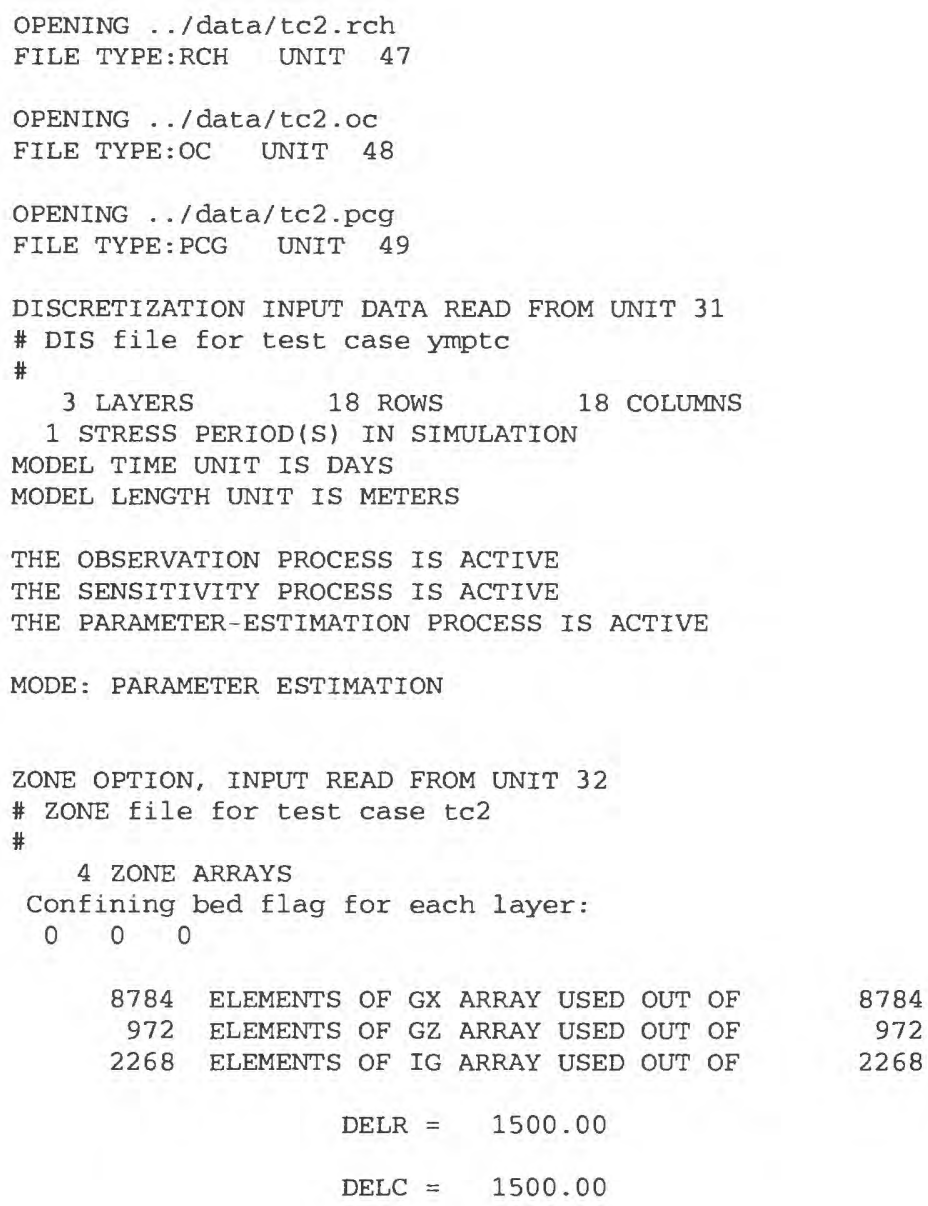

\begin{tabular}{|c|c|c|c|c|c|}
\hline & 1 & 2 & 3 & 4 & 5 \\
\hline & 6 & 7 & 8 & 9 & 10 \\
\hline & 11 & 12 & 13 & 14 & 15 \\
\hline & 16 & 17 & 18 & & \\
\hline$i$ & 0.0000 & 466.66 & 970.89 & 979.17 & 979.48 \\
\hline & 980.07 & 1025.0 & 1123.7 & 1184.3 & 1185.8 \\
\hline & 1186.5 & 9999.0 & 9999.0 & 9999.0 & 9999.0 \\
\hline & 9999.0 & 9999.0 & 9999.0 & & \\
\hline 2 & 0.0000 & 460.53 & 968.83 & 979.02 & 979.21 \\
\hline & 979.77 & 1015.1 & 1103.0 & 1170.6 & 1186.5 \\
\hline & 1187.3 & 1188.7 & 9999.0 & 9999.0 & 9999.0 \\
\hline & 9999.0 & 9999.0 & 9999.0 & & \\
\hline 3 & 0.0000 & 432.95 & 961.24 & 973.60 & 978.55 \\
\hline & 957.74 & 987.47 & 1088.8 & 1179.7 & 1186.8 \\
\hline & 1187.4 & 1190.1 & 1191.8 & 9999.0 & 9999.0 \\
\hline & 9999.0 & 9999.0 & 9999.0 & & \\
\hline 4 & 0.0000 & 291.69 & 752.49 & 967.22 & 971.47 \\
\hline & 964.35 & 990.43 & 1082.6 & 1176.5 & 1177.2 \\
\hline & 1159.7 & 1192.4 & 1193.5 & 1194.9 & 9999.0 \\
\hline & 9999.0 & 9999.0 & 9999.0 & & \\
\hline 5 & 0.0000 & 220.86 & 552.04 & 799.15 & 897.53 \\
\hline & 929.42 & 956.07 & 983.73 & 1077.6 & 1147.7 \\
\hline & 1154.3 & 1194.2 & 1195.1 & 1196.3 & 1197.3 \\
\hline & 9999.0 & 9999.0 & 9999.0 & & \\
\hline 6 & 0.0000 & 188.80 & 463.00 & 692.59 & 852.09 \\
\hline
\end{tabular}


APPENDIX A. EXAMPLE SIMULATIONS - Test Case 2 - GLOBAL Output File

\begin{tabular}{|c|c|c|c|c|c|}
\hline & 892.57 & 932.76 & 906.94 & 1007.6 & 1147.7 \\
\hline & 1201.2 & 1195.8 & 1196.4 & 1197.9 & 1198.3 \\
\hline & 1198.3 & 9999.0 & 9999.0 & & \\
\hline \multirow[t]{4}{*}{7} & 27.650 & 189.71 & 420.51 & 653.17 & 857.06 \\
\hline & 922.11 & 1014.7 & 951.16 & 1023.8 & 1184.0 \\
\hline & 1259.7 & 1242.4 & 1215.4 & 1200.6 & 1200.0 \\
\hline & 1198.8 & 1197.3 & 9999.0 & & \\
\hline \multirow[t]{4}{*}{8} & 50.330 & 209.99 & 431.34 & 642.47 & 850.77 \\
\hline & 944.38 & 1014.5 & 953.31 & 1036.8 & 1233.1 \\
\hline & 1337.1 & 1346.4 & 1256.8 & 1205.1 & 1203.7 \\
\hline & 1200.9 & 1197.3 & 1100.0 & & \\
\hline \multirow[t]{4}{*}{9} & 67.180 & 233.93 & 444.97 & 634.74 & 835.28 \\
\hline & 925.80 & 971.05 & 931.50 & 1049.6 & 1275.6 \\
\hline & 1407.2 & 1449.9 & 1356.6 & 1209.9 & 1209.1 \\
\hline & 1204.7 & 1176.9 & 1100.0 & & \\
\hline \multirow[t]{4}{*}{10} & 77.440 & 262.59 & 462.38 & 635.42 & 812.44 \\
\hline & 951.31 & 990.28 & 999.73 & 1107.8 & 1286.3 \\
\hline & 1395.3 & 1453.3 & 1424.8 & 1276.8 & 1214.3 \\
\hline & 1202.2 & 1159.1 & 1100.0 & & \\
\hline \multirow[t]{4}{*}{11} & 207.65 & 336.39 & 484.48 & 640.95 & 809.63 \\
\hline & 926.59 & 996.19 & 1045.8 & 1129.6 & 1312.3 \\
\hline & 1441.1 & 1457.0 & 1448.0 & 1315.5 & 1217.3 \\
\hline & 1204.8 & 1157.2 & 1100.0 & & \\
\hline \multirow[t]{4}{*}{12} & 9999.0 & 9999.0 & 9999.0 & 9999.0 & 871.62 \\
\hline & 949.88 & 1018.2 & 1062.9 & 1036.7 & 1312.1 \\
\hline & 1459.7 & 1459.8 & 1479.2 & 1376.0 & 1284.8 \\
\hline & 1218.5 & 1164.7 & 1100.0 & & \\
\hline \multirow[t]{4}{*}{13} & 9999.0 & 9999.0 & 9999.0 & 9999.0 & 9999.0 \\
\hline & 1000.4 & 1063.1 & 1123.8 & 1185.0 & 1336.6 \\
\hline & 1483.0 & 1513.5 & 1515.4 & 1419.2 & 1314.9 \\
\hline & 1228.8 & 1182.0 & 1153.7 & & \\
\hline \multirow[t]{4}{*}{14} & 9999.0 & 9999.0 & 9999.0 & 9999.0 & 9999.0 \\
\hline & 9999.0 & 1117.5 & 1183.2 & 1225.0 & 1283.5 \\
\hline & 1375.4 & 1405.0 & 1388.1 & 1333.3 & 1276.1 \\
\hline & 1215.9 & 1193.0 & 1177.7 & & \\
\hline \multirow[t]{4}{*}{15} & 9999.0 & 9999.0 & 9999.0 & 9999.0 & 9999.0 \\
\hline & 9999.0 & 9999.0 & 1239.2 & 1241.1 & 1242.5 \\
\hline & 1282.9 & 1303.6 & 1286.9 & 1219.0 & 1240.7 \\
\hline & 1206.7 & 1193.3 & 1188.8 & & \\
\hline \multirow[t]{4}{*}{16} & 9999.0 & 9999.0 & 9999.0 & 9999.0 & 9999.0 \\
\hline & 9999.0 & 9999.0 & 9999.0 & 1241.6 & 1242.1 \\
\hline & 1255.6 & 1262.5 & 1249.1 & 1206.2 & 1216.2 \\
\hline & 1197.5 & 1193.3 & 1192.3 & & \\
\hline \multirow[t]{4}{*}{17} & 9999.0 & 9999.0 & 9999.0 & 9999.0 & 9999.0 \\
\hline & 9999.0 & 9999.0 & 9999.0 & 9999.0 & 1242.2 \\
\hline & 1246.7 & 1247.3 & 1238.5 & 1221.5 & 1209.4 \\
\hline & 1195.8 & 1194.2 & 1193.7 & & \\
\hline \multirow{4}{*}{18} & 9999.0 & 9999.0 & 9999.0 & 9999.0 & 9999.0 \\
\hline & 9999.0 & 9999.0 & 9999.0 & 9999.0 & 9999.0 \\
\hline & 1244.5 & 1242.2 & 1234.8 & 1222.8 & 1208.1 \\
\hline & 1195.4 & 1194.6 & 1194.1 & & \\
\hline
\end{tabular}

MODEL LAYER BOTTOM EL. FOR LAYER 1

READING ON UNIT 31 WITH FORMAT: (18F10.2)

\begin{tabular}{|c|c|c|c|c|c|}
\hline & 1 & 2 & 3 & 4 & 5 \\
\hline & 6 & 7 & 8 & 9 & 10 \\
\hline & 11 & 12 & 13 & 14 & 15 \\
\hline & 16 & 17 & 18 & & \\
\hline 1 & -500.00 & -33.340 & 470.89 & 479.17 & 479.48 \\
\hline & 480.07 & 525.00 & 623.69 & 684.28 & 685.76 \\
\hline & 686.51 & 9999.0 & 9999.0 & 9999.0 & 9999.0 \\
\hline & 9999.0 & 9999.0 & 9999.0 & & \\
\hline 2 & -500.00 & -39.470 & 468.83 & 479.02 & 479.21 \\
\hline & 479.77 & 515.11 & 603.04 & 670.61 & 686.49 \\
\hline & 687.26 & 688.65 & 9999.0 & 9999.0 & 9999.0 \\
\hline & 9999.0 & 9999.0 & 9999.0 & & \\
\hline 3 & -500.00 & -67.050 & 461.24 & 473.60 & 478.55 \\
\hline
\end{tabular}


APPENDIX A. EXAMPLE SIMƯLATIONS - Test Case 2 - GLOBAL Output File

\begin{tabular}{|c|c|c|c|c|c|}
\hline & 457.74 & 487.47 & 588.84 & 679.69 & 686.78 \\
\hline & 687.39 & 690.05 & 691.79 & 9999.0 & 9999.0 \\
\hline & 9999.0 & 9999.0 & 9999.0 & & \\
\hline \multirow[t]{4}{*}{4} & -500.00 & -208.31 & 252.49 & 467.22 & 471.47 \\
\hline & 464.35 & 490.43 & 582.56 & 676.54 & 677.24 \\
\hline & 659.66 & 692.36 & 693.54 & 694.92 & 9999.0 \\
\hline & 9999.0 & 9999.0 & 9999.0 & & \\
\hline \multirow[t]{4}{*}{5} & -500.00 & -279.14 & 52.040 & 299.15 & 397.53 \\
\hline & 429.42 & 456.07 & 483.73 & 577.55 & 647.71 \\
\hline & 654.33 & 694.15 & 695.09 & 696.29 & 697.29 \\
\hline & 9999.0 & 9999.0 & 9999.0 & & \\
\hline \multirow[t]{4}{*}{6} & -500.00 & -311.20 & -37.000 & 192.59 & 352.09 \\
\hline & 392.57 & 432.76 & 406.94 & 507.63 & 647.73 \\
\hline & 701.15 & 695.77 & 696.37 & 697.88 & 698.28 \\
\hline & 698.34 & 9999.0 & 9999.0 & & \\
\hline \multirow[t]{4}{*}{7} & -472.35 & -310.29 & -79.490 & 153.17 & 357.06 \\
\hline & 422.11 & 514.73 & 451.16 & 523.76 & 683.96 \\
\hline & 759.68 & 742.39 & 715.40 & 700.60 & 700.03 \\
\hline & 698.83 & 697.33 & 9999.0 & & \\
\hline \multirow[t]{4}{*}{8} & -449.67 & -290.01 & -68.660 & 142.47 & 350.77 \\
\hline & 444.38 & 514.46 & 453.31 & 536.80 & 733.05 \\
\hline & 837.05 & 846.38 & 756.78 & 705.05 & 703.72 \\
\hline & 700.92 & 697.30 & 600.00 & & \\
\hline \multirow[t]{4}{*}{9} & -432.82 & -266.07 & -55.030 & 134.74 & 335.28 \\
\hline & 425.80 & 471.05 & 431.50 & 549.61 & 775.58 \\
\hline & 907.16 & 949.87 & 856.59 & 709.95 & 709.11 \\
\hline & 704.70 & 676.94 & 600.00 & & \\
\hline \multirow[t]{4}{*}{10} & -422.56 & -237.41 & -37.620 & 135.42 & 312.44 \\
\hline & 451.31 & 490.28 & 499.73 & 607.81 & 786.30 \\
\hline & 895.35 & 953.25 & 924.78 & 776.80 & 714.27 \\
\hline & 702.18 & 659.09 & 600.00 & & \\
\hline \multirow[t]{4}{*}{11} & -292.35 & -163.61 & -15.520 & 140.95 & 309.63 \\
\hline & 426.59 & 496.19 & 545.80 & 629.56 & 812.27 \\
\hline & 941.08 & 956.96 & 947.99 & 815.52 & 717.30 \\
\hline & 704.81 & 657.15 & 600.00 & & \\
\hline \multirow[t]{4}{*}{12} & 9999.0 & 9999.0 & 9999.0 & 9999.0 & 371.62 \\
\hline & 449.88 & 518.16 & 562.88 & 536.73 & 812.10 \\
\hline & 959.70 & 959.79 & 979.20 & 875.99 & 784.80 \\
\hline & 718.50 & 664.71 & 600.00 & & \\
\hline \multirow[t]{4}{*}{13} & 9999.0 & 9999.0 & 9999.0 & 9999.0 & 9999.0 \\
\hline & 500.38 & 563.05 & 623.83 & 684.97 & 836.58 \\
\hline & 982.97 & 1013.5 & 1015.4 & 919.18 & 814.91 \\
\hline & 728.81 & 681.96 & 653.66 & & \\
\hline \multirow[t]{4}{*}{14} & 9999.0 & 9999.0 & 9999.0 & 9999.0 & 9999.0 \\
\hline & 9999.0 & 617.51 & 683.17 & 725.02 & 783.48 \\
\hline & 875.39 & 904.99 & 888.08 & 833.35 & 776.05 \\
\hline & 715.86 & 693.01 & 677.67 & & \\
\hline \multirow[t]{4}{*}{15} & 9999.0 & 9999.0 & 9999.0 & 9999.0 & 9999.0 \\
\hline & 9999.0 & 9999.0 & 739.21 & 741.07 & 742.52 \\
\hline & 782.86 & 803.60 & 786.91 & 719.00 & 740.73 \\
\hline & 706.68 & 693.28 & 688.76 & & \\
\hline \multirow[t]{4}{*}{16} & 9999.0 & 9999.0 & 9999.0 & 9999.0 & 9999.0 \\
\hline & 9999.0 & 9999.0 & 9999.0 & 741.55 & 742.06 \\
\hline & 755.55 & 762.52 & 749.10 & 706.20 & 716.15 \\
\hline & 697.47 & 693.35 & 692.28 & & \\
\hline \multirow[t]{4}{*}{17} & 9999.0 & 9999.0 & 9999.0 & 9999.0 & 9999.0 \\
\hline & 9999.0 & 9999.0 & 9999.0 & 9999.0 & 742.22 \\
\hline & 746.68 & 747.25 & 738.52 & 721.48 & 709.43 \\
\hline & 695.85 & 694.18 & 693.66 & & \\
\hline \multirow[t]{4}{*}{18} & 9999.0 & 9999.0 & 9999.0 & 9999.0 & 9999.0 \\
\hline & 9999.0 & 9999.0 & 9999.0 & 9999.0 & 9999.0 \\
\hline & 744.51 & 742.16 & 734.80 & 722.75 & 708.12 \\
\hline & 695.45 & 694.60 & 694.10 & & \\
\hline
\end{tabular}

MODEL LAYER BOTTOM EL. FOR LAYER 2

READING ON UNIT 31 WITH FORMAT: (18F10.2)

$\begin{array}{rrrrr}1 & 2 & 3 & 4 & 5 \\ 6 & 7 & 8 & 9 & 10\end{array}$


APPENDIX A. EXAMPLE SIMULATIONS - Test Case 2 - GLOBAL Output File

\begin{tabular}{|c|c|c|c|c|c|}
\hline & 11 & 12 & 13 & \multirow[t]{2}{*}{14} & \multirow[t]{2}{*}{15} \\
\hline & 16 & 17 & 18 & & \\
\hline \multirow[t]{4}{*}{1} & -1250.0 & -783.34 & -279.11 & -270.83 & -270.52 \\
\hline & -269.93 & -225.00 & -126.31 & -65.720 & -64.240 \\
\hline & -63.490 & 9999.0 & 9999.0 & 9999.0 & 9999.0 \\
\hline & 9999.0 & 9999.0 & 9999.0 & & \\
\hline \multirow[t]{4}{*}{2} & -1250.0 & -789.47 & -281.17 & -270.98 & -270.79 \\
\hline & -270.23 & -234.89 & -146.96 & -79.390 & -63.510 \\
\hline & -62.740 & -61.350 & 9999.0 & 9999.0 & 9999.0 \\
\hline & 9999.0 & 9999.0 & 9999.0 & & \\
\hline \multirow[t]{4}{*}{3} & -1250.0 & -817.05 & -288.76 & -276.40 & -271.45 \\
\hline & -292.26 & -262.53 & -161.16 & -70.310 & -63.220 \\
\hline & -62.610 & -59.950 & -58.210 & 9999.0 & 9999.0 \\
\hline & 9999.0 & 9999.0 & 9999.0 & & \\
\hline \multirow[t]{4}{*}{4} & -1250.0 & -958.31 & -497.51 & -282.78 & -278.53 \\
\hline & -285.65 & -259.57 & -167.44 & -73.460 & -72.760 \\
\hline & -90.340 & -57.640 & -56.460 & -55.080 & 9999.0 \\
\hline & 9999.0 & 9999.0 & 9999.0 & & \\
\hline \multirow[t]{4}{*}{5} & -1250.0 & -1029.1 & -697.96 & -450.85 & -352.47 \\
\hline & -320.58 & -293.93 & -266.27 & -172.45 & -102.29 \\
\hline & -95.670 & -55.850 & -54.910 & -53.710 & -52.710 \\
\hline & 9999.0 & 9999.0 & 9999.0 & & \\
\hline \multirow[t]{4}{*}{6} & -1250.0 & -1061.2 & -787.00 & -557.41 & -397.91 \\
\hline & -357.43 & -317.24 & -343.06 & -242.37 & -102.27 \\
\hline & -48.850 & -54.230 & -53.630 & -52.120 & -51.720 \\
\hline & -51.660 & 9999.0 & 9999.0 & & \\
\hline \multirow[t]{4}{*}{7} & -1222.3 & -1060.3 & -829.49 & -596.83 & -392.94 \\
\hline & -327.89 & -235.27 & -298.84 & -226.24 & -66.040 \\
\hline & 9.6800 & -7.6100 & -34.600 & -49.400 & -49.970 \\
\hline & -51.170 & -52.670 & 9999.0 & & \\
\hline \multirow[t]{4}{*}{8} & -1199.7 & -1040.0 & -818.66 & -607.53 & -399.23 \\
\hline & -305.62 & -235.54 & -296.69 & -213.20 & -16.950 \\
\hline & 87.050 & 96.380 & 6.7800 & -44.950 & -46.280 \\
\hline & -49.080 & -52.700 & -150.00 & & \\
\hline 9 & -1182.8 & -1016.1 & -805.03 & -615.26 & -414.72 \\
\hline & -324.20 & -278.95 & -318.50 & -200.39 & 25.580 \\
\hline & 157.16 & 199.87 & 106.59 & -40.050 & -40.890 \\
\hline & -45.300 & -73.060 & -150.00 & & \\
\hline 10 & -1172.6 & -987.41 & -787.62 & -614.58 & -437.56 \\
\hline & -298.69 & -259.72 & -250.27 & -142.19 & 36.300 \\
\hline & 145.35 & 203.25 & 174.78 & 26.800 & -35.730 \\
\hline & -47.820 & -90.910 & -150.00 & & \\
\hline 11 & -1042.3 & -913.61 & -765.52 & -609.05 & -440.37 \\
\hline & -323.41 & -253.81 & -204.20 & -120.44 & 62.270 \\
\hline & 191.08 & 206.96 & 197.99 & 65.520 & -32.700 \\
\hline & -45.190 & -92.850 & -150.00 & & \\
\hline 12 & 9999.0 & 9999.0 & 9999.0 & 9999.0 & -378.38 \\
\hline & -300.12 & -231.84 & -187.12 & -213.27 & 62.100 \\
\hline & 209.70 & 209.79 & 229.20 & 125.99 & 34.800 \\
\hline & -31.500 & -85.290 & -150.00 & & \\
\hline 13 & 9999.0 & 9999.0 & 9999.0 & 9999.0 & 9999.0 \\
\hline & -249.62 & -186.95 & -126.17 & -65.030 & 86.580 \\
\hline & 232.97 & 263.53 & 265.39 & 169.18 & 64.910 \\
\hline & -21.190 & -68.040 & -96.340 & & \\
\hline 14 & 9999.0 & 9999.0 & 9999.0 & 9999.0 & 9999.0 \\
\hline & 9999.0 & -132.49 & -66.830 & -24.980 & 33.480 \\
\hline & 125.39 & 154.99 & 138.08 & 83.350 & 26.050 \\
\hline & -34.140 & -56.990 & -72.330 & & \\
\hline 15 & 9999.0 & 9999.0 & 9999.0 & 9999.0 & 9999.0 \\
\hline & 9999.0 & 9999.0 & -10.790 & -8.9300 & -7.4800 \\
\hline & 32.860 & 53.600 & 36.910 & -31.000 & -9.2700 \\
\hline & -43.320 & -56.720 & -61.240 & & \\
\hline 16 & 9999.0 & 9999.0 & 9999.0 & 9999.0 & 9999.0 \\
\hline & 9999.0 & 9999.0 & 9999.0 & -8.4500 & -7.9400 \\
\hline & 5.5500 & 12.520 & -0.90000 & -43.800 & -33.850 \\
\hline & -52.530 & -56.650 & -57.720 & & \\
\hline 17 & 9999.0 & 9999.0 & 9999.0 & 9999.0 & 9999.0 \\
\hline & 9999.0 & 9999.0 & 9999.0 & 9999.0 & -7.7800 \\
\hline & -3.3200 & -2.7500 & -11.480 & -28.520 & -40.570 \\
\hline & -54.150 & -55.820 & -56.340 & & \\
\hline
\end{tabular}


APPENDIX A. EXAMPLE SIMULATIONS - Test Case 2 - GLOBAL Output File

18

$\begin{array}{rr}9999.0 & 9999.0 \\ 9999.0 & 9999.0 \\ -5.4900 & -7.8400 \\ -54.550 & -55.400\end{array}$

\section{0}

9999.0

$-15.200$

$-55.900$

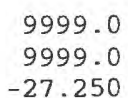

$-27.250$
9999.0

9999.0

41.880

MODEL LAYER BOTTOM EL. FOR LAYER 3

READING ON UNIT 31 WITH FORMAT: (18F10.2)

\begin{tabular}{|c|c|c|c|c|c|}
\hline & 1 & 2 & 3 & 4 & 5 \\
\hline & 6 & 7 & 8 & 9 & 10 \\
\hline & 11 & 12 & 13 & 14 & 15 \\
\hline & 16 & 17 & 18 & & \\
\hline 1 & -2750.0 & -2283.3 & -1779.1 & -1770.8 & -1770.5 \\
\hline & -1769.9 & -1725.0 & -1626.3 & -1565.7 & -1564.2 \\
\hline & -1563.5 & 9999.0 & 9999.0 & 9999.0 & 9999.0 \\
\hline & 9999.0 & 9999.0 & 9999.0 & & \\
\hline 2 & -2750.0 & -2289.5 & -1781.2 & -1771.0 & -1770.8 \\
\hline & -1770.2 & -1734.9 & -1647.0 & -1579.4 & -1563.5 \\
\hline & -1562.7 & -1561.3 & 9999.0 & 9999.0 & 9999.0 \\
\hline & 9999.0 & 9999.0 & 9999.0 & & \\
\hline 3 & -2750.0 & -2317.1 & -1788.8 & -1776.4 & -1771.4 \\
\hline & -1792.3 & -1762.5 & -1661.2 & -1570.3 & -1563.2 \\
\hline & -1562.6 & -1559.9 & -1558.2 & 9999.0 & 9999.0 \\
\hline & 9999.0 & 9999.0 & 9999.0 & & \\
\hline 4 & -2750.0 & -2458.3 & -1997.5 & -1782.8 & -1778.5 \\
\hline & -1785.7 & -1759.6 & -1667.4 & -1573.5 & -1572.8 \\
\hline & -1590.3 & -1557.6 & -1556.5 & -1555.1 & 9999.0 \\
\hline & 9999.0 & 9999.0 & 9999.0 & & \\
\hline 5 & -2750.0 & -2529.1 & -2198.0 & -1950.8 & -1852.5 \\
\hline & -1820.6 & -1793.9 & -1766.3 & -1672.4 & -1602.3 \\
\hline & -1595.7 & -1555.8 & -1554.9 & -1553.7 & -1552.7 \\
\hline & 9999.0 & 9999.0 & 9999.0 & & \\
\hline 6 & -2750.0 & -2561.2 & -2287.0 & -2057.4 & -1897.9 \\
\hline & -1857.4 & -1817.2 & -1843.1 & -1742.4 & -1602.3 \\
\hline & -1548.8 & -1554.2 & -1553.6 & -1552.1 & -1551.7 \\
\hline & -1551.7 & 9999.0 & 9999.0 & & \\
\hline 7 & -2722.4 & -2560.3 & -2329.5 & -2096.8 & -1892.9 \\
\hline & -1827.9 & -1735.3 & -1798.8 & -1726.2 & -1566.0 \\
\hline & -1490.3 & -1507.6 & -1534.6 & -1549.4 & -1550.0 \\
\hline & -1551.2 & -1552.7 & 9999.0 & & \\
\hline 8 & -2699.7 & -2540.0 & -2318.7 & -2107.5 & -1899.2 \\
\hline & -1805.6 & -1735.5 & -1796.7 & -1713.2 & -1516.9 \\
\hline & -1412.9 & -1403.6 & -1493.2 & -1544.9 & -1546.3 \\
\hline & -1549.1 & -1552.7 & -1650.0 & & \\
\hline 9 & -2682.8 & -2516.1 & -2305.0 & -2115.3 & -1914.7 \\
\hline & -1824.2 & -1778.9 & -1818.5 & -1700.4 & -1474.4 \\
\hline & -1342.8 & -1300.1 & -1393.4 & -1540.1 & -1540.9 \\
\hline & -1545.3 & -1573.1 & -1650.0 & & \\
\hline 10 & -2672.6 & -2487.4 & -2287.6 & -2114.6 & -1937.6 \\
\hline & -1798.7 & -1759.7 & -1750.3 & -1642.2 & -1463.7 \\
\hline & -1354.7 & -1296.8 & -1325.2 & -1473.2 & -1535.7 \\
\hline & -1547.8 & -1590.9 & -1650.0 & & \\
\hline 11 & -2542.4 & -2413.6 & -2265.5 & -2109.1 & -1940.4 \\
\hline & -1823.4 & -1753.8 & -1704.2 & -1620.4 & -1437.7 \\
\hline & -1308.9 & -1293.0 & -1302.0 & -1434.5 & -1532.7 \\
\hline & -1545.2 & -1592.8 & -1650.0 & & \\
\hline 12 & 9999.0 & 9999.0 & 9999.0 & 9999.0 & -1878.4 \\
\hline & -1800.1 & -1731.8 & -1687.1 & -1713.3 & -1437.9 \\
\hline & -1290.3 & -1290.2 & -1270.8 & -1374.0 & -1465.2 \\
\hline & -1531.5 & -1585.3 & -1650.0 & & \\
\hline 13 & 9999.0 & 9999.0 & 9999.0 & 9999.0 & 9999.0 \\
\hline & -1749.6 & -1686.9 & -1626.2 & -1565.0 & -1413.4 \\
\hline & -1267.0 & -1236.5 & -1234.6 & -1330.8 & -1435.1 \\
\hline & -1521.2 & -1568.0 & -1596.3 & & \\
\hline 14 & 9999.0 & 9999.0 & 9999.0 & 9999.0 & 9999.0 \\
\hline & 9999.0 & -1632.5 & -1566.8 & -1525.0 & -1466.5 \\
\hline & -1374.6 & -1345.0 & -1361.9 & -1416.7 & -1473.9 \\
\hline & -1534.1 & -1557.0 & -1572.3 & & \\
\hline
\end{tabular}


APPENDIX A. EXAMPLE SIMULATIONS - Test Case 2- GLOBAL Output File

\begin{tabular}{rrrrrr}
15 & 9999.0 & 9999.0 & 9999.0 & 9999.0 & 9999.0 \\
& 9999.0 & 9999.0 & -1510.8 & -1508.9 & -1507.5 \\
& -1467.1 & -1446.4 & -1463.1 & -1531.0 & -1509.3 \\
\multirow{3}{*}{16} & -1543.3 & -1556.7 & -1561.2 & & \\
& 9999.0 & 9999.0 & 9999.0 & 9999.0 & 9999.0 \\
& 9999.0 & 9999.0 & 9999.0 & -1508.4 & -1507.9 \\
& -1494.4 & -1487.5 & -1500.9 & -1543.8 & -1533.8 \\
17 & -1552.5 & -1556.7 & -1557.7 & & \\
& 9999.0 & 9999.0 & 9999.0 & 9999.0 & 9999.0 \\
& 9999.0 & 9999.0 & 9999.0 & 9999.0 & -1507.8 \\
& -1503.3 & -1502.8 & -1511.5 & -1528.5 & -1540.6 \\
18 & -1554.2 & -1555.8 & -1556.3 & & \\
& 9999.0 & 9999.0 & 9999.0 & 9999.0 & 9999.0 \\
& 9999.0 & 9999.0 & 9999.0 & 9999.0 & 9999.0 \\
& -1505.5 & -1507.8 & -1515.2 & -1527.3 & -1541.9
\end{tabular}

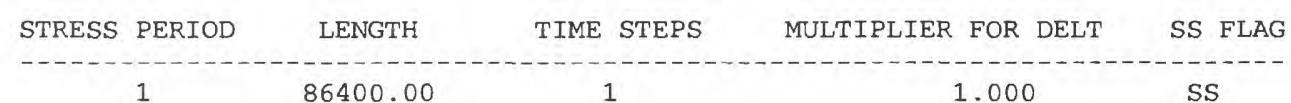

STEADY-STATE SIMULATION

ZONE ARRAY : ZLAYI

READING ON UNIT 32 WITH FORMAT: (I1, 17I2)

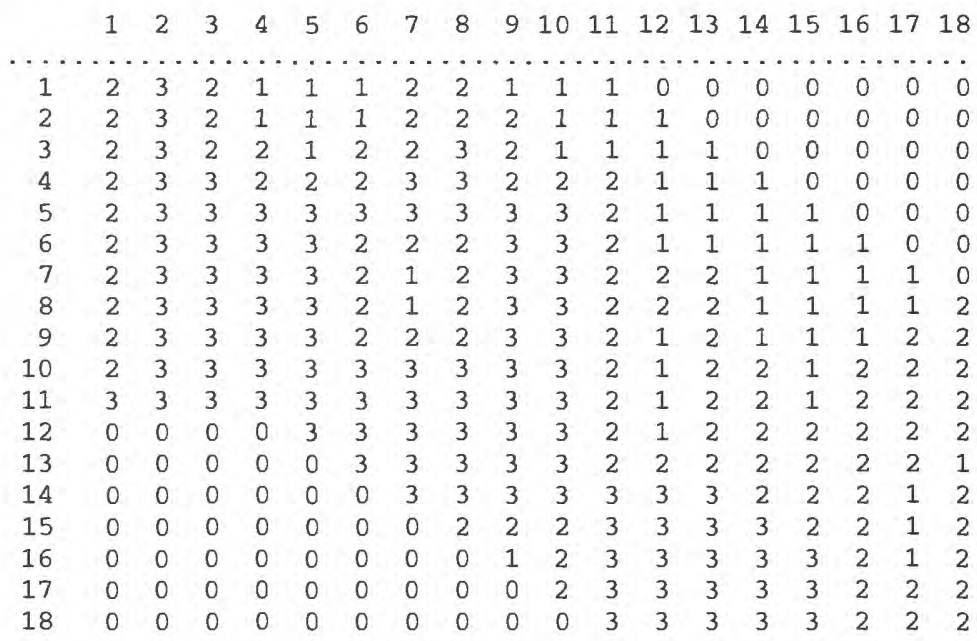

ZONE ARRAY: ZLAY2

READING ON UNIT 32 WITH FORMAT: (I1, 17I2)

$\begin{array}{llllllllllllllllll}1 & 2 & 3 & 4 & 5 & 6 & 7 & 8 & 9 & 10 & 11 & 12 & 13 & 14 & 15 & 16 & 17 & 18\end{array}$

$\begin{array}{ccccccccccccccccccc}1 & 3 & 3 & 3 & 3 & 3 & 3 & 3 & 3 & 3 & 3 & 3 & 0 & 0 & 0 & 0 & 0 & 0 & 0 \\ 2 & 3 & 3 & 3 & 3 & 3 & 3 & 3 & 4 & 4 & 4 & 3 & 3 & 0 & 0 & 0 & 0 & 0 & 0 \\ 3 & 3 & 3 & 3 & 3 & 3 & 3 & 3 & 4 & 4 & 4 & 3 & 3 & 3 & 0 & 0 & 0 & 0 & 0 \\ 4 & 3 & 3 & 3 & 3 & 3 & 3 & 4 & 4 & 4 & 4 & 4 & 4 & 3 & 3 & 0 & 0 & 0 & 0 \\ 5 & 3 & 3 & 3 & 3 & 3 & 3 & 3 & 3 & 3 & 4 & 4 & 4 & 3 & 3 & 3 & 0 & 0 & 0 \\ 6 & 3 & 3 & 3 & 3 & 3 & 2 & 2 & 2 & 3 & 4 & 4 & 4 & 3 & 3 & 2 & 2 & 0 & 0 \\ 7 & 3 & 3 & 3 & 4 & 3 & 2 & 1 & 2 & 3 & 4 & 4 & 3 & 3 & 2 & 2 & 2 & 2 & 0 \\ 8 & 3 & 3 & 3 & 3 & 3 & 2 & 1 & 2 & 3 & 4 & 4 & 3 & 2 & 2 & 2 & 2 & 2 & 3 \\ 9 & 3 & 3 & 3 & 3 & 3 & 2 & 1 & 2 & 3 & 4 & 4 & 3 & 3 & 2 & 1 & 2 & 3 & 3 \\ 10 & 3 & 3 & 3 & 3 & 3 & 2 & 2 & 2 & 3 & 3 & 3 & 3 & 3 & 2 & 1 & 2 & 3 & 3 \\ 11 & 3 & 3 & 3 & 3 & 3 & 3 & 3 & 3 & 3 & 3 & 3 & 3 & 3 & 2 & 1 & 2 & 3 & 2\end{array}$




$\begin{array}{lllllllllllllllllll}12 & 0 & 0 & 0 & 0 & 3 & 3 & 3 & 3 & 3 & 3 & 3 & 3 & 3 & 2 & 2 & 2 & 2 & 2 \\ 13 & 0 & 0 & 0 & 0 & 0 & 3 & 3 & 3 & 3 & 3 & 3 & 3 & 3 & 3 & 2 & 2 & 2 & 2 \\ 14 & 0 & 0 & 0 & 0 & 0 & 0 & 3 & 3 & 3 & 3 & 3 & 3 & 3 & 3 & 3 & 2 & 1 & 2 \\ 15 & 0 & 0 & 0 & 0 & 0 & 0 & 0 & 3 & 3 & 3 & 3 & 3 & 3 & 3 & 3 & 2 & 2 & 2 \\ 16 & 0 & 0 & 0 & 0 & 0 & 0 & 0 & 0 & 3 & 3 & 3 & 3 & 3 & 3 & 3 & 3 & 3 & 3 \\ 17 & 0 & 0 & 0 & 0 & 0 & 0 & 0 & 0 & 0 & 3 & 3 & 3 & 3 & 3 & 3 & 3 & 3 & 2 \\ 18 & 0 & 0 & 0 & 0 & 0 & 0 & 0 & 0 & 0 & 0 & 3 & 3 & 3 & 3 & 3 & 3 & 3 & 2\end{array}$

ZONE ARRAY: ZLAY3

READING ON UNIT 32 WITH FORMAT: (I1, 17I2)

$\begin{array}{rrrrrrrrrrrrrrrrrrr} & 1 & 2 & 3 & 4 & 5 & 6 & 7 & 8 & 9 & 10 & 11 & 12 & 13 & 14 & 15 & 16 & 17 & 18 \\ 1 & 3 & 4 & 4 & 3 & 3 & 3 & 3 & 3 & 3 & 3 & 3 & 0 & 0 & 0 & 0 & 0 & 0 & 0 \\ 2 & 3 & 4 & 4 & 4 & 3 & 3 & 3 & 4 & 4 & 4 & 3 & 3 & 0 & 0 & 0 & 0 & 0 & 0 \\ 3 & 3 & 4 & 4 & 4 & 4 & 3 & 4 & 4 & 4 & 4 & 4 & 3 & 3 & 0 & 0 & 0 & 0 & 0 \\ 4 & 3 & 4 & 4 & 4 & 4 & 4 & 4 & 4 & 4 & 4 & 4 & 4 & 3 & 3 & 0 & 0 & 0 & 0 \\ 5 & 3 & 4 & 4 & 4 & 4 & 4 & 4 & 4 & 4 & 4 & 4 & 4 & 4 & 3 & 3 & 0 & 0 & 0 \\ 6 & 3 & 4 & 4 & 4 & 4 & 4 & 4 & 4 & 4 & 4 & 4 & 4 & 4 & 4 & 4 & 4 & 0 & 0 \\ 7 & 3 & 4 & 4 & 4 & 4 & 4 & 1 & 1 & 1 & 1 & 1 & 1 & 1 & 1 & 1 & 1 & 1 & 0 \\ 8 & 3 & 3 & 4 & 4 & 4 & 4 & 1 & 3 & 3 & 3 & 4 & 4 & 4 & 4 & 4 & 4 & 1 & 1 \\ 9 & 3 & 3 & 3 & 3 & 4 & 4 & 4 & 4 & 4 & 3 & 3 & 3 & 3 & 3 & 3 & 3 & 3 & 3 \\ 10 & 3 & 3 & 3 & 3 & 3 & 4 & 4 & 4 & 4 & 4 & 4 & 4 & 3 & 3 & 3 & 3 & 3 & 3 \\ 11 & 3 & 3 & 3 & 3 & 3 & 3 & 4 & 4 & 4 & 3 & 3 & 3 & 3 & 3 & 3 & 3 & 3 & 3 \\ 12 & 0 & 0 & 0 & 0 & 3 & 3 & 4 & 4 & 4 & 4 & 3 & 3 & 3 & 3 & 3 & 4 & 3 & 3 \\ 13 & 0 & 0 & 0 & 0 & 0 & 3 & 3 & 4 & 4 & 4 & 4 & 4 & 4 & 4 & 4 & 4 & 4 & 4 \\ 14 & 0 & 0 & 0 & 0 & 0 & 0 & 3 & 3 & 4 & 4 & 4 & 4 & 4 & 4 & 4 & 4 & 4 & 4 \\ 15 & 0 & 0 & 0 & 0 & 0 & 0 & 0 & 3 & 3 & 3 & 3 & 3 & 3 & 3 & 3 & 3 & 3 & 3 \\ 16 & 0 & 0 & 0 & 0 & 0 & 0 & 0 & 0 & 3 & 3 & 3 & 3 & 3 & 3 & 3 & 3 & 3 & 3 \\ 17 & 0 & 0 & 0 & 0 & 0 & 0 & 0 & 0 & 0 & 3 & 3 & 3 & 3 & 3 & 3 & 3 & 3 & 3 \\ 18 & 0 & 0 & 0 & 0 & 0 & 0 & 0 & 0 & 0 & 0 & 3 & 3 & 3 & 3 & 3 & 3 & 3 & 3\end{array}$

ZONE ARRAY: RCHETM

READING ON UNIT 32 WITH FORMAT: (I1,17I2)

$\begin{array}{rcccccccccccccccccc} & 1 & 2 & 3 & 4 & 5 & 6 & 7 & 8 & 9 & 10 & 11 & 12 & 13 & 14 & 15 & 16 & 17 & 18 \\ 1 & 0 & 0 & 0 & 0 & 0 & 0 & 0 & 0 & 0 & 0 & 0 & 0 & 0 & 0 & 0 & 0 & 0 & 0 \\ 2 & 0 & 0 & 0 & 0 & 0 & 0 & 0 & 0 & 0 & 0 & 0 & 0 & 0 & 0 & 0 & 0 & 0 & 0 \\ 3 & 0 & 0 & 0 & 0 & 0 & 0 & 0 & 0 & 0 & 0 & 0 & 0 & 0 & 0 & 0 & 0 & 0 & 0 \\ 4 & 0 & 0 & 0 & 0 & 0 & 0 & 0 & 0 & 0 & 0 & 0 & 0 & 0 & 0 & 0 & 0 & 0 & 0 \\ 5 & 0 & 0 & 0 & 0 & 0 & 0 & 0 & 0 & 0 & 0 & 0 & 0 & 0 & 0 & 0 & 0 & 0 & 0 \\ 6 & 0 & 0 & 0 & 0 & 0 & 2 & 2 & 2 & 0 & 0 & 0 & 0 & 0 & 0 & 0 & 0 & 0 & 0 \\ 7 & 0 & 0 & 0 & 0 & 0 & 2 & 2 & 2 & 0 & 0 & 0 & 0 & 0 & 0 & 0 & 0 & 0 & 0 \\ 8 & 0 & 0 & 0 & 0 & 0 & 2 & 2 & 2 & 0 & 0 & 0 & 0 & 0 & 0 & 0 & 0 & 0 & 0 \\ 9 & 0 & 0 & 0 & 0 & 0 & 2 & 2 & 2 & 0 & 0 & 0 & 1 & 1 & 1 & 0 & 0 & 0 & 0 \\ 10 & 0 & 0 & 0 & 0 & 0 & 0 & 0 & 0 & 0 & 0 & 0 & 1 & 1 & 1 & 1 & 0 & 0 & 0 \\ 11 & 0 & 0 & 0 & 0 & 0 & 0 & 0 & 0 & 0 & 0 & 0 & 1 & 1 & 1 & 1 & 0 & 0 & 0 \\ 12 & 0 & 0 & 0 & 0 & 0 & 0 & 0 & 0 & 0 & 0 & 0 & 1 & 1 & 1 & 1 & 0 & 0 & 0 \\ 13 & 0 & 0 & 0 & 0 & 0 & 0 & 0 & 0 & 0 & 0 & 0 & 1 & 1 & 1 & 1 & 0 & 0 & 0 \\ 14 & 0 & 0 & 0 & 0 & 0 & 0 & 0 & 0 & 0 & 0 & 0 & 0 & 0 & 0 & 0 & 0 & 0 & 0 \\ 15 & 0 & 0 & 0 & 0 & 0 & 0 & 0 & 0 & 0 & 0 & 0 & 0 & 0 & 0 & 0 & 0 & 0 & 0 \\ 16 & 0 & 0 & 0 & 0 & 0 & 0 & 0 & 0 & 0 & 0 & 0 & 0 & 0 & 0 & 0 & 0 & 0 & 0 \\ 17 & 0 & 0 & 0 & 0 & 0 & 0 & 0 & 0 & 0 & 0 & 0 & 0 & 0 & 0 & 0 & 0 & 0 & 0 \\ 18 & 0 & 0 & 0 & 0 & 0 & 0 & 0 & 0 & 0 & 0 & 0 & 0 & 0 & 0 & 0 & 0 & 0 & 0\end{array}$

LPF1 -- LAYER PROPERTY FLOW PACKAGE, VERSION 1, 1/11/2000 INPUT READ FROM UNIT 42

\# LPF file for test case ymptc

\#

HEAD AT CELLS THAT CONVERT TO DRY $=-999.00$

6 Named Parameters

LAYER FLAGS:

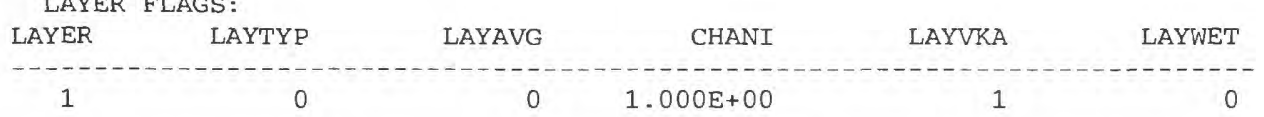


APPENDIX A. EXAMPLE SIMULATIONS - Test Case 2 - GLOBAL Output File

\begin{tabular}{|c|c|c|c|c|c|}
\hline 2 & 0 & 0 & $1.000 \mathrm{E}+00$ & 1 & 0 \\
\hline 3 & 0 & 0 & 1. $.000 \mathrm{E}+00$ & 1 & 0 \\
\hline INTE & PRETATION OF & $\begin{array}{l}\text { LAYER FLAGS: } \\
\text { INTERBLOCK }\end{array}$ & HORIZONTAL & DATA IN & \\
\hline LAYER & $\begin{array}{l}\text { LAYER TYPE } \\
\text { (LAYTYP) }\end{array}$ & $\begin{array}{c}\text { TRANSMISSIVITY } \\
\text { (LAYAVG) }\end{array}$ & $\begin{array}{c}\text { ANISOTROPY } \\
\text { (CHANI) }\end{array}$ & $\begin{array}{l}\text { ARRAY VKA } \\
\text { (LAYVKA) }\end{array}$ & $\begin{array}{l}\text { WETTABILITY } \\
\text { (LAYWET) }\end{array}$ \\
\hline 1 & CONFINED & HARMONIC & $1.000 E+00$ & ANISOTROPY & NON-WETTABLE \\
\hline 2 & CONFINED & HARMONIC & $1.000 E+00$ & ANISOTROPY & NON-WETTABLE \\
\hline 3 & CONEINED & HARMONIC & $1.000 E+00$ & ANISOTROPY & NON-WETTABLE \\
\hline
\end{tabular}

1944 ELEMENTS IN X ARRAY ARE USED BY LPF

18 ELEMENTS IN IX ARRAY ARE USED BY LPF

PCG2 - CONUUGATE GRADIENT SOLUTION PACKAGE, VERSION 2.4, 12/29/98

\# PCG file for test case tc2

MAXIMUM OF 60 CALLS OF SOLUTION ROUTINE

MAXIMUM OF 8 INTERNAL ITERATIONS PER CALL TO SOLUTION ROUTINE

MATRIX PRECONDITIONING TYPE : 1

3876 ELEMENTS IN X ARRAY ARE USED BY PCG

3360 ELEMENTS IN IX ARRAY ARE USED BY PCG

1944 ELEMENTS IN Z ARRAY ARE USED BY PCG

SEN1BAS6 - - SENSITIVITY PROCESS, VERSION 1.0, 10/15/98

INPUT READ FROM UNIT 25

\# SEN file for test case tc2

NUMBER OF PARAMETER VALUES TO BE READ FROM SEN FILE: 10

ISENALL . . . . . . . . . . . .

SENSITIVITIES WILL BE STORED IN MEMORY

FOR UP TO 10 PARAMETERS

1680 ELEMENTS IN X ARRAY ARE USED FOR SENSITIVITIES

972 ELEMENTS IN Z ARRAY ARE USED FOR SENSITIVITIES

20 ELEMENTS IN IX ARRAY ARE USED FOR SENSITIVITIES

PESIBAS6 - PARAMETER-ESTIMATION PROCESS, VERSION 1.0, 07/22/99

INPUT READ FROM UNIT 26

\# PES file for test case tc2

\#

MAXIMUM NUMBER OF PARAMETER-ESTIMATION ITERATIONS (MAX-ITER) $=30$

MAXIMUM PARAMETER CORRECTION (MAX-CHANGE) -

CLOSURE CRITERION (TOL) -

SUM OF SQUARES CLOSURE CRITERION (SOSC) $\ldots \ldots . . . . .$.

FLAG TO GENERATE INPUT NEEDED BY BEALE-2000 (IBEFLG) $\ldots \ldots \ldots$

FLAG TO GENERATE INPUT NEEDED BY YCINT-2000 (IYCFLG) $\ldots \ldots-\ldots$

OMIT PRINTING TO SCREEN (IF = 1) (IOSTAR)

ADJUST GAUSS-NEWTON MATRIX WITH NEWTON UPDATES $(I F=1)($ NOPT $)=0$

NUMBER OF FLETCHER-REEVES ITERATIONS (NFIT)

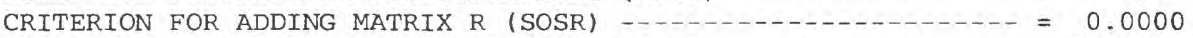

INITIAL VALUE OF MARQUARDT PARAMETER (RMAR)

MARQUARDT PARAMETER MULTIPLIER (RMARM) $\ldots \ldots \ldots$

APPLY MAX-CHANGE IN REGRESSION SPACE (IF = 1) (IAP) ...... =

FORMAT CODE FOR COVARIANCE AND CORRELATION MATRICES (IPRCOV) = 8 PRINT PARAMETER-ESTIMATION STATISTICS

EACH ITERATION (IF > 0) (IPRINT)

PRINT EIGENVALUES AND EIGENVECTORS OF

COVARIANCE MATRIX (IF > 0 ) (LPRINT)

SEARCH DIRECTION ADJUSTMENT PARAMETER (CSA) -.........

MODIFY CONVERGENCE CRITERIA (IF > 0) (FCONV)

CALCULATE SENSITIVITIES USING FINAL

PARAMETER ESTIMATES $(\mathrm{IF}>0)$ (LASTX) $\ldots$

NUMBER OF USUALLY POS. PARAMETERS THAT MAY BE NEG (NPNG) --- = 0 


\section{APPENDIX A. EXAMPLE SIMULATIONS - Test Case 2 - GLOBAL Output File}

NUMBER OF PARAMETERS WITH CORRELATED PRIOR INFORMATION (IPR) $=0$
NUMBER OF PRIOR-INFORMATION EQUATIONS (MPR)

533 ELEMENTS IN X ARRAY ARE USED FOR PARAMETER ESTIMATION

372 ELEMENTS IN $Z$ ARRAY ARE USED FOR PARAMETER ESTIMATION

32 ELEMENTS IN IX ARRAY ARE USED FOR PARAMETER ESTIMATION

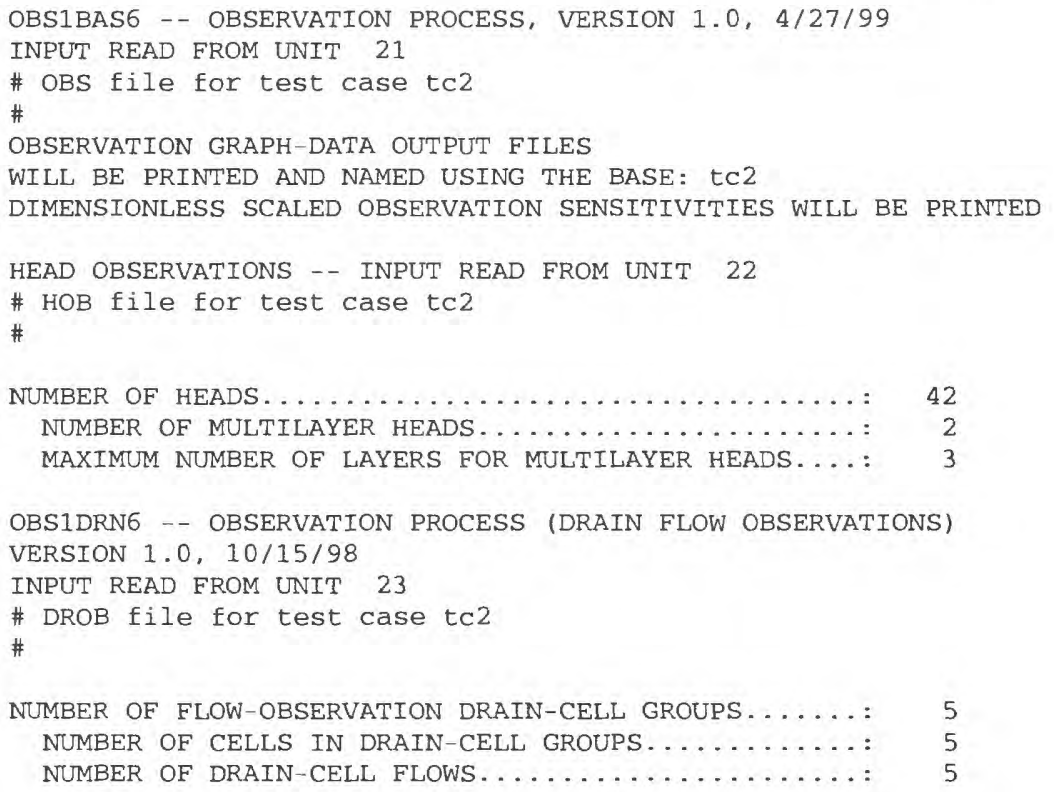

2572 ELEMENTS IN X ARRAY ARE USED FOR OBSERVATIONS

132 ELEMENTS IN Z ARRAY ARE USED FOR OBSERVATIONS

509 ELEMENTS IN IX ARRAY ARE USED FOR OBSERVATIONS

COMMON ERROR VARIANCE FOR ALL OBSERVATIONS SET TO:

1.000

$\begin{array}{rlll}10605 & \text { ELEMENTS OF X ARRAY USED OUT OF } & 10605 \\ 3420 & \text { ELEMENTS OF } & \text { Z ARRAY USED OUT OF } & 3420 \\ 3939 & \text { ELEMENTS OF IX ARRAY USED OUT OF } & 3939 \\ 9720 & \text { ELEMENTS OF XHS ARRAY USED OUT OF } & 9720\end{array}$

INFORMATION ON PARAMETERS LISTED IN SEN FILE

\begin{tabular}{lcccccc}
\multicolumn{1}{c}{ NAME } & ISENS & LN & $\begin{array}{c}\text { VALUE IN SEN } \\
\text { INPUT FILE }\end{array}$ & $\begin{array}{c}\text { LEWER } \\
\text { REANANABL } \\
\text { LIMIT }\end{array}$ & $\begin{array}{c}\text { UPPER } \\
\text { REASONABLE } \\
\text { LIMIT }\end{array}$ & $\begin{array}{c}\text { ALTERNATE } \\
\text { SCALING } \\
\text { FACTOR }\end{array}$ \\
HK_1 & 1 & 0 & 1.5000 & -1.4000 & -0.80000 & $0.10000 \mathrm{E}-02$ \\
HK_2 & 1 & 0 & $0.50000 \mathrm{E}-02$ & $0.20000 \mathrm{E}-08$ & $0.20000 \mathrm{E}-06$ & $0.10000 \mathrm{E}-04$ \\
HK_3 & 1 & 0 & $0.12000 \mathrm{E}-03$ & $0.10000 \mathrm{E}-08$ & $0.10000 \mathrm{E}-06$ & $0.10000 \mathrm{E}-06$ \\
HK_4 & 1 & 0 & $0.20000 \mathrm{E}-05$ & $0.12000 \mathrm{E}-03$ & $0.12000 \mathrm{E}-01$ & $0.10000 \mathrm{E}-08$ \\
ANIV_12 & 1 & 0 & 1.0000 & $0.13000 \mathrm{E}-03$ & $0.13000 \mathrm{E}-01$ & $0.10000 \mathrm{E}-02$ \\
ANIV_3 & 1 & 0 & 10.000 & $0.30000 \mathrm{E}-04$ & $0.30000 \mathrm{E}-02$ & $0.10000 \mathrm{E}-01$ \\
RCHRAT & 1 & 0 & $0.44000 \mathrm{E}-03$ & $0.40000 \mathrm{E}-05$ & $0.40000 \mathrm{E}-03$ & $0.10000 \mathrm{E}-06$ \\
ETMAX & 1 & 0 & $0.30000 \mathrm{E}-03$ & $0.40000 \mathrm{E}-05$ & $0.40000 \mathrm{E}-03$ & $0.10000 \mathrm{E}-06$ \\
C_GHB & 1 & 0 & 0.50000 & $0.20000 \mathrm{E}-04$ & $0.20000 \mathrm{E}-02$ & $0.10000 \mathrm{E}-03$ \\
C_DRN & 1 & 0 & 2.0000 & $0.10000 \mathrm{E}-07$ & $0.10000 \mathrm{E}-05$ & $0.10000 \mathrm{E}-02$ \\
\hline
\end{tabular}

FOR THE PARAMETERS LISTED IN THE TABLE ABOVE, PARAMETER VALUES IN INDIVIDUAL 
PACKAGE INPUT FILES ARE REPLACED BY THE VALUES FROM THE SEN INPUT FILE. THE ALTERNATE SCALING FACTOR IS USED TO SCALE SENSITIVITIES IF IT IS LARGER THAN THE PARAMETER VALUE IN ABSOLUTE VALUE AND THE PARAMETER IS NOT LOG-TRANSFORMED.

F STATISTIC FOR BEALE'S MEASURE SET TO (FSTAT) $\ldots \ldots \ldots$

HEAD OBSERVATION VARIANCES ARE MULTIPLIED BY: $\quad 1.000$

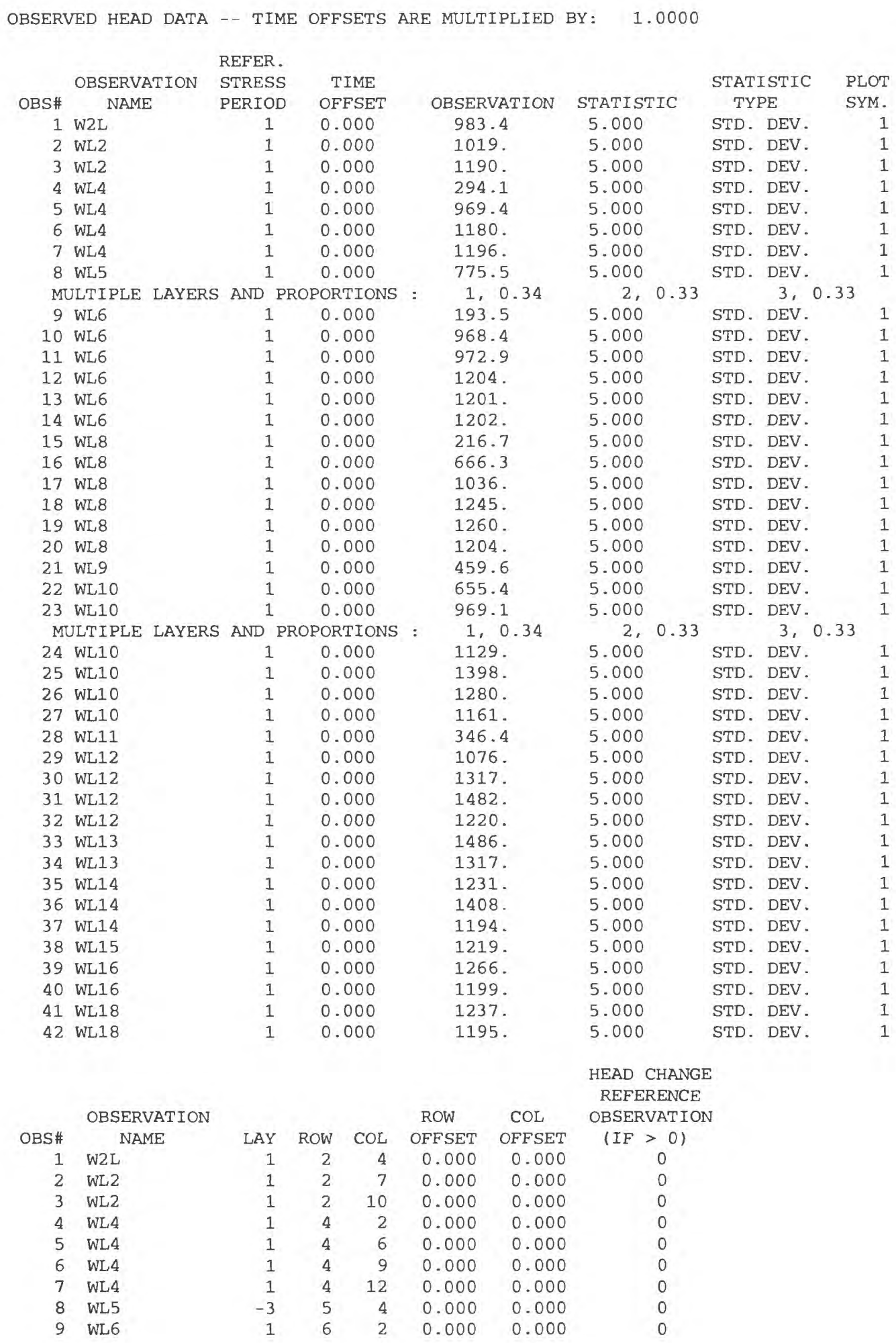


APPENDIX A. EXAMPLE SIMULATIONS - Test Case 2 - GLOBAL Output File

$\begin{array}{llrrrrrr}10 & \text { WL6 } & 1 & 6 & 6 & 0.000 & 0.000 & 0 \\ 11 & \text { WL6 } & 1 & 6 & 8 & 0.000 & 0.000 & 0 \\ 12 & \text { WL6 } & 1 & 6 & 11 & 0.000 & 0.000 & 0 \\ 13 & \text { WL6 } & 1 & 6 & 14 & 0.000 & 0.000 & 0 \\ 14 & \text { WL6 } & 1 & 6 & 16 & 0.000 & 0.000 & 0 \\ 15 & \text { WL8 } & 1 & 8 & 2 & 0.000 & 0.000 & 0 \\ 16 & \text { WL8 } & 1 & 8 & 4 & 0.000 & 0.000 & 0 \\ 17 & \text { WL8 } & 1 & 8 & 7 & 0.000 & 0.000 & 0 \\ 18 & \text { WL8 } & 1 & 8 & 10 & 0.000 & 0.000 & 0 \\ 19 & \text { WL8 } & 1 & 8 & 13 & 0.000 & 0.000 & 0 \\ 20 & \text { WL8 } & 1 & 8 & 16 & 0.000 & 0.000 & 0 \\ 21 & \text { WL9 } & 1 & 9 & 3 & 0.000 & 0.000 & 0 \\ 22 & \text { WL10 } & 1 & 10 & 4 & 0.000 & 0.000 & 0 \\ 23 & \text { WL10 } & -3 & 10 & 6 & 0.000 & 0.000 & 0 \\ 24 & \text { WL10 } & 1 & 10 & 9 & 0.000 & 0.000 & 0 \\ 25 & \text { WL10 } & 1 & 10 & 11 & 0.000 & 0.000 & 0 \\ 26 & \text { WL10 } & 1 & 10 & 14 & 0.000 & 0.000 & 0 \\ 27 & \text { WL10 } & 1 & 10 & 17 & 0.000 & 0.000 & 0 \\ 28 & \text { WL11 } & 1 & 11 & 2 & 0.000 & 0.000 & 0 \\ 29 & \text { WL12 } & 1 & 12 & 8 & 0.000 & 0.000 & 0 \\ 30 & \text { WL12 } & 1 & 12 & 10 & 0.000 & 0.000 & 0 \\ 31 & \text { WL12 } & 1 & 12 & 13 & 0.000 & 0.000 & 0 \\ 32 & \text { WL12 } & 1 & 12 & 16 & 0.000 & 0.000 & 0 \\ 33 & \text { WL13 } & 1 & 13 & 11 & 0.000 & 0.000 & 0 \\ 34 & \text { WL13 } & 1 & 13 & 15 & 0.000 & 0.000 & 0 \\ 35 & \text { WL14 } & 1 & 14 & 9 & 0.000 & 0.000 & 0 \\ 36 & \text { WL14 } & 1 & 14 & 12 & 0.000 & 0.000 & 0 \\ 37 & \text { WL14 } & 1 & 14 & 17 & 0.000 & 0.000 & 0 \\ 38 & \text { WL15 } & 1 & 15 & 14 & 0.000 & 0.000 & 0 \\ 39 & \text { WL16 } & 1 & 16 & 12 & 0.000 & 0.000 & 0 \\ 40 & \text { WL16 } & 1 & 16 & 16 & 0.000 & 0.000 & 0 \\ 41 & \text { WL18 } & 1 & 18 & 13 & 0.000 & 0.000 & 0 \\ 42 & \text { WL18 } & 1 & 18 & 18 & 0.000 & 0.000 & 0\end{array}$

DRAIN-CELL FLOW OBSERVATION VARIANCES ARE MULTIPLIED BY:

1.000

OBSERVED DRAIN-CELL FLOW DATA

-- TIME OFFSETS ARE MULTIPLIED BY: 1.0000

GROUP NUMBER: 1 BOUNDARY TYPE: DRN NUMBER OF CELLS IN GROUP: -1 NUMBER OF FLOW OBSERVATIONS: 1

$$
\begin{array}{ccccclcr}
\multicolumn{9}{c}{\text { OBSERVED }} \\
\text { OBSERVATION } & \text { REFER. } & \text { STRESS } & \text { TIME } & \text { DRAIN FLOW } & & \text { STATISTIC } & \text { PLOT } \\
\text { OBS\# NAME } & \text { PERIOD } & \text { OFFSET } & \text { GAIN }(-1 & \text { STATISTIC } & \text { TYPE } & \text { SYM. } \\
43 \text { DRN1 } & 1 & 0.000 & -573.4 & 0.3000 & \text { COEF. VAR. } & 4 \\
& & & & & & & \\
\text { LAYER ROW } & \text { COLUMN } & \text { FACTOR } & & & \\
1 . & 7 . & 6 . & 1.00 & &
\end{array}
$$

GROUP NUMBER: 2 BOUNDARY TYPE: DRN NUMBER OF CELLS IN GROUP: -1 NUMBER OF FLOW OBSERVATIONS: 1

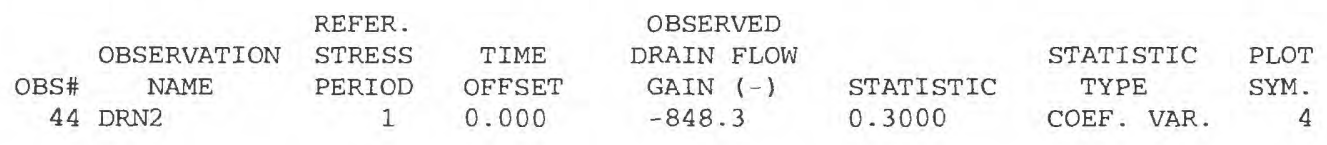

\begin{tabular}{|c|c|c|c|c|c|c|c|}
\hline & OBSERVATION & $\begin{array}{l}\text { REFER. } \\
\text { STRESS }\end{array}$ & & $\begin{array}{l}\text { OBSERVED } \\
\text { DRAIN FLOW }\end{array}$ & & STATTSTTC & PIOT \\
\hline OBS\# & NAME & PERIOD & OFFSET & GAIN $(-)$ & STATISTIC & TYPE & SYM. \\
\hline 45 & DRN3 & 1 & 0.000 & -135.2 & 0.3000 & COEF. VAR. & 4 \\
\hline & LAYER ROW & COLUMN & FACTOR & & & & \\
\hline
\end{tabular}

$$
\begin{aligned}
& \text { LAYER ROW COLUMN FACTOR } \\
& \text { 1. 10. } 11.1 .00
\end{aligned}
$$

GROUP NUMBER: 3 BOUNDARY TYPE: DRN NUMBER OF CELLS IN GROUP: -1 NUMBER OF FLOW OBSERVATIONS: 1 
GROUP NUMBER: 4 BOUNDARY TYPE: DRN NUMBER OF CELLS IN GROUP: - I NUMBER OF FLOW OBSERVATIONS: 1

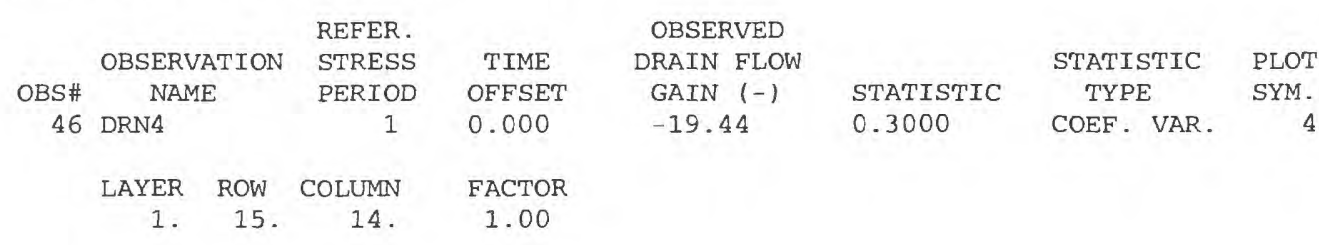

GROUP NUMBER: 5 BOUNDARY TYPE: DRN NUMBER OF CELLS IN GROUP: -1 NUMBER OF FLOW OBSERVATIONS: 1

\begin{tabular}{|c|c|c|c|c|c|c|c|}
\hline & OBSERVATION & $\begin{array}{l}\text { REFER. } \\
\text { STRESS }\end{array}$ & TIME & $\begin{array}{l}\text { OBSERVED } \\
\text { DRAIN FLOW }\end{array}$ & & STATISTIC & PLOT \\
\hline OBS\# & NAME & PERIOD & OFFSET & GAIN $(-)$ & STATISTIC & TYPE & SYM. \\
\hline 47 & DRN5 & 1 & 0.000 & -6.537 & 0.3000 & COEF. VAR. & 4 \\
\hline & LAYER ROW & COLUMN & FACTOR & & & & \\
\hline & 16. & 14. & 1.00 & & & & \\
\hline
\end{tabular}

GENERAL-HEAD-CELL FLOW OBSERVATION VARIANCES ARE MULTIPLIED BY: $\quad 1.000$

OBSERVED GENERAL-HEAD-CELL FLOW DATA

-- TIME OFFSETS ARE MULTIPLIED BY: 1.0000

GROUP NUMBER: 6 BOUNDARY TYPE: GHB NUMBER OF CELLS IN GROUP: -1 NUMBER OF FLOW OBSERVATIONS: 1

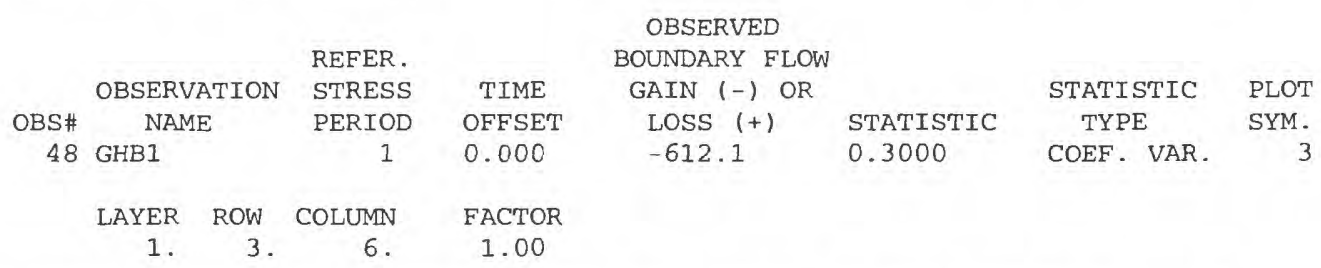

GROUP NUMBER: 7 BOUNDARY TYPE: GHB NUMBER OF CELLS IN GROUP: - 1 NUMBER OF FLOW OBSERVATIONS:

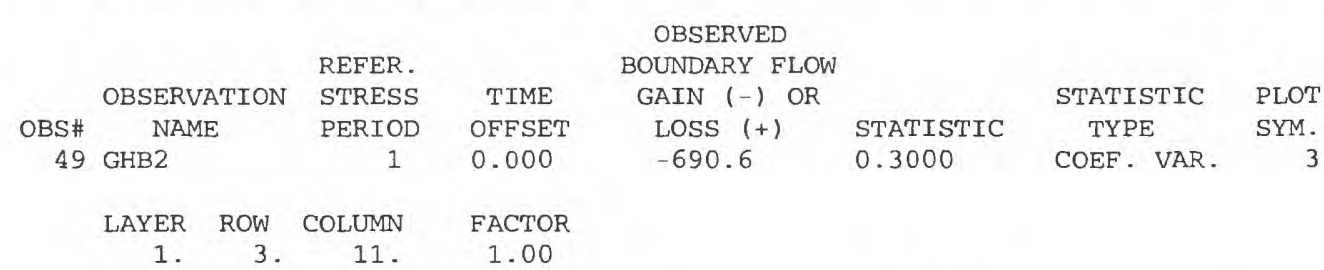

GROUP NUMBER: 8 BOUNDARY TYPE: GHB NUMBER OF CELLS IN GROUP: - I NUMBER OF FLOW OBSERVATIONS : 1

\begin{tabular}{cccccccr}
\multicolumn{10}{c}{ OBSERVED } \\
OBSERVATION & REFER. & STRESS & TIME & BOUNDARY FLOW & & \\
GAIN (-) OR & & STATISTIC & PLOT \\
OBS\# & NAME & PERIOD & OFFSET & LOSS (+) & STATISTIC & TYPE & SYM. \\
50 GHB3 & 1 & 0.000 & -662.7 & 0.3000 & COEF. VAR. & 3 \\
& & & & & & \\
LAYER ROW & COLUMN & FACTOR & & & \\
1. & 4. & 11. & 1.00 & &
\end{tabular}

GROUP NUMBER: 9 BOUNDARY TYPE: GHB NUMBER OF CELLS IN GROUP: -1 NUMBER OF FLOW OBSERVATIONS : TYPE:

\begin{tabular}{|c|c|c|c|c|c|c|c|}
\hline & & REFER. & & $\begin{array}{l}\text { OBSERVED } \\
\text { BOUNDARY FLOW }\end{array}$ & & & \\
\hline & OBSERVATION & STRESS & TIME & GAIN $(-)$ OR & & STATISTIC & PLOT \\
\hline OBS\# & NAME & PERIOD & OFFSET & Loss $(+)$ & STATISTIC & TYPE & SYM. \\
\hline
\end{tabular}


APPENDIX A. EXAMPLE SIMULATIONS - Test Case 2 - GLOBAL Output File
51 GHB4
1
0.000
$-657.4$
0.3000
COEF. VAR.
3
LAYER ROW COLUMN FACTOR

GROUP NUMBER: 10 BOUNDARY TYPE: GHB NUMBER OF CELLS IN GROUP: -1 NUMBER OF FLOW OBSERVATIONS

1

OBSERVED

\begin{tabular}{|c|c|c|c|c|c|c|c|c|}
\hline \multirow{3}{*}{$\begin{array}{r}\text { OBS\# } \\
52\end{array}$} & \multicolumn{2}{|c|}{ OBSERVATION } & $\begin{array}{l}\text { REFER. } \\
\text { STRESS }\end{array}$ & \multicolumn{3}{|c|}{ BOUNDARY FLOW } & \multirow{2}{*}{$\begin{array}{l}\text { STATISTIC } \\
\text { TYPE }\end{array}$} & \multirow{2}{*}{$\begin{array}{l}\text { PLOT } \\
\text { SYM. }\end{array}$} \\
\hline & NAN & & PERIOD & OFFSET & Loss $(+)$ & STATISTIC & & \\
\hline & GHB5 & & 1 & 0.000 & -38.76 & 0.3000 & COEF, VAR. & 3 \\
\hline & LAYER & ROW & COLUMN & FACTOR & & & & \\
\hline & 1. & 12. & 9. & 1.00 & & & & \\
\hline
\end{tabular}

SOLUTION BY THE CONJUGATE-GRADIENT METHOD

$\begin{array}{rr}\text { MAXIMUM NUMBER OF CALLS TO PCG ROUTINE }= & 60 \\ \text { MAXIMUM ITERATIONS PER CALL TO PCG }= & 8 \\ \text { MATRIX PRECONDITIONING TYPE }= & 1 \\ \text { FACTOR (ONLY USED WITH PRECOND. TYPE 1) }= & 0.10000 \mathrm{E}+01 \\ \text { OMIAL PRECOND. }=2 \text { (2) OR IS CALCULATED }: & 2 \\ \text { HEAD CHANGE CRITERION FOR CLOSURE }= & 0.10000 \mathrm{E}-01 \\ \text { RESIDUAL CHANGE CRITERION FOR CLOSURE }= & 0.80000 \mathrm{E}+02 \\ \text { AND RESIDUAL CHANGE PRINTOUT INTERVAL }= & 999 \\ \text { SOLVER IS LIMITED (1) OR SUPPRESSED (>1) }= & 2 \\ \text { DAMPING PARAMETER }= & 0.10000 \mathrm{E}+01\end{array}$

PRINTING FROM SOLVER IS LIMITED(1) OR SUPPRESSED $(>1)=$
DAMPING PARAMETER $=$

$0.10000 \mathrm{E}+01$

\begin{tabular}{|c|c|c|}
\hline CONVERGENCE & CRITERIA FOR & SENSITIVITIES \\
\hline PARAMETER & HCLOSE & RCLOSE \\
\hline HK_1 & $0.66667 \mathrm{E}-04$ & 0.53333 \\
\hline HK_2 & $0.20000 E-01$ & 160.00 \\
\hline HK_3 & 0.83333 & 6666.7 \\
\hline HK_4 & 50.000 & $0.40000 E+06$ \\
\hline ANIV_12 & $0.10000 \mathrm{E}-03$ & 0.80000 \\
\hline ANIV_3 & $0.10000 \mathrm{E}-04$ & $0.80000 \mathrm{E}-01$ \\
\hline RCHRAT & 0.22727 & 1818.2 \\
\hline ETMAX & 0.33333 & 2666.7 \\
\hline C_GHB & $0.20000 E-03$ & 1.6000 \\
\hline C_DRN & $0.50000 \mathrm{E}-04$ & 0.40000 \\
\hline
\end{tabular}

WETTING CAPABILITY IS NOT ACTIVE IN ANY LAYER

PARAMETERS DEFINED IN THE LPF PACKAGE

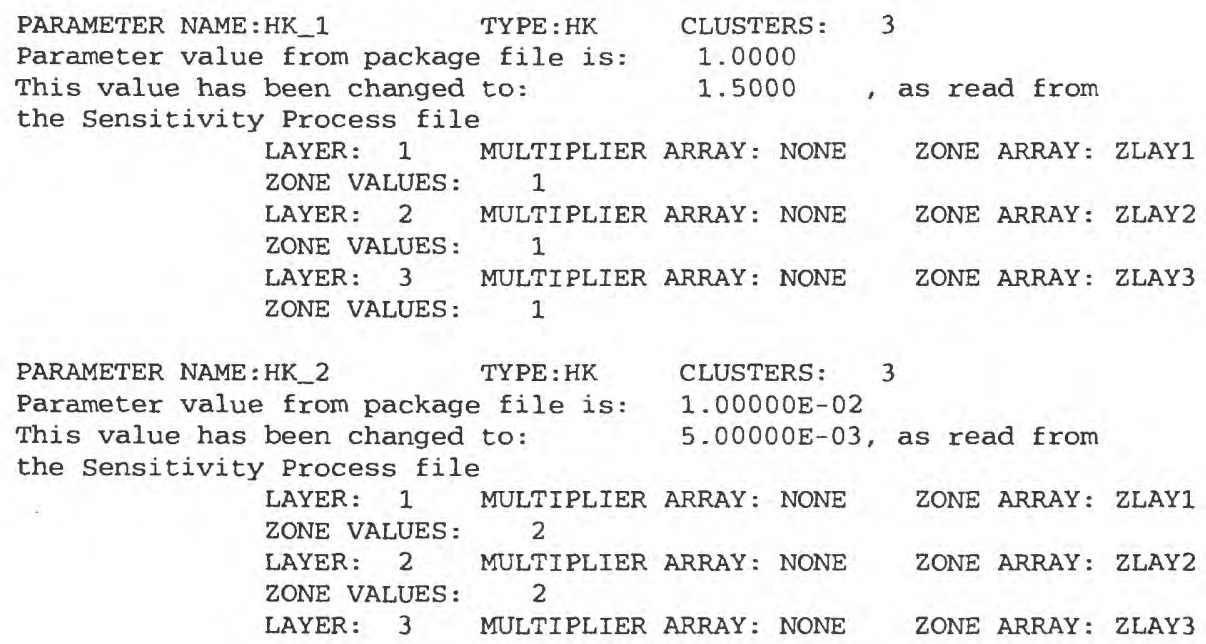




\section{APPENDIX A. EXAMPLE SIMULATIONS - Test Case 2 - GLOBAL Output File}

ZONE VALUES: $\quad 2$

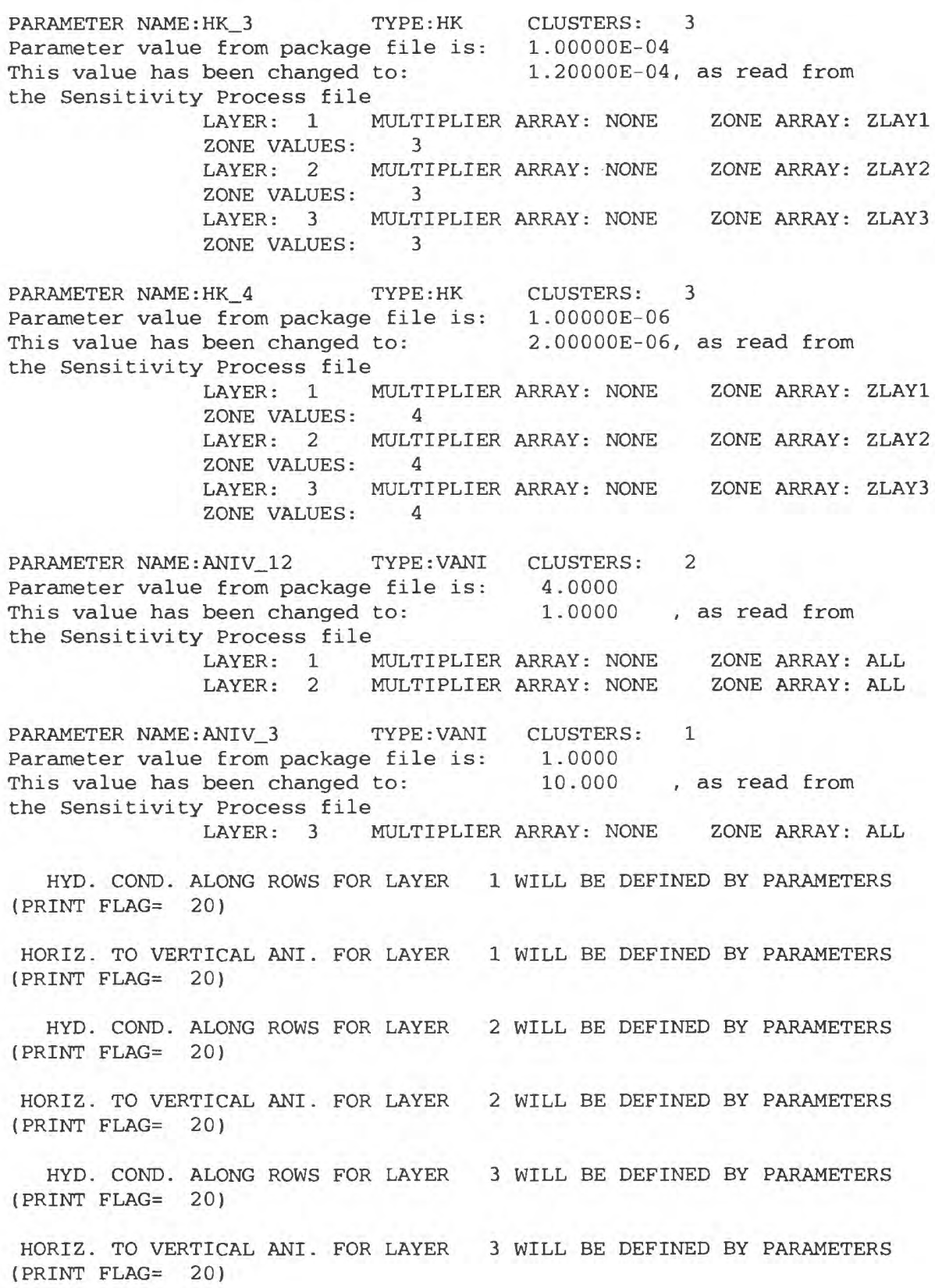


APPENDIX A. EXAMPLE SIMULATIONS - Test Case 2 - GLOBAL Output File

$\begin{array}{llllll}3 & 1 & 14 & 14 & 1200 . & 1.000 \\ 4 & 1 & 15 & 14 & 1200 . & 1.000 \\ 5 & 1 & 16 & 14 & 1200 . & 1.000\end{array}$

1 Evapotranspiration parameters

$\begin{array}{cl}\text { PARAMETER NAME:ETMAX TYPE:EVT } & \text { CLUSTERS: } 1 \\ \text { Parameter value from package file is: } & 4.00000 \mathrm{E}-04 \\ \text { This value has been changed to: } & 3.00000 \mathrm{E}-04 \text {, as read from } \\ \text { the Sensitivity Process file } & \\ \text { MULTIPLIER ARRAY: NONE } & \text { ZONE ARRAY: RCHETM } \\ \text { ZONE VALUES: 2 } & \end{array}$

1 GHB parameters

\begin{tabular}{|c|c|c|c|c|c|c|}
\hline \multicolumn{7}{|c|}{ PARAMETER NAME:C_GHB TYPE:GHB } \\
\hline \multirow{3}{*}{\multicolumn{5}{|c|}{$\begin{array}{l}\text { Paxameter value from package file is: } \\
\text { This value has been changed to: } \\
\text { the Sensitivity Process file }\end{array}$}} & 1.0000 & \\
\hline & & & & & 0.50000 & , as read from \\
\hline & & & & & & \\
\hline NUMBE & ER OF ENTRI & & 5 & & & \\
\hline BOUND. & NO. LAYER & ROW & $\mathrm{COL}$ & STAGE & STRESS & FACTOR \\
\hline 1 & 1 & 3 & 6 & 350.0 & & 1.000 \\
\hline 2 & 1 & 3 & 11 & 500.0 & & 1.000 \\
\hline 3 & 1 & 4 & 11 & 500.0 & & 1.000 \\
\hline 4 & 1 & 5 & 11 & 500.0 & & 1.000 \\
\hline 5 & 1 & 12 & 9 & 1000. & & 1.000 \\
\hline
\end{tabular}

1 Recharge parameters

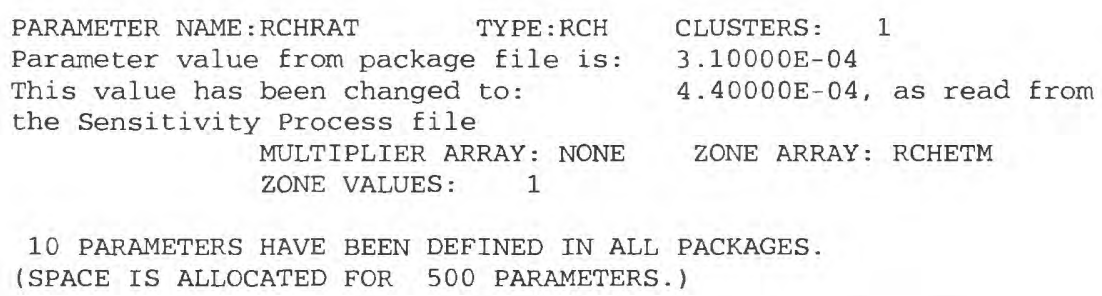

DIMENSIONLESS SCALED SENSITIVITIES (SCALED BY $B^{\star}\left(W T^{*}\right.$ *5))

\begin{tabular}{|c|c|c|c|c|c|c|}
\hline OBS \# & $\begin{array}{l}\text { PARAMETER: } \\
\text { OBSERVATION }\end{array}$ & HK_1 & HK_2 & HK_3 & HK_4 & ANIV__12 \\
\hline 1 & W2L & 0.371 & 18.6 & -20.6 & -0.214 & 13.9 \\
\hline 2 & WL2 2 & 0.240 & 10.9 & -16.8 & -0.180 & 15.0 \\
\hline 3 & WL2 2 & $0.146 \mathrm{E}-01$ & -24.1 & -1.99 & $-0.398 \mathrm{E}-01$ & 20.0 \\
\hline 4 & WL4 & 0.166 & 6.57 & -6.99 & -0.124 & 1.81 \\
\hline 5 & WL4 & 0.397 & 19.5 & -19.1 & -0.199 & 13.1 \\
\hline 6 & WL4 & $0.161 \mathrm{E}-01$ & -19.8 & -3.83 & $-0.672 \mathrm{E}-01$ & 19.3 \\
\hline 7 & WL4 & -0.493 & -24.0 & -1.87 & $-0.380 \mathrm{E}-01$ & 20.0 \\
\hline 8 & WL.5 & 0.712 & 10.6 & -11.2 & -0.236 & 6.76 \\
\hline 9 & WL6 & 0.288 & -0.216 & 0.103 & $0.401 \mathrm{E}-01$ & 0.404 \\
\hline 10 & WL6 & 1.06 & 3.88 & -0.385 & $-0.263 E-02$ & $0.169 \mathrm{E}-02$ \\
\hline 11 & WL6 & 1.41 & 4.43 & 0.181 & $0.318 \mathrm{E}-02$ & -1.28 \\
\hline 12 & WL6 & -0.794 & -26.4 & -2.95 & $-0.875 \mathrm{E}-01$ & 19.6 \\
\hline 13 & WL6 & -0.888 & -24.1 & -1.77 & $-0.362 \mathrm{E}-01$ & 20.1 \\
\hline 14 & WL6 & -0.733 & -24.1 & -1.70 & $-0.349 \mathrm{E}-01$ & 20.1 \\
\hline 15 & WL8 & 0.640 & -12.6 & 11.9 & 0.298 & 0.346 \\
\hline 16 & WL8 & 1.78 & -3.12 & 3.57 & 0.212 & 1.86 \\
\hline 17 & WL8 & 5.64 & -2.88 & $-0.368 \mathrm{E}-01$ & $0.217 \mathrm{E}-02$ & $-0.419 \mathrm{E}-01$ \\
\hline 18 & WL8 & 0.228 & -44.7 & -4.31 & -1.37 & 14.4 \\
\hline 19 & WL8 & -1.82 & -43.7 & -3.31 & $-0.628 \mathrm{E}-01$ & 20.4 \\
\hline 20 & WL8 & -1.17 & -24.1 & -1.67 & $-0.344 \mathrm{E}-01$ & 20.0 \\
\hline 21 & WL9 & 1.33 & -10.8 & 9.53 & 0.449 & 1.84 \\
\hline
\end{tabular}

180 
APPENDIX A. EXAMPLE SIMULATIONS - Test Case 2 - GLOBAL Output File

\begin{tabular}{|c|c|c|c|c|c|c|}
\hline 22 & WL10 & 2.05 & -8.71 & 6.27 & 0.518 & 2.14 \\
\hline 23 & WL10 & 3.39 & -3.88 & 0.366 & 0.563 & 1.10 \\
\hline 24 & WL10 & 2.05 & -22.5 & 0.196 & $-0.925 E-01$ & 6.73 \\
\hline 25 & WL10 & -1.69 & -55.1 & -8.20 & -0.140 & 14.9 \\
\hline 26 & WL10 & -3.44 & -49.9 & -2.31 & $-0.484 E-01$ & 22.8 \\
\hline 27 & WL10 & -1.35 & -14.0 & -0.916 & $-0.194 \mathrm{E}-01$ & 10.3 \\
\hline 28 & WL11 & 1.06 & -17.9 & 15.7 & 0.507 & 1.59 \\
\hline 29 & WL12 & 1.26 & -26.0 & 5.54 & 0.138 & 5.06 \\
\hline 30 & WL12 & -1.64 & -76.3 & -3.35 & -0.203 & 13.2 \\
\hline 31 & WL12 & -3.31 & -134 & -9.48 & -0.117 & 19.6 \\
\hline 32 & WL12 & -2.54 & -34.1 & -1.40 & $-0.293 E-01$ & 12.5 \\
\hline 33 & WL13 & -3.10 & -122 & $-15 \cdot 3$ & -0.152 & 18.1 \\
\hline 34 & WL13 & -2.60 & -68.5 & -2.14 & $-0.399 E-01$ & 16.0 \\
\hline 35 & WL14 & -0.815 & -56.8 & -1.46 & 0.110 & 9.07 \\
\hline 36 & WL14 & -2.37 & -101 & -8.47 & -0.117 & 14.1 \\
\hline 37 & WL1. 4 & -1.41 & -27.4 & -0.615 & $-0.177 \mathrm{E}-01$ & 7.02 \\
\hline 38 & WL15 & -0.287 & -7.56 & 5.90 & $0.179 E-02$ & 0.734 \\
\hline 39 & WL16 & -1.19 & -51.0 & -1.98 & 0.132 & 8.94 \\
\hline 40 & WL16 & -1.47 & -28.4 & -0.460 & $-0.162 E-01$ & 7.22 \\
\hline 41 & WL18 & -1.09 & -37.7 & -0.668 & 0.134 & 7.88 \\
\hline 42 & WL18 & -1.44 & -27.8 & -0.286 & $-0.149 E-01$ & 7.06 \\
\hline 43 & DRN1 & -0.120 & -0.437 & $0.111 E-01$ & $0.768 \mathrm{E}-04$ & $0.797 E-01$ \\
\hline 44 & DRN2 & $0.665 E-01$ & 2.16 & 0.322 & $0.550 \mathrm{E}-02$ & -0.586 \\
\hline 45 & DRN3 & 0.401 & 9.63 & 0.620 & $0.893 E-02$ & -2.36 \\
\hline 46 & DRN4 & 0.492 & 13.0 & -10.1 & $-0.307 E-02$ & -1.26 \\
\hline 47 & DRN5 & 0.864 & 25.0 & -17.9 & $-0.533 E-01$ & -2.28 \\
\hline 48 & GHB1 & $-0.450 \mathrm{E}-02$ & -0.287 & 0.259 & $0.274 \mathrm{E}-02$ & -0.182 \\
\hline 49 & GHB2 & $0.918 \mathrm{E}-03$ & 0.290 & $0.235 E-01$ & $0.474 E-03$ & -0.241 \\
\hline 50 & GHB3 & $0.338 E-02$ & 0.211 & $0.248 E-01$ & $0.503 E-03$ & -0.240 \\
\hline 51 & GHB4 & $0.693 E-02$ & 0.191 & $0.262 \mathrm{E}-01$ & $0.623 E-03$ & -0.237 \\
\hline 52 & GHB5 & $-0.156 \mathrm{E}-02$ & 4.68 & -3.86 & $0.221 \mathrm{E}-02$ & $-0.906 \mathrm{E}-01$ \\
\hline
\end{tabular}

COMPOSITE SCALED SENSITIVITIES ((SUM OF THE SQUARED VALUES)/ND) ** . 5
1. 64
39.7
7.40
0.258
11.5

DIMENSIONLESS SCALED SENSITIVITIES (SCALED BY B* $(W T * * .5)$ )

\begin{tabular}{rlrrrrr} 
& PARAMETER: & \multicolumn{1}{l}{ ANIV_3 } & RCHRAT & ETMAX & C_GHB & C_DRN \\
OBS \# & OBSERVATION & & & & & \\
1 & W2L & 0.372 & 23.3 & -0.874 & -17.6 & -2.44 \\
2 & WL2 & 0.444 & 25.2 & -0.867 & -15.4 & -2.62 \\
3 & WL2 & 0.800 & 34.6 & -0.796 & -3.50 & -3.46 \\
4 & WL4 & 0.138 & 6.80 & -0.367 & -5.15 & -0.765 \\
5 & WL4 & 0.335 & 22.0 & -0.989 & -18.8 & -2.35 \\
6 & WL4 & 0.763 & 33.5 & -0.912 & -4.77 & -3.38 \\
7 & WL4 & 0.802 & 34.7 & -0.796 & -3.25 & -3.47 \\
8 & WL5 & -2.54 & 12.9 & -1.43 & -9.36 & -1.76 \\
9 & WL6 & -0.176 & 3.00 & -0.577 & -2.05 & -0.524 \\
10 & WL6 & -0.231 & 0.738 & -3.70 & -0.176 & -1.39 \\
11 & WL6 & -0.232 & 1.07 & -6.64 & -0.120 & -0.287 \\
12 & WL6 & 0.796 & 43.0 & -0.825 & -5.42 & -5.64 \\
13 & WL6 & 0.805 & 34.7 & -0.796 & -2.91 & -3.45 \\
14 & WL6 & 0.805 & 34.3 & -0.791 & -2.75 & -3.39 \\
15 & WL8 & -1.37 & 2.44 & -1.16 & -0.848 & -0.619 \\
16 & WL8 & $0.680 E-01$ & 3.72 & -3.35 & -1.25 & -1.46 \\
17 & WL8 & -1.25 & 2.94 & -4.51 & -0.238 & -0.743 \\
18 & WL8 & 0.472 & 72.5 & -3.10 & -2.36 & -15.8 \\
19 & WL8 & 0.853 & 61.6 & -0.807 & -2.70 & -8.18 \\
20 & WL8 & 0.802 & 34.6 & -0.783 & -2.67 & -3.41 \\
21 & WL9 & -0.609 & 4.03 & -2.38 & -0.966 & -1.07 \\
22 & WL10 & 0.927 & 5.82 & -3.45 & -0.940 & -1.42 \\
23 & WL10 & -3.84 & 6.94 & -4.90 & -0.728 & -1.56 \\
24 & WL10 & -0.492 & 40.9 & -4.80 & -2.71 & -12.4 \\
25 & WL10 & 0.816 & 129. & -0.653 & -2.10 & -59.5 \\
26 & WL10 & 0.816 & 66.3 & -0.775 & -2.65 & -6.13 \\
27 & WL10 & 0.434 & 20.5 & -0.409 & -1.39 & -2.03 \\
28 & WL11 & -2.93 & 4.19 & -1.81 & -0.736 & -0.963 \\
29 & WL12 & 0.447 & 38.4 & -2.88 & -8.41 & -7.57 \\
30 & WL12 & 0.492 & 113. & -1.17 & -7.16 & -22.1 \\
31 & WL12 & 1.02 & 174. & -0.712 & -2.41 & -21.8 \\
32 & WL12 & 0.505 & 45.3 & -0.490 & -1.67 & -4.57 \\
& & & & & &
\end{tabular}

181 
APPENDIX A. EXAMPLE SIMULATIONS - Test Case 2 - GLOBAL Output File

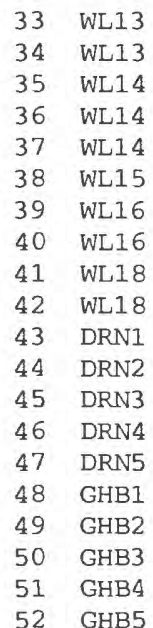

$\begin{array}{lc}1.08 & 174 . \\ 0.529 & 84.9 \\ 1.68 & 80.8 \\ 1.69 & 138 . \\ 0.228 & 36.1 \\ 0.594 \mathrm{E}-01 & 11.5 \\ 1.91 & 70.8 \\ 0.202 & 37.3 \\ 0.210 & 51.9 \\ 0.161 & 36.3 \\ 0.267 \mathrm{E}-01 & -0.652 \mathrm{E}-01 \\ -0.321 \mathrm{E}-01 & -5.07 \\ -0.948 \mathrm{E}-01 & -17.2 \\ -0.102 & -19.8 \\ 0.363 & -34.6 \\ -0.477 \mathrm{E}-02 & -0.305 \\ -0.966 \mathrm{E}-02 & -0.417 \\ -0.961 \mathrm{E}-02 & -0.420 \\ -0.955 \mathrm{E}-02 & -0.440 \\ -0.357 \mathrm{E}-01 & -7.00\end{array}$

-0.758
-0.497
-1.37
-0.712
-0.277
$-0.697 \mathrm{E}-01$
-0.697
-0.284
-0.535
-0.281
0.182
$0.256 \mathrm{E}-01$
$0.803 \mathrm{E}-01$
0.120
0.289
$0.118 \mathrm{E}-01$
$0.961 \mathrm{E}-02$
$0.969 \mathrm{E}-02$
$0.972 \mathrm{E}-02$
0.219

$-2.67$

$-1.70$

$-27.5$

$-5.46$

$-8.68$

$-2.49$

$-14.1$

$-0.947$

$-21.2$

$-5.00$

$-0.237$

$-9.16$

$-2.37$

$-12.8$

$-0.970$

$-1.80$

$-5.35$

$-0.959$

$-9.64$

$0.684 \mathrm{E}-02-6.06$

$0.826 \mathrm{E}-01 \quad-5.62$

$\begin{array}{ll}0.274 & -7.05\end{array}$

$0.407 \quad 2.11$

$\begin{array}{ll}0.978 & 4.36\end{array}$

$-1.29$

$0.321 E-01$

$-1.73$

$-1.65$

$0.418 \mathrm{E}-01$

$0.428 E-01$

$0.483 \mathrm{E}-01$

-1.62
-2.43

1.41

COMPOSITE SCALED SENSITIVITIES ((SUM OF THE SQUARED VALUES) /ND) * *.5
1.03
56.6
1.93
5.11
11.8

PARAMETER

$\begin{array}{lr}\text { HK_1 } & 1.64147 \mathrm{E}+00 \\ \text { HK_2 } & 3.96712 \mathrm{E}+01 \\ \text { HK_3 } & 7.39528 \mathrm{E}+00 \\ \text { HK_4 } & 2.58029 \mathrm{E}-01 \\ \text { ANIV_12 } & 1.14724 \mathrm{E}+01 \\ \text { ANIV_3 } & 1.02954 \mathrm{E}+00 \\ \text { RCHRAT } & 5.66007 \mathrm{E}+01 \\ \text { ETMAX } & 1.93453 \mathrm{E}+00 \\ \text { C_GHB } & 5.11281 \mathrm{E}+00 \\ \text { C_DRN } & 1.18290 \mathrm{E}+01\end{array}$

STARTING VALUES OF REGRESSION PARAMETERS :

$\begin{array}{llllll}\text { HK_1 } & \text { HK_2 } & \text { HK_3 } & \text { HK_4 } & \text { ANIV_12 } & \text { ANIV_3 } \\ \text { RCHRAT } & \text { ETMAX } & \text { C_GHB } & \text { C_DRN } & & \\ 1.5000 & 0.50000 \mathrm{E}-02 & 0.12000 \mathrm{E}-03 & 0.20000 \mathrm{E}-05 & 1.0000 & 10.000 \\ 0.44000 \mathrm{E}-03 & 0.30000 \mathrm{E}-03 & 0.50000 & 2.0000 & \end{array}$

SUMS OF SQUARED, WEIGHTED RESIDUALS:

ALL DEPENDENT VARIABLES: 29451.

DEP. VARIABLES PLUS PARAMETERS: 29451.

PARAMETER VALUES AND STATISTICS FOR ALL PARAMETER-ESTIMATION ITERATIONS

MODIFIED GAUSS-NEWTON CONVERGES IF THE ABSOLUTE VALUE OF THE MAXIMUM FRACTIONAL PARAMETER CHANGE (MAX CALC. CHANGE) IS LESS THAN TOL OR IF THE SUM OF SQUARED, WEIGHTED RESIDUALS CHANGES LESS THAN SOSC OVER TWO PARAMETER-ESTIMATION ITERATIONS.

MODIFIED GAUSS-NEWTON PROCEDURE FOR PARAMETER-ESTIMATION ITERATION NO. =

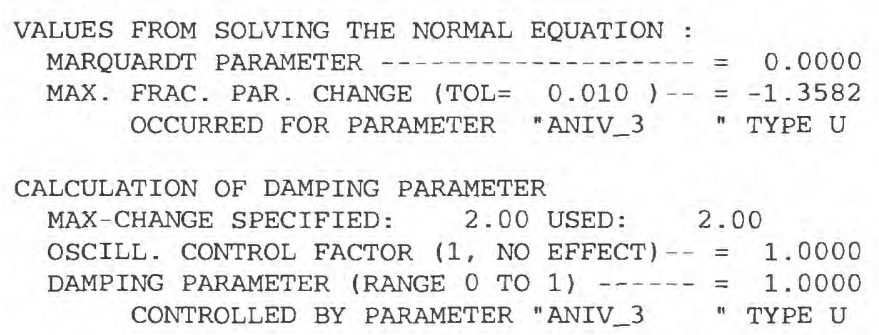




\section{APPENDIX A. EXAMPLE SIMULATIONS - Test Case 2 - GLOBAL Output File}

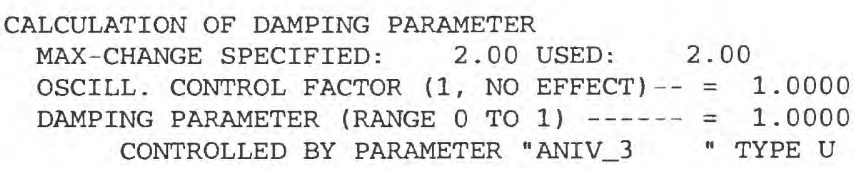

UPDATED ESTIMATES OF REGRESSION PARAMETERS :

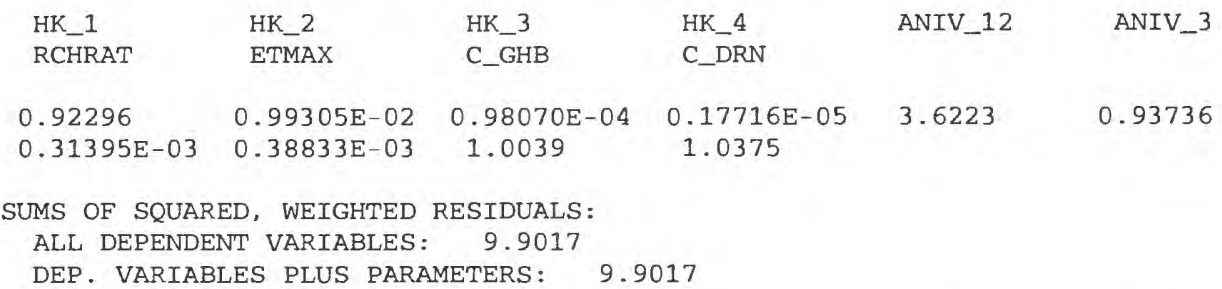

VALUES FROM SOLVING THE NORMAL EQUATION :

MARQUARDT PARAMETER -........... = 0.0000

MAX. FRAC. PAR. CHANGE $(T O L=0.010)--=-.42552$ OCCURRED FOR PARAMETER "HK 4 " TYPE U

CALCULATION OF DAMPING PARAMETER

MAX-CHANGE SPECIFIED: 2.00 USED: 2.00

OSCILL. CONTROL FACTOR ( 1 , NO EFFECT) $--=1.0000$

DAMPING PARAMETER (RANGE 0 TO 1 ) $\ldots \ldots=1.0000$ CONTROLLED BY PARAMETER "HK_4 "TYPE U

UPDATED ESTIMATES OF REGRESSION PARAMETERS :

\begin{tabular}{llllll} 
HK_1 & HK_2 & HK_3 & HK_4 & ANIV_12 & ANIV_3 \\
RCHRAT & ETMAX & C_GHB & C_DRN & \\
0.99548 & $0.99990 \mathrm{E}-02$ & $0.99831 \mathrm{E}-04$ & $0.10177 \mathrm{E}-05$ & 3.9789 & 1.0017 \\
$0.31006 \mathrm{E}-03$ & $0.39958 \mathrm{E}-03$ & 1.0007 & 1.0004 & \\
\multicolumn{7}{c}{} \\
SUMS OF SQUARED, WEIGHTED RESIDUALS: \\
ALL DEPENDENT VARIABLES: $0.65702 \mathrm{E}-01$ \\
DEP. VARIABLES PLUS PARAMETERS: $0.65702 \mathrm{E}-01$ &
\end{tabular}

MODIFIED GAUSS-NEWTON PROCEDURE FOR PARAMETER-ESTIMATION ITERATION NO. =

VALUES FROM SOLVING THE NORMAL EQUATION :

MARQUARDT PARAMETER -...

MAX. FRAC. PAR. CHANGE $(T O L=0.010)--=-.17448 \mathrm{E}-01$

OCCURRED FOR PARAMETER "HK_4 " TYPE U

CALCULATION OF DAMPING PARAMETER

MAX-CHANGE SPECIFIED: 2.00 USED: 2.00

OSCILL. CONTROL FACTOR (1, NO EFFECT) $--=1.0000$

DAMPING PARAMETER (RANGE 0 TO 1 ) $\ldots \ldots=1.0000$ CONTROLLED BY PARAMETER "HK_4 " TYPE U

UPDATED ESTIMATES OF REGRESSION PARAMETERS :

$\begin{array}{lllllr}\text { HK_1 } & \text { HK_2 } & \text { HK_3 } & \text { HK_4 } & \text { ANIV_12 } & \text { ANIV_3 } \\ \text { RCHRAT } & \text { ETMAX } & \text { C_GHB } & \text { C_DRN } & & \\ 0.99997 & 0.10000 \mathrm{E}-01 & 0.10000 \mathrm{E}-03 & 0.99998 \mathrm{E}-06 & 4.0000 & 1.0000 \\ 0.31000 \mathrm{E}-03 & 0.39997 \mathrm{E}-03 & 0.99999 & 1.0000 & & \end{array}$

SUMS OF SOUARED, WEIGHTED RESIDUALS:

ALL DEPENDENT VARIABLES: $0.81115 \mathrm{E}-06$

DEP. VARIABLES PLUS PARAMETERS: $0.81115 \mathrm{E}-06$

MODIFIED GAUSS-NEWTON PROCEDURE FOR PARAMETER-ESTIMATION ITERATION NO. = 
VALUES FROM SOLVING THE NORMAL EQUATION :

MARQUARDT PARAMETER _............ $=0.0000$

MAX. FRAC. PAR. CHANGE (TOL $=0.010)--=0.23497 \mathrm{E}-03$ OCCURRED FOR PARAMETER "HK_4 " TYPE U

CALCULATION OF DAMPING PARAMETER

MAX-CHANGE SPECIFIED: 2.00 USED: 2.00

OSCILL. CONTROL FACTOR (1, NO EFFECT) $--=0.99106$

DAMPING PARAMETER (RANGE 0 TO 1) $\ldots . .-=0.99106$ CONTROLLED BY PARAMETER "HK_4 " TYPE U

UPDATED ESTIMATES OF REGRESSION PARAMETERS :

$\begin{array}{llllll}\text { HK_1 } & \text { HK_2 } & \text { HK_3 } & \text { HK_4 } & \text { ANIV_12 } & \text { ANIV_3 } \\ \text { RCHRAT } & \text { ETMAX } & \text { C_GHB } & \text { C_DRN } & & \\ 0.99997 & 0.10000 \mathrm{E}-01 & 0.10000 \mathrm{E}-03 & 0.10002 \mathrm{E}-05 & 4.0000 & 1.0001 \\ 0.31000 \mathrm{E}-03 & 0.39997 \mathrm{E}-03 & 1.0000 & 1.0000 & \end{array}$

*** PARAMETER ESTIMATION CONVERGED BY SATISFYING THE TOL CRITERION ***

OBSERVATION SENSITIVITY TABLE(S) FOR PARAMETER-ESTIMATION ITERATION

7

DIMENSIONLESS SCALED SENSITIVITIES (SCALED BY B* (WT**.5))

\begin{tabular}{|c|c|c|c|c|c|c|}
\hline OBS \# & $\begin{array}{l}\text { PARAMETER: } \\
\text { OBSERVATION }\end{array}$ & HK_1 & HK_2 & HK_3 & HK_4 & ANIV_12 \\
\hline 1 & W2L & 3.05 & 13.4 & -11.6 & $-0.996 E-01$ & 9.28 \\
\hline 2 & WL2 & 2.99 & 7.58 & -9.55 & $-0.842 E-01$ & 9.62 \\
\hline 3 & WL2 & 3.26 & -20.5 & -1.72 & $-0.220 E-01$ & 11.4 \\
\hline 4 & WL4 & 0.944 & 4.11 & -3.41 & -0.164 & 0.102 \\
\hline 5 & WL4 & 3.02 & 15.5 & $-11 \cdot 2$ & $-0.973 E-01$ & 8.94 \\
\hline 6 & WL4 & 3.13 & -18.1 & -2.33 & $-0.262 E-01$ & 11.3 \\
\hline 7 & WL4 & 2.13 & -20.5 & -1.55 & $-0.205 E-01$ & 11.5 \\
\hline 8 & WL5 & 2.61 & 8.16 & -6.56 & -0.261 & 1.94 \\
\hline 9 & WL6 & 0.703 & $0.915 E-01$ & 0.117 & $-0.286 E-01$ & -1.02 \\
\hline 10 & WL6 & 1.82 & 2.85 & -0.170 & $-0.151 E-02$ & -0.653 \\
\hline 11 & WL6 & 1.85 & 2.80 & $0.546 \mathrm{E}-01$ & $.0 .883 E-03$ & -0.981 \\
\hline 12 & WL6 & 1.40 & -20.9 & -1.74 & $-0.242 \mathrm{E}-01$ & 11.4 \\
\hline 13 & WL6 & 1.07 & -20.6 & -1.43 & $-0.193 E-01$ & 11.6 \\
\hline 14 & WL6 & 0.989 & -20.6 & $-1 \cdot 36$ & $-0.185 E-01$ & 11.6 \\
\hline 15 & WL8 & 0.957 & -6.95 & 6.44 & 0.169 & -0.652 \\
\hline 16 & WL8 & 2.91 & -2.09 & 1.74 & 0.107 & 3.24 \\
\hline 17 & WL8 & 7.84 & -3.96 & -0.108 & $0.106 \mathrm{E}-03$ & 0.127 \\
\hline 18 & WL8 & 1.34 & $-31 \cdot 1$ & -1.92 & -0.204 & 9.12 \\
\hline 19 & WL8 & -0.143 & -30.2 & -1.76 & $-0.236 \mathrm{E}-01$ & 12.3 \\
\hline 20 & WL8 & 0.501 & -20.7 & -1.32 & $-0.180 E-01$ & 11.5 \\
\hline 21 & WL9 & 2.11 & -6.21 & 4.93 & 0.289 & 3.10 \\
\hline 22 & WL10 & 3.13 & -5.56 & 3.14 & 0.350 & 4.74 \\
\hline 23 & WL10 & 5.48 & -2.77 & -0.299 & 0.201 & $-0.182 E-01$ \\
\hline 24 & WL10 & 3.01 & $-17 \cdot 1$ & -0.319 & $-0.227 E-01$ & 4.94 \\
\hline 25 & WL10 & -0.831 & -50.4 & -3.27 & $-0.364 E-01$ & 11.7 \\
\hline 26 & WL10 & -1.75 & -32.9 & -1.56 & $-0.206 E-01$ & 13.2 \\
\hline 27 & WL10 & -0.406 & $-11 \cdot 3$ & -0.704 & $-0.945 E-02$ & 5.78 \\
\hline 28 & WL11 & 1.63 & -9.68 & 8.07 & 0.302 & 0.638 \\
\hline 29 & WL12 & 2.23 & -13.7 & 1.67 & $0.979 E-01$ & 2.14 \\
\hline 30 & WL12 & -0.822 & -43.7 & -1.12 & $-0.623 E-01$ & 8.40 \\
\hline 31 & WL12 & -2.09 & -69.5 & -3.00 & $-0.305 E-01$ & 13.1 \\
\hline 32 & WL12 & -1.46 & $-21 \cdot 4$ & -0.944 & $-0.127 E-01$ & 7.81 \\
\hline 33 & WL13 & -2.09 & -68.5 & -4.45 & $-0.384 E-01$ & 12.4 \\
\hline 34 & WL13 & -1.60 & -39.1 & -1.23 & $-0.158 E-01$ & 11.1 \\
\hline 35 & WL14 & $0.461 \mathrm{E}-01$ & -31.0 & -0.506 & $0.656 E-01$ & 5.15 \\
\hline 36 & WL14 & -1.53 & -55.2 & -2.51 & $-0.599 E-01$ & 10.2 \\
\hline 37 & WL14 & -0.899 & -16.5 & -0.485 & $-0.726 E-02$ & 4.39 \\
\hline 38 & WL15 & -0.267 & -6.95 & 2.63 & $0.177 \mathrm{E}-02$ & 1.27 \\
\hline 39 & WL16 & -0.614 & -29.2 & -0.665 & $0.747 E-01$ & 5.21 \\
\hline 40 & WL1 6 & -0.985 & -17.2 & -0.444 & $-0.693 E-02$ & 4.62 \\
\hline 41 & WL1 8 & -0.642 & -23.2 & -0.409 & $0.710 \mathrm{E}-01$ & 4.18 \\
\hline 42 & WL18 & -0.956 & -16.7 & -0.365 & $-0.608 E-02$ & 4.43 \\
\hline 43 & DRN1 & $-0.934 E-01$ & -0.110 & $0.391 E-02$ & $0.292 \mathrm{E}-04$ & $0.298 \mathrm{E}-01$ \\
\hline
\end{tabular}


APPENDIX A. EXAMPLE SIMÜLATIONS - Test Case 2 - GLOBAL Output File

$\begin{array}{lllrlrr}44 & \text { DRN2 } & 0.163 \mathrm{E}-01 & 0.990 & 0.643 \mathrm{E}-01 & 0.716 \mathrm{E}-03 & -0.229 \\ 45 & \text { DRN3 } & 0.171 & 4.56 & 0.197 & 0.207 \mathrm{E}-02 & -1.12 \\ 46 & \text { DRN4 } & 0.229 & 5.96 & -2.25 & -0.151 \mathrm{E}-02 & -1.08 \\ 47 & \text { DRN5 } & 0.345 & 9.64 & -2.78 & -0.195 \mathrm{E}-01 & -1.20 \\ 48 & \text { GHB1 } & -0.801 \mathrm{E}-01 & -0.463 & 0.295 & 0.256 \mathrm{E}-02 & -0.242 \\ 49 & \text { GHB2 } & -0.745 \mathrm{E}-01 & 0.494 & 0.401 \mathrm{E}-01 & 0.520 \mathrm{E}-03 & -0.276 \\ 50 & \text { GHB3 } & -0.629 \mathrm{E}-01 & 0.346 & 0.397 \mathrm{E}-01 & 0.518 \mathrm{E}-03 & -0.276 \\ 51 & \text { GHB4 } & -0.461 \mathrm{E}-01 & 0.298 & 0.384 \mathrm{E}-01 & 0.522 \mathrm{E}-03 & -0.274 \\ 52 & \text { GHB5 } & -0.878 \mathrm{E}-01 & 2.40 & -2.66 & -0.290 \mathrm{E}-02 & 0.115\end{array}$

COMPOSITE SCALED SENSITIVITIES ((SUM OF THE SQUARED VALUES)/ND) **. 5

$$
\begin{array}{ccccc}
2.10 & 23.9 & 3.56 & 0.106 & 7.15
\end{array}
$$

\begin{tabular}{|c|c|c|c|c|c|c|}
\hline OBS \# & $\begin{array}{l}\text { PARAMETER: } \\
\text { OBSERVATION }\end{array}$ & ANIV_3 & RCHRAT & ETMAX & C_GHB & C_DRN \\
\hline 1 & W2L & $0.407 \mathrm{E}-01$ & 23.6 & -0.627 & -25.5 & -1.67 \\
\hline 2 & WL2 & $0.434 \mathrm{E}-01$ & 25.0 & -0.627 & -22.8 & -1.76 \\
\hline 3 & WL2 & $0.583 \mathrm{E}-01$ & 31.4 & -0.620 & -8.68 & -2.20 \\
\hline 4 & WL4 & $0.610 \mathrm{E}-01$ & 6.53 & -0.282 & -7.06 & -0.479 \\
\hline 5 & WL4 & $0.377 \mathrm{E}-01$ & 22.8 & -0.670 & -27.1 & -1.62 \\
\hline 6 & WL4 & $0.566 \mathrm{E}-01$ & 30.8 & -0.651 & -9.78 & -2.17 \\
\hline 7 & WL4 & $0.586 \mathrm{E}-01$ & 31.6 & -0.621 & -7.95 & -2.22 \\
\hline 8 & WL5 & -1.90 & 13.2 & -1.43 & -14.2 & -1.10 \\
\hline 9 & WL6 & $0.579 E-02$ & 2.57 & -0.497 & -2.63 & -0.249 \\
\hline 10 & WL6 & $-0.410 \mathrm{E}-01$ & 0.500 & -4.23 & -0.175 & -0.566 \\
\hline 11 & WL6 & $-0.356 E-01$ & 0.534 & -4.95 & -0.133 & -0.123 \\
\hline 12 & WL6 & $0.592 \mathrm{E}-01$ & 35.3 & -0.621 & -9.65 & -2.84 \\
\hline 13 & WL6 & $0.589 E-01$ & 31.7 & -0.624 & -7.02 & -2.21 \\
\hline 14 & WL6 & $0.588 E-01$ & 31.3 & -0.621 & -6.57 & -2.16 \\
\hline 15 & WL8 & -0.144 & 1.38 & -0.865 & -0.885 & -0.201 \\
\hline 16 & WL8 & 0.159 & 2.65 & -2.98 & -1.66 & -0.572 \\
\hline 17 & WL8 & -0.165 & 1.72 & -4.65 & -0.355 & -0.378 \\
\hline 18 & WL8 & $0.197 \mathrm{E}-01$ & 45.6 & -1.89 & -5.18 & -5.55 \\
\hline 19 & WL8 & $0.659 \mathrm{E}-01$ & 43.9 & -0.632 & -6.42 & -3.63 \\
\hline 20 & WL8 & $0.583 E-01$ & 31.5 & -0.611 & -6.29 & -2.16 \\
\hline 21 & WL9 & $-0.876 \mathrm{E}-01$ & 2.32 & -1.97 & -1.01 & -0.372 \\
\hline 22 & WL10 & 0.146 & 3.22 & -2.83 & -0.843 & -0.497 \\
\hline 23 & WL10 & -2.98 & 2.91 & -4.37 & -0.514 & -0.492 \\
\hline 24 & WL10 & -0.115 & 24.8 & -3.27 & -2.52 & -3.99 \\
\hline 25 & WL10 & $0.749 \mathrm{E}-01$ & 80.2 & -0.572 & -5.70 & -17.7 \\
\hline 26 & WL10 & $0.586 \mathrm{E}-01$ & 47.2 & -0.604 & -6.20 & -3.12 \\
\hline 27 & WL10 & $0.333 E-01$ & 17.6 & -0.314 & -3.22 & -1.19 \\
\hline 28 & WL11 & -0.668 & 2.08 & -1.43 & -0.599 & -0.300 \\
\hline 29 & WL12 & $-0.940 E-01$ & 18.0 & -2.15 & -3.63 & -2.09 \\
\hline 30 & WL12 & $-0.301 \mathrm{E}-01$ & 60.2 & -0.807 & -5.63 & -6.86 \\
\hline 31 & WL12 & $0.775 E-01$ & 90.1 & -0.566 & -5.73 & -7.35 \\
\hline 32 & WL12 & $0.458 \mathrm{E}-01$ & 30.8 & -0.382 & -3.92 & -2.00 \\
\hline 33 & WL13 & $0.815 \mathrm{E}-01$ & 92.2 & -0.589 & -5.81 & -9.06 \\
\hline 34 & WL13 & $0.476 \mathrm{E}-01$ & 50.4 & -0.404 & -4.11 & -3.20 \\
\hline 35 & WL14 & $-0.432 \mathrm{E}-01$ & 41.6 & -1.09 & -3.96 & $-4 \cdot 30$ \\
\hline 36 & WL14 & 0.177 & 72.6 & -0.590 & -4.71 & -6.76 \\
\hline 37 & WL14 & $0.264 \mathrm{E}-01$ & 22.6 & -0.228 & -2.30 & -1.69 \\
\hline 38 & WL15 & $0.249 E-01$ & 9.48 & $-0.828 E-01$ & -0.736 & -3.92 \\
\hline 39 & WL16 & $0.828 E-01$ & 38.9 & -0.593 & -3.11 & -4.05 \\
\hline 40 & WL16 & $0.264 \mathrm{E}-01$ & 23.5 & -0.234 & -2.36 & -1.79 \\
\hline 41 & WL1 8 & $-0.522 E-01$ & 31.0 & -0.462 & -2.66 & -3.16 \\
\hline 42 & WL18 & $0.253 \mathrm{E}-01$ & 22.7 & -0.230 & -2.31 & -1.73 \\
\hline 43 & DRN1 & $0.209 E-02$ & $-0.216 \mathrm{E}-01$ & 0.134 & $0.495 E-02$ & -3.25 \\
\hline 44 & DRN2 & $-0.147 \mathrm{E}-02$ & -1.58 & $0.112 \mathrm{E}-01$ & 0.112 & -2.98 \\
\hline 45 & DRN3 & $-0.580 \mathrm{E}-02$ & -6.31 & $0.438 \mathrm{E}-01$ & 0.443 & -2.54 \\
\hline 46 & DRN4 & $-0.213 E-01$ & -8.13 & $0.710 \mathrm{E}-01$ & 0.631 & $0.258 \mathrm{E}-01$ \\
\hline 47 & DRN5 & $-0.480 E-02$ & -13.0 & 0.156 & 1.11 & 0.944 \\
\hline 48 & GHB1 & $-0.104 \mathrm{E}-02$ & -0.620 & $0.167 E-01$ & -2.55 & $0.439 \mathrm{E}-01$ \\
\hline 49 & GHB2 & $-0.141 \mathrm{E}-02$ & -0.758 & $0.149 E-01$ & -3.13 & $0.531 \mathrm{E}-01$ \\
\hline 50 & GHB3 & $-0.140 E-02$ & -0.762 & $0.150 \mathrm{E}-01$ & -2.99 & $0.538 \mathrm{E}-01$ \\
\hline 51 & GHB4 & $-0.140 E-02$ & -0.779 & $0.149 E-01$ & -2.94 & $0.569 \mathrm{E}-01$ \\
\hline 52 & GHB5 & $0.957 \mathrm{E}-02$ & -3.27 & 0.147 & -0.307 & 0.381 \\
\hline
\end{tabular}

DIMENSIONLESS SCALED SENSITIVITIES (SCALED BY $B *(W T * * .5)$ )

COMPOSITE SCALED SENSITIVITIES ((SUM OF THE SQUARED VALUES)/ND) **. 5 


\section{APPENDIX A. EXAMPLE SIMULATIONS - Test Case 2 - GLOBAL Output File}
0.503
33.5
1.62
7.64
3.87

$\begin{array}{lc}\text { PARAMETER } & \text { COMPOSITE SCALED SENSITIVITY } \\ \text { HK_1 } & 2.10022 \mathrm{E}+00 \\ \text { HK_2 } & 2.38897 \mathrm{E}+01 \\ \text { HK_3 } & 3.56124 \mathrm{E}+00 \\ \text { HK_4 } & 1.05956 \mathrm{E}-01 \\ \text { ANIV_12 } & 7.15238 \mathrm{E}+00 \\ \text { ANIV_3 } & 5.03201 \mathrm{E}-01 \\ \text { RCHRAT } & 3.34578 \mathrm{E}+01 \\ \text { ETMAX } & 1.62396 \mathrm{E}+00 \\ \text { C_GHB } & 7.63518 \mathrm{E}+00 \\ \text { C_DRN } & 3.86683 \mathrm{E}+00\end{array}$

FINAL PARAMETER VALUES AND STATISTICS:

PARAMETER NAME (S) AND VALUE (S):

$\begin{array}{llllll}\text { HK_1 } & \text { HK_2 } & \text { HK_3 } & \text { HK_4 } & \text { ANIV_12 } & \text { ANIV_3 } \\ \text { RCHRAT } & \text { ETMAX } & \text { C_GHB } & \text { C_DRN } & & \\ 0.100 \mathrm{E}+01 & 0.100 \mathrm{E}-01 & 0.100 \mathrm{E}-03 & 0.100 \mathrm{E}-05 & 0.400 \mathrm{E}+01 & 0.100 \mathrm{E}+01 \\ 0.310 \mathrm{E}-03 & 0.400 \mathrm{E}-03 & 0.100 \mathrm{E}+01 & 0.100 \mathrm{E}+01 & & \end{array}$

SUMS OF SQUARED WEIGHTED RESIDUALS:
OBSERVATIONS
PRIOR INFO.
TOTAL
$0.410 \mathrm{E}-05$
0.00
$0.410 \mathrm{E}-05$

SELECTED STATISTICS FROM MODIFIED GAUSS-NEWTON ITERATIONS

\begin{tabular}{ccccc} 
& MAX. PARAMETER CALC. CHANGE & MAX. CHANGE & DAMPING \\
ITER. & PARNAM & MAX. CHANGE & ALLOWED & PARAMETER \\
\hline 1 & ANIV_3 & -1.36000 & 2.00000 & 1.0000 \\
2 & ANIV_3 & 4.86000 & 2.00000 & 0.14000 \\
3 & ANIV_3 & 3.05000 & 2.00000 & 0.65600 \\
4 & ANIV_3 & 0.861000 & 2.00000 & 1.0000 \\
5 & HK_4 & -0.426000 & 2.00000 & 1.0000 \\
6 & HK_4 & $-0.174000 \mathrm{E}-01$ & 2.00000 & 1.0000 \\
7 & HK_4 & $0.235000 \mathrm{E}-03$ & 2.00000 & 0.99100
\end{tabular}

SUMS OF SQUARED WEIGHTED RESIDUALS FOR EACH ITERATION

\begin{tabular}{rccc}
\multicolumn{4}{c}{ SUMS OF SQUARED WEIGHTED RESIDUALS } \\
ITER. & OBSERVATIONS & PRIOR INFO. & TOTAL \\
1 & 29451. & 0.0000 & 29451. \\
2 & 1812.9 & 0.0000 & 1812.9 \\
3 & 1361.3 & 0.0000 & 1361.3 \\
4 & 262.36 & 0.0000 & 262.36 \\
5 & 9.9017 & 0.0000 & 9.9017 \\
6 & $0.65702 \mathrm{E}-01$ & 0.0000 & $0.65702 \mathrm{E}-01$ \\
7 & $0.81115 \mathrm{E}-06$ & 0.0000 & $0.81115 \mathrm{E}-06$ \\
FINAL & $0.41025 \mathrm{E}-05$ & 0.0000 & $0.41025 \mathrm{E}-05$
\end{tabular}

*** PARAMETER ESTIMATION CONVERGED BY SATISFYING THE TOL CRITERION ***

\begin{tabular}{|c|c|c|c|c|c|}
\hline \multicolumn{6}{|c|}{ COVARIANCE MATRIX FOR THE PARAMETERS } \\
\hline & HK_1 & HK_2 & HK_3 & HK_4 & ANIV_12 \\
\hline & ANIV_3 & RCHRAT & ETMAX & C_GHB & C_DRN \\
\hline \multirow[t]{2}{*}{ HK_1 } & $2.86701 \mathrm{E}-09$ & $7.76145 \mathrm{E}-12$ & $6.78091 \mathrm{E}-14$ & $2.18372 \mathrm{E}-15$ & $-1.28874 E-09$ \\
\hline & $1.09082 \mathrm{E}-09$ & $2.73615 E-13$ & $1.06302 \mathrm{E}-12$ & $1.00825 \mathrm{E}-09$ & $8.06626 \mathrm{E}-10$ \\
\hline \multirow[t]{2}{*}{ HK_2 } & $7.76145 \mathrm{E}-12$ & $1.00961 \mathrm{E}-13$ & $9.11305 \mathrm{E}-16$ & $3.05533 \mathrm{E}-17$ & 1. $66685 \mathrm{E}-12$ \\
\hline & $-6.27254 \mathrm{E}-13$ & $2.91140 \mathrm{E}-15$ & $3.41987 \mathrm{E}-15$ & $9.86347 \mathrm{E}-12$ & $7.21171 \mathrm{E}-12$ \\
\hline
\end{tabular}


APPENDIX A. EXAMPLE SIMULATIONS - Test Case 2 - GLOBAL Output File

$\begin{array}{lrrrrr}\text { HK_3 } & 6.78091 \mathrm{E}-14 & 9.11305 \mathrm{E}-16 & 1.64551 \mathrm{E}-17 & -8.79877 \mathrm{E}-19 & -1.44657 \mathrm{E}-14 \\ & -2.42452 \mathrm{E}-14 & 2.64987 \mathrm{E}-17 & 3.35160 \mathrm{E}-17 & 6.01727 \mathrm{E}-14 & 6.43074 \mathrm{E}-14 \\ \text { HK_4 } & 2.18372 \mathrm{E}-15 & 3.05533 \mathrm{E}-17 & -8.79877 \mathrm{E}-19 & 4.11776 \mathrm{E}-19 & -5.37673 \mathrm{E}-15 \\ & 5.41411 \mathrm{E}-15 & 9.90218 \mathrm{E}-19 & 2.73188 \mathrm{E}-18 & 4.92704 \mathrm{E}-15 & 2.60470 \mathrm{E}-15 \\ \text { ANIV_12 } & -1.28874 \mathrm{E}-09 & 1.66685 \mathrm{E}-12 & -1.44657 \mathrm{E}-14 & -5.37673 \mathrm{E}-15 & 7.53322 \mathrm{E}-09 \\ & -2.63963 \mathrm{E}-09 & -8.19376 \mathrm{E}-14 & -3.80990 \mathrm{E}-13 & 6.03050 \mathrm{E}-10 & -6.48214 \mathrm{E}-10 \\ \text { ANIV_3 } & 1.09082 \mathrm{E}-09 & -6.27254 \mathrm{E}-13 & -2.42452 \mathrm{E}-14 & 5.41411 \mathrm{E}-15 & -2.63963 \mathrm{E}-09 \\ & 1.04257 \mathrm{E}-08 & 2.52097 \mathrm{E}-14 & -1.55551 \mathrm{E}-13 & -8.85222 \mathrm{E}-11 & 2.33532 \mathrm{E}-10 \\ \text { RCHRAT } & 2.73615 \mathrm{E}-13 & 2.91140 \mathrm{E}-15 & 2.64987 \mathrm{E}-17 & 9.90218 \mathrm{E}-19 & -8.19376 \mathrm{E}-14 \\ & 2.52097 \mathrm{E}-14 & 8.75464 \mathrm{E}-17 & 1.16081 \mathrm{E}-16 & 2.84967 \mathrm{E}-13 & 2.44646 \mathrm{E}-13 \\ \text { ETMAX } & 1.06302 \mathrm{E}-12 & 3.41987 \mathrm{E}-15 & 3.35160 \mathrm{E}-17 & 2.73188 \mathrm{E}-18 & -3.80990 \mathrm{E}-13 \\ & -1.55551 \mathrm{E}-13 & 1.16081 \mathrm{E}-16 & 5.82719 \mathrm{E}-16 & 3.93976 \mathrm{E}-13 & 2.99916 \mathrm{E}-13 \\ \text { C_GHB } & 1.00825 \mathrm{E}-09 & 9.86347 \mathrm{E}-12 & 6.01727 \mathrm{E}-14 & 4.92704 \mathrm{E}-15 & 6.03050 \mathrm{E}-10 \\ & -8.85222 \mathrm{E}-11 & 2.84967 \mathrm{E}-13 & 3.93976 \mathrm{E}-13 & 1.18791 \mathrm{E}-09 & 7.28316 \mathrm{E}-10 \\ \text { C_DRN } & 8.06626 \mathrm{E}-10 & 7.21171 \mathrm{E}-12 & 6.43074 \mathrm{E}-14 & 2.60470 \mathrm{E}-15 & -6.48214 \mathrm{E}-10 \\ & 2.33532 \mathrm{E}-10 & 2.44646 \mathrm{E}-13 & 2.99916 \mathrm{E}-13 & 7.28316 \mathrm{E}-10 & 1.24762 \mathrm{E}-09\end{array}$

PARAMETER SUMMARY

PHYSICAL PARAMETER VALUES --- NONE OF THE PARAMETERS IS LOG TRANSFORMED

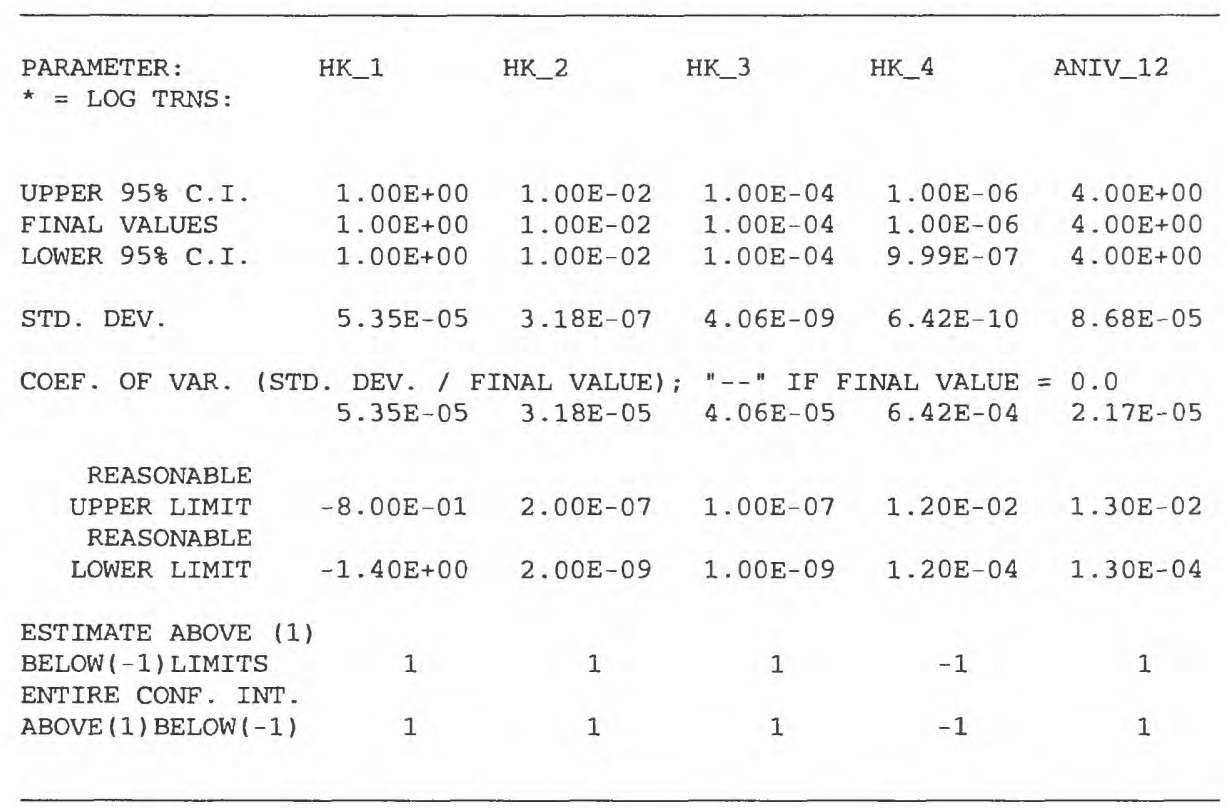

PHYSICAL PARAMETER VALUES -.- NONE OF THE PARAMETERS IS LOG TRANSFORMED

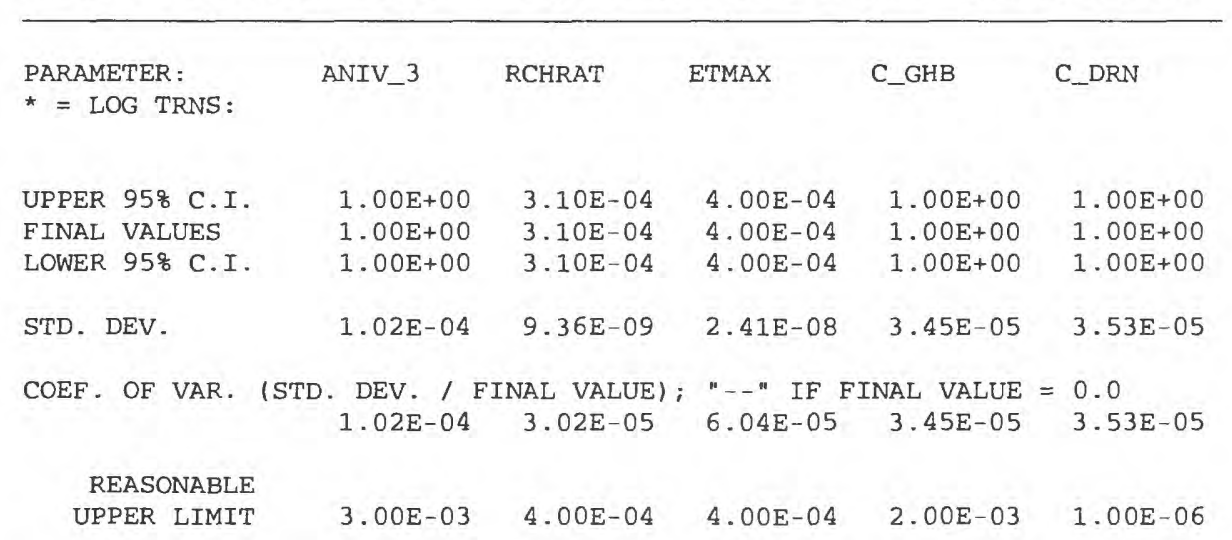




\section{APPENDIX A. EXAMPLE SIMULATIONS - Test Case 2 - GLOBAL Output File}

REASONABLE

LOWER IIMIT
3. $00 \mathrm{E}-05$

4.00E-06
4.00E-06

$2.00 \mathrm{E}-05$
$1.00 \mathrm{E}-08$

ESTIMATE ABOVE (1) BELOW (-1) LIMITS

ENTIRE CONF. INT. ABOVE (1) BELOW (-1)

\begin{abstract}
1
\end{abstract}
1
0

0
1

1
1

1

SOME PARAMETER VALUES ARE OUTSIDE THEIR USER-SPECIFIED REASONABLE RANGES TO A STATISTICALLY SIGNIFICANT EXTENT, BASED ON LINEAR THEORY. THIS IMPLIES THAT THERE ARE PROBLEMS WITH THE OBSERVATIONS, THE MODEL DOES NOT ADEQUATELY REPRESENT THE PHYSICAL SYSTEM, THE DATA ARE NOT CONSISTENT WITH THEIR SIMULATED EQUIVALENTS, OR THE SPECIFIED MINIMUM AND/OR MAXIMUM ARE NOT REASONABLE. CHECK YOUR DATA, CONCEPTUAL MODEL, AND MODEL DESIGN.
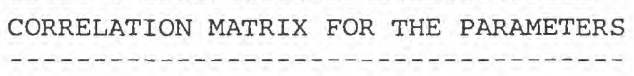

$\begin{array}{lllll}\text { HK_1 } & \text { HK_2 } & \text { HK_3 } & \text { HK_4 } & \text { ANIV_12 } \\ \text { ANIV 3 } & \text { RCHRAT } & \text { ETMAX } & \text { C_GHB } & \text { C DRN }\end{array}$

\begin{tabular}{|c|c|c|c|c|c|}
\hline \multirow[t]{2}{*}{ HK_1 } & 1.0000 & 0.45620 & 0.31219 & $6.35555 E-02$ & -0.27731 \\
\hline & 0.19952 & 0.54614 & 0.82243 & 0.54634 & 0.42650 \\
\hline \multirow[t]{2}{*}{ HK_2 } & 0.45620 & 1.0000 & 0.70703 & 0.14985 & $6.04407 E-02$ \\
\hline & $-1.93337 \mathrm{E}-02$ & 0.97928 & 0.44586 & 0.90066 & 0.64257 \\
\hline \multirow[t]{2}{*}{$\mathrm{HK}_{-} 3$} & 0.31219 & 0.70703 & 1.0000 & -0.33802 & $-4.10866 \mathrm{E}-02$ \\
\hline & $-5.85359 E-02$ & 0.69816 & 0.34227 & 0.43038 & 0.44882 \\
\hline \multirow[t]{2}{*}{ HK_4 } & $6.35555 E-02$ & 0.14985 & -0.33802 & 1.0000 & $-9.65378 \mathrm{E}-02$ \\
\hline & $8.26311 E-02$ & 0.16492 & 0.17636 & 0.22277 & 0.11492 \\
\hline \multirow[t]{2}{*}{ ANIV_12 } & -0.27731 & $6.04407 \mathrm{E}-02$ & $-4.10866 E-02$ & $-9.65378 \mathrm{E}-02$ & 1.0000 \\
\hline & -0.29785 & -0.10090 & -0.18184 & 0.20159 & -0.21144 \\
\hline \multirow[t]{2}{*}{ ANIV__3 } & 0.19952 & $-1.93337 \mathrm{E}-02$ & $-5.85359 E-02$ & $8.26311 E-02$ & -0.29785 \\
\hline & 1.0000 & $2.63873 E-02$ & $-6.31086 E-02$ & $-2.51540 E-02$ & $6.47517 E-02$ \\
\hline \multirow[t]{2}{*}{ RCHRAT } & 0.54614 & 0.97928 & 0.69816 & 0.16492 & -0.10090 \\
\hline & $2.63873 E-02$ & 1.0000 & 0.51394 & 0.88366 & 0.74025 \\
\hline \multirow[t]{2}{*}{ ETMAX } & 0.82243 & 0.44586 & 0.34227 & 0.17636 & -0.18184 \\
\hline & $-6.31086 E-02$ & 0.51394 & 1.0000 & 0.47353 & 0.35175 \\
\hline \multirow[t]{2}{*}{ C_GHB } & 0.54634 & 0.90066 & 0.43038 & 0.22277 & 0.20159 \\
\hline & $-2.51540 E-02$ & 0.88366 & 0.47353 & 1.0000 & 0.59825 \\
\hline \multirow[t]{2}{*}{ C_DRN } & 0.42650 & 0.64257 & 0.44882 & 0.11492 & -0.21144 \\
\hline & $6.47517 \mathrm{E}-02$ & 0.74025 & 0.35175 & 0.59825 & 1.0000 \\
\hline
\end{tabular}

THE CORRELATION OF THE FOLLOWING PARAMETER PAIRS $>=.95$ PARAMETER PARAMETER CORRELATION $\begin{array}{llr}\text { HK_2 } & \text { RCHRAT } & 0.98\end{array}$

THE CORRELATION OF THE FOLLOWING PARAMETER PAIRS IS BETWEEN .90 AND .95 PARAMETER PARAMETER CORRELATION $\begin{array}{lll}\text { HK_2 } & \text { C_GHB } & 0.90\end{array}$

THE CORRELATION OF THE FOLLOWING PARAMETER PAIRS IS BETWEEN .85 AND .90 PARAMETER PARAMETER CORRELATION $\begin{array}{lll}\text { RCHRAT } & \text { C_GHB } & 0.88\end{array}$

CORRELATIONS GREATER THAN 0.95 COULD INDICATE THAT THERE IS NOT ENOUGH INFORMATION IN THE OBSERVATIONS AND PRIOR USED IN THE REGRESSION TO ESTIMATE PARAMETER VALUES INDIVIDUALLY.

TO CHECK THIS, START THE REGRESSION FROM SETS OF INITIAL PARAMETER VALUES THAT DIFFER BY MORE THAT TWO STANDARD DEVIATIONS FROM THE ESTIMATED VALUES. IF THE RESULTING ESTIMATES ARE WELL WITHIN ONE STANDARD DEVIATION OF THE PREVIOUSLY ESTIMATED VALUE, THE ESTIMATES ARE PROBABLY DETERMINED INDEPENDENTLY WITH THE OBSERVATIONS AND PRIOR USED IN THE REGRESSION. OTHERWISE, YOU MAY ONLY BE ESTIMATING THE RATIO OR SUM OF THE HIGHLY CORRELATED PARAMETERS. THE INITIAL PARAMETER VALUES ARE IN THE SEN FILE. 


\section{APPENDIX A. EXAMPLE SIMULATIONS - Test Case 2 - GLOBAL Output File}

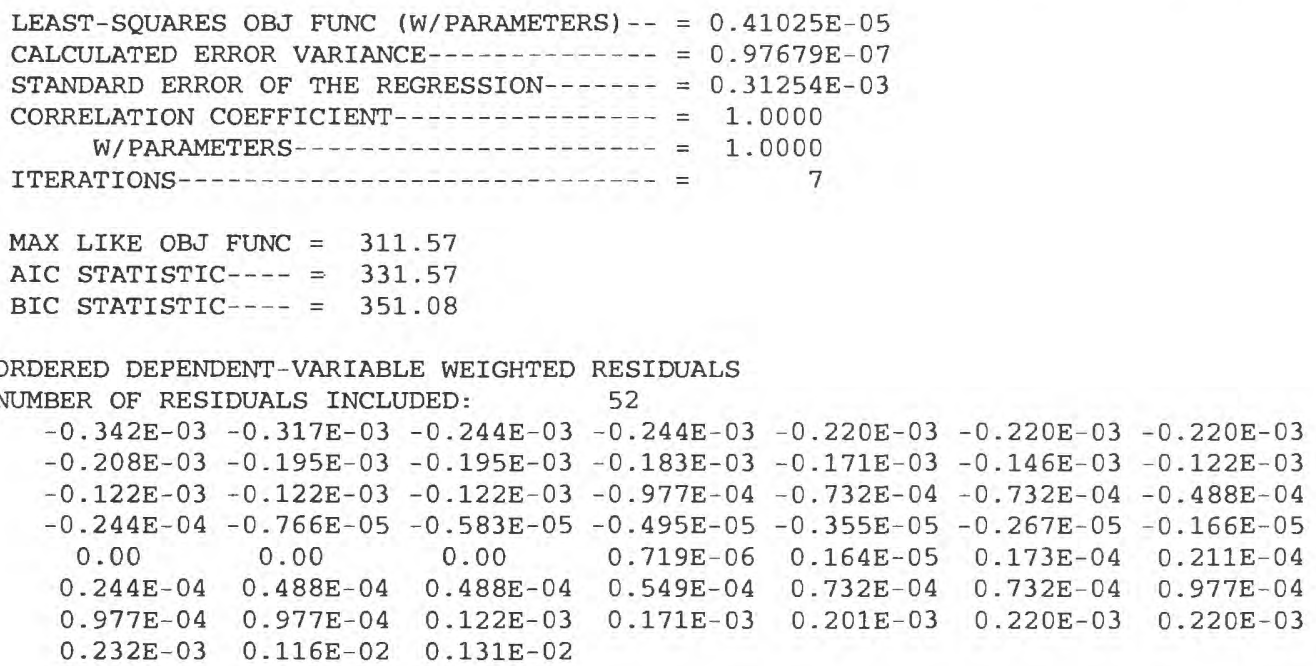

SMALLEST AND LARGEST DEPENDENT-VARIABLE WEIGHTED RESIDUALS

\begin{tabular}{|c|c|c|c|c|c|}
\hline \multicolumn{2}{|c|}{ SMALLEST WEIGHTED } & D RESIDUALS & \multicolumn{2}{|c|}{ LARGEST WEIGHTED } & RESIDUALS \\
\hline & OBSERVATION & WEIGHTED & & OBSERVATION & WEIGHTED \\
\hline OBS\# & NAME & RESIDUAL & OBS\# & NAME & RESIDUAL \\
\hline 11 & WL6 & $-0.34180 E-03$ & 10 & WL6 & $0.13062 E-02$ \\
\hline 33 & WL13 & $-0.31738 E-03$ & 8 & WL5 & $0.11597 \mathrm{E}-02$ \\
\hline 26 & WL10 & $-0.24414 \mathrm{E}-03$ & 16 & WL8 & $0.23193 E-03$ \\
\hline 25 & WL10 & $-0.24414 E-03$ & 35 & WL14 & $0.21973 E-03$ \\
\hline 19 & WL8 & $-0.21973 E-03$ & 24 & WL10 & $0.21973 E-03$ \\
\hline
\end{tabular}

CORRELATION BETWEEN ORDERED WEIGHTED RESIDUALS AND NORMAL ORDER STATISTICS $(E Q .38$ OF TEXT) $=0.659$

COMMENTS ON THE INTERPRETATION OF THE CORRELATION BETWEEN WEIGHTED RESIDUALS AND NORMAL ORDER STATISTICS:

The critical value for correlation at the $5 \%$ significance level is 0.956

IF the reported CORRELATION is GREATER than the $5 \%$ critical value, ACCEPT the hypothesis that the weighted residuals are INDEPENDENT AND NORMALLY DISTRIBUTED at the 5 \% significance level. The probability that this conclusion is wrong is less than 58.

IF the reported correlation IS LESS THAN the $5 \%$ critical value REJECT the, hypothesis that the weighted residuals are INDEPENDENT AND NORMALLY DISTRIBUTED at the $5 \%$ significance level.

The analysis can also be done using the $10 \%$ significance level. The associated critical value is 0.964

*** PARAMETER ESTIMATION CONVERGED BY SATISFYING THE TOL CRITERION * * 


\section{LIST Output File}

The LIST file, tc2.1st, follows:

MODFLOW-2000

U.S. GEOLOGICAL SURVEY MODULAR FINITE-DIFFERENCE GROUND-WATER FLOW MODEL VERSION $1.0 \quad 06 / 13 / 2000$

This model run produced both GLOBAL and LIST files. This is the LIST file.

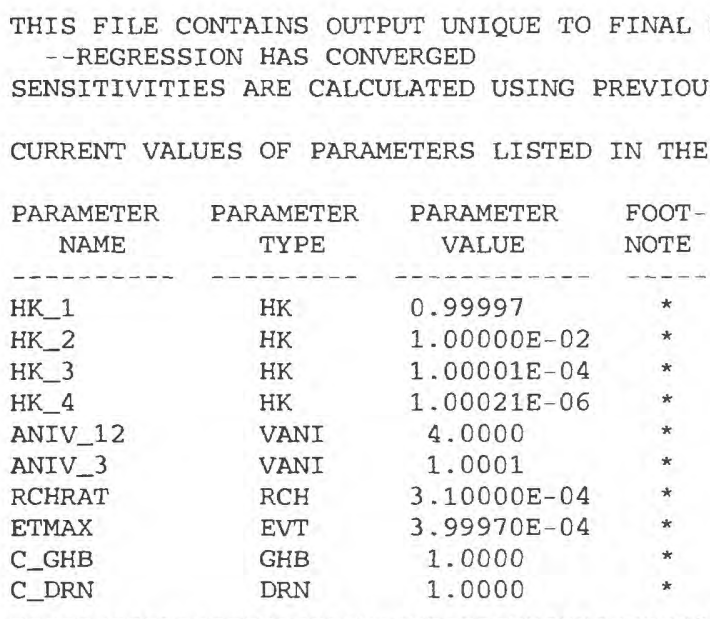

* INDICATES VAlUe aDJUSTABle by PARAMETERESTIMATION PROCESS

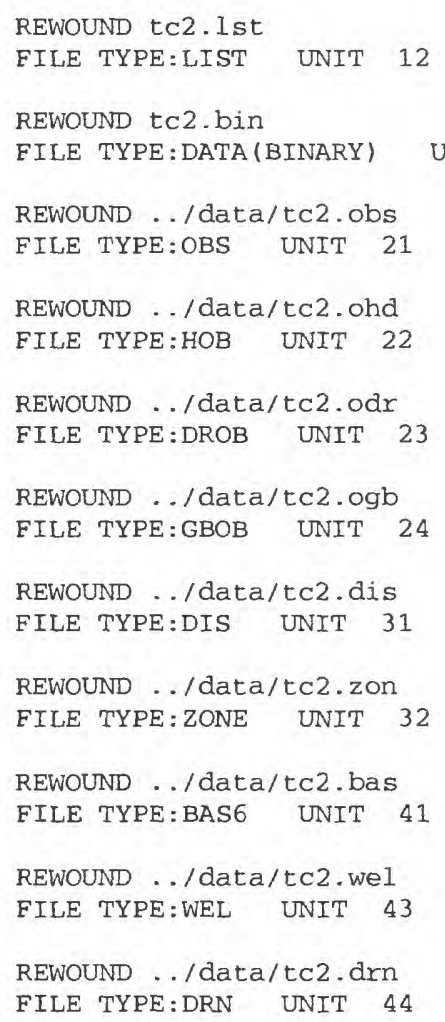




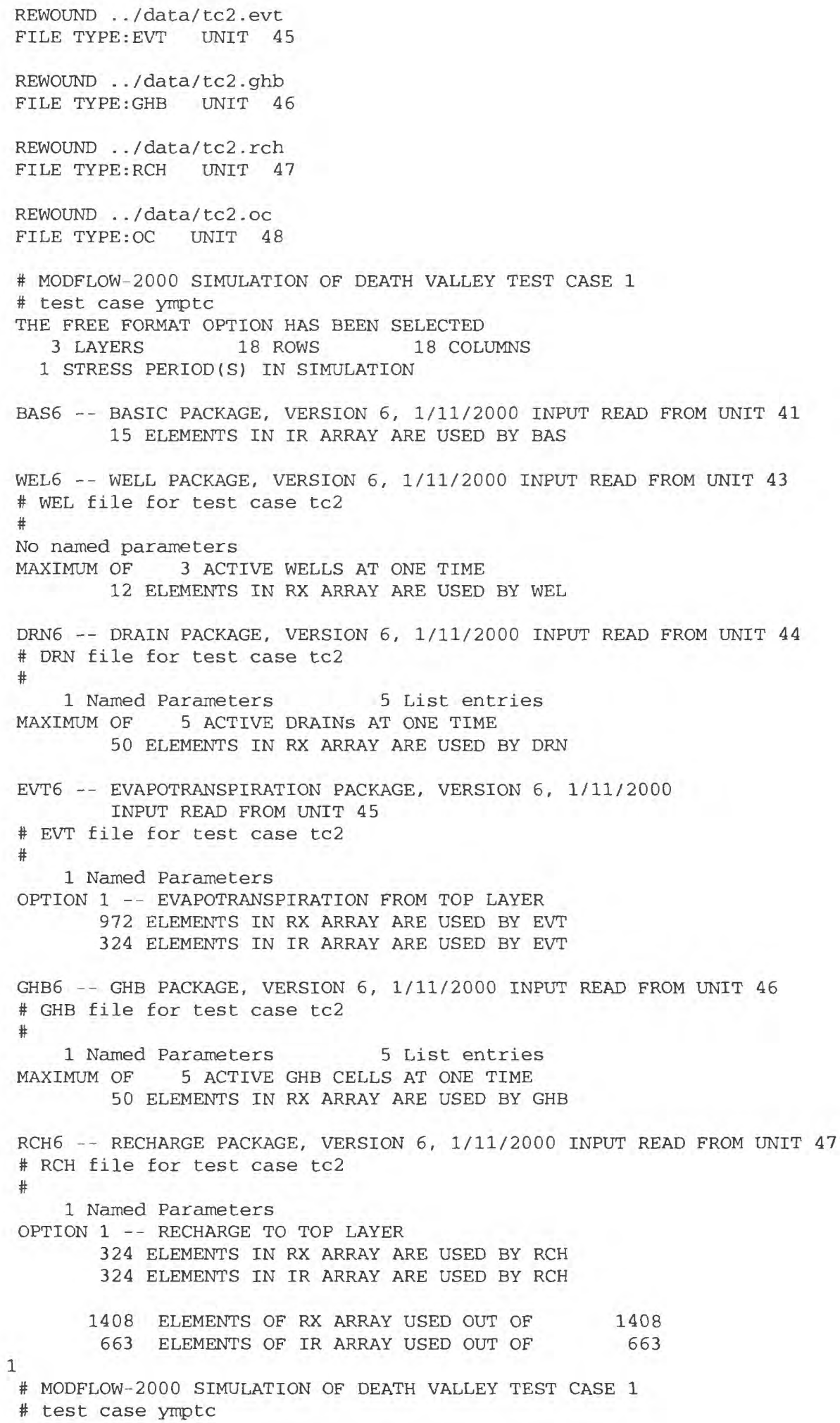


READING ON UNIT 41 WITH FORMAT: $(18 \mathrm{I} 3$ )

BOUNDARY ARRAY FOR LAYER 2

READING ON UNIT 41 WITH FORMAT: (18I3)

BOUNDARY ARRAY FOR LAYER 3

READING ON UNIT 41 WITH FORMAT: (18I3)

AQUIFER HEAD WILL BE SET TO 9999.0 AT ALL NO-FLOW NODES (IBOUND=0).

INITIAL HEAD FOR LAYER 1

READING ON UNIT 41 WITH FORMAT: (18F10.2)

\begin{tabular}{|c|c|c|c|c|c|c|}
\hline & 1 & 2 & 3 & 4 & 5 & 6 \\
\hline & 7 & 8 & 9 & 10 & 11 & 12 \\
\hline & 13 & 14 & 15 & 16 & 17 & 18 \\
\hline$i$ & 0.000 & 466.7 & 970.9 & 979.2 & 979.5 & 980.1 \\
\hline & 1025. & 1124. & 1184. & 1186. & 1187. & 9999. \\
\hline & 9999. & 9999. & 9999. & 9999. & 9999. & 9999. \\
\hline 2 & 0.000 & 460.5 & 968.8 & 979.0 & 979.2 & 979.8 \\
\hline & 1015. & 1103. & 1171. & 1186. & 1187. & 1189. \\
\hline & 9999. & 9999. & 9999. & 9999. & 9999. & 9999. \\
\hline & & & & - & & \\
\hline & & & & 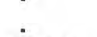 & & \\
\hline 17 & 9999. & 9999. & 9999. & 9999. & 9999. & 9999. \\
\hline & 9999. & 9999. & 9999. & 1242 . & 1247. & 1247. \\
\hline & 1239. & 1221. & 1209. & 1196. & 1194. & 1194. \\
\hline 18 & 9999. & 9999. & 9999. & 9999. & 9999. & 9999. \\
\hline & 9999. & 9999. & 9999. & 9999. & 1245. & 1242 . \\
\hline & 1235. & 1223. & 1208. & 1195. & 1195. & 1194 \\
\hline
\end{tabular}

INITIAL HEAD FOR LAYER 2

READING ON UNIT 41 WITH FORMAT: (18F10.2)

\begin{tabular}{|c|c|c|c|c|c|c|}
\hline & 1 & 2 & 3 & 4 & 5 & 6 \\
\hline & 7 & 8 & 9 & 10 & 11 & 12 \\
\hline & 13 & 14 & 15 & 16 & 17 & 18 \\
\hline 1 & 0.000 & 466.7 & 970.9 & 979.2 & 979.5 & 980.1 \\
\hline & 1025. & 1124. & 1184. & 1186. & 1187. & 9999. \\
\hline & 9999. & 9999. & 9999. & 9999. & 9999. & 9999. \\
\hline 2 & 0.000 & 460.5 & 968.8 & 979.0 & 979.2 & 979.8 \\
\hline & 1015. & 1103. & 1171. & 1186. & 1187. & 1189. \\
\hline & 9999. & 9999. & 9999. & 9999. & 9999. & 9999. \\
\hline & & & & . & & \\
\hline & & & & - & & \\
\hline 17 & 9999. & 9999. & 9999. & 9999. & 9999. & 9999. \\
\hline & 9999. & 9999. & 9999. & 1242 . & 1247. & 1247 \\
\hline & 1239. & 1221 . & 1209. & 1196. & 1194. & 1194. \\
\hline 18 & 9999. & 9999. & 9999. & 9999. & 9999. & 9999. \\
\hline & 9999. & 9999. & 9999. & 9999. & 1245. & 1242. \\
\hline & 1235. & 1223. & 1208. & 1195. & 1195. & 1194 \\
\hline
\end{tabular}

INITIAL HEAD FOR LAYER 3

READING ON UNIT 41 WITH FORMAT: (18F10.2)

4

5

6 
APPENDIX A. EXAMPLE SIMULATIONS - Test Case 2 - LIST Output File

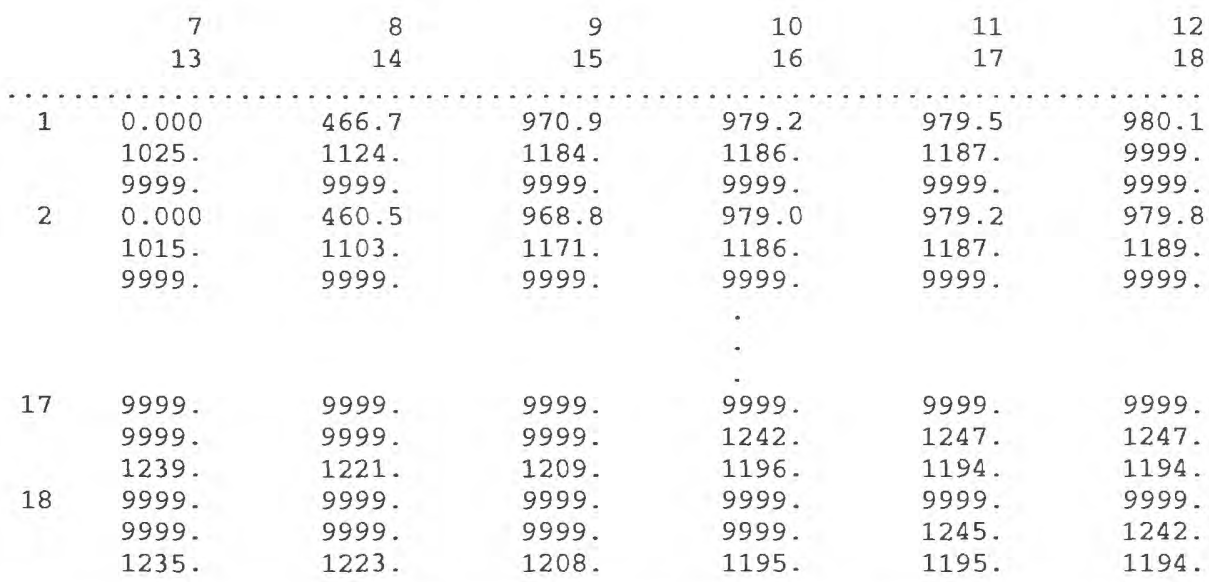

OUTPUT CONTROL IS SPECIFIED ONLY AT TIME STEPS FOR WHICH OUTPUT IS DESIRED HEAD PRINT FORMAT CODE IS 20 DRAWDOWN PRINT FORMAT CODE IS 0 HEADS WILL BE SAVED ON UNIT 13 DRAWDOWNS WILL BE SAVED ON UNIT 0 HK_1

HYD. COND. ALONG ROWS is defined by the following parameters:

HK_ 2

HK_3

HYD. COND. ALONG ROWS FOR LAYER

\begin{tabular}{|c|c|c|c|c|c|c|}
\hline & 1 & 2 & 3 & 4 & 5 & 6 \\
\hline & 7 & 8 & 9 & 10 & 11 & 12 \\
\hline & 13 & 14 & 15 & 16 & 17 & 18 \\
\hline 1 & $1.0000 \mathrm{E}-02$ & $1.0000 \mathrm{E}-04$ & $1.0000 \mathrm{E}-02$ & 1.000 & 1.000 & 1.000 \\
\hline & $1.0000 \mathrm{E}-02$ & $1.0000 \mathrm{E}-02$ & 1.000 & 1.000 & 1.000 & 0.000 \\
\hline & 0.000 & 0.000 & 0.000 & 0.000 & 0.000 & 0.000 \\
\hline 2 & $1.0000 \mathrm{E}-02$ & $1.0000 \mathrm{E}-04$ & $1.0000 \mathrm{E}-02$ & 1.000 & 1.000 & 1.000 \\
\hline & $1.0000 \mathrm{E}-02$ & $1.0000 \mathrm{E}-02$ & $1.0000 \mathrm{E}-02$ & 1.000 & 1.000 & 1.000 \\
\hline & 0.000 & 0.000 & 0.000 & 0.000 & 0.000 & 0.000 \\
\hline & & & & - & & \\
\hline & & & & . & & \\
\hline 17 & 0.000 & 0.000 & 0.000 & 0.000 & 0.000 & 0.000 \\
\hline & 0.000 & 0.000 & 0.000 & $1.0000 \mathrm{E}-02$ & $1.0000 \mathrm{E}-04$ & $1.0000 \mathrm{E}$ \\
\hline & 1. $.0000 \mathrm{E}-04$ & $1.0000 E-04$ & $1.0000 \mathrm{E}-04$ & $1.0000 \mathrm{E}-02$ & $1.0000 E-02$ & 1. $.0000 \mathrm{E}-02$ \\
\hline 18 & 0.000 & 0.000 & 0.000 & 0.000 & 0.000 & 0.000 \\
\hline & 0.000 & 0.000 & 0.000 & 0.000 & $1.0000 \mathrm{E}-04$ & $1.0000 \mathrm{E}-04$ \\
\hline & $1.0000 \mathrm{E}-04$ & $1.0000 \mathrm{E}-04$ & $1.0000 \mathrm{E}-04$ & $1.0000 \mathrm{E}-02$ & $1.0000 \mathrm{E}-02$ & $1.0000 \mathrm{E}-02$ \\
\hline
\end{tabular}

HORIZ. TO VERTICAL ANI. is defined by the following parameters: ANIV_12

HORIZ. TO VERTICAL ANI. $=4.00002$ FOR LAYER 1 HK_1

HYD. COND. ALONG ROWS is defined by the following parameters:

HK_2

HK_3

HK_4

HYD. COND. ALONG ROWS FOR LAYER 2

\begin{tabular}{|c|c|c|c|c|c|}
\hline 1 & 2 & 3 & 4 & 5 & 6 \\
\hline 7 & 8 & 9 & 10 & 11 & 12 \\
\hline 13 & 14 & 15 & 16 & 17 & 18 \\
\hline $\begin{array}{l}1.0000 \mathrm{E}-04 \\
1.0000 \mathrm{E}-04\end{array}$ & $\begin{array}{l}1.0000 \mathrm{E}-04 \\
1.0000 \mathrm{E}-04\end{array}$ & $\begin{array}{l}1.0000 E-04 \\
1.0000 E-04\end{array}$ & $\begin{array}{l}1.0000 \mathrm{E}-04 \\
1.0000 \mathrm{E}-04\end{array}$ & $\begin{array}{l}1.0000 \mathrm{E}-04 \\
1.0000 \mathrm{E}-04\end{array}$ & $\begin{array}{c}1.0000 \mathrm{E}-04 \\
0.000\end{array}$ \\
\hline
\end{tabular}




\section{APPENDIX A. EXAMPLE SIMULATIONS - Test Case 2 - LIST Output File}

$\begin{array}{ccccccc} & 0.000 & 0.000 & 0.000 & 0.000 & 0.000 & 0.000 \\ 1.0000 \mathrm{E}-04 & 1.0000 \mathrm{E}-04 & 1.0000 \mathrm{E}-04 & 1.0000 \mathrm{E}-04 & 1.0000 \mathrm{E}-04 & 1.0000 \mathrm{E}-04 \\ & 1.0000 \mathrm{E}-04 & 1.0002 \mathrm{E}-06 & 1.0002 \mathrm{E}-06 & 1.0002 \mathrm{E}-06 & 1.0000 \mathrm{E}-04 & 1.0000 \mathrm{E}-04 \\ & 0.000 & 0.000 & 0.000 & 0.000 & 0.000 & 0.000 \\ & & & & . & & \\ & & & & & & \\ 17 & 0.000 & 0.000 & 0.000 & 0.000 & 0.000 & 0.000 \\ & 0.000 & 0.000 & 0.000 & 1.0000 \mathrm{E}-04 & 1.0000 \mathrm{E}-04 & 1.0000 \mathrm{E}-04 \\ & 1.0000 \mathrm{E}-04 & 1.0000 \mathrm{E}-04 & 1.0000 \mathrm{E}-04 & 1.0000 \mathrm{E}-04 & 1.0000 \mathrm{E}-04 & 1.0000 \mathrm{E}-02 \\ 18 & 0.000 & 0.000 & 0.000 & 0.000 & 0.000 & 0.000 \\ & 0.000 & 0.000 & 0.000 & 0.000 & 1.0000 \mathrm{E}-04 & 1.0000 \mathrm{E}-04 \\ & 1.0000 \mathrm{E}-04 & 1.0000 \mathrm{E}-04 & 1.0000 \mathrm{E}-04 & 1.0000 \mathrm{E}-04 & 1.0000 \mathrm{E}-04 & 1.0000 \mathrm{E}-02\end{array}$

HORIZ. TO VERTICAL ANI. is defined by the following parameters: ANIV_12

HORIZ. TO VERTICAL ANI. $=4.00002$ FOR LAYER 2

HK_1

HYD. COND. ALONG ROWS is defined by the following parameters:

HK_3

HK_4

HYD. COND. ALONG ROWS FOR LAYER 3

\begin{tabular}{|c|c|c|c|c|c|c|}
\hline & 1 & 2 & 3 & 4 & 5 & 6 \\
\hline & 7 & 8 & 9 & 10 & 11 & 12 \\
\hline & 13 & 14 & 15 & 16 & 17 & 18 \\
\hline 1 & $1.0000 \mathrm{E}-04$ & $1.0002 \mathrm{E}-06$ & $1.0002 \mathrm{E}-06$ & $1.0000 \mathrm{E}-04$ & $1.0000 \mathrm{E}-04$ & $1.0000 E-04$ \\
\hline & $\begin{array}{l}1.0000 \mathrm{E}-04 \\
0.000\end{array}$ & $\begin{array}{l}1.0000 \mathrm{E}-04 \\
0.000\end{array}$ & $\begin{array}{l}1.0000 \mathrm{E}-04 \\
0.000\end{array}$ & $\begin{array}{l}1.0000 E-04 \\
0.000\end{array}$ & $\begin{array}{l}1.0000 E-04 \\
0.000\end{array}$ & $\begin{array}{l}0.000 \\
0.000\end{array}$ \\
\hline 2 & $1.0000 \mathrm{E}-04$ & $1.0002 \mathrm{E}-06$ & $1.0002 \mathrm{E}-06$ & $1.0002 \mathrm{E}-06$ & $1.0000 \mathrm{E}-04$ & $1.0000 E-04$ \\
\hline & $1.0000 \mathrm{E}-04$ & $1.0002 \mathrm{E}-06$ & $1.0002 \mathrm{E}-06$ & $1.0002 \mathrm{E}-06$ & $\begin{array}{l}1.0000 \mathrm{E}-04 \\
0.000\end{array}$ & $\begin{array}{c}1.0000 E-04 \\
0.000\end{array}$ \\
\hline & & & & . & & \\
\hline 17 & 0.000 & 0.000 & 0.000 & 0.000 & 0.000 & 0.000 \\
\hline & 0.000 & 0.000 & 0.000 & $1.0000 \mathrm{E}-04$ & $1.0000 \mathrm{E}-04$ & $1.0000 E-04$ \\
\hline & $1.0000 \mathrm{E}-04$ & $1.0000 \mathrm{E}-04$ & $1.0000 \mathrm{E}-04$ & $1.0000 \mathrm{E}-04$ & $1.0000 \mathrm{E}-04$ & $1.0000 \mathrm{E}-04$ \\
\hline 18 & 0.000 & 0.000 & 0.000 & 0.000 & 0.000 & 0.000 \\
\hline & 0.000 & 0.000 & 0.000 & 0.000 & $1.0000 \mathrm{E}-04$ & $1.0000 \mathrm{E}-04$ \\
\hline & $1.0000 \mathrm{E}-04$ & $1.0000 \mathrm{E}-04$ & $1.0000 \mathrm{E}-04$ & $1.0000 \mathrm{E}-04$ & $1.0000 E-04$ & $1.0000 E-04$ \\
\hline
\end{tabular}

HORIZ. TO VERTICAL ANI. is defined by the following parameters: ANIV_3

HORIZ. TO VERTICAL ANI. $=1.00006$ FOR LAYER 3

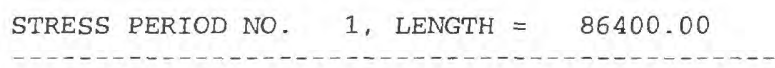

NUMBER OF TIME STEPS $=1$

MULTIPLIER FOR DELT $=1.000$

INITIAL TIME STEP SIZE $=86400.00$

\begin{tabular}{ccccr} 
WELL NO. LAYER & ROW & COL & STRESS RA \\
\hline 1 & 1 & 9 & 7 & -100.0 \\
2 & 1 & 8 & 16 & -200.0 \\
3 & 1 & 11 & 13 & -150.0
\end{tabular}

3 WELLS 


\begin{tabular}{cccccc}
$\begin{array}{l}\text { Parameter: } \\
\text { DRAIN NO. }\end{array}$ & $\begin{array}{l}\text { C_DRN } \\
\text { LAYER }\end{array}$ & ROW & COL & DRAIN EL. & CONDUCTANCE \\
\hline 1 & 1 & 7 & 6 & 400.0 & 1.000 \\
2 & 1 & 10 & 11 & 550.0 & 1.000 \\
3 & 1 & 14 & 14 & 1200. & 1.000 \\
4 & 1 & 15 & 14 & 1200. & 1.000 \\
5 & 1 & 16 & 14 & 1200. & 1.000
\end{tabular}

5 DRAINS

ET SURFACE $=1000.00$

EVTR array defined by the following parameters:

Parameter: ETMAX

EVAPOTRANSPIRATION RATE

\begin{tabular}{|c|c|c|c|c|c|c|}
\hline & 1 & 2 & 3 & 4 & 5 & 6 \\
\hline & 7 & 8 & 9 & 10 & 11 & 12 \\
\hline & 13 & 14 & 15 & 16 & 17 & 18 \\
\hline & $\ldots \ldots$ & $\ldots \ldots$ & $\ldots \ldots$ & $\ldots \ldots$ & $\ldots \ldots$ & $\ldots \ldots$ \\
\hline \multirow[t]{3}{*}{1} & 0.000 & 0.000 & 0.000 & 0.000 & 0.000 & 0.000 \\
\hline & 0.000 & 0.000 & 0.000 & 0.000 & 0.000 & 0.000 \\
\hline & 0.000 & 0.000 & 0.000 & 0.000 & 0.000 & 0.000 \\
\hline \multirow[t]{3}{*}{2} & 0.000 & 0.000 & 0.000 & 0.000 & 0.000 & 0.000 \\
\hline & 0.000 & 0.000 & 0.000 & 0.000 & 0.000 & 0.000 \\
\hline & 0.000 & 0.000 & 0.000 & 0.000 & 0.000 & 0.000 \\
\hline \multirow[t]{3}{*}{3} & 0.000 & 0.000 & 0.000 & 0.000 & 0.000 & 0.000 \\
\hline & 0.000 & 0.000 & 0.000 & 0.000 & 0.000 & 0.000 \\
\hline & 0.000 & 0.000 & 0.000 & 0.000 & 0.000 & 0.000 \\
\hline \multirow[t]{3}{*}{4} & 0.000 & 0.000 & 0.000 & 0.000 & 0.000 & 0.000 \\
\hline & 0.000 & 0.000 & 0.000 & 0.000 & 0.000 & 0.000 \\
\hline & 0.000 & 0.000 & 0.000 & 0.000 & 0.000 & 0.000 \\
\hline \multirow[t]{3}{*}{5} & 0.000 & 0.000 & 0.000 & 0.000 & 0.000 & 0.000 \\
\hline & 0.000 & 0.000 & 0.000 & 0.000 & 0.000 & 0.000 \\
\hline & 0.000 & 0.000 & 0.000 & 0.000 & 0.000 & 0.000 \\
\hline \multirow[t]{3}{*}{6} & 0.000 & 0.000 & 0.000 & 0.000 & 0.000 & $3.9997 E-04$ \\
\hline & $3.9997 E-04$ & $3.9997 E-04$ & 0.000 & 0.000 & 0.000 & 0.000 \\
\hline & 0.000 & 0.000 & 0.000 & 0.000 & 0.000 & 0.000 \\
\hline \multirow[t]{3}{*}{7} & 0.000 & 0.000 & 0.000 & 0.000 & 0.000 & $3.9997 E-04$ \\
\hline & $3.9997 E-04$ & $3.9997 E-04$ & 0.000 & 0.000 & 0.000 & 0.000 \\
\hline & 0.000 & 0.000 & 0.000 & 0.000 & 0.000 & 0.000 \\
\hline \multirow[t]{3}{*}{8} & 0.000 & 0.000 & 0.000 & 0.000 & 0.000 & $3.9997 E-04$ \\
\hline & $3.9997 \mathrm{E}-04$ & $3.9997 \mathrm{E}-04$ & 0.000 & 0.000 & 0.000 & 0.000 \\
\hline & 0.000 & 0.000 & 0.000 & 0.000 & 0.000 & 0.000 \\
\hline \multirow[t]{3}{*}{9} & 0.000 & 0.000 & 0.000 & 0.000 & 0.000 & $3.9997 \mathrm{E}-04$ \\
\hline & $3.9997 E-04$ & $3.9997 E-04$ & 0.000 & 0.000 & 0.000 & 0.000 \\
\hline & 0.000 & 0.000 & 0.000 & 0.000 & 0.000 & 0.000 \\
\hline \multirow[t]{3}{*}{10} & 0.000 & 0.000 & 0.000 & 0.000 & 0.000 & 0.000 \\
\hline & 0.000 & 0.000 & 0.000 & 0.000 & 0.000 & 0.000 \\
\hline & 0.000 & 0.000 & 0.000 & 0.000 & 0.000 & 0.000 \\
\hline \multirow[t]{3}{*}{11} & 0.000 & 0.000 & 0.000 & 0.000 & 0.000 & 0.000 \\
\hline & 0.000 & 0.000 & 0.000 & 0.000 & 0.000 & 0.000 \\
\hline & 0.000 & 0.000 & 0.000 & 0.000 & 0.000 & 0.000 \\
\hline \multirow[t]{3}{*}{12} & 0.000 & 0.000 & 0.000 & 0.000 & 0.000 & 0.000 \\
\hline & 0.000 & 0.000 & 0.000 & 0.000 & 0.000 & 0.000 \\
\hline & 0.000 & 0.000 & 0.000 & 0.000 & 0.000 & 0.000 \\
\hline \multirow[t]{3}{*}{13} & 0.000 & 0.000 & 0.000 & 0.000 & 0.000 & 0.000 \\
\hline & 0.000 & 0.000 & 0.000 & 0.000 & 0.000 & 0.000 \\
\hline & 0.000 & 0.000 & 0.000 & 0.000 & 0.000 & 0.000 \\
\hline \multirow[t]{3}{*}{14} & 0.000 & 0.000 & 0.000 & 0.000 & 0.000 & 0.000 \\
\hline & 0.000 & 0.000 & 0.000 & 0.000 & 0.000 & 0.000 \\
\hline & 0.000 & 0.000 & 0.000 & 0.000 & 0.000 & 0.000 \\
\hline \multirow[t]{3}{*}{15} & 0.000 & 0.000 & 0.000 & 0.000 & 0.000 & 0.000 \\
\hline & 0.000 & 0.000 & 0.000 & 0.000 & 0.000 & 0.000 \\
\hline & 0.000 & 0.000 & 0.000 & 0.000 & 0.000 & 0.000 \\
\hline 16 & 0.000 & 0.000 & 0.000 & 0.000 & 0.000 & 0.000 \\
\hline
\end{tabular}


APPENDIX A. EXAMPLE SIMULATIONS - Test Case 2 - LIST Output File

\begin{tabular}{lllllll} 
& 0.000 & 0.000 & 0.000 & 0.000 & 0.000 & 0.000 \\
\multirow{3}{*}{17} & 0.000 & 0.000 & 0.000 & 0.000 & 0.000 & 0.000 \\
& 0.000 & 0.000 & 0.000 & 0.000 & 0.000 & 0.000 \\
& 0.000 & 0.000 & 0.000 & 0.000 & 0.000 & 0.000 \\
& 0.000 & 0.000 & 0.000 & 0.000 & 0.000 & 0.000 \\
& 0.000 & 0.000 & 0.000 & 0.000 & 0.000 & 0.000 \\
& 0.000 & 0.000 & 0.000 & 0.000 & 0.000 & 0.000 \\
& 0.000 & 0.000 & 0.000 & 0.000 & 0.000 & 0.000
\end{tabular}

EXTINCTION DEPTH $=50.0000$

\begin{tabular}{cccccc} 
Parameter: & C_GHB & & \\
BOUND. NO. LAYER & ROW & COL & STAGE & CONDUCTANCE \\
\hline 1 & 1 & 3 & 6 & 350.0 & 1.000 \\
2 & 1 & 3 & 11 & 500.0 & 1.000 \\
3 & 1 & 4 & 11 & 500.0 & 1.000 \\
4 & 1 & 5 & 11 & 500.0 & 1.000 \\
5 & 1 & 12 & 9 & 1000. & 1.000
\end{tabular}

5 GHB CELLS

RECH array defined by the following parameters:

Parameter: RCHRAT

RECHARGE

\begin{tabular}{|c|c|c|c|c|c|c|}
\hline & 1 & 2 & 3 & 4 & 5 & 6 \\
\hline & 7 & 8 & 9 & 10 & 11 & 12 \\
\hline & 13 & 14 & 15 & 16 & 17 & 18 \\
\hline 1 & 0.000 & 0.000 & 0.000 & 0.000 & 0.000 & 0.000 \\
\hline & 0.000 & 0.000 & 0.000 & 0.000 & 0.000 & 0.000 \\
\hline & 0.000 & 0.000 & 0.000 & 0.000 & 0.000 & 0.000 \\
\hline 2 & 0.000 & 0.000 & 0.000 & 0.000 & 0.000 & 0.000 \\
\hline & 0.000 & 0.000 & 0.000 & 0.000 & 0.000 & 0.000 \\
\hline & 0.000 & 0.000 & 0.000 & 0.000 & 0.000 & 0.000 \\
\hline 3 & 0.000 & 0.000 & 0.000 & 0.000 & 0.000 & 0.000 \\
\hline & 0.000 & 0.000 & 0.000 & 0.000 & 0.000 & 0.000 \\
\hline & 0.000 & 0.000 & 0.000 & 0.000 & 0.000 & 0.000 \\
\hline 4 & 0.000 & 0.000 & 0.000 & 0.000 & 0.000 & 0.000 \\
\hline & 0.000 & 0.000 & 0.000 & 0.000 & 0.000 & 0.000 \\
\hline & 0.000 & 0.000 & 0.000 & 0.000 & 0.000 & 0.000 \\
\hline 5 & 0.000 & 0.000 & 0.000 & 0.000 & 0.000 & 0.000 \\
\hline & 0.000 & 0.000 & 0.000 & 0.000 & 0.000 & 0.000 \\
\hline & 0.000 & 0.000 & 0.000 & 0.000 & 0.000 & 0.000 \\
\hline 6 & 0.000 & 0.000 & 0.000 & 0.000 & 0.000 & 0.000 \\
\hline & 0.000 & 0.000 & 0.000 & 0.000 & 0.000 & 0.000 \\
\hline & 0.000 & 0.000 & 0.000 & 0.000 & 0.000 & 0.000 \\
\hline 7 & 0.000 & 0.000 & 0.000 & 0.000 & 0.000 & 0.000 \\
\hline & 0.000 & 0.000 & 0.000 & 0.000 & 0.000 & 0.000 \\
\hline & 0.000 & 0.000 & 0.000 & 0.000 & 0.000 & 0.000 \\
\hline 8 & 0.000 & 0.000 & 0.000 & 0.000 & 0.000 & 0.000 \\
\hline & 0.000 & 0.000 & 0.000 & 0.000 & 0.000 & 0.000 \\
\hline & 0.000 & 0.000 & 0.000 & 0.000 & 0.000 & 0.000 \\
\hline 9 & 0.000 & 0.000 & 0.000 & 0.000 & 0.000 & 0.000 \\
\hline & 0.000 & 0.000 & 0.000 & 0.000 & 0.000 & $3.1000 \mathrm{E}-04$ \\
\hline & $3.1000 \mathrm{E}-04$ & $3.1000 E-04$ & 0.000 & 0.000 & 0.000 & 0.000 \\
\hline 10 & 0.000 & 0.000 & 0.000 & 0.000 & 0.000 & 0.000 \\
\hline & 0.000 & 0.000 & 0.000 & 0.000 & 0.000 & $3.1000 \mathrm{E}-04$ \\
\hline & $3.1000 \mathrm{E}-04$ & $3.1000 E-04$ & $3.1000 \mathrm{E}-04$ & 0.000 & 0.000 & 0.000 \\
\hline 11 & 0.000 & 0.000 & 0.000 & 0.000 & 0.000 & 0.000 \\
\hline & 0.000 & 0.000 & 0.000 & 0.000 & 0.000 & $3.1000 \mathrm{E}-04$ \\
\hline & $3.1000 \mathrm{E}-04$ & $3.1000 \mathrm{E}-04$ & $3.1000 \mathrm{E}-04$ & 0.000 & 0.000 & 0.000 \\
\hline 12 & 0.000 & 0.000 & 0.000 & 0.000 & 0.000 & 0.000 \\
\hline & 0.000 & 0.000 & 0.000 & 0.000 & 0.000 & $3.1000 \mathrm{E}-04$ \\
\hline & $3.1000 E-04$ & $3.1000 E-04$ & $3.1000 \mathrm{E}-04$ & 0.000 & 0.000 & 0.000 \\
\hline 13 & 0.000 & 0.000 & 0.000 & 0.000 & 0.000 & 0.000 \\
\hline
\end{tabular}


APPENDIX A. EXAMPLE SIMULATIONS - Test Case 2 - LIST Output File

\begin{tabular}{ccccccc} 
& 0.000 & 0.000 & 0.000 & 0.000 & 0.000 & $3.1000 \mathrm{E}-04$ \\
\multirow{1}{*}{14} & $3.1000 \mathrm{E}-04$ & $3.1000 \mathrm{E}-04$ & $3.1000 \mathrm{E}-04$ & 0.000 & 0.000 & 0.000 \\
& 0.000 & 0.000 & 0.000 & 0.000 & 0.000 & 0.000 \\
& 0.000 & 0.000 & 0.000 & 0.000 & 0.000 & 0.000 \\
15 & 0.000 & 0.000 & 0.000 & 0.000 & 0.000 & 0.000 \\
& 0.000 & 0.000 & 0.000 & 0.000 & 0.000 & 0.000 \\
& 0.000 & 0.000 & 0.000 & 0.000 & 0.000 & 0.000 \\
& 0.000 & 0.000 & 0.000 & 0.000 & 0.000 & 0.000 \\
16 & 0.000 & 0.000 & 0.000 & 0.000 & 0.000 & 0.000 \\
& 0.000 & 0.000 & 0.000 & 0.000 & 0.000 & 0.000 \\
17 & 0.000 & 0.000 & 0.000 & 0.000 & 0.000 & 0.000 \\
& 0.000 & 0.000 & 0.000 & 0.000 & 0.000 & 0.000 \\
& 0.000 & 0.000 & 0.000 & 0.000 & 0.000 & 0.000 \\
& 0.000 & 0.000 & 0.000 & 0.000 & 0.000 & 0.000 \\
& 0.000 & 0.000 & 0.000 & 0.000 & 0.000 & 0.000 \\
& 0.000 & 0.000 & 0.000 & 0.000 & 0.000 & 0.000 \\
& 0.000 & 0.000 & 0.000 & 0.000 & 0.000 & 0.000
\end{tabular}

SOLVING FOR HEAD

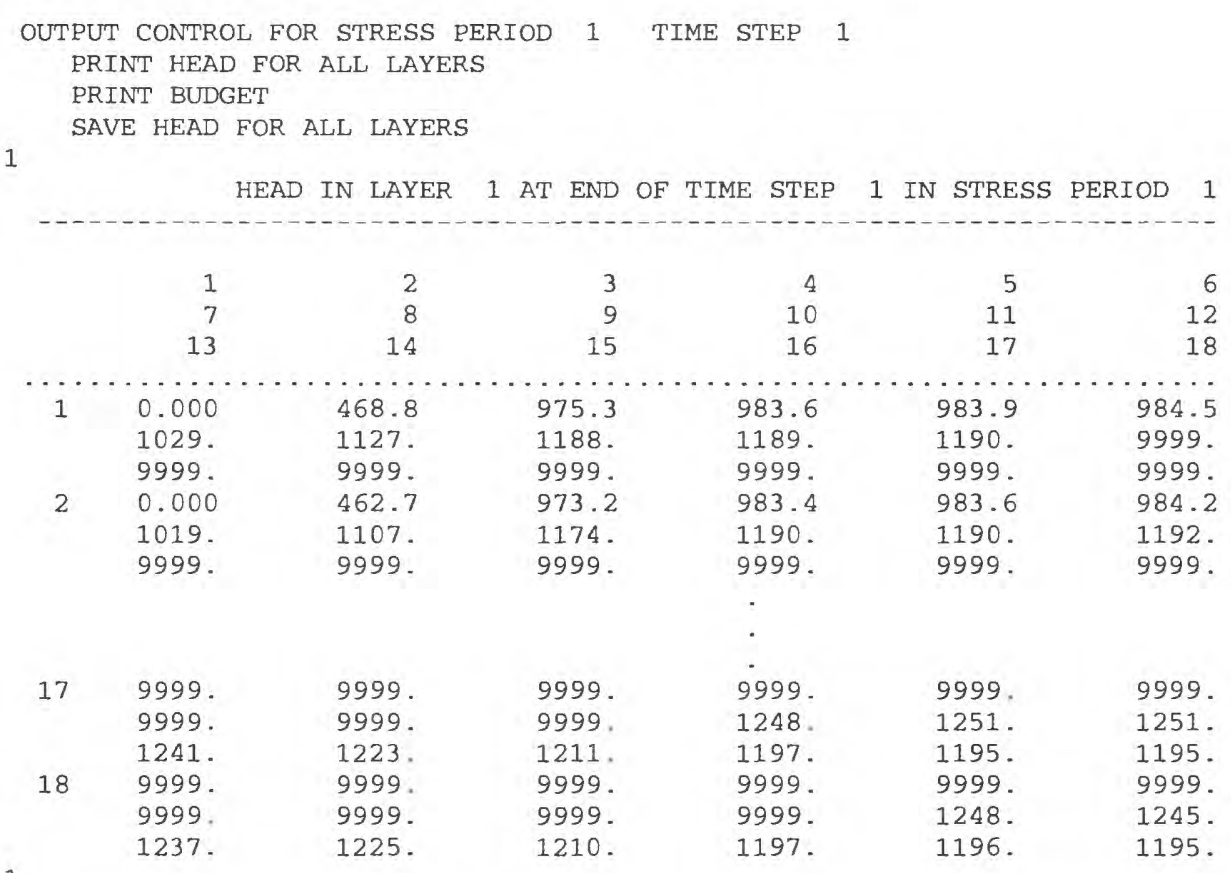

1 HEAD IN LAYER 2 AT END OF TIME STEP 1 IN STRESS PERIOD 1

\begin{tabular}{|c|c|c|c|c|c|c|}
\hline & 1 & 2 & 3 & 4 & 5 & 6 \\
\hline & 7 & 8 & 9 & 10 & 11 & 12 \\
\hline & 13 & 14 & 15 & 16 & 17 & 18 \\
\hline \multirow[t]{3}{*}{1} & 0.000 & 430.8 & 828.8 & 947.7 & 979.0 & 996.6 \\
\hline & 1035. & 1105. & 1155. & 1173. & 1182. & 9999. \\
\hline & 9999. & 9999. & 9999. & 9999. & 9999. & 9999. \\
\hline \multirow[t]{4}{*}{2} & 0.000 & 422.0 & 819.2 & 937.8 & 975.3 & 989.8 \\
\hline & 1013. & 1080 . & 1145. & 1174. & 1187. & 1189. \\
\hline & 9999. & 9999. & 9999. & 9999. & 9999. & 9999. \\
\hline & & & & - & & \\
\hline \multirow{3}{*}{17} & 9999. & 9999. & 9999. & 9999. & 9999. & 9999. \\
\hline & 9999. & 9999. & 9999. & 1250. & 1252. & 1250. \\
\hline & 1242. & 1228 . & 1216. & 1204. & 1198. & 1195. \\
\hline \multirow[t]{3}{*}{18} & 9999. & 9999. & 9999. & 9999. & 9999. & 9999. \\
\hline & 9999. & 9999. & 9999. & 9999. & 1248. & 1245. \\
\hline & 1238. & 1227. & 1215. & 1203. & 1198. & 1195. \\
\hline
\end{tabular}




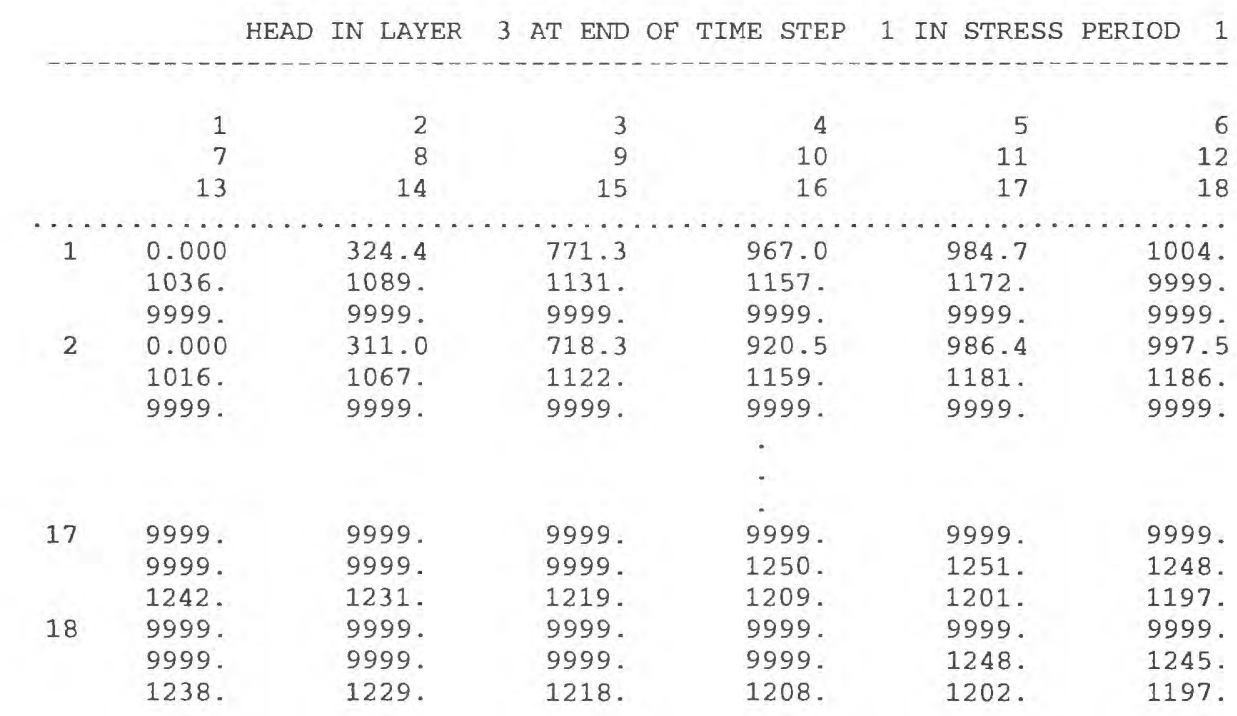

VOLUMETRIC BUDGET FOR ENTIRE MODEL AT END OF TIME STEP 1 IN STRESS PERIOD 1

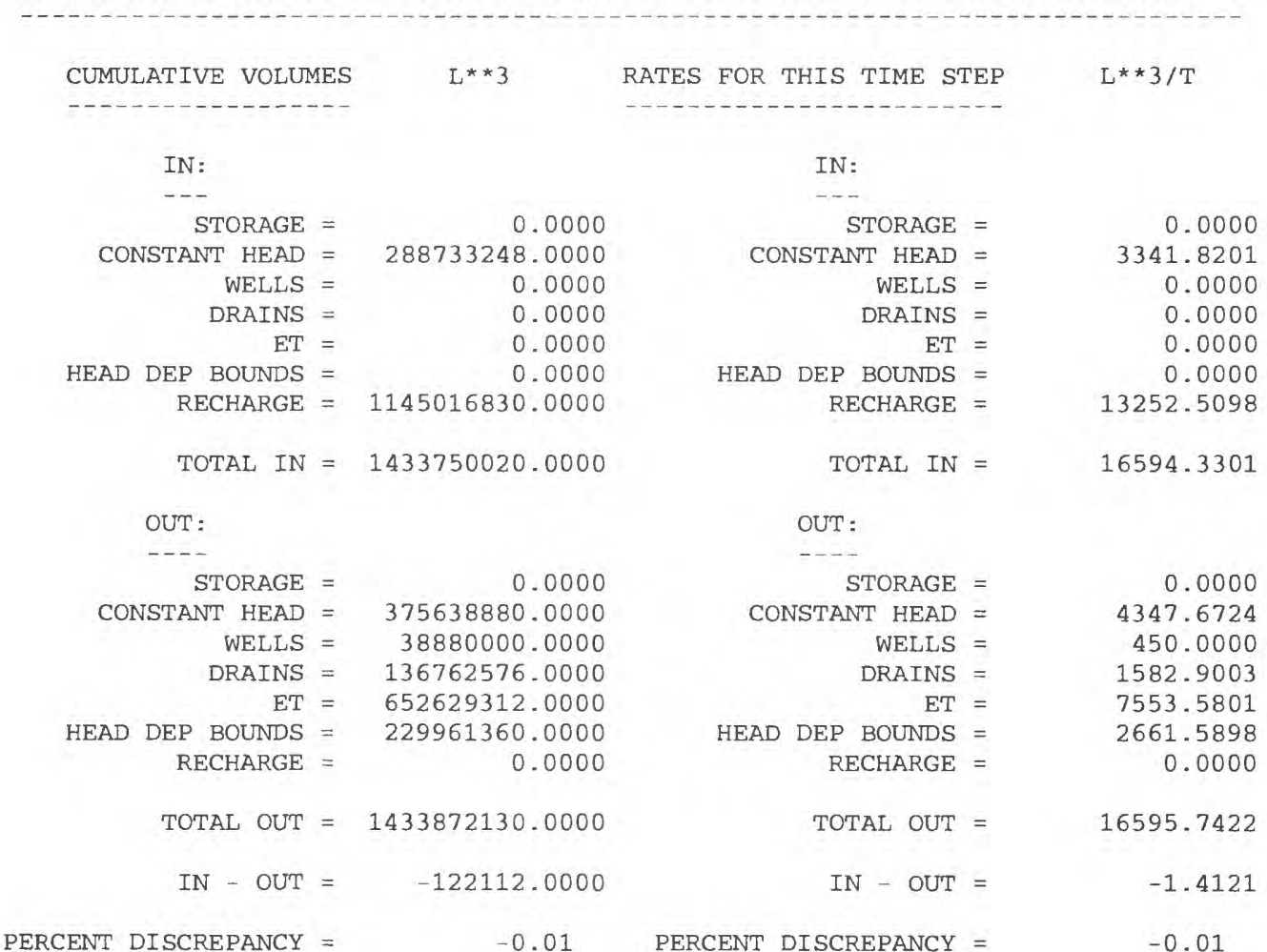

PERCENT DISCREPANCY =

$-0.01$

ERCENT DISCREPANCY = 
APPENDIX A. EXAMPLE SIMULATIONS - Test Case 2 - LIST Output File

DATA AT HEAD LOCATIONS

\begin{tabular}{|c|c|c|c|c|c|c|}
\hline OBS\# & $\begin{array}{l}\text { OBSERVATION } \\
\text { NAME }\end{array}$ & $\begin{array}{l}\text { MEAS. } \\
\text { HEAD }\end{array}$ & $\begin{array}{l}\text { CALC. } \\
\text { HEAD }\end{array}$ & RESIDUAL & WEIGHT** .5 & $\begin{array}{l}\text { WEIGHTED } \\
\text { RESIDUAL }\end{array}$ \\
\hline 1 & W2L & 983.424 & 983.425 & $-0.104 \mathrm{E}-02$ & 0.200 & $-0.208 E-03$ \\
\hline 2 & WL2 & 1019.315 & 1019.316 & $-0.916 \mathrm{E}-03$ & 0.200 & $-0.183 E-03$ \\
\hline 3 & WL2 & 1189.710 & 1189.711 & $-0.977 E-03$ & 0.200 & $-0.195 \mathrm{E}-03$ \\
\hline 4 & WL4 & 294.081 & 294.080 & $0.854 E-03$ & 0.200 & $0.171 \mathrm{E}-03$ \\
\hline 5 & WL4 & 969.420 & 969.421 & $-0.110 \mathrm{E}-02$ & 0.200 & $-0.220 \mathrm{E}-03$ \\
\hline 6 & WL4 & 1180.158 & 1180.158 & $-0.366 \mathrm{E}-03$ & 0.200 & $-0.732 \mathrm{E}-04$ \\
\hline 7 & WL4 & 1195.562 & 1195.563 & $-0.977 \mathrm{E}-03$ & 0.200 & $-0.195 E-03$ \\
\hline 8 & WL5 & 775.509 & 775.503 & $0.580 \mathrm{E}-02$ & 0.200 & $0.116 \mathrm{E}-02$ \\
\hline 9 & WL6 & 193.527 & 193.527 & $0.275 E-03$ & 0.200 & $0.549 E-04$ \\
\hline 10 & WL6 & 968.365 & 968.359 & $0.653 \mathrm{E}-02$ & 0.200 & $0.131 \mathrm{E}-02$ \\
\hline 11 & WL 6 & 972.933 & 972.935 & $-0.171 \mathrm{E}-02$ & 0.200 & $-0.342 \mathrm{E}-03$ \\
\hline 12 & WL 6 & 1204.389 & 1204.390 & $-0.732 \mathrm{E}-03$ & 0.200 & $-0.146 E-03$ \\
\hline 13 & WL 6 & 1201.081 & 1201.082 & $-0.610 \mathrm{E}-03$ & 0.200 & $-0.122 \mathrm{E}-03$ \\
\hline 14 & WL6 & 1201.518 & 1201.519 & $-0.110 \mathrm{E}-02$ & 0.200 & $-0.220 \mathrm{E}-03$ \\
\hline 15 & WL8 & 216.710 & 216.709 & $0.244 \mathrm{E}-03$ & 0.200 & $0.488 E-04$ \\
\hline 16 & WL 8 & 666.268 & 666.267 & $0.116 \mathrm{E}-02$ & 0.200 & $0.232 E-03$ \\
\hline 17 & WL8 & 1036.441 & 1036.441 & 0.00 & 0.200 & 0.00 \\
\hline 18 & WL8 & 1244.779 & 1244.780 & $-0.610 \mathrm{E}-03$ & 0.200 & $-0.122 \mathrm{E}-03$ \\
\hline 19 & WL8 & 1260.021 & 1260.022 & $-0.110 \mathrm{E}-02$ & 0.200 & $-0.220 \mathrm{E}-03$ \\
\hline 20 & WL8 & 1204.042 & 1204.042 & $-0.122 \mathrm{E}-03$ & 0.200 & $-0.244 E-04$ \\
\hline 21 & WL9 & 459.601 & 459.601 & $0.488 \mathrm{E}-03$ & 0.200 & $0.977 \mathrm{E}-04$ \\
\hline 22 & WL10 & 655.416 & 655.415 & $0.610 \mathrm{E}-03$ & 0.200 & $0.122 \mathrm{E}-03$ \\
\hline 23 & WL10 & 969.059 & 969.058 & $0.488 \mathrm{E}-03$ & 0.200 & $0.977 \mathrm{E}-04$ \\
\hline 24 & WL10 & 1128.703 & 1128.702 & $0.110 \mathrm{E}-02$ & 0.200 & $0.220 \mathrm{E}-03$ \\
\hline 25 & WL10 & 1398.338 & 1398.339 & $-0.122 \mathrm{E}-02$ & 0.200 & $-0.244 \mathrm{E}-03$ \\
\hline 26 & WL10 & 1279.890 & 1279.891 & $-0.122 \mathrm{E}-02$ & 0.200 & $-0.244 \mathrm{E}-03$ \\
\hline 27 & WL10 & 1160.692 & 1160.692 & $0.122 \mathrm{E}-03$ & 0.200 & $0.244 \mathrm{E}-04$ \\
\hline 28 & WL11 & 346.381 & 346.380 & $0.101 \mathrm{E}-02$ & 0.200 & $0.201 \mathrm{E}-03$ \\
\hline 29 & WL12 & 1075.812 & 1075.812 & $0.366 \mathrm{E}-03$ & 0.200 & $0.732 \mathrm{E}-04$ \\
\hline 30 & WL12 & 1316.665 & 1316.665 & 0.00 & 0.200 & 0.00 \\
\hline 31 & WL12 & 1482.124 & 1482.124 & $0.366 \mathrm{E}-03$ & 0.200 & $0.732 \mathrm{E}-04$ \\
\hline 32 & WL12 & 1220.460 & 1220.461 & $-0.854 \mathrm{E}-03$ & 0.200 & $-0.171 E-03$ \\
\hline 33 & WL13 & 1486.043 & 1486.045 & $-0.159 E-02$ & 0.200 & $-0.317 E-03$ \\
\hline 34 & WL13 & 1316.981 & 1316.982 & $-0.610 \mathrm{E}-03$ & 0.200 & $-0.122 \mathrm{E}-03$ \\
\hline 35 & WL14 & 1231.437 & 1231.436 & $0.110 \mathrm{E}-02$ & 0.200 & $0.220 \mathrm{E}-03$ \\
\hline 36 & WL14 & 1408.171 & 1408.171 & $-0.366 E-03$ & 0.200 & $-0.732 \mathrm{E}-04$ \\
\hline 37 & WL14 & 1194.175 & 1194.176 & $-0.610 \mathrm{E}-03$ & 0.200 & $-0.122 \mathrm{E}-03$ \\
\hline 38 & WL15 & 1219.439 & 1219.439 & $-0.244 E-03$ & 0.200 & $-0.488 E-04$ \\
\hline 39 & WL16 & 1265.893 & 1265.892 & $0.488 \mathrm{E}-03$ & 0.200 & $0.977 \mathrm{E}-04$ \\
\hline 40 & WL16 & 1198.671 & 1198.671 & $0.244 \mathrm{E}-03$ & 0.200 & $0.488 \mathrm{E}-04$ \\
\hline 41 & WL18 & 1237.400 & 1237.400 & 0.00 & 0.200 & 0.00 \\
\hline 42 & WL18 & 1195.280 & 1195.281 & $-0.488 E-03$ & 0.200 & $-0.977 \mathrm{E}-04$ \\
\hline
\end{tabular}

STATISTICS FOR HEAD RESIDUALS :

MAXIMUM WEIGHTED RESIDUAL : $0.131 \mathrm{E}-02$ OBS\# 10

MINIMUM WEIGHTED RESIDUAL :-0.342E-03 OBS\# 11

AVERAGE WEIGHTED RESIDUAL : $0.128 \mathrm{E}-04$

\# RESIDUALS $>=0$. : 20

\# RESIDUALS < $0 . \quad$ : $\quad 22$

NUMBER OF RUNS : 13 IN 42 OBSERVATIONS

SUM OF SQUARED WEIGHTED RESIDUALS (HEADS ONLY) $0.41016 \mathrm{E}-05$

DATA FOR FLOWS REPRESENTED USING THE DRAIN PACKAGE

\begin{tabular}{|c|c|c|c|c|c|c|}
\hline OBS\# & $\begin{array}{l}\text { OBSERVATION } \\
\text { NAME }\end{array}$ & $\begin{array}{l}\text { MEAS. } \\
\text { FLOW }\end{array}$ & $\begin{array}{l}\text { CALC. } \\
\text { FLOW }\end{array}$ & RESIDUAL & WEIGHT** .5 & $\begin{array}{l}\text { WEIGHTED } \\
\text { RESIDUAL }\end{array}$ \\
\hline 43 & DRN1 & -573 & -573 & $-0.610 \mathrm{E}-03$ & $0.581 E-02$ & $-0.355 E-05$ \\
\hline 44 & DRN2 & 848 & -848 & $0.183 E-03$ & $0.393 \mathrm{E}-02$ & $0.719 \mathrm{E}-06$ \\
\hline 45 & DRN3 & -135 & -135 & $0.854 E-03$ & $0.247 E-01$ & $0.211 \mathrm{E}-04$ \\
\hline 46 & DRN4 & -19.4 & -19.4 & $0.101 E-03$ & 0.171 & $0.173 E-04$ \\
\hline 7 & DRN5 & .54 & -6.54 & $-0.525 E-05$ & 0.510 & $-0.267 \mathrm{E}-05$ \\
\hline
\end{tabular}


APPENDIX A. EXAMPLE SIMULATIONS - Test Case 2 - LIST Output File

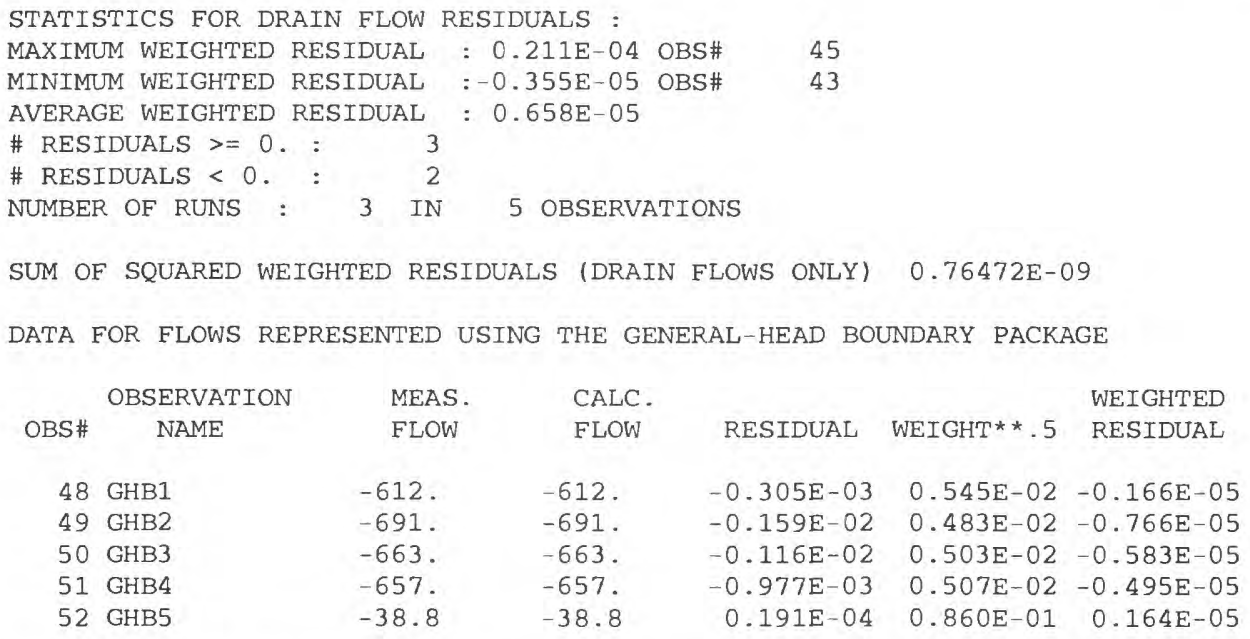

STATISTICS FOR GENERAL-HEAD BOUNDARY FLOW RESIDUALS :

MAXIMUM WEIGHTED RESIDUAL : 0.164E-05 OBS\# 52

MINIMUM WEIGHTED RESIDUAL :-0.766E-05 OBS\# 49

AVERAGE WEIGHTED RESIDUAL :-0.369E-05

\# RESIDUALS $>=0$. : 1

\# RESIDUALS < 0 . : 4

NUMBER OF RUNS : 2 IN 5 OBSERVATIONS

SUM OF SQUARED WEIGHTED RESIDUALS

(GENERAL-HEAD BOUNDARY FLOWS ONLY) $0.12266 \mathrm{E}-09$

SUM OF SQUARED WEIGHTED RESIDUALS (ALL DEPENDENT VARIABLES) 0.41025E-05

STATISTICS FOR ALL RESIDUALS :

AVERAGE WEIGHTED RESIDUAL : 0.106E-04

\# RESIDUALS > = 0

\# RESIDUALS < 0 . : $\quad 28$

NUMBER OF RUNS : 16 IN 52 OBSERVATIONS

INTERPRETTING THE CALCULATED RUNS STATISTIC VALUE OF -2.92 NOTE: THE FOLLOWING APPLIES ONLY IF

\# RESIDUALS $>=0$. IS GREATER THAN 10 AND

\# RESIDUALS < 0 . IS GREATER THAN 10

THE NEGATIVE VALUE MAY INDICATE TOO FEW RUNS:

IF THE VALUE IS LESS THAN - 1.28 , THERE IS LESS THAN A 10 PERCENT CHANCE THE VALUES ARE RANDOM,

IF THE VALUE IS LESS THAN -1.645, THERE IS LESS THAN A 5 PERCENT CHANCE THE VALUES ARE RANDOM,

IF THE VALUE IS LESS THAN -1.96, THERE IS LESS THAN A 2.5 PERCENT CHANCE THE VALUES ARE RANDOM. 


\section{APPENDIX B. PROGRAM DISTRIBUTION, INSTALLATION, AND A HINT FOR EXECUTION}

\section{Distributed Files and Directories}

MODFLOW-2000 can be downloaded from the Internet site listed in the preface. Executable files compiled for several popular operating systems are available for download. When uncompressed, a directory and six subdirectories are created (table B1).

Table BI: Contents of the subdirectories distributed with MODFLOW-2000.

\begin{tabular}{|c|l|}
\hline Subdirectory & \multicolumn{1}{|c|}{ Contents } \\
\hline bin & $\begin{array}{l}\text { Executable files of MODFLOW-2000 and the three post-processing programs, } \\
\text { RESAN-2000, YCINT-2000, and BEALE-2000. These files can be executed by } \\
\text { typing the file name at the operating-system command prompt. The platform } \\
\text { required for the executables is stated on the distribution site. }\end{array}$ \\
\hline data & $\begin{array}{l}\text { Input and output files for test cases, including the two described in Appendix A. } \\
\text { See the cases.txt file in this directory for brief descriptions of other test cases. }\end{array}$ \\
\hline doc & Documentation files, in PDF format. \\
\hline source & $\begin{array}{l}\text { Source-code files for MODFLOW-2000 named with the extension "f'. Files } \\
\text { "param.inc" and "parallel.inc" also contain source code and are referenced from } \\
\text { other source files. }\end{array}$ \\
\hline src-post & $\begin{array}{l}\text { Source-code files for the post-processing programs RESAN-2000, YCINT-2000, } \\
\text { and BEALE-2000. }\end{array}$ \\
\hline test-os & $\begin{array}{l}\text { The contents of this directory are operating-system dependent. The directory } \\
\text { may contain utility programs and files that can be used to run the test cases. The } \\
\text { directory name is formed by substituting the operating-system name for "os". }\end{array}$ \\
\hline
\end{tabular}

\section{Compiling and Linking}

If changes to the source code are required, or if MODFLOW-2000 and the postprocessors will be used with an operating system other than those for which executable files are distributed, the programs need to be compiled. For MODFLOW-2000, all files with the extension ".f" in the "source" directory need to be included in the compilation, with the following exception: Either "para-mpi.f" or "para-non.f" needs to be excluded (see the Parallel Processing section).

The distributed source code is compatible with standard Fortran 90 and Fortran 95, and it complies with the fixed source form, where specific columns are reserved for statement labels, indicator of a continuation line, and Fortran statements. Columns after 72 may be used for comments. See the Memory Requirements section for instructions for converting MODFLOW2000 to conform with FORTRAN 77.

The object files created during compilation must be linked to create an executable program. The linker program commonly is invoked as part of the compilation procedure. Note that the object file created from either "para-mpi.f" or "para-non.f' need to be included in the linking procedure, but not both. 


\section{APPENDIX B. PROGRAM DISTRIBUTION, INSTALLATION, AND A HINT FOR EXECUTION}

\section{Parallel Processing}

The parallel computing capabilities provided with MODFLOW-2000 involve the sensitivity loop of figure 1. Sensitivity and related Observation Process calculations for each parameter are assigned to different processors for simultaneous execution.

Parallelization available with this version of MODFLOW-2000 is implemented with Message Passing Interface (MPI), which is described in several texts, including Pacheco (1997). To use this capability, two things are required. First, MPI needs to be available on the computer being used, and the methods used to compile, load, and run the program need to be coordinated with MPI. The version of MPI needed, and the changes required in the compiling, loading, and running of the program, including the way that MODFLOW-2000 is told how many processors to use, is platform dependent. Users will need to read the relevant MPI documentation or consult their computer personnel for this information. The Compiling and Linking section provides some information related to compiling the program.

Once MPI is available, the parallel-processing capabilities of MODFLOW-2000 need to be enabled. This is accomplished by exchanging one file for another during compilation, by activating one statement in each of two files, and by making sure that the file "mpif.h" exists as needed. If more than 40 processors are to be requested, an additional change is needed. These steps are accomplished as follows.

1. As distributed, the program is compiled with the file "para-non.f", and without "para-mpi.f". To enable parallel processing, the program needs to be compiled with the file "para-mpi.f", and without the file "para-non.f".

2. The statements that need to be activated are in files "mf2k.f" and "obsbas 1.f", and reference the file "mpif.h" in INCLUDE statements. To activate these lines search for "mpif.h" and then remove the "C" in column 1 . The program will search for file "mpif.h", and find it in the MPI library, as needed, as long as no file of that name is present in the local directory. The file "mpif.h" contains specific information that is dependent on the particular implementation of MPI used.

3. File "parallel.inc" is distributed with MODFLOW-2000 and only needs to be changed if the number of processors that might be used is greater than 40 . In this circumstance, variable MAXNP, which is defined on the fourth line of "parallel.inc" in a "PARAMETER" statement needs to be increased in value.

Once MODFLOW-2000 is set up for parallel execution, the computational speedup attained depends on the relation between the number of parameters for which sensitivities are being calculated (equivalent to the number of parameters being estimated if the ParameterEstimation Process is active) and the number of processors used. The greatest possible speedup occurs when the number of processors equals the number of parameters for which sensitivities are to be calculated; additional processors will not improve performance. In this situation, the sensitivities for the entire grid for each parameter are calculated on a separate processor, and execution times are reduced from approximately being proportional to (Hill, 1998, p. 66):

[the time required for a forward solution $] \times[1+$ the number of parameters $]$,

to

[the time required for a forward solution] $\times 2$. 
For twenty parameters, execution times would be reduced by about a factor of 10 . If fewer processors are available, execution times are approximately proportional to

[the time required for a forward solution] $x$

$[1+$ (number of parameters/number of processors) $]$,

with any fraction in the number in parentheses resulting in the number being equal to the next larger integer.

If all processors have the same execution speed, they are used most efficiently if the number of processors divides evenly into the number of parameters. For example, if three processors are used and nine parameters are defined, the number in parentheses will be three and all processors will be used nearly continuously. However, for the same nine parameters and four processors, the number in parentheses will still be three so that the expected time of solution will be the same, but three of the processors will be idle while one performs calculations for the ninth parameter. The situation is shown in table B2.

Table B2: The sequence of calculations performed by MODFLOW-2000 given nine parameters and (A) three and (B) four computer processors.

(A)

\begin{tabular}{|c|c|c|c|}
\hline $\begin{array}{c}\text { Processor } \\
\text { Number }\end{array}$ & \multicolumn{3}{|c|}{$\begin{array}{c}\text { Each processor sequentially calculates sensitivities for the indicated parameters. } \\
\text { The sensitivity loop is executed simultaneously for all parameters in the first } \\
\text { column of parameters, then for all parameters in the second column, and so on. }\end{array}$} \\
\hline 1 & Parameter 1 & Parameter 4 & Parameter 7 \\
\hline 2 & Parameter 2 & Parameter 5 & Parameter 8 \\
\hline 3 & Parameter 3 & Parameter 6 & Parameter 9 \\
\hline
\end{tabular}

(B)

\begin{tabular}{|c|c|c|c|}
\hline $\begin{array}{c}\text { Processor } \\
\text { Number }\end{array}$ & \multicolumn{3}{|c|}{$\begin{array}{c}\text { Each processor sequentially calculates sensitivities for the indicated parameters. } \\
\text { The sensitivity loop is executed simultaneously for all parameters in the first } \\
\text { column of parameters, then for all parameters in the second column, and so on. }\end{array}$} \\
\hline 1 & Parameter 1 & Parameter 5 & Parameter 9 \\
\hline 2 & Parameter 2 & Parameter 6 & (idle) \\
\hline 3 & Parameter 3 & Parameter 7 & (idle) \\
\hline 4 & Parameter 4 & Parameter 9 & (idle) \\
\hline
\end{tabular}

\section{Error Reporting with Parallel Processing Enabled}

Support for parallel processing includes a method for reporting certain errors that MODFLOW-2000 is programmed to recognize. These errors may be caused by problems related to input data or to the nature of the system of equations being solved. The error-reporting method in MODFLOW-2000 is designed to ensure that the user can determine the cause of an error when 
more than one MPI process is used. Note that an "MPI process" is an element of the MessagePassing Interface and is distinct from "process" as used elsewhere in this report.

When MODFLOW-2000 recognizes a fatal error, it writes an error message to the GLOBAL file, the LIST file, or both, and the program stops. In addition, for errors that occur in the sensitivity loop or below in the flow chart (fig. 1), MODFLOW-2000 writes the error message to an error file and informs the user of its existence by writing a message to the screen. If parallel processing is not enabled or if parallel processing is enabled and only one MPI process is being used, one error file named "mf2kerr.p00" is created. If parallel processing is enabled and more than one MPI process is being used, one error file is created for each MPI process. The error files are named "mf2kerr.p\#\#", where "\#\#" is an MPI process number. MPI processes are numbered sequentially starting at zero. Warnings also are written to the "mf 2 kerr.p\#\#" file(s), but do not cause the program to stop. If MODFLOW-2000 runs to completion without encountering an error or warning condition, the mf2kerr.p\#\# files are deleted.

When more than one MPI process is being used, MPI process zero produces the GLOBAL and LIST files. However, MPI processes with numbers greater than zero may produce output that would be written to the GLOBAL or LIST file when parallel processing is not enabled or only one MPI process is being used. Output produced by MPI processes with numbers greater than zero is written to additional files, which are created to facilitate identification of problems. The additional files are called "mf2kglob.p\#\#" and "mf2klist.p\#\#", where "\#\#" is the MPI process number (greater than zero). The "mf2kglob.p\#\#" file contains a subset of the output that would be written to the GLOBAL file when the program is not using multiple MPI processes, and the "mf2klist.p\#\#" file contains a subset of the output that would be written to the LIST file. These files may be of assistance in determining the cause of an error or warning. If MODFLOW2000 runs to completion without encountering an error or warning, the $\mathrm{mf} 2 \mathrm{kglob}$.p\#\# and $\mathrm{mf} 2 \mathrm{klist}$.p\#\# files are deleted. If an error or warning condition is encountered, the existence of these files is made known to the user by a message written to the screen.

\section{Parallel Processing Hints}

In the absence of warnings and errors, when more than one MPI process is used by MODFLOW-2000, the output files are produced by only one MPI process, called the master process. When more than one MPI process is used, individual iterations of the sensitivity loop are executed by different MPI processes. In this situation, output from within the sensitivity loop, for iterations executed by MPI processes other than the master process, is not written to userdefined output files. This behavior is acceptable when the program is used in the ParameterEstimation mode, but not when the program is used to generate full-grid sensitivity arrays. If the program is being used in either the Parameter-Sensitivity mode or the Parameter Sensitivity with Observations mode and the user needs the grid sensitivity arrays, the program needs to be run using only one MPI process or with parallel processing disabled. The same is true if the user needs to see the solver balance from the sensitivity-equation solution for all parameters.

\section{Portability}

The Observation, Sensitivity, and Parameter-Estimation Processes were written in standard Fortran 90. The modular style used is similar to that of previous versions of MODFLOW. Portability is discussed in detail by Harbaugh and others (2000).

\section{Memory Requirements}

As distributed, the source files and executable file dynamically allocate memory for arrays GX, RX, X, IG, IR, IX, GZ, Z, and XHS. Dynamic memory allocation is standard in 
Fortran 90 and Fortran 95, but not in FORTRAN 77. If a user needs to recompile the program but does not have access to a Fortran 90 or Fortran 95 (or later) compiler, the program easily may be converted to standard FORTRAN 77 and compiled. To convert the code to standard FORTRAN 77, the main program unit (file "mf2k.f") must be modified in four locations. The locations in the file where modifications are required may be found by searching for the string "STATIC".

Location 1. Change three lines: (1) Uncomment the PARAMETER statement that declares the Fortran parameters LENGX, LENIG, LENGZ, LENX, LENIX, LENZ, LENRX, LENIR, and LENXHS. (2) Uncomment the DIMENSION statement that dimensions arrays GX, IG, X, IX, RX, IR, GZ, Z, and XHS. (3) Comment out the ALLOCATABLE statement that declares as allocatable arrays GX, IG, X, IX, RX, IR, GZ, Z, and XHS. If, after compilation, the program indicates one or more arrays is dimensioned too small, edit the PARAMETER statement in step 1 to increase the array dimension(s) appropriately, and recompile the program. Note that a "Fortran parameter" is an element of the Fortran programming language and is distinct from "parameter" as used elsewhere in this report.

Location 2. After the call to subroutine GLO1BAS6AL: Comment out the three arithmetic assignment statements that assign values to LENGX, LENGZ, and LENIG and the ALLOCATE statement that dynamically allocates memory for arrays GX, GZ, and IG.

Location 3. After the call to subroutine OBSIBAS6AC: Comment out: (1) the assignment statements that assign LENX, LENZ, and LENIX; (2) the IF...ELSE...ENDIF block in which LENXHS is assigned; and (3) the ALLOCATE statement that dynamically allocates memory for arrays X, Z, IX, and XHS.

Location 4. After the call to subroutine GWF1HFB6AL: Comment out the IF...ENDIF block in which LENRX and LENIR are assigned and the ALLOCATE statement that dynamically allocates memory for arrays RX, and IR.

When these modifications are made, MODFLOW-2000 uses only static memory allocation, and the arrays GX, RX, X, IG, IR, IX, GZ, Z, and XHS need to be dimensioned large enough to provide adequate memory to accommodate the needs of any particular program run the user may want to make. The Fortran parameters LENGX, LENRX, LENX, LENIG, LENIR, LENIX, LENGX, LENZ, and LENXHS provide dimensions for these arrays, as indicated in table B3. If any array is not dimensioned large enough, the program stops with an error message indicating how many elements are required for the array. In this case, set the appropriate dimensioning Fortran parameter to a number at least as large as the number of elements required and recompile the program.

If IUHEAD of the Sensitivity Process input file is less than or equal to zero, sensitivities are stored in memory from one time step to the next and the dimension of XHS, LENXHS, needs to be at least NROW $\times$ NCOL $\times$ NLAY $\times$ MXSEN, where NROW is the number of rows in the model, NCOL is the number of columns, NLAY is the number of layers, and MXSEN is read from item 1 of the Sensitivity Process input file. Specifying IUHEAD less than or equal to zero will minimize the number of file units in simultaneous use by the program and will result in faster execution times. Specifying IUHEAD greater than zero and compiling the program with LENXHS=l will minimize memory usage, but will require that MXSEN sequentially numbered file units be available for use by the program (in addition to those listed in the name file). 
Table B3: Arrays and corresponding dimensioning Fortran parameters in MODFLOW-2000. When the program is converted to FORTRAN 77, only static memory allocation is allowed. In this circumstance, the listed arrays are dimensioned using the listed Fortran parameters prior to compilation.

\begin{tabular}{|c|c|}
\hline Array name & Dimensioning Fortran parameter \\
\hline IG & LENIG \\
\hline IR & LENIR \\
\hline IX & LENIX \\
\hline GX & LENGX \\
\hline RX & LENRX \\
\hline$X$ & LENX \\
\hline XHS & LENXHS \\
\hline GZ & LENGX \\
\hline Z & LENZ \\
\hline
\end{tabular}

Regardless of whether the main program unit is converted to use only static memory allocation or is left as distributed to use dynamic memory allocation, Fortran parameters are used to set static dimensions for some arrays. File "param.inc" specifies dimensions for arrays that contain information related to named MODFLOW-2000 parameters, as noted in table B4.

Table B4: Fortran parameters specified in file "param.inc" that could require adjustment for some problems.

\begin{tabular}{|c|c|c|}
\hline $\begin{array}{c}\text { Fortran } \\
\text { parameter }\end{array}$ & Value as distributed & Description \\
\hline MXPAR & 500 & $\begin{array}{c}\text { Maximum number of parameters that can be defined in all } \\
\text { input files. }\end{array}$ \\
\hline MXCLST & 1000 & $\begin{array}{l}\text { Maximum number of clusters that can be used to define all } \\
\text { array-type parameters (parameters that control model input } \\
\text { specified as NCOL } \times \text { NROW arrays) }\end{array}$ \\
\hline MXZON & 200 & Maximum number of zone arrays that may be defined \\
\hline MXMLT & 200 & Maximum number of multiplier arrays \\
\hline
\end{tabular}




\section{A Hint for Execution}

The programs described in this work all query for the name of the NAME FILE when executed. To avoid the tedium of repeatedly typing this name, on most computer operating systems the program can be executed in a manner that automatically provides the file name to the program. The exact setup is computer dependent. As an example, in a Windows environment the files in table B5 would be needed. In this example, the program could be executed from Windows Explorer by double-clicking on modflow.bat.

Table B5: The files needed to automatically answer MODFLOW-2000's query for the NAME

FILE using, as an example, a Windows computer operating system. All file names could be different.

\begin{tabular}{|c|c|}
\hline \hline File name & $\begin{array}{c}\text { CONTENTS (Literal file names are italicized. Include paths to the files if they } \\
\text { are not located in the currnt directory.) }\end{array}$ \\
\hline modflow.bat & $\mathrm{mf} 2 \mathrm{k}<$ namefile. $f n$ \\
\hline namefile.fn & Name file name \\
\hline
\end{tabular}




\section{APPENDIX C. SUGGESTIONS FOR GRAPHICAL INTERFACE DESIGN}

The Observation, Sensitivity, and Parameter-Estimation Processes produce important information that needs to be displayed graphically to achieve its full potential. Thus, the design of graphical interfaces is important. A variety of display suggestions are mentioned in Hill (1998), and in table 16. Files that are formatted to facilitate plotting are produced by MODFLOW-2000 and postprocessors RESAN-2000, YCINT-2000, AND BEALE-2000, as listed in tables 5, 6, and 16. 